\title{
Fingerprint Vendor Technology Evaluation
}

Craig Watson

Gregory Fiumara

Elham Tabassi

Su Lan Cheng

Patricia Flanagan

Wayne Salamon

http://dx.doi.org/10.6028/NIST.IR.8034

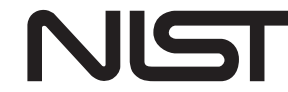

National Institute of Standards and Technology U.S. Department of Commerce 


\title{
Fingerprint Vendor Technology Evaluation
}

\author{
Craig Watson \\ Gregory Fiumara \\ Elham Tabassi \\ Su Lan Cheng \\ Patricia Flanagan \\ Wayne Salamon \\ Information Access Division \\ Information Technology Laboratory
}

This publication is available free of charge from:

http://dx.doi.org/10.6028/NIST.IR.8034

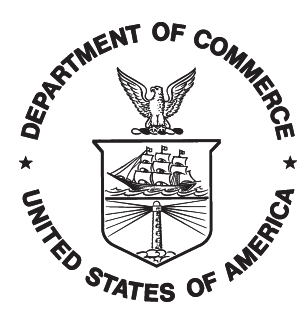

U.S. Department of Commerce

Penny Pritzker, Secretary

National Institute of Standards and Technology 


\title{
Fingerprint Vendor Technology Evaluation
}

\author{
Evaluation of Fingerprint Matching Algorithms \\ NIST Interagency Report 8034
}

Craig Watson • Gregory Fiumara • Elham Tabassi

Su Lan Cheng • Patricia Flanagan • Wayne Salamon 


\section{Acknowledgements}

$\triangle$ Sponsors: The authors would like to thank the sponsors of this project: the Department of Homeland Security (DHS) and the Federal Bureau of Investigation (FBI).

$\triangleright$ Data Providers: The authors would like to thank all the organizations that have shared biometric data with NIST for this and other evaluations. This data has proven invaluable for benchmarking and advancement of biometric matching technologies through these evaluations. Data used in FpVTE came from the FBI, DHS, Los Angeles County Sheriff's Department (LACNTY), Arizona Department of Public Safety (AZDPS), and Texas Department of Public Safety (TXDPS).

$\triangleright$ Participants: The authors would also like to thank the participants for their time and contribution to this evaluation. We know that preparing the software for the evaluation is not a trivial task and the validation process can be stressful when problems occur. Thank you for your time and participation as this evaluation would not be possible without it.

\section{Disclaimer}

Certain commercial equipment, instruments, or materials are identified in this paper in order to specify the experimental procedure adequately. Such identification is not intended to imply recommendation or endorsement by the National Institute of Standards and Technology, nor is it intended to imply that the materials or equipment identified are necessarily the best available for the purpose.

\begin{tabular}{l|l|l|l|ll|l}
$\mathrm{C}=$ afis team & $\mathrm{D}=3 \mathrm{M}$ Cogent & $\mathrm{E}=$ Neurotechnology & $\mathrm{F}=$ Papillon & $\mathrm{G}=$ Dermalog & $\mathrm{H}=$ Hisign Bio-Info Institute \\
$\mathrm{I}=\mathrm{NEC}$ & $\mathrm{J}=$ Sonda & $\mathrm{K}=$ Tiger IT & $\mathrm{L}=$ Innovatrics & $\mathrm{M}=$ SPEX & $\mathrm{O}=$ ID Solutions \\
$\mathrm{P}=\mathrm{id} 3$ & $\mathrm{Q}=$ Morpho & $\mathrm{S}=$ Decatur Industries & $\mathrm{T}=$ BIO-key & $\mathrm{U}=$ Aware & $\mathrm{V}=$ AA Technology
\end{tabular}




\section{Contents}

Acknowledgements

Disclaimer

Executive Summary

xiii

Caveats

Release Notes

xvi

1 Introduction

2 History and Motivation 3

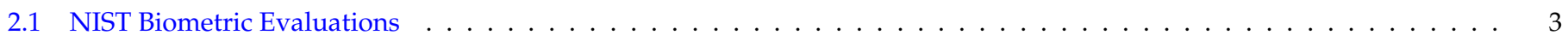

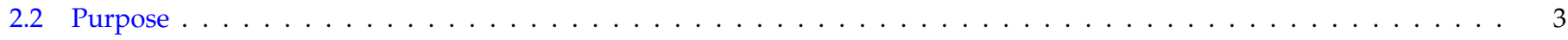

3 Data 5

3.1 Classes of Participation $\ldots \ldots \ldots \ldots$

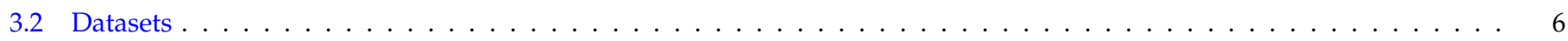

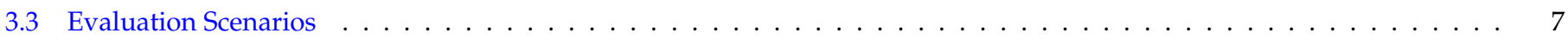

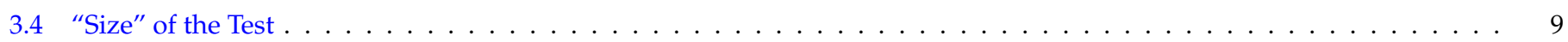

4 Experiment and Test Protocol 10

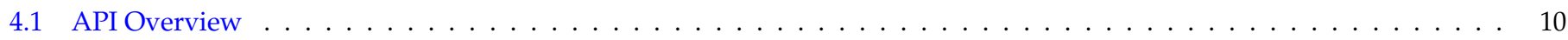

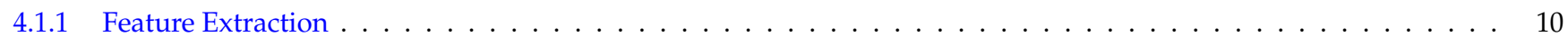

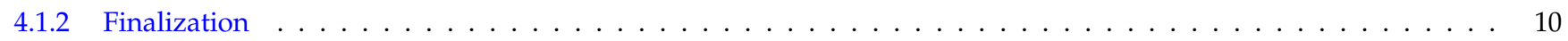

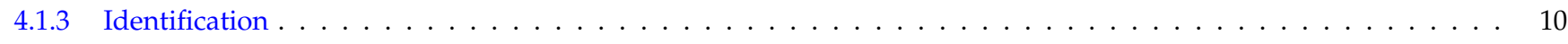

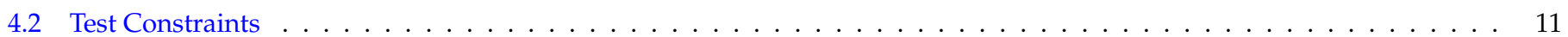

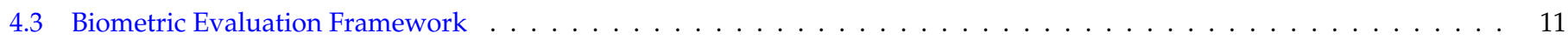

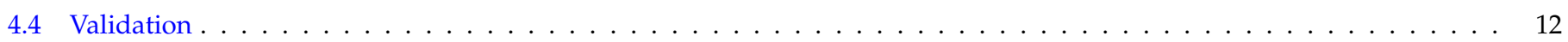

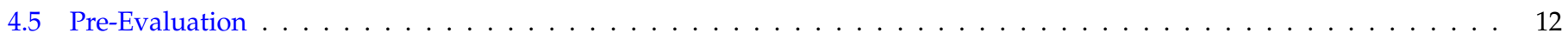

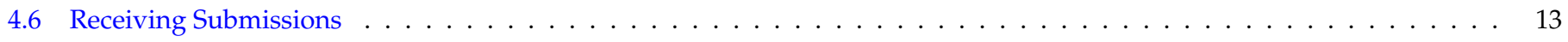

5 Two-Stage Matching 16

5.1 Enrollment Set Partitioning . . . . . . . . . . . . . . . . . . . . . . . . . . . . . . . . . .

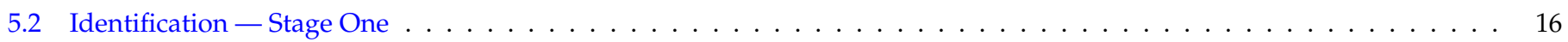

5.3 Identification - Stage Two $\ldots \ldots \ldots \ldots$

6 Metrics 18

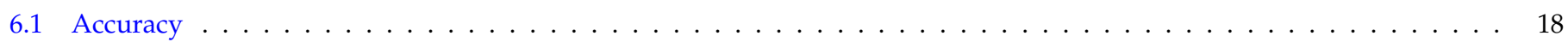

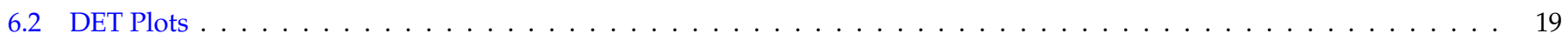

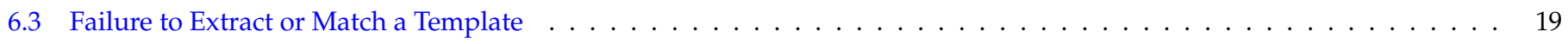

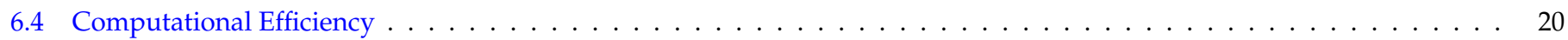

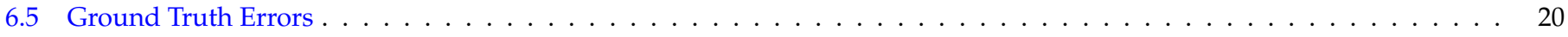

\begin{tabular}{l|l|l|l|l|l}
$\mathrm{C}=$ afis team & $\mathrm{D}=$ = 3M Cogent & $\mathrm{E}=$ Neurotechnology & $\mathrm{F}=$ Papillon & $\mathrm{G}=$ Dermalog & $\mathrm{H}=$ Hisign Bio-Info Institute \\
$\mathrm{I}=$ NEC & $\mathrm{J}=$ Sonda & $\mathrm{K}=$ Tiger IT & $\mathrm{L}=$ Innovatrics & $\mathrm{M}=$ SPEX & $\mathrm{O}=$ ID Solutions \\
$\mathrm{P}=\mathrm{id} 3$ & $\mathrm{Q}=$ Morpho & $\mathrm{S}=$ Decatur Industries & $\mathrm{T}=$ BIO-key & $\mathrm{U}=$ Aware & $\mathrm{V}=$ AA Technology
\end{tabular}


7 Accuracy Results 22

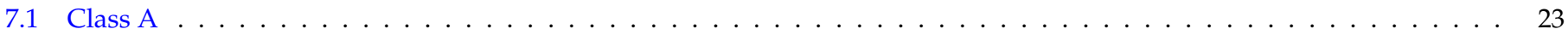

7.1 .1 Single-Index Finger Identification . . . . . . . . . . . . . . . . . . . . . . . . 23

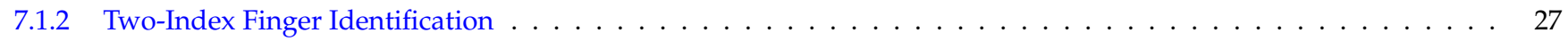

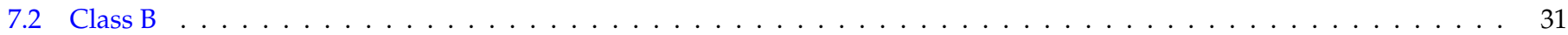

7.3 Class $\ldots \ldots \ldots \ldots \ldots \ldots$

8 Accuracy/Search Time Tradeoff 41

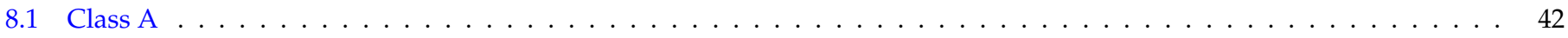

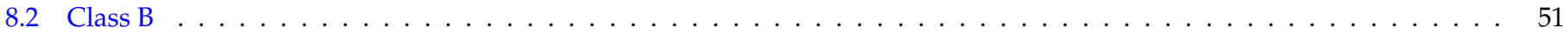

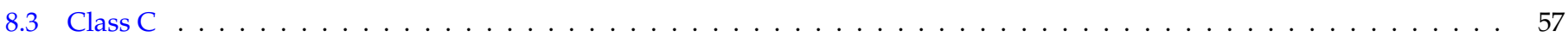

9 Accuracy Computational Resources Tradeoff 61

9.1 Storage and Memory $\ldots \ldots \ldots \ldots \ldots$

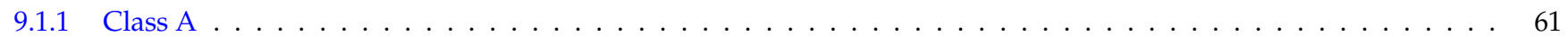

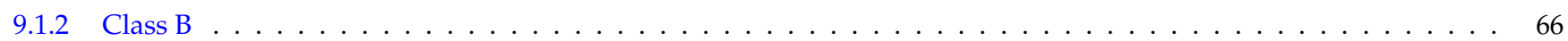

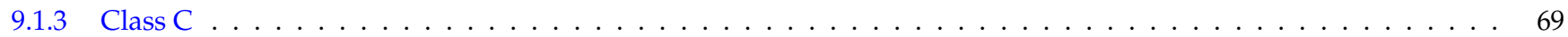

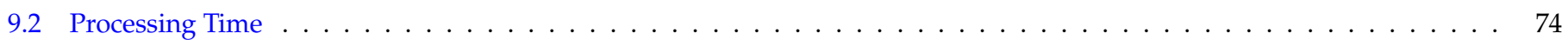

10 Ranked Results 78

11 How Many Fingers are Needed 82

12 FpVTE 2003 Comparison 83

13 Lessons Learned for Large-Scale Testing 86

14 Way Forward 88

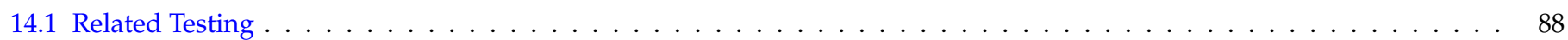

14.1 Forensic Palmprint $\ldots \ldots \ldots \ldots$

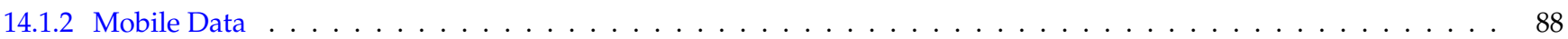

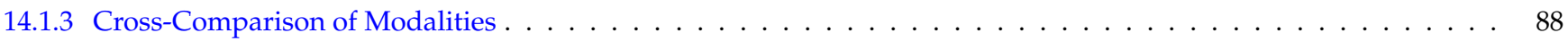

References 89

A Individual Participant FNIR Plots 90

B Combined Class DETs and CMCs 109

C Accuracy Time Tradeoff Detailed Tables with Median Values 114

D Accuracy Time Tradeoff Detailed Tables with Mean Values 128

E Progression for Last Two Submissions 142

F Enrollment Size 153

G Search Template Sizes 160

\begin{tabular}{l|l|l|l|l|l}
$\mathrm{C}=$ afis team & $\mathrm{D}=3 \mathrm{M}$ Cogent & $\mathrm{E}=$ Neurotechnology & $\mathrm{F}=$ Papillon & $\mathrm{G}=$ Dermalog & $\mathrm{H}=$ Hisign Bio-Info Institute \\
$\mathrm{I}=$ NEC & $\mathrm{J}=$ Sonda & $\mathrm{K}=$ Tiger IT & $\mathrm{L}=$ Innovatrics & $\mathrm{M}=$ SPEX & $\mathrm{O}=$ ID Solutions \\
$\mathrm{P}=\mathrm{id} 3$ & $\mathrm{Q}=$ Morpho & $\mathrm{S}=$ Decatur Industries & $\mathrm{T}=$ BIO-key & $\mathrm{U}=$ Aware & $\mathrm{V}=$ AA Technology
\end{tabular}


G.1 Mean Values . . . . . . . . . . . . . . . . . . . . . . . . . . . . . . . 160

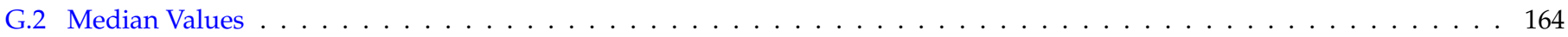

H Template Creation Times 168

I Ranked Results 178

J Relative Combined Results 188

K Relative Accuracy and Number of Fingers 198

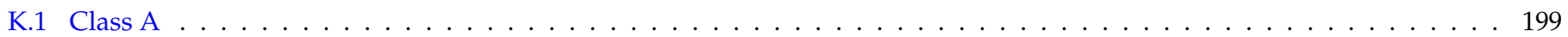

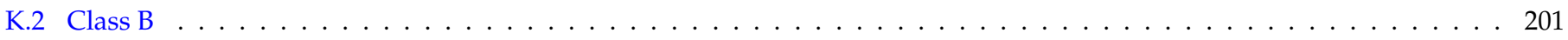

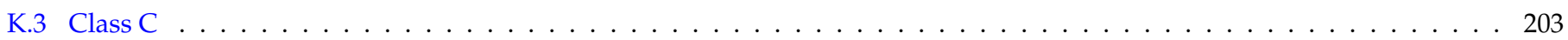

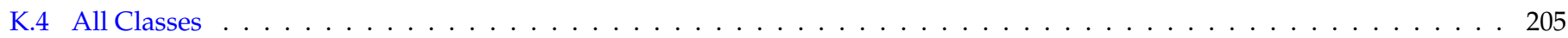

L Combined Sorted Rankings for a Theoretical Use Case 207

\section{List of Figures}

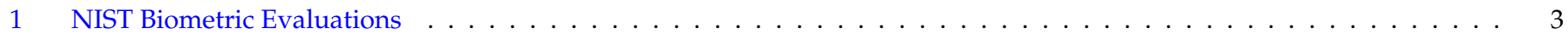

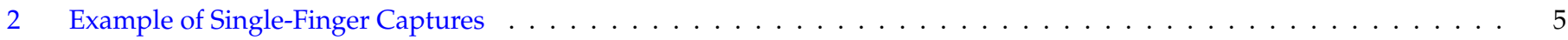

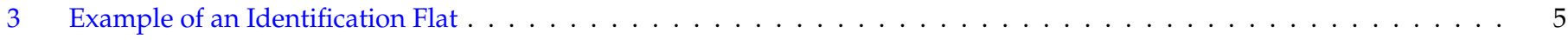

4 Examples of Live-Scan and Rescanned Ink . . . . . . . . . . . . . . . . . . . . . . . . . . . 5

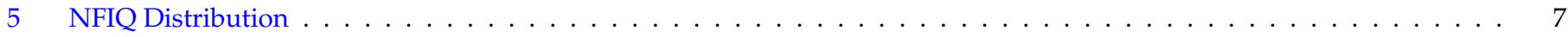

6 Number of Submissions Received . . . . . . . . . . . . . . . . . . . . . . . . . . . 13

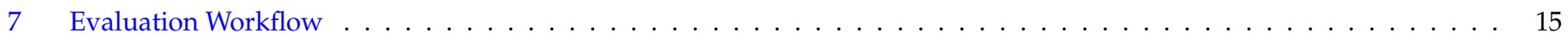

9 Example of a Flipped Single-Index Finger Image . . . . . . . . . . . . . . . . . . . . . . . . . . . .

8 Example of a DET showing a Spike in FPIR . . . . . . . . . . . . . . . . . . . . . . . . . . 20

10 Example of a Flipped Left Slap Image . . . . . . . . . . . . . . . . . . . . . . . . . . . . . . 21

11 Example of a Flipped Right Slap Image . . . . . . . . . . . . . . . . . . . . . . . . . . . . . .

12 Rank-sorted FNIR for Class A - Single Index Finger . . . . . . . . . . . . . . . . . . . . . . . . 23

13 DET for Class A - Single Index Finger . . . . . . . . . . . . . . . . . . . . . . . . . . . 24

$14 \mathrm{CMC}$ for Class $\mathrm{A}-$ Single Index Finger $\ldots \ldots \ldots \ldots$

15 Rank-sorted FNIR for Class A - Two Index Fingers . . . . . . . . . . . . . . . . . . . . . . . . . . 27

16 DET for Class $\mathrm{A}-$ Two Index Fingers $\ldots \ldots \ldots$

17 CMC for Class A - Two Index Fingers . . . . . . . . . . . . . . . . . . . . . . . . . . . . . . . . 29

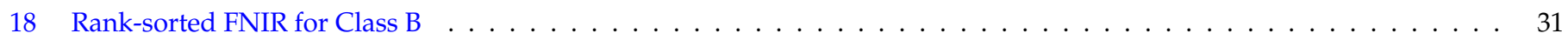

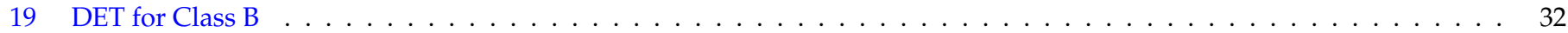

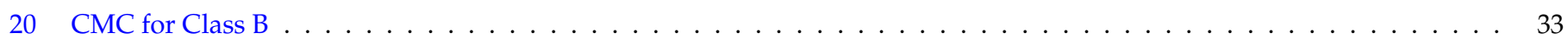

21 Rank-sorted FNIR for Class $\mathrm{f} \ldots \ldots \ldots \ldots$

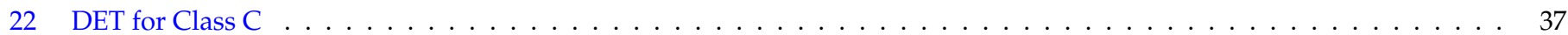

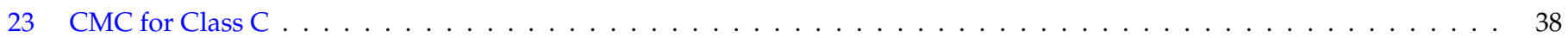

24 FNIR and Search Time Scatter Plot - Class A — Left Index - Less Than 20-Second Searches . . . . . . . . . . . . . . 43

\begin{tabular}{l|l|l|l|ll|l}
$\mathrm{C}=$ afis team & $\mathrm{D}=3 \mathrm{M}$ Cogent & $\mathrm{E}=$ Neurotechnology & $\mathrm{F}=$ Papillon & $\mathrm{G}=$ Dermalog & $\mathrm{H}=$ Hisign Bio-Info Institute \\
$\mathrm{I}=\mathrm{NEC}$ & $\mathrm{J}=$ Sonda & $\mathrm{K}=$ Tiger IT & $\mathrm{L}=$ Innovatrics & $\mathrm{M}=$ SPEX & $\mathrm{O}=$ ID Solutions \\
$\mathrm{P}=\mathrm{id} 3$ & $\mathrm{Q}=$ Morpho & $\mathrm{S}=$ Decatur Industries & $\mathrm{T}=$ BIO-key & $\mathrm{U}=$ Aware & $\mathrm{V}=$ AA Technology
\end{tabular}


FNIR and Search Time Scatter Plot — Class A — Left Index — Greater Than or Equal To 20-Second Searches . . . . . . . 43

FNIR and Search Time Scatter Plot - Class A — Right Index — Less Than 20-Second Searches . . . . . . . . . . . 44

FNIR and Search Time Scatter Plot - Class A — Right Index — Greater Than or Equal To 20-Second Searches . . . . . . 44

FNIR and Search Time Scatter Plot - Class A — Left and Right Index — Less Than 20-Second Searches . . . . . . . . . . 45

FNIR and Search Time Scatter Plot — Class A — Left and Right Index — Greater Than or Equal To 20-Second Searches . 45

Submission Round Progression - Class A — Left Index — Less Than 20-Second Searches . . . . . . . . . . . . . . . . . 46

Submission Round Progression - Class A — Left Index — Greater Than or Equal To 20-Second Searches . . . . . . . . . 46

Submission Round Progression - Class A — Right Index — Less Than 20-Second Searches . . . . . . . . . . . . 47

Submission Round Progression — Class A — Right Index — Greater Than or Equal To 20-Second Searches . . . . . . . . 47

Submission Round Progression - Class A — Left and Right Index — Less Than 20-Second Searches . . . . . . . . . . . . 48

Submission Round Progression — Class A — Left and Right Index — Greater Than or Equal To 20-Second Searches . . . 48

FNIR and Search Time Scatter Plot - Class B - Left Slap . . . . . . . . . . . . . . . . . . . . 52

FNIR and Search Time Scatter Plot - Class B - Right Slap . . . . . . . . . . . . . . . . 5 . . .

FNIR and Search Time Scatter Plot - Class B - Left and Right Slap . . . . . . . . . . . . . . . . . 53

FNIR and Search Time Scatter Plot - Class B — Identification Flats . . . . . . . . . . . . . . . . . . . . . . 53

Submission Round Progression - Class B - Left Slap . . . . . . . . . . . . . . . . . . . . 54

Submission Round Progression - Class B - Right Slap . . . . . . . . . . . . . . . . . . 54

Submission Round Progression - Class B — Left and Right Slap . . . . . . . . . . . . . . . . . . . 55

Submission Round Progression - Class B - IDFlat . . . . . . . . . . . . . . . . . . 55

FNIR and Search Time Scatter Plot - Class C . . . . . . . . . . . . . . . . . . . . . 58

Submission Round Progression - Class C . . . . . . . . . . . . . . . . . . . . . . 59

FNIR and RAM Used for Enrollment Set Tradeoff Scatter Plot for Class A — Left Index . . . . . . . . . . . . . . . 62

FNIR and RAM Used for Enrollment Set Tradeoff Scatter Plot for Class A — Right Index . . . . . . . . . . . . . . . . 62

FNIR and Search Template Size in RAM Tradeoff Scatter Plot for Class A — Left Index . . . . . . . . . . . . . . . 63

FNIR and Search Template Size in RAM Tradeoff Scatter Plot for Class A - Right Index . . . . . . . . . . . . . . 63

FNIR and RAM Used for Enrollment Set Scatter Plot for Class A — Left and Right Index . . . . . . . . . . . . . . . . . 64

FNIR and Search Template Size in RAM Tradeoff Scatter Plot for Class A — Left and Right Index . . . . . . . . . . . 64

Enrollment Size in RAM Compared to On Disk for Class A — Left and Right Index . . . . . . . . . . . . . . . . . . . 65

Enrollment Size and Search Template Size in RAM Compared to On Disk for Class A — Left and Right Index . . . . . . . 65

FNIR and RAM Used for Enrollment Set for Class B - Identification Flats . . . . . . . . . . . . . . . . 67

FNIR and Search Template Size in RAM Tradeoff Scatter Plot for Class B — Identification Flats . . . . . . . . . . . . . 67

Enrollment Size in RAM Compared to On Disk for Class B - Identification Flats . . . . . . . . . . . . . . . . . . 68

Search Template Size in RAM Compared to On Disk for Class B — Identification Flats . . . . . . . . . . . . . . 68

FNIR and RAM Used for Enrollment Set Tradeoff Scatter Plot for Class C - Ten-Finger Plain-to-Plain . . . . . . . . . . . 70

FNIR and RAM Used for Enrollment Set Tradeoff Scatter Plot for Class C — Ten-Finger Rolled-to-Rolled . . . . . . . . . 70

FNIR and Search Template Size in RAM Tradeoff Scatter Plot for Class C - Ten-Finger Plain-to-Plain . . . . . . . . . . 71

FNIR and Search Template Size in RAM Tradeoff Scatter Plot for Class C — Ten-Finger Rolled-to-Rolled . . . . . . . . . . 71

Enrollment Size and Search Template Size in RAM Compared to On Disk for Class C — Plain Impression . . . . . . . . . 72

Enrollment Size and Search Template Size in RAM Compared to On Disk for Class C - Plain Impression . . . . . . . . . 72

Enrollment Size and Search Template Size in RAM Compared to On Disk for Class C — Rolled Impression . . . . . . . . 73

Enrollment Size and Search Template Size in RAM Compared to On Disk for Class C — Rolled Impression . . . . . . . . 73

\begin{tabular}{l|l|l|l|l|l|l}
$\mathrm{C}=$ afis team & $\mathrm{D}=$ = 3M Cogent & $\mathrm{E}=$ Neurotechnology & $\mathrm{F}=$ Papillon & $\mathrm{G}=$ Dermalog & $\mathrm{H}=$ Hisign Bio-Info Institute \\
$\mathrm{I}=$ NEC & $\mathrm{J}=$ Sonda & $\mathrm{K}=$ Tiger IT & $\mathrm{L}=$ Innovatrics & $\mathrm{M}=$ SPEX & $\mathrm{O}=$ ID Solutions \\
$\mathrm{P}=\mathrm{id} 3$ & $\mathrm{Q}=$ Morpho & $\mathrm{S}=$ Decatur Industries & $\mathrm{T}=$ BIO-key & $\mathrm{U}=$ Aware & $\mathrm{V}=$ AA Technology
\end{tabular}


FNIR and Template Creation Time Tradeoff Scatter Plot for Class A — Left Index

FNIR and Template Creation Time Tradeoff Scatter Plot for Class A - Right Index . . . . . . . . . . . . . . . 75

FNIR and Template Creation Time Tradeoff Scatter Plot for Class A - Left and Right Index . . . . . . . . . . . . . . . 76

FNIR and Template Creation Time Tradeoff Scatter Plot for Class B - Identification Flats . . . . . . . . . . . . . . 76

FNIR and Template Creation Time Tradeoff Scatter Plot for Class C - Ten-Finger Plain-to-Plain . . . . . . . . . . . . 77

FNIR and Template Creation Time Tradeoff Scatter Plot for Class C - Ten-Finger Rolled-to-Rolled . . . . . . . . . . . . . 77

Rank-sorted FNIR for All Classes . . . . . . . . . . . . . . . . . . . . . . . . . 82

FpVTE 2003 SST Results . . . . . . . . . . . . . . . . . . . . . . . . . . . . . . . . .

FpVTE 2003 MST Results . . . . . . . . . . . . . . . . . . . . . . . . . . . . . . . . . 884

FpVTE 2003 LST Results . . . . . . . . . . . . . . . . . . . . . . . . . . . . . .

FpVTE 2003 LST Number of Fingers Results . . . . . . . . . . . . . . . . . . . . . . 85

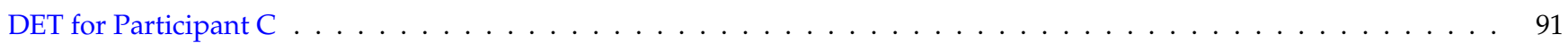

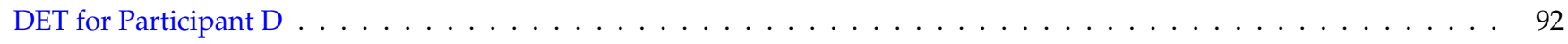

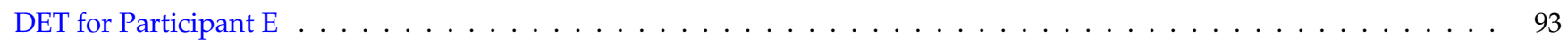

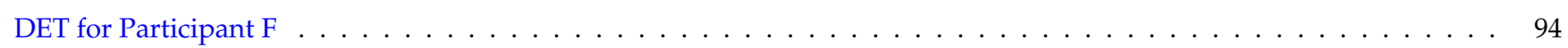

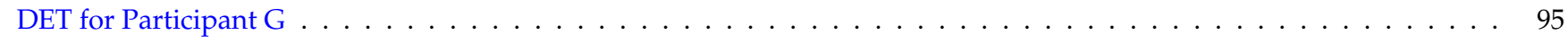

DET for Participant $\mathrm{H} \ldots \ldots \ldots \ldots \ldots \ldots \ldots$

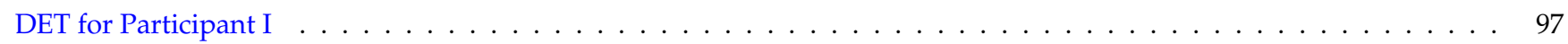

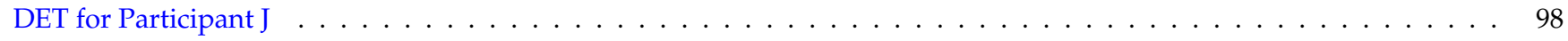

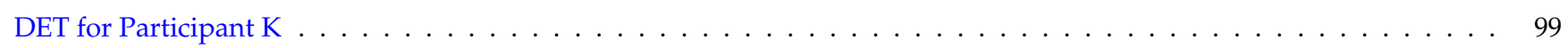

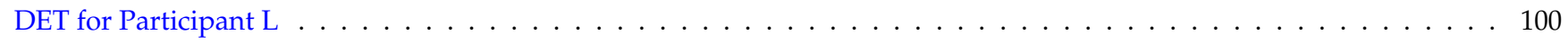

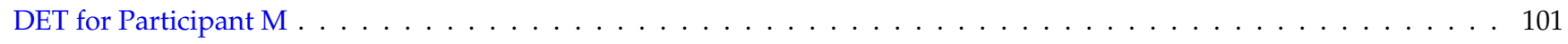

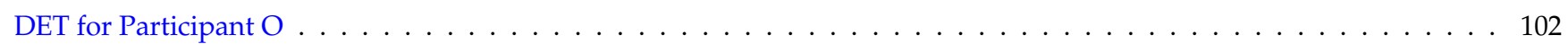

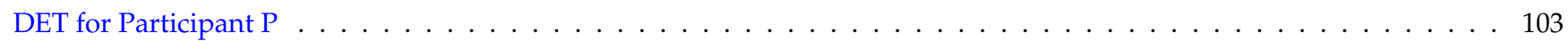

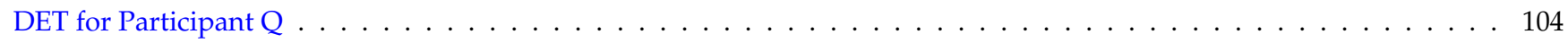

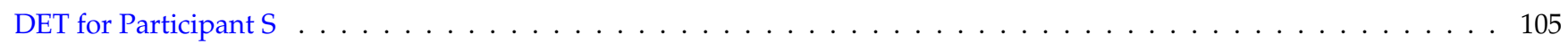

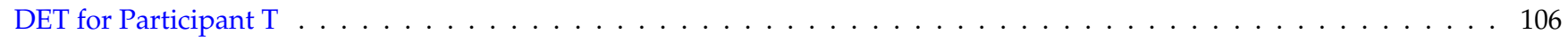

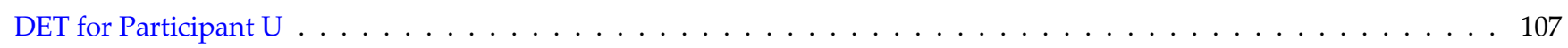

DET for Participant $\mathrm{V} \ldots \ldots \ldots \ldots \ldots \ldots$

DET for All Participants in All Classes - First Submissions . . . . . . . . . . . . . . . . . . . 110

DET for All Participants in All Classes — Second Submissions . . . . . . . . . . . . . . . . . . . . . 111

CMC for All Participants in All Classes — First Submissions . . . . . . . . . . . . . . . . . . . . 112

CMC for All Participants in All Classes — Second Submissions . . . . . . . . . . . . . . . . . . . . 113

Relative Combined Results - Left Index - Class A . . . . . . . . . . . . . . . . . . . . . . . . 189

100 Relative Combined Results - Right Index - Class A . . . . . . . . . . . . . . . . . . . . . . . . 190

1 Relative Combined Results - Left and Right Index - Class A . . . . . . . . . . . . . . . . . . 191

Relative Combined Results - Left Slap - Class B . . . . . . . . . . . . . . . . . . . . . . . . . . . . 192

3 Relative Combined Results - Right Slap - Class B . . . . . . . . . . . . . . . . . . . . . 193

4 Relative Combined Results - Left and Right Slap - Class B . . . . . . . . . . . . . . . . . . . 194

Relative Combined Results - Identification Flats - Class B . . . . . . . . . . . . . . . . . . 195

\begin{tabular}{l|l|l|l|l|l}
$\mathrm{C}=$ afis team & $\mathrm{D}=3 \mathrm{M}$ Cogent & $\mathrm{E}=$ Neurotechnology & $\mathrm{F}=$ Papillon & $\mathrm{G}=$ Dermalog & $\mathrm{H}=$ Hisign Bio-Info Institute \\
$\mathrm{I}=\mathrm{NEC}$ & $\mathrm{J}=$ Sonda & $\mathrm{K}=$ Tiger IT & $\mathrm{L}=$ Innovatrics & $\mathrm{M}=$ SPEX & $\mathrm{O}=$ ID Solutions \\
$\mathrm{P}=\mathrm{id3}$ & $\mathrm{Q}=$ Morpho & $\mathrm{S}=$ Decatur Industries & $\mathrm{T}=$ BIO-key & $\mathrm{U}=$ Aware & $\mathrm{V}=$ AA Technology
\end{tabular}


107 Relative Combined Results - Ten-Finger Rolled-to-Rolled - Class C . . . . . . . . . . . . . . . . . . . . . . 197

108 Relative Accuracy - Class A . . . . . . . . . . . . . . . . . . . . . . . . 200

109 Relative Accuracy - Class B . . . . . . . . . . . . . . . . . . . . . . . . . . 202

110 Relative Accuracy - Class C . . . . . . . . . . . . . . . . . . . . . . . . . . 204

111 Relative Accuracy - All Classes (Select Fingers) . . . . . . . . . . . . . . . . . . . . . . . . 206

\section{List of Tables}

$1 \quad$ List of Participants . . . . . . . . . . . . . . . . . . . . . . . . . .

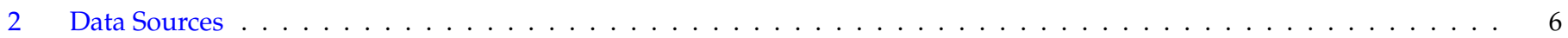

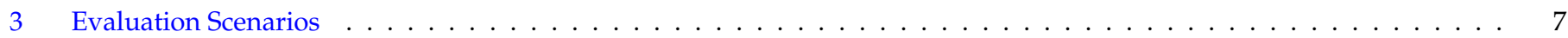

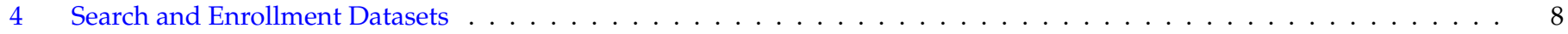

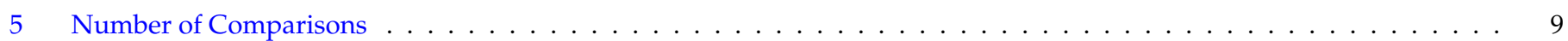

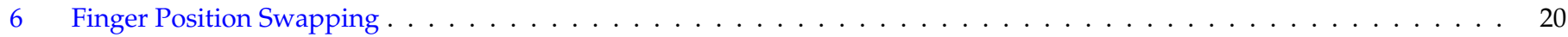

$7 \quad$ FNIR @ FPIR $=10^{-3}-$ Class A - Left Index . . . . . . . . . . . . . . . . . . . . . . . . . 26

8 FNIR $@$ FPIR $=10^{-3}-$ Class A - Right Index . . . . . . . . . . . . . . . . . . . . . 26

9 FNIR @ FPIR $=10^{-3}-$ Class A - Left and Right Index . . . . . . . . . . . . . . . . . 30

10 FNIR $@$ FPIR $=10^{-3}-$ Class B $-\operatorname{Left} \operatorname{Slap} \ldots \ldots \ldots$

11 FNIR @ FPIR $=10^{-3}-$ Class B - Right Slap $\ldots \ldots \ldots \ldots$

12 FNIR @ FPIR $=10^{-3}-$ Class B - Left and Right Slap . . . . . . . . . . . . . . . . . . . . . . 35

13 FNIR $@$ FPIR $=10^{-3}-$ Class B - Identification Flats $\ldots \ldots \ldots$

14 FNIR @ FPIR $=10^{-3}-$ Class $\mathrm{C}-$ Ten-Finger Plain-to-Plain . . . . . . . . . . . . . . . . . . . . . . 39

15 FNIR @ FPIR $=10^{-3}-$ Class C - Ten-Finger Rolled-to-Rolled . . . . . . . . . . . . . . . . . . . . . . . . 39

16 FNIR @ FPIR $=10^{-3}-$ Class $\mathrm{C}-$ Ten-Finger Plain-to-Rolled . . . . . . . . . . . . . . . . . . . . . . 40

17 Median Identification Times for Class A . . . . . . . . . . . . . . . . . . . . . . . . 49

18 Median Identification Times for Class A . . . . . . . . . . . . . . . . . . . . . . . . . . 50

19 Median Identification Times for Class B . . . . . . . . . . . . . . . . . . . . . . . . . . . . 56

20 Median Identification Times for Class C . . . . . . . . . . . . . . . . . . . . . . . . 60

21 Ranked Results for Class A — Left and Right Index . . . . . . . . . . . . . . . . . . . . . . . . . . 79

22 Ranked Results for Class B - Identification Flats . . . . . . . . . . . . . . . . . . . . . . . 80

23 Ranked Results for Class C - Ten-Finger Rolled-to-Rolled . . . . . . . . . . . . . . . . . . . . . . 81

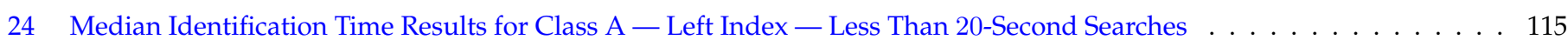

25 Median Identification Time Results for Class A — Left Index — Greater Than or Equal To 20-Second Searches . . . . . . 116

26 Median Identification Time Results for Class A — Right Index — Less Than 20-Second Searches . . . . . . . . . . . . . . 117

27 Median Identification Time Results for Class A — Right Index — Greater Than or Equal To 20-Second Searches . . . . . . 118

28 Median Identification Time Results for Class A — Left and Right Index — Less Than 20-Second Searches . . . . . . . . . 119

29 Median Identification Time Results for Class A — Left and Right Index — Greater Than or Equal To 20-Second Searches

30 Median Identification Time Results for Class B - Left Slap . . . . . . . . . . . . . . . . . . . . . . . . . 121

31 Median Identification Time Results for Class B - Right Slap . . . . . . . . . . . . . . . . . . 122

32 Median Identification Time Results for Class B - Left and Right Slap . . . . . . . . . . . . . . . . . . . . . . . . 123

33 Median Identification Time Results for Class B - Identification Flats . . . . . . . . . . . . . . . . . . . . . 124

\begin{tabular}{l|l|l|l|l|l}
$\mathrm{C}=$ afis team & $\mathrm{D}=$ = 3M Cogent & $\mathrm{E}=$ Neurotechnology & $\mathrm{F}=$ Papillon & $\mathrm{G}=$ Dermalog & $\mathrm{H}=$ Hisign Bio-Info Institute \\
$\mathrm{I}=$ NEC & $\mathrm{J}=$ Sonda & $\mathrm{K}=$ Tiger IT & $\mathrm{L}=$ Innovatrics & $\mathrm{M}=$ SPEX & $\mathrm{O}=$ ID Solutions \\
$\mathrm{P}=\mathrm{id} 3$ & $\mathrm{Q}=$ Morpho & $\mathrm{S}=$ Decatur Industries & $\mathrm{T}=$ BIO-key & $\mathrm{U}=$ Aware & $\mathrm{V}=$ AA Technology
\end{tabular}


34 Median Identification Time Results for Class C - Ten-Finger Plain-to-Plain

Mean Identification Time Results for Class A — Right Index — Greater Than or Equal To 20-Second Searches . . . . . . .

Mean Search Template Sizes for Class B . . . . . . . . . . . . . . . . . . . . . . . 162

Mean Search Template Sizes for Class C . . . . . . . . . . . . . . . . . . . . . . 163

Median Search Template Sizes for Class A . . . . . . . . . . . . . . . . . . . . . 165

Median Search Template Sizes for Class B . . . . . . . . . . . . . . . . . . . . . . . . . . . 166

Median Search Template Sizes for Class C . . . . . . . . . . . . . . . . . . . . . 167

Enrollment Time Results for Class A — Left Index . . . . . . . . . . . . . . . . . . . . . . . . 169

Enrollment Time Results for Class A - Right Index . . . . . . . . . . . . . . . . . . . . . . 170

Enrollment Time Results for Class A - Left and Right Index . . . . . . . . . . . . . . . . . . . . 171

\begin{tabular}{l|l|l|l|l|l}
$\mathrm{C}=$ afis team & $\mathrm{D}=$ = 3M Cogent & $\mathrm{E}=$ Neurotechnology & $\mathrm{F}=$ Papillon & $\mathrm{G}=$ Dermalog & $\mathrm{H}=$ Hisign Bio-Info Institute \\
$\mathrm{I}=$ NEC & $\mathrm{J}=$ Sonda & $\mathrm{K}=$ Tiger IT & $\mathrm{L}=$ Innovatrics & $\mathrm{M}=$ SPEX & $\mathrm{O}=$ ID Solutions \\
$\mathrm{P}=\mathrm{id} 3$ & $\mathrm{Q}=$ Morpho & $\mathrm{S}=$ Decatur Industries & $\mathrm{T}=$ BIO-key & $\mathrm{U}=$ Aware & $\mathrm{V}=$ AA Technology
\end{tabular}


Enrollment Time Results for Class B - Left Slap . . . . . . . . . . . . . . . . . . . . . . . . . . . . . . . . . . . . . . 172

Enrollment Time Results for Class B — Right Slap ～. . . . . . . . . . . . . . . . . . . . . . . . . . . 173

Enrollment Time Results for Class B — Left and Right Slap . . . . . . . . . . . . . . . . . . . . . . . . . 174

Enrollment Time Results for Class B — Identification Flats ～. . . . . . . . . . . . . . . . . . . . . . . . . . . 175

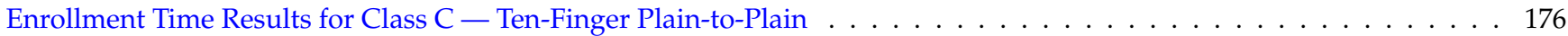

Enrollment Time Results for Class C - Ten-Finger Rolled-to-Rolled . . . . . . . . . . . . . . . . . . . . . . . . . 177

Ranked Results for Class A — Left Index . . . . . . . . . . . . . . . . . . . . . . . . . . . . . . . . 179

Ranked Results for Class A — Right Index ～. . . . . . . . . . . . . . . . . . . . . . . . . . . 180

Ranked Results for Class A — Left and Right Index ～. . . . . . . . . . . . . . . . . . . . . . . . . . . . 181

Ranked Results for Class B - Left Slap ～. . . . . . . . . . . . . . . . . . . . . . . . . . . . . . . . . 182

Ranked Results for Class B — Right Slap ． . . . . . . . . . . . . . . . . . . . . . . . . . . . 183

Ranked Results for Class B — Left and Right Slap . . . . . . . . . . . . . . . . . . . . . . . . . . . . 184

Ranked Results for Class B — Identification Flats ． . . . . . . . . . . . . . . . . . . . . . . . . . . . . . . 185

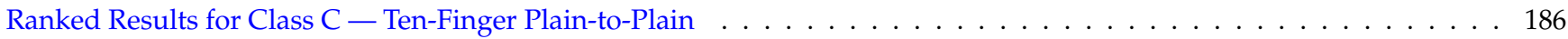

Ranked Results for Class C - Ten-Finger Rolled-to-Rolled ～. . . . . . . . . . . . . . . . . . . . . . . . . . . . . . 187

Operational Ranking for Class A - Left Index ～. . . . . . . . . . . . . . . . . . . . . . . . . . . . . . . . . . . . 209

Operational Ranking for Class A — Right Index . . . . . . . . . . . . . . . . . . . . . . . . . . . . . . . . 210

Operational Ranking for Class A — Left and Right Index . . . . . . . . . . . . . . . . . . . . . . . . . . . 211

Operational Ranking for Class B — Left Slap . . . . . . . . . . . . . . . . . . . . . . . . . . . . . . . . . . 212

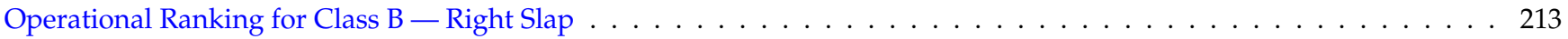

Operational Ranking for Class B — Left and Right Slap ～. . . . . . . . . . . . . . . . . . . . . . . . . 214

Operational Ranking for Class B - Identification Flats . . . . . . . . . . . . . . . . . . . . . 215

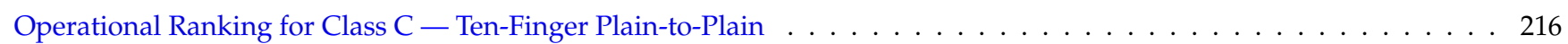

Operational Ranking for Class $\mathrm{C}-$ Ten-Finger Rolled-to-Rolled $\ldots \ldots \ldots \ldots \ldots \ldots \ldots \ldots \ldots \ldots$

\begin{tabular}{l|l|l|l|l|l|l}
$\mathrm{C}=$ afis team & $\mathrm{D}=3 \mathrm{M}$ Cogent & $\mathrm{E}=$ Neurotechnology & $\mathrm{F}=$ Papillon & $\mathrm{G}=$ Dermalog & $\mathrm{H}=$ Hisign Bio-Info Institute \\
$\mathrm{I}=\mathrm{NEC}$ & $\mathrm{J}=$ Sonda & $\mathrm{K}=$ Tiger IT & $\mathrm{L}=$ Innovatrics & $\mathrm{M}=$ SPEX & $\mathrm{O}=$ ID Solutions \\
$\mathrm{P}=\mathrm{id} 3$ & $\mathrm{Q}=$ Morpho & $\mathrm{S}=$ Decatur Industries & $\mathrm{T}=$ BIO-key & $\mathrm{U}=$ Aware & $\mathrm{V}=$ AA Technology
\end{tabular}




\section{Executive Summary}

FpVTE was conducted primarily to assess the current capabilities of fingerprint matching algorithms using operational datasets containing several million subjects. There were three classes of participation that examined one-to-many identification using various finger combinations from single finger up to ten fingers. Class $\mathbf{A}$ used single-index finger capture data and evaluated single index finger (right or left) and two index finger (right and left) identification. Class B used identification flat (IDFlat) captures (4-4-2; left slap, right slap, and two thumbs simultaneously) and evaluated ten-finger, eight-finger (right and left slap), and four-finger (right or left slap) identification. Class $\mathbf{C}$ used rolled and plain impression (4-4-1-1; left slap, right slap, left thumb, and right thumb) captures and evaluated ten-finger rolled-to-rolled, ten-finger plain-to-plain, and ten-finger plain-to-rolled identification. Enrollment sets used for one-to-many identification varied in size from 5000 up to 5000000 enrolled subjects. Any segmentation of four-finger slap images or two-thumb captures was performed by the submitted software. All data used was sequestered operational data that was not shared with any of the participants.

The evaluation allowed each participant to make two submissions per class (A, B, and C) of participation over three rounds. After each of the first two rounds of submissions, feedback was provided to the participants and they were allowed to evaluate their performance, make adjustments to their submissions, and resubmit for the next round. The results of the third and final round of submissions are reported in this document.

The evaluation was conducted at the National Institute of Standards and Technology (NIST) using commodity NISTowned hardware. Participant submissions were compliant to the testing Application Programming Interface (API), which were linked to a NIST-developed test driver and run by NIST employees. All submissions went through validation testing to ensure that results generated on NIST's hardware matched results participants generated on their own hardware.

This was the first large-scale one-to-many fingerprint evaluation since FpVTE 2003. In 2003, participants brought their own hardware to NIST to process the evaluation data. The datasets in 2003 had approximately 25000 subjects and required millions of single subject-to-subject matches. The current FpVTE used a testing model closer to real one-to-many identification systems by allowing the submitted software to control how it does the one-to-many search and return a candidate list of potential matches. The number of subjects used was also significantly higher, as the current FpVTE had $\approx 10$ million subjects in the testing datasets.

The results in this report are based on 30000 (10000 mates and 20000 nonmates) search subjects. There will be an additional report with results (lower errors rates) using 350000 (50 000 mates and 300000 nonmates) search subjects.

In addition to measuring current performance capabilities of one-to-many identification algorithms, FpVTE was conducted to:

$\triangleright$ study open-set identification versus enrolled sample sizes extending into the multiple millions;

$\triangleright$ provide a testing framework and API for enrollment sizes that must be spread across the memory of multiple compute nodes;

$\triangleright$ evaluate on operational datasets containing newer data from live-scan ten-finger "identification flat" capture systems, other live-scan capture devices (e.g., single-finger and multi-finger), and historically significant scanned inked fingerprints;

$\triangleright$ analyze one-to-many identification accuracy, speed, template size, number of fingers, enrollment set sizes, and computational resources;

$\triangleright$ create a fingerprint testing data repository with a vast majority of data errors corrected.

FpVTE was not intended to:

$\triangleright$ measure performance of an operational Automated Fingerprint Identification System (AFIS);

$\triangleright$ evaluate scanners or acquisition devices;

$\triangleright$ evaluate latent fingerprint or mobile-captured data.

\begin{tabular}{l|l|l|l|ll|l}
$\mathrm{C}=$ afis team & $\mathrm{D}=3 \mathrm{M}$ Cogent & $\mathrm{E}=$ Neurotechnology & $\mathrm{F}=$ Papillon & $\mathrm{G}=$ Dermalog & $\mathrm{H}=$ Hisign Bio-Info Institute \\
$\mathrm{I}=$ NEC & $\mathrm{J}=$ Sonda & $\mathrm{K}=$ Tiger IT & $\mathrm{L}=$ Innovatrics & $\mathrm{M}=$ SPEX & $\mathrm{O}=$ ID Solutions \\
$\mathrm{P}=\mathrm{id} 3$ & $\mathrm{Q}=$ Morpho & $\mathrm{S}=$ Decatur Industries & $\mathrm{T}=$ BIO-key & $\mathrm{U}=$ Aware & $\mathrm{V}=$ AA Technology
\end{tabular}


Key results from FpVTE were:

$\triangleright$ Fingerprint Identification Accuracy: The most accurate fingerprint identification submissions achieved false negative identification rates (FNIR, or "miss rates") of $1.9 \%$ for single index fingers, $0.27 \%$ for two index fingers, $0.45 \%$ for four-finger identification flats (IDFlats), $0.15 \%$ for eight-finger IDFlats, $0.09 \%$ for ten-finger IDFlats, $0.1 \%$ for tenfinger rolled-to-rolled, $0.13 \%$ for ten-finger plain-to-plain, and $0.11 \%$ for ten-finger plain-to-rolled. These numbers are reported at a false positive identification rate (FPIR) of $10^{-3} .30000$ search subjects were used for these results (10 000 mates and 20000 nonmates). The number of enrolled subjects used for single index fingers was 100 000, 1.6 million for two index fingers, 3 million for IDFlats, and 5 million for ten finger plains and rolled. A larger search set (50 000 mates and 300000 nonmates) is being completed so that an even smaller FPIR can be reported. Those results, when available, will be included in an additional report. Section 7.

$\triangleright$ Accuracy versus Speed: The fastest submissions were not the most accurate. The most accurate submissions showed the ability to decrease search times with minimal loss in accuracy. The format of this evaluation may not have fully explored the lower limit of search speed with minimal loss in accuracy. Section 8 and Appendix E.

$\triangleright$ Number of Fingers: Not surprisingly, using more fingers improved accuracy. In fact, the most accurate results were achieved with ten fingers, searching against the largest enrollment sets of 3 and 5 million subjects. An interesting result that needs further study and analysis is that two-index finger accuracy was better than four-finger IDFlats. Based on some analysis of missed mates, it appears image quality may have played some roll in this result. Section 11.

$\triangleright$ Computation Resources: The most accurate submissions were able to achieve their results with similar Random Access Memory (RAM) usage to other submissions. These same accurate submissions typically took longer to enroll (i.e., extract features) the fingerprint images used in the evaluation. Section 9.

$\triangleright$ Candidate Lists: For most top performers, a majority of the time, the mate appeared within the top three candidates of the candidate list or did not appear at all. Section 7.

$\triangleright$ Ranked Results: Results that group FNIR, enrollment time, search time, and RAM usage all in a single table are shown in Section 10 and Appendix I. The tables are rank-sorted on FNIR, but include ranks in all the other categories for cross-category comparison.

$\triangleright$ Number of Subjects in Enrollment Set: Results for different enrollment set population sizes will not be available until the larger search sets are complete, but the initial results across classes of participation showed that eight- and ten-finger accuracy was superior versus larger enrollment sets. In fact, the most accurate identification results were always achieved with the ten-finger search sets. Section 7.

$\triangleright$ Data Type: There were three classes of participation in which data from single fingers, IDFlats, and "legacy" tenfinger rolled and plain impression data types were evaluated. Results of IDFlats and ten-finger rolled and plain showed little variation in the accuracy of the top-performing submissions. This means the best performers could tune their submissions to accurately match all the data types evaluated. Section 10.

$\triangleright$ Accuracy Gap: The "gap" between the most accurate submissions and the "next tier" appears to be much closer than in FpVTE 2003. Sections 10 and 12.

\begin{tabular}{l|l|l|l|ll|l}
$\mathrm{C}=$ afis team & $\mathrm{D}=3 \mathrm{M}$ Cogent & $\mathrm{E}=$ Neurotechnology & $\mathrm{F}=$ Papillon & $\mathrm{G}=$ Dermalog & $\mathrm{H}=$ Hisign Bio-Info Institute \\
$\mathrm{I}=$ NEC & $\mathrm{J}=$ Sonda & $\mathrm{K}=$ Tiger IT & $\mathrm{L}=$ Innovatrics & $\mathrm{M}=$ SPEX & $\mathrm{O}=$ ID Solutions \\
$\mathrm{P}=\mathrm{id} 3$ & $\mathrm{Q}=$ Morpho & $\mathrm{S}=$ Decatur Industries & $\mathrm{T}=$ BIO-key & $\mathrm{U}=$ Aware & $\mathrm{V}=$ AA Technology
\end{tabular}




\section{Caveats}

1. Specific nature of the biometric data: The absolute error rates quoted here were measured over a very large fixed corpus of operational fingerprint images. The error rates measured here are realistic if the submissions are applied to this kind of data. However, in other applications, the applicability of the results may differ due to a number of factors legitimately not reflected in the FpVTE experimental design. Among these are:

$\triangleright$ how much slap fingerprint segmentation errors contribute to core matching accuracy;

$\triangleright$ algorithmic limitations caused by the FpVTE API;

$\triangleright$ unknown bugs in the submission;

$\triangleright$ image quality from using a different data source.

2. Not an Automated Fingerprint Identification System (AFIS) test: While this evaluation is intended to measure the core capabilities of matching algorithms in a large one-to-many scenario, it is not intended to be a full assessment of an operational AFIS.

3. Timing of the submissions: While every attempt was made to perform timing on the exact same hardware with the exact same conditions, it is possible that certain functions of the operating system could have had an unintended negative effect on timing results. The timing operations reported for each submission submitted were performed in the exact same manner. Generous "cutoff" times were employed to prevent wasting compute cycles on submissions whose API functions never returned a value.

4. Aggregate finger positions: When reporting estimated template RAM usage statistics, participants were permitted to return an aggregate template size when the input image contained more than one finger (e.g., "slaps"). Any statistics over these types of images were reported as-is and the values were not divided by the number of fingers expected for the capture type. While these participants are not denoted in the report, the actual RAM usage may be a better statistic for comparison.

\begin{tabular}{l|l|l|l|ll|l}
$\mathrm{C}=$ afis team & $\mathrm{D}=3 \mathrm{M}$ Cogent & $\mathrm{E}=$ Neurotechnology & $\mathrm{F}=$ Papillon & $\mathrm{G}=$ Dermalog & $\mathrm{H}=$ Hisign Bio-Info Institute \\
$\mathrm{I}=$ NEC & $\mathrm{J}=$ Sonda & $\mathrm{K}=$ Tiger IT & $\mathrm{L}=$ Innovatrics & $\mathrm{M}=$ SPEX & $\mathrm{O}=$ ID Solutions \\
$\mathrm{P}=\mathrm{id} 3$ & $\mathrm{Q}=$ Morpho & $\mathrm{S}=$ Decatur Industries & $\mathrm{T}=$ BIO-key & $\mathrm{U}=$ Aware & $\mathrm{V}=$ AA Technology
\end{tabular}




\section{Release Notes}

\section{All FpVTE related reports, drafts, announcements, and news items may be found on the website http://fingerprint.nist.gov.}

$\triangle$ Application Program Interface and Test Plan: The FpVTE API [15] contains additional details about creating a submission compatible with the FpVTE test driver. All submissions tested in FpVTE from 2012-2014 were fully compatible with the FpVTE API and linked without modification to the FpVTE test driver.

$\triangleright$ Appendices: Appendix A has full-scale plots for individual participant results that some may prefer to the grouped plots in the main body of the report. Appendices B through L have complete sets of tables for various analyses to help reduce the number of tables in the main body of the report.

$\triangleright$ Submission identifiers: Throughout this report, submissions are identified by letter code. For reference, the letters are associated with the providers' names in a running footnote.

$\triangleright$ Typesetting and Graphics: Virtually all of the content in this report was produced automatically. This involved the use of scripting tools to generate LTEX content and $\mathbb{R}$ graphs directly from the FpVTE test driver's output. Other graphics were produced with TikZ. Use of these technologies improved timeliness, flexibility, maintainability, and reduced transcription errors.

$\triangleright$ Evaluation Data Ground Truth: Unknown mates within and across datasets create a significant problem and time delay for large one-to-many data testing.

$\triangleright$ Contact: Correspondence regarding this report should be directed to FPVTE at NIST dot GOV.

\begin{tabular}{l|l|l|l|l|l}
$\mathrm{C}=$ afis team & $\mathrm{D}=3 \mathrm{M}$ Cogent & $\mathrm{E}=$ Neurotechnology & $\mathrm{F}=$ Papillon & $\mathrm{G}=$ Dermalog & $\mathrm{H}=$ Hisign Bio-Info Institute \\
$\mathrm{I}=\mathrm{NEC}$ & $\mathrm{J}=$ Sonda & $\mathrm{K}=$ Tiger IT & $\mathrm{L}=$ Innovatrics & $\mathrm{M}=$ SPEX & $\mathrm{O}=$ ID Solutions \\
$\mathrm{P}=\mathrm{id} 3$ & $\mathrm{Q}=$ Morpho & $\mathrm{S}=$ Decatur Industries & $\mathrm{T}=$ BIO-key & $\mathrm{U}=$ Aware & $\mathrm{V}=$ AA Technology
\end{tabular}




\section{Introduction}

In 2012, NIST launched a new Fingerprint Vendor Technology Evaluation (FpVTE) with two main goals. The first goal was to assess the current capabilities of matching algorithms using operational datasets with several million subjects. The second goal was to evaluate different operational considerations that could impact matching accuracy. These considerations included number of fingers used, data types (live-scan, single-finger capture, slap capture requiring segmentation, and rolled), number of enrolled subjects, and matching speeds.

Evaluating biometric capabilities is an important task, particularly for fingerprint identification, given its widespread applications. Large-scale evaluations of core accuracy and functionality of biometric recognition algorithms using operational data will not only reveal the capabilities of the current state of the art but can also identify the limitations and gaps of the current algorithms. The former sets realistic operational expectations and the latter directs future research to improve and enhance current technologies.

FpVTE was conducted by NIST and sponsored by the Department of Homeland Security (DHS) and the Federal Bureau of Investigation (FBI). All work was performed at the NIST Gaithersburg facility using hardware owned by NIST. FpVTE includes three rounds of submissions, with participants making algorithm adjustments based on performance reports after the first two submissions. Participant submissions were required to conform to the test plan API [15].

The evaluation had three classes of participation. Class A used single-index finger capture data and evaluated single index fingers searched against 5000 up to 100000 enrolled subjects ("single-finger identification"), and two index fingers searched against 10000 up to 1600000 enrolled subjects ("two-finger identification"). Class B used IDFlat captures (44-2) and evaluated ten-finger, eight-finger (right and left slap), and four-finger (right or left slap) identification searched against 500000 up to 3000000 enrolled subjects. Class $C$ used rolled and plain impression (4-4-1-1) captures and evaluated ten-finger rolled impression, ten-finger plain impression, and ten-finger plain impression matched to rolled impression identification searched against 500000 up to 5000000 enrolled subjects. Any segmentation of four-finger slap images or two-thumb captures was performed by the submission.

Biometric identification is defined as, "[the] process of searching against a biometric enrollment database to find and return the biometric reference identifier(s) attributable to a single individual" [7]. Biometric identification is broadly categorized into closed-set and open-set identification.

Closed-set identification refers to cases where all searches have a corresponding enrolled mate in the biometric enrollment database. An example of a closed-set identification application is a cruise ship on which all passengers are enrolled. The outcome of a closed-set identification subsystem is a candidate list that contains the identity of one or more enrolled individuals whose enrolled samples are most similar to the search (query) sample. Ideally, the correct mate appears in the first rank. As such, the primary accuracy metric for closed-set identification is hit rate (or its complement, miss rate $=$ 1.0 - hit rate), which is the fraction of times the system returns the correct identity within the specified top ranks.

In open-set identification, not all searches have a corresponding enrolled mate in the biometric enrollment database [3]. The expected outcome of an open-set identification subsystem is a candidate list of $L$ closest (or most similar) enrolled identities when the search sample is from an enrolled individual, or an indication that the search sample is from an individual not in the biometric enrollment database. Therefore, primary accuracy metrics for an open-set identification are false positive identification (false alarm or Type I error) rate and false negative identification (miss or Type II error) rate. These metrics are described in Section 6.

Closed-set identification applications are very limited because in the majority of real-world identification applications, not all individuals are or can be enrolled. Most real-world biometric identification applications, such as searches against a watch-list or searches for first-time arrestees, are open-set identification. For that reason, FpVTE only evaluated open-set identification algorithms.

This document reviews metrics for evaluating the performance of open-set identification algorithms and reports performance for submissions from 18 participants. Three participated in Class A only and the other 15 submitted for all three classes. Four participants withdrew before making a submission for testing. Table 1 shows the list of participants and in which classes they made submissions for evaluation. These participants are referenced at the bottom of every page with their assigned identification letter.

\begin{tabular}{l|l|l|l|ll|l}
$\mathrm{C}=$ afis team & $\mathrm{D}=3 \mathrm{M}$ Cogent & $\mathrm{E}=$ Neurotechnology & $\mathrm{F}=$ Papillon & $\mathrm{G}=$ Dermalog & $\mathrm{H}=$ Hisign Bio-Info Institute \\
$\mathrm{I}=$ NEC & $\mathrm{J}=$ Sonda & $\mathrm{K}=$ Tiger IT & $\mathrm{L}=$ Innovatrics & $\mathrm{M}=$ SPEX & $\mathrm{O}=$ ID Solutions \\
$\mathrm{P}=\mathrm{id} 3$ & $\mathrm{Q}=$ Morpho & $\mathrm{S}=$ Decatur Industries & $\mathrm{T}=$ BIO-key & $\mathrm{U}=$ Aware & $\mathrm{V}=$ AA Technology
\end{tabular}




\begin{tabular}{|c|c|c|}
\hline ID & Name & Participation Class \\
\hline \hline C & afis team & A, B, C \\
\hline D & 3M Cogent & A, B, C \\
\hline E & Neurotechnology & A, B, C \\
\hline F & Papillon & A, B, C \\
\hline G & Dermalog & A, B, C \\
\hline H & Hisign Bio-Info Institute & A, B, C \\
\hline I & NEC & A, B, C \\
\hline J & Sonda & A, B, C \\
\hline K & Tiger IT & A \\
\hline L & Innovatrics & A, B, C \\
\hline M & SPEX & A, B, C \\
\hline O & ID Solutions & A, B, C \\
\hline P & id3 & A \\
\hline Q & Morpho & A, B, C \\
\hline S & Decatur Industries & A, B, C \\
\hline T & BIO-key & A \\
\hline U & Aware & A, B, C \\
\hline V & AA Technology & A, B, C \\
\hline
\end{tabular}

Table 1: Participant IDs, names, and classes of submission for evaluation.

Section 2 has some history on fingerprint evaluations performed at NIST. Section 3 describes the data used in FpVTE. Section 4 describes the protocol used for submission acceptance and testing. Section 5 gives details on the two-stage matching approach used in FpVTE. Sections 6 through 11 talk about the metrics used to measure accuracy and report the results from testing. While recognition error rates are important and widely reported, computational resources required by algorithms are a significant aspect of performance, especially for large-scale operations. To that end, we report the computation time, storage requirements, and their accuracy tradeoffs for each of the submissions. Finally, Section 12 examines some FpVTE 2003 results, then Section 13 and Section 14 talk about some lessons learned and future plans.

\begin{tabular}{l|l|l|l|ll|l}
$\mathrm{C}=$ afis team & $\mathrm{D}=3 \mathrm{M}$ Cogent & $\mathrm{E}=$ Neurotechnology & $\mathrm{F}=$ Papillon & $\mathrm{G}=$ Dermalog & $\mathrm{H}=$ Hisign Bio-Info Institute \\
$\mathrm{I}=$ NEC & $\mathrm{J}=$ Sonda & $\mathrm{K}=$ Tiger IT & $\mathrm{L}=$ Innovatrics & $\mathrm{M}=$ SPEX & $\mathrm{O}=$ ID Solutions \\
$\mathrm{P}=\mathrm{id} 3$ & $\mathrm{Q}=$ Morpho & $\mathrm{S}=$ Decatur Industries & $\mathrm{T}=$ BIO-key & $\mathrm{U}=$ Aware & $\mathrm{V}=$ AA Technology
\end{tabular}




\section{History and Motivation}

\subsection{NIST Biometric Evaluations}

The first one-to-many fingerprint evaluation conducted at NIST was FpVTE 2003. This evaluation required that participants bring their own hardware and software to NIST for the evaluation. NIST supplied the data and retained all matching results for final analysis. FpVTE 2003 had three classes of participation: Small-Scale, Medium-Scale, and LargeScale. Small-Scale testing used 1000 single-finger capture images resulting in 1 million subject-to-subject comparisons. Medium-Scale testing used 10000 single-finger captures resulting in 100 million comparisons. Large-Scale testing used 25000 subjects and various combinations of fingers resulting in 1.044 billion comparisons. The evaluation had 18 participants that submitted 34 systems for testing.

NIST has conducted several fingerprint-related evaluations in the last decade (Figure 1). The first fingerprint evaluation was called Proprietary Fingerprint Template 2003 (PFT 2003). PFT 2003 was a one-to-one matching evaluation that looked at the core matching capabilities of fingerprint matching software. It did not evaluate one-to-many capabilities. In 2010, NIST changed the name of PFT 2003 to PFTII, utilizing newer, larger datasets and reporting information on timing and template sizes, in addition to accuracy.

Minutiae Exchange (MINEX), also a one-to-one matching evaluation, began in 2005. MINEX was started to support testing of fingerprint matching technologies using INCITS 378 standard interoperable templates [6]. About a year later, Ongoing MINEX was created to support Personal Identity Verification (PIV) by establishing guidelines and measuring accuracy for interoperable template encoders and matchers.

In addition to fingerprint-related evaluations, NIST has performed evaluations for other biometrics such as face and iris. Additional information and links for all the NIST biometric related evaluations can be found on the NIST biometric evaluations website [5].

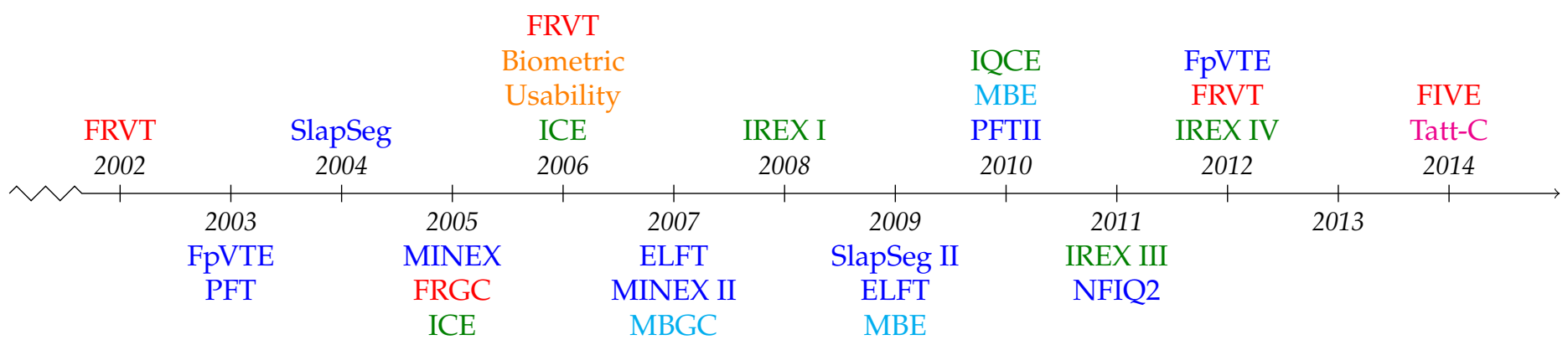

Figure 1: NIST biometric evaluations.

\subsection{Purpose}

One of the main purposes of this FpVTE evaluation was to provide a refresh on the testing performed in 2003 and allow an opportunity for participation by organizations that missed the previous evaluation. There had been many inquiries in the past several years on when NIST would perform a similar evaluation. Additionally, the dataset size for FpVTE 2003 was around 25000 total subjects. The current FpVTE testing used enrollment sets ranging from 10000 subjects to 5 million subjects.

NIST has already conducted one-to-many biometric evaluations with enrollment set sizes over 1 million subjects for other modalities (e.g., Face Recognition Vendor Test (FRVT)/Multiple Biometric Evaluation (MBE) for face and Iris Exchange Evaluation (IREX III) for iris), in which the sizes of the enrollment templates allowed the entire enrollment set to fit into the RAM of a single compute node. FpVTE was the first biometric evaluation at NIST that added the ability to partition the enrollment set across multiple compute nodes, expanding the possibilities in size and breadth of enrollment sets. In addition to broadening the enrollment set, FpVTE strived to:

\begin{tabular}{l|l|l|l|ll|l}
$\mathrm{C}=$ afis team & $\mathrm{D}=3 \mathrm{M}$ Cogent & $\mathrm{E}=$ Neurotechnology & $\mathrm{F}=$ Papillon & $\mathrm{G}=$ Dermalog & $\mathrm{H}=$ Hisign Bio-Info Institute \\
$\mathrm{I}=$ NEC & $\mathrm{J}=$ Sonda & $\mathrm{K}=$ Tiger IT & $\mathrm{L}=$ Innovatrics & $\mathrm{M}=$ SPEX & $\mathrm{O}=$ ID Solutions \\
$\mathrm{P}=\mathrm{id} 3$ & $\mathrm{Q}=$ Morpho & $\mathrm{S}=$ Decatur Industries & $\mathrm{T}=$ BIO-key & $\mathrm{U}=$ Aware & $\mathrm{V}=$ AA Technology
\end{tabular}


$\triangleright$ assess the current performance of one-to-many fingerprint matching software using operational fingerprint data;

$\triangleright$ provide a testing framework and API for enrollment sizes that must be spread across the memory of multiple compute nodes;

$\triangleright$ support US Government and other sponsors in setting operational thresholds;

$\triangleright$ evaluate on operational datasets containing newer data from live-scan ten-finger IDFlat capture systems, other livescan capture devices (e.g., single-finger and multi-finger), and historically significant scanned inked fingerprints;

$\triangleright$ analyze one-to-many identification accuracy versus, speed, template size, number of fingers, enrollment set sizes, and computational resources.

\begin{tabular}{l|l|l|l|ll|l|l}
$\mathrm{C}=$ afis team & $\mathrm{D}=3 \mathrm{M}$ Cogent & $\mathrm{E}=$ Neurotechnology & $\mathrm{F}=$ Papillon & $\mathrm{G}=$ Dermalog & $\mathrm{H}=$ Hisign Bio-Info Institute \\
$\mathrm{I}=$ NEC & $\mathrm{J}=$ Sonda & $\mathrm{K}=$ Tiger IT & $\mathrm{L}=$ Innovatrics & $\mathrm{M}=$ SPEX & $\mathrm{O}=$ ID Solutions \\
$\mathrm{P}=\mathrm{id} 3$ & $\mathrm{Q}=$ Morpho & $\mathrm{S}=$ Decatur Industries & $\mathrm{T}=$ BIO-key & $\mathrm{U}=$ Aware & $\mathrm{V}=$ AA Technology
\end{tabular}




\section{Data}

\subsection{Classes of Participation}

FpVTE was separated into three classes of participation: A, B, and C. All participants were required to make a Class A submission. Along with the Class A submission, a participant could additionally participate in Class B, or both Class B and Class $\mathrm{C}$. These were the only three participation combinations available.

Images were captured via live-scan sensor and rescanned ink. A live-scan sensor refers to the type of sensor that digitally records the friction ridges of a finger through techniques such as electrical or optical sensing. Scanned ink is the process of creating a digital image by using an image scanner to optically capture from paper images of friction ridges created by a finger covered with ink.

Class A consisted of live-scan single-finger captures of the left and right index fingers (Figure 2).

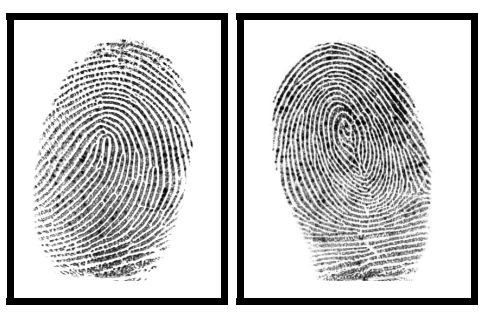

Figure 2: Example of single-finger captures of left and right index fingers.

Class B consisted of live-scan IDFlats, which captured left and right four-finger slaps and simultaneous left and right thumbs, known as "4-4-2" (Figure 3).

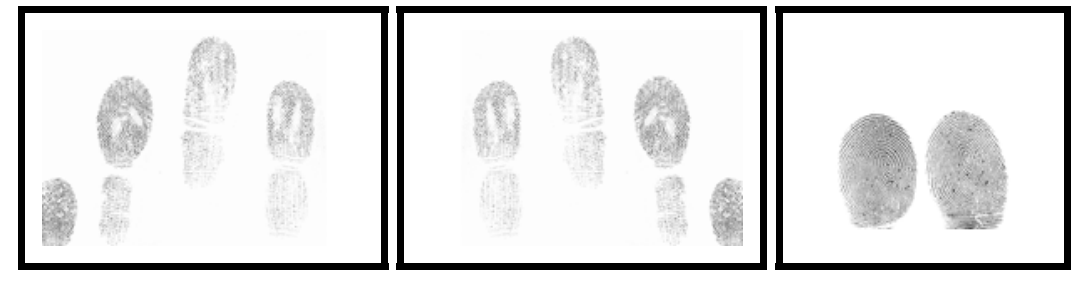

Figure 3: Example of an identification flat (4-4-2) capture of a left and right slap and left and right thumbs.

Class $C$ consisted of the rolled and plain impressions from a more traditional 14 print card/record and was a mix of live-scan and rescanned ink (Figure 4).
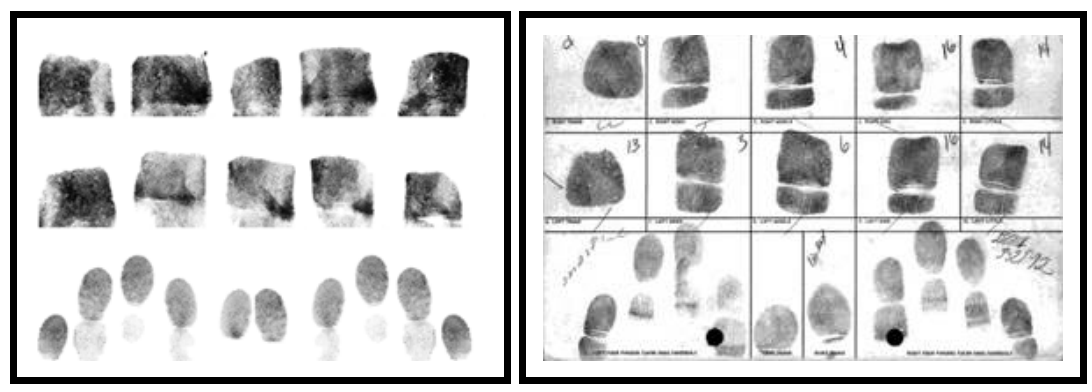

Figure 4: Example of live-scan and rescanned ink 14 print card/record that include rolled and plain impressions.

\begin{tabular}{l|l|l|l|ll|l}
$\mathrm{C}=$ afis team & $\mathrm{D}=3 \mathrm{M}$ Cogent & $\mathrm{E}=$ Neurotechnology & $\mathrm{F}=$ Papillon & $\mathrm{G}=$ Dermalog & $\mathrm{H}=$ Hisign Bio-Info Institute \\
$\mathrm{I}=\mathrm{NEC}$ & $\mathrm{J}=$ Sonda & $\mathrm{K}=$ Tiger IT & $\mathrm{L}=$ Innovatrics & $\mathrm{M}=$ SPEX & $\mathrm{O}=$ ID Solutions \\
$\mathrm{P}=\mathrm{id} 3$ & $\mathrm{Q}=$ Morpho & $\mathrm{S}=$ Decatur Industries & $\mathrm{T}=$ BIO-key & $\mathrm{U}=$ Aware & $\mathrm{V}=$ AA Technology
\end{tabular}




\subsection{Datasets}

The evaluation datasets used in FpVTE were from anonymized operational datasets and made available to NIST for fingerprint evaluations. The datasets are for government use only and will not be released to the public. The datasets will, to the extent permitted by law, be protected under the Freedom of Information Act (5 U.S.C. § 522) and the Privacy Act (5 U.S.C. § 522a) as applicable.

The datasets were comprised of several fingerprint impression types, including rolled, multi-finger plains, and singlefinger plains. Rolled images were all individual captures that attempted to record the full width of the fingerprint by rolling from side-to-side during capture. Multi-finger plains captured the four right and four left fingers at the same time. For identification flats, the two thumbs were captured at the same time. Single-finger plains were individual captures of the right and left index fingers on a single-finger capture device.

Many of the datasets were larger samples of data used in previous NIST evaluations, such as PFT, MINEX, NIST Fingerprint Image Quality (NFIQ), and FpVTE 2003. The single-finger capture and identification flat fingerprint images were provided by DHS. The ten-finger rolled and slap fingerprint images included data from the FBI, DHS, Los Angeles County Sheriff's Department (LACNTY), Arizona Department of Public Safety (AZDPS), and Texas Department of Public Safety (TXDPS). Table 2 shows the source of data for each class in the evaluation.

\begin{tabular}{|c|c|c|c|c|c|}
\hline Class & Dataset & Enroll Mate & Enroll Nonmate & Search Mate & Search Nonmate \\
\hline \hline \multirow{3}{*}{ A } & VISIT-I/POEBVA & $25 \%$ & $5 \%$ & $25 \%$ & $25 \%$ \\
\cline { 2 - 6 } & DHS2 & $25 \%$ & $5 \%$ & $25 \%$ & $25 \%$ \\
\cline { 2 - 6 } & VISIT-II & $50 \%$ & $90 \%$ & $50 \%$ & $50 \%$ \\
\hline \hline \multirow{2}{*}{ B } & VISIT-II & $96 \%$ & $85 \%$ & $96 \%$ & $62.5 \%$ \\
\cline { 2 - 6 } & PDR_IDF & $4 \%$ & $15 \%$ & $4 \%$ & $37.5 \%$ \\
\hline \hline & AZDPS & $33.3 \%$ & $4 \%$ & $33.3 \%$ & $18.75 \%$ \\
\cline { 2 - 6 } & INSBEN & $0 \%$ & $11.5 \%$ & $0 \%$ & $12.5 \%$ \\
\cline { 2 - 6 } C & LACNTY & $33.3 \%$ & $30 \%$ & $33.3 \%$ & $18.75 \%$ \\
\cline { 2 - 6 } & TXDPS & $0 \%$ & $11.5 \%$ & $0 \%$ & $12.5 \%$ \\
\cline { 2 - 6 } & PDR-Roll & $33.3 \%$ & $43 \%$ & $33.3 \%$ & $37.5 \%$ \\
\hline
\end{tabular}

Table 2: Percentage of data used from each source.

All images in the datasets were 8-bit grayscale. Images were previously compressed using Wavelet Scalar Quantization (WSQ) compression [2], but were passed to the submitted software as reconstructed raw pixel images, decompressed using libwsq from NIST's NIST Biometric Image Software (NBIS) distribution [14]. All images were scanned at 500 pixels per inch. The dimensions of the images varied, but were provided as input information to the participant's submission. The distribution of NIST Fingerprint Image Quality (NFIQ) algorithm [12,13] values for the evaluation datasets is shown in Figure 5. NIST Fingerprint Segmentation algorithm (NFSEG) was used to segment slap impression images into individual fingers before computing NFIQ values.

Multiple-finger plain captures were not segmented. Submissions were required to perform segmentation of fingerprints, if necessary. Subjects with missing fingers were not removed from the dataset.

\begin{tabular}{l|l|l|l|ll|l}
$\mathrm{C}=$ afis team & $\mathrm{D}=3 \mathrm{M}$ Cogent & $\mathrm{E}=$ Neurotechnology & $\mathrm{F}=$ Papillon & $\mathrm{G}=$ Dermalog & $\mathrm{H}=$ Hisign Bio-Info Institute \\
$\mathrm{I}=\mathrm{NEC}$ & $\mathrm{J}=$ Sonda & $\mathrm{K}=$ Tiger IT & $\mathrm{L}=$ Innovatrics & $\mathrm{M}=$ SPEX & $\mathrm{O}=$ ID Solutions \\
$\mathrm{P}=\mathrm{id} 3$ & $\mathrm{Q}=$ Morpho & $\mathrm{S}=$ Decatur Industries & $\mathrm{T}=$ BIO-key & $\mathrm{U}=$ Aware & $\mathrm{V}=$ AA Technology
\end{tabular}




\begin{tabular}{|c|c|c|c|c|c|c|c|}
\hline Class & Fingers & Type & $\mathbf{1}$ & $\mathbf{2}$ & $\mathbf{3}$ & $\mathbf{4}$ & $\mathbf{5}$ \\
\hline \hline \multirow{2}{*}{$\mathrm{A}$} & L/R Index & Enroll & $42.8 \%$ & $32.5 \%$ & $20.2 \%$ & $2.2 \%$ & $2.3 \%$ \\
& L/R Index & Search & $39.5 \%$ & $32.9 \%$ & $20.7 \%$ & $2.2 \%$ & $4.7 \%$ \\
\hline \hline \multirow{3}{*}{$\mathrm{B}$} & Left Slap & Search & $44.9 \%$ & $26.8 \%$ & $16.0 \%$ & $6.9 \%$ & $5.4 \%$ \\
& Right Slap & Search & $50.2 \%$ & $24.4 \%$ & $13.0 \%$ & $5.8 \%$ & $6.6 \%$ \\
& L/R Slap & Search & $47.6 \%$ & $25.6 \%$ & $14.5 \%$ & $6.3 \%$ & $6.0 \%$ \\
& IDFlats & Search & $48.3 \%$ & $25.7 \%$ & $14.2 \%$ & $6.4 \%$ & $5.4 \%$ \\
\hline \hline \multirow{3}{*}{ C } & Ten Plain & Search & $47.8 \%$ & $26.6 \%$ & $16.2 \%$ & $5.5 \%$ & $3.9 \%$ \\
& Ten Rolled & Enroll & $34.2 \%$ & $19.5 \%$ & $25.0 \%$ & $8.6 \%$ & $12.7 \%$ \\
& Ten Rolled & Search & $35.4 \%$ & $21.3 \%$ & $23.0 \%$ & $9.1 \%$ & $11.2 \%$ \\
\hline
\end{tabular}

Figure 5: NFIQ distribution for datasets, after segmentation, where 1 is highest quality and 5 is lowest quality.

\subsection{Evaluation Scenarios}

The three classes of participation had various data type and fingerprint combinations that could be evaluated as summarized in autoreftab:data-scenarios. The contents of Table 4 shows details of the search and enrollment sets used during the evaluation for each class of participation. The final column of Table 4 shows the various sizes of the datasets that were tested. The subjects reserved for the search sets contained 200000 with a known mate and 400000 with no mate in the enrollment set. This report is based on a random sample of 10000 mated and 20000 nonmated searches from the full search set.

Table 3 shows the various combinations in which the search sets were searched against the enrollment sets listed in Table 4. Those combinations are also described for each class as follows.

\section{$\triangleright$ Class A - Index Fingers}

- One plain index finger searched against an enrollment set of one plain index fingers. The plain images were from single-finger captures of left and right index fingers.

- Two plain index fingers searched against an enrollment set of two plain index codes. fingers. The plain images were from single-finger captures of left and right index fingers.

$\triangleright$ Class B - Identification Flats

- Four-, eight-, and ten-finger identification flats (4-4-2, left/right four-finger plain impressions, and a two-thumb plain impression) searched against an enrollment set of ten-finger identification flats. Any segmentation was performed by the submission.

\section{$\triangleright$ Class C - Ten-Finger Rolled/Slap}

- Ten-finger rolled impressions searched against an enrollment set of ten-finger rolled impressions.

- Ten-finger plain impressions searched against an enrollment set of ten-finger plain impressions. Plain impression images were 4-4-1-1 (left/right four-finger plain impression and left/right single-thumb plain impressions). Any segmentation was performed by the submission.

- Ten-finger plain impressions searched against an enrollment set of ten-finger rolled impressions. Plain impression images were 4-4-1-1 (left/right four-finger plain impressions and left/right single-thumb plain impressions). Any segmentation was performed by the submission.

\begin{tabular}{l|l|l|l|ll|l}
$\mathrm{C}=$ afis team & $\mathrm{D}=$ = 3M Cogent & $\mathrm{E}=$ Neurotechnology & $\mathrm{F}=$ Papillon & $\mathrm{G}=$ Dermalog & $\mathrm{H}=$ Hisign Bio-Info Institute \\
$\mathrm{I}=$ NEC & $\mathrm{J}=$ Sonda & $\mathrm{K}=$ Tiger IT & $\mathrm{L}=$ Innovatrics & $\mathrm{M}=$ SPEX & $\mathrm{O}=$ ID Solutions \\
$\mathrm{P}=\mathrm{id} 3$ & $\mathrm{Q}=$ Morpho & $\mathrm{S}=$ Decatur Industries & $\mathrm{T}=$ BIO-key & $\mathrm{U}=$ Aware & $\mathrm{V}=$ AA Technology
\end{tabular}




\begin{tabular}{|c|c|c|c|c|c|c|c|}
\hline & Class & Set & Description & \# Images & \# Fingers & Impression & \# Subjects \\
\hline \multirow{9}{*}{$\begin{array}{l}\tilde{D} \\
\text { Jँ } \\
\text { ஸे }\end{array}$} & \multirow{3}{*}{$\mathrm{A}$} & S1 & Right Index & 1 & 1 & Plain & $\begin{array}{r}\text { Mate: } 10000 \\
\text { Nonmate: } 20000\end{array}$ \\
\hline & & S2 & Left Index & 1 & 1 & Plain & $\begin{array}{r}\text { Mate: } 10000 \\
\text { Nonmate: } 20000\end{array}$ \\
\hline & & S3 & Left and Right Index & 2 & 2 & Plain & $\begin{array}{r}\text { Mate: } 10000 \\
\text { Nonmate: } 20000\end{array}$ \\
\hline & \multirow{4}{*}{ B } & $\mathrm{S} 4$ & Right Slap IDFlat & 1 & 4 & Plain & $\begin{array}{r}\text { Mate: } 10000 \\
\text { Nonmate: } 20000\end{array}$ \\
\hline & & S5 & Left Slap IDFlat & 1 & 4 & Plain & $\begin{array}{r}\text { Mate: } 10000 \\
\text { Nonmate: } 20000\end{array}$ \\
\hline & & S6 & Left and Right Slap IDFlat & 2 & $\begin{array}{c}8 \\
(4,4)\end{array}$ & Plain & $\begin{array}{r}\text { Mate: } 10000 \\
\text { Nonmate: } 20000\end{array}$ \\
\hline & & S7 & Identification Flats & 3 & $\begin{array}{c}10 \\
(4,4,2)\end{array}$ & Plain & $\begin{array}{r}\text { Mate: } 10000 \\
\text { Nonmate: } 20000\end{array}$ \\
\hline & \multirow{2}{*}{$\mathrm{C}$} & S8 & Ten Finger Rolled & 10 & 10 & Rolled & $\begin{array}{r}\text { Mate: } 10000 \\
\text { Nonmate: } 20000\end{array}$ \\
\hline & & S9 & Ten Finger Plain & 4 & $\begin{array}{c}10 \\
(4,4,1,1)\end{array}$ & Plain & $\begin{array}{r}\text { Mate: } 10000 \\
\text { Nonmate: } 20000\end{array}$ \\
\hline \multirow{6}{*}{ 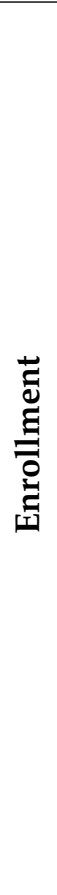 } & \multirow{3}{*}{ A } & E1 & Right Index & 1 & 1 & Plain & $\begin{array}{r}10000 \\
100000\end{array}$ \\
\hline & & E2 & Left Index & 1 & 1 & Plain & $\begin{array}{r}10000 \\
100000\end{array}$ \\
\hline & & E3 & Left and Right Index & 2 & 2 & Plain & $\begin{array}{r}100000 \\
500000 \\
1600000 \\
\end{array}$ \\
\hline & B & $\mathrm{E} 4$ & Identification Flats & 3 & $\begin{array}{c}10 \\
(4,4,2)\end{array}$ & Plain & $\begin{array}{r}500000 \\
1600000 \\
3600000 \\
\end{array}$ \\
\hline & \multirow{2}{*}{$\mathrm{C}$} & E5 & Ten Finger Rolled & 10 & 10 & Rolled & $\begin{array}{r}500000 \\
1600000 \\
3000000 \\
5000000\end{array}$ \\
\hline & & E6 & Ten Finger Plain & 4 & $\begin{array}{c}10 \\
(4,4,1,1)\end{array}$ & Plain & $\begin{array}{r}500000 \\
1600000 \\
3000000 \\
5000000\end{array}$ \\
\hline
\end{tabular}

Table 4: Search and enrollment datasets used in FpVTE. Class refers to the class of participation, as defined in Subsection 3.1. Set is an identifier used to uniquely identify the various FpVTE datasets in a concise manner. Description indicates the finger combinations composing each dataset. \# Images specifies the number of images per subject. \# Fingers shows the maximum number of fingers per subject. If a subject's fingers were spread across multiple images, the maximum number of fingers for each image is also shown. Impression refers to the impression type of the imagery in each dataset. Impressions could be either plain or rolled, as defined in Subsection 3.2. \# Subjects shows the number of subjects in each dataset. For 'Search' datasets, the value has been split into mate and nonmate subjects, referring to whether or not a known mate for the subject exists in the corresponding enrollment set. For 'Enrollment' datasets, the \# Subjects show the various enrollment set sizes planned for evaluation.

\begin{tabular}{l|l|l|l|ll|l}
$\mathrm{C}=$ afis team & $\mathrm{D}=3 \mathrm{M}$ Cogent & $\mathrm{E}=$ Neurotechnology & $\mathrm{F}=$ Papillon & $\mathrm{G}=$ Dermalog & $\mathrm{H}=$ Hisign Bio-Info Institute \\
$\mathrm{I}=$ NEC & $\mathrm{J}=$ Sonda & $\mathrm{K}=$ Tiger IT & $\mathrm{L}=$ Innovatrics & $\mathrm{M}=$ SPEX & $\mathrm{O}=$ ID Solutions \\
$\mathrm{P}=\mathrm{id} 3$ & $\mathrm{Q}=$ Morpho & $\mathrm{S}=$ Decatur Industries & $\mathrm{T}=$ BIO-key & $\mathrm{U}=$ Aware & $\mathrm{V}=$ AA Technology
\end{tabular}




\section{4 "Size" of the Test}

The overall size of the test had implications on total run time. Table 5 shows the number of enrollments and comparisons that were completed for each submission. Time limits allowed for a maximum of 3 seconds per finger for each enrollment, and up to 500 seconds for Class A and 90 seconds for Class B and C to complete each search. Participants could make two submissions for each class of participation. Three of the 18 participants submitted for Class A only, while the other 15 submitted for all three classes, creating a total of 96 submissions analyzed in this report. There were three rounds of submissions, with participants receiving results after the first two submissions to analyze before making their next submission. Most submissions shared enrollment templates between both submissions within a class, but two or three submissions in

\begin{tabular}{ccc}
\hline Class & $\begin{array}{c}\text { Enrollments } \\
\text { Performed }\end{array}$ & $\begin{array}{c}\text { Subject to Subject } \\
\text { Comparisons }\end{array}$ \\
\hline A & 5.8 & 54 \\
B & 45.5 & 362 \\
C & 113 & 452 \\
\hline
\end{tabular}

Table 5: Approximate number (in millions) of enrollments and comparisons that were completed to evaluate each submission for that class of participation. each round required generating enrollment templates separately.

One unexpected event was that the majority of the submissions required re-enrolling the data for each round of submissions. Each round of submissions required approximately 2.5 billion fingerprint enrollments. It took two to three months for each round of submissions to enroll all the data for all the submissions before searching could begin. The 30000 (20000 nonmate and 10000 mate) searches took another one to two months to complete for each round of submissions for all classes across all submissions and finger combinations. The first two rounds resulted in approximately 43 trillion subjectto-subject comparisons, and the final round will have approximately 470 trillion subject-to-subject comparisons. The final submission will be the only one to do searching against variable enrollment set sizes. Total enrollment and search times were slightly longer when accounting for submissions that failed in the middle of enrollment or matching and had to be fixed and restarted (Subsection 4.4).

\begin{tabular}{l|l|l|l|ll|l}
$\mathrm{C}=$ afis team & $\mathrm{D}=3 \mathrm{M}$ Cogent & $\mathrm{E}=$ Neurotechnology & $\mathrm{F}=$ Papillon & $\mathrm{G}=$ Dermalog & $\mathrm{H}=$ Hisign Bio-Info Institute \\
$\mathrm{I}=$ NEC & $\mathrm{J}=$ Sonda & $\mathrm{K}=$ Tiger IT & $\mathrm{L}=$ Innovatrics & $\mathrm{M}=$ SPEX & $\mathrm{O}=$ ID Solutions \\
$\mathrm{P}=\mathrm{id} 3$ & $\mathrm{Q}=$ Morpho & $\mathrm{S}=$ Decatur Industries & $\mathrm{T}=$ BIO-key & $\mathrm{U}=$ Aware & $\mathrm{V}=$ AA Technology
\end{tabular}




\section{Experiment and Test Protocol}

\subsection{API Overview}

The FpVTE API consisted of three major steps: feature extraction, finalization, and identification. During all steps of the evaluation, participants had read-only access to a configuration directory, where configuration files, models, or other algorithm-specific data could be stored. Not all participants made use of the configuration directory. It was made available as a convenience to those participants who did not wish to store customized traits about their software inside the submission itself. In practice, many participants were able to perform changes, such as adjusting identification speed, simply by changing a configuration file within this directory instead of needing to recompile their submission.

\subsubsection{Feature Extraction}

The first step in the FpVTE evaluation pipeline was feature extraction. During this step, software submissions were given the opportunity to turn one or more images of fingerprints into a single fingerprint template. There were no requirements on the format of the fingerprint template, as it was expected that the majority of participants would make use of proprietary template formats. The FpVTE API provided a way to distinguish between the images that would be used for searching and the images used to compose the enrollment set, though participants were free to treat all images in the same fashion.

For each instance of feature extraction, the FpVTE test driver called an initialization method a single time, giving the submission an opportunity to load information that might be needed for feature extraction. After the initialization call, the feature extraction method was called $N$ times. The FpVTE API provided the finger position, impression type, NFIQ value (no value provided for slap images), image dimensions, and raw image bytes for each input image during the call to extract features. As output, participants were to return the finger position, an image quality value, a fingerprint template, and the size of the fingerprint template as it would be stored in RAM. The RAM size was important to determine the number of compute nodes needed during identification, because the size of the template on disk might be significantly larger or smaller than the size of the template in RAM. Participants could optionally return a core and delta coordinate for the image.

\subsubsection{Finalization}

As fingerprint templates were returned from feature extraction, the FpVTE test driver added them to a RecordStore (a key-value pair storage mechanism of the Biometric Evaluation Framework). This allowed I/O operations to be excluded when calculating the runtime of feature extraction, as well as to allow NIST to store the large quantity of template data in the most efficient way on NIST's hardware. Once all features were extracted from enrollment set fingerprint images, the FpVTE test driver called an API method to "finalize" the enrollment set. Based on the sum of the sizes of templates in RAM, NIST calculated the appropriate number of compute nodes and passed this information, along with the RecordStore of all templates, to the finalization method. During this method, the submission was to divide the enrollment set into a partition for each compute node, as well as perform any sort of indexing, statistics, or other pre-identification tasks required.

Unlike how NIST controlled the storage mechanism for templates in feature extraction, participants had full control of how to store their finalized enrollment sets. In many cases, the size of the finalized enrollment set was either much larger or much smaller than the sum of the fingerprint templates sizes returned from feature extraction as stored on disk.

\subsubsection{Identification}

Once the finalized set was created, its read-only root directory was provided to the identification methods. Searching the finalized set for candidates occurred over two stages. The first stage was performed on separate compute nodes (if necessary, as determined by the amount of RAM needed), potentially searching subsets of the enrollment set. Output from

\begin{tabular}{l|l|l|l|ll|l}
$\mathrm{C}=$ afis team & $\mathrm{D}=3 \mathrm{M}$ Cogent & $\mathrm{E}=$ Neurotechnology & $\mathrm{F}=$ Papillon & $\mathrm{G}=$ Dermalog & $\mathrm{H}=$ Hisign Bio-Info Institute \\
$\mathrm{I}=$ NEC & $\mathrm{J}=$ Sonda & $\mathrm{K}=$ Tiger IT & $\mathrm{L}=$ Innovatrics & $\mathrm{M}=$ SPEX & $\mathrm{O}=$ ID Solutions \\
$\mathrm{P}=\mathrm{id} 3$ & $\mathrm{Q}=$ Morpho & $\mathrm{S}=$ Decatur Industries & $\mathrm{T}=$ BIO-key & $\mathrm{U}=$ Aware & $\mathrm{V}=$ AA Technology
\end{tabular}


this first stage was coalesced and provided to the second stage, which took place on a single compute node. These two stages of identification are described at length in Section 5.

\subsection{Test Constraints}

In order to complete the evaluation in a reasonable amount of time, as well as to mimic potential operational requirements, certain constraints were placed on FpVTE submissions. All times are maximum averages over the pre-evaluation datasets (Section 3), though NIST employed reasonable "cutoff" times to prevent processes from never completing. See Caveats for additional information about timing.

$\triangleright$ Operating System and Compilation Environment: All submitted implementations required 64-bit linkage and were tested on CentOS 6.2 (kernel version 2.6.32-220.7.1.el6.x86_64). NIST linked participant submissions to the FpVTE test driver written in $\mathrm{C}^{+}$with GNU g++ 4.4.6-3, using glibcxx 3.4.13 and glibc 2.12.

$\triangleright$ Evaluation Hardware: Timing computations were performed on a Dell M610 with two Intel X5690 3.47 GHz processors and 192 GB of RAM.

$\triangleright$ Threaded Computations: Threaded computations were only allowed for the finalization step. All other functions were not to perform multithreaded computations, as the FpVTE test driver handled parallelism efficiently on the NIST compute nodes.

$\triangleright$ Feature Extraction Time: Feature extraction was required to complete in $\mathbf{3}$ seconds or less for each input fingerprint. A four-finger slap was counted as four input fingerprints.

$\triangleright$ Finalization Time: Finalization of the enrollment set of fingerprint templates was required to complete in 12 hours or less on a single compute node.

$\triangleright$ Search Time: Search time is the combined time measurement for stage one and stage two identification (not including initialization times). For Class A data, searches needed to complete in under $\mathbf{5 0 0}$ seconds, though implementations that searched in under 20 seconds were reported separately. For Class B and Class C, all searches needed to complete in under $\mathbf{9 0}$ seconds.

\subsection{Biometric Evaluation Framework}

The FpVTE test driver made use of several C++ classes that are part of the NIST Image Group's Biometric Evaluation Framework [11] designed to make writing code for running biometric evaluations easier and more efficient, especially on NIST-owned equipment. Classes from the framework used in the FpVTE API included key-value pair file storage, safe dynamic arrays, error handling, and more. While the framework was mainly provided to participants as a means of interacting with the FPVTE API, many participants chose to use some classes internally in their submissions.

In order to process the massive amount of data required for the evaluation, the submission was executed as a scalable parallel job. Within the NIST testbed, an implementation of the Message Passing Interface (MPI) [10] was used to execute the evaluation test programs. By using the MPI software, the size of the parallel job (in terms of participating compute nodes) can be matched to the size of the input dataset.

The framework supports parallelism by abstracting and hiding the lower-level communication, error handling, and other facets of the MPI library. The framework application need only implement a few functions in order to become an MPI parallel job. One feature of the framework is a set of classes that support the distribution of record keys, or key-value pairs, across the computation cluster. Key distribution allows for driving the test where all nodes have local access to the data. Key-value distribution has the advantage of running the job with the data source present only on a single node.

Configuration of the job is managed with a simple text file that specifies the input data source, logging system (either files or a log server), and the number of data consumers assigned to each compute node. By using a configuration file, test scripts were simpler to invoke, the probability of error was reduced, and replication of the test scenario was implicitly provided.

\begin{tabular}{l|l|l|l|ll|l}
$\mathrm{C}=$ afis team & $\mathrm{D}=3 \mathrm{M}$ Cogent & $\mathrm{E}=$ Neurotechnology & $\mathrm{F}=$ Papillon & $\mathrm{G}=$ Dermalog & $\mathrm{H}=$ Hisign Bio-Info Institute \\
$\mathrm{I}=$ NEC & $\mathrm{J}=$ Sonda & $\mathrm{K}=$ Tiger IT & $\mathrm{L}=$ Innovatrics & $\mathrm{M}=$ SPEX & $\mathrm{O}=$ ID Solutions \\
$\mathrm{P}=\mathrm{id} 3$ & $\mathrm{Q}=$ Morpho & $\mathrm{S}=$ Decatur Industries & $\mathrm{T}=$ BIO-key & $\mathrm{U}=$ Aware & $\mathrm{V}=$ AA Technology
\end{tabular}




\subsection{Validation}

Participants were provided imagery from publicly available datasets in the same format as the full evaluation datasets. These validation datasets were used to test implementation functionality before NIST performed the evaluation on a much larger dataset. Participants ran a validation test with the provided data on their own systems and provided NIST with the resulting candidate lists and scores from a set of pre-selected searches. NIST did the same on the machines used for the full evaluation to confirm that both NIST and the participant produced the same results. Validation also provided a way to make sure that participants were following the conventions of the evaluation, such as returning appropriate quality values and numbers of candidates, among others.

Care was taken to make the validation process as simple as possible, to let the participant focus more on their submission than about intricacies specific to FpVTE. To assist, NIST provided a minimal version of the FpVTE test driver software used in the evaluation to aid participants through the expected calling structure of the evaluation API. Scripts were provided to compile the test driver and run the validation test. All a participant needed to do was create a properly named software submission, run the script, and submit the generated results file to NIST. Additionally, a build of the Biometric Evaluation Framework was provided, and many participants made use of features found in the framework in their API implementations.

\subsection{Pre-Evaluation}

A timing test was performed to make sure no submission was in violation of the required time limits. First, a sample of the evaluation dataset was enrolled using ten processes per compute node and the timing of those enrollments were evaluated to make sure they did not exceed the average of three seconds per fingerprint. I/O time was not included during this step, as all data was passed to and from the FpVTE test driver in memory. If the submission passed the enrollment timing test, the full set of data was enrolled and finalized for matching. After enrollment, the next timing test was a random sample of mated and nonmated searches matched against the maximum enrollment set for each evaluation class. If the average search time on this sample test set, using a single process on each compute node, completed under the maximum time limit, the full search set of 30000 searches was performed. The formula used to compute the total search time was:

$$
T_{s}=\left(t_{1} \times b_{1}\right)+t_{2}
$$

where $t_{1}=$ average identification stage one time

$b_{1}=$ number of blades required for identification stage one

$t_{2}=$ identification stage two time

This formula allowed for a fair comparison between submissions that used one compute node for identification stage one versus others that may have used two or more compute nodes to store the enrollment set. The formula was vetted with the participants because it assumes that the enrollment set is evenly distributed across all identification stage one blades and that search time is fairly linear versus enrollment set size.

Every attempt was made to use the same hardware and number of processes for every submission when reporting timing results. Additionally, the hardware used for timing was dedicated to the FpVTE test driver process with no other jobs running on the system except OS related processes.

\begin{tabular}{l|l|l|l|ll|l}
$\mathrm{C}=$ afis team & $\mathrm{D}=3 \mathrm{M}$ Cogent & $\mathrm{E}=$ Neurotechnology & $\mathrm{F}=$ Papillon & $\mathrm{G}=$ Dermalog & $\mathrm{H}=$ Hisign Bio-Info Institute \\
$\mathrm{I}=$ NEC & $\mathrm{J}=$ Sonda & $\mathrm{K}=$ Tiger IT & $\mathrm{L}=$ Innovatrics & $\mathrm{M}=$ SPEX & $\mathrm{O}=$ ID Solutions \\
$\mathrm{P}=\mathrm{id} 3$ & $\mathrm{Q}=$ Morpho & $\mathrm{S}=$ Decatur Industries & $\mathrm{T}=$ BIO-key & $\mathrm{U}=$ Aware & $\mathrm{V}=$ AA Technology
\end{tabular}




\subsection{Receiving Submissions}

Every FpVTE submission received by NIST went through the FpVTE Validation and Pre-Evaluation processes before committing resources to complete the full evaluation. Figure 7 visualizes the submission process with a flowchart. Many submissions underwent the process multiple times due to defects in the initial submission. Potential FpVTE software submission defects that would require a resubmission include:

$\triangleright$ submission incorrectly signed or encrypted;

$\triangleright$ participant's validation results differ from NIST's;

$\triangleright$ software errors during evaluation;

$\triangleright$ maximum average time limit exceeded;

$\triangleright$ invalid API implementation.

NIST provided no upper bound on the number of times that a participant could resubmit software in the event of a defect. Unfortunately, this created an unpredictably large amount of additional work for NIST, through managing submissions, helping participants debug, reporting status, and the like. Figure 6 details the number of non-debugging submissions received from participants over the course of the evaluation. The general trend of participants not achieving a "valid" submission on the first or second attempt ultimately played a part in forcing NIST to extend the original evaluation deadlines.

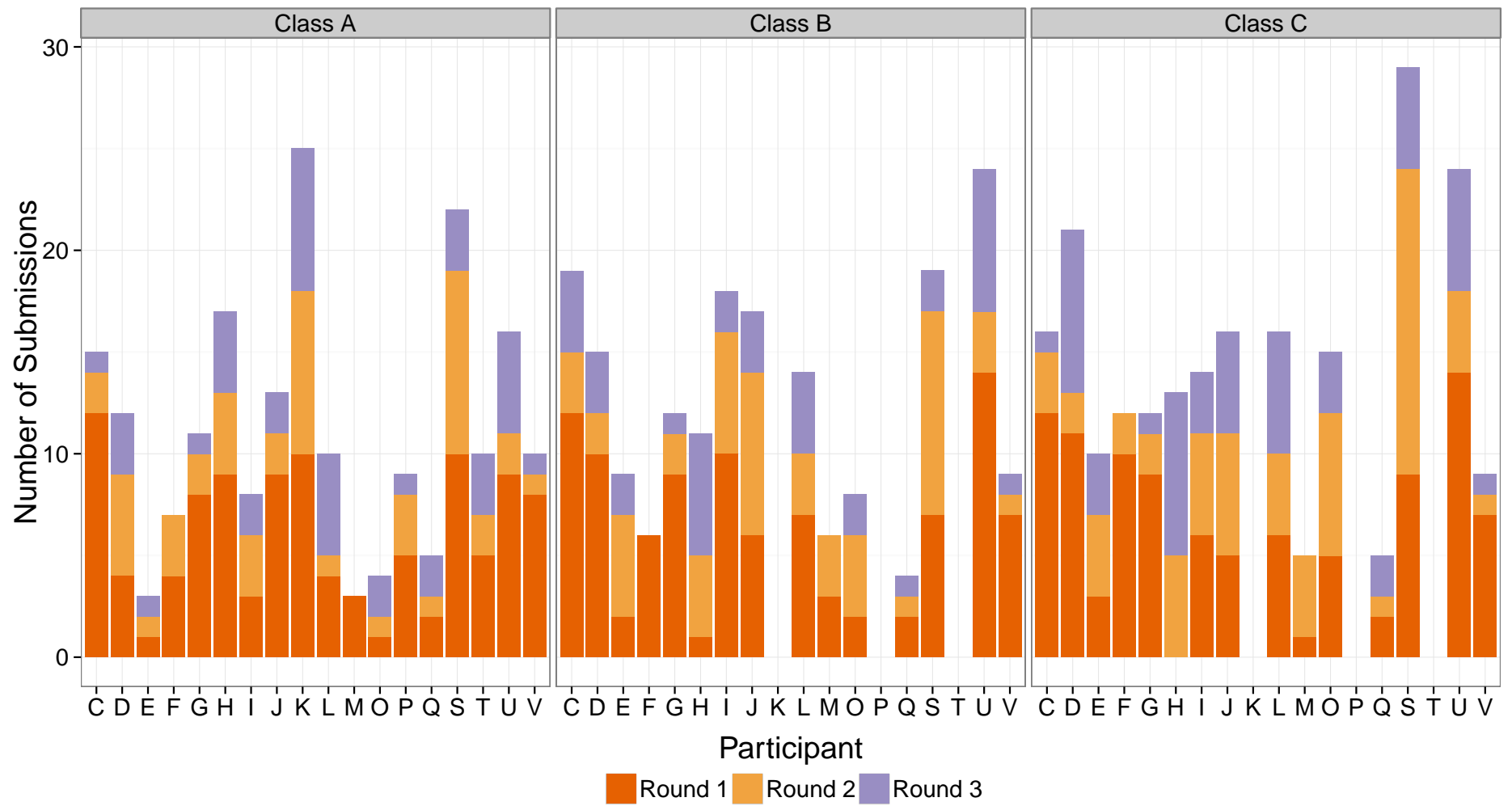

Figure 6: Total number of non-debugging submissions received before the final deadline.

At the conclusion of the evaluation, 733 FpVTE validation submissions were received by NIST, including validation submissions from participants who ultimately withdrew from the evaluation. For this total, a submission is considered a discrete transmission of non-debugging software to NIST that required action by a NIST employee, on a per-class basis. There were three rounds during which participants could make submissions. Participants were given an opportunity to update their submissions at the end of the first two submission rounds. Because FpVTE was a black-box evaluation, NIST

\begin{tabular}{l|l|l|l|ll|l}
$\mathrm{C}=$ afis team & $\mathrm{D}=3 \mathrm{M}$ Cogent & $\mathrm{E}=$ Neurotechnology & $\mathrm{F}=$ Papillon & $\mathrm{G}=$ Dermalog & $\mathrm{H}=$ Hisign Bio-Info Institute \\
$\mathrm{I}=$ NEC & $\mathrm{J}=$ Sonda & $\mathrm{K}=$ Tiger IT & $\mathrm{L}=$ Innovatrics & $\mathrm{M}=$ SPEX & $\mathrm{O}=$ ID Solutions \\
$\mathrm{P}=\mathrm{id} 3$ & $\mathrm{Q}=$ Morpho & $\mathrm{S}=$ Decatur Industries & $\mathrm{T}=$ BIO-key & $\mathrm{U}=$ Aware & $\mathrm{V}=$ AA Technology
\end{tabular}


ultimately had no idea what changes to the submissions were made. A number of submissions majorly regressed in their lack of defects, so it can be anecdotally inferred that large portions or even entire submissions were changed by some participants.

NIST imposed certain timing requirements on participants to ensure that the evaluation could be completed on NIST hardware in a reasonable amount of time, as well as to mimic a possible operational constraint. Several participants tried to tune their submissions to make use of the maximum amount of time, giving themselves a potential increase in overall accuracy. While the back-and-forth between NIST and the participants did increase the runtime of the evaluation, timing defects were the most understandable defect encountered in the evaluation and was primarily caused by NIST-based evaluation restrictions, not participant error.

During the final submission, participants $\mathbf{C}, \mathbf{D}, \mathbf{E}, \mathbf{G}, \mathbf{H}, \mathbf{I}, \mathbf{O}, \mathbf{P}, \mathbf{Q}$, and $\mathbf{V}$ provided one or more submissions that validated and ran to completion without NIST encountering defects other than timing restrictions. Participants C, E, G, I, O, and $\mathbf{V}$ met this requirement for all classes of participation in the final submission. Participant $\mathbf{E}$ was the only participant to achieve no defects other than timing restrictions, for every class during all three submission periods.

\begin{tabular}{l|l|l|l|ll|l}
$\mathrm{C}=$ afis team & $\mathrm{D}=3 \mathrm{M}$ Cogent & $\mathrm{E}=$ Neurotechnology & $\mathrm{F}=$ Papillon & $\mathrm{G}=$ Dermalog & $\mathrm{H}=$ Hisign Bio-Info Institute \\
$\mathrm{I}=\mathrm{NEC}$ & $\mathrm{J}=$ Sonda & $\mathrm{K}=$ Tiger IT & $\mathrm{L}=$ Innovatrics & $\mathrm{M}=$ SPEX & $\mathrm{O}=$ ID Solutions \\
$\mathrm{P}=\mathrm{id} 3$ & $\mathrm{Q}=$ Morpho & $\mathrm{S}=$ Decatur Industries & $\mathrm{T}=$ BIO-key & $\mathrm{U}=$ Aware & $\mathrm{V}=$ AA Technology
\end{tabular}




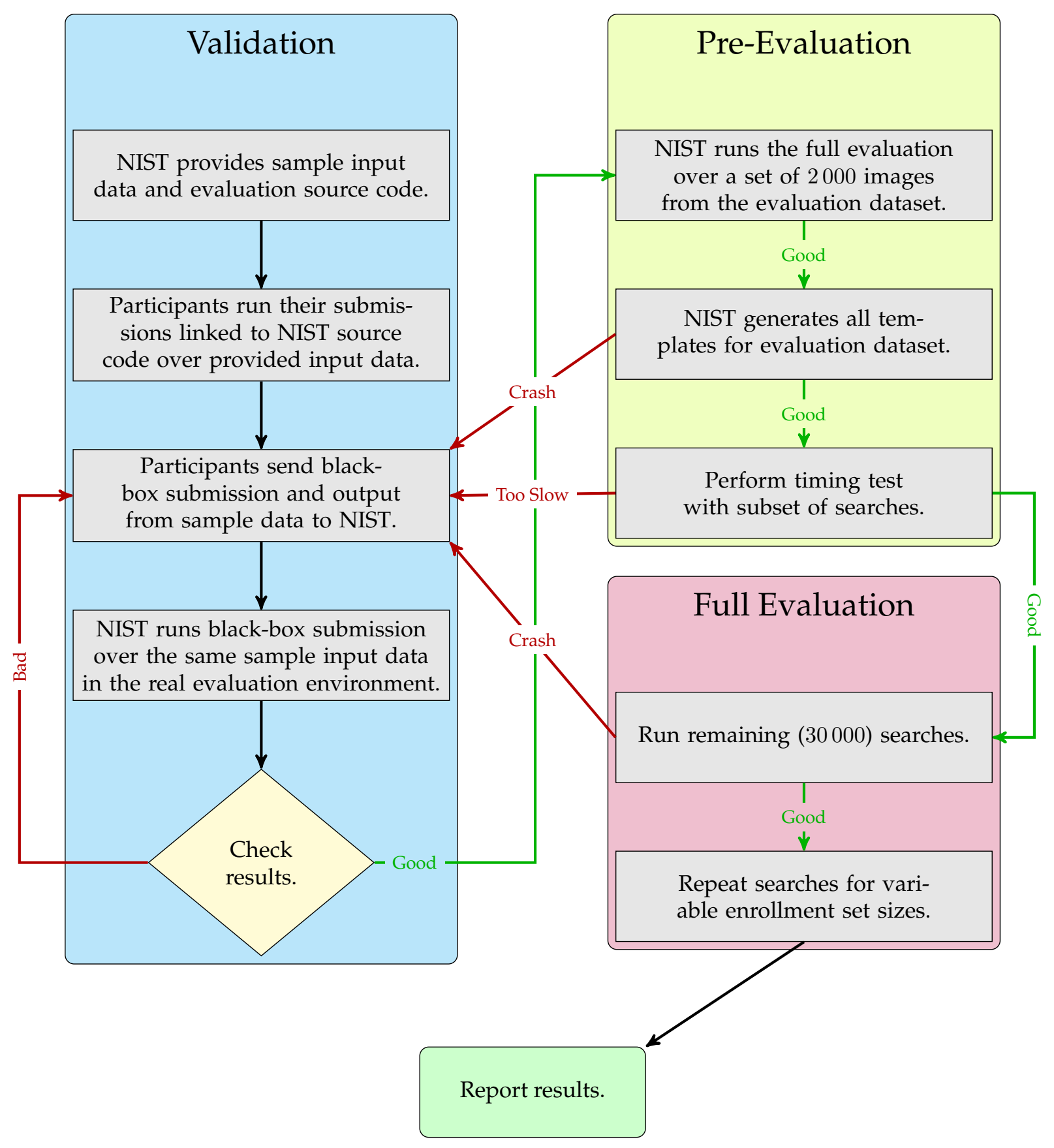

Figure 7: Evaluation workflow.

\begin{tabular}{l|l|l|l|ll|l}
$\mathrm{C}=$ afis team & $\mathrm{D}=3 \mathrm{M}$ Cogent & $\mathrm{E}=$ Neurotechnology & $\mathrm{F}=$ Papillon & $\mathrm{G}=$ Dermalog & $\mathrm{H}=$ Hisign Bio-Info Institute \\
$\mathrm{I}=$ NEC & $\mathrm{J}=$ Sonda & $\mathrm{K}=$ Tiger IT & $\mathrm{L}=$ Innovatrics & $\mathrm{M}=$ SPEX & $\mathrm{O}=$ ID Solutions \\
$\mathrm{P}=\mathrm{id} 3$ & $\mathrm{Q}=$ Morpho & $\mathrm{S}=$ Decatur Industries & $\mathrm{T}=$ BIO-key & $\mathrm{U}=$ Aware & $\mathrm{V}=$ AA Technology
\end{tabular}




\section{Two-Stage Matching}

The one-to-many identification step of FpVTE was divided into two distinct phases, under the expectation that storing the entire enrollment set in RAM on a single compute node would not have been possible. While this was true for many submissions, it did not hold true for all, especially with Class A data. Even if the submission's enrollment set fit in the RAM of a single compute node, the two-stage match technique was still used.

\subsection{Enrollment Set Partitioning}

NIST determined the amount of RAM needed to hold the entire enrollment set based on a size value returned per finger during enrollment. In some cases, unique forms of compression were employed, which allowed submissions to use significantly less RAM during identification than reported during enrollment. In other cases, submissions reported very small RAM requirements per finger, but underlying implementation details required significantly more RAM. This information was volunteered by participants and documented in Section 9.

After the amount of required RAM was determined, NIST identified the number of compute nodes necessary to support the RAM requirements. Compute nodes had 192 GB of RAM each (see Subsection 4.2 for more information). Submissions were then invoked with a method asking them to "finalize" the set of enrollment templates for $B$ compute nodes with a maximum of 192 GB of RAM per node. This gave submissions an opportunity to partition or subdivide the enrollment set into more manageable pieces on a per-compute node basis.

\subsection{Identification - Stage One}

Once the enrollment set had been partitioned, or "finalized," the next step was to perform searches on each of the partitions. First, the submission's identification initialization method was called before the first stage of matching. It was expected that submissions would iterate over their enrollment set partition and load it into the RAM of the compute node for faster access. Some submissions spent much longer than anticipated in this initialization method, and may have performed some additional binning or pruning that wasn't otherwise executed during the partitioning step. It's important to note that the NIST evaluation compute nodes did not have swap enabled, and so the only memory that could have been allocated was physical RAM.

After initialization, each search template was matched on each compute node specified during partitioning. To speed up this process, the FpVTE test driver forked into multiple processes. Under Linux, fork is implemented using copy-on-write pages, so as long as the submission's child processes did not write to the RAM allocated during initialization, multiple identification processes could run in parallel with access to the enrollment set in RAM without fear of RAM being over allocated. Submissions were allocated a 4 GB RAM disk file system per compute node, where free-form data could be written. A RAM disk was used to avoid timing I/O as part of the identification process. The FpVTE test driver later persisted the data to a permanent storage device, and provided this data to the submission during the second stage of identification.

If an submission failed to perform identification for any reason in the first stage of identification, it was marked as a miss in stage two, which increased false negative identification rate but slightly decreased false positive identification rate (Section 6).

\subsection{Identification - Stage Two}

After all compute nodes had finished the first stage of identification, the second stage was invoked. The submission's initialization method for the second stage was called, pointing the submission to the location of its partitioned enrollment sets (Subsection 5.1) and the RAM disk data from identification stage one. Then, each search template was submitted to stage two for final identification matching. This search method was to return a candidate list, not exceeding 100 candidates, with corresponding similarity scores in descending order (where the candidate at rank 1 was the most similar). Each call

\begin{tabular}{l|l|l|l|ll|l}
$\mathrm{C}=$ afis team & $\mathrm{D}=3 \mathrm{M}$ Cogent & $\mathrm{E}=$ Neurotechnology & $\mathrm{F}=$ Papillon & $\mathrm{G}=$ Dermalog & $\mathrm{H}=$ Hisign Bio-Info Institute \\
$\mathrm{I}=$ NEC & $\mathrm{J}=$ Sonda & $\mathrm{K}=$ Tiger IT & $\mathrm{L}=$ Innovatrics & $\mathrm{M}=$ SPEX & $\mathrm{O}=$ ID Solutions \\
$\mathrm{P}=\mathrm{id} 3$ & $\mathrm{Q}=$ Morpho & $\mathrm{S}=$ Decatur Industries & $\mathrm{T}=$ BIO-key & $\mathrm{U}=$ Aware & $\mathrm{V}=$ AA Technology
\end{tabular}


to perform a search included a path to a directory rooted on a RAM disk file system, in which the FpVTE test driver placed the submission's output from stage one (Subsection 5.2).

There was no intended control on how the second stage of identification reached the final candidate list, but the search time limit imposed on submissions combined both stage one and stage two search times (see Section 4 for detailed information on timing constraints and calculations). Stage two could have been as simple as a sort of stage one results, or as complex as an additional level of matching involving templates. Based on the timing figures observed during the evaluation, both trivial and complex stage two implementations were used by FpVTE participants.

Stage two took place on an individual compute node, though the pool of search templates may have been partitioned and searched on multiple compute nodes independently by the FpVTE test driver to increase throughput.

\begin{tabular}{l|l|l|l|ll|l}
$\mathrm{C}=$ afis team & $\mathrm{D}=3 \mathrm{M}$ Cogent & $\mathrm{E}=$ Neurotechnology & $\mathrm{F}=$ Papillon & $\mathrm{G}=$ Dermalog & $\mathrm{H}=$ Hisign Bio-Info Institute \\
$\mathrm{I}=$ NEC & $\mathrm{J}=$ Sonda & $\mathrm{K}=$ Tiger IT & $\mathrm{L}=$ Innovatrics & $\mathrm{M}=$ SPEX & $\mathrm{O}=$ ID Solutions \\
$\mathrm{P}=\mathrm{id} 3$ & $\mathrm{Q}=$ Morpho & $\mathrm{S}=$ Decatur Industries & $\mathrm{T}=$ BIO-key & $\mathrm{U}=$ Aware & $\mathrm{V}=$ AA Technology
\end{tabular}




\section{Metrics}

The detection error tradeoff (DET) characteristic curve [9] was the primary metric used for comparing accuracy in FpVTE. Specific points along the DET were examined for making comparisons between submissions. For this initial results report, the number of mate searches was limited to 10000 and the number of nonmate searches was limited to 20000 subjects, restraining the smallest error rate that could be used with statistical confidence to $10^{-3}$.

In addition to the accuracy of the submission, this report compares speed and computational resource usage. Comparisons between all submission are made and reported.

\subsection{Accuracy}

Open-set identification algorithms can make two types of recognition error:

$\triangleright$ Search of a biometric sample of an individual not enrolled in the biometric enrollment set (a nonmated search) returns the biometric reference identifier(s) attributable to one or more enrolled person. This is considered Type I, or false alarm, because it returns a false identity.

$\triangleright$ Search of a biometric sample of an enrolled individual (a mated search) returns an incorrect enrolled identity. This is considered Type II, or miss, because it misses the correct identity.

FpVTE quantified the accuracy of the open-set identification algorithms as follows:

$\triangleright$ False positive identification rate (FPIR), or Type I error rate, is the fraction of the nonmated searches where one or more enrolled identities are returned at or above threshold (T) [4]. FPIR is a function of: the size of the enrollment set $(N)$, length of candidate lists $(L)$, and score threshold $(T)$. In the general case, this can be summarized as

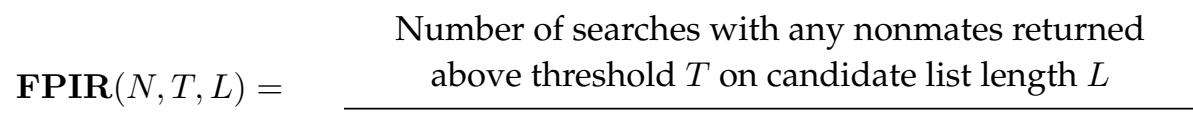

Number of nonmated searches conducted

and more precisely notated for this evaluation as

$$
\operatorname{FPIR}(T)=\frac{\sum_{q=1}^{Q} H\left(d_{q 1}-T\right)}{Q}
$$

where $Q$ is the number of searches performed for which there exists no mate in the enrollment set, $d_{q 1}$ is the highest similarity score reported by the algorithm for the $q$-th search. The function $H(x)$ is the Heaviside step function

$$
H(x)= \begin{cases}0, & \text { if } x<0 \\ 1, & \text { if } x \geq 0\end{cases}
$$

$\triangleright$ False negative identification rate (FNIR), or Type II error rate, is the fraction of the mated searches where the enrolled mate is outside the top $R$ rank or comparison score is below threshold $(T)$. FNIR is a function of: the size of the enrollment set $(N)$, length of candidate lists $(L)$, score threshold $(T)$, and the number of top candidates being considered $(R)$. This is summarized in the general case as

Number of mates outside top $R$ ranks or below

$$
\operatorname{FNIR}(N, R, T, L)=\quad \text { threshold } T \text { on candidate list length } L
$$

Number of mated searches conducted

\begin{tabular}{l|l|l|l|ll|l}
$\mathrm{C}=$ afis team & $\mathrm{D}=3 \mathrm{M}$ Cogent & $\mathrm{E}=$ Neurotechnology & $\mathrm{F}=$ Papillon & $\mathrm{G}=$ Dermalog & $\mathrm{H}=$ Hisign Bio-Info Institute \\
$\mathrm{I}=$ NEC & $\mathrm{J}=$ Sonda & $\mathrm{K}=$ Tiger IT & $\mathrm{L}=$ Innovatrics & $\mathrm{M}=$ SPEX & $\mathrm{O}=$ ID Solutions \\
$\mathrm{P}=\mathrm{id} 3$ & $\mathrm{Q}=$ Morpho & $\mathrm{S}=$ Decatur Industries & $\mathrm{T}=$ BIO-key & $\mathrm{U}=$ Aware & $\mathrm{V}=$ AA Technology
\end{tabular}


and is defined formally for this evaluation as

$$
\operatorname{FNIR}(T)=1-\frac{1}{P} \sum_{p=1}^{P} \sum_{r=1}^{L} I_{p r}\left[1-H\left(d_{p r}-T\right)\right]
$$

where $P$ is the number of searches performed for which there exists a mate in the enrollment set, $d_{p r}$ is the $r$-th lowest similarity score reported by the algorithm for the $p$-th search, and $I_{p r}$ is 1 if the identity of the $r$-th candidate is the same as the identity of search $p$, or 0 otherwise.

Note that FNIR computation does not care about the cause of a miss: failure to correctly identify a sample (e.g., due to poor quality), failure to extract a template, failure to generate a comparison score, and software crashes are all dealt with similarly.

The terms "hit rate," "reliability," and "sensitivity" that have been mentioned in some literature on automated fingerprint identification systems (AFIS) $[8,16]$ are just the complement of FNIR, computed as 1 - FNIR.

Another widely used accuracy metric is cumulative match characteristic (CMC), which is the fraction of the mated searches where the enrolled mate is at rank $R$ or better, regardless of its comparison score. CMC is a special case of FNIR, or more precisely, hit rate, when the constraint on threshold is removed, as shown in Equation 7.

$$
\operatorname{CMC}(N, L, R)=1-\operatorname{FNIR}(N, L, T=0, R)
$$

Rank-one hit rate, $\mathrm{CMC}(N, L, R=1)$, is the most common accuracy metric reported in academic and AFIS-related literature. While CMC is reported for the tested submissions, it is an inadequate accuracy metric because its makes strong or weak hits indistinguishable by ignoring similarity scores, and does not report Type I errors.

\subsection{DET Plots}

DET characteristic curves are the primary accuracy metric for offline testing of biometric recognition algorithms. Each point on a DET curve exhibits the false positive identification and false negative identification rates associated with a certain threshold value. The DET curve spans the entire range of possible threshold values, which is normally the range of the comparison scores. To reveal the dependence of FNIR and FPIR at a fixed threshold, the DET curves are connected at points where FNIRs and FPIRs are observed at the same threshold values.

As it is conventional, DET curves are presented for FpVTE submission. In a DET curve, Type I error rates are plotted on the $x$-axis and Type II error rates are plotted on the $y$-axis, giving uniform treatment to both types of error. Both axes use a logarithmic scale, which spreads out the plot and better distinguishes different well-performing systems. When calculating FPIR and FNIR, all ranks were considered $(L=R=100)$.

\subsection{Failure to Extract or Match a Template}

Failure to extract is the fraction of images for which a template is not generated. Template generation can fail for the enrollment sample or the search sample. In both cases, failure to extract a template is included as a miss in the computation of FNIR (see section 6.1).

Additionally, recognition algorithms fail to execute one-to-many searches to produce comparison scores. The result is that a valid candidate list is not produced. Such failures might be voluntary (e.g., refusal to process a poor quality image) or involuntary (e.g., software crashes). Either way, it is an undesirable behavior, and should be included in computation of recognition errors, particularly to allow for fair comparison of submissions. FpVTE treated such failure cases as a miss and added them to the Type II errors.

\begin{tabular}{l|l|l|l|l|l}
$\mathrm{C}=$ afis team & $\mathrm{D}=3 \mathrm{M}$ Cogent & $\mathrm{E}=$ Neurotechnology & $\mathrm{F}=$ Papillon & $\mathrm{G}=$ Dermalog & $\mathrm{H}=$ Hisign Bio-Info Institute \\
$\mathrm{I}=$ NEC & $\mathrm{J}=$ Sonda & $\mathrm{K}=$ Tiger IT & $\mathrm{L}=$ Innovatrics & $\mathrm{M}=$ SPEX & $\mathrm{O}=$ ID Solutions \\
$\mathrm{P}=\mathrm{id} 3$ & $\mathrm{Q}=$ Morpho & $\mathrm{S}=$ Decatur Industries & $\mathrm{T}=$ BIO-key & $\mathrm{U}=$ Aware & $\mathrm{V}=$ AA Technology
\end{tabular}




\begin{tabular}{|c|c|}
\hline Right Hand & Left Hand \\
\hline \hline 1 & 6 \\
\hline 2 & 7 \\
\hline 3 & 8 \\
\hline 4 & 9 \\
\hline 5 & 10 \\
\hline 11 & 12 \\
\hline 13 & 14 \\
\hline
\end{tabular}

Table 6: Table showing which finger positions were swapped when the images were flipped for nonmate searches.

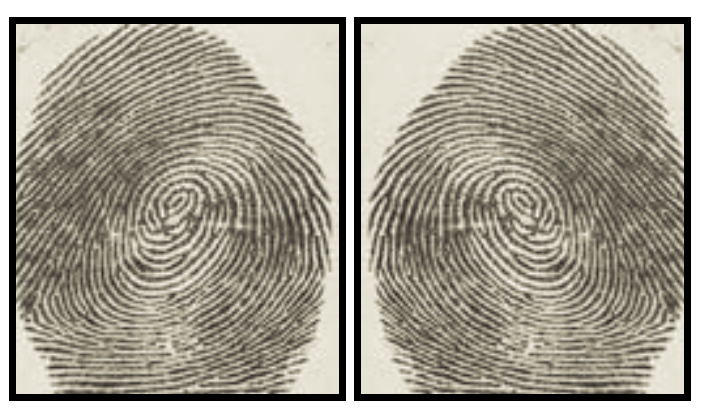

Figure 9: Example of flipped single-index finger image.

\subsection{Computational Efficiency}

Another aspect of performance is the computational resources required by a submission. This report includes a comparison of template generation times, one-to-many search times, and template sizes for the submissions, along with their accuracy at a set FPIR point of $10^{-3}$. The timing numbers are based on data samples for which all submissions were run under identical conditions on the same hardware.

\subsection{Ground Truth Errors}

There were two types of ground truth errors that had to be resolved for FpVTE datasets. The first involved nonmate searches that had an unknown mate in the enrollment set. These errors would wrongly increase FPIR (see Section 6). The second involved mated searches that did not match the presumed mate or had other unknown mates in the enrollment set. Either of these mated search errors could wrongly increase FNIR. An example of the effect on a DET curve is shown in Figure 8. Once the errors show up at a certain threshold, the error rate sharply increases and remains erroneously high.

The nonmate search errors were resolved by flipping fingerprint images on the vertical axis for nonmated searches. In order to have the correct topography of the finger, left/right hand labelings and finger positions were reversed after flipping. Table 6 shows how the finger positions were swapped to keep the correct finger topography. Example images are shown in Figure 9, Figure 10, and Figure 11.

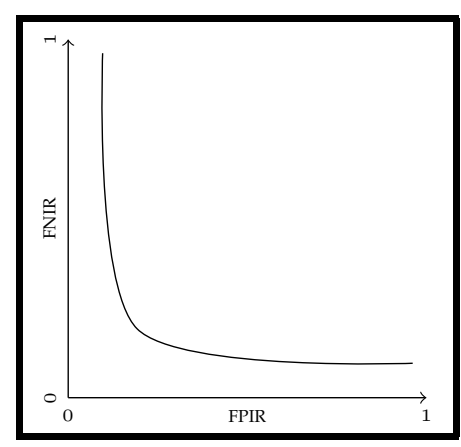

Figure 8: Example of a DET showing a spike in False Positive Identification Rate. This is usually a sign of ground truth errors in nonmate searches.

The mated search errors had to be resolved through manual inspection. A large cause of the unknown mates resulted from FpVTE using data from multiple sources. These ground truth errors had to be detected by examining the unexpected high-scoring alleged nonmates produced by the submissions. The results from the submissions were grouped together to determine which unexpected high-scoring alleged nonmates needed manual inspection. The first step was to look at searches where all the submissions had an unexpected high-scoring alleged nonmate above a certain threshold. Next, cases were examined for which only some of the submissions had a high-scoring alleged nonmate. After examining these cases, if the majority were true mates, the thresholds used were decreased and the process repeated until very few or no more true mates were found. Not all submissions were used for this process as some produced results that would have required too much manual work to inspect all of the potential errors produced. This process was repeated for low scoring alleged mates. The mate searches with low scores were examined to determine if the alleged mate was truly a mate or if there was a ground truth error. Very low quality images were not removed from the datasets.

\begin{tabular}{l|l|l|l|ll|l}
$\mathrm{C}=$ afis team & $\mathrm{D}=3 \mathrm{M}$ Cogent & $\mathrm{E}=$ Neurotechnology & $\mathrm{F}=$ Papillon & $\mathrm{G}=$ Dermalog & $\mathrm{H}=$ Hisign Bio-Info Institute \\
$\mathrm{I}=$ NEC & $\mathrm{J}=$ Sonda & $\mathrm{K}=$ Tiger IT & $\mathrm{L}=$ Innovatrics & $\mathrm{M}=$ SPEX & $\mathrm{O}=$ ID Solutions \\
$\mathrm{P}=\mathrm{id} 3$ & $\mathrm{Q}=$ Morpho & $\mathrm{S}=$ Decatur Industries & $\mathrm{T}=$ BIO-key & $\mathrm{U}=$ Aware & $\mathrm{V}=$ AA Technology
\end{tabular}




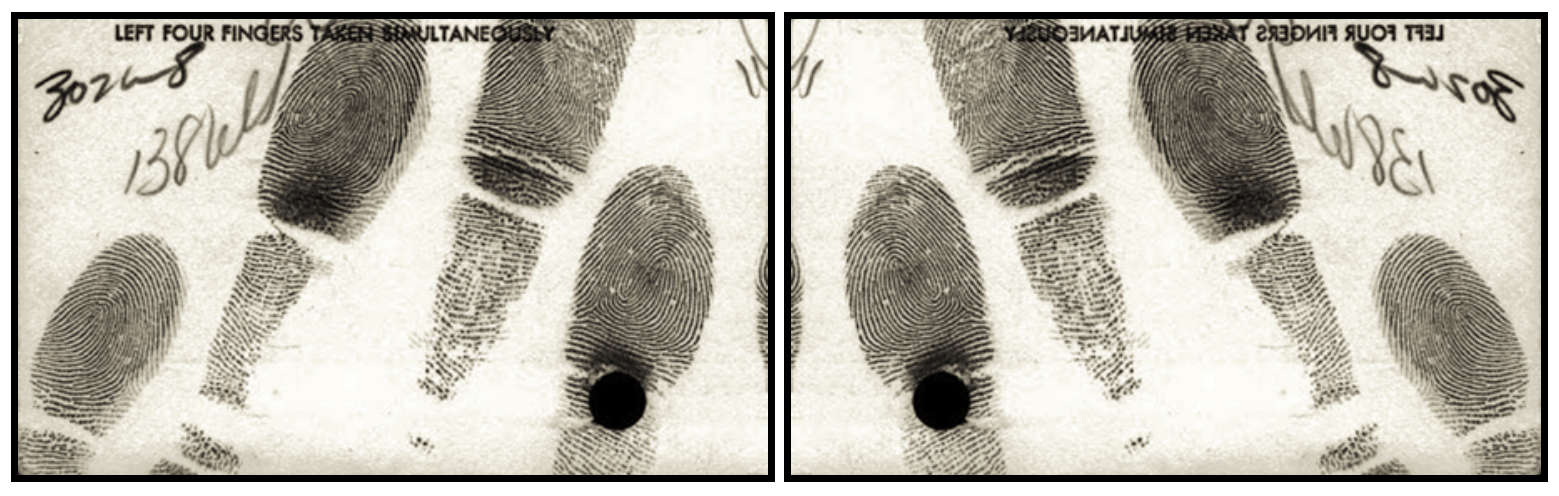

Figure 10: Example of flipped left slap image. Note that the flipped image was used as a right slap.
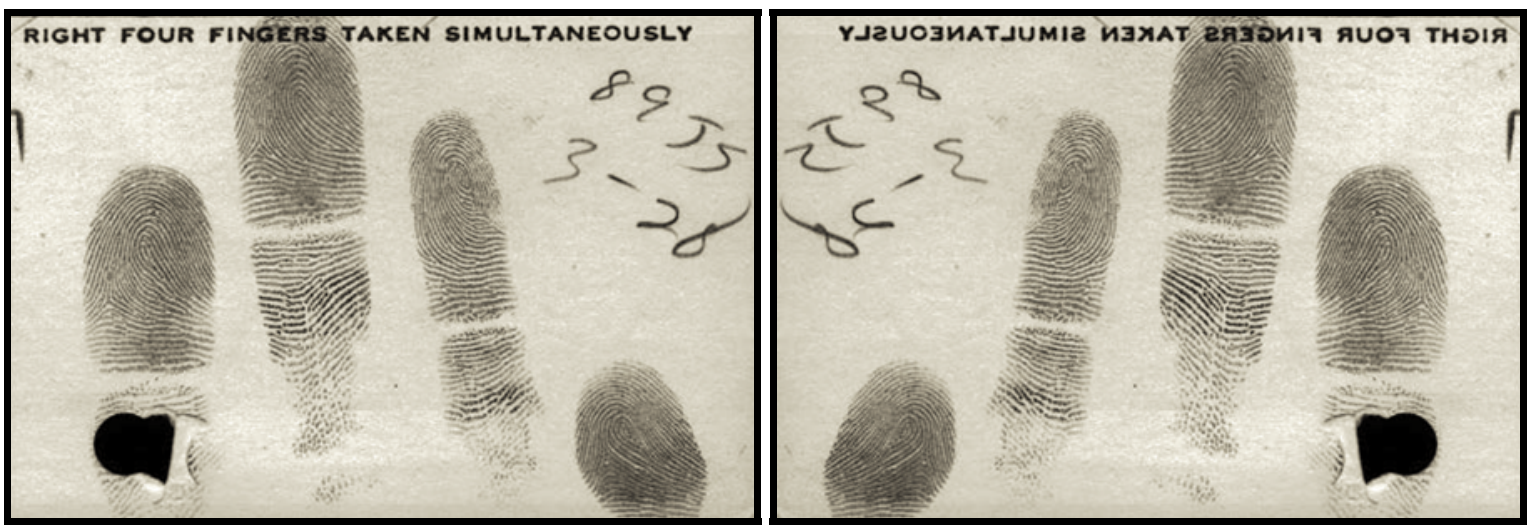

Figure 11: Example of flipped right slap image. Note that the flipped image was used as a left slap.

\begin{tabular}{l|l|l|l|ll|l}
$\mathrm{C}=$ afis team & $\mathrm{D}=3 \mathrm{M}$ Cogent & $\mathrm{E}=$ Neurotechnology & $\mathrm{F}=$ Papillon & $\mathrm{G}=$ Dermalog & $\mathrm{H}=$ Hisign Bio-Info Institute \\
$\mathrm{I}=$ NEC & $\mathrm{J}=$ Sonda & $\mathrm{K}=$ Tiger IT & $\mathrm{L}=$ Innovatrics & $\mathrm{M}=$ SPEX & $\mathrm{O}=$ ID Solutions \\
$\mathrm{P}=\mathrm{id} 3$ & $\mathrm{Q}=$ Morpho & $\mathrm{S}=$ Decatur Industries & $\mathrm{T}=$ BIO-key & $\mathrm{U}=$ Aware & $\mathrm{V}=$ AA Technology
\end{tabular}




\section{Accuracy Results}

Subsections 7.1 through 3 show the identification accuracy results, from round 3 submissions, for all three classes of participation. The sections include plots sorted by rank for FNIR at a fixed FPIR of $10^{-3}$, Detection Error Tradeoff (DET) curves showing accuracy over a range of threshold values, Cumulative Match Characteristic (CMC) curves showing accuracy over a range of candidate list ranks, and tables with FNIR values at a fixed FPIR. A complete set of full-size DET curves for each participant are included in Appendix A. Appendix B shows DET and CMC plots for all submissions and classes grouped on a single page.

This section (7) is followed by sections showing accuracy tradeoff results. Section 8 shows FNIR compared to search time statistics. Appendix C and Appendix D have a complete set of tables for search time statistics. Appendix E shows the progression of timing and results from the second to third round of submissions. Section 9 shows FNIR compared to computational resources such as RAM usage and enrollment (i.e., template creation) times. Appendices $\mathrm{F}$ to $\mathrm{H}$ have detailed tables on templates sizes and creation times.

These results are coalesced in Section 10, with tables combining ranked results for each category (FNIR, search time, RAM, enrollment time). Appendix I has the complete set of tables for ranked results in all classes of participation. Appendix $\mathrm{K}$ plots relative comparisons of FNIR, RAM usage, template creation times, and search times.

Section 11 combines results across classes of participation showing how accuracy varied based on the number of fingers available for searching. Appendix J plots relative comparisons, by class, for each search set used in FpVTE.

\begin{tabular}{l|l|l|l|ll|l}
$\mathrm{C}=$ afis team & $\mathrm{D}=3 \mathrm{M}$ Cogent & $\mathrm{E}=$ Neurotechnology & $\mathrm{F}=$ Papillon & $\mathrm{G}=$ Dermalog & $\mathrm{H}=$ Hisign Bio-Info Institute \\
$\mathrm{I}=$ NEC & $\mathrm{J}=$ Sonda & $\mathrm{K}=$ Tiger IT & $\mathrm{L}=$ Innovatrics & $\mathrm{M}=$ SPEX & $\mathrm{O}=$ ID Solutions \\
$\mathrm{P}=\mathrm{id} 3$ & $\mathrm{Q}=$ Morpho & $\mathrm{S}=$ Decatur Industries & $\mathrm{T}=$ BIO-key & $\mathrm{U}=$ Aware & $\mathrm{V}=$ AA Technology
\end{tabular}




\subsection{Class A}

\subsubsection{Single-Index Finger Identification}

The accuracy of single-finger identification is shown with rank-sorted FNIR points (based on right index finger results) in Figure 12, DET curves in Figure 13, CMC plots in Figure 14, and tables of FNIR points at a fixed FPIR in Tables 7 through 8.

Some observations for Class A single-finger identification include (all FNIR values are at FPIR $=10^{-3}$ ):

$\triangleright$ The most accurate submissions were $\mathbf{D}, \mathbf{Q}, \mathbf{I}, \mathbf{V}$ and $\mathbf{L} 2$.

$\triangleright$ The right index finger was more accurate than the left index finger.

$\triangleright$ The most accurate submission D achieved a FNIR of $1.97 \%$ for the left index finger and $1.9 \%$ for the right index finger searched against an enrollment set of 100000 subjects.

$\triangleright$ There is a measurable accuracy gap between the top performers and the next level of performers.

$\triangleright$ The CMC plots in Figure 14 are not as flat as the other classes. This indicates that while most mates appear within the top three candidates on the list, there are some that appear further down the list when using single-finger identification.

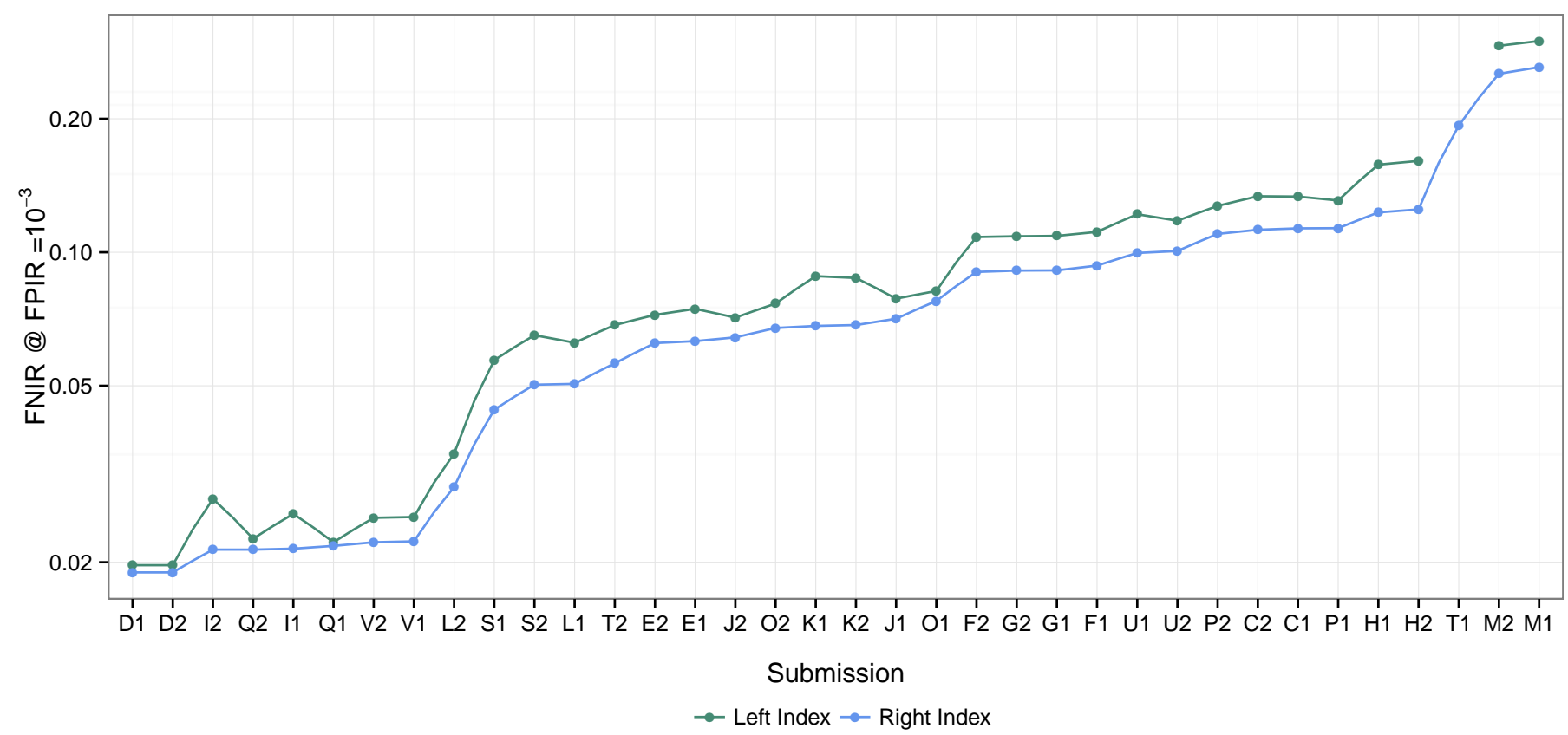

Figure 12: Rank-sorted FNIR @ FPIR $=10^{-3}$ for Class A — Single Index Finger searching 30000 subjects against 100000 subjects. Submissions " 1 " and "2" from round 3.

\begin{tabular}{l|l|l|l|ll|l}
$\mathrm{C}=$ afis team & $\mathrm{D}=3 \mathrm{M}$ Cogent & $\mathrm{E}=$ Neurotechnology & $\mathrm{F}=$ Papillon & $\mathrm{G}=$ Dermalog & $\mathrm{H}=$ Hisign Bio-Info Institute \\
$\mathrm{I}=$ NEC & $\mathrm{J}=$ Sonda & $\mathrm{K}=$ Tiger IT & $\mathrm{L}=$ Innovatrics & $\mathrm{M}=$ SPEX & $\mathrm{O}=$ ID Solutions \\
$\mathrm{P}=\mathrm{id} 3$ & $\mathrm{Q}=$ Morpho & $\mathrm{S}=$ Decatur Industries & $\mathrm{T}=$ BIO-key & $\mathrm{U}=$ Aware & $\mathrm{V}=$ AA Technology
\end{tabular}




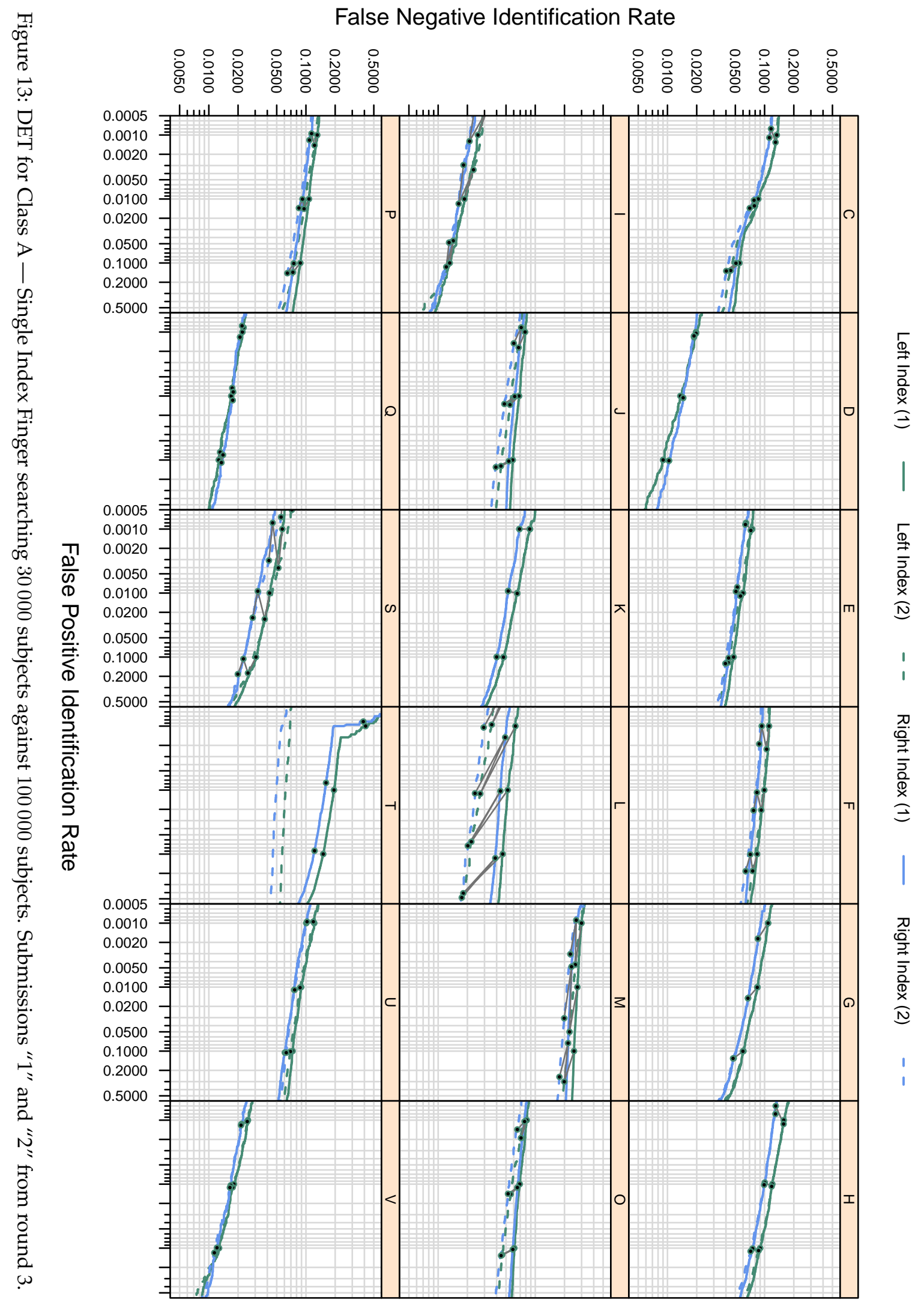

\footnotetext{
$\mathrm{C}=$ afis team $\mathrm{D}=3 \mathrm{M}$ Cogent $\mid \mathrm{E}=$ Neurotechnology $\mid \mathrm{F}=$ Papillon $\quad \mathrm{G}=$ Dermalog $\mathrm{H}=$ Hisign Bio-Info Institute

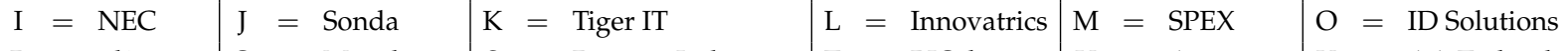

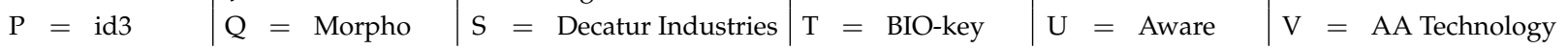




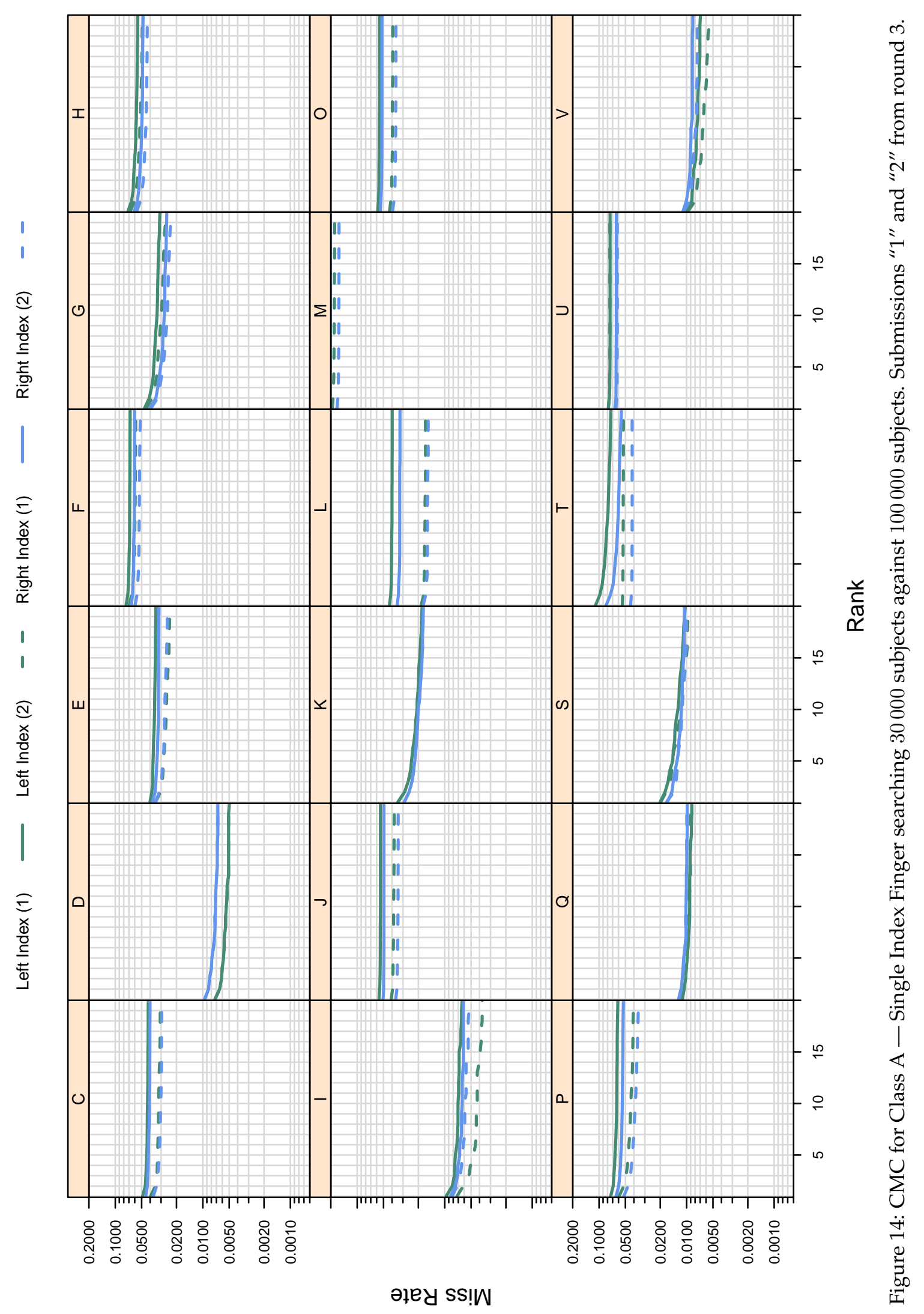

\footnotetext{
$\mathrm{C}=$ afis team $\mathrm{D}=3 \mathrm{M}$ Cogent $\mid \mathrm{E}=$ Neurotechnology $\mid \mathrm{F}=$ Papillon $\quad \mathrm{G}=$ Dermalog $\mathrm{H}=$ Hisign Bio-Info Institute

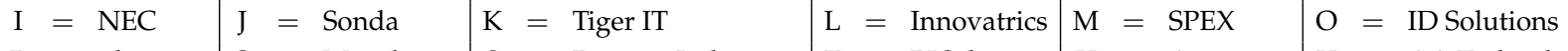

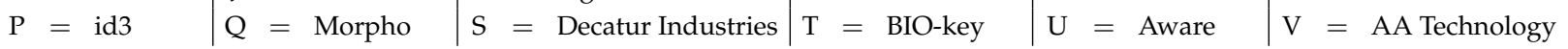




\begin{tabular}{|c|c|c|c|}
\hline \multicolumn{2}{|c|}{ Participant } & \multirow{2}{*}{\multicolumn{2}{|c|}{ FNIR $@$ FPIR $=10^{-3}$}} \\
\hline Letter & Sub.\# & & \\
\hline \multirow{2}{*}{$\mathrm{C}$} & 1 & 30 & 0.1335 \\
\hline & 2 & 31 & 0.1337 \\
\hline \multirow{2}{*}{ D } & 1 & 1 & 0.0197 \\
\hline & 2 & 1 & 0.0197 \\
\hline \multirow{2}{*}{ E } & 1 & 16 & 0.0745 \\
\hline & 2 & 15 & 0.0723 \\
\hline \multirow{2}{*}{$\mathrm{F}$} & 1 & 25 & 0.1111 \\
\hline & 2 & 22 & 0.1082 \\
\hline \multirow{2}{*}{ G } & 1 & 24 & 0.1089 \\
\hline & 2 & 23 & 0.1086 \\
\hline \multirow{2}{*}{$\mathrm{H}$} & 1 & 32 & 0.1576 \\
\hline & 2 & 33 & 0.1607 \\
\hline \multirow{2}{*}{ I } & 1 & 7 & 0.0257 \\
\hline & 2 & 8 & 0.0278 \\
\hline \multirow{2}{*}{$\mathrm{J}$} & 1 & 18 & 0.0786 \\
\hline & 2 & 14 & 0.0712 \\
\hline \multirow{2}{*}{ K } & 1 & 21 & 0.0883 \\
\hline & 2 & 20 & 0.0875 \\
\hline \multirow{2}{*}{$\mathrm{L}$} & 1 & 11 & 0.0625 \\
\hline & 2 & 9 & 0.0351 \\
\hline \multirow{2}{*}{ M } & 1 & 35 & 0.2995 \\
\hline & 2 & 34 & 0.2921 \\
\hline \multirow{2}{*}{$\mathrm{O}$} & 1 & 19 & 0.0818 \\
\hline & 2 & 17 & 0.0766 \\
\hline \multirow{2}{*}{$\mathrm{P}$} & 1 & 29 & 0.1308 \\
\hline & 2 & 28 & 0.1272 \\
\hline \multirow{2}{*}{ Q } & 1 & 3 & 0.0222 \\
\hline & 2 & 4 & 0.0226 \\
\hline \multirow{2}{*}{ S } & 1 & 10 & 0.0571 \\
\hline & 2 & 12 & 0.0650 \\
\hline \multirow{2}{*}{$\mathrm{T}$} & 1 & 36 & NA \\
\hline & 2 & 13 & 0.0685 \\
\hline \multirow{2}{*}{ U } & 1 & 27 & 0.1218 \\
\hline & 2 & 26 & 0.1178 \\
\hline \multirow{2}{*}{ V } & 1 & 6 & 0.0253 \\
\hline & 2 & 5 & 0.0252 \\
\hline
\end{tabular}

Table 7: Tabulation of results for Class A - Left Index, with an enrollment set size of 100000 . Letter refers to the participant's letter code found on the footer of this page. Sub. \# is an identifier used to differentiate between the two submissions each participant could make. FNIR was computed at the score threshold that gave FPIR $=10^{-3}$. NA indicates that the operations required to produce the value could not be performed. The number to the left of a value provides the value's column-wise ranking, with the best performance shaded in green and the worst in pink.

\begin{tabular}{|c|c|c|c|}
\hline \multicolumn{2}{|c|}{ Participant } & \multirow{2}{*}{\multicolumn{2}{|c|}{ FNIR $@$ FPIR $=10^{-3}$}} \\
\hline Letter & Sub.\# & & \\
\hline \multirow{2}{*}{ C } & 1 & 30 & 0.1132 \\
\hline & 2 & 29 & 0.1124 \\
\hline \multirow{2}{*}{$\mathrm{D}$} & 1 & 1 & 0.0190 \\
\hline & 2 & 1 & 0.0190 \\
\hline \multirow{2}{*}{ E } & 1 & 15 & 0.0630 \\
\hline & 2 & 14 & 0.0624 \\
\hline \multirow{2}{*}{$\mathrm{F}$} & 1 & 25 & 0.0933 \\
\hline & 2 & 22 & 0.0903 \\
\hline \multirow{2}{*}{ G } & 1 & 24 & 0.0910 \\
\hline & 2 & 23 & 0.0909 \\
\hline \multirow{2}{*}{$\mathrm{H}$} & 1 & 32 & 0.1230 \\
\hline & 2 & 33 & 0.1249 \\
\hline \multirow{2}{*}{ I } & 1 & 5 & 0.0215 \\
\hline & 2 & 3 & 0.0214 \\
\hline \multirow{2}{*}{$\mathrm{J}$} & 1 & 20 & 0.0708 \\
\hline & 2 & 16 & 0.0643 \\
\hline \multirow{2}{*}{ K } & 1 & 18 & 0.0682 \\
\hline & 2 & 19 & 0.0685 \\
\hline \multirow{2}{*}{$\mathrm{L}$} & 1 & 12 & 0.0505 \\
\hline & 2 & 9 & 0.0295 \\
\hline \multirow{2}{*}{ M } & 1 & 36 & 0.2615 \\
\hline & 2 & 35 & 0.2526 \\
\hline \multirow{2}{*}{$\mathrm{O}$} & 1 & 21 & 0.0776 \\
\hline & 2 & 17 & 0.0675 \\
\hline \multirow{2}{*}{ P } & 1 & 31 & 0.1133 \\
\hline & 2 & 28 & 0.1100 \\
\hline \multirow{2}{*}{$\mathrm{Q}$} & 1 & 6 & 0.0218 \\
\hline & 2 & 3 & 0.0214 \\
\hline \multirow{2}{*}{$\mathrm{S}$} & 1 & 10 & 0.0442 \\
\hline & 2 & 11 & 0.0503 \\
\hline \multirow{2}{*}{$\mathrm{T}$} & 1 & 34 & 0.1929 \\
\hline & 2 & 13 & 0.0562 \\
\hline \multirow{2}{*}{ U } & 1 & 26 & 0.0996 \\
\hline & 2 & 27 & 0.1007 \\
\hline \multirow{2}{*}{ V } & 1 & 8 & 0.0223 \\
\hline & 2 & 7 & 0.0222 \\
\hline
\end{tabular}

Table 8: Tabulation of results for Class A - Right Index, with an enrollment set size of 100000 . Letter refers to the participant's letter code found on the footer of this page. Sub. \# is an identifier used to differentiate between the two submissions each participant could make. FNIR was computed at the score threshold that gave FPIR $=$ $10^{-3}$. The number to the left of a value provides the value's columnwise ranking, with the best performance shaded in green and the worst in pink.

\footnotetext{
$\mathrm{C}=$ afis team $\mathrm{D}=3 \mathrm{M}$ Cogent $\mathrm{E}=$ Neurotechnology $\mid \mathrm{F}=$ Papillon $\quad \mathrm{G}=$ Dermalog $\mathrm{H}=$ Hisign Bio-Info Institute

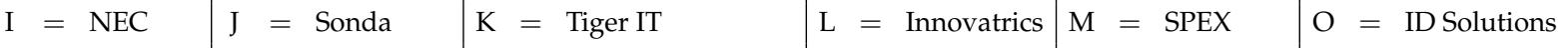

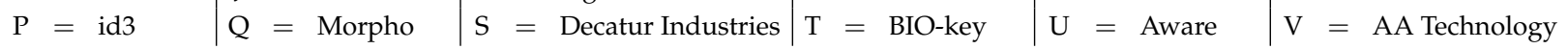




\subsubsection{Two-Index Finger Identification}

The accuracy of two-finger identification is shown with rank-sorted FNIR points in Figure 15, DET curves in Figure 16, CMC plots in Figure 17, and a table of FNIR points at a fixed FPIR in Table 9.

Some observations for Class A two-finger identification include (all FNIR values are at FPIR $=10^{-3}$ ):

$\triangleright$ The most accurate submissions were $\mathbf{Q}, \mathbf{V}, \mathbf{D}$, and $\mathbf{I}$.

$\triangleright$ The most accurate submission $\mathbf{Q}$ achieved a FNIR of $0.27 \%$ searched against an enrollment set of 1.6 million subjects.

$\triangleright$ The accuracy gap between the top performers and the second tier, while still measurable, was not as large as singlefinger identification.

$\triangleright$ Two-finger identification was far superior to single-finger identification and scaled to much larger enrollment sets.

$\triangleright$ The CMC plots in Figure 17 flatten out faster than in single-finger identification. In most cases, the mate is within the top three candidates of the list or doesn't appear at all. There were a few submissions that extend down to the top five to ten candidates.

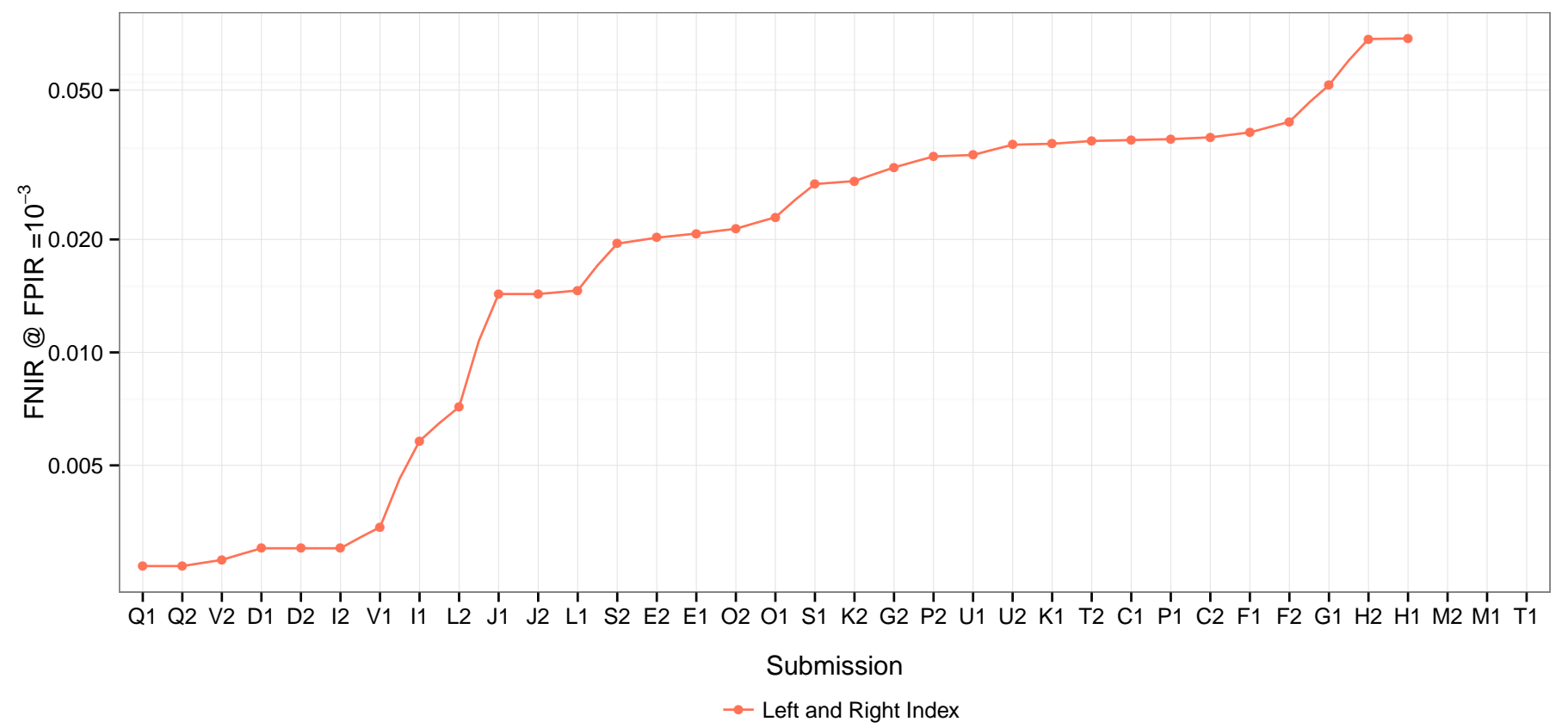

Figure 15: Rank-sorted FNIR @ FPIR =10 ${ }^{-3}$ for Class A — Two Index Fingers searching 30000 subjects against 1600000 subjects. Submissions " 1 " and "2" from round 3.

\begin{tabular}{l|l|l|l|l|l|l}
$\mathrm{C}=$ afis team & $\mathrm{D}=$ = 3M Cogent & $\mathrm{E}=$ Neurotechnology & $\mathrm{F}=$ Papillon & $\mathrm{G}=$ Dermalog & $\mathrm{H}=$ Hisign Bio-Info Institute \\
$\mathrm{I}=$ NEC & $\mathrm{J}=$ Sonda & $\mathrm{K}=$ Tiger IT & $\mathrm{L}=$ Innovatrics & $\mathrm{M}=$ SPEX & $\mathrm{O}=$ ID Solutions \\
$\mathrm{P}=\mathrm{id} 3$ & $\mathrm{Q}=$ Morpho & $\mathrm{S}=$ Decatur Industries & $\mathrm{T}=$ BIO-key & $\mathrm{U}=$ Aware & $\mathrm{V}=$ AA Technology
\end{tabular}




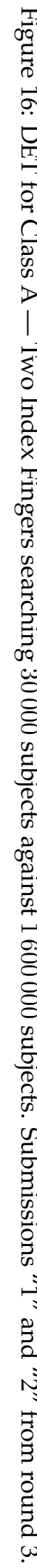

False Negative Identification Rate
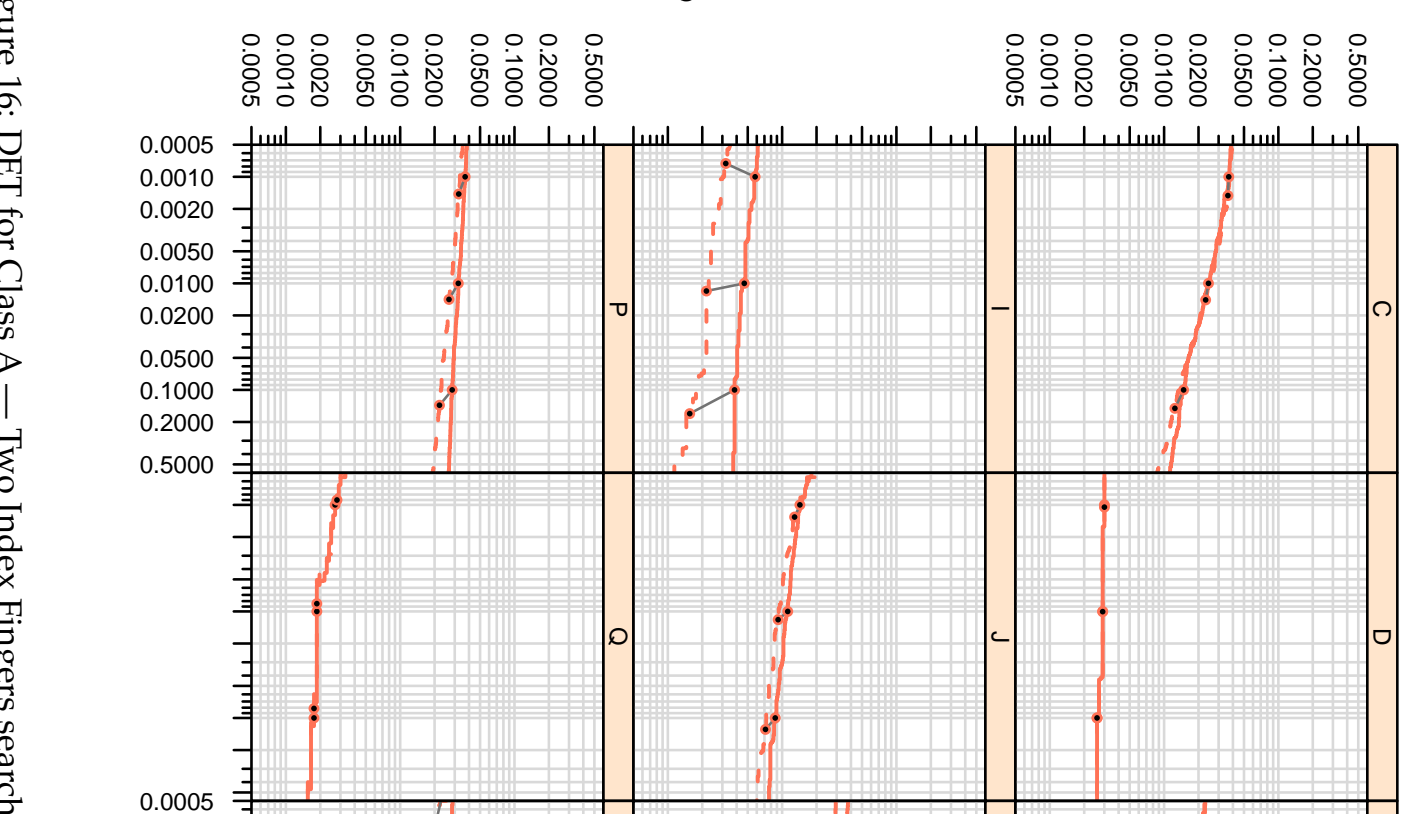

要

8
0
0

क 0.0200

. 0.0500

का 0.2000

$\begin{array}{lll}00 & \overline{2} & 0.5000\end{array}$

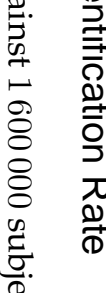

0.0005
0.0010

in 0.0020

0.0050

0.0100

के

$0.0500=$

0.1000

0.5000

ș

웅

3

己

$\omega$

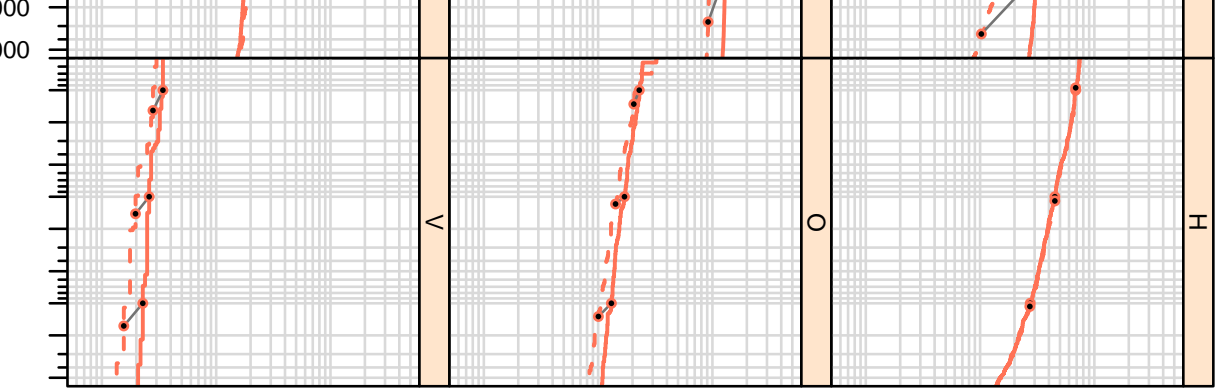

$\mathrm{C}=$ afis team $\mathrm{D}=3 \mathrm{M}$ Cogent $\mid \mathrm{E}=$ Neurotechnology

$\mathrm{F}=$ Papillon

$\mathrm{G}=$ Dermalog $\mathrm{H}=$ Hisign Bio-Info Institute

$\mathrm{I}=\mathrm{NEC}$

$\mathrm{J}=$ Sonda

$\mathrm{K}=$ Tiger IT

$\mathrm{L}=$ Innovatrics $\mathrm{M}=$ SPEX

$\mathrm{O}=$ ID Solutions

$\mathrm{P}=\mathrm{id} 3$

$\mathrm{Q}=$ Morpho

$\mathrm{S}=$ Decatur Industries $\mathrm{T}=$ BIO-key $\quad \mathrm{U}=$ Aware

$\mathrm{V}=\mathrm{AA}$ Technology 


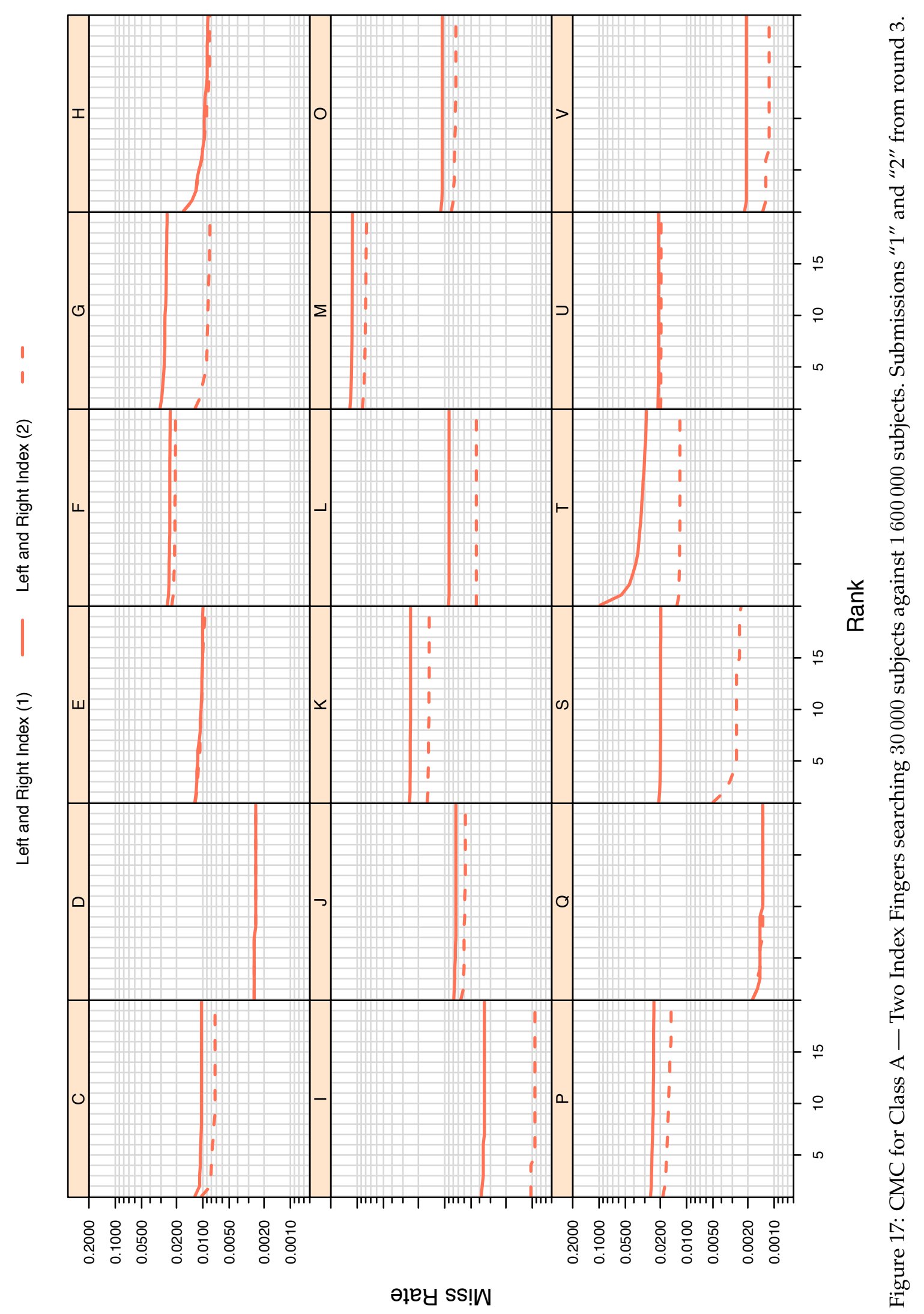

\footnotetext{
$\mathrm{C}=$ afis team $\mathrm{D}=3 \mathrm{M}$ Cogent $\mid \mathrm{E}=$ Neurotechnology $\mid \mathrm{F}=$ Papillon $\quad \mathrm{G}=$ Dermalog $\mathrm{H}=$ Hisign Bio-Info Institute

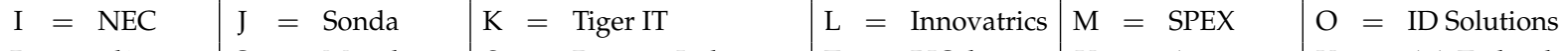

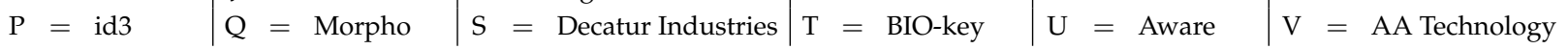




\begin{tabular}{|c|c|c|c|}
\hline \multicolumn{2}{|c|}{ Participant } & \multirow{2}{*}{\multicolumn{2}{|c|}{ FNIR @ FPIR $=10^{-3}$}} \\
\hline Letter & Sub. \# & & \\
\hline \multirow{2}{*}{$\mathrm{C}$} & 1 & 26 & 0.0368 \\
\hline & 2 & 28 & 0.0374 \\
\hline \multirow{2}{*}{$\mathrm{D}$} & 1 & 4 & 0.0030 \\
\hline & 2 & 4 & 0.0030 \\
\hline \multirow{2}{*}{$\mathrm{E}$} & 1 & 15 & 0.0207 \\
\hline & 2 & 14 & 0.0202 \\
\hline \multirow{2}{*}{$\mathrm{F}$} & 1 & 29 & 0.0386 \\
\hline & 2 & 30 & 0.0412 \\
\hline \multirow{2}{*}{ G } & 1 & 31 & 0.0515 \\
\hline & 2 & 20 & 0.0311 \\
\hline \multirow{2}{*}{$\mathrm{H}$} & 1 & 33 & 0.0686 \\
\hline & 2 & 32 & 0.0684 \\
\hline \multirow{2}{*}{ I } & 1 & 8 & 0.0058 \\
\hline & 2 & 4 & 0.0030 \\
\hline \multirow{2}{*}{$\mathrm{J}$} & 1 & 10 & 0.0143 \\
\hline & 2 & 10 & 0.0143 \\
\hline \multirow{2}{*}{$\mathrm{K}$} & 1 & 24 & 0.0360 \\
\hline & 2 & 19 & 0.0286 \\
\hline \multirow{2}{*}{$\mathrm{L}$} & 1 & 12 & 0.0146 \\
\hline & 2 & 9 & 0.0072 \\
\hline \multirow{2}{*}{$\mathrm{M}$} & 1 & 35 & NA \\
\hline & 2 & 34 & NA \\
\hline \multirow{2}{*}{$\mathrm{O}$} & 1 & 17 & 0.0229 \\
\hline & 2 & 16 & 0.0214 \\
\hline \multirow{2}{*}{$\mathrm{P}$} & 1 & 27 & 0.0370 \\
\hline & 2 & 21 & 0.0333 \\
\hline \multirow{2}{*}{ Q } & 1 & 1 & 0.0027 \\
\hline & 2 & 1 & 0.0027 \\
\hline \multirow{2}{*}{$S$} & 1 & 18 & 0.0281 \\
\hline & 2 & 13 & 0.0195 \\
\hline \multirow{2}{*}{$\mathrm{T}$} & 1 & 36 & NA \\
\hline & 2 & 25 & 0.0366 \\
\hline \multirow{2}{*}{$\mathrm{U}$} & 1 & 22 & 0.0336 \\
\hline & 2 & 23 & 0.0358 \\
\hline \multirow{2}{*}{ V } & 1 & 7 & 0.0034 \\
\hline & 2 & 3 & 0.0028 \\
\hline
\end{tabular}

Table 9: Tabulation of results for Class A — Left and Right Index, with an enrollment set size of 1600000 . Letter refers to the participant's letter code found on the footer of this page. Sub. \# is an identifier used to differentiate between the two submissions each participant could make. FNIR was computed at the score threshold that gave FPIR $=10^{-3}$. NA indicates that the operations required to produce the value could not be performed. The number to the left of a value provides the value's column-wise ranking, with the best performance shaded in green and the worst in pink.

\begin{tabular}{l|l|l|l|ll|l}
$\mathrm{C}=$ afis team & $\mathrm{D}=3 \mathrm{M}$ Cogent & $\mathrm{E}=$ Neurotechnology & $\mathrm{F}=$ Papillon & $\mathrm{G}=$ Dermalog & $\mathrm{H}=$ Hisign Bio-Info Institute \\
$\mathrm{I}=$ NEC & $\mathrm{J}=$ Sonda & $\mathrm{K}=$ Tiger IT & $\mathrm{L}=$ Innovatrics & $\mathrm{M}=$ SPEX & $\mathrm{O}=$ ID Solutions \\
$\mathrm{P}=\mathrm{id} 3$ & $\mathrm{Q}=$ Morpho & $\mathrm{S}=$ Decatur Industries & $\mathrm{T}=$ BIO-key & $\mathrm{U}=$ Aware & $\mathrm{V}=$ AA Technology
\end{tabular}




\subsection{Class B}

The accuracy results for Class B, which included four-finger, eight-finger, and ten-finger identification, are shown with rank-sorted FNIR points (based on ten finger results) in Figure 18, DET curves in Figure 19, CMC plots in Figure 20, and tables of FNIR points at fixed FPIR in Tables 10 through 13.

Some observations for Class B include (all FNIR values are at FPIR $=10^{-3}$ ):

$\triangleright$ The most accurate submissions with all ten fingers were $\mathbf{I}, \mathbf{Q}$, and $\mathbf{D}$, with FNIRs ranging from $0.09 \%$ to $0.2 \%$.

$\triangleright$ The next level of submissions were $\mathbf{V}, \mathbf{E}, \mathbf{L}, \mathbf{J}, \mathbf{O}$, and $\mathbf{G}$, with FNIRs ranging from $0.24 \%$ to $0.4 \%$. The separation between the top performers and next level of performers was noticeably lower as more fingers are used.

$\triangleright$ Right slaps (FNIR $=0.45 \%$, I2) were more accurate than left slaps (FNIR $=0.94 \%$, I2).

$\triangleright$ Four-finger identification (FNIR $=0.45 \%$ ) performed worse than two-finger identification (FNIR $=0.27 \%$ ), as reported in Subsubsection 7.1.2. Two potential causes for this result are that the submissions in Class B had to perform segmentation as part of the feature extraction process, and possibly a variation in image quality between the datasets. Further study will be needed to determine the primary reason four-finger slaps performed worse than two index fingers.

$\triangleright$ The matching accuracy improved significantly going from four fingers to eight fingers.

$\triangleright$ The best submission I2 achieved FNIRs as follows: left slap (0.94\%), right slap (0.45\%), left and right slaps $(0.15 \%)$, and identification slaps (0.09\%).

$\triangleright$ The CMC plots in Figure 20 generally show very flat responses. This indicates that for most submissions, the mate is within the top three candidates on the list or the mate doesn't appear on the list at all.

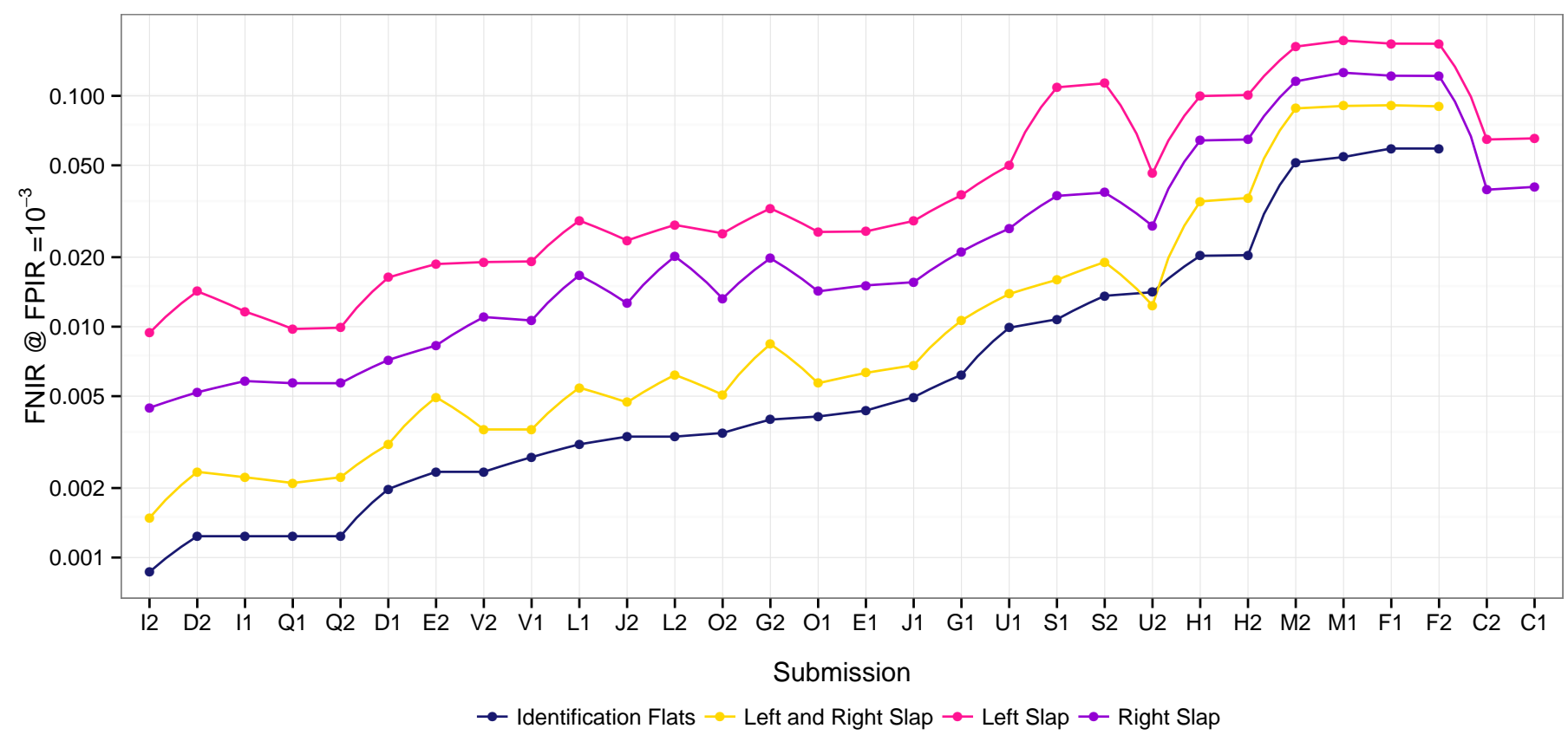

Figure 18: Rank-sorted FNIR @ FPIR $=10^{-3}$ for Class B — Left Slap, Right Slap, Left and Right Slap, and IDFlats searching 30000 subjects against 3000000 subjects. Submissions " 1 " and "2" from round 3.

\begin{tabular}{l|l|l|l|ll|l}
$\mathrm{C}=$ afis team & $\mathrm{D}=3 \mathrm{M}$ Cogent & $\mathrm{E}=$ Neurotechnology & $\mathrm{F}=$ Papillon & $\mathrm{G}=$ Dermalog & $\mathrm{H}=$ Hisign Bio-Info Institute \\
$\mathrm{I}=$ NEC & $\mathrm{J}=$ Sonda & $\mathrm{K}=$ Tiger IT & $\mathrm{L}=$ Innovatrics & $\mathrm{M}=$ SPEX & $\mathrm{O}=$ ID Solutions \\
$\mathrm{P}=\mathrm{id} 3$ & $\mathrm{Q}=$ Morpho & $\mathrm{S}=$ Decatur Industries & $\mathrm{T}=$ BIO-key & $\mathrm{U}=$ Aware & $\mathrm{V}=$ AA Technology
\end{tabular}




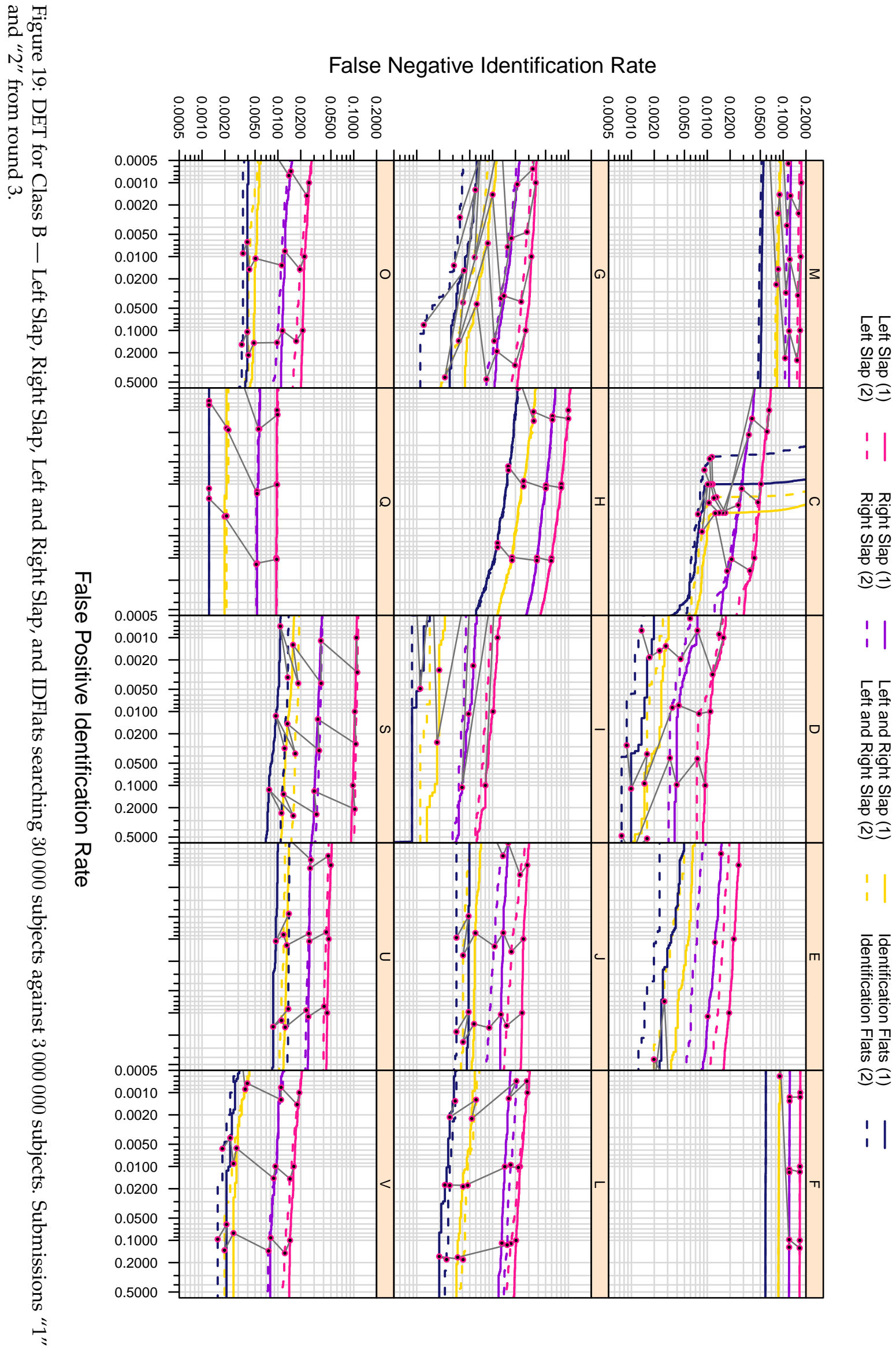

\footnotetext{
$\mathrm{C}=$ afis team $\mathrm{D}=3 \mathrm{M}$ Cogent $\mid \mathrm{E}=$ Neurotechnology $\mid \mathrm{F}=$ Papillon $\mid \mathrm{G}=$ Dermalog $\mathrm{H}=$ Hisign Bio-Info Institute

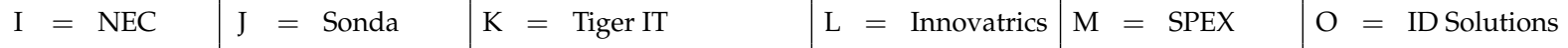

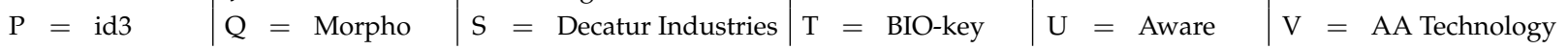




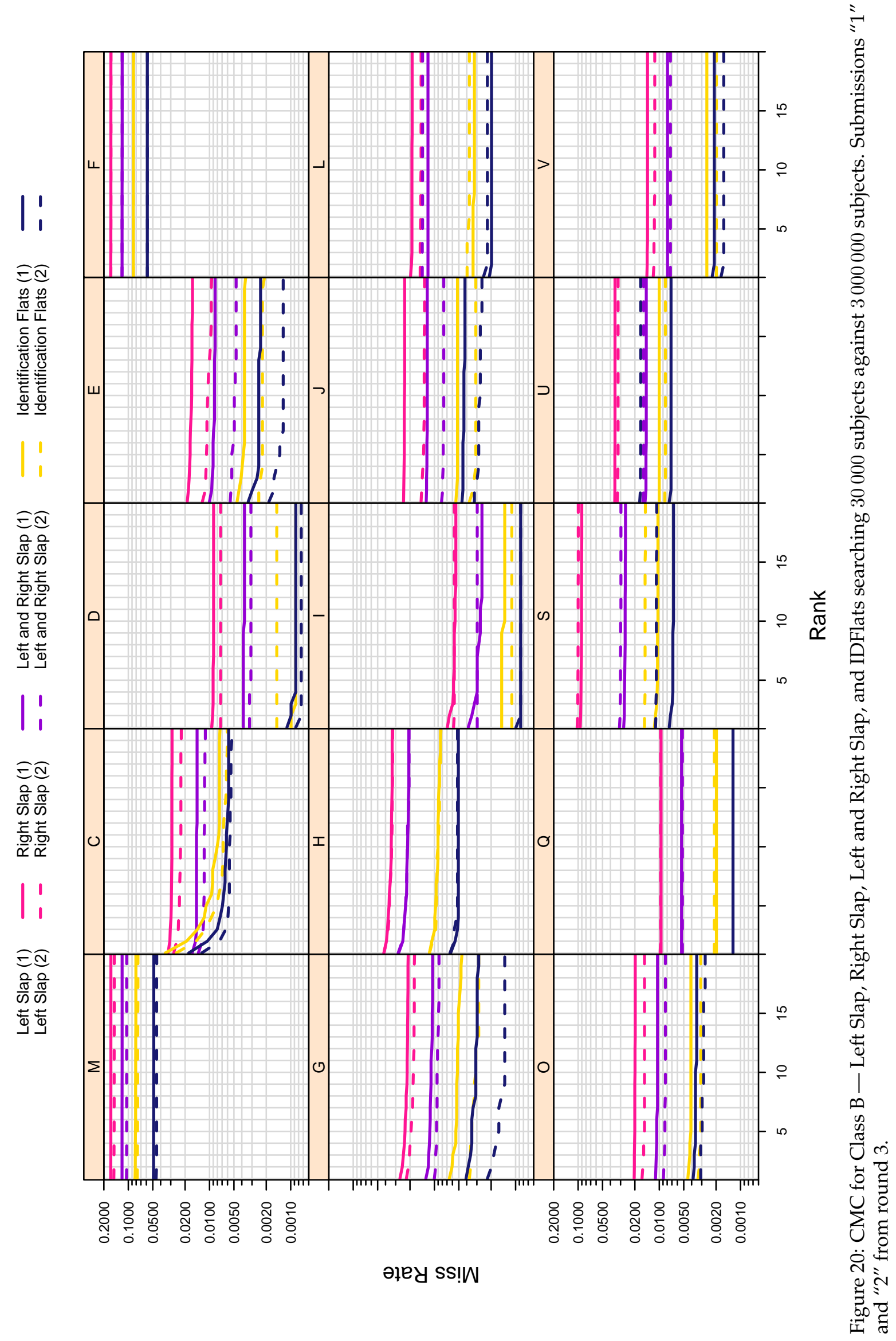

\footnotetext{
$\mathrm{C}=$ afis team $\mathrm{D}=3 \mathrm{M}$ Cogent $\mid \mathrm{E}=$ Neurotechnology $\mid \mathrm{F}=$ Papillon $\quad \mathrm{G}=$ Dermalog $\mathrm{H}=$ Hisign Bio-Info Institute

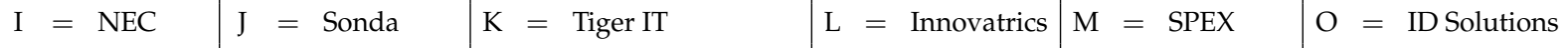

$\mathrm{P}=\mathrm{id} 3 \quad \mathrm{Q}=$ Morpho $\quad \mathrm{S}=$ Decatur Industries $\mid \mathrm{T}=$ BIO-key $\quad \mathrm{U}=$ Aware $\quad \mathrm{V}=$ AA Technology
} 


\begin{tabular}{|c|c|c|c|}
\hline \multicolumn{2}{|c|}{ Participant } & \multirow{2}{*}{\multicolumn{2}{|c|}{ FNIR @ FPIR $=10^{-3}$}} \\
\hline Letter & Sub.\# & & \\
\hline \multirow{2}{*}{$\mathrm{C}$} & 1 & 22 & 0.0654 \\
\hline & 2 & 21 & 0.0647 \\
\hline \multirow{2}{*}{$\mathrm{D}$} & 1 & 6 & 0.0163 \\
\hline & 2 & 5 & 0.0142 \\
\hline \multirow{2}{*}{$\mathrm{E}$} & 1 & 13 & 0.0259 \\
\hline & 2 & 7 & 0.0187 \\
\hline \multirow{2}{*}{$\mathrm{F}$} & 1 & 29 & 0.1684 \\
\hline & 2 & 28 & 0.1681 \\
\hline \multirow{2}{*}{ G } & 1 & 18 & 0.0371 \\
\hline & 2 & 17 & 0.0325 \\
\hline \multirow{2}{*}{$\mathrm{H}$} & 1 & 23 & 0.0998 \\
\hline & 2 & 24 & 0.1008 \\
\hline \multirow{2}{*}{ I } & 1 & 4 & 0.0116 \\
\hline & 2 & 1 & 0.0094 \\
\hline \multirow{2}{*}{$\mathrm{J}$} & 1 & 15 & 0.0287 \\
\hline & 2 & 10 & 0.0236 \\
\hline \multirow{2}{*}{$\mathrm{L}$} & 1 & 16 & 0.0288 \\
\hline & 2 & 14 & 0.0276 \\
\hline \multirow{2}{*}{ M } & 1 & 30 & 0.1736 \\
\hline & 2 & 27 & 0.1634 \\
\hline \multirow{2}{*}{$\mathrm{O}$} & 1 & 12 & 0.0257 \\
\hline & 2 & 11 & 0.0254 \\
\hline \multirow{2}{*}{ Q } & 1 & 2 & 0.0098 \\
\hline & 2 & 3 & 0.0099 \\
\hline \multirow{2}{*}{$S$} & 1 & 25 & 0.1089 \\
\hline & 2 & 26 & 0.1133 \\
\hline \multirow{2}{*}{$\mathrm{U}$} & 1 & 20 & 0.0500 \\
\hline & 2 & 19 & 0.0461 \\
\hline \multirow{2}{*}{ V } & 1 & 9 & 0.0192 \\
\hline & 2 & 8 & 0.0190 \\
\hline
\end{tabular}

Table 10: Tabulation of results for Class B - Left Slap, with an enrollment set size of 3000000 . Letter refers to the participant's letter code found on the footer of this page. Sub. \# is an identifier used to differentiate between the two submissions each participant could make. FNIR was computed at the score threshold that gave FPIR $=10^{-3}$. The number to the left of a value provides the value's column-wise ranking, with the best performance shaded in green and the worst in pink.

\begin{tabular}{|c|c|c|c|}
\hline \multicolumn{2}{|c|}{ Participant } & \multirow{2}{*}{\multicolumn{2}{|c|}{ FNIR $@$ FPIR $=10^{-3}$}} \\
\hline Letter & Sub.\# & & \\
\hline \multirow{2}{*}{$\mathrm{C}$} & 1 & 24 & 0.0403 \\
\hline & 2 & 23 & 0.0392 \\
\hline \multirow{2}{*}{$\mathrm{D}$} & 1 & 6 & 0.0072 \\
\hline & 2 & 2 & 0.0052 \\
\hline \multirow{2}{*}{$\mathrm{E}$} & 1 & 13 & 0.0151 \\
\hline & 2 & 7 & 0.0083 \\
\hline \multirow{2}{*}{$\mathrm{F}$} & 1 & 29 & 0.1222 \\
\hline & 2 & 28 & 0.1220 \\
\hline \multirow{2}{*}{ G } & 1 & 18 & 0.0212 \\
\hline & 2 & 16 & 0.0198 \\
\hline \multirow{2}{*}{$\mathrm{H}$} & 1 & 25 & 0.0641 \\
\hline & 2 & 26 & 0.0647 \\
\hline \multirow{2}{*}{ I } & 1 & 5 & 0.0058 \\
\hline & 2 & 1 & 0.0045 \\
\hline \multirow{2}{*}{$\mathrm{J}$} & 1 & 14 & 0.0156 \\
\hline & 2 & 10 & 0.0126 \\
\hline \multirow{2}{*}{$\mathrm{L}$} & 1 & 15 & 0.0167 \\
\hline & 2 & 17 & 0.0202 \\
\hline \multirow{2}{*}{ M } & 1 & 30 & 0.1259 \\
\hline & 2 & 27 & 0.1155 \\
\hline \multirow{2}{*}{$\mathrm{O}$} & 1 & 12 & 0.0142 \\
\hline & 2 & 11 & 0.0132 \\
\hline \multirow{2}{*}{$\mathrm{Q}$} & 1 & 3 & 0.0057 \\
\hline & 2 & 3 & 0.0057 \\
\hline \multirow{2}{*}{$\mathrm{S}$} & 1 & 21 & 0.0369 \\
\hline & 2 & 22 & 0.0381 \\
\hline \multirow{2}{*}{$\mathrm{U}$} & 1 & 19 & 0.0266 \\
\hline & 2 & 20 & 0.0273 \\
\hline \multirow{2}{*}{ V } & 1 & 8 & 0.0106 \\
\hline & 2 & 9 & 0.0110 \\
\hline
\end{tabular}

Table 11: Tabulation of results for Class B - Right Slap, with an enrollment set size of 3000000 . Letter refers to the participant's letter code found on the footer of this page. Sub. \# is an identifier used to differentiate between the two submissions each participant could make. FNIR was computed at the score threshold that gave FPIR $=$ $10^{-3}$. The number to the left of a value provides the value's columnwise ranking, with the best performance shaded in green and the worst in pink.

\begin{tabular}{l|l|l|l|l|l|l}
$\mathrm{C}=$ afis team & $\mathrm{D}=3 \mathrm{M}$ Cogent & $\mathrm{E}=$ Neurotechnology & $\mathrm{F}=$ Papillon & $\mathrm{G}=$ Dermalog & $\mathrm{H}=$ Hisign Bio-Info Institute \\
$\mathrm{I}=\mathrm{NEC}$ & $\mathrm{J}=$ Sonda & $\mathrm{K}=$ Tiger IT & $\mathrm{L}=$ Innovatrics & $\mathrm{M}=$ SPEX & $\mathrm{O}=$ ID Solutions \\
$\mathrm{P}=\mathrm{id} 3$ & $\mathrm{Q}=$ Morpho & $\mathrm{S}=$ Decatur Industries & $\mathrm{T}=$ BIO-key & $\mathrm{U}=$ Aware & $\mathrm{V}=$ AA Technology
\end{tabular}

$\mathrm{C}=$ afis team $\mid \mathrm{D}=3 \mathrm{M}$ Cogent $\mid \mathrm{E}=$ Neurotechnology $\mid \mathrm{F}=$ Papillon $\mid \mathrm{G}=$ Dermalog $\mid \mathrm{H}=$ Hisign Bio-Info Institute

$\mathrm{P}=\mathrm{id} 3 \quad \mathrm{Q}=$ Morpho $\mid \mathrm{S}=$ Decatur Industries $\mid \mathrm{T}=$ BIO-key $\mid \mathrm{U}=$ Aware $\quad \mathrm{V}=$ AA Technology 


\begin{tabular}{|c|c|c|c|}
\hline \multicolumn{2}{|c|}{ Participant } & \multirow{2}{*}{\multicolumn{2}{|c|}{ FNIR @ FPIR $=10^{-3}$}} \\
\hline Letter & Sub.\# & & \\
\hline \multirow{2}{*}{$\mathrm{C}$} & 1 & 30 & NA \\
\hline & 2 & 29 & NA \\
\hline \multirow{2}{*}{$\mathrm{D}$} & 1 & 6 & 0.0031 \\
\hline & 2 & 5 & 0.0024 \\
\hline \multirow{2}{*}{ E } & 1 & 15 & 0.0063 \\
\hline & 2 & 10 & 0.0049 \\
\hline \multirow{2}{*}{$\mathrm{F}$} & 1 & 28 & 0.0910 \\
\hline & 2 & 26 & 0.0901 \\
\hline \multirow{2}{*}{ G } & 1 & 18 & 0.0106 \\
\hline & 2 & 17 & 0.0084 \\
\hline \multirow{2}{*}{$\mathrm{H}$} & 1 & 23 & 0.0349 \\
\hline & 2 & 24 & 0.0361 \\
\hline \multirow{2}{*}{ I } & 1 & 3 & 0.0022 \\
\hline & 2 & 1 & 0.0015 \\
\hline \multirow{2}{*}{$\mathrm{J}$} & 1 & 16 & 0.0068 \\
\hline & 2 & 9 & 0.0047 \\
\hline \multirow{2}{*}{$\mathrm{L}$} & 1 & 12 & 0.0054 \\
\hline & 2 & 14 & 0.0062 \\
\hline \multirow{2}{*}{ M } & 1 & 27 & 0.0904 \\
\hline & 2 & 25 & 0.0882 \\
\hline \multirow{2}{*}{$\mathrm{O}$} & 1 & 13 & 0.0057 \\
\hline & 2 & 11 & 0.0051 \\
\hline \multirow{2}{*}{ Q } & 1 & 2 & 0.0021 \\
\hline & 2 & 3 & 0.0022 \\
\hline \multirow{2}{*}{$S$} & 1 & 21 & 0.0160 \\
\hline & 2 & 22 & 0.0190 \\
\hline \multirow{2}{*}{$\mathrm{U}$} & 1 & 20 & 0.0139 \\
\hline & 2 & 19 & 0.0124 \\
\hline \multirow{2}{*}{ V } & 1 & 7 & 0.0036 \\
\hline & 2 & 7 & 0.0036 \\
\hline
\end{tabular}

Table 12: Tabulation of results for Class B — Left and Right Slap, with an enrollment set size of 3000000 . Letter refers to the participant's letter code found on the footer of this page. Sub. \# is an identifier used to differentiate between the two submissions each participant could make. FNIR was computed at the score threshold that gave FPIR $=10^{-3}$. NA indicates that the operations required to produce the value could not be performed. The number to the left of a value provides the value's column-wise ranking, with the best performance shaded in green and the worst in pink.

\begin{tabular}{|c|c|c|c|}
\hline \multicolumn{2}{|c|}{ Participant } & \multirow{2}{*}{\multicolumn{2}{|c|}{ FNIR $@$ FPIR $=10^{-3}$}} \\
\hline Letter & Sub.\# & & \\
\hline \multirow{2}{*}{$\mathrm{C}$} & 1 & 30 & NA \\
\hline & 2 & 29 & NA \\
\hline \multirow{2}{*}{ D } & 1 & 6 & 0.0020 \\
\hline & 2 & 2 & 0.0012 \\
\hline \multirow{2}{*}{ E } & 1 & 16 & 0.0043 \\
\hline & 2 & 7 & 0.0024 \\
\hline \multirow{2}{*}{$\mathrm{F}$} & 1 & 27 & 0.0591 \\
\hline & 2 & 27 & 0.0591 \\
\hline \multirow{2}{*}{ G } & 1 & 18 & 0.0062 \\
\hline & 2 & 14 & 0.0040 \\
\hline \multirow{2}{*}{$\mathrm{H}$} & 1 & 23 & 0.0203 \\
\hline & 2 & 24 & 0.0204 \\
\hline \multirow{2}{*}{ I } & 1 & 2 & 0.0012 \\
\hline & 2 & 1 & 0.0009 \\
\hline \multirow{2}{*}{$\mathrm{J}$} & 1 & 17 & 0.0049 \\
\hline & 2 & 11 & 0.0033 \\
\hline \multirow{2}{*}{$\mathrm{L}$} & 1 & 10 & 0.0031 \\
\hline & 2 & 11 & 0.0033 \\
\hline \multirow{2}{*}{ M } & 1 & 26 & 0.0543 \\
\hline & 2 & 25 & 0.0515 \\
\hline \multirow{2}{*}{$\mathrm{O}$} & 1 & 15 & 0.0041 \\
\hline & 2 & 13 & 0.0035 \\
\hline \multirow{2}{*}{ Q } & 1 & 2 & 0.0012 \\
\hline & 2 & 2 & 0.0012 \\
\hline \multirow{2}{*}{ S } & 1 & 20 & 0.0108 \\
\hline & 2 & 21 & 0.0136 \\
\hline \multirow{2}{*}{ U } & 1 & 19 & 0.0099 \\
\hline & 2 & 22 & 0.0141 \\
\hline \multirow{2}{*}{ V } & 1 & 9 & 0.0027 \\
\hline & 2 & 7 & 0.0024 \\
\hline
\end{tabular}

Table 13: Tabulation of results for Class B - Identification Flats, with an enrollment set size of 3000000 . Letter refers to the participant's letter code found on the footer of this page. Sub. \# is an identifier used to differentiate between the two submissions each participant could make. FNIR was computed at the score threshold that gave FPIR $=10^{-3}$. NA indicates that the operations required to produce the value could not be performed. The number to the left of a value provides the value's column-wise ranking, with the best performance shaded in green and the worst in pink.

\begin{tabular}{l|l|l|l|l|l|l}
$\mathrm{C}=$ afis team & $\mathrm{D}=3 \mathrm{M}$ Cogent & $\mathrm{E}=$ Neurotechnology & $\mathrm{F}=$ Papillon & $\mathrm{G}=$ Dermalog & $\mathrm{H}=$ Hisign Bio-Info Institute \\
$\mathrm{I}=\mathrm{NEC}$ & $\mathrm{J}=$ Sonda & $\mathrm{K}=$ Tiger IT & $\mathrm{L}=$ Innovatrics & $\mathrm{M}=$ SPEX & $\mathrm{O}=$ ID Solutions \\
$\mathrm{P}=\mathrm{id} 3$ & $\mathrm{Q}=$ Morpho & $\mathrm{S}=$ Decatur Industries & $\mathrm{T}=$ BIO-key & $\mathrm{U}=$ Aware & $\mathrm{V}=$ AA Technology
\end{tabular}

$\mathrm{C}=$ afis team $\mid \mathrm{D}=3 \mathrm{M}$ Cogent $\mid \mathrm{E}=$ Neurotechnology $\mid \mathrm{F}=$ Papillon $\mid \mathrm{G}=$ Dermalog $\mid \mathrm{H}=$ Hisign Bio-Info Institute

$\mathrm{P}=\mathrm{id} 3 \quad \mathrm{Q}=$ Morpho $\quad \mathrm{S}=$ Decatur Industries $\mid \mathrm{T}=$ BIO-key $\mid \mathrm{U}=$ Aware $\quad \mathrm{V}=$ AA Technology 


\subsection{Class C}

The accuracy results for Class $\mathrm{C}$, which included ten-finger identification for plain and rolled impression types using scanned ink and livescan data, are shown with rank-sorted FNIR points (based on ten-finger rolled-to-rolled impression results) in Figure 21, DET curves in Figure 22, CMC plots in Figure 23, and tables of FNIR points at a fixed FPIR in Tables 14 through 16.

Some observations for Class $C$ include (all FNIR values are at FPIR $=10^{-3}$ ):

$\triangleright$ The most accurate submissions were I, Q, D, and $\mathbf{V}$ (ten-finger rolled-to-rolled), with FNIRs ranging from $0.1 \%$ to $0.19 \%$ for ten-finger plain impressions and ten-finger rolled impressions.

$\triangleright$ There was not a clear difference between ten-finger plain-to-plain and ten-finger rolled-to-rolled results. This is a bit of a surprise, as the ten-finger plain data had to be segmented by the submission and the ten-finger rolled data did not. It also is a surprise after the observations of lower accuracy seen in Class B four-finger slaps versus the Class A two index finger accuracy (Subsection 7.2). It might be interesting to perform a similar Class $C$ test with only four-finger slaps to see if the results are similar to Class B four-finger slap results.

$\triangleright$ The second level of performers were E2, J, O, and V (ten-finger plain) with FNIRs ranging from $0.25 \%$ to $0.50 \%$ for ten-finger plain-to-plain and ten-finger rolled-to-rolled impressions.

$\triangleright$ The best performers were able to handle both rolled and plain impression images with little variation in FNIR, there was a slight decrease when comparing plain-to-rolled impressions.

$\triangleright$ Similar to Class B, the Class C CMC plots in Figure 23 are very flat, indicating the mate is within the top two positions on the candidate list or it is completely missed.

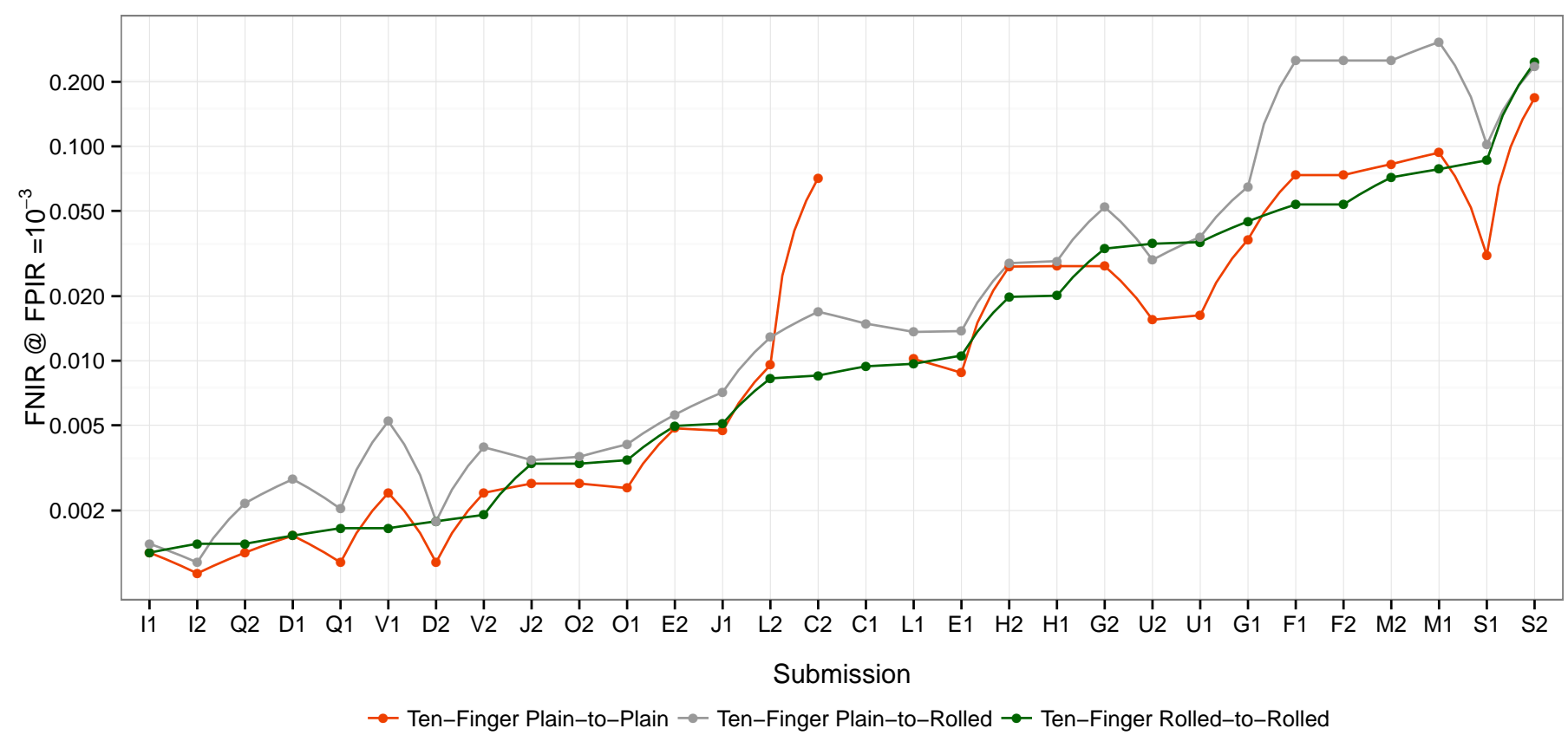

Figure 21: Rank-sorted FNIR @ FPIR $=10^{-3}$ for Class C - Ten-Finger plain-to-plain, rolled-to-rolled, and plain-to-rolled searching 30000 subjects against 5000000 subjects. Submissions " 1 " and " 2 " from round 3.

\begin{tabular}{l|l|l|l|l|l|l}
$\mathrm{C}=$ afis team & $\mathrm{D}=$ = 3M Cogent & $\mathrm{E}=$ Neurotechnology & $\mathrm{F}=$ Papillon & $\mathrm{G}=$ Dermalog & $\mathrm{H}=$ Hisign Bio-Info Institute \\
$\mathrm{I}=$ NEC & $\mathrm{J}=$ Sonda & $\mathrm{K}=$ Tiger IT & $\mathrm{L}=$ Innovatrics & $\mathrm{M}=$ SPEX & $\mathrm{O}=$ ID Solutions \\
$\mathrm{P}=\mathrm{id} 3$ & $\mathrm{Q}=$ Morpho & $\mathrm{S}=$ Decatur Industries & $\mathrm{T}=$ BIO-key & $\mathrm{U}=$ Aware & $\mathrm{V}=$ AA Technology
\end{tabular}




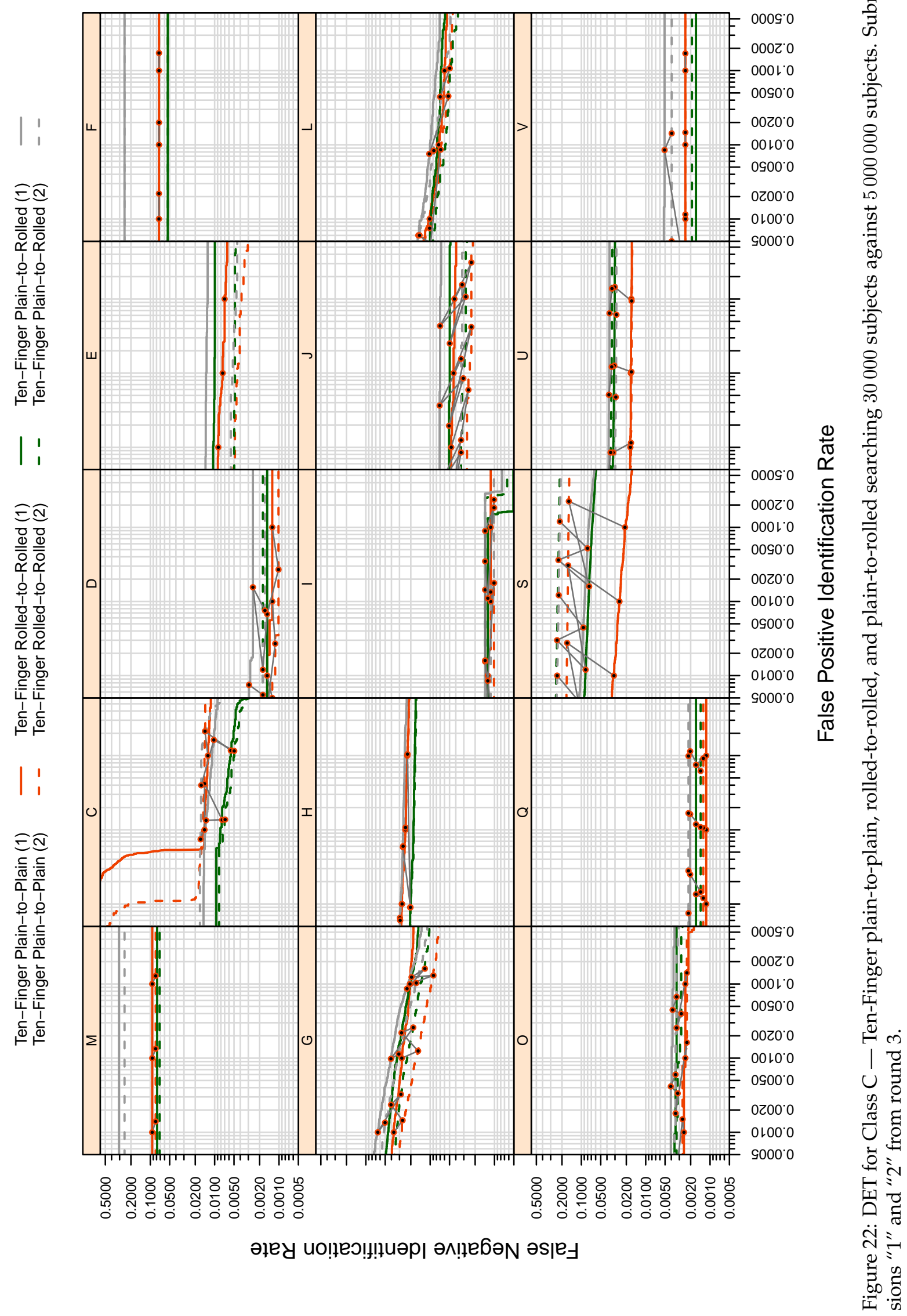

\footnotetext{
$\mathrm{C}=$ afis team $\mathrm{D}=3 \mathrm{M}$ Cogent $\mid \mathrm{E}=$ Neurotechnology $\mid \mathrm{F}=$ Papillon $\quad \mathrm{G}=$ Dermalog $\mathrm{H}=$ Hisign Bio-Info Institute

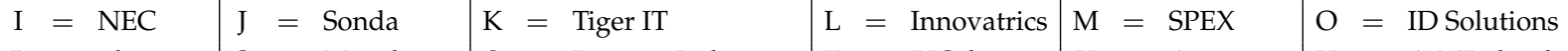

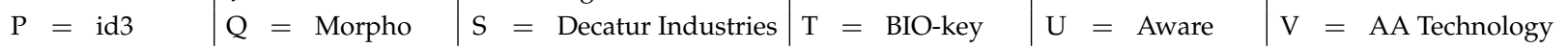



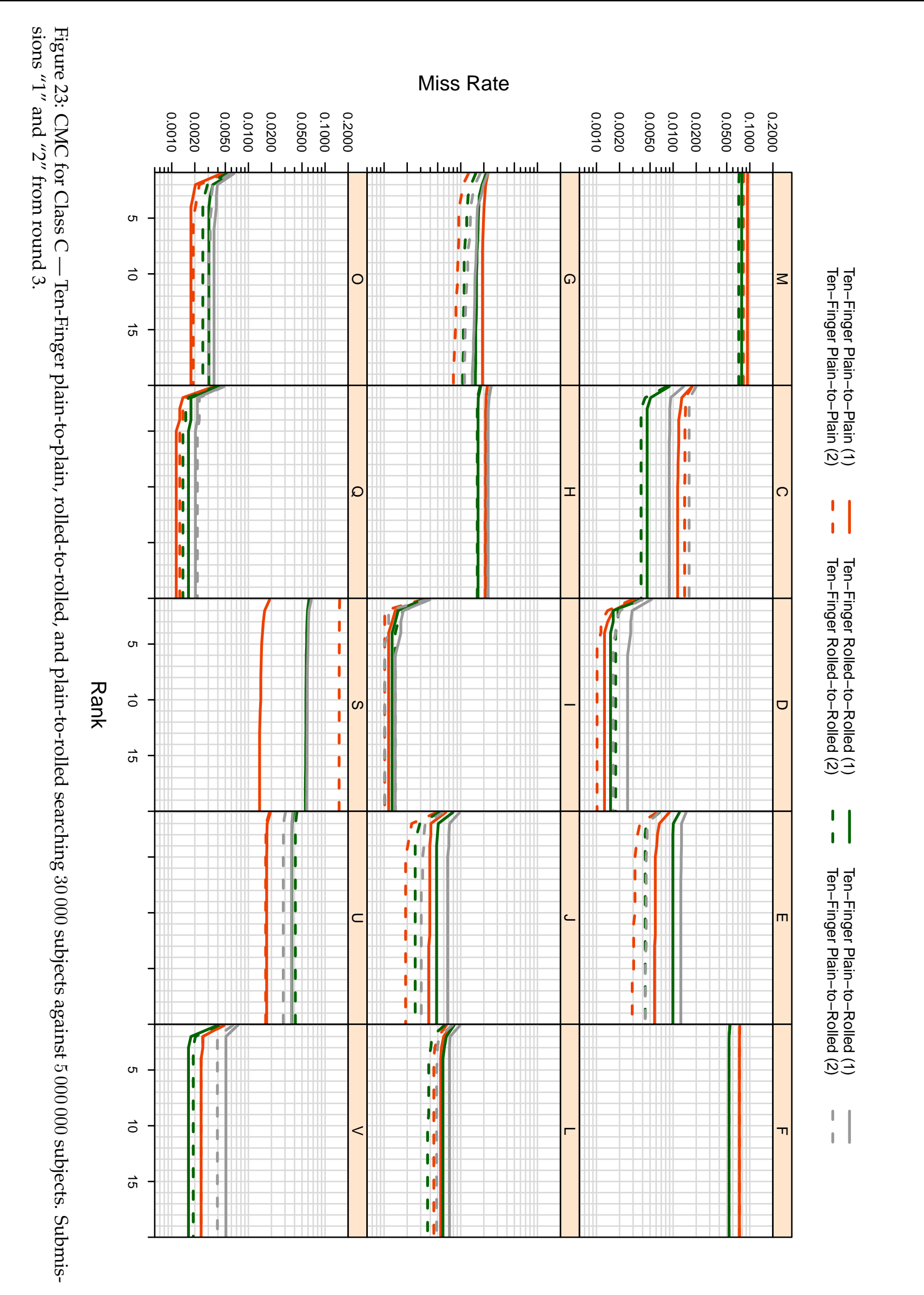


\begin{tabular}{|c|c|c|c|}
\hline \multicolumn{2}{|c|}{ Participant } & \multirow{2}{*}{\multicolumn{2}{|c|}{ FNIR @ FPIR $=10^{-3}$}} \\
\hline Letter & Sub.\# & & \\
\hline \multirow{2}{*}{$\mathrm{C}$} & 1 & 30 & NA \\
\hline & 2 & 24 & 0.0711 \\
\hline \multirow{2}{*}{$\mathrm{D}$} & 1 & 6 & 0.0015 \\
\hline & 2 & 2 & 0.0011 \\
\hline \multirow{2}{*}{ E } & 1 & 14 & 0.0088 \\
\hline & 2 & 13 & 0.0048 \\
\hline \multirow{2}{*}{$\mathrm{F}$} & 1 & 25 & 0.0734 \\
\hline & 2 & 25 & 0.0734 \\
\hline \multirow{2}{*}{ G } & 1 & 23 & 0.0368 \\
\hline & 2 & 20 & 0.0276 \\
\hline \multirow{2}{*}{$\mathrm{H}$} & 1 & 20 & 0.0276 \\
\hline & 2 & 19 & 0.0275 \\
\hline \multirow{2}{*}{ I } & 1 & 4 & 0.0013 \\
\hline & 2 & 1 & 0.0010 \\
\hline \multirow{2}{*}{$\mathrm{J}$} & 1 & 12 & 0.0047 \\
\hline & 2 & 10 & 0.0027 \\
\hline \multirow{2}{*}{$\mathrm{L}$} & 1 & 16 & 0.0102 \\
\hline & 2 & 15 & 0.0095 \\
\hline \multirow{2}{*}{ M } & 1 & 28 & 0.0934 \\
\hline & 2 & 27 & 0.0826 \\
\hline \multirow{2}{*}{$\mathrm{O}$} & 1 & 9 & 0.0025 \\
\hline & 2 & 10 & 0.0027 \\
\hline \multirow{2}{*}{ Q } & 1 & 2 & 0.0011 \\
\hline & 2 & 4 & 0.0013 \\
\hline \multirow{2}{*}{$S$} & 1 & 22 & 0.0311 \\
\hline & 2 & 29 & 0.1680 \\
\hline \multirow{2}{*}{$\mathrm{U}$} & 1 & 18 & 0.0163 \\
\hline & 2 & 17 & 0.0155 \\
\hline \multirow{2}{*}{ V } & 1 & 7 & 0.0024 \\
\hline & 2 & 7 & 0.0024 \\
\hline
\end{tabular}

Table 14: Tabulation of results for Class C - Ten-Finger Plain-toPlain, with an enrollment set size of 5000000 . Letter refers to the participant's letter code found on the footer of this page. Sub. \# is an identifier used to differentiate between the two submissions each participant could make. FNIR was computed at the score threshold that gave FPIR $=10^{-3}$. NA indicates that the operations required to produce the value could not be performed. The number to the left of a value provides the value's column-wise ranking, with the best performance shaded in green and the worst in pink.

\begin{tabular}{|c|c|c|c|}
\hline \multicolumn{2}{|c|}{ Participant } & \multirow{2}{*}{\multicolumn{2}{|c|}{ FNIR @ FPIR $=10^{-3}$}} \\
\hline Letter & Sub. \# & & \\
\hline \multirow{2}{*}{$\mathrm{C}$} & 1 & 16 & 0.0094 \\
\hline & 2 & 15 & 0.0085 \\
\hline \multirow{2}{*}{ D } & 1 & 4 & 0.0015 \\
\hline & 2 & 7 & 0.0018 \\
\hline \multirow{2}{*}{$\mathrm{E}$} & 1 & 18 & 0.0106 \\
\hline & 2 & 12 & 0.0050 \\
\hline \multirow{2}{*}{$\mathrm{F}$} & 1 & 25 & 0.0536 \\
\hline & 2 & 25 & 0.0536 \\
\hline \multirow{2}{*}{ G } & 1 & 24 & 0.0447 \\
\hline & 2 & 21 & 0.0333 \\
\hline \multirow{2}{*}{$\mathrm{H}$} & 1 & 20 & 0.0201 \\
\hline & 2 & 19 & 0.0199 \\
\hline \multirow{2}{*}{ I } & 1 & 1 & 0.0013 \\
\hline & 2 & 2 & 0.0014 \\
\hline \multirow{2}{*}{$\mathrm{J}$} & 1 & 13 & 0.0051 \\
\hline & 2 & 9 & 0.0033 \\
\hline \multirow{2}{*}{$\mathrm{L}$} & 1 & 17 & 0.0097 \\
\hline & 2 & 14 & 0.0083 \\
\hline \multirow{2}{*}{ M } & 1 & 28 & 0.0783 \\
\hline & 2 & 27 & 0.0716 \\
\hline \multirow{2}{*}{$\mathrm{O}$} & 1 & 11 & 0.0034 \\
\hline & 2 & 9 & 0.0033 \\
\hline \multirow{2}{*}{ Q } & 1 & 5 & 0.0017 \\
\hline & 2 & 2 & 0.0014 \\
\hline \multirow{2}{*}{ S } & 1 & 29 & 0.0860 \\
\hline & 2 & 30 & 0.2462 \\
\hline \multirow{2}{*}{$\mathrm{U}$} & 1 & 23 & 0.0358 \\
\hline & 2 & 22 & 0.0351 \\
\hline \multirow{2}{*}{ V } & 1 & 5 & 0.0017 \\
\hline & 2 & 8 & 0.0019 \\
\hline
\end{tabular}

Table 15: Tabulation of results for Class C - Ten-Finger Rolled-toRolled, with an enrollment set size of 5000000 . Letter refers to the participant's letter code found on the footer of this page. Sub. \# is an identifier used to differentiate between the two submissions each participant could make. FNIR was computed at the score threshold that gave FPIR $=10^{-3}$. The number to the left of a value provides the value's column-wise ranking, with the best performance shaded in green and the worst in pink.

\begin{tabular}{l|l|l|l|l|l|l}
$\mathrm{C}=$ afis team & $\mathrm{D}=3 \mathrm{M}$ Cogent & $\mathrm{E}=$ Neurotechnology & $\mathrm{F}=$ Papillon & $\mathrm{G}=$ Dermalog & $\mathrm{H}=$ Hisign Bio-Info Institute \\
$\mathrm{I}=\mathrm{NEC}$ & $\mathrm{J}=$ Sonda & $\mathrm{K}=$ Tiger IT & $\mathrm{L}=$ Innovatrics & $\mathrm{M}=$ SPEX & $\mathrm{O}=$ ID Solutions \\
$\mathrm{P}=\mathrm{id} 3$ & $\mathrm{Q}=$ Morpho & $\mathrm{S}=$ Decatur Industries & $\mathrm{T}=$ BIO-key & $\mathrm{U}=$ Aware & $\mathrm{V}=$ AA Technology
\end{tabular}

$\mathrm{C}=$ afis team $\mid \mathrm{D}=3 \mathrm{M}$ Cogent $\mid \mathrm{E}=$ Neurotechnology $\mid \mathrm{F}=$ Papillon $\mid \mathrm{G}=$ Dermalog $\mid \mathrm{H}=$ Hisign Bio-Info Institute

$\mathrm{P}=\mathrm{id} 3 \quad \mathrm{Q}=$ Morpho $\mid \mathrm{S}=$ Decatur Industries $\mid \mathrm{T}=$ BIO-key $\mid \mathrm{U}=$ Aware $\quad \mathrm{V}=$ AA Technology 


\begin{tabular}{|c|c|c|c|}
\hline \multicolumn{2}{|c|}{ Participant } & \multirow{2}{*}{\multicolumn{2}{|c|}{ FNIR $@$ FPIR $=10^{-3}$}} \\
\hline Letter & Sub. \# & & \\
\hline \multirow{2}{*}{ C } & 1 & 17 & 0.0149 \\
\hline & 2 & 18 & 0.0169 \\
\hline \multirow{2}{*}{$\mathrm{D}$} & 1 & 6 & 0.0028 \\
\hline & 2 & 3 & 0.0018 \\
\hline \multirow{2}{*}{$\mathrm{E}$} & 1 & 16 & 0.0137 \\
\hline & 2 & 12 & 0.0056 \\
\hline \multirow{2}{*}{ F } & 1 & 27 & 0.2514 \\
\hline & 2 & 27 & 0.2514 \\
\hline \multirow{2}{*}{ G } & 1 & 24 & 0.0649 \\
\hline & 2 & 23 & 0.0521 \\
\hline \multirow{2}{*}{$\mathrm{H}$} & 1 & 20 & 0.0291 \\
\hline & 2 & 19 & 0.0285 \\
\hline \multirow{2}{*}{ I } & 1 & 2 & 0.0014 \\
\hline & 2 & 1 & 0.0011 \\
\hline \multirow{2}{*}{$\mathrm{J}$} & 1 & 13 & 0.0071 \\
\hline & 2 & 7 & 0.0034 \\
\hline \multirow{2}{*}{ L } & 1 & 15 & 0.0136 \\
\hline & 2 & 14 & 0.0129 \\
\hline \multirow{2}{*}{ M } & 1 & 30 & 0.3067 \\
\hline & 2 & 27 & 0.2514 \\
\hline \multirow{2}{*}{$\mathrm{O}$} & 1 & 10 & 0.0041 \\
\hline & 2 & 8 & 0.0036 \\
\hline \multirow{2}{*}{ Q } & 1 & 4 & 0.0020 \\
\hline & 2 & 5 & 0.0022 \\
\hline \multirow{2}{*}{$S$} & 1 & 25 & 0.1017 \\
\hline & 2 & 26 & 0.2366 \\
\hline \multirow{2}{*}{$\mathrm{U}$} & 1 & 22 & 0.0378 \\
\hline & 2 & 21 & 0.0295 \\
\hline \multirow{2}{*}{ V } & 1 & 11 & 0.0052 \\
\hline & 2 & 9 & 0.0039 \\
\hline
\end{tabular}

Table 16: Tabulation of results for Class C - Ten-Finger Plain-to-Rolled, with an enrollment set size of 5000000 . Letter refers to the participant's letter code found on the footer of this page. Sub. \# is an identifier used to differentiate between the two submissions each participant could make. FNIR was computed at the score threshold that gave FPIR $=10^{-3}$. The number to the left of a value provides the value's column-wise ranking, with the best performance shaded in green and the worst in pink.

\begin{tabular}{l|l|l|l|l|l|l}
$\mathrm{C}=$ afis team & $\mathrm{D}=3 \mathrm{M}$ Cogent & $\mathrm{E}=$ Neurotechnology & $\mathrm{F}=$ Papillon & $\mathrm{G}=$ Dermalog & $\mathrm{H}=$ Hisign Bio-Info Institute \\
$\mathrm{I}=$ NEC & $\mathrm{J}=$ Sonda & $\mathrm{K}=$ Tiger IT & $\mathrm{L}=$ Innovatrics & $\mathrm{M}=$ SPEX & $\mathrm{O}=$ ID Solutions \\
$\mathrm{P}=\mathrm{id} 3$ & $\mathrm{Q}=$ Morpho & $\mathrm{S}=$ Decatur Industries & $\mathrm{T}=$ BIO-key & $\mathrm{U}=$ Aware & $\mathrm{V}=$ AA Technology
\end{tabular}




\section{Accuracy/Search Time Tradeoff}

This section examines the tradeoff between FNIR and the amount of time each submission needed to perform identification searches. The only time restriction placed on the submissions were they must complete searches before a maximum time limit (Class A: 500 seconds, Class B/C: 90 seconds). NIST allowed for and encouraged two submissions per round. The intent of this decision was to demonstrate the tradeoff between accuracy and speed, but there was no requirement that the same basic algorithm be used for the intended "fast" and "slow" submission. It was possible that two completely different algorithmic approaches were used by a participant, which could introduce other factors when comparing accuracy to search time for a given participant. As such, the two submissions are simply labeled " 1 " and " 2 " in this report. The timing tables shown in this section are based on the detailed timing tables in Appendix $C$ that show the timing for each stage of identification.

In addition to the accuracy/timing tradeoffs plots and timing tables in this section, Appendix E contains a full set of tables that show timing changes that occurred between the last two rounds of submissions. Those tables provide more data to analyze tradeoffs between search time and accuracy. Again, the algorithm could have changed from one round of submissions to the next, but it is generally assumed that the basic algorithmic approach stayed the same, while "controls" were tweaked to improve accuracy.

One general observation noticed across classes of participation was that increased search times are not a guarantee of increased accuracy. This turned out to be inconsistent across the test. In general, most submissions obtain some improvement in accuracy with increased search times, but most gains are modest, and a few cases had no gain or a slight loss in accuracy. There are other cases where submissions decreased search time yet still improved accuracy.

It might be useful in future evaluations to encourage competition over extremely fast searches and see which submissions have a search time vs accuracy advantage, as opposed to tweaking for maximum accuracy, as was done in the current FpVTE protocol.

\begin{tabular}{l|l|l|l|ll|l}
$\mathrm{C}=$ afis team & $\mathrm{D}=3 \mathrm{M}$ Cogent & $\mathrm{E}=$ Neurotechnology & $\mathrm{F}=$ Papillon & $\mathrm{G}=$ Dermalog & $\mathrm{H}=$ Hisign Bio-Info Institute \\
$\mathrm{I}=$ NEC & $\mathrm{J}=$ Sonda & $\mathrm{K}=$ Tiger IT & $\mathrm{L}=$ Innovatrics & $\mathrm{M}=$ SPEX & $\mathrm{O}=$ ID Solutions \\
$\mathrm{P}=\mathrm{id} 3$ & $\mathrm{Q}=$ Morpho & $\mathrm{S}=$ Decatur Industries & $\mathrm{T}=$ BIO-key & $\mathrm{U}=$ Aware & $\mathrm{V}=$ AA Technology
\end{tabular}




\subsection{Class A}

Tabulated comparisons of identification times for index finger identification submissions are shown in Tables 17 and 18. The search times shown in these tables are from the "Total/One" column in Tables 24 through 29 included in Appendix C. For reference, the FNIR values from Section 7 are reprinted to the right of the search times. Class A tables were split into two groups. The first group includes submissions that performed searches on average in less than 20 seconds, and the second includes those that took, on average, 20 seconds or longer.

The tables were used to create scatter plots showing accuracy, search times, and search template creation times. Those plots are shown in Figures 24 through 29.

Some observations for Class A identification times include:

$\triangleright$ For single index fingers, there was essentially no accuracy improvement observed with larger search times.

$\triangleright$ For two index fingers, there was improvement shown by some participants with increased search time. The overall benefit might depend on the application.

$\triangleright$ It is difficult to compare single-finger identification results to two-finger identification results here, as the enrollment set sizes used were not the same (100000 and 1.6 million, respectively).

$\triangleright$ The number of processes running (one or ten) didn't appear to have a major effect on throughput for Class A single index fingers, and only a slight increase in processing time was observed for two-finger searches. See Appendix $\mathrm{C}$ and Appendix D for complete details.

$\triangleright$ Tables 50 through 52 in Appendix E and Figures 30 through 35 show differences between the last two rounds of submissions. Most submissions lowered FNIR for single finger but required longer search times. The results for two index fingers were not as consistent. Most lowered FNIR, but some had a significant increase in search time for little gain in FNIR. While it is not known what changes were made between submissions, there is some indication that high accuracy can be achieved with some of the fast submissions. The absolute best accuracy is achieved by slightly slower submissions.

\begin{tabular}{l|l|l|l|ll|l}
$\mathrm{C}=$ afis team & $\mathrm{D}=3 \mathrm{M}$ Cogent & $\mathrm{E}=$ Neurotechnology & $\mathrm{F}=$ Papillon & $\mathrm{G}=$ Dermalog & $\mathrm{H}=$ Hisign Bio-Info Institute \\
$\mathrm{I}=$ NEC & $\mathrm{J}=$ Sonda & $\mathrm{K}=$ Tiger IT & $\mathrm{L}=$ Innovatrics & $\mathrm{M}=$ SPEX & $\mathrm{O}=$ ID Solutions \\
$\mathrm{P}=\mathrm{id} 3$ & $\mathrm{Q}=$ Morpho & $\mathrm{S}=$ Decatur Industries & $\mathrm{T}=$ BIO-key & $\mathrm{U}=$ Aware & $\mathrm{V}=$ AA Technology
\end{tabular}




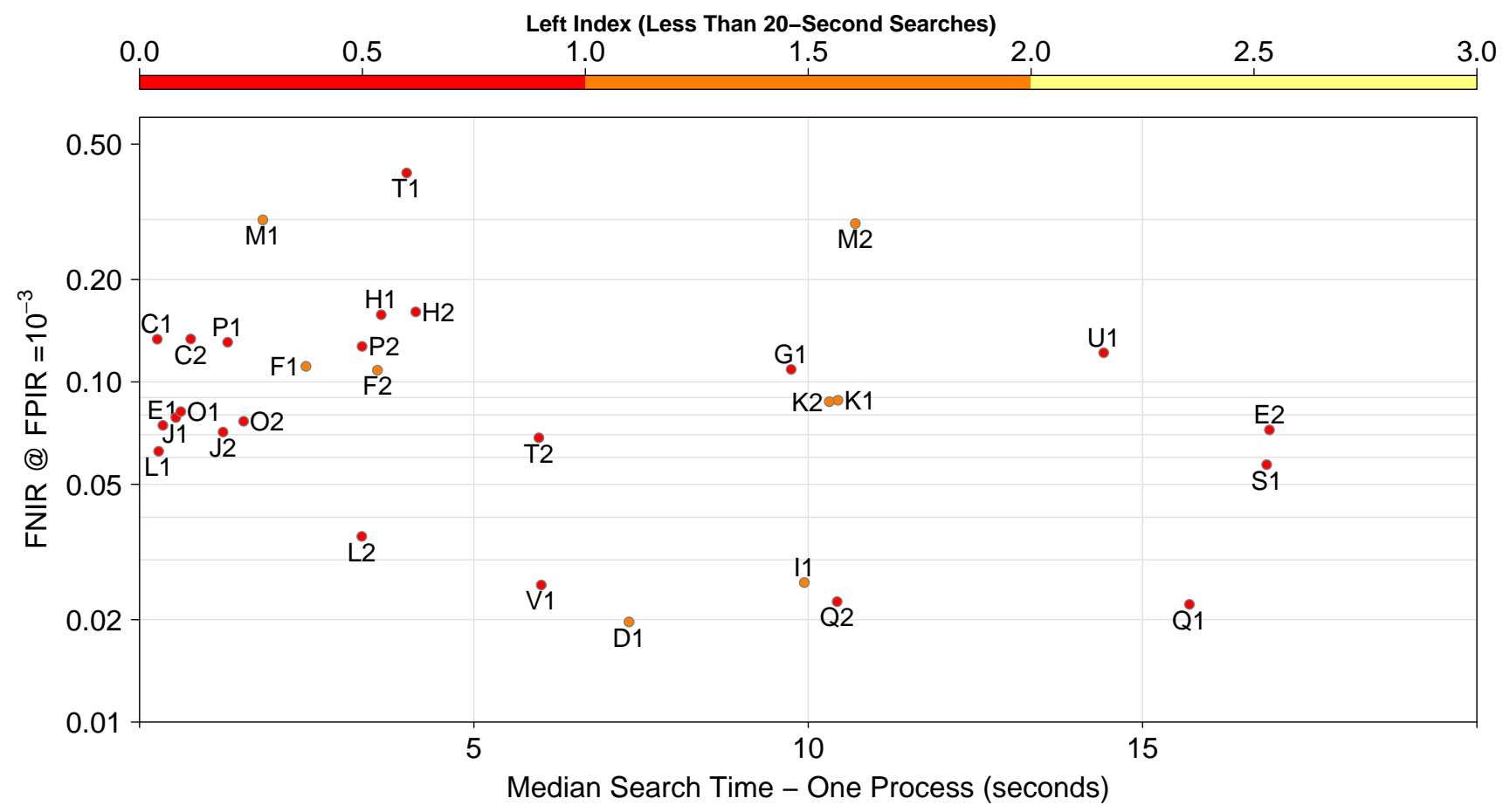

Figure 24: Scatter plot of FNIR @ FPIR $=10^{-3}$ searching 30000 subjects against 100000 subjects and median search time (less than 20 seconds) for a single process for Class A - Left Index. The color of the data point is used to show the search template creation time. The color scale for search template creation time is at the top of the plot. Median search times are plotted in seconds. The FNIR and median search time data are from Table 17 and search template creation times can be found in Table 72 in Appendix $\mathrm{H}$.

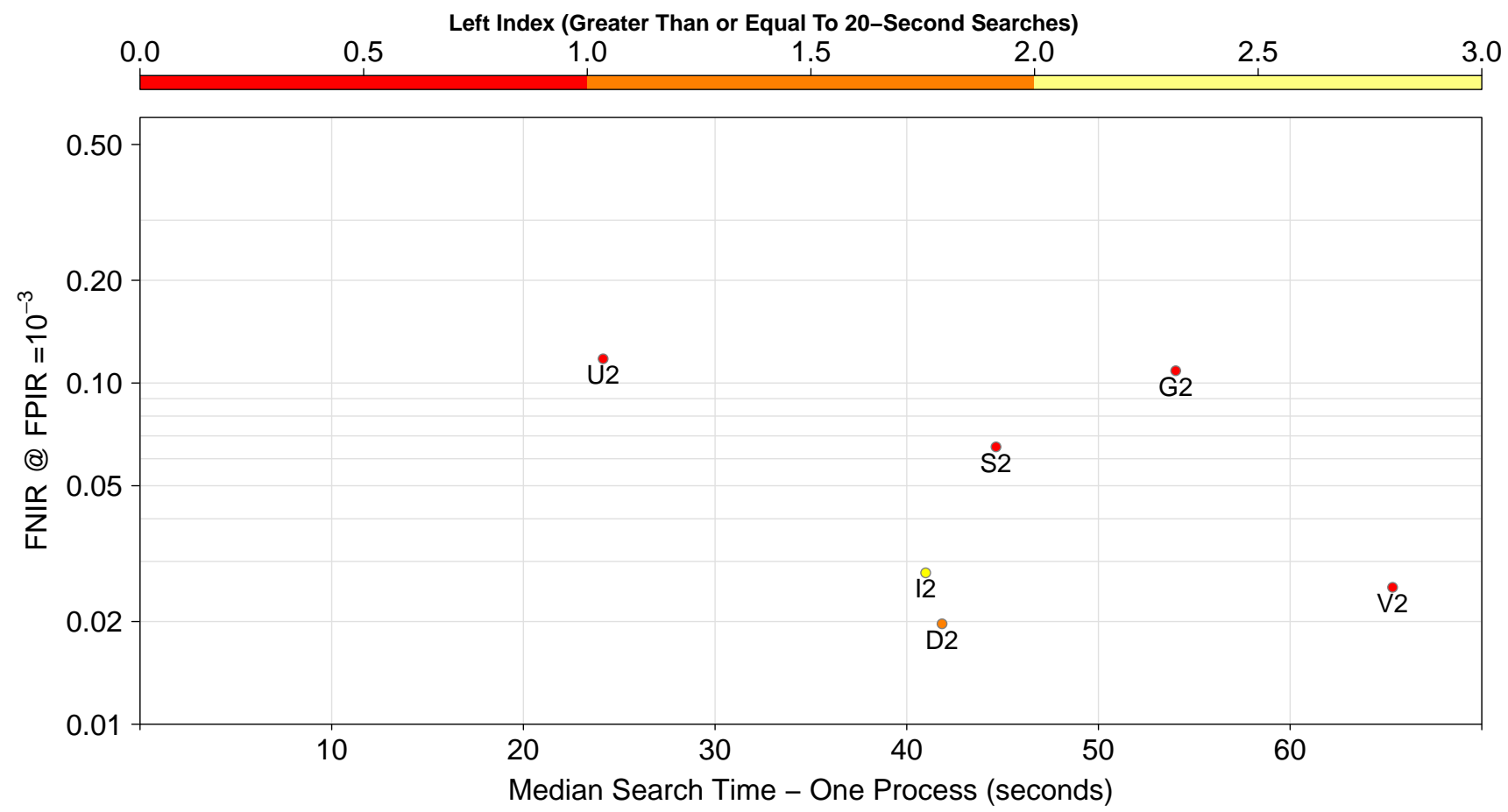

Figure 25: Scatter plot of FNIR @ FPIR $=10^{-3}$ searching 30000 subjects against 100000 subjects and median search time (greater than or equal to 20 seconds) for a single process for Class A - Left Index. The color of the data point is used to show the search template creation time. The color scale for search template creation time is at the top of the plot. Median search times are plotted in seconds. The FNIR and median search time data are from Table 17 and search template creation times can be found in Table 72 in Appendix H.

\begin{tabular}{l|l|l|l|l|l|l}
$\mathrm{C}=$ afis team & $\mathrm{D}=$ = 3M Cogent & $\mathrm{E}=$ Neurotechnology & $\mathrm{F}=$ Papillon & $\mathrm{G}=$ Dermalog & $\mathrm{H}=$ Hisign Bio-Info Institute \\
$\mathrm{I}=$ NEC & $\mathrm{J}=$ Sonda & $\mathrm{K}=$ Tiger IT & $\mathrm{L}=$ Innovatrics & $\mathrm{M}=$ SPEX & $\mathrm{O}=$ ID Solutions \\
$\mathrm{P}=\mathrm{id} 3$ & $\mathrm{Q}=$ Morpho & $\mathrm{S}=$ Decatur Industries & $\mathrm{T}=$ BIO-key & $\mathrm{U}=$ Aware & $\mathrm{V}=$ AA Technology
\end{tabular}




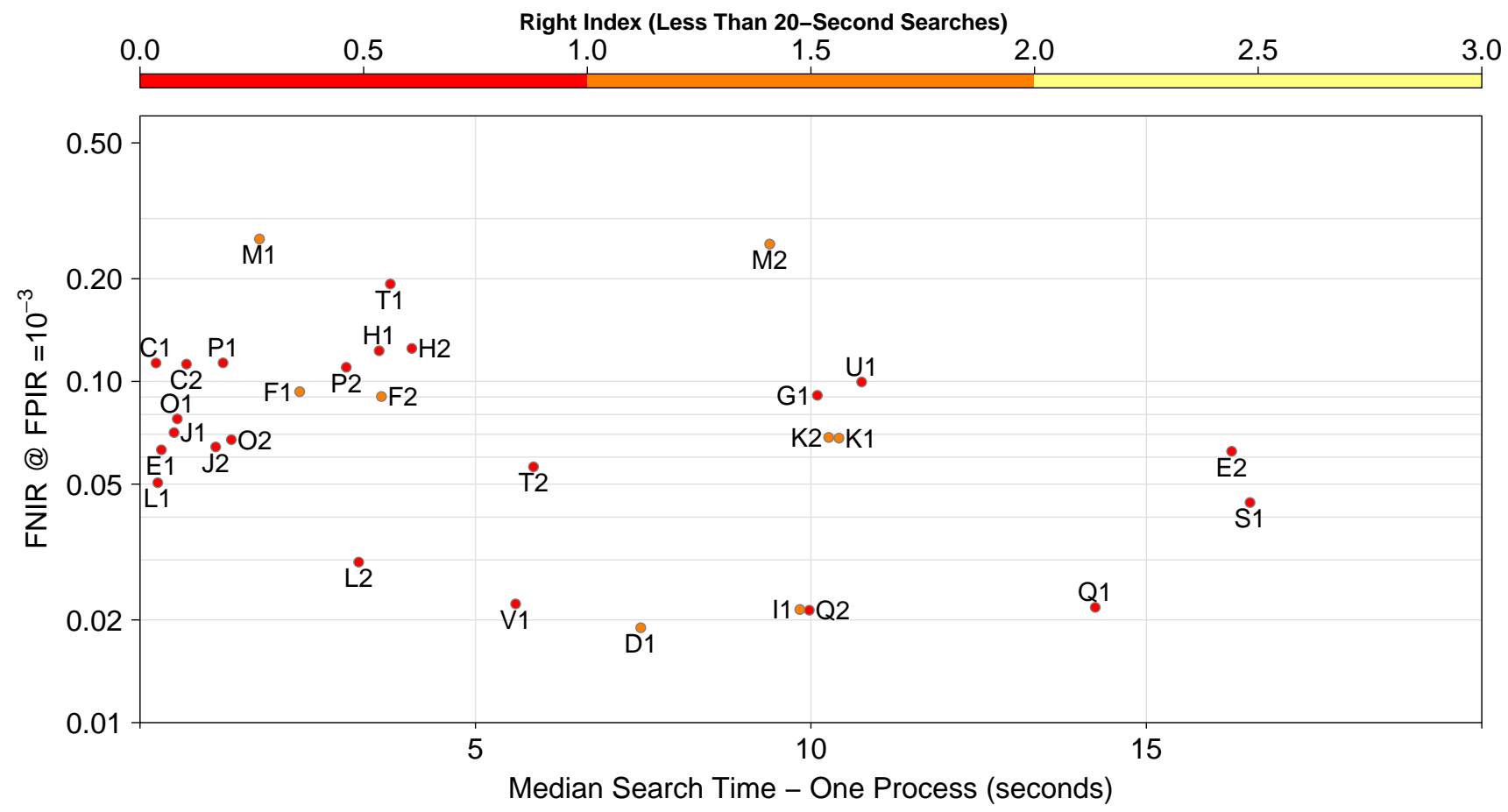

Figure 26: Scatter plot of FNIR @ FPIR $=10^{-3}$ searching 30000 subjects against 100000 subjects and median search time (less than 20 seconds) for a single process. The color of the data point is used to show the search template creation time. The color scale for search template creation time is at the top of the plot. Median search times are plotted in seconds. The FNIR and median search time data are from Table 17 and search template creation times can be found in Table 73 in Appendix H.

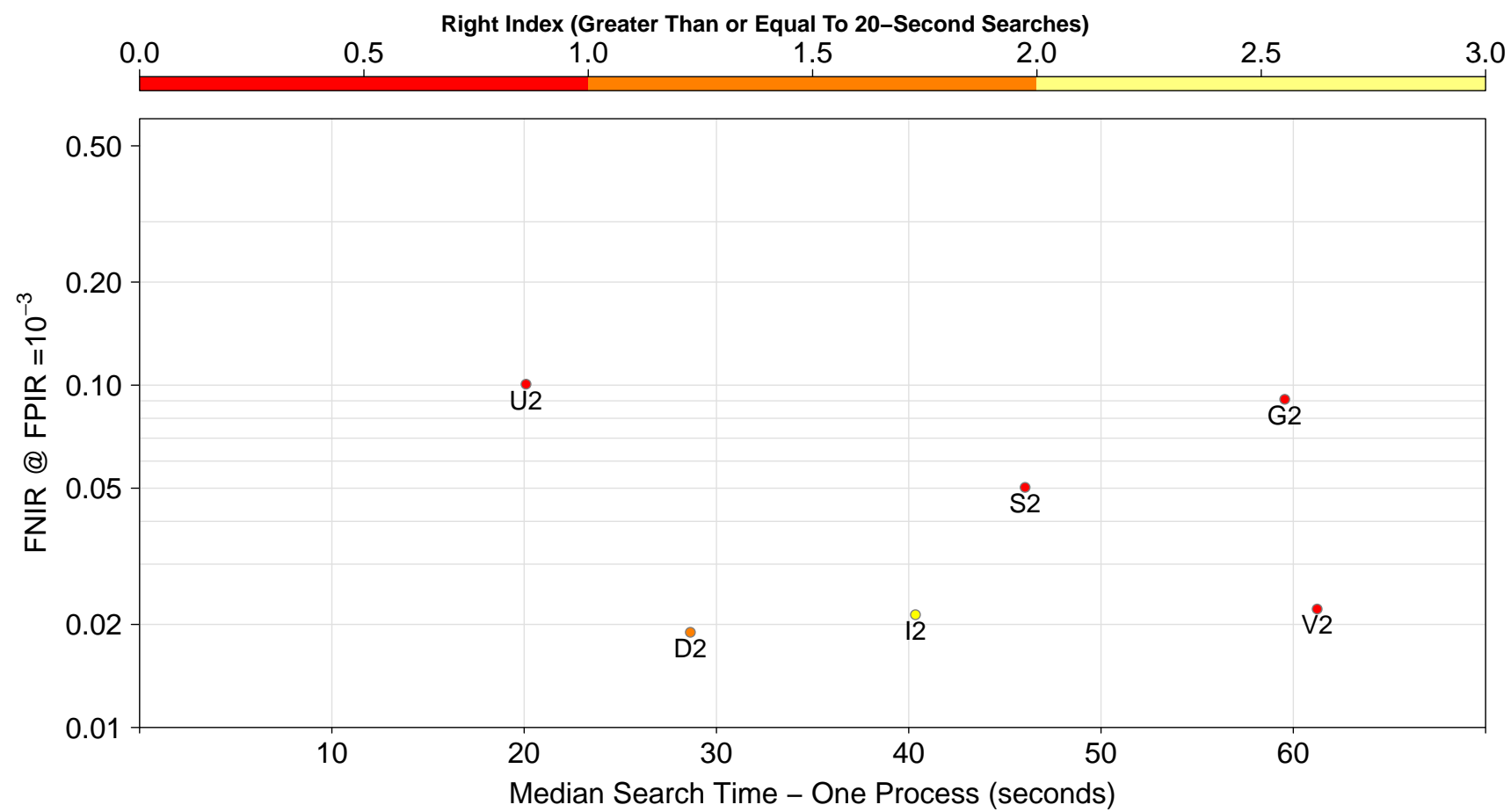

Figure 27: Scatter plot of FNIR @ FPIR $=10^{-3}$ searching 30000 subjects against 100000 subjects and median search time (greater than or equal to 20 seconds) for a single process for Class A - Right Index. The color of the data point is used to show the search template creation time. The color scale for search template creation time is at the top of the plot. Median search times are plotted in seconds. The FNIR and median search time data are from Table 17 and search template creation times can be found in Table 73 in Appendix H.

\begin{tabular}{l|l|l|l|l|l|l}
$\mathrm{C}=$ afis team & $\mathrm{D}=3 \mathrm{M}$ Cogent & $\mathrm{E}=$ Neurotechnology & $\mathrm{F}=$ Papillon & $\mathrm{G}=$ Dermalog & $\mathrm{H}=$ Hisign Bio-Info Institute \\
$\mathrm{I}=$ NEC & $\mathrm{J}=$ Sonda & $\mathrm{K}=$ Tiger IT & $\mathrm{L}=$ Innovatrics & $\mathrm{M}=$ SPEX & $\mathrm{O}=$ ID Solutions \\
$\mathrm{P}=\mathrm{id} 3$ & $\mathrm{Q}=$ Morpho & $\mathrm{S}=$ Decatur Industries & $\mathrm{T}=$ BIO-key & $\mathrm{U}=$ Aware & $\mathrm{V}=$ AA Technology
\end{tabular}




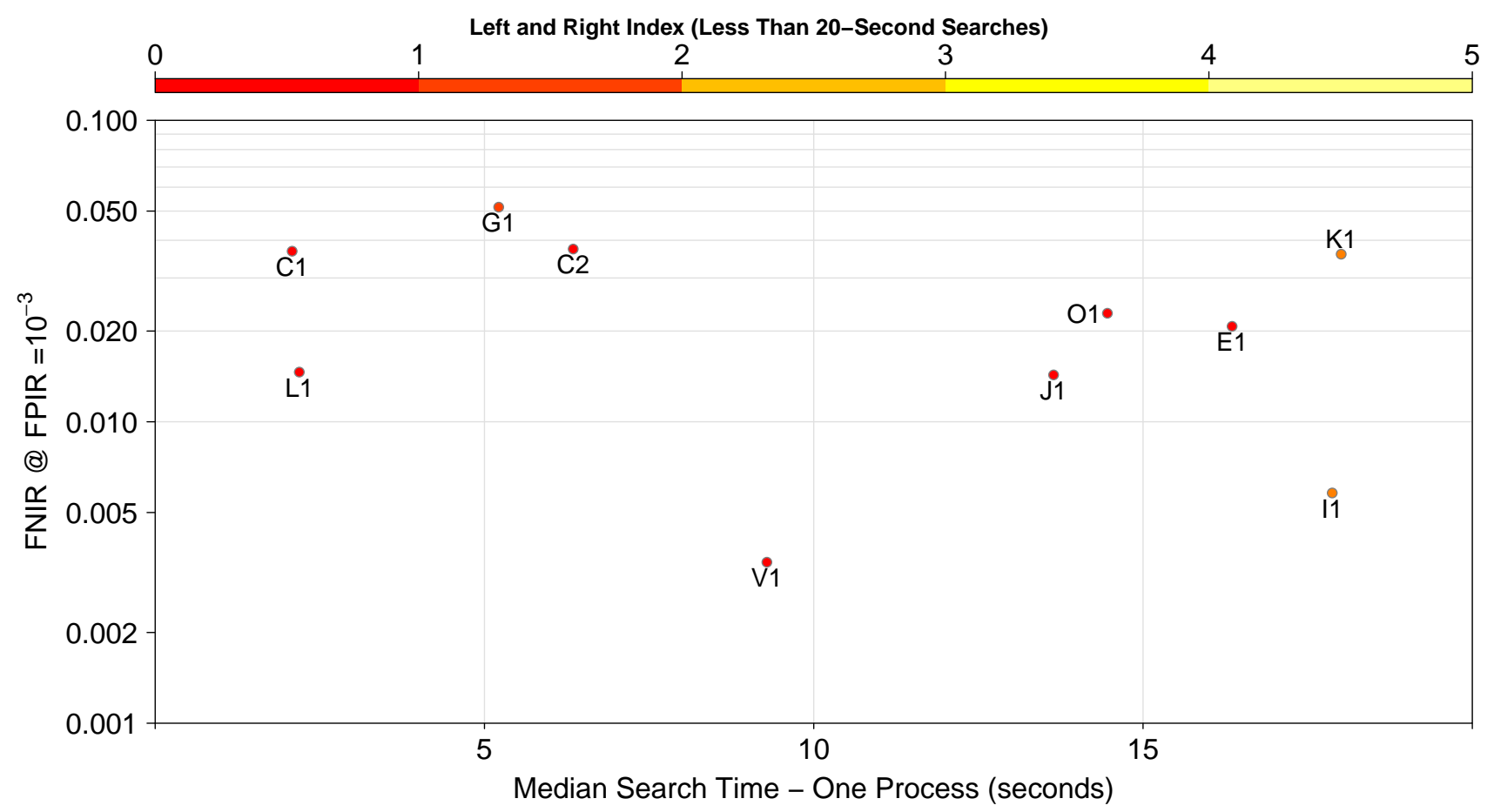

Figure 28: Scatter plot of FNIR @ FPIR $=10^{-3}$ searching 30000 subjects against 1600000 subjects and median search time (less than 20 seconds) for a single process for Class A - Left and Right Index. The color of the data point is used to show the search template creation time. The color scale for search template creation time is at the top of the plot. Median search times are plotted in seconds. The FNIR and median search time data are from Table 18 and search template creation times can be found in Table 74 in Appendix $\mathrm{H}$.

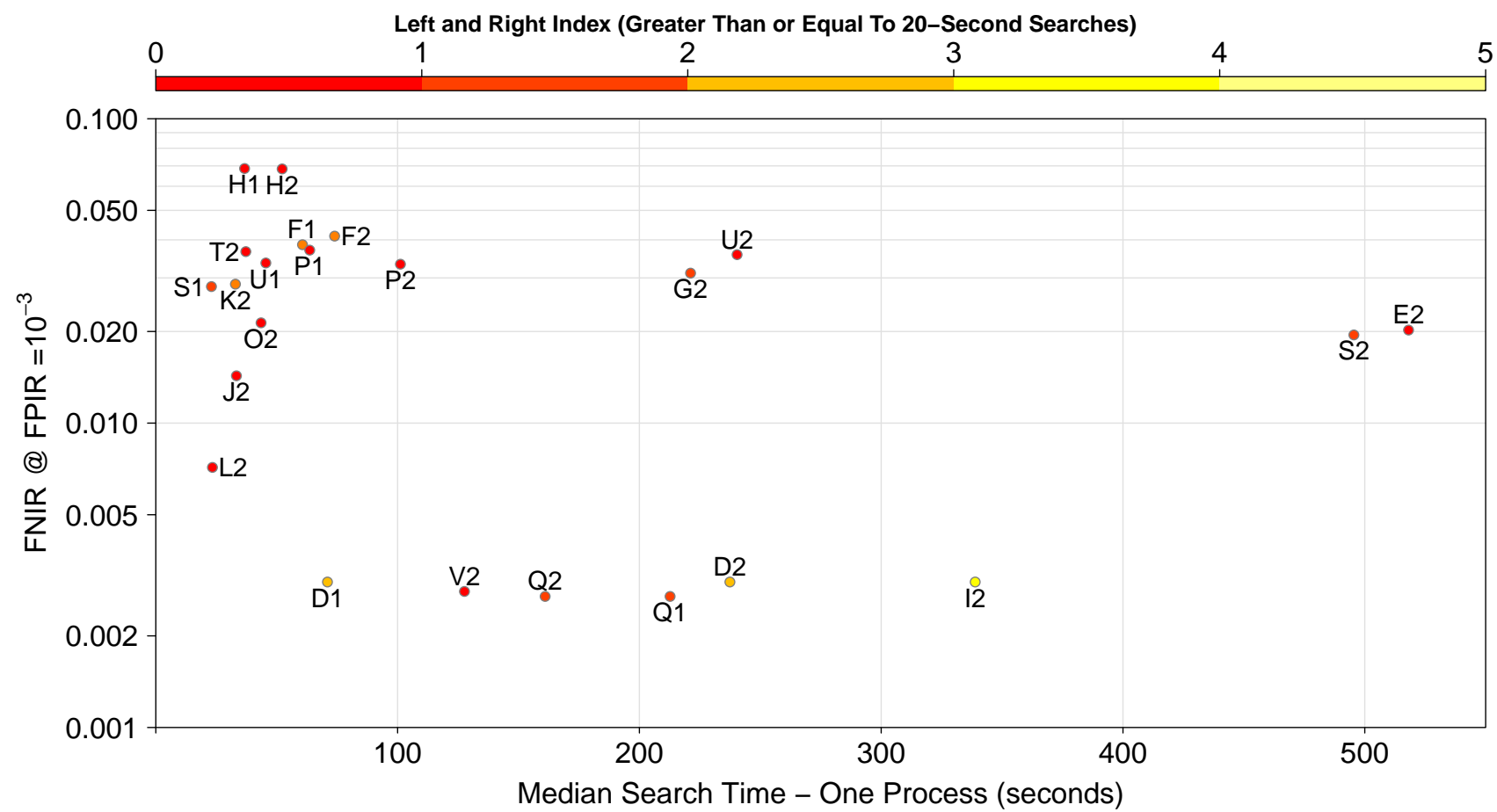

Figure 29: Scatter plot of FNIR @ FPIR $=10^{-3}$ searching 30000 subjects against 1600000 subjects and median search time (greater than or equal to 20 seconds) for a single process for Class A - Left and Right Index. The color of the data point is used to show the search template creation time. The color scale for search template creation time is at the top of the plot. Median search times are plotted in seconds. The FNIR and median search time data are from Table 18 and search template creation times can be found in Table 74 in Appendix H.

\begin{tabular}{l|l|l|l|l|l|l}
$\mathrm{C}=$ afis team & $\mathrm{D}=3 \mathrm{M}$ Cogent & $\mathrm{E}=$ Neurotechnology & $\mathrm{F}=$ Papillon & $\mathrm{G}=$ Dermalog & $\mathrm{H}=$ Hisign Bio-Info Institute \\
$\mathrm{I}=$ NEC & $\mathrm{J}=$ Sonda & $\mathrm{K}=$ Tiger IT & $\mathrm{L}=$ Innovatrics & $\mathrm{M}=$ SPEX & $\mathrm{O}=$ ID Solutions \\
$\mathrm{P}=\mathrm{id} 3$ & $\mathrm{Q}=$ Morpho & $\mathrm{S}=$ Decatur Industries & $\mathrm{T}=$ BIO-key & $\mathrm{U}=$ Aware & $\mathrm{V}=$ AA Technology
\end{tabular}




\section{Left Index (Less Than 20-Second Searches)}

5

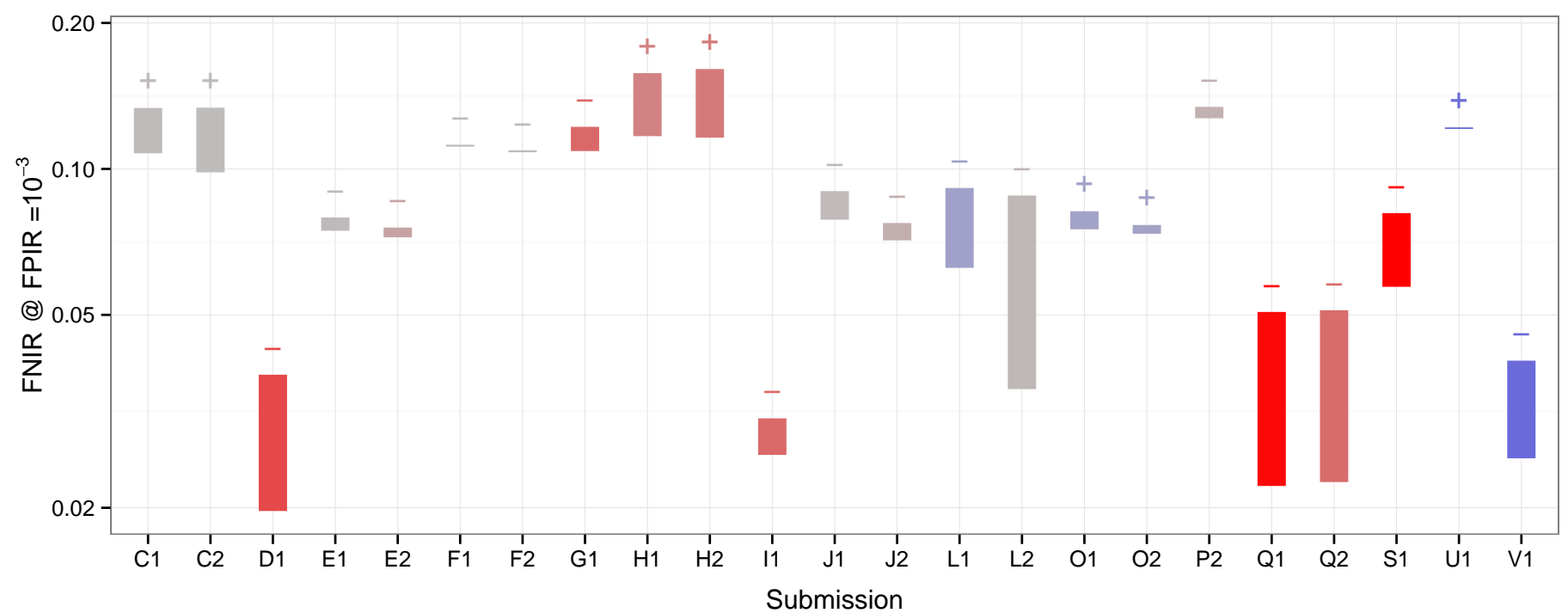

Figure 30: Plots showing difference in FNIR @ FPIR $=10^{-3}$ searching 30000 subjects against 1600000 subjects and difference in search times, for a single search process, between round 2 and round 3 submissions for Class A - Left Index. The "+" symbol indicates that FNIR increased from round 2 to round 3 and "-" indicates a decrease in FNIR. The color of the bar shows the change in search time. The color scale for difference in search time is at the top of the plot and the units are in seconds. The data for the plots are taken from the tables in Appendix E.

\section{Left Index (Greater Than or Equal To 20-Second Searches)}

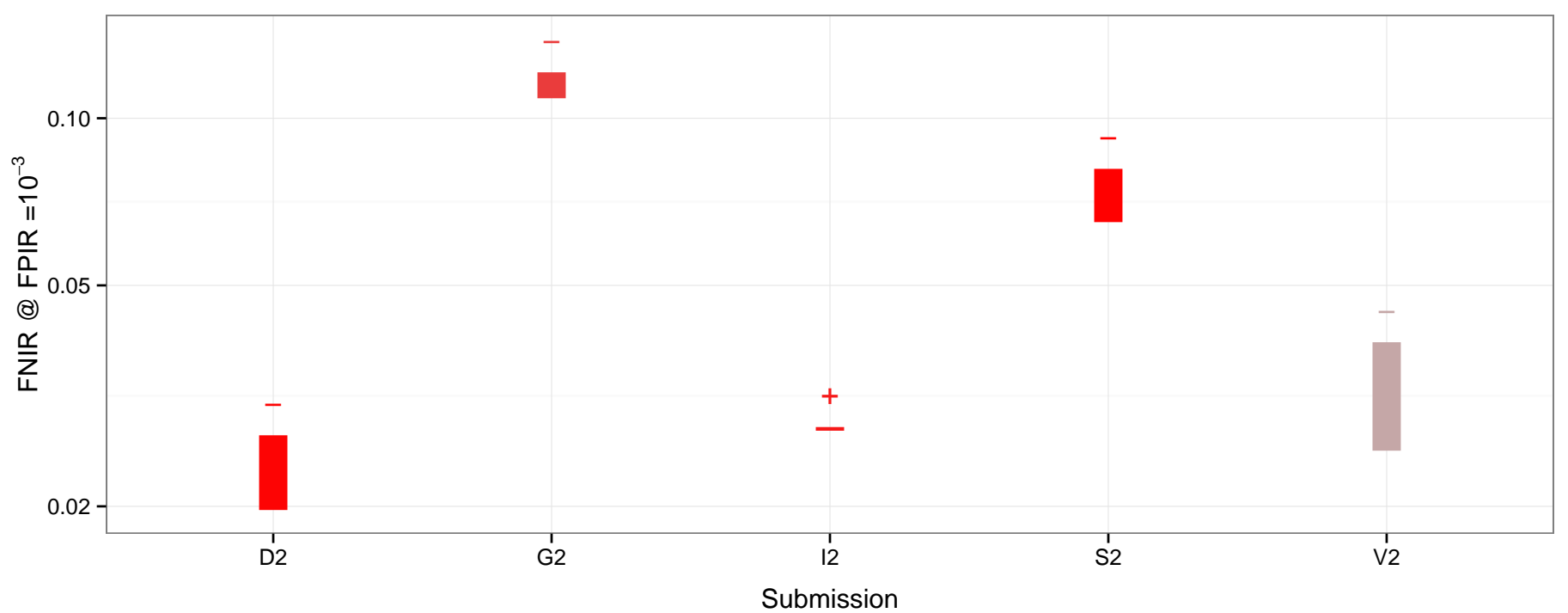

Figure 31: Plots showing difference in FNIR @ FPIR $=10^{-3}$ searching 30000 subjects against 1600000 subjects and difference in search times, for a single search process, between round 2 and round 3 submissions for Class A - Left Index. The " + " symbol indicates that FNIR increased from round 2 to round 3 and "-" indicates a decrease in FNIR. The color of the bar shows the change in search time. The color scale for difference in search time is at the top of the plot and the units are in seconds. The data for the plots are taken from the tables in Appendix E.

\begin{tabular}{l|l|l|l|l|l|l}
$\mathrm{C}=$ afis team & $\mathrm{D}=3 \mathrm{M}$ Cogent & $\mathrm{E}=$ Neurotechnology & $\mathrm{F}=$ Papillon & $\mathrm{G}=$ Dermalog & $\mathrm{H}=$ Hisign Bio-Info Institute \\
$\mathrm{I}=$ NEC & $\mathrm{J}=$ Sonda & $\mathrm{K}=$ Tiger IT & $\mathrm{L}=$ Innovatrics & $\mathrm{M}=$ SPEX & $\mathrm{O}=$ ID Solutions \\
$\mathrm{P}=\mathrm{id} 3$ & $\mathrm{Q}=$ Morpho & $\mathrm{S}=$ Decatur Industries & $\mathrm{T}=$ BIO-key & $\mathrm{U}=$ Aware & $\mathrm{V}=$ AA Technology
\end{tabular}




\section{Right Index (Less Than 20-Second Searches)}

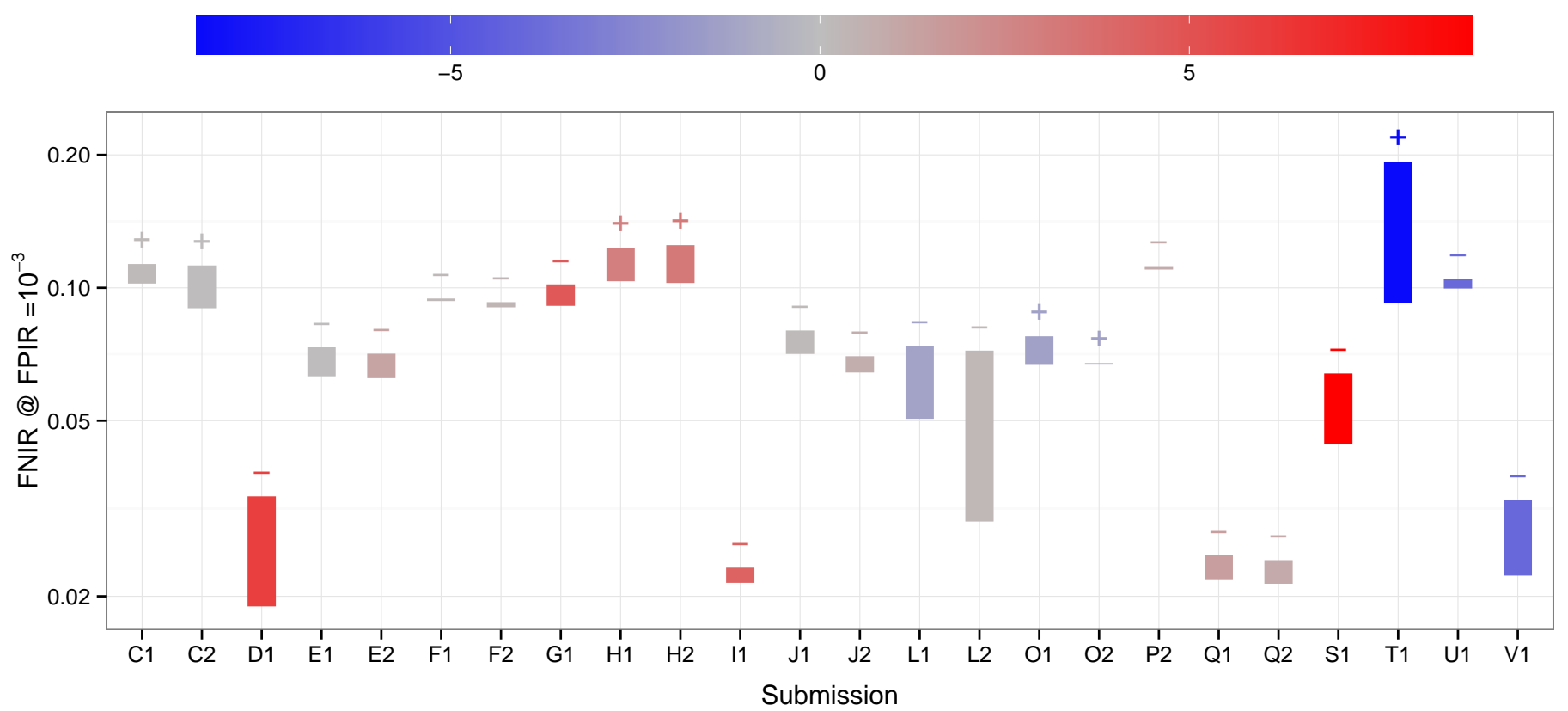

Figure 32: Plots showing difference in FNIR @ FPIR $=10^{-3}$ searching 30000 subjects against 1600000 subjects and difference in search times, for a single search process, between round 2 and round 3 submissions for Class A - Right Index. The "+" symbol indicates that FNIR increased from round 2 to round 3 and "-" indicates a decrease in FNIR. The color of the bar shows the change in search time. The color scale for difference in search time is at the top of the plot and the units are in seconds. The data for the plots are taken from the tables in Appendix E.

\section{Right Index (Greater Than or Equal To 20-Second Searches)}

10

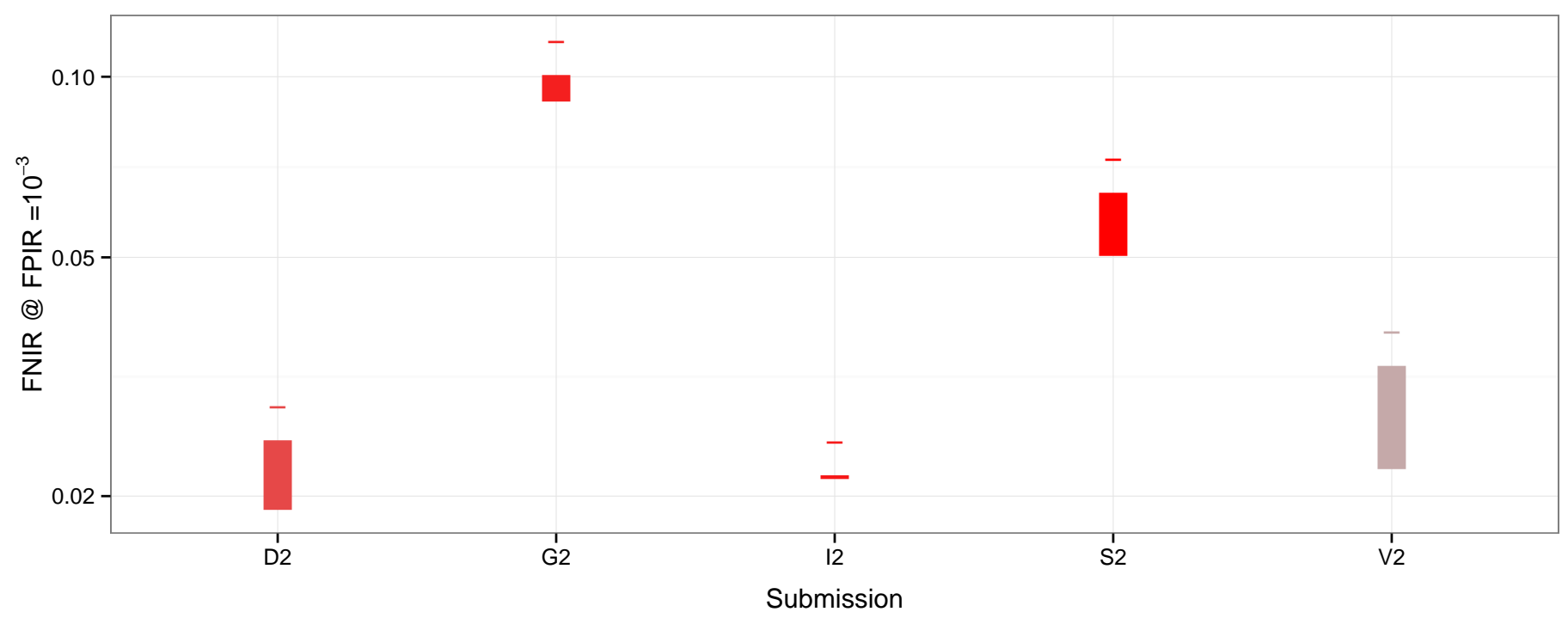

Figure 33: Plots showing difference in FNIR @ FPIR $=10^{-3}$ searching 30000 subjects against 1600000 subjects and difference in search times, for a single search process, between round 2 and round 3 submissions for Class A - Right Index. The " + " symbol indicates that FNIR increased from round 2 to round 3 and "-" indicates a decrease in FNIR. The color of the bar shows the change in search time. The color scale for difference in search time is at the top of the plot and the units are in seconds. The data for the plots are taken from the tables in Appendix E. 


\section{Left and Right Index (Less Than 20-Second Searches)}

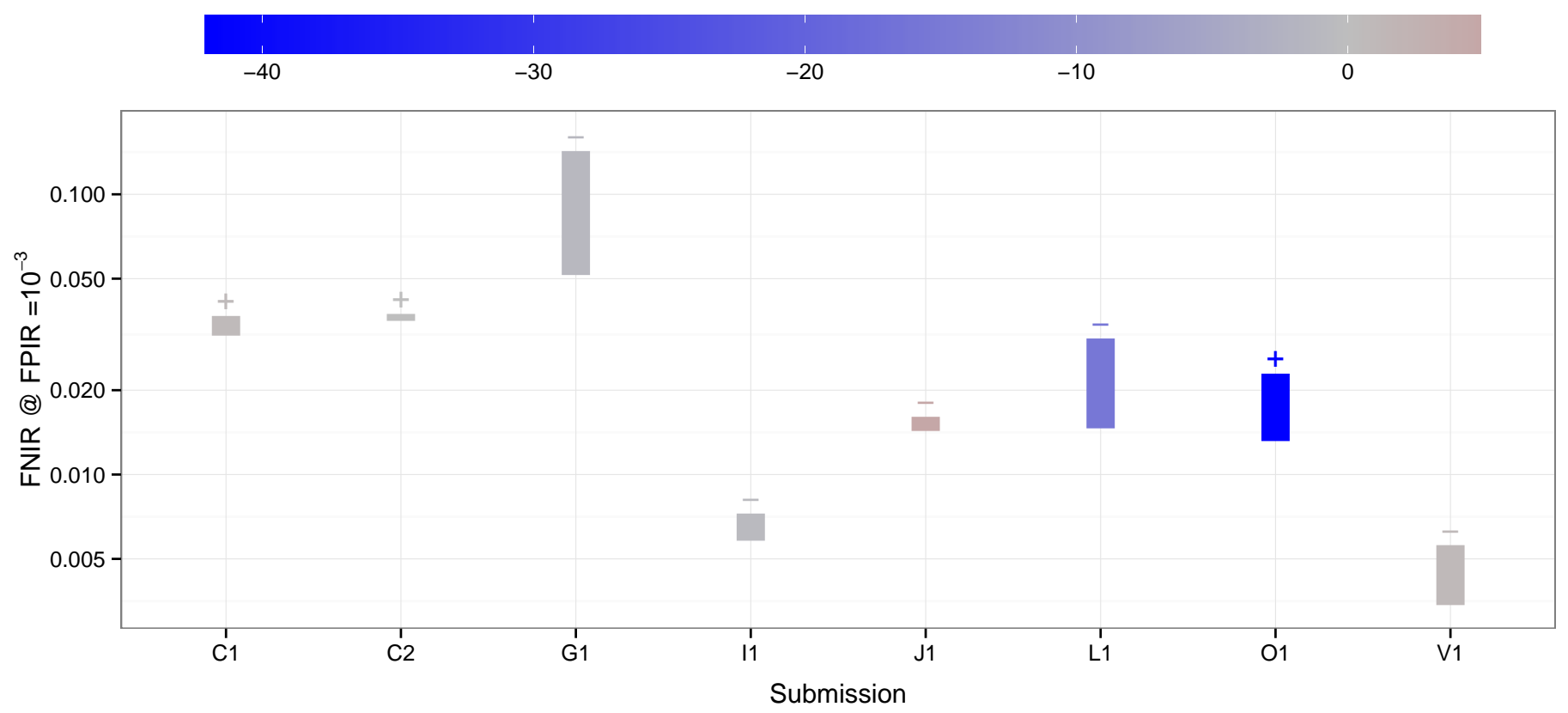

Figure 34: Plots showing difference in FNIR @ FPIR $=10^{-3}$ searching 30000 subjects against 1600000 subjects and difference in search times, for a single search process, between round 2 and round 3 submissions for Class A - Left and Right Index. The "+" symbol indicates that FNIR increased from round 2 to round 3 and "-" indicates a decrease in FNIR. The color of the bar shows the change in search time. The color scale for difference in search time is at the top of the plot and the units are in seconds. The data for the plots are taken from the tables in Appendix E.

\section{Left and Right Index (Greater Than or Equal To 20-Second Searches)}

$\begin{array}{rrr}100 & 200 & 300\end{array}$

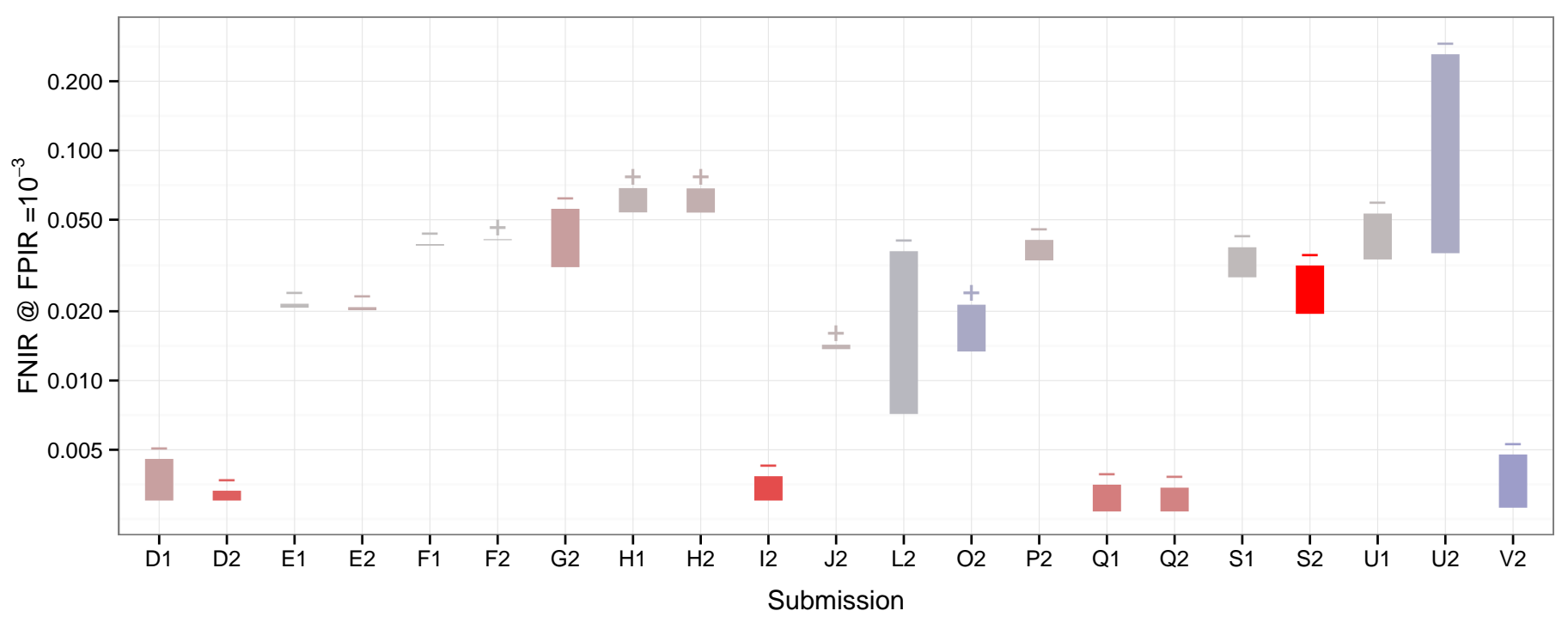

Figure 35: Plots showing difference in FNIR @ FPIR $=10^{-3}$ searching 30000 subjects against 1600000 subjects and difference in search times, for a single search process, between round 2 and round 3 submissions for Class A - Left and Right Index. The "+" symbol indicates that FNIR increased from round 2 to round 3 and "-" indicates a decrease in FNIR. The color of the bar shows the change in search time. The color scale for difference in search time is at the top of the plot and the units are in seconds. The data for the plots are taken from the tables in Appendix E.

\begin{tabular}{l|l|l|l|l|l|l}
$\mathrm{C}=$ afis team & $\mathrm{D}=3 \mathrm{M}$ Cogent & $\mathrm{E}=$ Neurotechnology & $\mathrm{F}=$ Papillon & $\mathrm{G}=$ Dermalog & $\mathrm{H}=$ Hisign Bio-Info Institute \\
$\mathrm{I}=$ NEC & $\mathrm{J}=$ Sonda & $\mathrm{K}=$ Tiger IT & $\mathrm{L}=$ Innovatrics & $\mathrm{M}=$ SPEX & $\mathrm{O}=$ ID Solutions \\
$\mathrm{P}=\mathrm{id} 3$ & $\mathrm{Q}=$ Morpho & $\mathrm{S}=$ Decatur Industries & $\mathrm{T}=$ BIO-key & $\mathrm{U}=$ Aware & $\mathrm{V}=$ AA Technology
\end{tabular}




\begin{tabular}{|c|c|c|c|c|c|c|c|c|c|c|}
\hline & \multicolumn{2}{|c|}{ Participant } & \multicolumn{4}{|c|}{ Left Index } & \multicolumn{4}{|c|}{ Right Index } \\
\hline & \multirow{3}{*}{$\frac{\text { Letter }}{\mathrm{C}}$} & \multirow{2}{*}{$\frac{\text { Sub.\# }}{1}$} & \multicolumn{2}{|c|}{ Time } & \multicolumn{2}{|c|}{ FNIR } & \multicolumn{2}{|c|}{ Time } & \multicolumn{2}{|c|}{ FNIR } \\
\hline \multirow{30}{*}{ 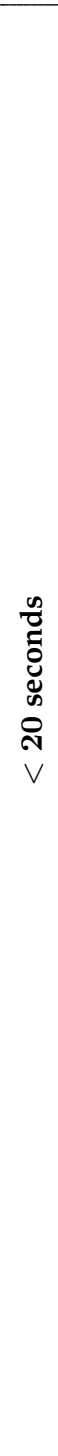 } & & & 1 & 0.26 & 24 & 0.1335 & 1 & 0.24 & 24 & 0.1132 \\
\hline & & 2 & 6 & 0.76 & 25 & 0.1337 & 6 & 0.69 & 23 & 0.1124 \\
\hline & $\mathrm{D}$ & 1 & 20 & 7.32 & 1 & 0.0197 & 20 & 7.46 & 1 & 0.0190 \\
\hline & \multirow{2}{*}{$\mathrm{E}$} & 1 & 3 & 0.35 & 12 & 0.0745 & 3 & 0.32 & 11 & 0.0630 \\
\hline & & 2 & 30 & 16.90 & 11 & 0.0723 & 29 & 16.27 & 10 & 0.0624 \\
\hline & \multirow{2}{*}{$\mathrm{F}$} & 1 & 11 & 2.49 & 20 & 0.1111 & 11 & 2.38 & 20 & 0.0933 \\
\hline & & 2 & 14 & 3.56 & 18 & 0.1082 & 15 & 3.60 & 18 & 0.0903 \\
\hline & G & 1 & 21 & 9.74 & 19 & 0.1089 & 24 & 10.10 & 19 & 0.0910 \\
\hline & \multirow{2}{*}{$\mathrm{H}$} & 1 & 15 & 3.61 & 26 & 0.1576 & 14 & 3.56 & 26 & 0.1230 \\
\hline & & 2 & 17 & 4.13 & 27 & 0.1607 & 17 & 4.05 & 27 & 0.1249 \\
\hline & I & 1 & 22 & 9.94 & 5 & 0.0257 & 22 & 9.83 & 3 & 0.0215 \\
\hline & \multirow{2}{*}{$\mathrm{J}$} & 1 & 4 & 0.54 & 14 & 0.0786 & 4 & 0.51 & 16 & 0.0708 \\
\hline & & 2 & 7 & 1.25 & 10 & 0.0712 & 7 & 1.13 & 12 & 0.0643 \\
\hline & \multirow{2}{*}{ K } & 1 & 25 & 10.44 & 17 & 0.0883 & 26 & 10.42 & 14 & 0.0682 \\
\hline & & 2 & 23 & 10.32 & 16 & 0.0875 & 25 & 10.27 & 15 & 0.0685 \\
\hline & \multirow{2}{*}{$\mathrm{L}$} & 1 & 2 & 0.29 & 8 & 0.0625 & 2 & 0.26 & 8 & 0.0505 \\
\hline & & 2 & 12 & 3.32 & 6 & 0.0351 & 13 & 3.26 & 6 & 0.0295 \\
\hline & \multirow{2}{*}{$\mathrm{M}$} & 1 & 10 & 1.84 & 29 & 0.2995 & 10 & 1.78 & 30 & 0.2615 \\
\hline & & 2 & 26 & 10.70 & 28 & 0.2921 & 21 & 9.39 & 29 & 0.2526 \\
\hline & \multirow{2}{*}{$\mathrm{O}$} & 1 & 5 & 0.62 & 15 & 0.0818 & 5 & 0.56 & 17 & 0.0776 \\
\hline & & 2 & 9 & 1.56 & 13 & 0.0766 & 9 & 1.36 & 13 & 0.0675 \\
\hline & \multirow{2}{*}{$\mathrm{P}$} & 1 & 8 & 1.32 & 23 & 0.1308 & 8 & 1.24 & 25 & 0.1133 \\
\hline & & 2 & 13 & 3.33 & 22 & 0.1272 & 12 & 3.07 & 22 & 0.1100 \\
\hline & \multirow{2}{*}{ Q } & 1 & 28 & 15.70 & 2 & 0.0222 & 28 & 14.24 & 4 & 0.0218 \\
\hline & & 2 & 24 & 10.43 & 3 & 0.0226 & 23 & 9.98 & 2 & 0.0214 \\
\hline & $S$ & 1 & 29 & 16.86 & 7 & 0.0571 & 30 & 16.55 & 7 & 0.0442 \\
\hline & \multirow{2}{*}{$\mathrm{T}$} & 1 & 16 & 3.99 & 30 & NA & 16 & 3.73 & 28 & 0.1929 \\
\hline & & 2 & 18 & 5.97 & 9 & 0.0685 & 19 & 5.87 & 9 & 0.0562 \\
\hline & $\mathrm{U}$ & 1 & 27 & 14.42 & 21 & 0.1218 & 27 & 10.76 & 21 & 0.0996 \\
\hline & $\mathrm{V}$ & 1 & 19 & 6.01 & 4 & 0.0253 & 18 & 5.60 & 5 & 0.0223 \\
\hline \multirow{6}{*}{ 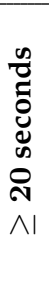 } & $\mathrm{D}$ & 2 & 3 & 41.84 & 1 & 0.0197 & 2 & 28.64 & 1 & 0.0190 \\
\hline & G & 2 & 5 & 54.03 & 5 & 0.1086 & 5 & 59.55 & 5 & 0.0909 \\
\hline & I & 2 & 2 & 40.99 & 3 & 0.0278 & 3 & 40.35 & 2 & 0.0214 \\
\hline & $S$ & 2 & 4 & 44.66 & 4 & 0.0650 & 4 & 46.05 & 4 & 0.0503 \\
\hline & $\mathrm{U}$ & 2 & 1 & 24.16 & 6 & 0.1178 & 1 & 20.10 & 6 & 0.1007 \\
\hline & $\mathrm{V}$ & 2 & 6 & 65.35 & 2 & 0.0252 & 6 & 61.24 & 3 & 0.0222 \\
\hline
\end{tabular}

Table 17: Tabulation of median identification times for Class A. Submissions were split into two groups. The first group includes submissions that performed searches on average in less than 20 seconds, and the second includes those that took, on average, 20 seconds or longer. Letter refers to the participant's letter code found on the footer of this page. Sub. \# is an identifier used to differentiate between the two submissions each participant could make. The Time column shows the time used to perform a search over an enrollment set of 100000 . Time values are median times reported in seconds, but were originally recorded to microsecond precision. The FNIR column shows FNIR for each submission at FPIR $=10^{-3}$. NA indicates that the operations required to produce the value could not be performed. The number to the left of a value provides the value's column-wise ranking, with the best performance shaded in green and the worst in pink.

\begin{tabular}{l|l|l|l|ll|l}
$\mathrm{C}=$ afis team & $\mathrm{D}=3 \mathrm{M}$ Cogent & $\mathrm{E}=$ Neurotechnology & $\mathrm{F}=$ Papillon & $\mathrm{G}=$ Dermalog & $\mathrm{H}=$ Hisign Bio-Info Institute \\
$\mathrm{I}=$ NEC & $\mathrm{J}=$ Sonda & $\mathrm{K}=$ Tiger IT & $\mathrm{L}=$ Innovatrics & $\mathrm{M}=$ SPEX & $\mathrm{O}=$ ID Solutions \\
$\mathrm{P}=\mathrm{id} 3$ & $\mathrm{Q}=$ Morpho & $\mathrm{S}=$ Decatur Industries & $\mathrm{T}=$ BIO-key & $\mathrm{U}=$ Aware & $\mathrm{V}=$ AA Technology
\end{tabular}




\begin{tabular}{|c|c|c|c|c|c|c|}
\hline & \multicolumn{2}{|c|}{ Participant } & \multicolumn{4}{|c|}{ Left and Right Index } \\
\hline & Letter & Sub.\# & \multicolumn{2}{|c|}{ Time } & \multicolumn{2}{|c|}{ FNIR } \\
\hline \multirow{9}{*}{ 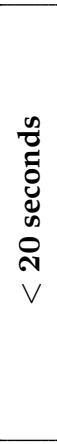 } & \multirow{2}{*}{$\mathrm{C}$} & 1 & 1 & 2.08 & 7 & 0.0368 \\
\hline & & 2 & 4 & 6.35 & 8 & 0.0374 \\
\hline & G & 1 & 3 & 5.22 & 9 & 0.0515 \\
\hline & I & 1 & 8 & 17.87 & 2 & 0.0058 \\
\hline & $\mathrm{J}$ & 1 & 6 & 13.64 & 3 & 0.0143 \\
\hline & $\mathrm{K}$ & 1 & 9 & 18.01 & 6 & 0.0360 \\
\hline & $\mathrm{L}$ & 1 & 2 & 2.19 & 4 & 0.0146 \\
\hline & $\mathrm{O}$ & 1 & 7 & 14.46 & 5 & 0.0229 \\
\hline & $\mathrm{V}$ & 1 & 5 & 9.29 & 1 & 0.0034 \\
\hline \multirow{27}{*}{ 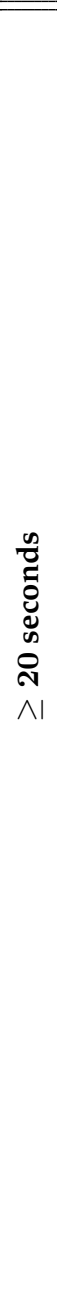 } & \multirow{2}{*}{$\mathrm{D}$} & 1 & 15 & 70.99 & 4 & 0.0030 \\
\hline & & 2 & 23 & 237.43 & 4 & 0.0030 \\
\hline & \multirow{2}{*}{$\mathrm{E}$} & 1 & 1 & 16.35 & 11 & 0.0207 \\
\hline & & 2 & 27 & 518.11 & 10 & 0.0202 \\
\hline & \multirow{2}{*}{$\mathrm{F}$} & 1 & 13 & 60.66 & 21 & 0.0386 \\
\hline & & 2 & 16 & 73.95 & 22 & 0.0412 \\
\hline & G & 2 & 22 & 221.16 & 15 & 0.0311 \\
\hline & \multirow{2}{*}{$\mathrm{H}$} & 1 & 8 & 36.71 & 24 & 0.0686 \\
\hline & & 2 & 12 & 52.25 & 23 & 0.0684 \\
\hline & I & 2 & 25 & 338.88 & 4 & 0.0030 \\
\hline & $\mathrm{J}$ & 2 & 7 & 33.35 & 8 & 0.0143 \\
\hline & $\mathrm{K}$ & 2 & 6 & 32.93 & 14 & 0.0286 \\
\hline & $\mathrm{L}$ & 2 & 3 & 23.42 & 7 & 0.0072 \\
\hline & \multirow{2}{*}{ M } & 1 & 5 & 31.44 & 26 & NA \\
\hline & & 2 & 20 & 171.24 & 25 & NA \\
\hline & $\mathrm{O}$ & 2 & 10 & 43.53 & 12 & 0.0214 \\
\hline & \multirow{2}{*}{$\mathrm{P}$} & 1 & 14 & 63.65 & 20 & 0.0370 \\
\hline & & 2 & 17 & 101.16 & 16 & 0.0333 \\
\hline & \multirow{2}{*}{ Q } & 1 & 21 & 212.69 & 1 & 0.0027 \\
\hline & & 2 & 19 & 161.02 & 1 & 0.0027 \\
\hline & \multirow{2}{*}{ S } & 1 & 2 & 23.00 & 13 & 0.0281 \\
\hline & & 2 & 26 & 495.50 & 9 & 0.0195 \\
\hline & \multirow{2}{*}{$\mathrm{T}$} & 1 & 4 & 25.68 & 27 & NA \\
\hline & & 2 & 9 & 37.23 & 19 & 0.0366 \\
\hline & \multirow{2}{*}{$\mathrm{U}$} & 1 & 11 & 45.51 & 17 & 0.0336 \\
\hline & & 2 & 24 & 240.40 & 18 & 0.0358 \\
\hline & $\mathrm{V}$ & 2 & 18 & 127.65 & 3 & 0.0028 \\
\hline
\end{tabular}

Table 18: Tabulation of median identification times for Class A. Submissions were split into two groups. The first group includes submissions that performed searches on average in less than 20 seconds, and the second includes those that took, on average, 20 seconds or longer. Letter refers to the participant's letter code found on the footer of this page. Sub. \# is an identifier used to differentiate between the two submissions each participant could make. The Time column shows the time used to perform a search over an enrollment set of 1600000 . Time values are median times reported in seconds, but were originally recorded to microsecond precision. The FNIR column shows FNIR for each submission at FPIR $=10^{-3}$. NA indicates that the operations required to produce the value could not be performed. The number to the left of a value provides the value's column-wise ranking, with the best performance shaded in green and the worst in pink.

\begin{tabular}{l|l|l|l|ll|l}
$\mathrm{C}=$ afis team & $\mathrm{D}=3 \mathrm{M}$ Cogent & $\mathrm{E}=$ Neurotechnology & $\mathrm{F}=$ Papillon & $\mathrm{G}=$ Dermalog & $\mathrm{H}=$ Hisign Bio-Info Institute \\
$\mathrm{I}=$ NEC & $\mathrm{J}=$ Sonda & $\mathrm{K}=$ Tiger IT & $\mathrm{L}=$ Innovatrics & $\mathrm{M}=$ SPEX & $\mathrm{O}=$ ID Solutions \\
$\mathrm{P}=\mathrm{id} 3$ & $\mathrm{Q}=$ Morpho & $\mathrm{S}=$ Decatur Industries & $\mathrm{T}=$ BIO-key & $\mathrm{U}=$ Aware & $\mathrm{V}=$ AA Technology
\end{tabular}




\subsection{Class B}

Tabulated comparisons of identification times for IDFlat submissions are shown in Table 19. The search times shown in this table are from the "Total/One" column in Tables 30 through 33 included in Appendix C. For reference, the FNIR values from Section 7 are reprinted to the right of the identification times.

The tables were used to create scatter plots showing accuracy, search times, and search template creation times. Those plots are shown in Figures 36 through 39.

Some observations for Class B identification times include:

$\triangleright$ Most submissions had some improvement in accuracy with longer search times.

$\triangleright$ Results vary, but some submissions (D, G, I1, Q1) performed searches faster when more fingers were available, while others $(\mathbf{H}, \mathbf{L}, \mathbf{S})$ required longer search times with more fingers.

$\triangleright$ Most search times increased modestly when ten processes were running in parallel, compared to the single process timing test. See Appendix C and Appendix D for complete details.

$\triangleright$ Tables 30 through 33 in Appendix E and Figures 40 through 43 show differences between the last two rounds of submissions. There are a variety of results in these tables. Again, most had improvement in FNIRs, but some with longer search times and some with dramatically shorter search times. Like Class A, there are certainly indications that high accuracy can be achieved with some fast submissions.

\begin{tabular}{l|l|l|l|l|l|l}
$\mathrm{C}=$ afis team & $\mathrm{D}=3 \mathrm{M}$ Cogent & $\mathrm{E}=$ Neurotechnology & $\mathrm{F}=$ Papillon & $\mathrm{G}=$ Dermalog & $\mathrm{H}=$ Hisign Bio-Info Institute \\
$\mathrm{I}=\mathrm{NEC}$ & $\mathrm{J}=$ Sonda & $\mathrm{K}=$ Tiger IT & $\mathrm{L}=$ Innovatrics & $\mathrm{M}=$ SPEX & $\mathrm{O}=$ ID Solutions \\
$\mathrm{P}=\mathrm{id} 3$ & $\mathrm{Q}=$ Morpho & $\mathrm{S}=$ Decatur Industries & $\mathrm{T}=$ BIO-key & $\mathrm{U}=$ Aware & $\mathrm{V}=$ AA Technology
\end{tabular}




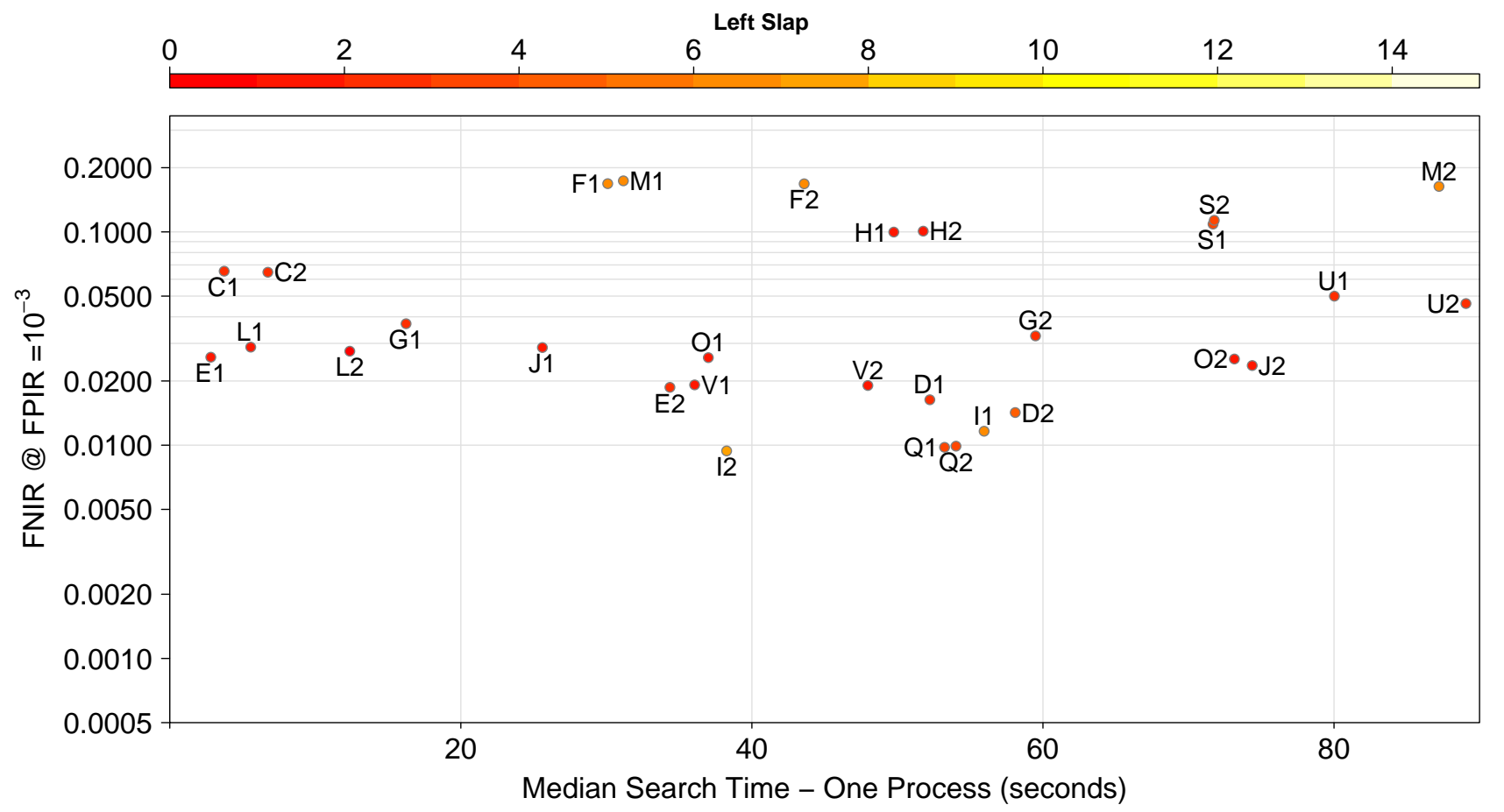

Figure 36: Scatter plot of FNIR @ FPIR $=10^{-3}$ searching 30000 subjects against 3000000 subjects and median search time for a single process for Class B - Left Slap. The color of the data point is used to show the search template creation time. The color scale for search template creation time is at the top of the plot. Median search times are plotted in seconds. The FNIR and median search time data are from Table 19 and search template creation times can be found in Table 75 in Appendix H.

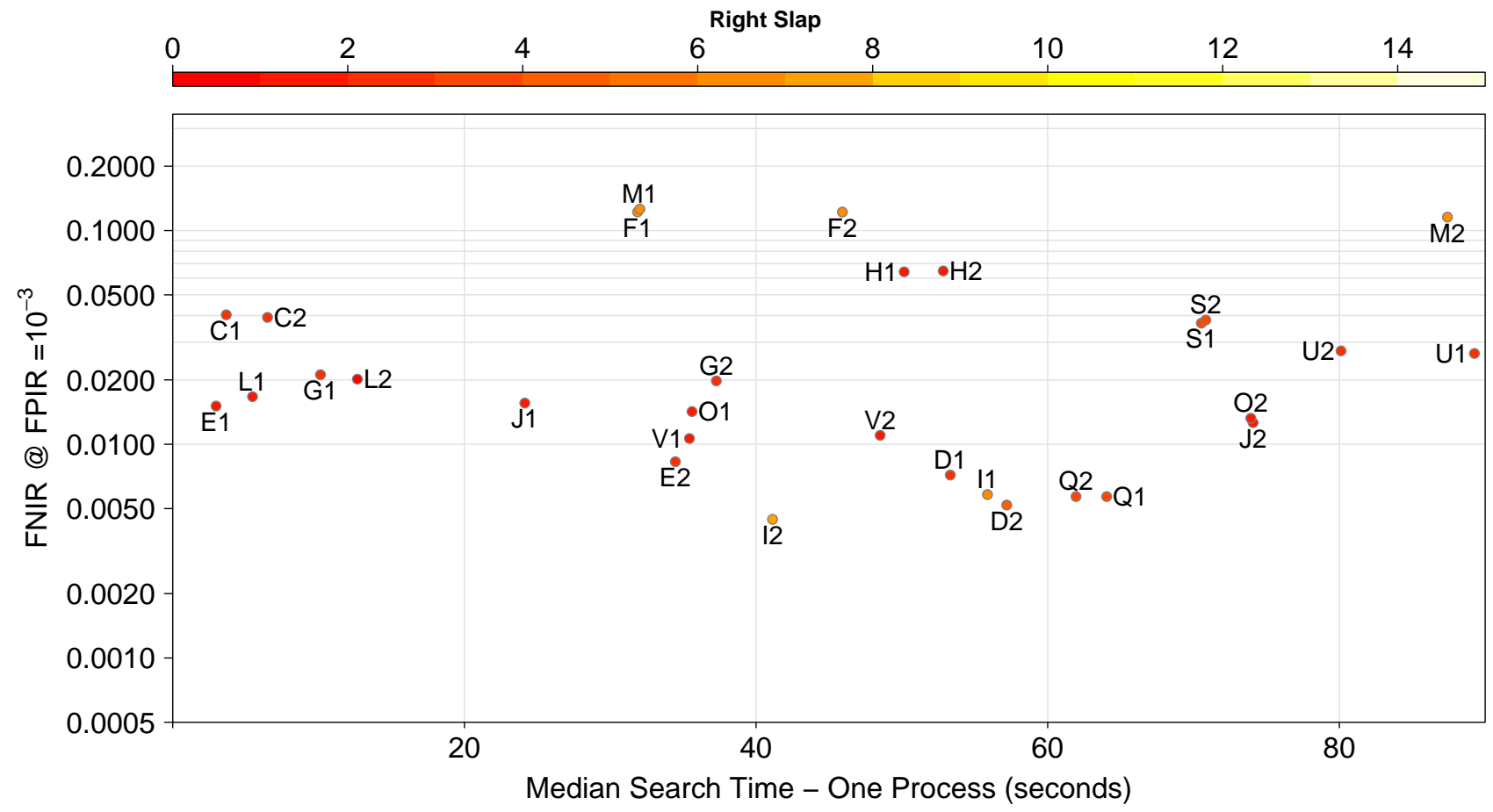

Figure 37: Scatter plot of FNIR @ FPIR $=10^{-3}$ searching 30000 subjects against 3000000 subjects and median search time for a single process for Class B - Right Slap. The color of the data point is used to show the search template creation time. The color scale for search template creation time is at the top of the plot. Median search times are plotted in seconds. The FNIR and median search time data are from Table 19 and search template creation times can be found in Table 76 in Appendix H.

\begin{tabular}{l|l|l|l|l|l|l}
$\mathrm{C}=$ afis team & $\mathrm{D}=3 \mathrm{M}$ Cogent & $\mathrm{E}=$ Neurotechnology & $\mathrm{F}=$ Papillon & $\mathrm{G}=$ Dermalog & $\mathrm{H}=$ Hisign Bio-Info Institute \\
$\mathrm{I}=$ NEC & $\mathrm{J}=$ Sonda & $\mathrm{K}=$ Tiger IT & $\mathrm{L}=$ Innovatrics & $\mathrm{M}=$ SPEX & $\mathrm{O}=$ ID Solutions \\
$\mathrm{P}=\mathrm{id} 3$ & $\mathrm{Q}=$ Morpho & $\mathrm{S}=$ Decatur Industries & $\mathrm{T}=$ BIO-key & $\mathrm{U}=$ Aware & $\mathrm{V}=$ AA Technology
\end{tabular}




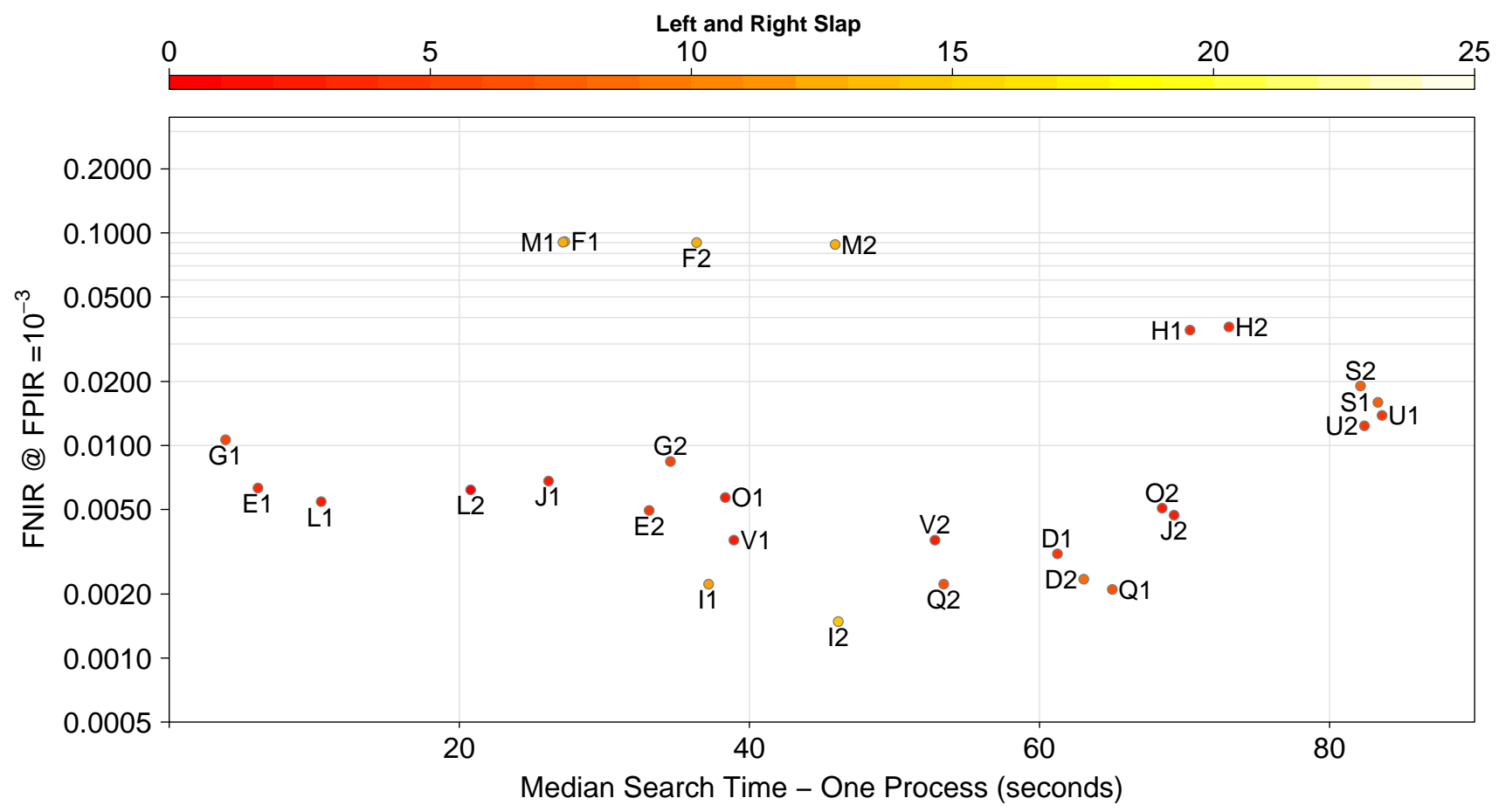

Figure 38: Scatter plot of FNIR @ FPIR $=10^{-3}$ searching 30000 subjects against 3000000 subjects and median search time for a single process for Class B - Left and Right Slap. The color of the data point is used to show the search template creation time. The color scale for search template creation time is at the top of the plot. Median search times are plotted in seconds. The FNIR and median search time data are from Table 19 and search template creation times can be found in Table 77 in Appendix H.

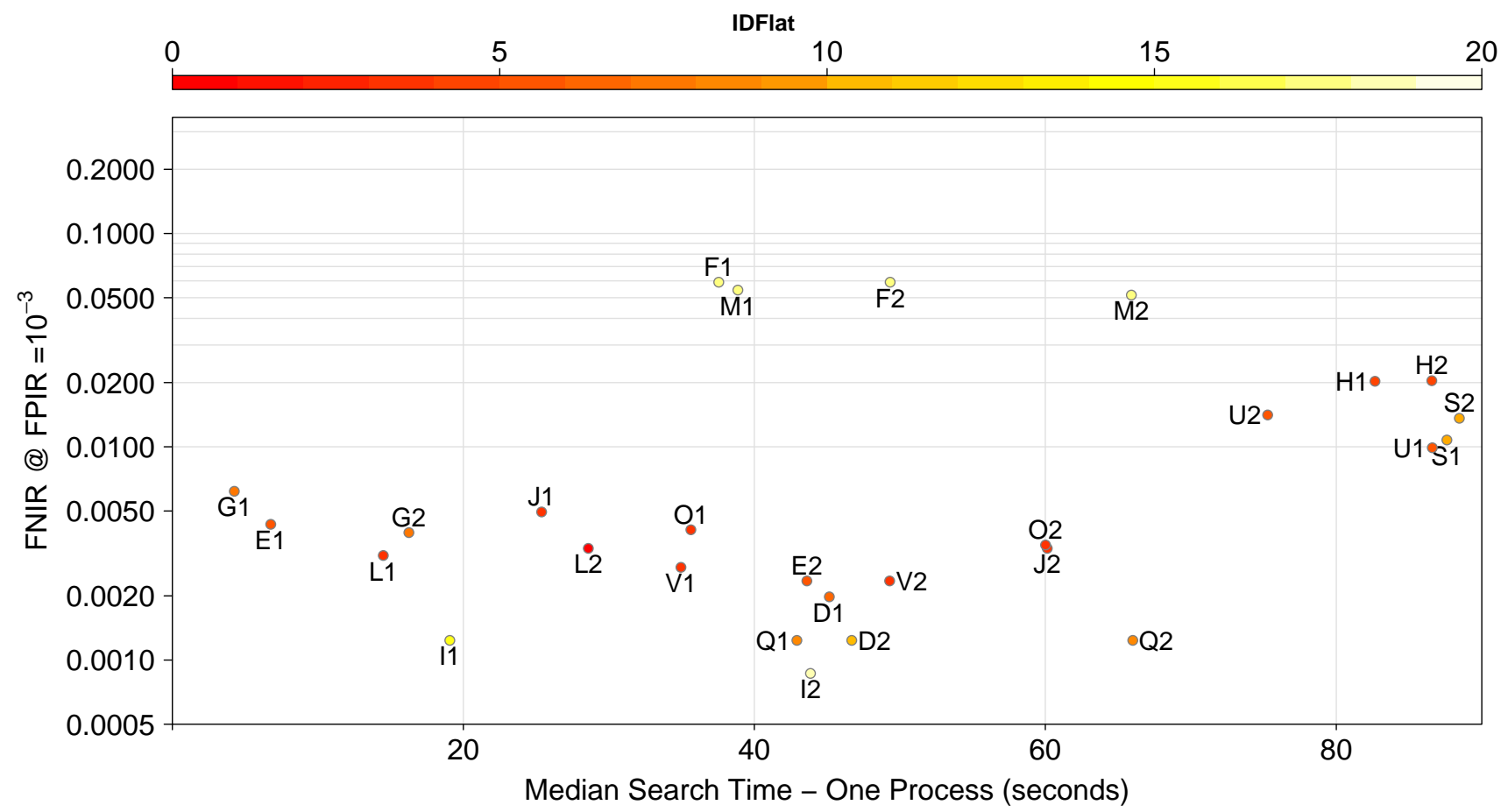

Figure 39: Scatter plot of FNIR @ FPIR $=10^{-3}$ searching 30000 subjects against 3000000 subjects and median search time for a single process for Class $\mathrm{B}$ - Identification Flats. The color of the data point is used to show the search template creation time. The color scale for search template creation time is at the top of the plot. Median search times are plotted in seconds. The FNIR and median search time data are from Table 19 and search template creation times can be found in Table 78 in Appendix $\mathrm{H}$.

\begin{tabular}{l|l|l|l|l|l|l}
$\mathrm{C}=$ afis team & $\mathrm{D}=$ = 3M Cogent & $\mathrm{E}=$ Neurotechnology & $\mathrm{F}=$ Papillon & $\mathrm{G}=$ Dermalog & $\mathrm{H}=$ Hisign Bio-Info Institute \\
$\mathrm{I}=$ NEC & $\mathrm{J}=$ Sonda & $\mathrm{K}=$ Tiger IT & $\mathrm{L}=$ Innovatrics & $\mathrm{M}=$ SPEX & $\mathrm{O}=$ ID Solutions \\
$\mathrm{P}=\mathrm{id} 3$ & $\mathrm{Q}=$ Morpho & $\mathrm{S}=$ Decatur Industries & $\mathrm{T}=$ BIO-key & $\mathrm{U}=$ Aware & $\mathrm{V}=$ AA Technology
\end{tabular}




\section{Left Slap}

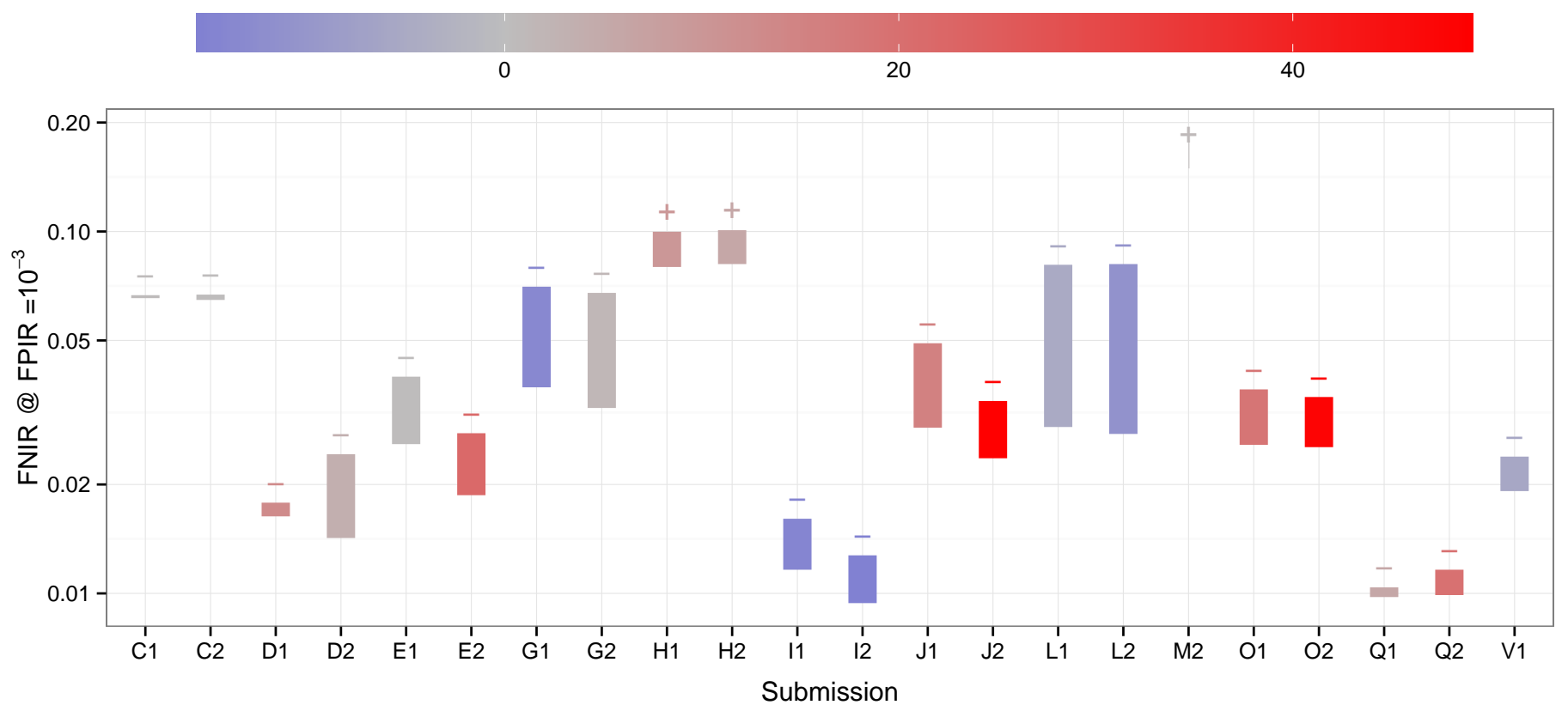

Figure 40: Plots showing difference in FNIR @ FPIR $=10^{-3}$ searching 30000 subjects against 3000000 subjects and difference in search times, for a single search process, between round 2 and round 3 submissions for Class B - Left Slap. The " + " symbol indicates that FNIR increased from round 2 to round 3 and "-" indicates a decrease in FNIR. The color of the bar shows the change in search time. The color scale for difference in search time is at the top of the plot and the units are in seconds. The data for the plots are taken from the tables in Appendix E.

\section{Right Slap}

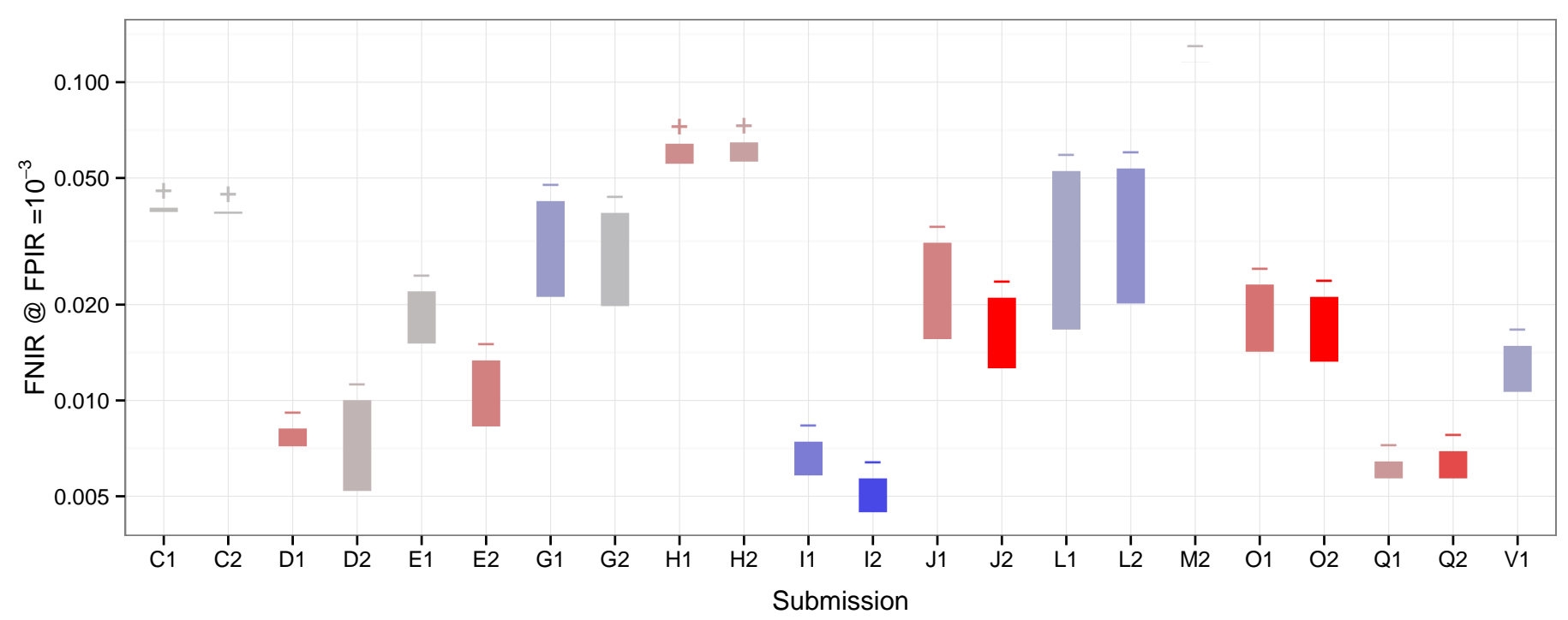

Figure 41: Plots showing difference in FNIR @ FPIR $=10^{-3}$ searching 30000 subjects against 3000000 subjects and difference in search times, for a single search process, between round 2 and round 3 submissions for Class B - Right Slap. The " + " symbol indicates that FNIR increased from round 2 to round 3 and "-" indicates a decrease in FNIR. The color of the bar shows the change in search time. The color scale for difference in search time is at the top of the plot and the units are in seconds. The data for the plots are taken from the tables in Appendix E.

\begin{tabular}{l|l|l|l|l|l|l}
$\mathrm{C}=$ afis team & $\mathrm{D}=$ = 3M Cogent & $\mathrm{E}=$ Neurotechnology & $\mathrm{F}=$ Papillon & $\mathrm{G}=$ Dermalog & $\mathrm{H}=$ Hisign Bio-Info Institute \\
$\mathrm{I}=$ NEC & $\mathrm{J}=$ Sonda & $\mathrm{K}=$ Tiger IT & $\mathrm{L}=$ Innovatrics & $\mathrm{M}=$ SPEX & $\mathrm{O}=$ ID Solutions \\
$\mathrm{P}=\mathrm{id} 3$ & $\mathrm{Q}=$ Morpho & $\mathrm{S}=$ Decatur Industries & $\mathrm{T}=$ BIO-key & $\mathrm{U}=$ Aware & $\mathrm{V}=$ AA Technology
\end{tabular}




\section{Left and Right Slap}

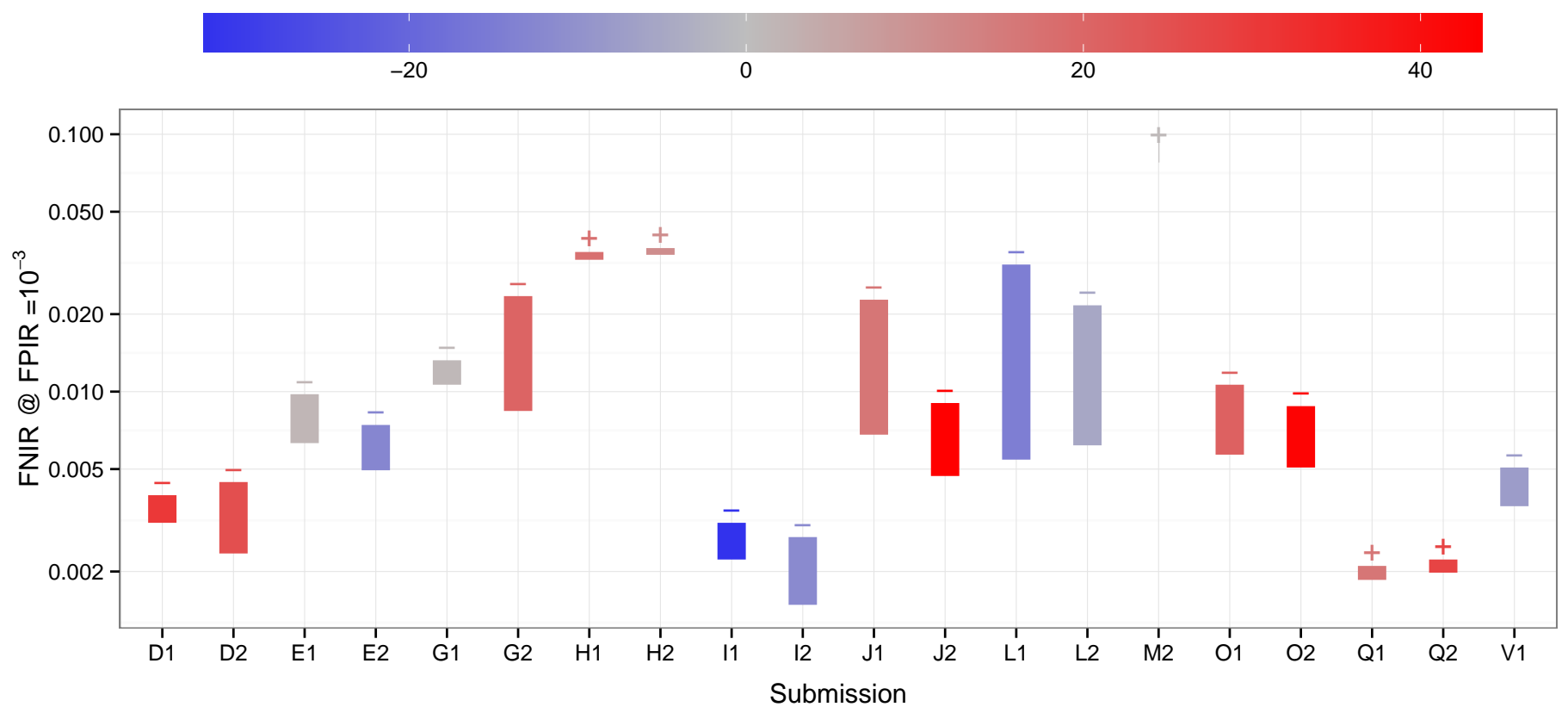

Figure 42: Plots showing difference in FNIR @ FPIR $=10^{-3}$ searching 30000 subjects against 3000000 subjects and difference in search times, for a single search process, between round 2 and round 3 submissions for Class B - Left and Right Slap. The "+" symbol indicates that FNIR increased from round 2 to round 3 and "-" indicates a decrease in FNIR. The color of the bar shows the change in search time. The color scale for difference in search time is at the top of the plot and the units are in seconds. The data for the plots are taken from the tables in Appendix E.

\section{Identification Flats}

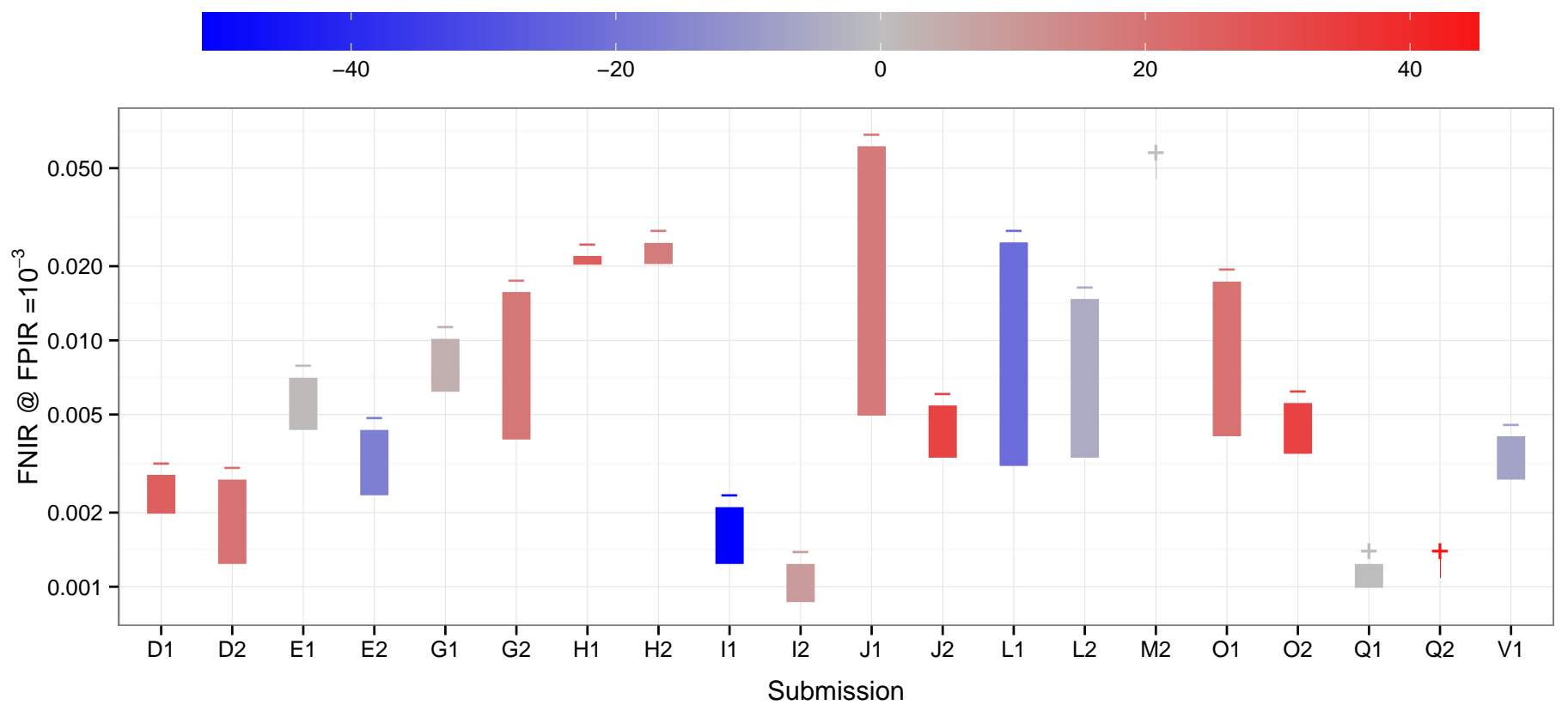

Figure 43: Plots showing difference in FNIR @ FPIR $=10^{-3}$ searching 30000 subjects against 3000000 subjects and difference in search times, for a single search process, between round 2 and round 3 submissions for Class B - IDFlat. The " + " symbol indicates that FNIR increased from round 2 to round 3 and " - " indicates a decrease in FNIR. The color of the bar shows the change in search time. The color scale for difference in search time is at the top of the plot and the units are in seconds. The data for the plots are taken from the tables in Appendix E.

\begin{tabular}{l|l|l|l|l|l|l}
$\mathrm{C}=$ afis team & $\mathrm{D}=3 \mathrm{M}$ Cogent & $\mathrm{E}=$ Neurotechnology & $\mathrm{F}=$ Papillon & $\mathrm{G}=$ Dermalog & $\mathrm{H}=$ Hisign Bio-Info Institute \\
$\mathrm{I}=$ NEC & $\mathrm{J}=$ Sonda & $\mathrm{K}=$ Tiger IT & $\mathrm{L}=$ Innovatrics & $\mathrm{M}=$ SPEX & $\mathrm{O}=$ ID Solutions \\
$\mathrm{P}=\mathrm{id} 3$ & $\mathrm{Q}=$ Morpho & $\mathrm{S}=$ Decatur Industries & $\mathrm{T}=$ BIO-key & $\mathrm{U}=$ Aware & $\mathrm{V}=$ AA Technology
\end{tabular}




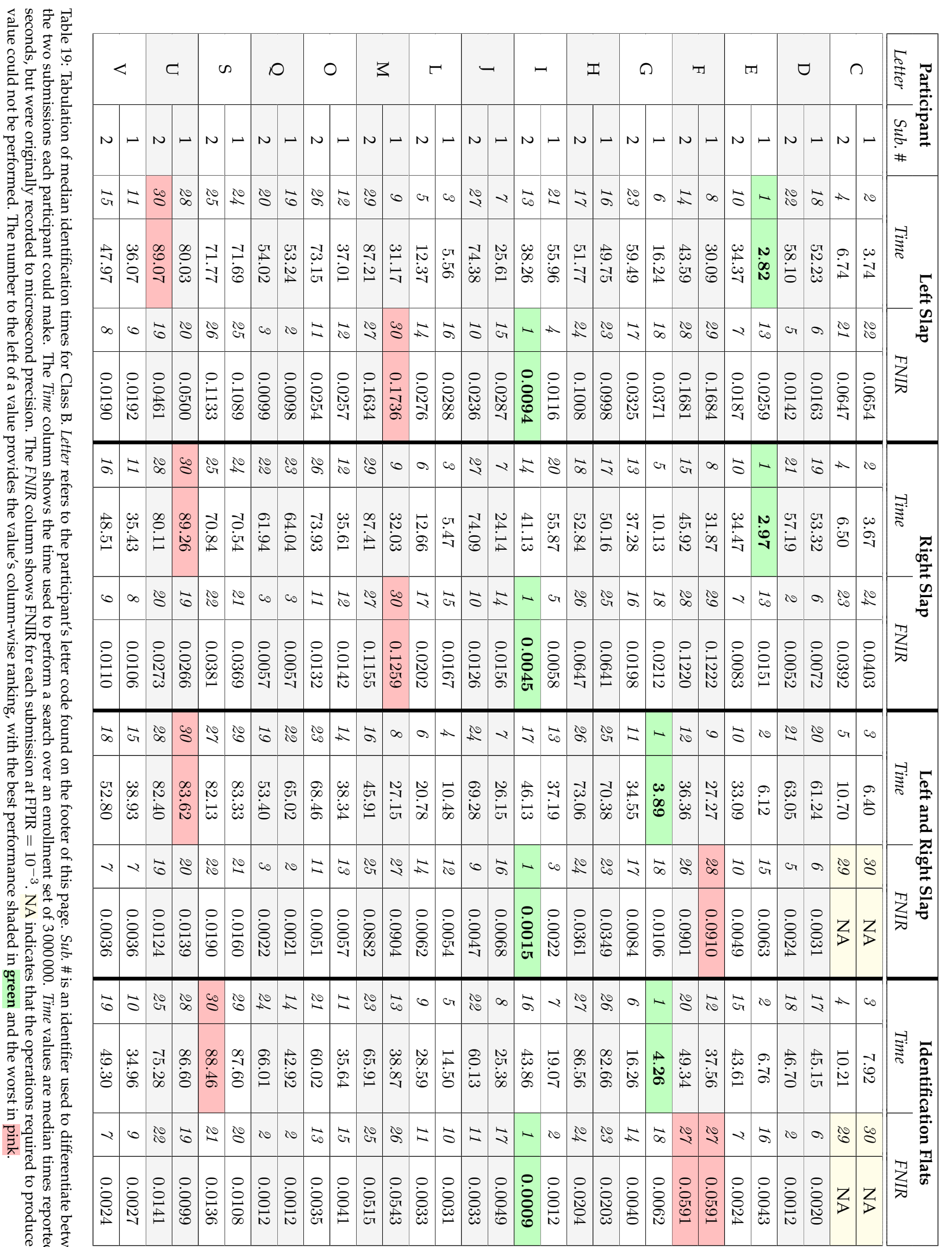

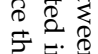

$\mathrm{C}=$ afis team $\mathrm{D}=3 \mathrm{M}$ Cogent $\mid \mathrm{E}=$ Neurotechnology $\mid \mathrm{F}=$ Papillon $\mid \mathrm{G}=$ Dermalog $\mid \mathrm{H}=$ Hisign Bio-Info Institute

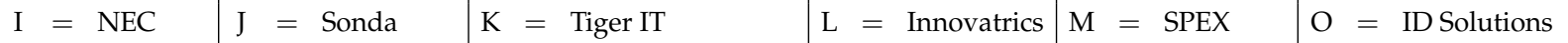

\begin{tabular}{l|l|l|l|l}
$\mathrm{P}=\mathrm{id} 3$ & $\mathrm{Q}=$ Morpho & $\mathrm{S}=$ Decatur Industries $\mathrm{T}=$ BIO-key & $\mathrm{U}=$ Aware & $\mathrm{V}=$ AA Technology
\end{tabular} 


\subsection{Class C}

Tabulated comparisons of identification times for ten-finger identification submissions are shown in Table 20. The search times shown in this table are from the "Total/One" column in Tables 34 through 36 included in Appendix C. For reference, FNIR values from Section 7 are reprinted to the right of the identification times.

The tables were used to create scatter plots showing accuracy, search times, and search template creation times. Those plots are shown in Figure 44.

Some observations for Class $\mathrm{C}$ identification times include:

$\triangleright$ Like classes A and B, gains varied across the participants, but most had some level of improvement in accuracy with longer search times.

$\triangleright$ Results for some submissions varied between ten-finger plain-to-plain and ten-finger rolled-to-rolled impressions. Some were faster with plain impressions and others were faster with rolled impressions. The most accurate submissions appeared to match both types accurately.

$\triangleright$ Tables 34 through 36 in Appendix E and Figure 45 show differences between the last two rounds of submissions. Like classes A and B, these results indicate that high accuracy can be achieved with some fast submissions, but the absolute best accuracy was not the fastest submission.

\begin{tabular}{l|l|l|l|l|l|l}
$\mathrm{C}=$ afis team & $\mathrm{D}=3 \mathrm{M}$ Cogent & $\mathrm{E}=$ Neurotechnology & $\mathrm{F}=$ Papillon & $\mathrm{G}=$ Dermalog & $\mathrm{H}=$ Hisign Bio-Info Institute \\
$\mathrm{I}=\mathrm{NEC}$ & $\mathrm{J}=$ Sonda & $\mathrm{K}=$ Tiger IT & $\mathrm{L}=$ Innovatrics & $\mathrm{M}=$ SPEX & $\mathrm{O}=$ ID Solutions \\
$\mathrm{P}=$ id3 & $\mathrm{Q}=$ Morpho & $\mathrm{S}=$ Decatur Industries & $\mathrm{T}=$ BIO-key & $\mathrm{U}=$ Aware & $\mathrm{V}=$ AA Technology
\end{tabular}



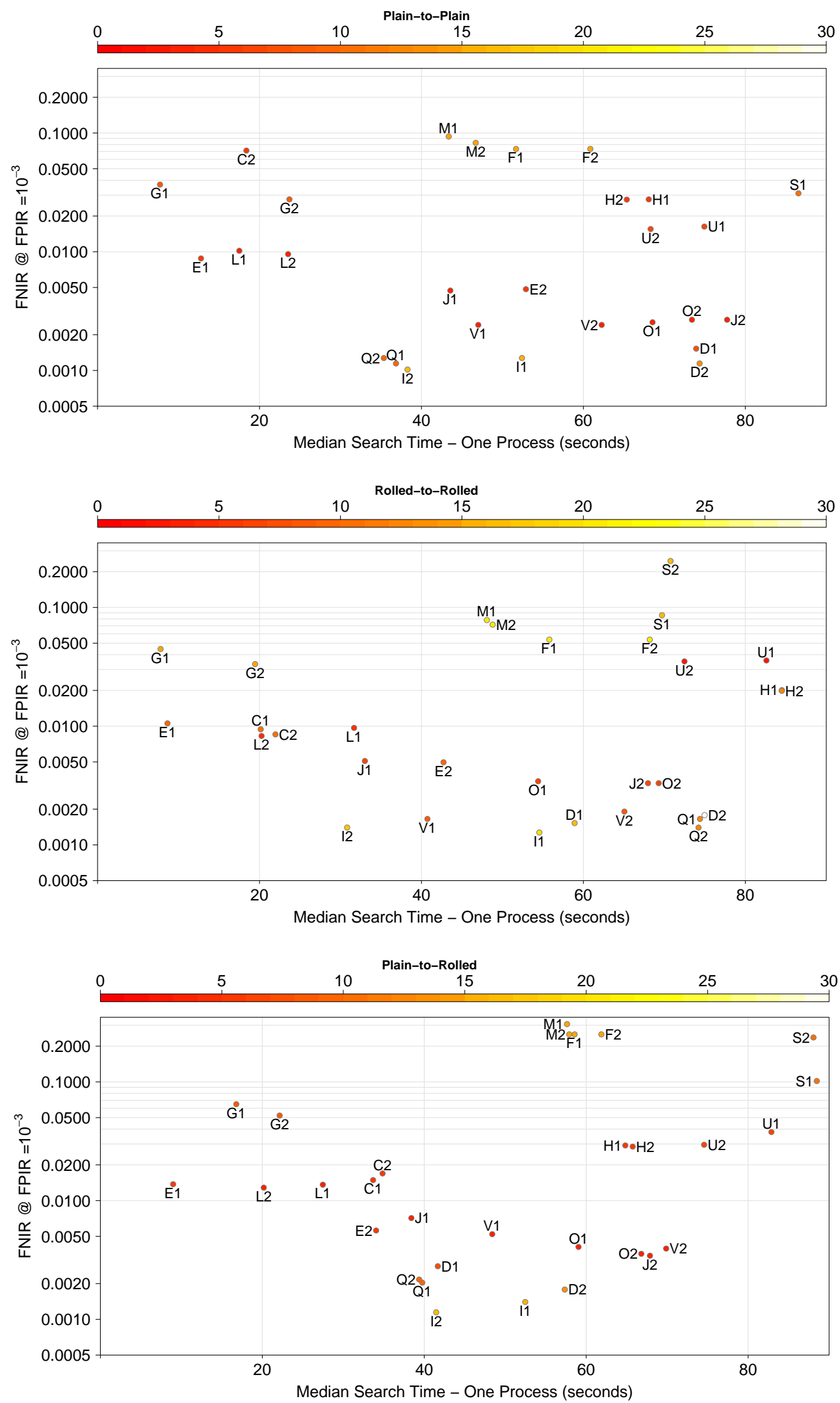

Figure 44: Scatter plot of FNIR @ FPIR $=10^{-3}$ searching 30000 subjects against 5000000 subjects and median search time for a single process for Class C. The color of the data point is used to show the search template creation time. The color scale for search template creation time is at the top of the plot. Median search times are plotted in seconds. The FNIR and median search time data are from Table 20 and search template creation times can be found in Tables 79 and 80 in Appendix H.

\begin{tabular}{l|l|l|l|ll|l}
$\mathrm{C}=$ afis team & $\mathrm{D}=3 \mathrm{M}$ Cogent & $\mathrm{E}=$ Neurotechnology & $\mathrm{F}=$ Papillon & $\mathrm{G}=$ Dermalog & $\mathrm{H}=$ Hisign Bio-Info Institute \\
$\mathrm{I}=$ NEC & $\mathrm{J}=$ Sonda & $\mathrm{K}=$ Tiger IT & $\mathrm{L}=$ Innovatrics & $\mathrm{M}=$ SPEX & $\mathrm{O}=$ ID Solutions \\
$\mathrm{P}=\mathrm{id} 3$ & $\mathrm{Q}=$ Morpho & $\mathrm{S}=$ Decatur Industries & $\mathrm{T}=$ BIO-key & $\mathrm{U}=$ Aware & $\mathrm{V}=$ AA Technology
\end{tabular}



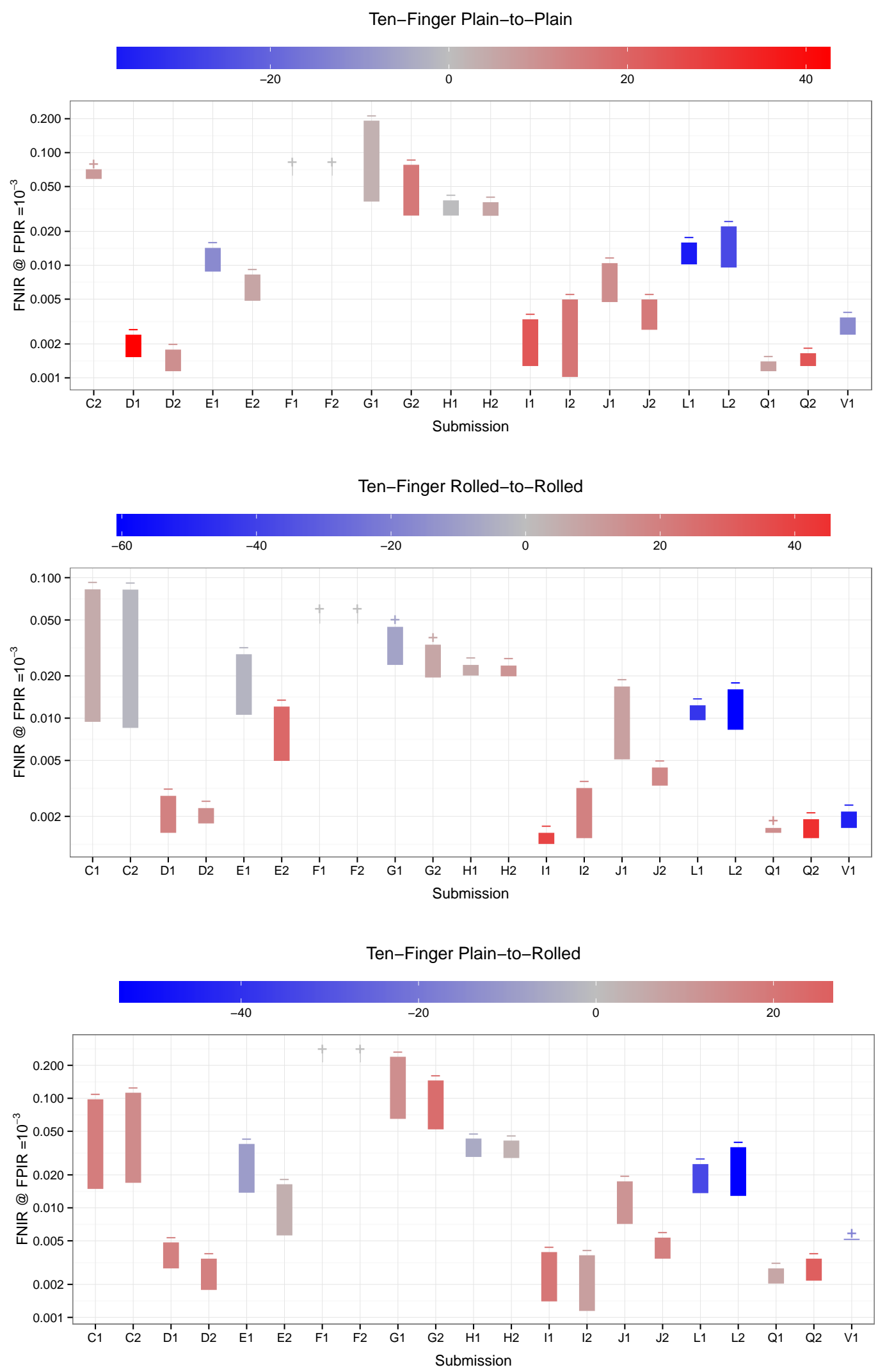

Figure 45: Plots showing difference in FNIR @ FPIR $=10^{-3}$ searching 30000 subjects against 5000000 subjects and difference in search times, for a single search process, between round 2 and round 3 submissions for Class $C$. The " + " symbol indicates that FNIR increased from round 2 to round 3 and " - " indicates a decrease in FNIR. The color of the bar shows the change in search time. The color scale for difference in search time is at the top of the plot and the units are in seconds. The data for the plots are taken from the tables in Appendix E.

\begin{tabular}{l|l|l|l|ll|l}
$\mathrm{C}=$ afis team & $\mathrm{D}=3 \mathrm{M}$ Cogent & $\mathrm{E}=$ Neurotechnology & $\mathrm{F}=$ Papillon & $\mathrm{G}=$ Dermalog & $\mathrm{H}=$ Hisign Bio-Info Institute \\
$\mathrm{I}=$ NEC & $\mathrm{J}=$ Sonda & $\mathrm{K}=$ Tiger IT & $\mathrm{L}=$ Innovatrics & $\mathrm{M}=$ SPEX & $\mathrm{O}=$ ID Solutions \\
$\mathrm{P}=\mathrm{id} 3$ & $\mathrm{Q}=$ Morpho & $\mathrm{S}=$ Decatur Industries & $\mathrm{T}=$ BIO-key & $\mathrm{U}=$ Aware & $\mathrm{V}=$ AA Technology
\end{tabular}




\begin{tabular}{|c|c|c|c|c|c|c|c|c|c|c|c|c|c|}
\hline \multicolumn{2}{|c|}{ Participant } & \multicolumn{4}{|c|}{ Ten-Finger Plain-to-Plain } & \multicolumn{4}{|c|}{ Ten-Finger Rolled-to-Rolled } & \multicolumn{4}{|c|}{ Ten-Finger Plain-to-Rolled } \\
\hline Letter & Sub. \# & \multicolumn{2}{|c|}{ Time } & \multicolumn{2}{|c|}{ FNIR } & \multicolumn{2}{|c|}{ Time } & \multicolumn{2}{|c|}{ FNIR } & \multicolumn{2}{|c|}{ Time } & \multicolumn{2}{|c|}{ FNIR } \\
\hline \multirow{2}{*}{ C } & 1 & 4 & 18.30 & 30 & NA & 4 & 20.15 & 16 & 0.0094 & 6 & 33.68 & 17 & 0.0149 \\
\hline & 2 & 5 & 18.38 & 24 & 0.0711 & 6 & 21.97 & 15 & 0.0085 & 8 & 34.85 & 18 & 0.0169 \\
\hline \multirow{2}{*}{$\mathrm{D}$} & 1 & 25 & 73.93 & 6 & 0.0015 & 17 & 58.92 & 4 & 0.0015 & 13 & 41.67 & 6 & 0.0028 \\
\hline & 2 & 26 & 74.36 & 2 & 0.0011 & 27 & 74.97 & 7 & 0.0018 & 16 & 57.36 & 3 & 0.0018 \\
\hline \multirow{2}{*}{$\mathrm{E}$} & 1 & 2 & 12.77 & 14 & 0.0088 & 2 & 8.63 & 18 & 0.0106 & 1 & 8.99 & 16 & 0.0137 \\
\hline & 2 & 17 & 52.91 & 13 & 0.0048 & 11 & 42.73 & 12 & 0.0050 & 7 & 34.06 & 12 & 0.0056 \\
\hline \multirow{2}{*}{$\mathrm{F}$} & 1 & 15 & 51.69 & 25 & 0.0734 & 16 & 55.79 & 25 & 0.0536 & 19 & 58.57 & 27 & 0.2514 \\
\hline & 2 & 18 & 60.86 & 25 & 0.0734 & 20 & 68.20 & 25 & 0.0536 & 21 & 61.87 & 27 & 0.2514 \\
\hline \multirow{2}{*}{ G } & 1 & 1 & 7.71 & 23 & 0.0368 & 1 & 7.78 & 24 & 0.0447 & 2 & 16.78 & 24 & 0.0649 \\
\hline & 2 & 7 & 23.69 & 20 & 0.0276 & 3 & 19.46 & 21 & 0.0333 & 4 & 22.14 & 23 & 0.0521 \\
\hline \multirow{2}{*}{$\mathrm{H}$} & 1 & 21 & 68.07 & 20 & 0.0276 & 30 & 84.51 & 20 & 0.0201 & 22 & 64.83 & 20 & 0.0291 \\
\hline & 2 & 20 & 65.36 & 19 & 0.0275 & 29 & 84.50 & 19 & 0.0199 & 23 & 65.74 & 19 & 0.0285 \\
\hline \multirow{2}{*}{ I } & 1 & 16 & 52.41 & 4 & 0.0013 & 15 & 54.56 & 1 & 0.0013 & 15 & 52.47 & 2 & 0.0014 \\
\hline & 2 & 10 & 38.28 & 1 & 0.0010 & 7 & 30.82 & 2 & 0.0014 & 12 & 41.48 & 1 & 0.0011 \\
\hline \multirow{2}{*}{$\mathrm{J}$} & 1 & 12 & 43.57 & 12 & 0.0047 & 9 & 33.02 & 13 & 0.0051 & 9 & 38.41 & 13 & 0.0071 \\
\hline & 2 & 28 & 77.75 & 10 & 0.0027 & 19 & 67.98 & 9 & 0.0033 & 25 & 67.88 & 7 & 0.0034 \\
\hline \multirow{2}{*}{$\mathrm{L}$} & 1 & 3 & 17.51 & 16 & 0.0102 & 8 & 31.68 & 17 & 0.0097 & 5 & 27.47 & 15 & 0.0136 \\
\hline & 2 & 6 & 23.53 & 15 & 0.0095 & 5 & 20.25 & 14 & 0.0083 & 3 & 20.18 & 14 & 0.0129 \\
\hline \multirow{2}{*}{ M } & 1 & 11 & 43.37 & 28 & 0.0934 & 12 & 48.08 & 28 & 0.0783 & 17 & 57.63 & 30 & 0.3067 \\
\hline & 2 & 13 & 46.70 & 27 & 0.0826 & 13 & 48.80 & 27 & 0.0716 & 18 & 57.91 & 27 & 0.2514 \\
\hline \multirow{2}{*}{$\mathrm{O}$} & 1 & 23 & 68.54 & 9 & 0.0025 & 14 & 54.42 & 11 & 0.0034 & 20 & 59.05 & 10 & 0.0041 \\
\hline & 2 & 24 & 73.41 & 10 & 0.0027 & 21 & 69.30 & 9 & 0.0033 & 24 & 66.80 & 8 & 0.0036 \\
\hline \multirow{2}{*}{ Q } & 1 & 9 & 36.86 & 2 & 0.0011 & 26 & 74.40 & 5 & 0.0017 & 11 & 39.76 & 4 & 0.0020 \\
\hline & 2 & 8 & 35.35 & 4 & 0.0013 & 25 & 74.22 & 2 & 0.0014 & 10 & 39.37 & 5 & 0.0022 \\
\hline \multirow{2}{*}{ S } & 1 & 29 & 86.56 & 22 & 0.0311 & 22 & 69.71 & 29 & 0.0860 & 30 & 88.48 & 25 & 0.1017 \\
\hline & 2 & 30 & 91.74 & 29 & 0.1680 & 23 & 70.77 & 30 & 0.2462 & 29 & 88.07 & 26 & 0.2366 \\
\hline \multirow{2}{*}{$\mathrm{U}$} & 1 & 27 & 74.94 & 18 & 0.0163 & 28 & 82.61 & 23 & 0.0358 & 28 & 82.88 & 22 & 0.0378 \\
\hline & 2 & 22 & 68.30 & 17 & 0.0155 & 24 & 72.48 & 22 & 0.0351 & 27 & 74.56 & 21 & 0.0295 \\
\hline \multirow{2}{*}{ V } & 1 & 14 & 47.02 & 7 & 0.0024 & 10 & 40.74 & 5 & 0.0017 & 14 & 48.40 & 11 & 0.0052 \\
\hline & 2 & 19 & 62.26 & 7 & 0.0024 & 18 & 65.07 & 8 & 0.0019 & 26 & 69.87 & 9 & 0.0039 \\
\hline
\end{tabular}

Table 20: Tabulation of median identification times for Class C. Letter refers to the participant's letter code found on the footer of this page. Sub. \# is an identifier used to differentiate between the two submissions each participant could make. The Time column shows the time used to perform a search over an enrollment set of 5000000 . Time values are median times reported in seconds, but were originally recorded to microsecond precision. The FNIR column shows FNIR for each submission at FPIR $=10^{-3}$. NA indicates that the operations required to produce the value could not be performed. The number to the left of a value provides the value's column-wise ranking, with the best performance shaded in green and the worst in pink. 


\section{Accuracy Computational Resources Tradeoff}

This section discusses the computational resources used by each submission, mainly looking for trends in accuracy of a submission versus the load it created on the compute nodes. Statistics include how large the stored/finalized templates are on disk versus in memory and how much time it took to create feature templates. Detailed tables in Appendix F show enrollment set sizes, in Appendix G show search template sizes, and in Appendix H show template creation times. All these tables were used to create the scatter plots used in this section. Appendix K plots relative comparisons of FNIR, RAM usage, template creation times, and search times.

Addtionally, all the numbers in Tables 60 through 65 in Appendix F are based on the maximum enrollment set sizes for each class shown in Table 4. The RAM values reported are the best estimate based on the information recorded. It is possible that a submission used more or less RAM depending on the internal operations of the submitted software. See the Lessons Learned for Large-Scale Testing section for more details.

\subsection{Storage and Memory}

It is important to note that the Actual RAM used (Appendix F) is the sum of the resident enrollment set sizes of identification stage one processes after returning from the identification stage one initialization method. More information on this can be found in the FpVTE API [15].

Every attempt was made to run each submission on the minimum number of compute nodes needed to successfully complete the evaluation. This generally meant multiple passes of running enrollment set finalization and redoing timing validation tests to determine the minimum number of compute nodes needed. If too few compute nodes were used, the submission would crash and not work properly.

When looking at the results, there are submissions like those from participant $\mathbf{Q}$ that had large finalized enrollment set, but used a lot less Actual RAM during stage one identification. In fact, $\mathbf{Q}$ always ran on a single compute node despite the Finalized storage size. Participant L, on the other hand, clearly compressed templates, so they used more Actual RAM than Finalized storage. This behavior was pre-reported to NIST, which made it easier to plan ahead when testing the submission.

\subsubsection{Class A}

Scatter plots comparing FNIR and computational resources used by index finger identification submissions are shown in Figures 46 through 51.

Figures 52 through 53 shows a comparison of the templates (right and left index) as stored on disk with the actual size used in RAM. For the majority of participants, the numbers were very similar but there were exceptions such as T, P, G, $\mathbf{L}$, and $\mathbf{Q}$.

Some observations for Class A computational resources include:

$\triangleright$ The most accurate submissions were $\mathbf{Q}, \mathbf{V}, \mathbf{I}, \mathbf{D}$ and $\mathbf{L}$.

$\triangleright$ The most accurate submissions used the same or less RAM as other submissions with $\mathbf{2} 2$ being an exception.

$\triangleright$ It appears that high accuracy can be achieved without using a large amount of storage.

$\triangleright$ Participant $\mathbf{T}^{\prime}$ 's submissions consistently used the least computational resources but with the least accuracy.

$\triangleright$ Of the most accurate submissions, participant $\mathbf{V}$ used the least amount of storage space.

$\triangleright$ Participant K consumed the most RAM and disk space, significantly higher than all other participants.

$\triangleright$ Participant $\mathbf{Q}$ used the least amount of RAM and achieved the highest accuracy of the most accurate submissions.

\begin{tabular}{l|l|l|l|l|l|l}
$\mathrm{C}=$ afis team & $\mathrm{D}=$ = 3M Cogent & $\mathrm{E}=$ Neurotechnology & $\mathrm{F}=$ Papillon & $\mathrm{G}=$ Dermalog & $\mathrm{H}=$ Hisign Bio-Info Institute \\
$\mathrm{I}=$ NEC & $\mathrm{J}=$ Sonda & $\mathrm{K}=$ Tiger IT & $\mathrm{L}=$ Innovatrics & $\mathrm{M}=$ SPEX & $\mathrm{O}=$ ID Solutions \\
$\mathrm{P}=\mathrm{id} 3$ & $\mathrm{Q}=$ Morpho & $\mathrm{S}=$ Decatur Industries & $\mathrm{T}=$ BIO-key & $\mathrm{U}=$ Aware & $\mathrm{V}=$ AA Technology
\end{tabular}




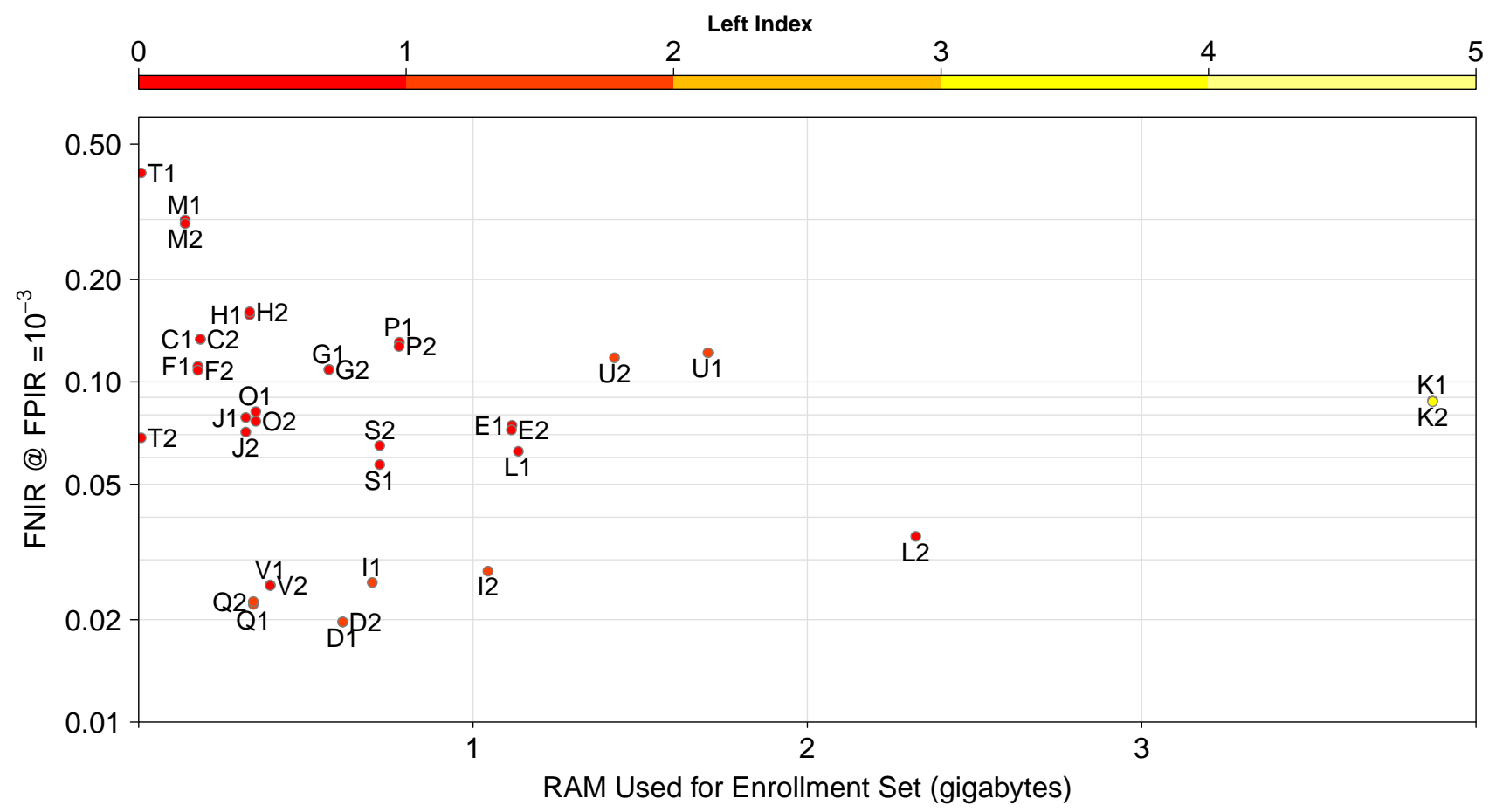

Figure 46: Scatter plot of FNIR @ FPIR $=10^{-3}$ searching 30000 subjects against 100000 subjects and RAM used for enrollment set for Class A — Left Index. The color of the data point is used to show the On Disk Finalized Enrollment Size. The color scale for the On Disk Finalized Enrollment Size is at the top of the plot and the units are in gigabytes. The data for the scatter plot comes from Table 60 in Appendix F. RAM Used for Enrollment Set is from the RAM/Actual column and On Disk Finalized Enrollment is from the On Disk/Finalized column.

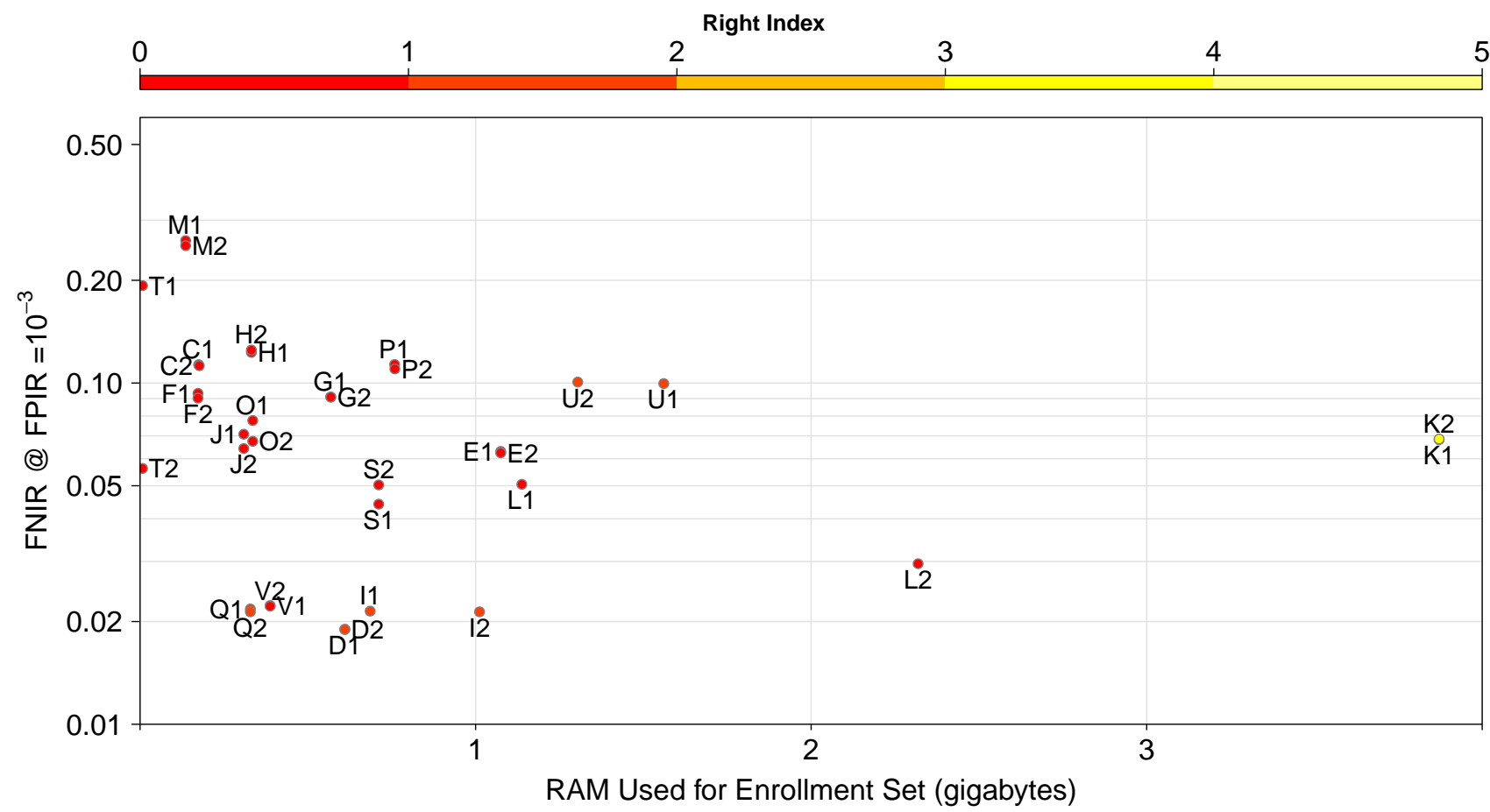

Figure 47: Scatter plot of FNIR @ FPIR $=10^{-3}$ searching 30000 subjects against 100000 subjects and RAM used for enrollment set for Class A — Right Index. The color of the data point is used to show the On Disk Finalized Enrollment Size. The color scale for the On Disk Finalized Enrollment Size is at the top of the plot and the units are in gigabytes. The data for the scatter plot comes from Table 61 in Appendix F. RAM Used for Enrollment Set is from the RAM/Actual column and On Disk Finalized Enrollment is from the On Disk/Finalized column.

\begin{tabular}{l|l|l|l|l|l|l}
$\mathrm{C}=$ afis team & $\mathrm{D}=3 \mathrm{M}$ Cogent & $\mathrm{E}=$ Neurotechnology & $\mathrm{F}=$ Papillon & $\mathrm{G}=$ Dermalog & $\mathrm{H}=$ Hisign Bio-Info Institute \\
$\mathrm{I}=$ NEC & $\mathrm{J}=$ Sonda & $\mathrm{K}=$ Tiger IT & $\mathrm{L}=$ Innovatrics & $\mathrm{M}=$ SPEX & $\mathrm{O}=$ ID Solutions \\
$\mathrm{P}=\mathrm{id} 3$ & $\mathrm{Q}=$ Morpho & $\mathrm{S}=$ Decatur Industries & $\mathrm{T}=$ BIO-key & $\mathrm{U}=$ Aware & $\mathrm{V}=$ AA Technology
\end{tabular}




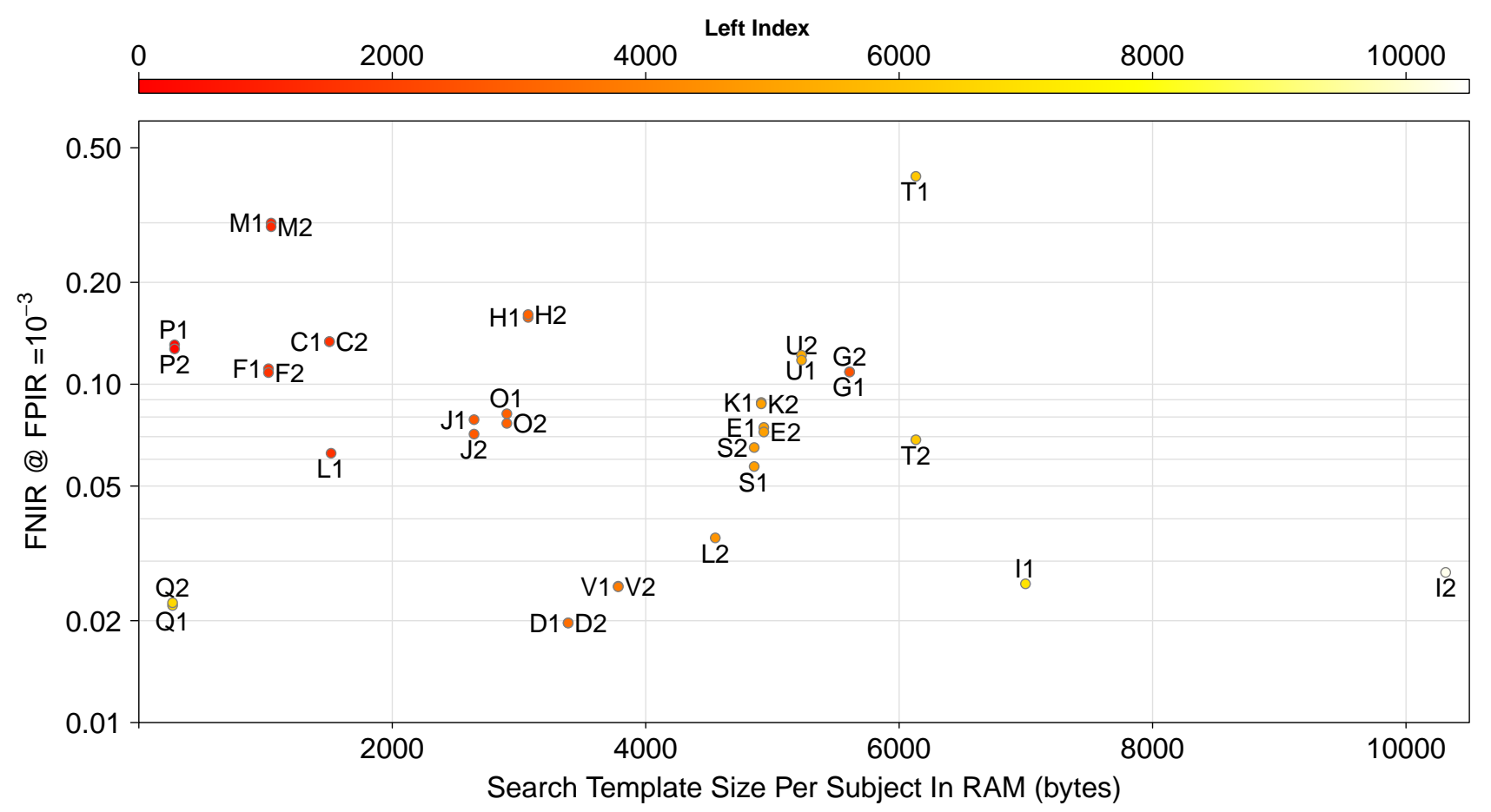

Figure 48: Scatter plot of FNIR @ FPIR $=10^{-3}$ searching 30000 subjects against 100000 subjects and search template size in RAM for Class A — Left Index. The color of the data point is used to show Search Template Size On Disk. The color scale for Search Template Size On Disk is at the top of the plot and the units are in bytes. On Disk comes from table Table 66 in Appendix G.

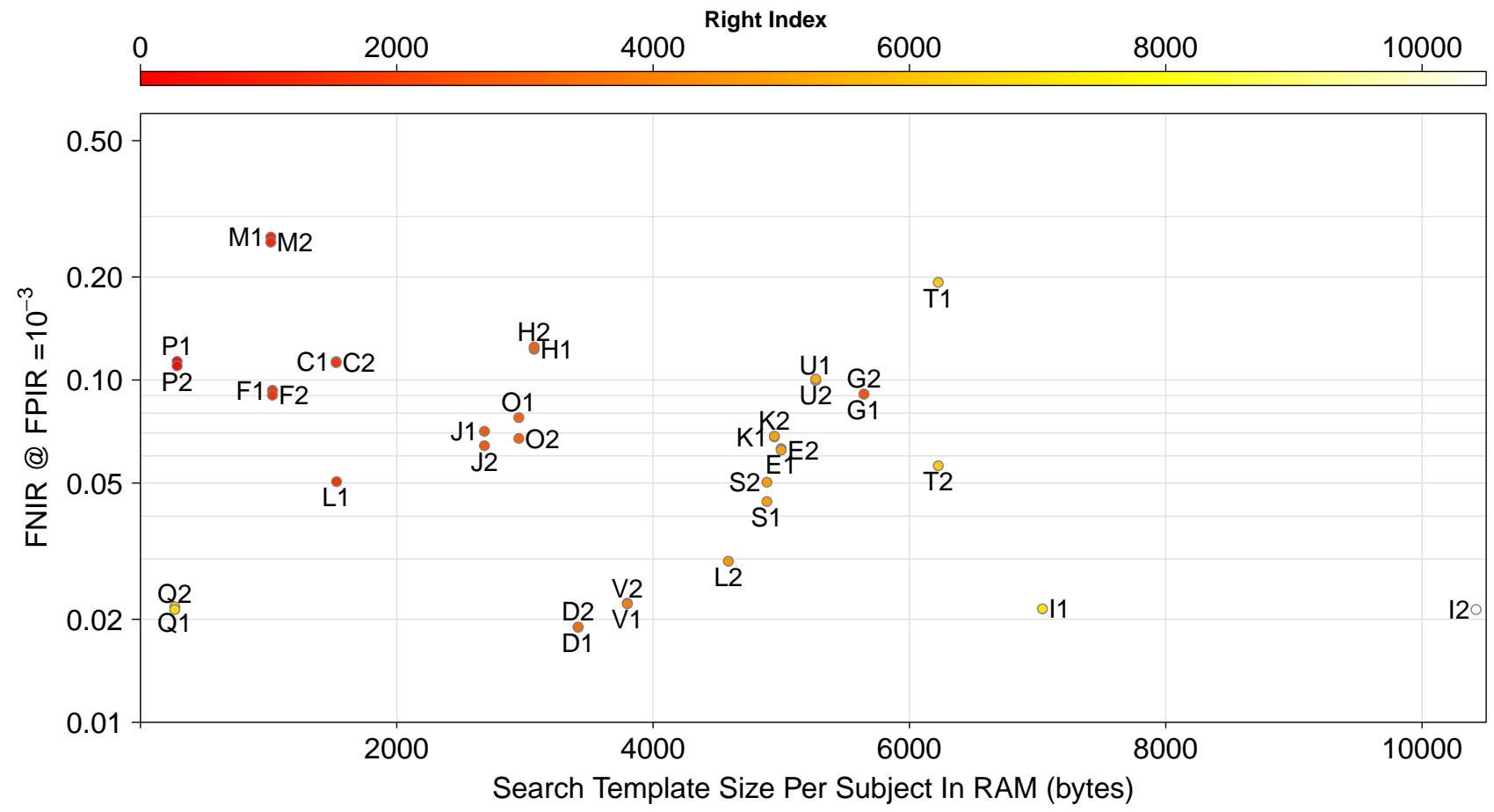

Figure 49: Scatter plot of FNIR @ FPIR $=10^{-3}$ searching 30000 subjects against 100000 subjects and search template size in RAM for Class A — Right Index. The color of the data point is used to show Search Template Size On Disk. The color scale for Search Template Size On Disk is at the top of the plot and the units are in bytes. On Disk comes from table Table 66 in Appendix G.

\begin{tabular}{l|l|l|l|l|l|l}
$\mathrm{C}=$ afis team & $\mathrm{D}=3 \mathrm{M}$ Cogent & $\mathrm{E}=$ Neurotechnology & $\mathrm{F}=$ Papillon & $\mathrm{G}=$ Dermalog & $\mathrm{H}=$ Hisign Bio-Info Institute \\
$\mathrm{I}=$ NEC & $\mathrm{J}=$ Sonda & $\mathrm{K}=$ Tiger IT & $\mathrm{L}=$ Innovatrics & $\mathrm{M}=$ SPEX & $\mathrm{O}=$ ID Solutions \\
$\mathrm{P}=\mathrm{id} 3$ & $\mathrm{Q}=$ Morpho & $\mathrm{S}=$ Decatur Industries & $\mathrm{T}=$ BIO-key & $\mathrm{U}=$ Aware & $\mathrm{V}=$ AA Technology
\end{tabular}




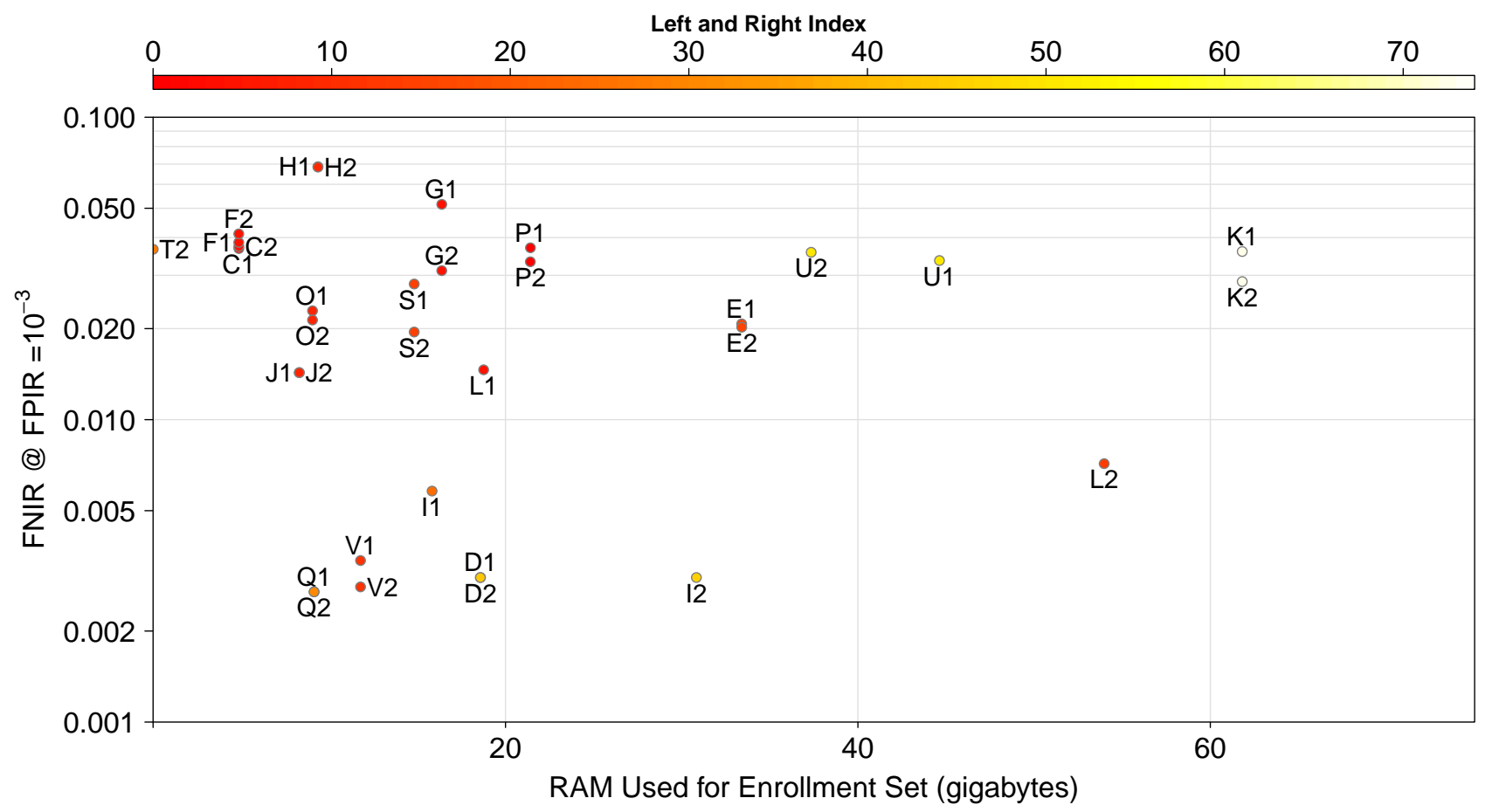

Figure 50: Scatter plot of FNIR @ FPIR $=10^{-3}$ searching 30000 subjects against 1600000 subjects and RAM used for enrollment set for Class A — Left and Right Index. The color of the data point is used to show the On Disk Finalized Enrollment Set size. The color scale for the On Disk Finalized Enrollment Set is at the top of the plot and the units are in gigabytes. The data for the enrollment set size comes from Table 62 in Appendix F. RAM used for Enrollment Set is from the RAM/Actual column and On Disk Finalized Enrollment Set is from the On Disk/Finalized column.

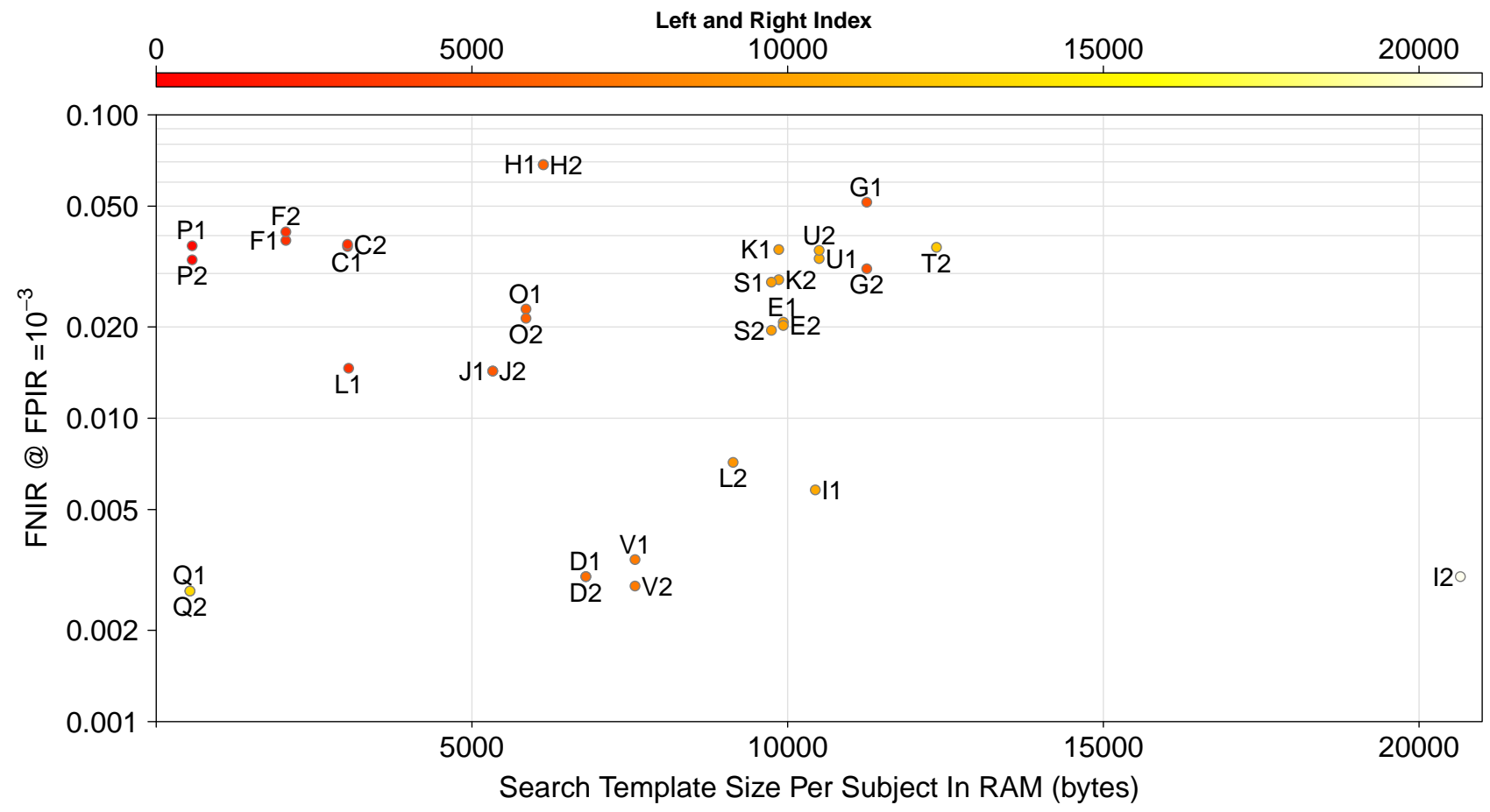

Figure 51: Scatter plot of FNIR @ FPIR $=10^{-3}$ searching 30000 subjects against 1600000 subjects and search template size in RAM. The color of the data point is used to show Search Template Size On Disk. The color scale for Search Template Size On Disk is at the top of the plot and the units are in bytes. On Disk comes from Table 66 in Appendix G.

\begin{tabular}{l|l|l|l|ll|l}
$\mathrm{C}=$ afis team & $\mathrm{D}=3 \mathrm{M}$ Cogent & $\mathrm{E}=$ Neurotechnology & $\mathrm{F}=$ Papillon & $\mathrm{G}=$ Dermalog & $\mathrm{H}=$ Hisign Bio-Info Institute \\
$\mathrm{I}=$ NEC & $\mathrm{J}=$ Sonda & $\mathrm{K}=$ Tiger IT & $\mathrm{L}=$ Innovatrics & $\mathrm{M}=$ SPEX & $\mathrm{O}=$ ID Solutions \\
$\mathrm{P}=\mathrm{id} 3$ & $\mathrm{Q}=$ Morpho & $\mathrm{S}=$ Decatur Industries & $\mathrm{T}=$ BIO-key & $\mathrm{U}=$ Aware & $\mathrm{V}=$ AA Technology
\end{tabular}




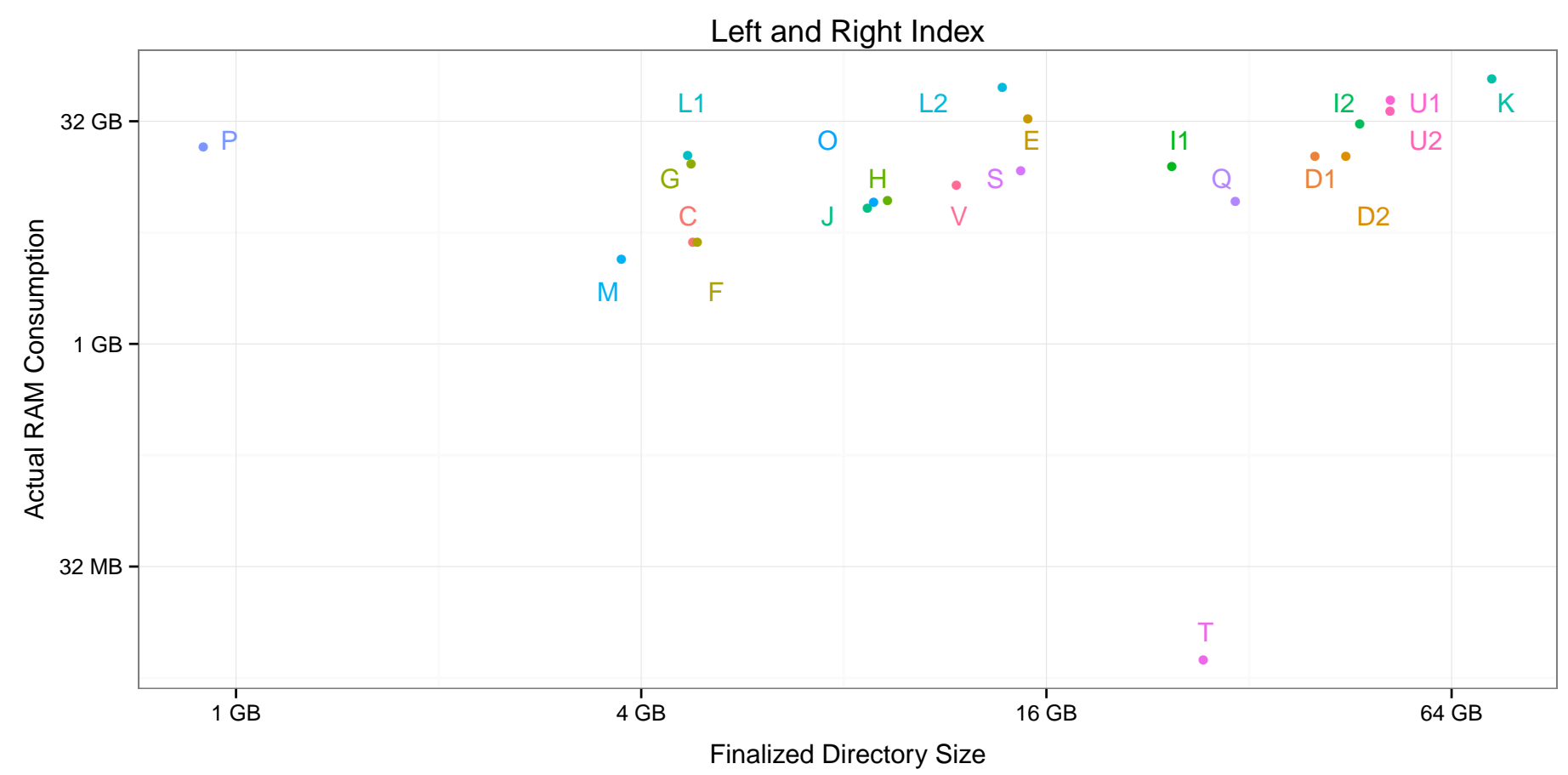

Figure 52: Comparison of enrollment size in RAM and on disk. The $x$ - and $y$-axes use log scales. The data for enrollment size comes from Table 62 in Appendix F. Actual RAM Consumption is from the RAM/Actual column and Finalized Directory Size is from the On Disk/Finalized column.

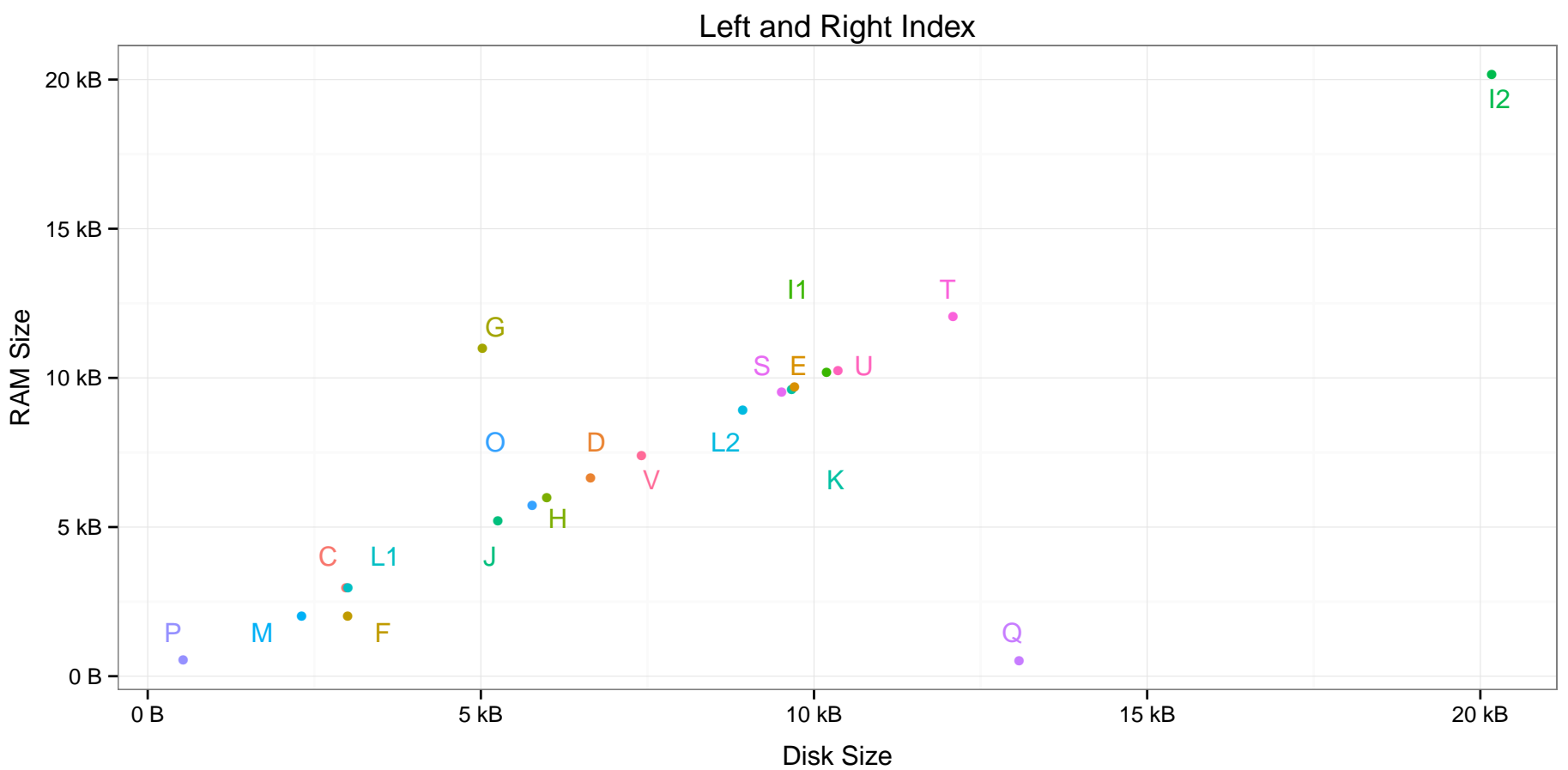

Figure 53: Comparison of search template size in RAM and on disk. On Disk comes from Table 66 in Appendix G.

\begin{tabular}{l|l|l|l|l|l|l}
$\mathrm{C}=$ afis team & $\mathrm{D}=$ = 3M Cogent & $\mathrm{E}=$ Neurotechnology & $\mathrm{F}=$ Papillon & $\mathrm{G}=$ Dermalog & $\mathrm{H}=$ Hisign Bio-Info Institute \\
$\mathrm{I}=$ NEC & $\mathrm{J}=$ Sonda & $\mathrm{K}=$ Tiger IT & $\mathrm{L}=$ Innovatrics & $\mathrm{M}=$ SPEX & $\mathrm{O}=$ ID Solutions \\
$\mathrm{P}=\mathrm{id} 3$ & $\mathrm{Q}=$ Morpho & $\mathrm{S}=$ Decatur Industries & $\mathrm{T}=$ BIO-key & $\mathrm{U}=$ Aware & $\mathrm{V}=$ AA Technology
\end{tabular}




\subsubsection{Class B}

Scatter plots comparing FNIR and computational resources used by IDFlat identification submissions are shown in Figures 54 through 55.

Figures 56 through 57 shows a comparison of the templates as stored on disk with the actual size used in RAM. Like class A results, this plot highlights submissions where on disk and in RAM usage differed such as $\mathbf{E}, \mathbf{G}, \mathbf{L}$, and $\mathbf{Q}$.

Some observations for Class B computational resources include:

$\triangleright$ The lowest RAM usage is also one of the top performers $(\mathbf{Q})$.

$\triangleright$ Other top performers (participants D and I) do not have the largest RAM usage.

$\triangleright$ Participant I cut RAM usage in half with minimal drop in accuracy.

$\triangleright$ Like Class A, high accuracy can be achieved while keeping RAM usage relatively low.

\begin{tabular}{l|l|l|l|l|l|l}
$\mathrm{C}=$ afis team & $\mathrm{D}=3 \mathrm{M}$ Cogent & $\mathrm{E}=$ Neurotechnology & $\mathrm{F}=$ Papillon & $\mathrm{G}=$ Dermalog & $\mathrm{H}=$ Hisign Bio-Info Institute \\
$\mathrm{I}=\mathrm{NEC}$ & $\mathrm{J}=$ Sonda & $\mathrm{K}=$ Tiger IT & $\mathrm{L}=$ Innovatrics & $\mathrm{M}=$ SPEX & $\mathrm{O}=$ ID Solutions \\
$\mathrm{P}=\mathrm{id} 3$ & $\mathrm{Q}=$ Morpho & $\mathrm{S}=$ Decatur Industries & $\mathrm{T}=$ BIO-key & $\mathrm{U}=$ Aware & $\mathrm{V}=$ AA Technology
\end{tabular}




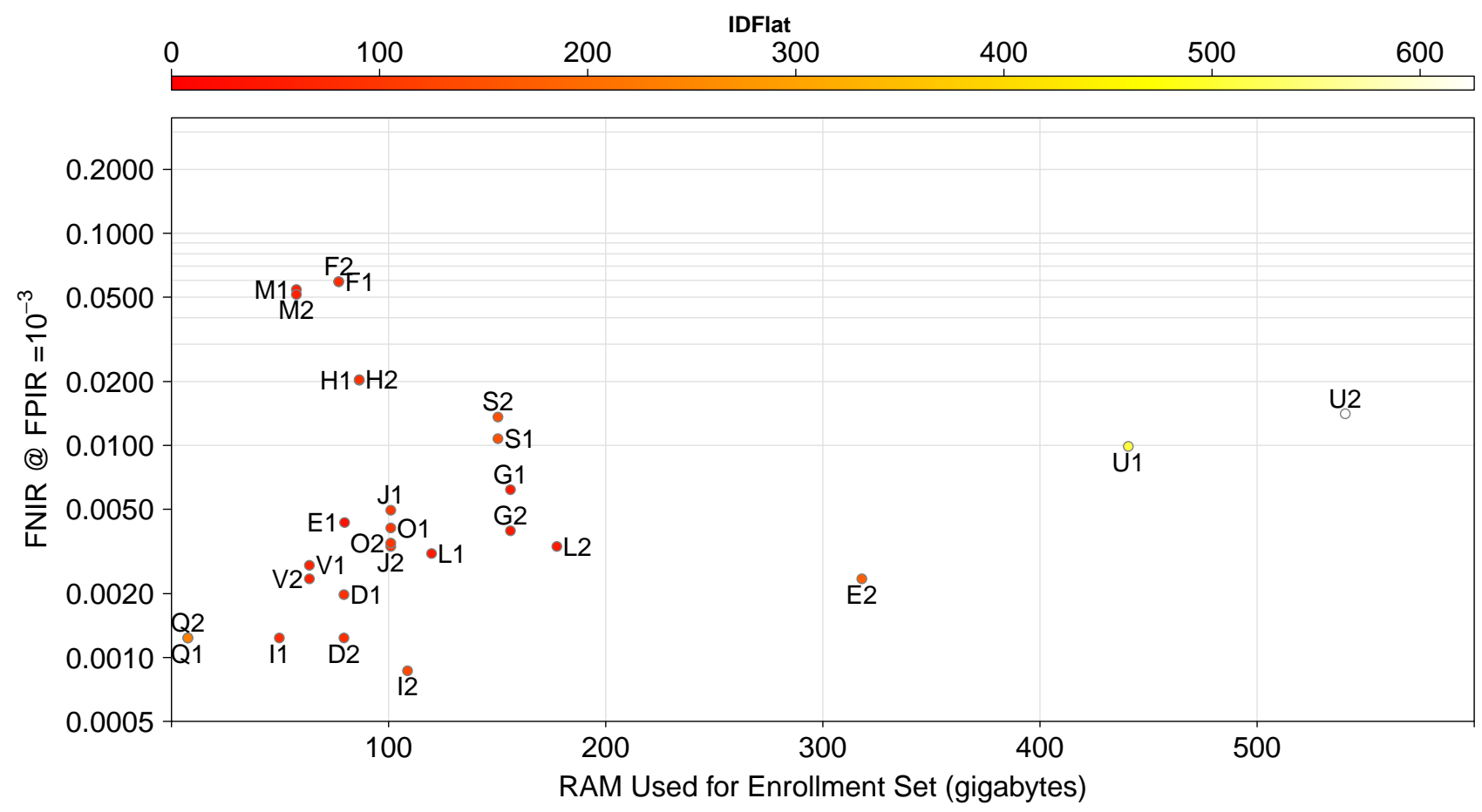

Figure 54: Scatter plot of FNIR @ FPIR $=10^{-3}$ searching 30000 subjects against 3000000 subjects and RAM used for enrollment set for Class B Identification Flats. The color of the data point is used to show On Disk Finalized Enrollment Set size. The color scale for the on disk finalized enrollment size is at the top of the plot and the units are in gigabytes. The data for the enrollment set size comes from Table 63 in Appendix F. RAM Used for Enrollment Set is from the RAM/Actual column and On Disk Finalized Enrollment Set is from the On Disk/Finalized column.

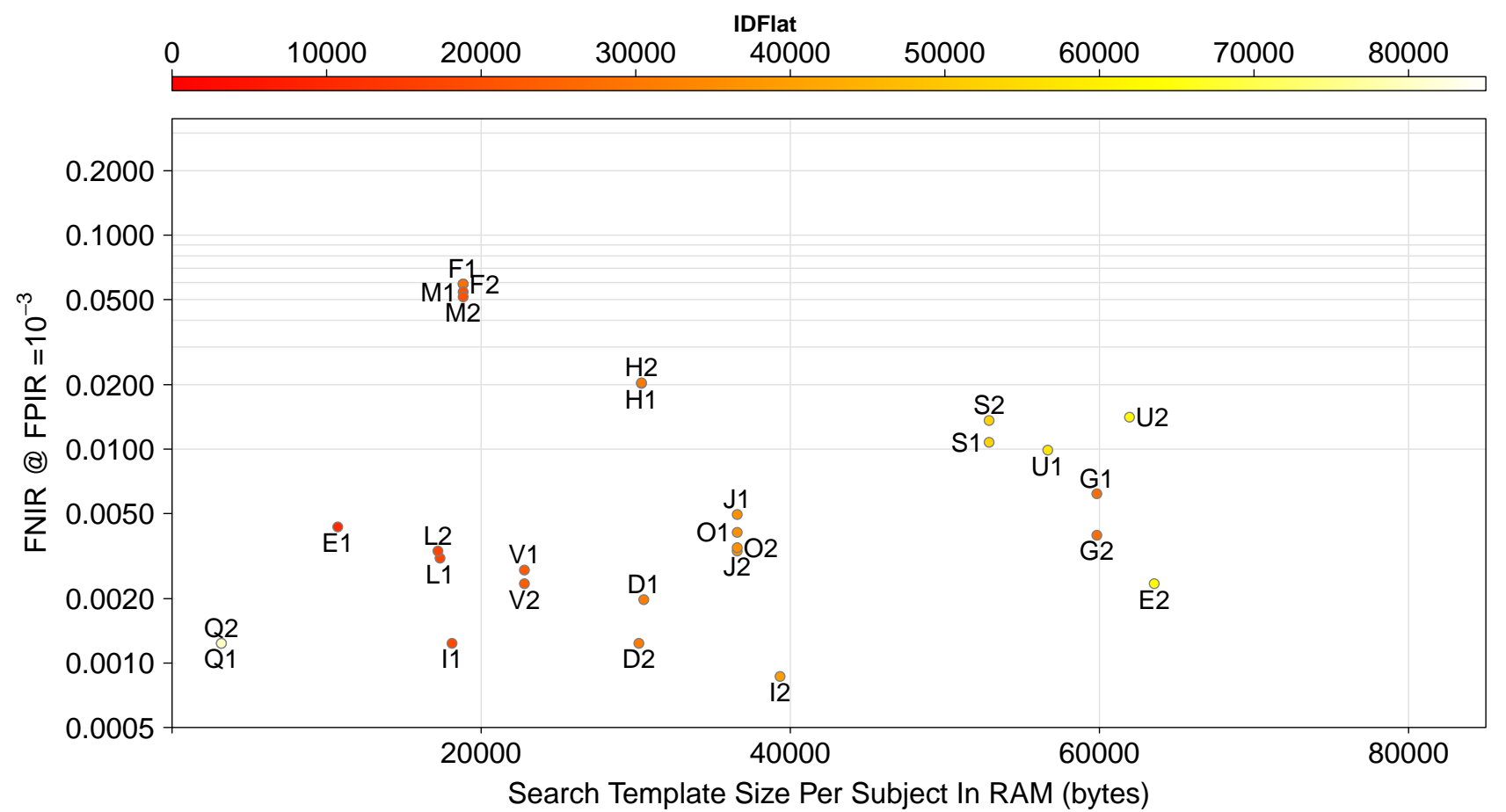

Figure 55: Scatter plot of FNIR @ FPIR $=10^{-3}$ searching 30000 subjects against 3000000 subjects and search template size in RAM for Class B Identification Flats. The color of the data point is used to show Search Template Size On Disk. The color scale for Search Template Size On Disk is at the top of the plot and the units are in bytes. On Disk comes from Table 67in Appendix G.

\begin{tabular}{l|l|l|l|l|l|l}
$\mathrm{C}=$ afis team & $\mathrm{D}=3 \mathrm{M}$ Cogent & $\mathrm{E}=$ Neurotechnology & $\mathrm{F}=$ Papillon & $\mathrm{G}=$ Dermalog & $\mathrm{H}=$ Hisign Bio-Info Institute \\
$\mathrm{I}=$ NEC & $\mathrm{J}=$ Sonda & $\mathrm{K}=$ Tiger IT & $\mathrm{L}=$ Innovatrics & $\mathrm{M}=$ SPEX & $\mathrm{O}=$ ID Solutions \\
$\mathrm{P}=\mathrm{id} 3$ & $\mathrm{Q}=$ Morpho & $\mathrm{S}=$ Decatur Industries & $\mathrm{T}=$ BIO-key & $\mathrm{U}=$ Aware & $\mathrm{V}=$ AA Technology
\end{tabular}




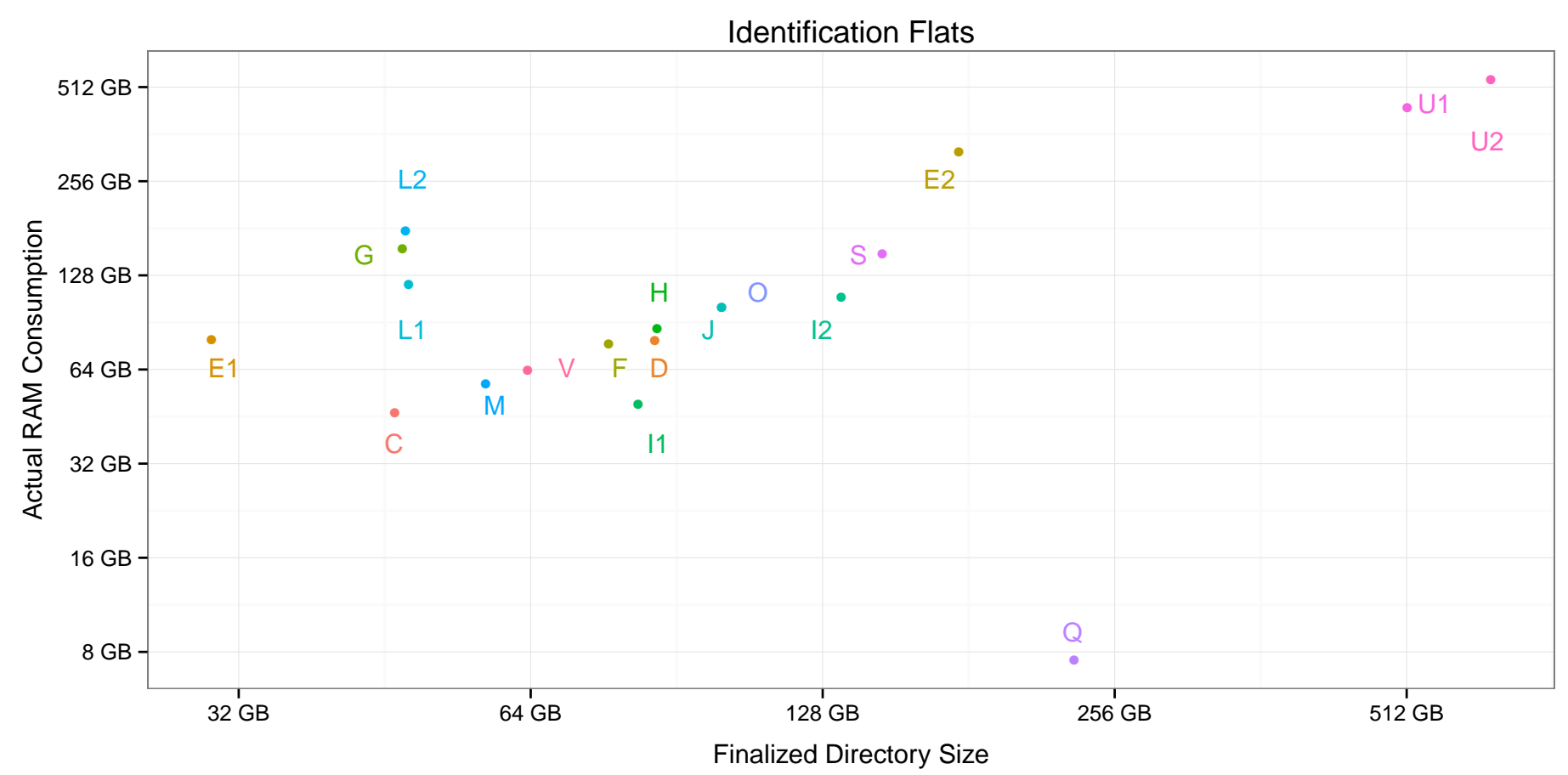

Figure 56: Comparison of enrollment size in RAM and on disk for Class B - Identification Flats. The $x$ - and $y$-axes use log scales. The data for enrollment size comes from Table 63 in Appendix F. Actual RAM Consumption is from the RAM/Actual column and Finalized Directory Size is from the On Disk/Finalized column.

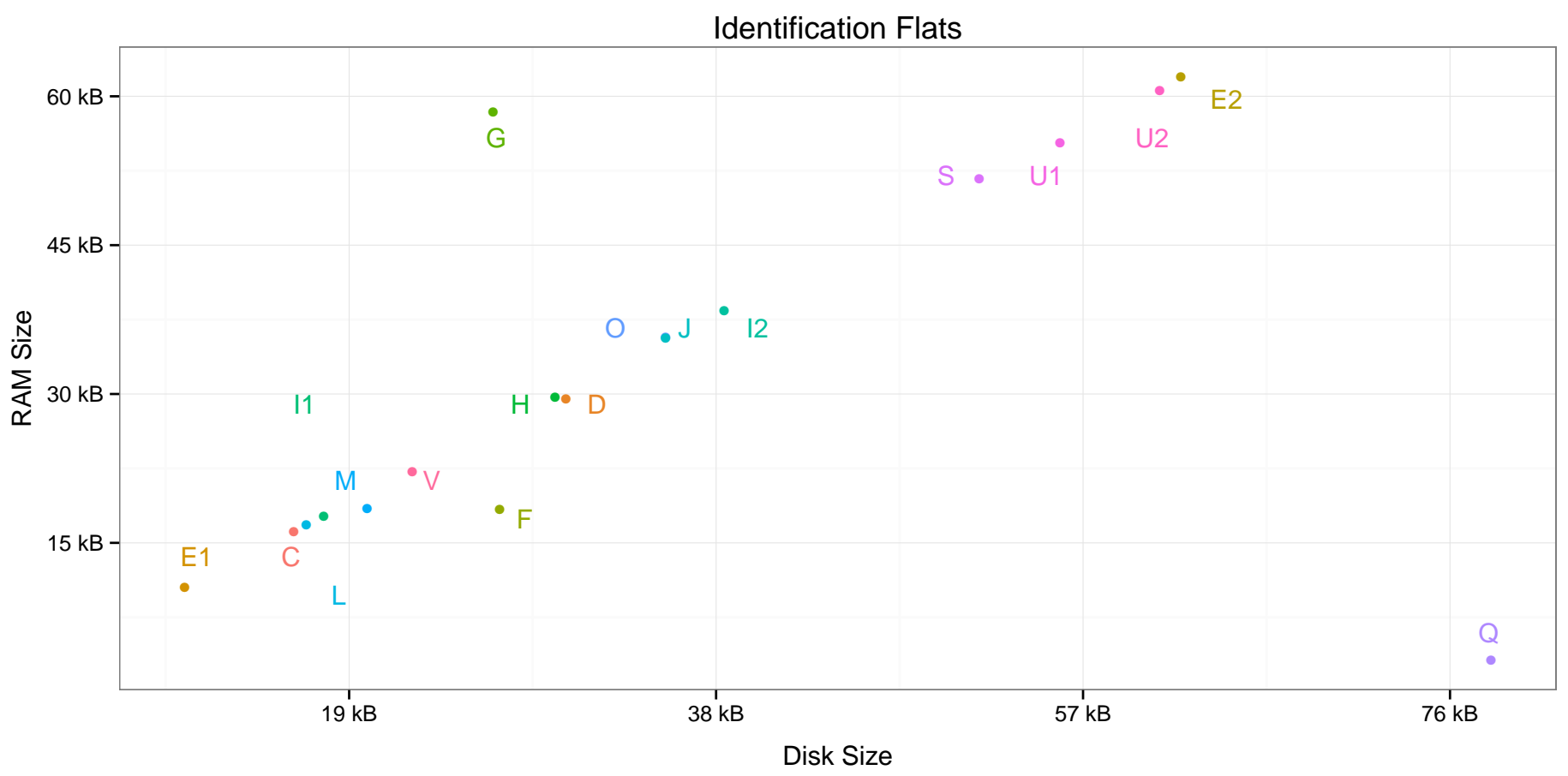

Figure 57: Comparison of search template size in RAM and on disk for Class B — Identification Flats. On Disk comes from Table 67 in Appendix G.

\begin{tabular}{l|l|l|l|l|l|l}
$\mathrm{C}=$ afis team & $\mathrm{D}=3 \mathrm{M}$ Cogent & $\mathrm{E}=$ Neurotechnology & $\mathrm{F}=$ Papillon & $\mathrm{G}=$ Dermalog & $\mathrm{H}=$ Hisign Bio-Info Institute \\
$\mathrm{I}=$ NEC & $\mathrm{J}=$ Sonda & $\mathrm{K}=$ Tiger IT & $\mathrm{L}=$ Innovatrics & $\mathrm{M}=$ SPEX & $\mathrm{O}=$ ID Solutions \\
$\mathrm{P}=\mathrm{id} 3$ & $\mathrm{Q}=$ Morpho & $\mathrm{S}=$ Decatur Industries & $\mathrm{T}=$ BIO-key & $\mathrm{U}=$ Aware & $\mathrm{V}=$ AA Technology
\end{tabular}




\subsubsection{Class C}

Scatter plots comparing FNIR and computational resources used by plain and rolled impression submissions are shown in Figures 58 through 61.

Figures 62 through 65 show a comparison of the templates as stored on disk with the actual size used in RAM. Like class $A$ and $B$ results, these plot highlight submissions where on disk and in RAM usage differed for several submissions.

Some observations for Class C computational resources include:

$\triangleright$ Like classes A and B, the top performers do not use the largest amount of RAM.

$\triangleright$ High accuracy can be achieved with relatively low RAM usage.

$\triangleright$ Ten-finger rolled data used more RAM than ten-finger plain data, but is not more accurate.

\begin{tabular}{l|l|l|l|l|l|l}
$\mathrm{C}=$ afis team & $\mathrm{D}=3 \mathrm{M}$ Cogent & $\mathrm{E}=$ Neurotechnology & $\mathrm{F}=$ Papillon & $\mathrm{G}=$ Dermalog & $\mathrm{H}=$ Hisign Bio-Info Institute \\
$\mathrm{I}=$ NEC & $\mathrm{J}=$ Sonda & $\mathrm{K}=$ Tiger IT & $\mathrm{L}=$ Innovatrics & $\mathrm{M}=$ SPEX & $\mathrm{O}=$ ID Solutions \\
$\mathrm{P}=$ id3 & $\mathrm{Q}=$ Morpho & $\mathrm{S}=$ Decatur Industries & $\mathrm{T}=$ BIO-key & $\mathrm{U}=$ Aware & $\mathrm{V}=$ AA Technology
\end{tabular}




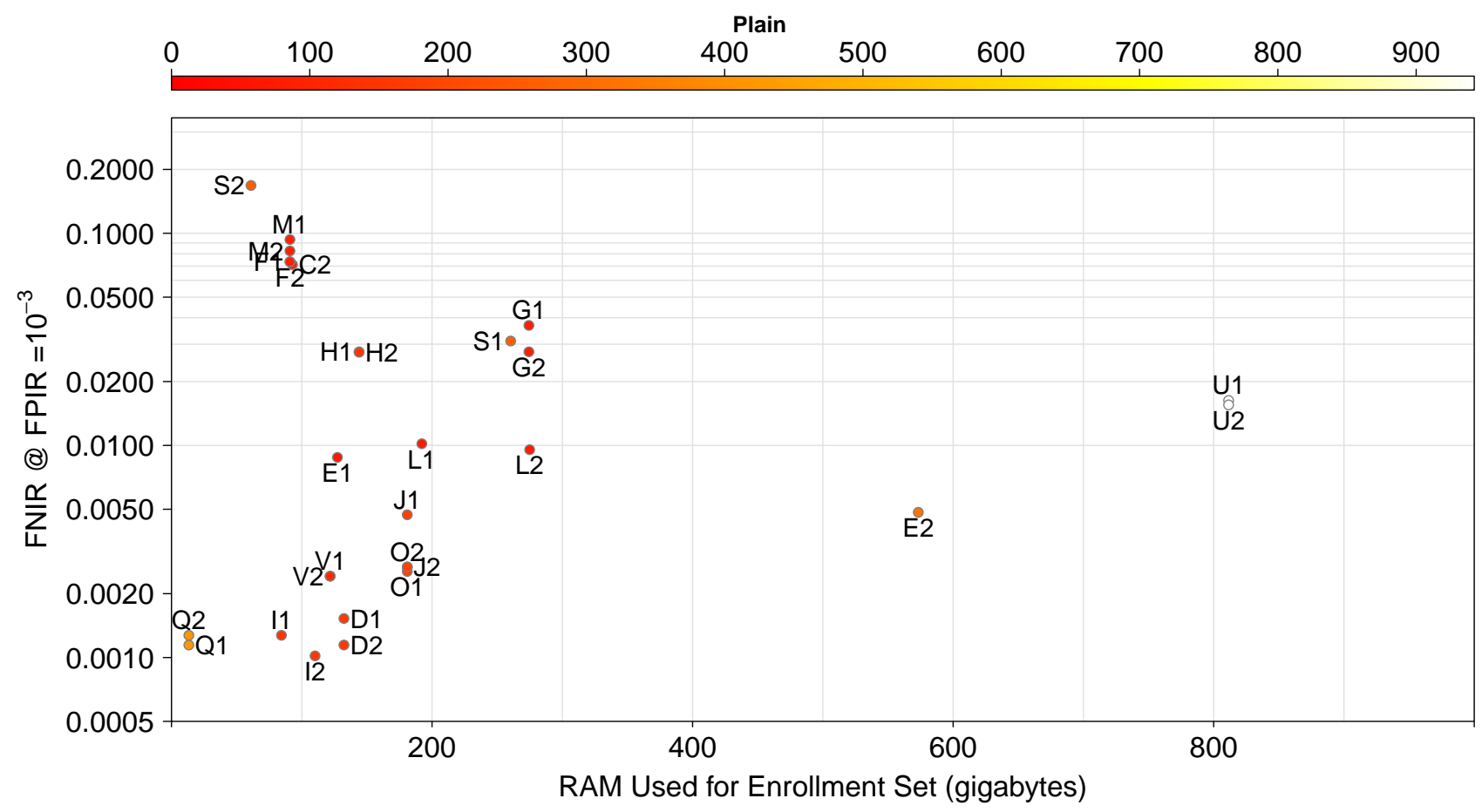

Figure 58: Scatter plot of FNIR @ FPIR $=10^{-3}$ searching 30000 subjects against 5000000 subjects and RAM used for enrollment set for Class C Ten-Finger Rolled-to-Rolled. The color of the data point is used to show the On Disk Finalized Enrollment Set size. The color scale for the On Disk Finalized Enrollment Size is at the top of the plot and the units are in gigabytes. The data for the scatter plot comes from Table 64 in Appendix F. RAM used for Enrollment Set is from the RAM/Actual column and On Disk Finalized Enrollment Set is from the On Disk/Finalized column.

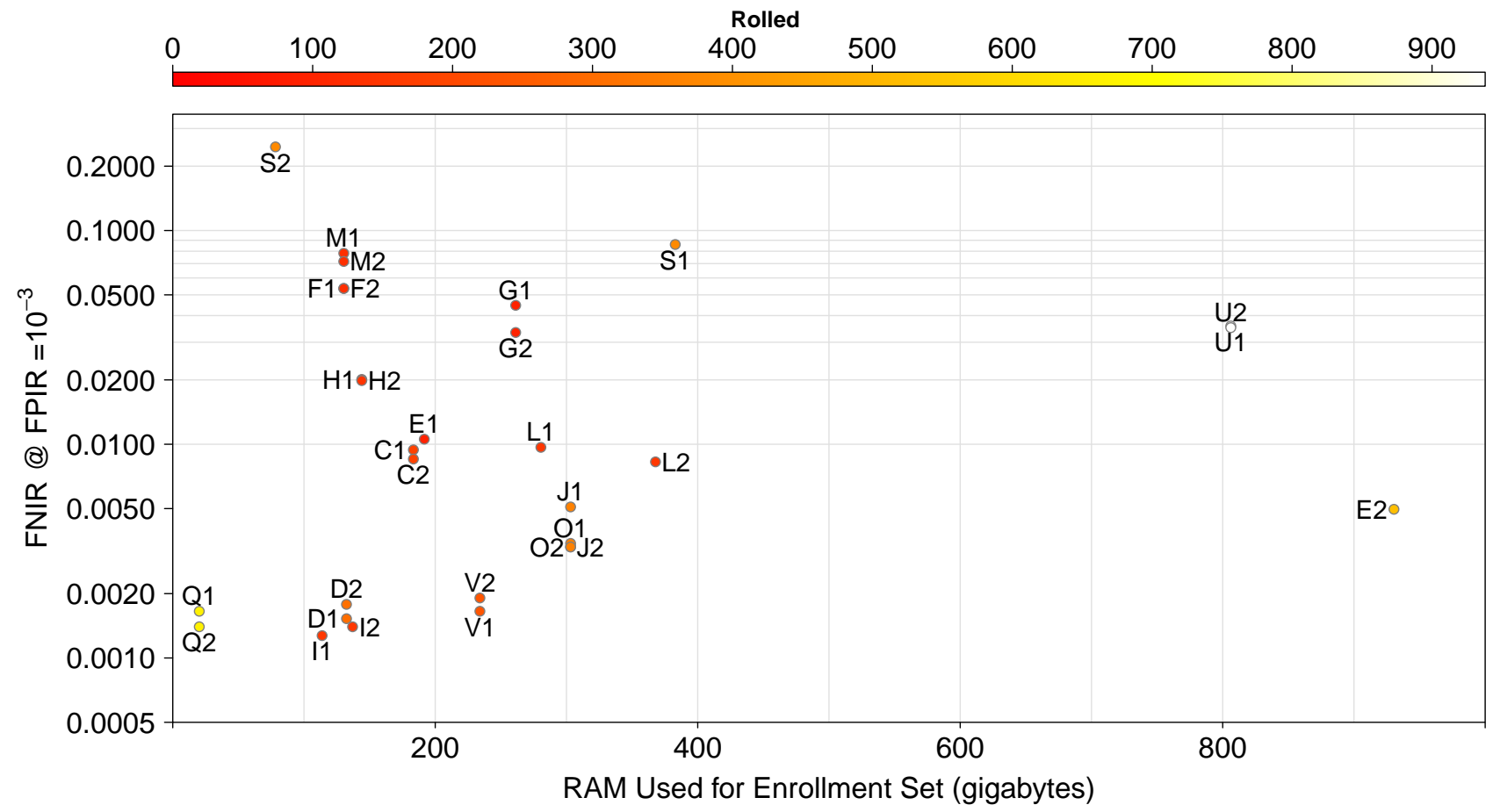

Figure 59: Scatter plot of FNIR @ FPIR $=10^{-3}$ searching 30000 subjects against 5000000 subjects and RAM used for enrollment set for Class C Ten-Finger Rolled-to-Rolled. The color of the data point is used to show the On Disk Finalized Enrollment Set size. The color scale for the On Disk Finalized Enrollment Size is at the top of the plot and the units are in gigabytes. The data for the scatter plot comes from Table 65 in Appendix F. RAM used for Enrollment Set is from the RAM/Actual column and On Disk Finalized Enrollment Set is from the On Disk/Finalized column.

\begin{tabular}{l|l|l|l|ll|l}
$\mathrm{C}=$ afis team & $\mathrm{D}=3 \mathrm{M}$ Cogent & $\mathrm{E}=$ Neurotechnology & $\mathrm{F}=$ Papillon & $\mathrm{G}=$ Dermalog & $\mathrm{H}=$ Hisign Bio-Info Institute \\
$\mathrm{I}=$ NEC & $\mathrm{J}=$ Sonda & $\mathrm{K}=$ Tiger IT & $\mathrm{L}=$ Innovatrics & $\mathrm{M}=$ SPEX & $\mathrm{O}=$ ID Solutions \\
$\mathrm{P}=\mathrm{id} 3$ & $\mathrm{Q}=$ Morpho & $\mathrm{S}=$ Decatur Industries & $\mathrm{T}=$ BIO-key & $\mathrm{U}=$ Aware & $\mathrm{V}=$ AA Technology
\end{tabular}




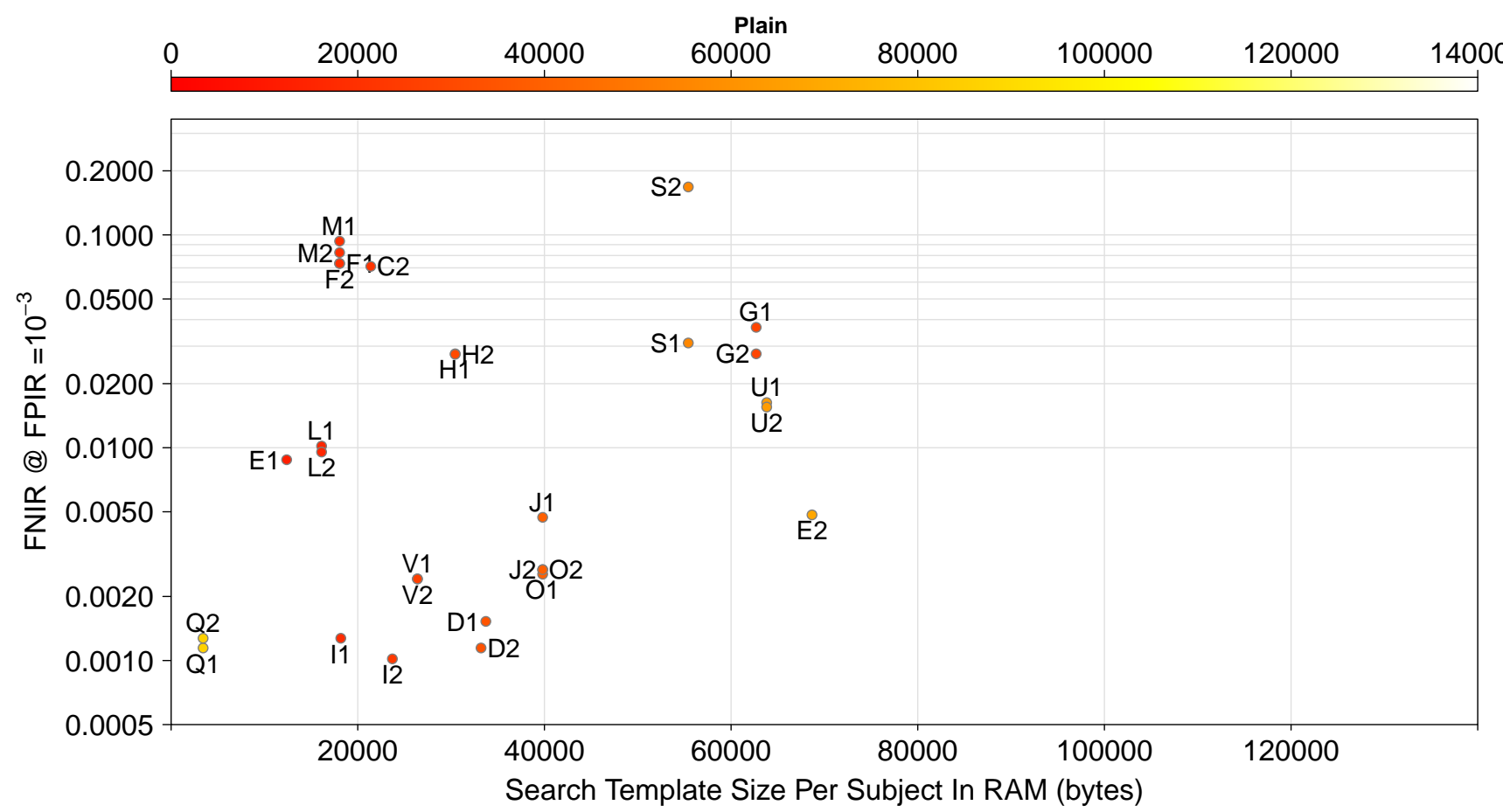

Figure 60: Scatter plot of FNIR @ FPIR $=10^{-3}$ searching 30000 subjects against 5000000 subjects and search template size in RAM for Class C Ten-Finger Plain-to-Plain. The color of the data point is used to show Search Template Size On Disk. The color scale for Search Template Size On Disk is at the top of the plot and the units are in bytes. On Disk comes from Table 68 in Appendix G.

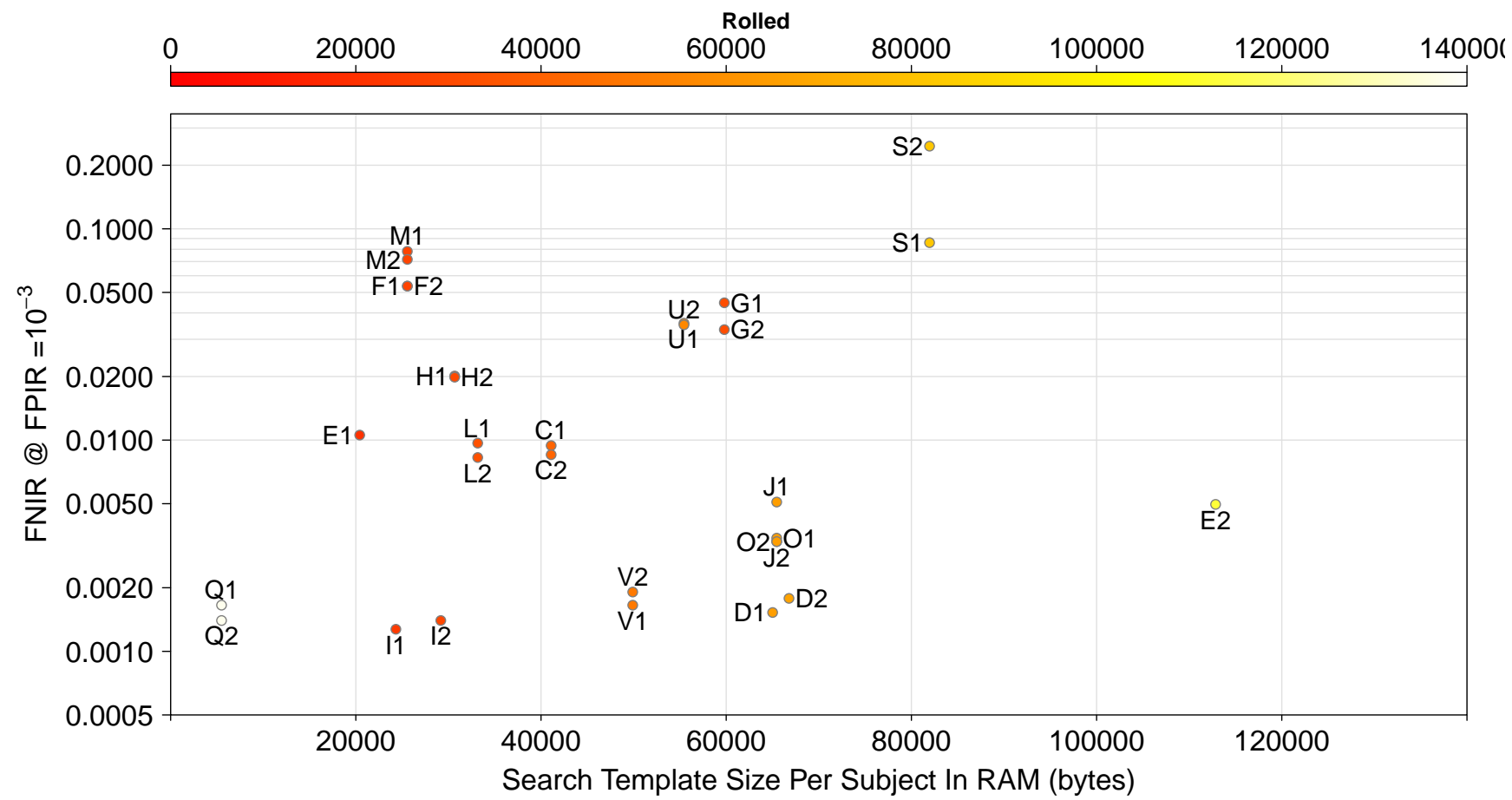

Figure 61: Scatter plot of FNIR @ FPIR $=10^{-3}$ searching 30000 subjects against 5000000 subjects and search template size in RAM for Class C Ten-Finger Rolled-to-Rolled. The color of the data point is used to show Search Template Size On Disk. The color scale for Search Template Size On Disk is at the top of the plot and the units are in bytes. On Disk comes from Table 68 in Appendix G.

\begin{tabular}{l|l|l|l|ll|l}
$\mathrm{C}=$ afis team & $\mathrm{D}=3 \mathrm{M}$ Cogent & $\mathrm{E}=$ Neurotechnology & $\mathrm{F}=$ Papillon & $\mathrm{G}=$ Dermalog & $\mathrm{H}=$ Hisign Bio-Info Institute \\
$\mathrm{I}=$ NEC & $\mathrm{J}=$ Sonda & $\mathrm{K}=$ Tiger IT & $\mathrm{L}=$ Innovatrics & $\mathrm{M}=$ SPEX & $\mathrm{O}=$ ID Solutions \\
$\mathrm{P}=\mathrm{id} 3$ & $\mathrm{Q}=$ Morpho & $\mathrm{S}=$ Decatur Industries & $\mathrm{T}=$ BIO-key & $\mathrm{U}=$ Aware & $\mathrm{V}=$ AA Technology
\end{tabular}




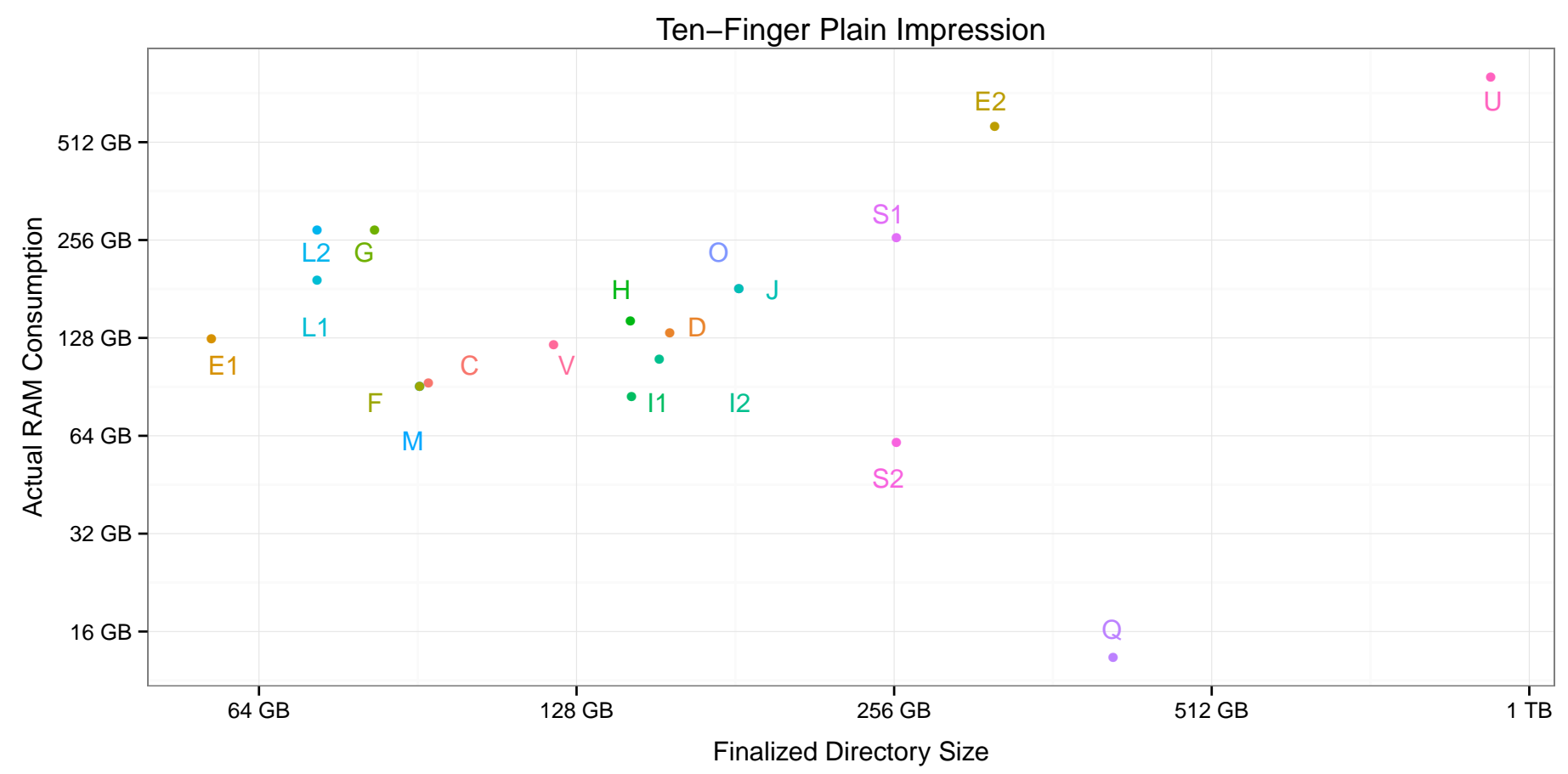

Figure 62: Comparison of enrollment size in RAM and on disk for Class C - Plain Impression. The $x$ - and $y$-axes use log scales. The data for enrollment size comes from Table 64 in Appendix F. Actual RAM Consumption is from the RAM/Actual column and Finalized Directory Size is from the On Disk/Finalized column.

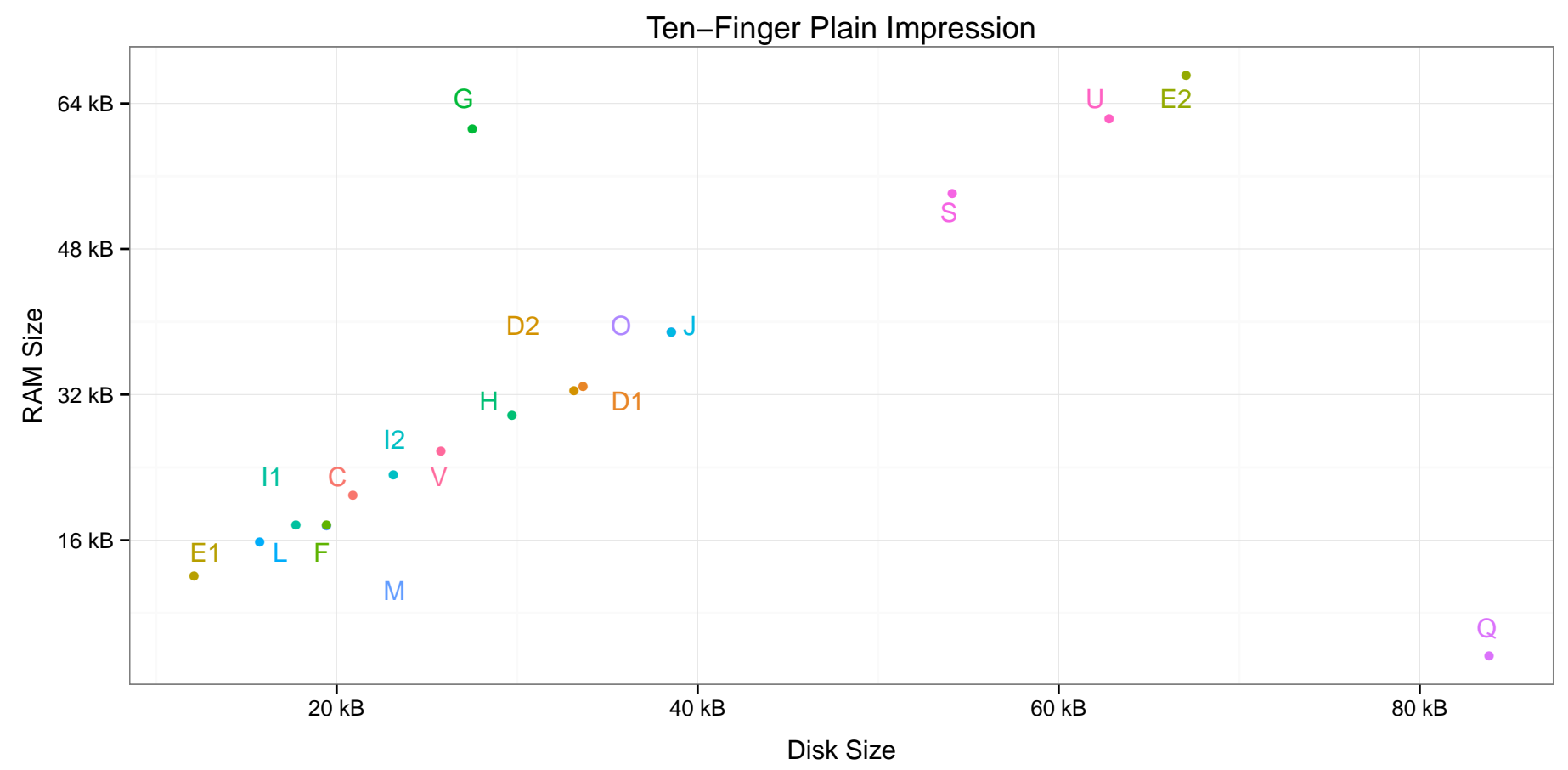

Figure 63: Comparison of search template size in RAM and on disk for Class C — Plain Impression. On Disk comes from Table 68 in Appendix G.

\begin{tabular}{l|l|l|l|l|l|l}
$\mathrm{C}=$ afis team & $\mathrm{D}=$ = 3M Cogent & $\mathrm{E}=$ Neurotechnology & $\mathrm{F}=$ Papillon & $\mathrm{G}=$ Dermalog & $\mathrm{H}=$ Hisign Bio-Info Institute \\
$\mathrm{I}=$ NEC & $\mathrm{J}=$ Sonda & $\mathrm{K}=$ Tiger IT & $\mathrm{L}=$ Innovatrics & $\mathrm{M}=$ SPEX & $\mathrm{O}=$ ID Solutions \\
$\mathrm{P}=\mathrm{id} 3$ & $\mathrm{Q}=$ Morpho & $\mathrm{S}=$ Decatur Industries & $\mathrm{T}=$ BIO-key & $\mathrm{U}=$ Aware & $\mathrm{V}=$ AA Technology
\end{tabular}


Ten-Finger Rolled Impression

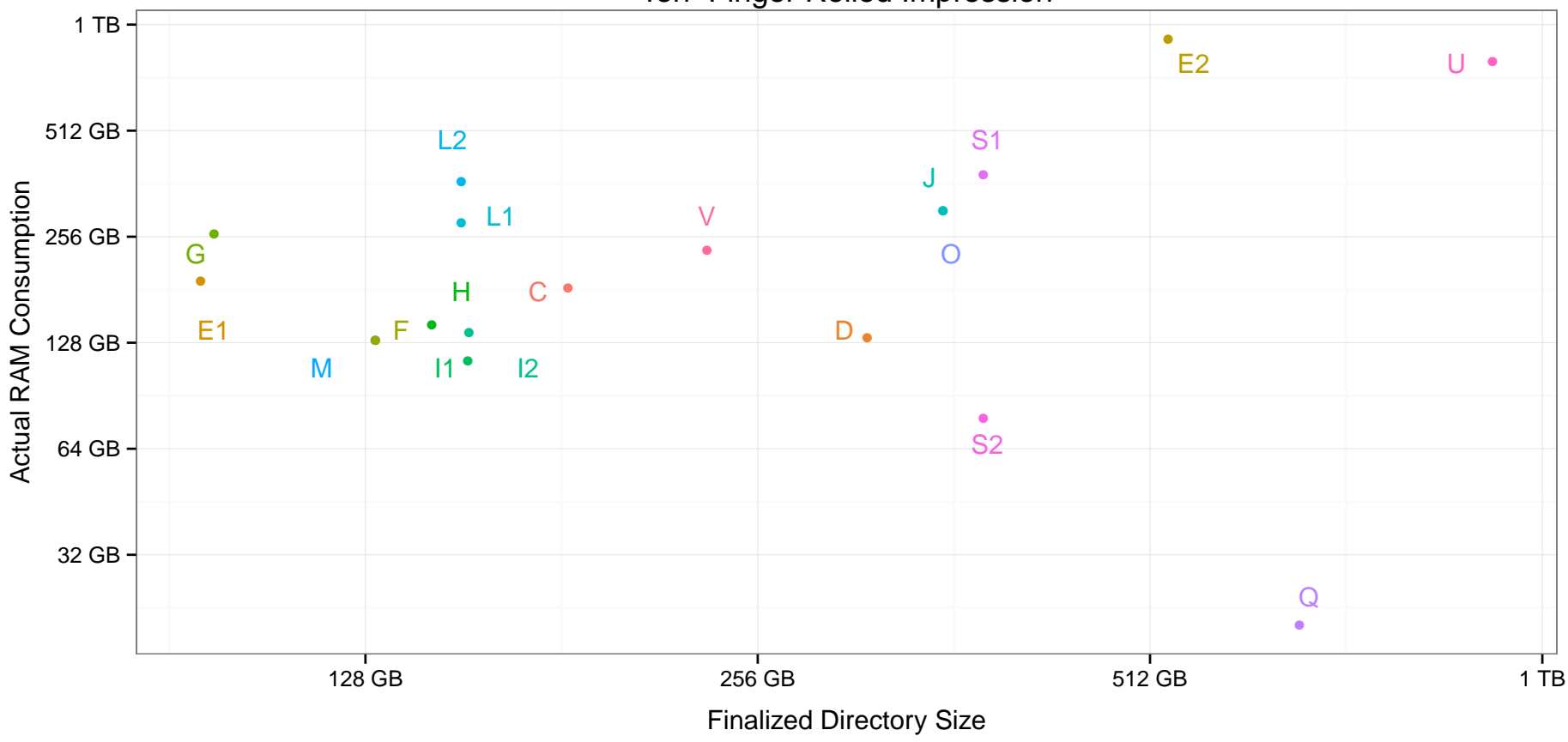

Figure 64: Comparison of enrollment size in RAM and on disk for Class C - Rolled Impression. The $x$ - and $y$-axes use log scales. The data for enrollment size comes from Table 65 in Appendix F. Actual RAM Consumption is from the RAM/Actual column and Finalized Directory Size is from the On Disk/Finalized column.

\section{Ten-Finger Rolled Impression}

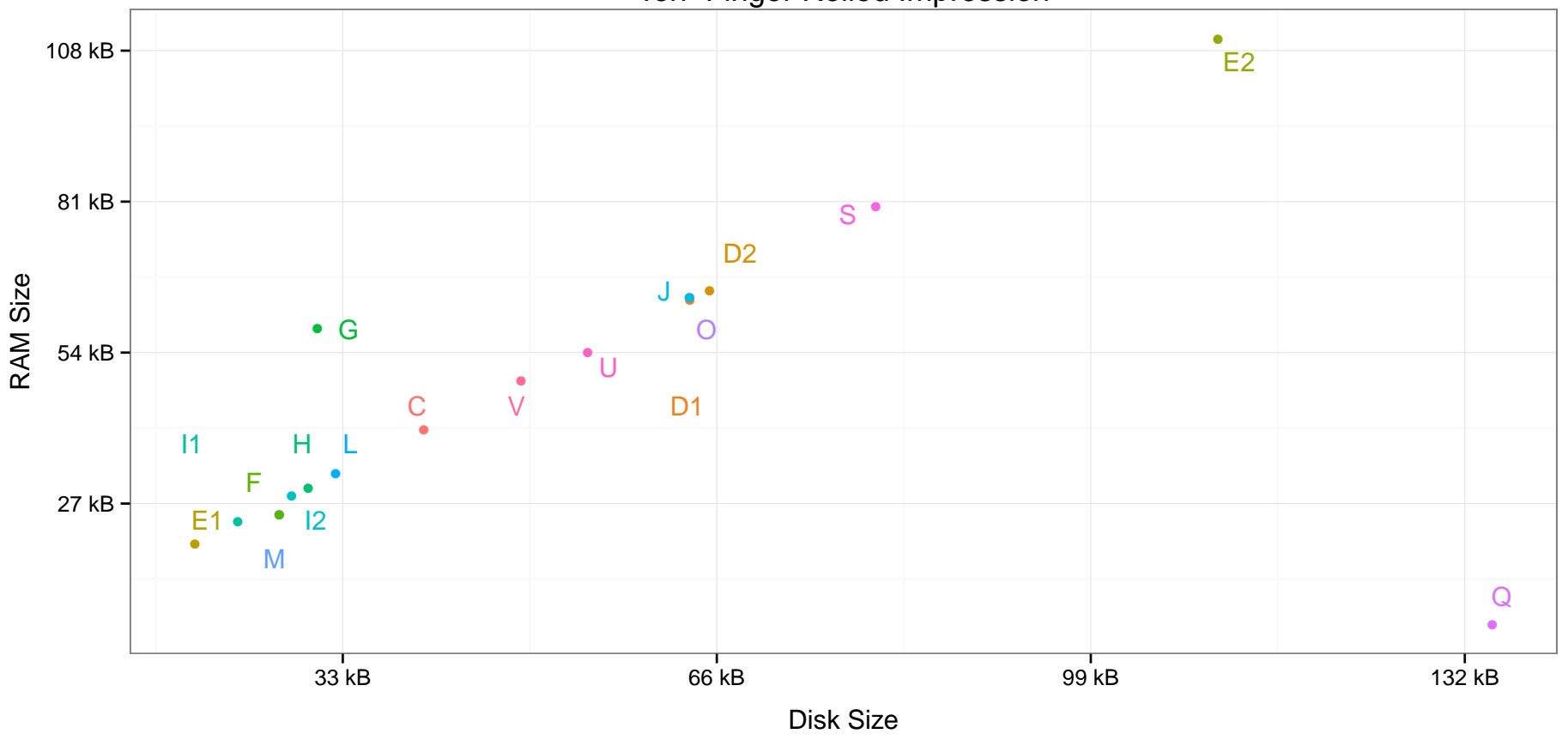

Figure 65: Comparison of search template size in RAM and on disk for Class C - Rolled Impression. On Disk comes from Table 68 in Appendix G.

\begin{tabular}{l|l|l|l|l|l|l}
$\mathrm{C}=$ afis team & $\mathrm{D}=3 \mathrm{M}$ Cogent & $\mathrm{E}=$ Neurotechnology & $\mathrm{F}=$ Papillon & $\mathrm{G}=$ Dermalog & $\mathrm{H}=$ Hisign Bio-Info Institute \\
$\mathrm{I}=$ NEC & $\mathrm{J}=$ Sonda & $\mathrm{K}=$ Tiger IT & $\mathrm{L}=$ Innovatrics & $\mathrm{M}=$ SPEX & $\mathrm{O}=$ ID Solutions \\
$\mathrm{P}=\mathrm{id} 3$ & $\mathrm{Q}=$ Morpho & $\mathrm{S}=$ Decatur Industries & $\mathrm{T}=$ BIO-key & $\mathrm{U}=$ Aware & $\mathrm{V}=$ AA Technology
\end{tabular}




\subsection{Processing Time}

This section shows the time required to enroll fingerprints images also referred to as template creation time. The template creation times were recorded by enrolling a common sample of the datasets on common hardware, with 100 processes running in parallel across 10 compute nodes (10 processes per compute node). Any segmentation time for slap captures was included in the template creation times.

The detailed tables (Tables 72 and 80) used to make the scatter plots (Figures 66 through 71) in this section are included in Appendix H. The Enrollment columns give some idea as to the required system capacity needed to enroll a large dataset in a reasonable time frame. As an example, if a submission takes the full time allowed per image (3 seconds) and 16 compute nodes are used for the enrollment process, it will take approximately 16 days to process all the enrollment sets for all three classes.

In an operational sense, enrollment only occurs a single time for the entire dataset, and then on an as-needed basis when new subjects are added to the dataset. The Search columns are different, as they show the time needed to create a template every time a new search is performed. This time would be factored in as part of the overall search time process.

Some observations from tables and plots include:

$\triangleright$ The most accurate submissions did not have the fastest enrollment times. In fact, the best performers tend to have longer enrollment times.

$\triangleright$ Enrollment time appears to be proportional to finger type and impression. For example, single-finger captures are faster than ten-finger plain impressions, which are faster than ten-finger rolled impressions.

$\triangleright$ Segmentation does not appear to significantly increase enrollment times, as noted by comparing ten-finger plain impressions to ten-finger rolled impressions.

\begin{tabular}{l|l|l|l|l|l}
$\mathrm{C}=$ afis team & $\mathrm{D}=3 \mathrm{M}$ Cogent & $\mathrm{E}=$ Neurotechnology & $\mathrm{F}=$ Papillon & $\mathrm{G}=$ Dermalog & $\mathrm{H}=$ Hisign Bio-Info Institute \\
$\mathrm{I}=$ NEC & $\mathrm{J}=$ Sonda & $\mathrm{K}=$ Tiger IT & $\mathrm{L}=$ Innovatrics & $\mathrm{M}=$ SPEX & $\mathrm{O}=$ ID Solutions
\end{tabular}

$\mathrm{P}=\mathrm{id3} \quad \mathrm{Q}=$ Morpho $\mid \mathrm{S}=$ Decatur Industries $\mathrm{T}=$ BIO-key $\mid \mathrm{U}=$ Aware $\quad \mathrm{V}=$ AA Technology 


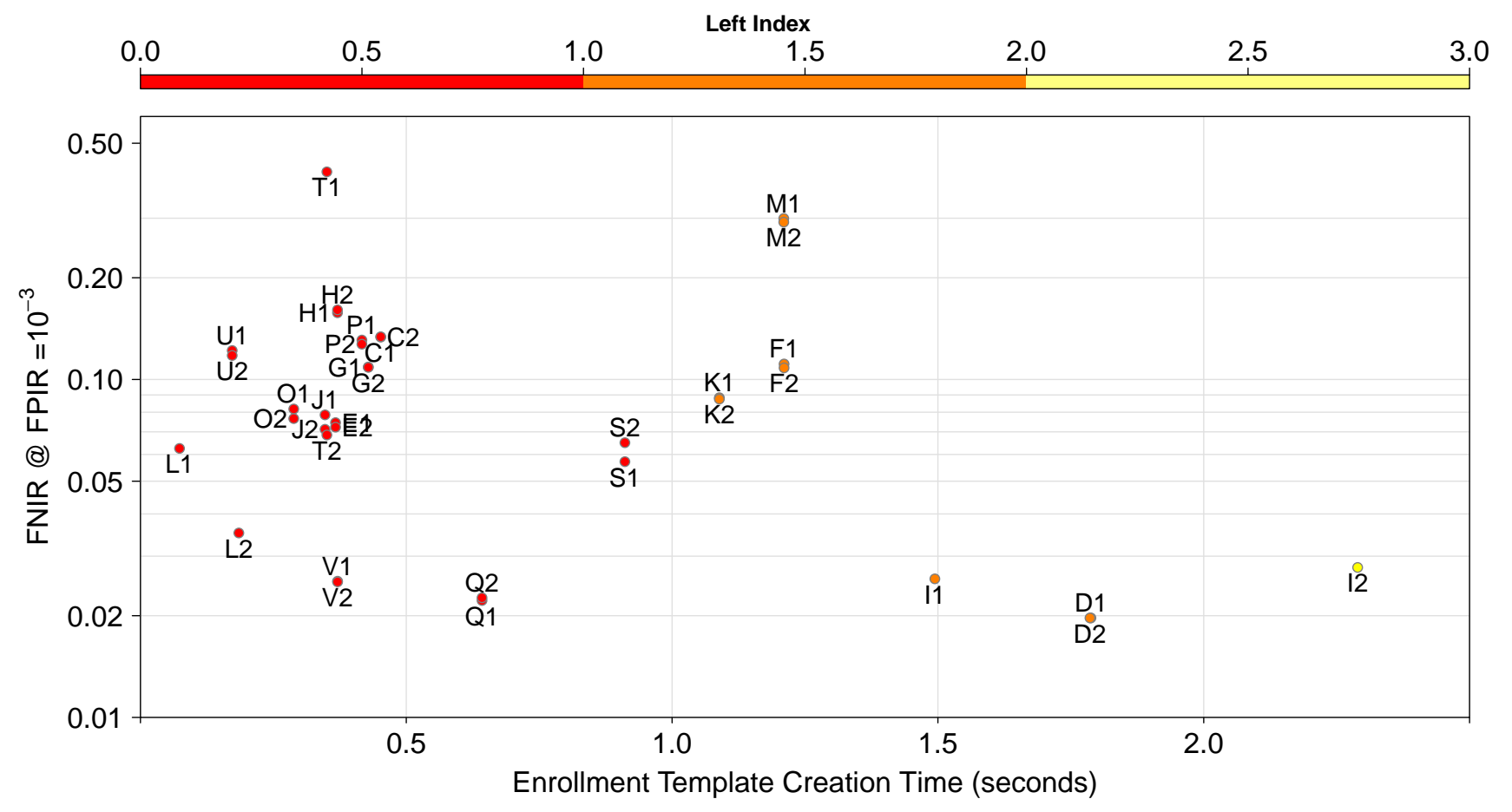

Figure 66: Scatter plot of FNIR @ FPIR $=10^{-3}$ searching 30000 subjects against 100000 subjects and enrollment template creation time for Class A Left Index. The color of the data point is used to show the search template creation time. The color scale for search template creation time is at the top of the plot and the units are in seconds. The template creation time data is from Table 72 in Appendix H.

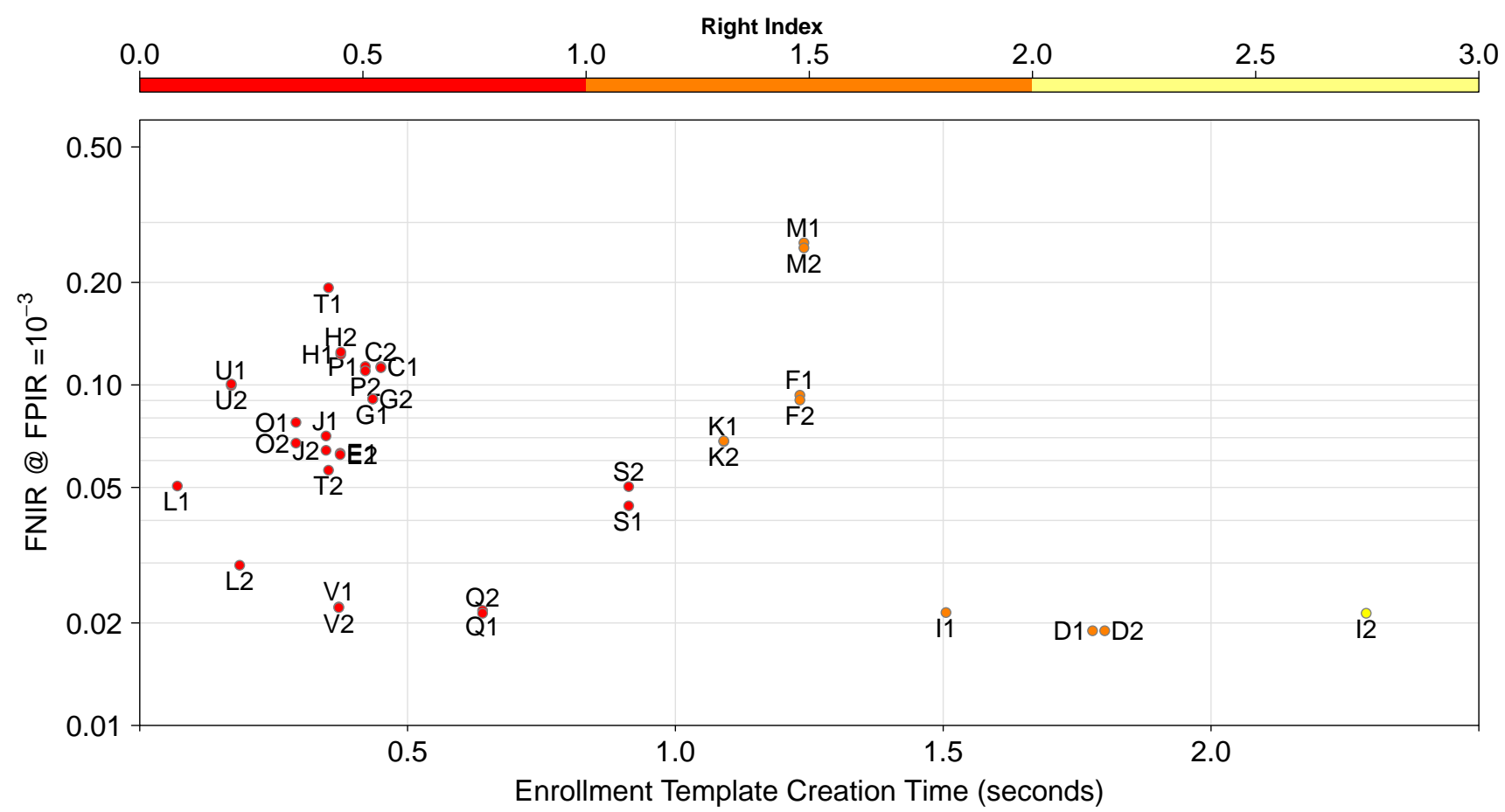

Figure 67: Scatter plot of FNIR @ FPIR $=10^{-3}$ searching 30000 subjects against 100000 subjects and enrollment template creation time for Class A Right Index. The color of the data point is used to show the search template creation time. The color scale for search template creation time is at the top of the plot and the units are in seconds. The template creation time data is from Table 73 in Appendix H.

\begin{tabular}{l|l|l|l|l|l|l}
$\mathrm{C}=$ afis team & $\mathrm{D}=$ = 3M Cogent & $\mathrm{E}=$ Neurotechnology & $\mathrm{F}=$ Papillon & $\mathrm{G}=$ Dermalog & $\mathrm{H}=$ Hisign Bio-Info Institute \\
$\mathrm{I}=$ NEC & $\mathrm{J}=$ Sonda & $\mathrm{K}=$ Tiger IT & $\mathrm{L}=$ Innovatrics & $\mathrm{M}=$ SPEX & $\mathrm{O}=$ ID Solutions \\
$\mathrm{P}=\mathrm{id} 3$ & $\mathrm{Q}=$ Morpho & $\mathrm{S}=$ Decatur Industries & $\mathrm{T}=$ BIO-key & $\mathrm{U}=$ Aware & $\mathrm{V}=$ AA Technology
\end{tabular}




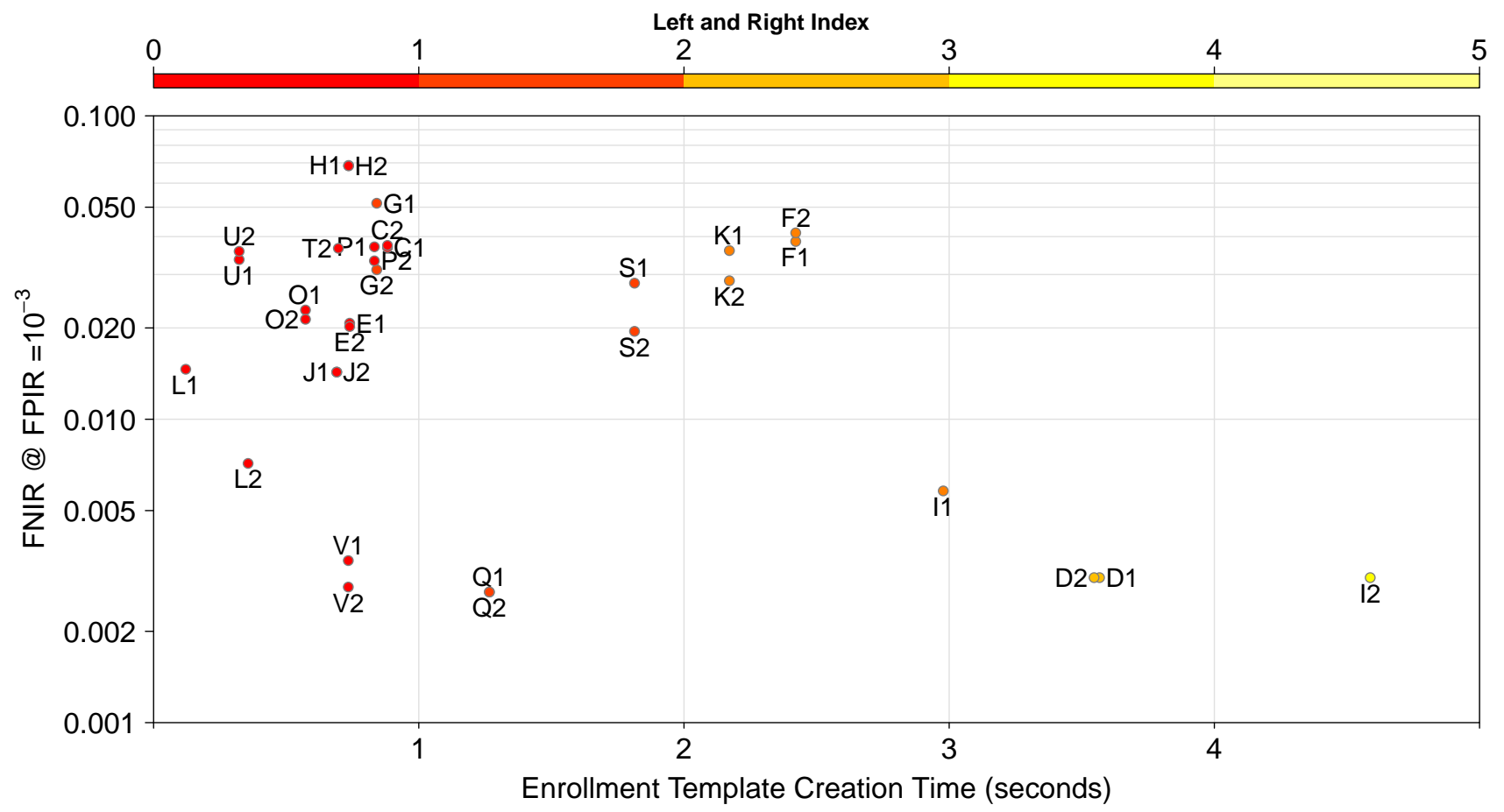

Figure 68: Scatter plot of FNIR @ FPIR $=10^{-3}$ searching 30000 subjects against 1600000 subjects and enrollment template creation time for Class A Left and Right Index. The color of the data point is used to show the search template creation time. The color scale for search template creation time is at the top of the plot and the units are in seconds. The template creation time data is from Table 74 in Appendix $\mathrm{H}$.

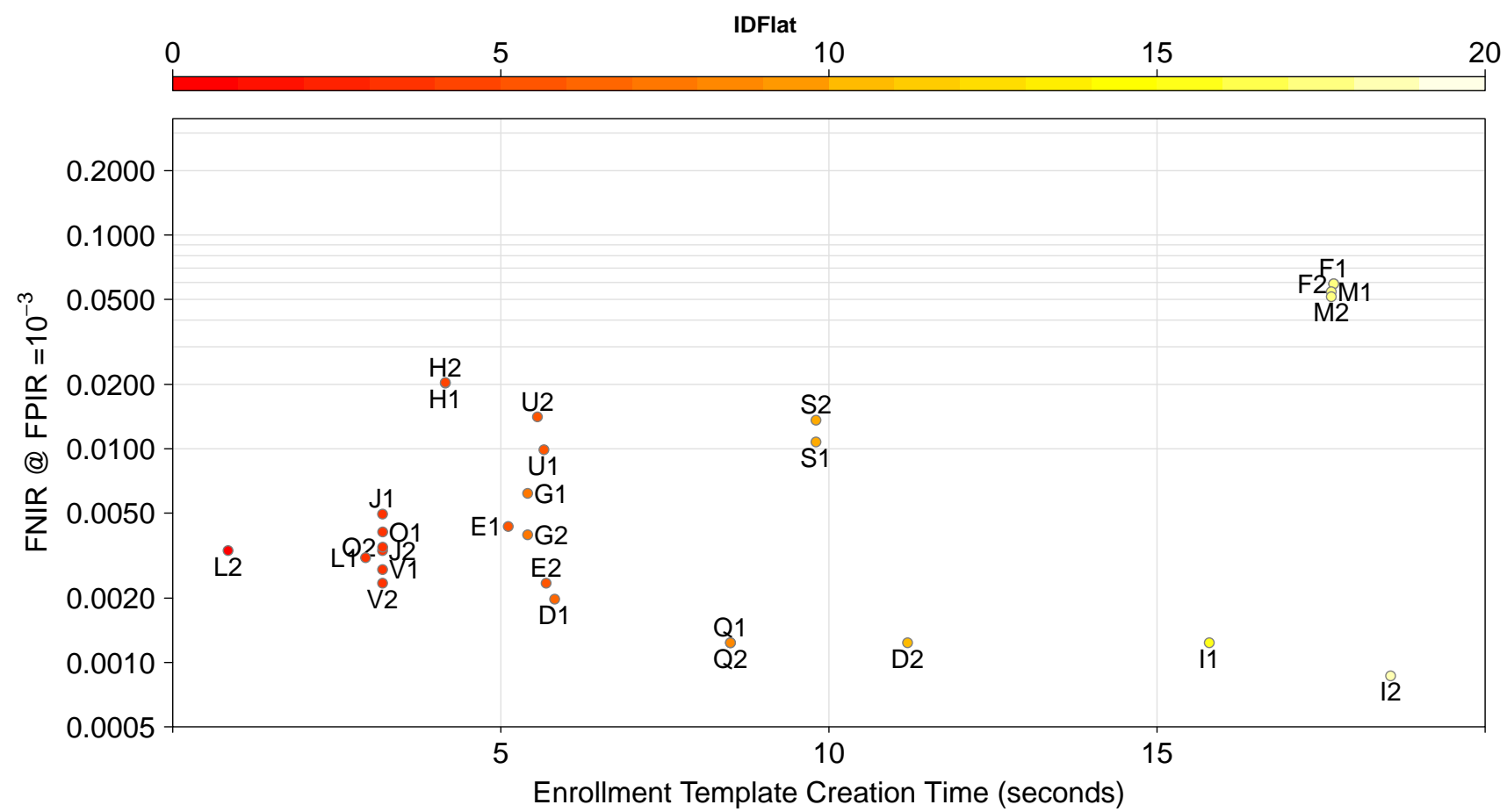

Figure 69: Scatter plot of FNIR @ FPIR $=10^{-3}$ searching 30000 subjects against 3000000 subjects and enrollment template creation time for Class B Identification Flats. The color of the data point is used to show the search template creation time. The color scale for search template creation time is at the top of the plot and the units are in seconds. The template creation time data is from Table 78 in Appendix $\mathrm{H}$.

\begin{tabular}{l|l|l|l|l|l|l}
$\mathrm{C}=$ afis team & $\mathrm{D}=3 \mathrm{M}$ Cogent & $\mathrm{E}=$ Neurotechnology & $\mathrm{F}=$ Papillon & $\mathrm{G}=$ Dermalog & $\mathrm{H}=$ Hisign Bio-Info Institute \\
$\mathrm{I}=$ NEC & $\mathrm{J}=$ Sonda & $\mathrm{K}=$ Tiger IT & $\mathrm{L}=$ Innovatrics & $\mathrm{M}=$ SPEX & $\mathrm{O}=$ ID Solutions \\
$\mathrm{P}=\mathrm{id} 3$ & $\mathrm{Q}=$ Morpho & $\mathrm{S}=$ Decatur Industries & $\mathrm{T}=$ BIO-key & $\mathrm{U}=$ Aware & $\mathrm{V}=$ AA Technology
\end{tabular}




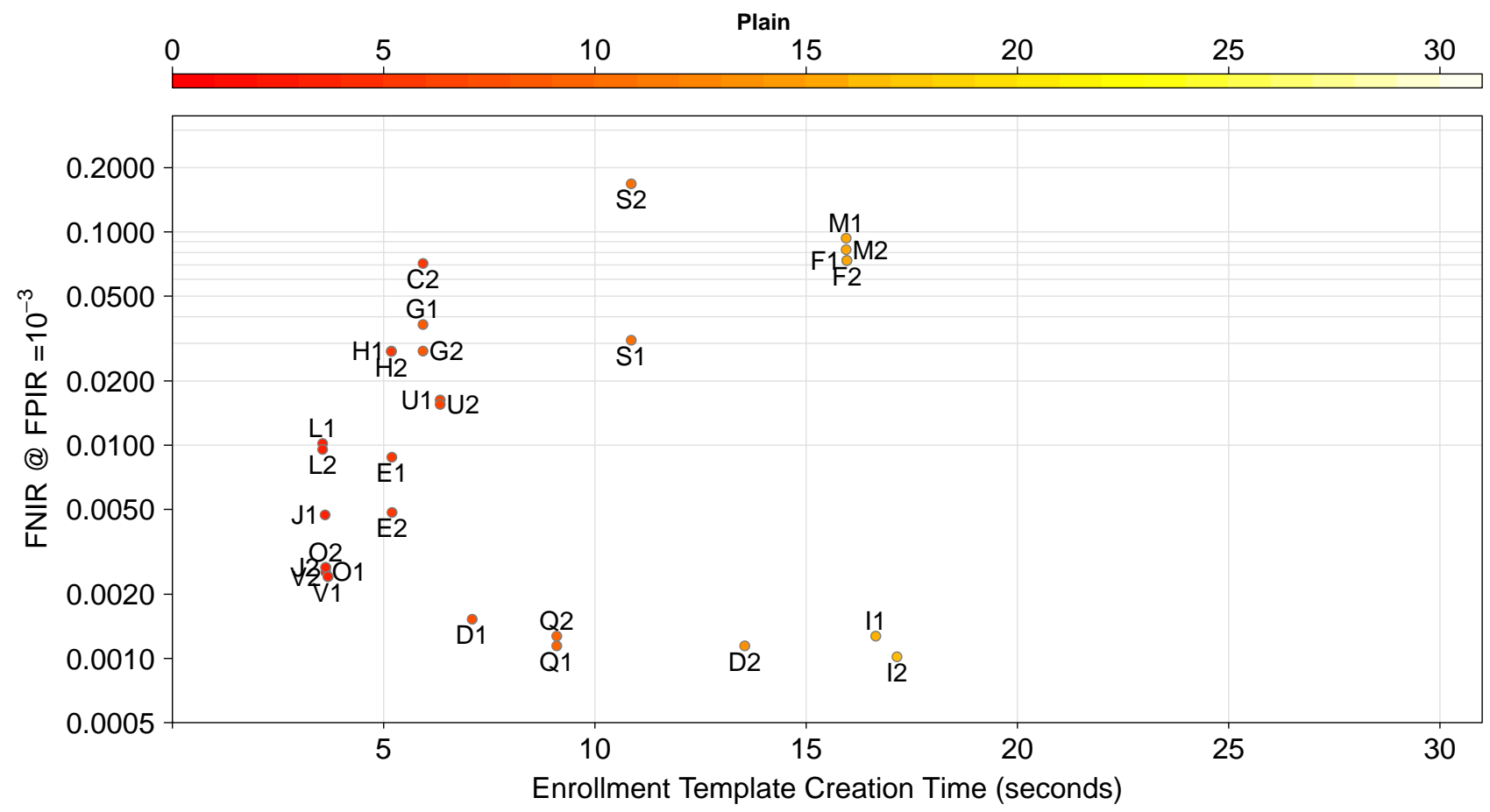

Figure 70: Scatter plot of FNIR @ FPIR $=10^{-3}$ searching 30000 subjects against 5000000 subjects and enrollment template creation time for Class C Ten-Finger Plain-to-Plain. The color of the data point is used to show the search template creation time. The color scale for search template creation time is at the top of the plot and the units are in seconds. The template creation time data is from Table 79 in Appendix H.

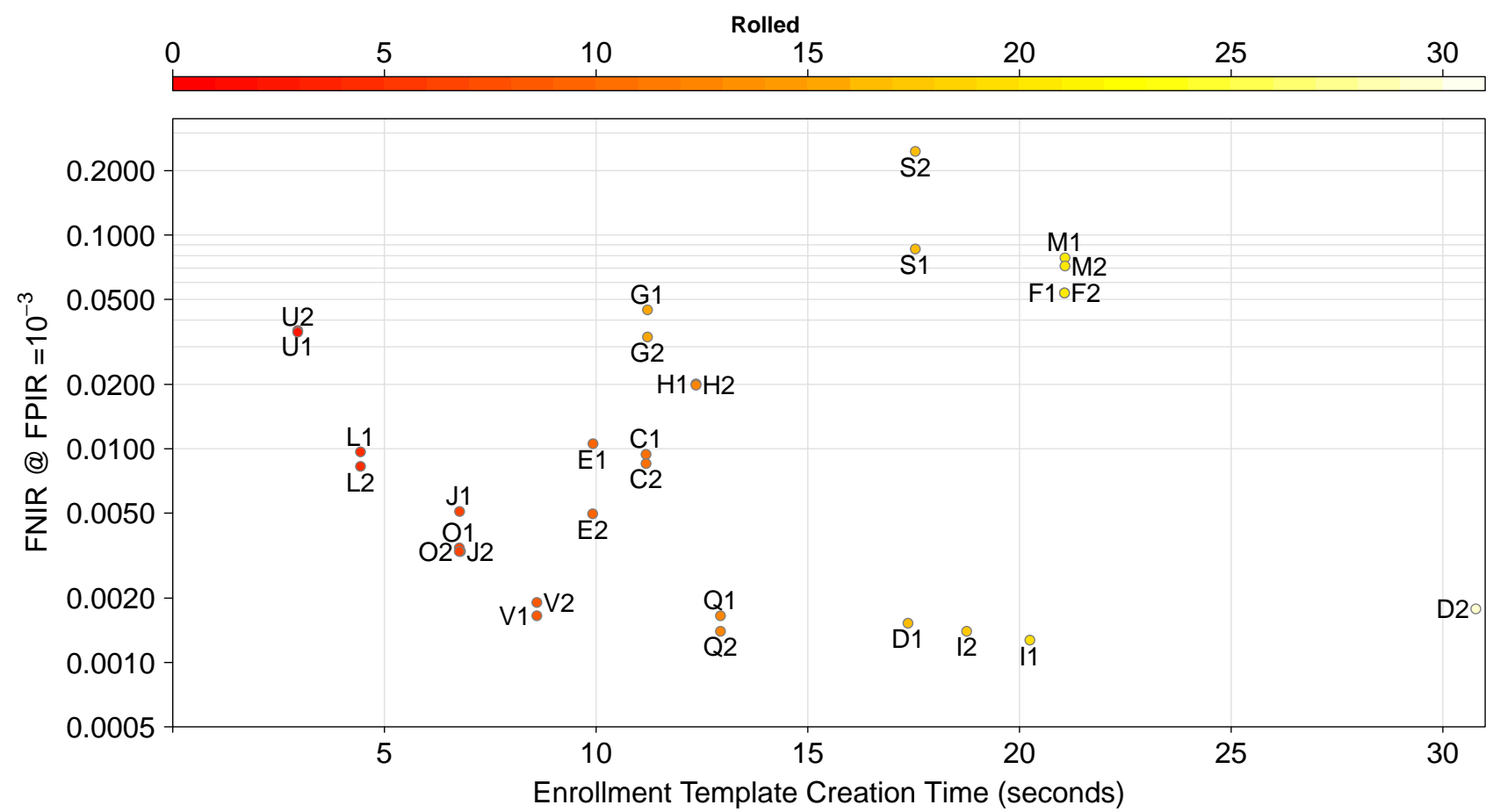

Figure 71: Scatter plot of FNIR @ FPIR =10 $10^{-3}$ searching 30000 subjects against 5000000 subjects and enrollment template creation time for Class C Ten-Finger Rolled-to-Rolled. The color of the data point is used to show the search template creation time. The color scale for search template creation time is at the top of the plot and the units are in seconds. The template creation time data is from Table 80 in Appendix $\mathrm{H}$.

\begin{tabular}{l|l|l|l|l|l|l}
$\mathrm{C}=$ afis team & $\mathrm{D}=3 \mathrm{M}$ Cogent & $\mathrm{E}=$ Neurotechnology & $\mathrm{F}=$ Papillon & $\mathrm{G}=$ Dermalog & $\mathrm{H}=$ Hisign Bio-Info Institute \\
$\mathrm{I}=$ NEC & $\mathrm{J}=$ Sonda & $\mathrm{K}=$ Tiger IT & $\mathrm{L}=$ Innovatrics & $\mathrm{M}=$ SPEX & $\mathrm{O}=$ ID Solutions \\
$\mathrm{P}=\mathrm{id} 3$ & $\mathrm{Q}=$ Morpho & $\mathrm{S}=$ Decatur Industries & $\mathrm{T}=$ BIO-key & $\mathrm{U}=$ Aware & $\mathrm{V}=$ AA Technology
\end{tabular}




\section{Ranked Results}

This section combines tables from Sections 7 and 9 into a single table. The resulting tables are rank-sorted based on FNIR values.

There is one table from each class of participation included in the main body of this report. The full set of tables for all classes and search set scenarios are included in Appendix I.

These tables are useful because they combine all the high-level information in a single table. They are rank-sorted on FNIR, as accuracy is generally considered the most important goal for an identification algorithm to achieve. The reader can then look across and see how a participant ranked in other areas such as search time (Identification), search template creation time (Search Enrollment), and memory usage (RAM). As stated in previous sections, the most accurate submissions are not the fastest. In all three classes, there is a two to three times increase in the error rate when comparing the most accurate submission with one of the top three fastest in search speed.

Appendix K plots relative comparisons of FNIR, RAM usage, template creation times, and search times.

Appendix $\mathrm{L}$ is one attempt to take the tables in this section and apply some relative weight or importance to each column. The tables in Appendix L use these weights to produce a score for each submission and then sort the results based on those scores. Refer to Appendix L for more details.

\begin{tabular}{l|l|l|l|ll|l}
$\mathrm{C}=$ afis team & $\mathrm{D}=3 \mathrm{M}$ Cogent & $\mathrm{E}=$ Neurotechnology & $\mathrm{F}=$ Papillon & $\mathrm{G}=$ Dermalog & $\mathrm{H}=$ Hisign Bio-Info Institute \\
$\mathrm{I}=$ NEC & $\mathrm{J}=$ Sonda & $\mathrm{K}=$ Tiger IT & $\mathrm{L}=$ Innovatrics & $\mathrm{M}=$ SPEX & $\mathrm{O}=$ ID Solutions \\
$\mathrm{P}=\mathrm{id} 3$ & $\mathrm{Q}=$ Morpho & $\mathrm{S}=$ Decatur Industries & $\mathrm{T}=$ BIO-key & $\mathrm{U}=$ Aware & $\mathrm{V}=$ AA Technology
\end{tabular}




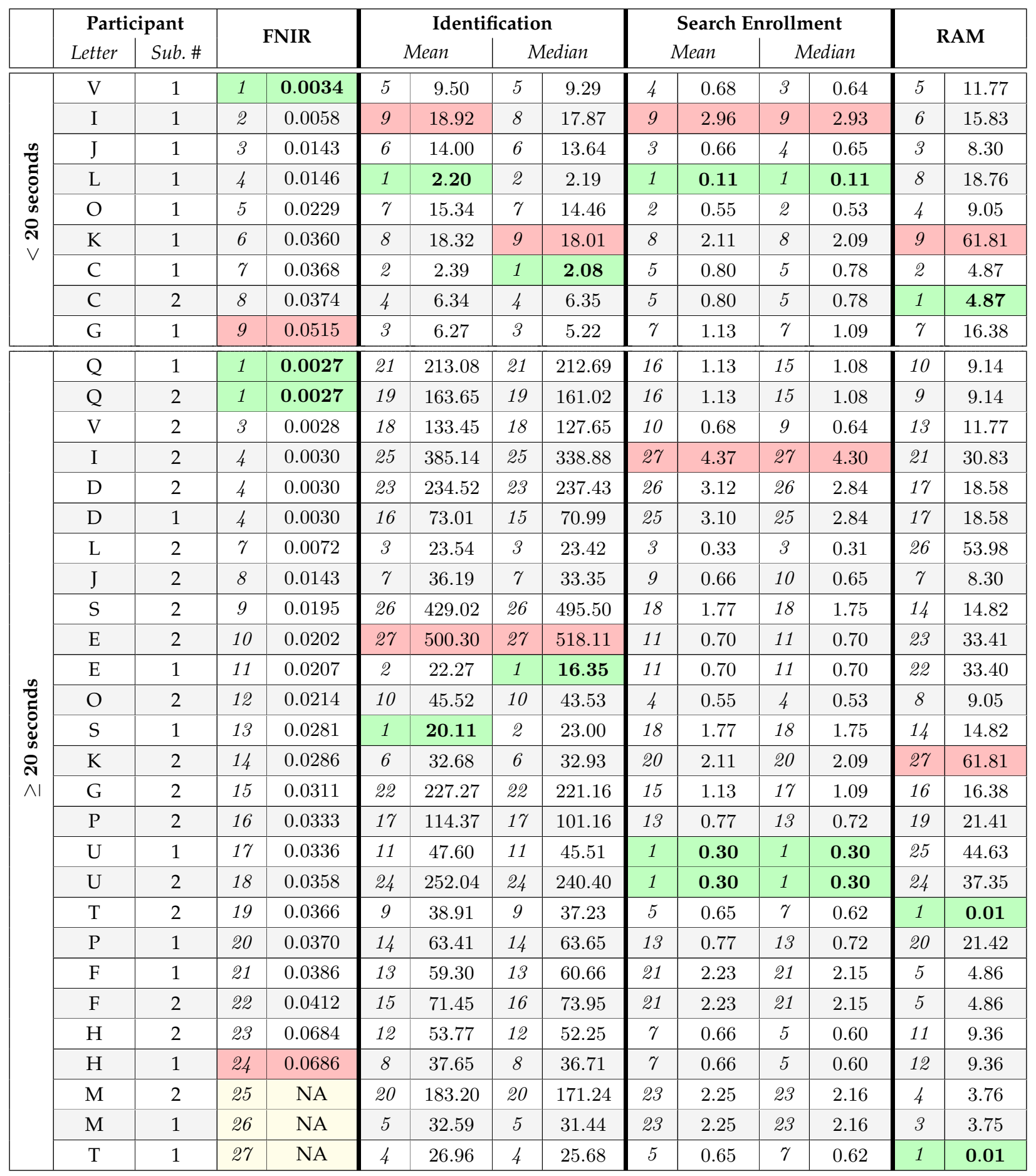

Table 21: Tabulation of ranked results for Class A - Left and Right Index. Submissions were split into two groups. The first group includes submissions that performed searches on average in less than 20 seconds, and the second includes those that took, on average, 20 seconds or longer. Letter refers to the participant's letter code found on the footer of this page. Sub. \# is an identifier used to differentiate between the two submissions each participant could make. The FNIR column was computed at the score threshold that gave FPIR $=10^{-3}$. NA indicates that the operations required to produce the value could not be performed. The Identification column shows the time used to perform a search over an enrollment set of 1600000 , as seen in Table 18 . The Search Enrollment column shows the time used to create a search template to be used for a query, as seen in Table 74. Identification and Search Enrollment durations are reported in seconds, but were originally recorded to microsecond precision. RAM refers to the sum of the resident set sizes of the stage one identification processes over all compute nodes after returning from the identification stage one initialization method, as seen in Table 62. $R A M$ is reported in gigabytes, where 1 GB is equal to 1073741824 bytes. The number to the left of a value provides the value's column-wise ranking, with the best performance shaded in green and the worst in pink. The table is sorted on the FNIR column-wise ranking.

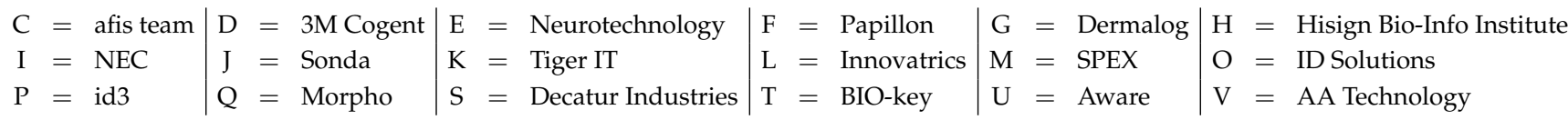




\begin{tabular}{|c|c|c|c|c|c|c|c|c|c|c|c|c|c|}
\hline \multicolumn{2}{|c|}{ Participant } & \multirow{2}{*}{\multicolumn{2}{|c|}{ FNIR }} & \multicolumn{4}{|c|}{ Identification } & \multicolumn{4}{|c|}{ Search Enrollment } & \multirow{2}{*}{\multicolumn{2}{|c|}{ RAM }} \\
\hline Letter & Sub. \# & & & \multicolumn{2}{|c|}{ Mean } & \multicolumn{2}{|c|}{ Median } & \multicolumn{2}{|c|}{ Mean } & \multicolumn{2}{|c|}{ Median } & & \\
\hline I & 2 & 1 & 0.0009 & 20 & 60.01 & 16 & 43.86 & 30 & 19.36 & 30 & 19.36 & 21 & 108.71 \\
\hline$Q$ & 1 & 2 & 0.0012 & 14 & 48.85 & 14 & 42.92 & 20 & 8.89 & 20 & 8.92 & 1 & 7.54 \\
\hline $\mathrm{Q}$ & 2 & 2 & 0.0012 & 24 & 71.67 & 24 & 66.01 & 20 & 8.89 & 20 & 8.92 & 2 & 7.54 \\
\hline $\mathrm{I}$ & 1 & 2 & 0.0012 & 6 & 24.57 & 7 & 19.07 & 25 & 16.50 & 25 & 16.52 & 5 & 49.68 \\
\hline $\mathrm{D}$ & 2 & 2 & 0.0012 & 19 & 54.37 & 18 & 46.70 & 24 & 11.90 & 24 & 11.86 & 13 & 79.43 \\
\hline $\mathrm{D}$ & 1 & 6 & 0.0020 & 17 & 52.42 & 17 & 45.15 & 17 & 6.19 & 17 & 6.16 & 12 & 79.43 \\
\hline $\mathrm{V}$ & 2 & 7 & 0.0024 & 15 & 49.72 & 19 & 49.30 & 3 & 3.35 & 7 & 3.36 & 9 & 63.53 \\
\hline$E$ & 2 & 7 & 0.0024 & 18 & 52.88 & 15 & 43.61 & 16 & 5.95 & 16 & 5.93 & 28 & 317.95 \\
\hline $\mathrm{V}$ & 1 & 9 & 0.0027 & 10 & 35.51 & 10 & 34.96 & 3 & 3.35 & 7 & 3.36 & 8 & 63.53 \\
\hline $\mathrm{L}$ & 1 & 10 & 0.0031 & 5 & 14.42 & 5 & 14.50 & 2 & 3.05 & 2 & 3.05 & 22 & 119.79 \\
\hline $\mathrm{L}$ & 2 & 11 & 0.0033 & 8 & 28.56 & 9 & 28.59 & 1 & 0.88 & 1 & 0.88 & 27 & 177.48 \\
\hline $\mathrm{J}$ & 2 & 11 & 0.0033 & 22 & 64.38 & 22 & 60.13 & 7 & 3.38 & 3 & 3.35 & 17 & 101.00 \\
\hline $\mathrm{O}$ & 2 & 13 & 0.0035 & 21 & 63.87 & 21 & 60.02 & 5 & 3.38 & 5 & 3.36 & 20 & 101.00 \\
\hline G & 2 & 14 & 0.0040 & 9 & 31.67 & 6 & 16.26 & 18 & 7.89 & 18 & 7.90 & 26 & 156.12 \\
\hline $\mathrm{O}$ & 1 & 15 & 0.0041 & 11 & 37.04 & 11 & 35.64 & 5 & 3.38 & 5 & 3.36 & 19 & 101.00 \\
\hline E & 1 & 16 & 0.0043 & 2 & 8.76 & 2 & 6.76 & 13 & 5.35 & 13 & 5.34 & 14 & 79.73 \\
\hline $\mathrm{J}$ & 1 & 17 & 0.0049 & 7 & 26.31 & 8 & 25.38 & 7 & 3.38 & 3 & 3.35 & 18 & 101.00 \\
\hline $\mathrm{G}$ & 1 & 18 & 0.0062 & 1 & 6.33 & 1 & 4.26 & 18 & 7.89 & 18 & 7.90 & 25 & 156.12 \\
\hline $\mathrm{U}$ & 1 & 19 & 0.0099 & 30 & 88.83 & 28 & 86.60 & 15 & 5.90 & 15 & 5.82 & 29 & 440.68 \\
\hline$S$ & 1 & 20 & 0.0108 & 28 & 86.50 & 29 & 87.60 & 22 & 10.24 & 22 & 10.31 & 24 & 150.35 \\
\hline $\mathrm{S}$ & 2 & 21 & 0.0136 & 29 & 88.70 & 30 & 88.46 & 22 & 10.24 & 22 & 10.31 & 23 & 150.35 \\
\hline $\mathrm{U}$ & 2 & 22 & 0.0141 & 25 & 80.14 & 25 & 75.28 & 14 & 5.80 & 14 & 5.73 & 30 & 540.60 \\
\hline $\mathrm{H}$ & 1 & 23 & 0.0203 & 26 & 82.43 & 26 & 82.66 & 9 & 4.37 & 9 & 4.37 & 15 & 86.46 \\
\hline $\mathrm{H}$ & 2 & 24 & 0.0204 & 27 & 85.74 & 27 & 86.56 & 9 & 4.37 & 9 & 4.37 & 16 & 86.46 \\
\hline $\mathrm{M}$ & 2 & 25 & 0.0515 & 23 & 70.45 & 23 & 65.91 & 26 & 18.36 & 28 & 18.11 & 6 & 57.53 \\
\hline $\mathrm{M}$ & 1 & 26 & 0.0543 & 13 & 41.37 & 13 & 38.87 & 26 & 18.36 & 28 & 18.11 & 6 & 57.53 \\
\hline $\mathrm{F}$ & 1 & 27 & 0.0591 & 12 & 39.12 & 12 & 37.56 & 28 & 18.36 & 26 & 18.07 & 10 & 77.02 \\
\hline $\mathrm{F}$ & 2 & 27 & 0.0591 & 16 & 51.59 & 20 & 49.34 & 28 & 18.36 & 26 & 18.07 & 10 & 77.02 \\
\hline $\mathrm{C}$ & 2 & 29 & NA & 4 & 10.28 & 4 & 10.21 & 11 & 5.16 & 11 & 5.13 & 4 & 46.49 \\
\hline C & 1 & 30 & NA & 3 & 9.00 & 3 & 7.92 & 11 & 5.16 & 11 & 5.13 & 3 & 46.49 \\
\hline
\end{tabular}

Table 22: Tabulation of ranked results for Class B - Identification Flats. Letter refers to the participant's letter code found on the footer of this page. Sub. \# is an identifier used to differentiate between the two submissions each participant could make. The FNIR column was computed at the score threshold that gave FPIR $=10^{-3}$. NA indicates that the operations required to produce the value could not be performed. The Identification column shows the time used to perform a search over an enrollment set of 3000 000, as seen in Table 19. The Search Enrollment column shows the time used to create a search template to be used for a query, as seen in Table 78. Identification and Search Enrollment durations are reported in seconds, but were originally recorded to microsecond precision. RAM refers to the sum of the resident set sizes of the stage one identification processes over all compute nodes after returning from the identification stage one initialization method, as seen in Table 63. RAM is reported in gigabytes, where 1 GB is equal to 1073741824 bytes. The number to the left of a value provides the value's column-wise ranking, with the best performance shaded in green and the worst in pink. The table is sorted on the FNIR column-wise ranking. 


\begin{tabular}{|c|c|c|c|c|c|c|c|c|c|c|c|c|c|}
\hline \multicolumn{2}{|c|}{ Participant } & \multirow{2}{*}{\multicolumn{2}{|c|}{ FNIR }} & \multicolumn{4}{|c|}{ Identification } & \multicolumn{4}{|c|}{ Search Enrollment } & \multirow{2}{*}{\multicolumn{2}{|c|}{ RAM }} \\
\hline Letter & Sub.\# & & & \multicolumn{2}{|c|}{ Mean } & \multicolumn{2}{|c|}{ Median } & \multicolumn{2}{|c|}{ Mean } & \multicolumn{2}{|c|}{ Median } & & \\
\hline I & 1 & 1 & 0.0013 & 24 & 79.04 & 15 & 54.56 & 25 & 20.21 & 25 & 20.23 & 4 & 113.80 \\
\hline$Q$ & 2 & 2 & 0.0014 & 27 & 83.35 & 25 & 74.22 & 17 & 12.95 & 17 & 13.06 & 2 & 20.22 \\
\hline I & 2 & 2 & 0.0014 & 9 & 40.53 & 7 & 30.82 & 24 & 18.72 & 24 & 18.73 & 11 & 137.03 \\
\hline $\mathrm{D}$ & 1 & 4 & 0.0015 & 17 & 65.97 & 17 & 58.92 & 23 & 17.44 & 21 & 17.39 & 10 & 132.40 \\
\hline Q & 1 & 5 & 0.0017 & 26 & 83.25 & 26 & 74.40 & 17 & 12.95 & 17 & 13.06 & 1 & 20.22 \\
\hline $\mathrm{V}$ & 1 & 5 & 0.0017 & 10 & 40.94 & 10 & 40.74 & 9 & 8.48 & 9 & 8.50 & 17 & 234.03 \\
\hline $\mathrm{D}$ & 2 & 7 & 0.0018 & 30 & 86.39 & 27 & 74.97 & 30 & 30.43 & 30 & 30.47 & 9 & 132.37 \\
\hline V & 2 & 8 & 0.0019 & 16 & 65.47 & 18 & 65.07 & 9 & 8.48 & 9 & 8.50 & 18 & 234.03 \\
\hline $\mathrm{O}$ & 2 & 9 & 0.0033 & 19 & 72.55 & 21 & 69.30 & 6 & 6.80 & 6 & 6.71 & 23 & 303.13 \\
\hline $\mathrm{J}$ & 2 & 9 & 0.0033 & 20 & 72.62 & 19 & 67.98 & 8 & 6.82 & 8 & 6.74 & 24 & 303.13 \\
\hline $\mathrm{O}$ & 1 & 11 & 0.0034 & 15 & 59.01 & 14 & 54.42 & 6 & 6.80 & 6 & 6.71 & 25 & 303.13 \\
\hline $\mathrm{E}$ & 2 & 12 & 0.0050 & 14 & 57.02 & 11 & 42.73 & 11 & 9.89 & 11 & 9.91 & 30 & 930.48 \\
\hline $\mathrm{J}$ & 1 & 13 & 0.0051 & 8 & 36.09 & 9 & 33.02 & 5 & 6.78 & 5 & 6.69 & 22 & 303.13 \\
\hline $\mathrm{L}$ & 2 & 14 & 0.0083 & 3 & 19.52 & 5 & 20.25 & 3 & 4.36 & 3 & 4.36 & 26 & 367.82 \\
\hline $\mathrm{C}$ & 2 & 15 & 0.0085 & 5 & 25.91 & 6 & 21.97 & 13 & 10.79 & 13 & 10.69 & 15 & 183.36 \\
\hline C & 1 & 16 & 0.0094 & 4 & 25.25 & 4 & 20.15 & 13 & 10.79 & 13 & 10.69 & 14 & 183.36 \\
\hline $\mathrm{L}$ & 1 & 17 & 0.0097 & 7 & 31.42 & 8 & 31.68 & 3 & 4.36 & 3 & 4.36 & 21 & 280.49 \\
\hline $\mathrm{E}$ & 1 & 18 & 0.0106 & 1 & 9.52 & 2 & 8.63 & 12 & 9.90 & 12 & 9.92 & 16 & 191.66 \\
\hline $\mathrm{H}$ & 2 & 19 & 0.0199 & 29 & 84.26 & 29 & 84.50 & 15 & 12.08 & 15 & 12.17 & 12 & 144.02 \\
\hline $\mathrm{H}$ & 1 & 20 & 0.0201 & 28 & 84.14 & 30 & 84.51 & 15 & 12.08 & 15 & 12.17 & 13 & 144.02 \\
\hline G & 2 & 21 & 0.0333 & 6 & 30.61 & 3 & 19.46 & 19 & 15.55 & 19 & 15.50 & 19 & 261.29 \\
\hline $\mathrm{U}$ & 2 & 22 & 0.0351 & 23 & 74.39 & 24 & 72.48 & 1 & 2.94 & 1 & 2.87 & 28 & 806.17 \\
\hline $\mathrm{U}$ & 1 & 23 & 0.0358 & 25 & 82.76 & 28 & 82.61 & 1 & 2.94 & 1 & 2.87 & 28 & 806.17 \\
\hline$G$ & 1 & 24 & 0.0447 & 2 & 11.67 & 1 & 7.78 & 19 & 15.55 & 19 & 15.50 & 20 & 261.29 \\
\hline $\mathrm{F}$ & 1 & 25 & 0.0536 & 13 & 55.63 & 16 & 55.79 & 28 & 21.07 & 28 & 20.92 & 7 & 130.29 \\
\hline $\mathrm{F}$ & 2 & 25 & 0.0536 & 18 & 68.61 & 20 & 68.20 & 28 & 21.07 & 28 & 20.92 & 6 & 130.29 \\
\hline$M$ & 2 & 27 & 0.0716 & 12 & 48.71 & 13 & 48.80 & 26 & 21.02 & 26 & 20.89 & 5 & 130.29 \\
\hline $\mathrm{M}$ & 1 & 28 & 0.0783 & 11 & 47.38 & 12 & 48.08 & 26 & 21.02 & 26 & 20.89 & 8 & 130.29 \\
\hline $\mathrm{S}$ & 1 & 29 & 0.0860 & 22 & 74.01 & 22 & 69.71 & 21 & 17.43 & 22 & 17.57 & 27 & 382.88 \\
\hline$S$ & 2 & 30 & 0.2462 & 21 & 72.95 & 23 & 70.77 & 21 & 17.43 & 22 & 17.57 & 3 & 78.28 \\
\hline
\end{tabular}

Table 23: Tabulation of ranked results for Class C - Ten-Finger Rolled-to-Rolled. Letter refers to the participant's letter code found on the footer of this page. Sub. \# is an identifier used to differentiate between the two submissions each participant could make. The FNIR column was computed at the score threshold that gave FPIR $=10^{-3}$. The Identification column shows the time used to perform a search over an enrollment set of 5000000 , as seen in Table 20. The Search Enrollment column shows the time used to create a search template to be used for a query, as seen in Table 80. Identification and Search Enrollment durations are reported in seconds, but were originally recorded to microsecond precision. RAM refers to the sum of the resident set sizes of the stage one identification processes over all compute nodes after returning from the identification stage one initialization method, as seen in Table 65. RAM is reported in gigabytes, where 1 GB is equal to 1073741824 bytes. The number to the left of a value provides the value's column-wise ranking, with the best performance shaded in green and the worst in pink. The table is sorted on the FNIR column-wise ranking.

\begin{tabular}{l|l|l|l|ll|l}
$\mathrm{C}=$ afis team & $\mathrm{D}=3 \mathrm{M}$ Cogent & $\mathrm{E}=$ Neurotechnology & $\mathrm{F}=$ Papillon & $\mathrm{G}=$ Dermalog & $\mathrm{H}=$ Hisign Bio-Info Institute \\
$\mathrm{I}=$ NEC & $\mathrm{J}=$ Sonda & $\mathrm{K}=$ Tiger IT & $\mathrm{L}=$ Innovatrics & $\mathrm{M}=$ SPEX & $\mathrm{O}=$ ID Solutions \\
$\mathrm{P}=\mathrm{id} 3$ & $\mathrm{Q}=$ Morpho & $\mathrm{S}=$ Decatur Industries & $\mathrm{T}=$ BIO-key & $\mathrm{U}=$ Aware & $\mathrm{V}=$ AA Technology
\end{tabular}




\section{How Many Fingers are Needed}

It is already well known that using more fingers results in a lower FNIR [17]. This section combines the results from Figures 12, 15, 18, and 21 into a single plot in Figure 72.

The reader is reminded that enrollment set sizes were 100000 subjects for single index fingers, 1.6 million subjects for two index fingers, 3 million subjects for IDFlats, and 5 million for ten-finger rolled and plain impressions. The search set size was 30000 that included 20000 nonmate and 10000 mate searches.

Additionally, Appendix J plots relative comparisons, by class, for each search set used in FpVTE.

Some observations regarding numbers of fingers include:

$\triangleright$ More fingers were better and produced the most accurate results.

$\triangleright$ More fingers took more time to enroll (Subsection 9.2)

$\triangleright$ Ten-finger plain-to-plain impressions were as accurate as ten-finger rolled-to-rolled impressions with higher performing submissions.

$\triangleright$ More fingers generally produce faster search times against very large enrollment sets (Section 8).

$\triangleright$ Class B four-finger slap identification appeared to be less accurate than Class A two-finger identification. This needs further investigation as to the cause. Two possibilities are slap segmentation errors or fingerprint image quality. After manually inspecting some of the errors and considering the ten finger IDFlat and plain-to-plain results, it would appear that image quality may have been the largest contributing factor.

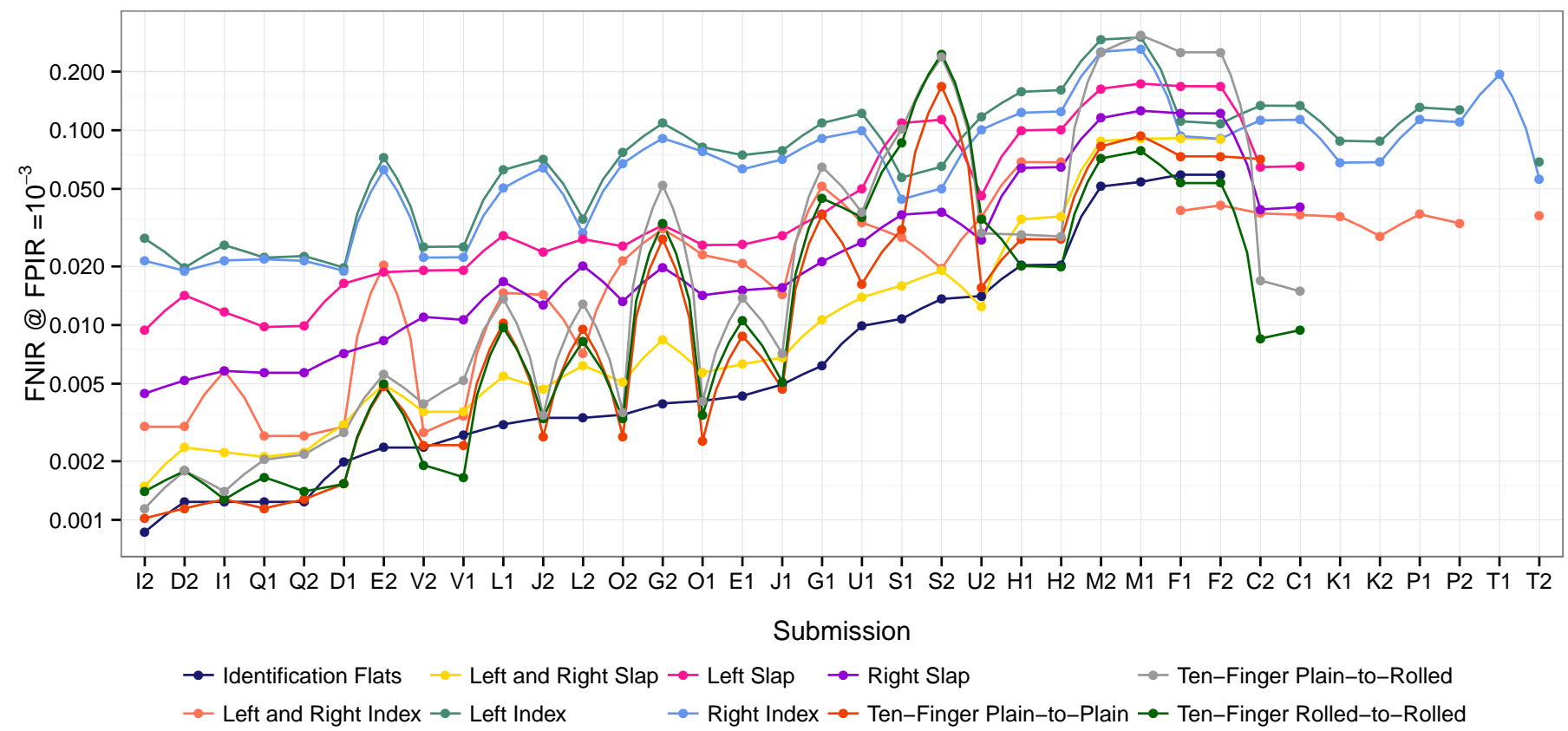

Figure 72: Rank-sorted FNIR @ FPIR $=10^{-3}$ for All Classes. Submissions "1" and "2" from round 3.

\begin{tabular}{l|l|l|l|l|l|l}
$\mathrm{C}=$ afis team & $\mathrm{D}=$ = 3M Cogent & $\mathrm{E}=$ Neurotechnology & $\mathrm{F}=$ Papillon & $\mathrm{G}=$ Dermalog & $\mathrm{H}=$ Hisign Bio-Info Institute \\
$\mathrm{I}=$ NEC & $\mathrm{J}=$ Sonda & $\mathrm{K}=$ Tiger IT & $\mathrm{L}=$ Innovatrics & $\mathrm{M}=$ SPEX & $\mathrm{O}=$ ID Solutions \\
$\mathrm{P}=\mathrm{id} 3$ & $\mathrm{Q}=$ Morpho & $\mathrm{S}=$ Decatur Industries & $\mathrm{T}=$ BIO-key & $\mathrm{U}=$ Aware & $\mathrm{V}=$ AA Technology
\end{tabular}




\section{FpVTE 2003 Comparison}

FpVTE 2003 [17] was composed of three separate tests, the Large-Scale Test (LST), the Medium-Scale Test (MST), and the Small-Scale Test (SST). SST and MST tested matching accuracy using individual fingerprints, all of which were images from right index fingers. This contrasts with LST, which evaluated matching accuracy using sets of fingerprint images, where each set includes one to ten finger positions collected from an individual subject at one time.

LST used 64000 fingerprint sets from 25000 subjects. These fingerprint sets comprised multiple test sets with varying combinations of one, two, four, eight, and ten fingers. MST used 10000 right index fingers and SST used a subset of 1000 right index fingers.

A significant difference between FpVTE 2012 and FpVTE 2003 testing procedures was that FpVTE 2003 required participants to match all subjects in the datasets against each other and return all 1-to-1 match scores. Therefore, while a direct comparison of results from the two FpVTE evaluations is not possible, this section will look at some of the observations from 2003 and note changes that have occurred in FpVTE 2012.

Looking at Figures 73 through 75 (focusing on the "Standard Partition" and "Average TAR") , a notable observation is that the accuracy gap in 2003 between the most accurate and least accurate systems was very significant. In current results, there is still a measurable accuracy gap, but it doesn't seem to be nearly as large.

In 2003, the accuracy results (as shown in Figure 76) indicated some difficulty measuring the accuracy difference when using four-, eight-, and ten-finger datasets. While it was clear that more fingers produced higher accuracy, it was not clear if ten fingers was significantly better than four fingers. The current FpVTE results used large enough datasets to allow a more accurate measurement of four-, eight-, and ten-finger search sets. There was a noticeable improvement in matching accuracy going from a four-finger search set to a ten-finger search set in FpVTE 2012.

Effects of fingerprint quality will be analyzed in a different FpVTE report to see if current technologies have improved when using low quality fingerprint data.

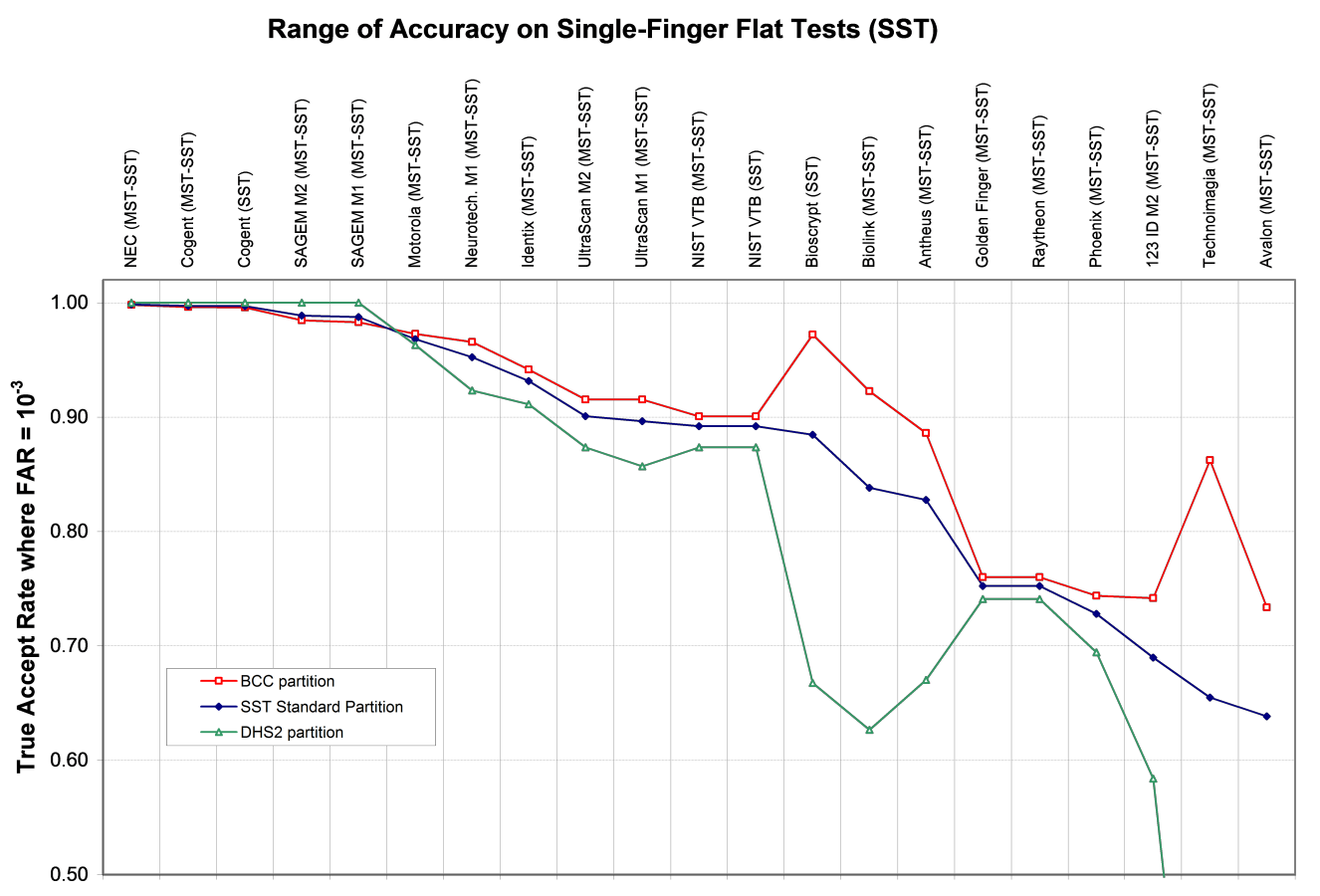

Figure 73: FpVTE 2003 SST Results - Range of accuracy on single-finger flats (SST). These systems are sorted by accuracy on the SST standard partition. Note that these results are reported at FAR $=10^{-3}$ [17].

\begin{tabular}{l|l|l|l|ll|l}
$\mathrm{C}=$ afis team & $\mathrm{D}=$ = 3M Cogent & $\mathrm{E}=$ Neurotechnology & $\mathrm{F}=$ Papillon & $\mathrm{G}=$ Dermalog & $\mathrm{H}=$ Hisign Bio-Info Institute \\
$\mathrm{I}=$ NEC & $\mathrm{J}=$ Sonda & $\mathrm{K}=$ Tiger IT & $\mathrm{L}=$ Innovatrics & $\mathrm{M}=$ SPEX & $\mathrm{O}=$ ID Solutions \\
$\mathrm{P}=\mathrm{id} 3$ & $\mathrm{Q}=$ Morpho & $\mathrm{S}=$ Decatur Industries & $\mathrm{T}=$ BIO-key & $\mathrm{U}=$ Aware & $\mathrm{V}=$ AA Technology
\end{tabular}




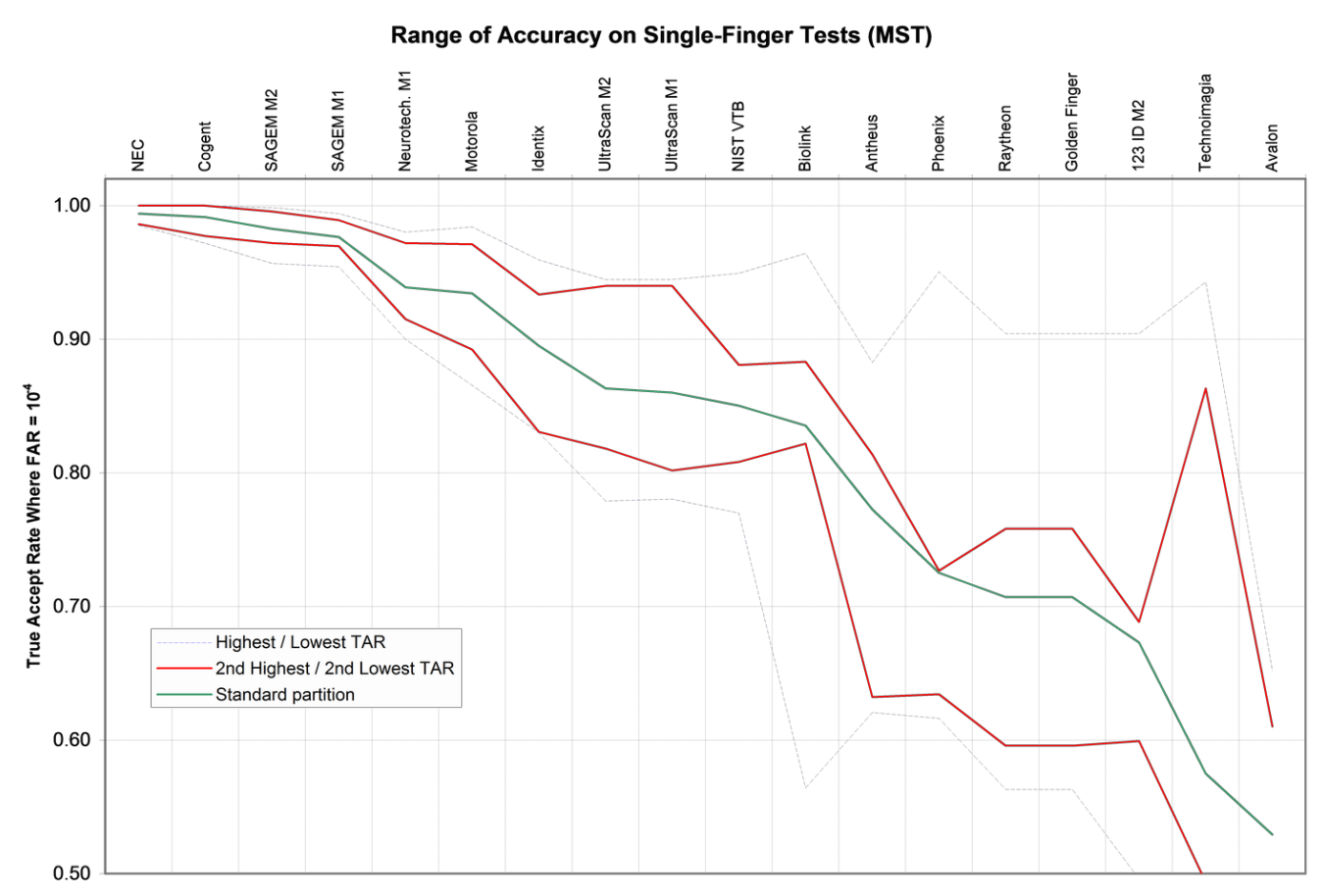

Figure 74: FpVTE 2003 MST Results - Range of accuracy across 7 MST partitions. These systems are sorted by accuracy on the standard MST, which is simply the combination of the other partitions [17].

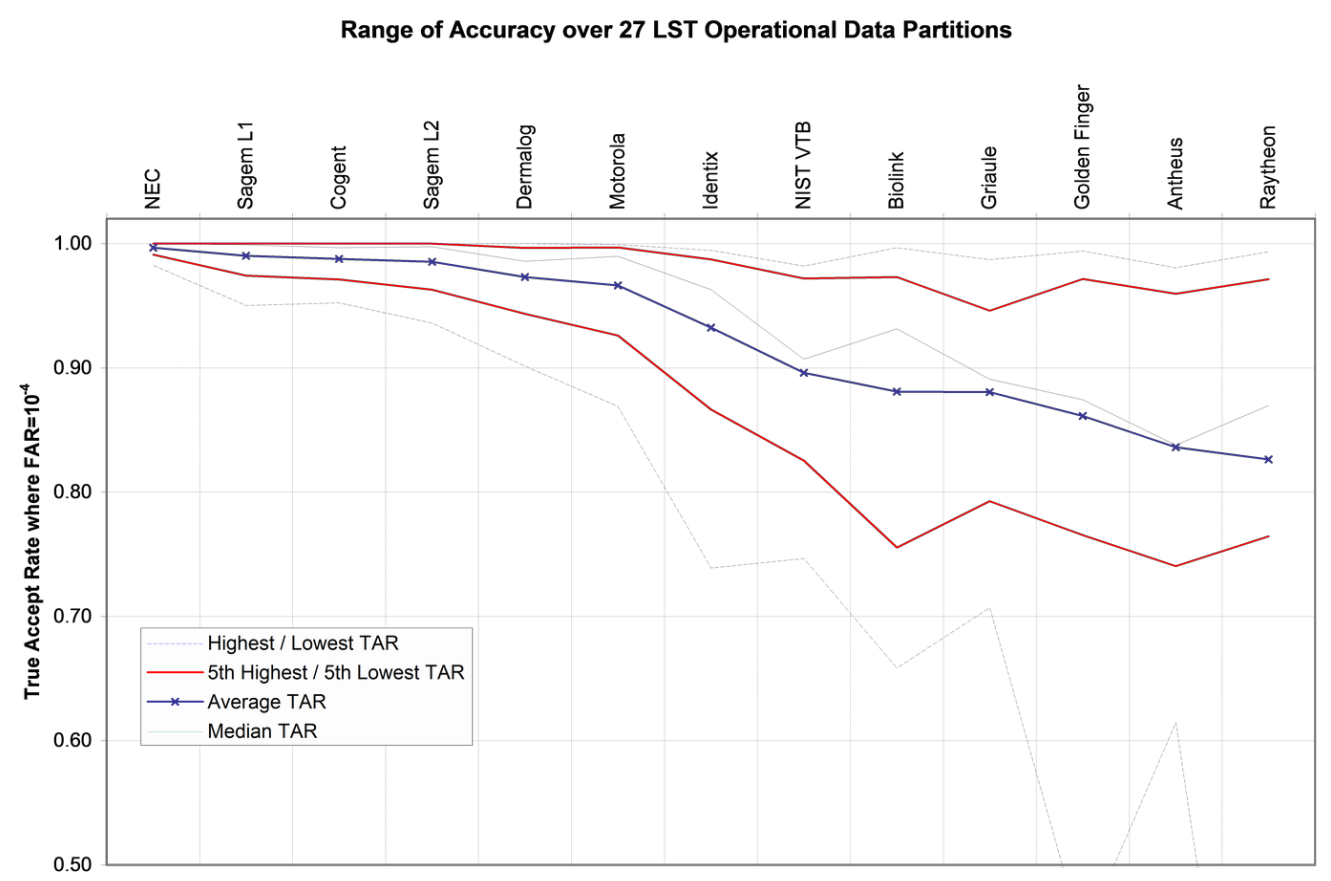

Figure 75: FpVTE 2003 LST Results - Range of accuracy over 27 operational LST partitions. The systems are sorted by their average accuracy over the 27 partitions. Note that sorting by median accuracy would change the order for some systems [17].

\begin{tabular}{l|l|l|l|l|l|l}
$\mathrm{C}=$ afis team & $\mathrm{D}=$ = 3M Cogent & $\mathrm{E}=$ Neurotechnology & $\mathrm{F}=$ Papillon & $\mathrm{G}=$ Dermalog & $\mathrm{H}=$ Hisign Bio-Info Institute \\
$\mathrm{I}=$ NEC & $\mathrm{J}=$ Sonda & $\mathrm{K}=$ Tiger IT & $\mathrm{L}=$ Innovatrics & $\mathrm{M}=$ SPEX & $\mathrm{O}=$ ID Solutions \\
$\mathrm{P}=\mathrm{id} 3$ & $\mathrm{Q}=$ Morpho & $\mathrm{S}=$ Decatur Industries & $\mathrm{T}=$ BIO-key & $\mathrm{U}=$ Aware & $\mathrm{V}=$ AA Technology
\end{tabular}




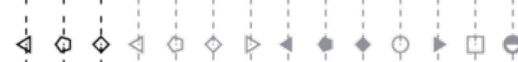

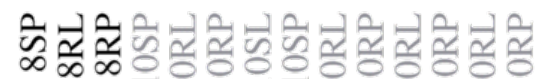

$x \times x-1$.

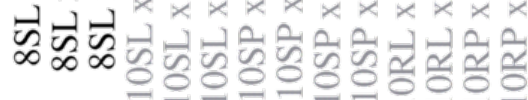

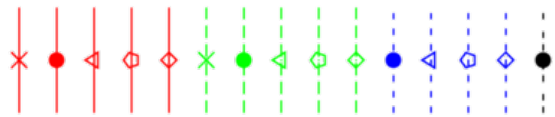

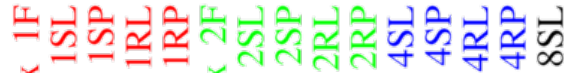

$\times x_{x} \times \bar{x} \times x \times x \times x \times x \times$

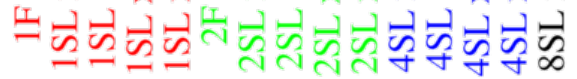

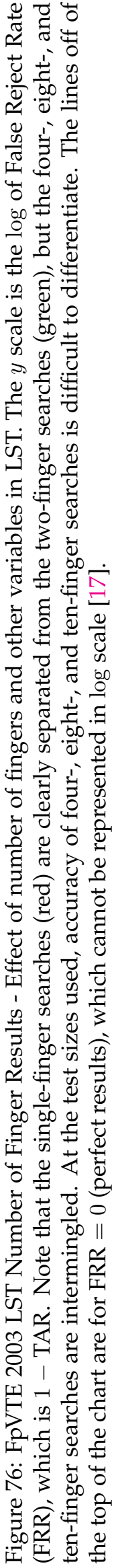

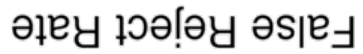

(ISา) snәчıน

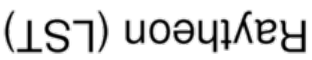

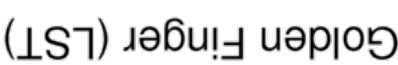

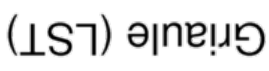

(เSา) צи!!|ำ

( $\perp S 7) ~ g \perp \wedge \perp S I N$

(LSา) x!!uәр।

(LSา) ej01010W

(ıSา) борешхә

(เSา)

(เSา) 1นә6оว

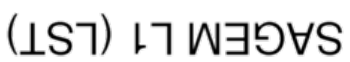

( $\perp S\urcorner) ~ Э \exists N$

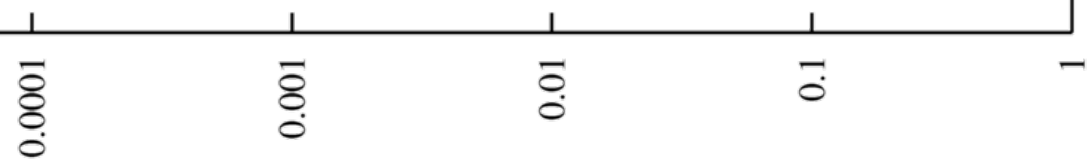

1 [य

\footnotetext{
$\mathrm{C}=$ afis team $\mathrm{D}=3 \mathrm{M}$ Cogent $\mid \mathrm{E}=$ Neurotechnology $\mid \mathrm{F}=$ Papillon $\quad \mathrm{G}=$ Dermalog $\mathrm{H}=$ Hisign Bio-Info Institute

\begin{tabular}{l|l|l|l|l}
$\mathrm{I}=$ NEC & $\mathrm{J}=$ Sonda & $\mathrm{K}=$ Tiger IT & $\mathrm{L}=$ Innovatrics $\mathrm{M}=$ SPEX & $\mathrm{O}=$ ID Solutions
\end{tabular}

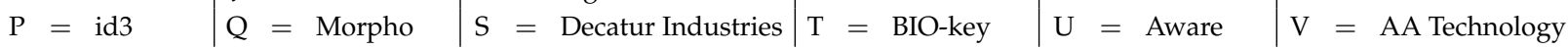




\section{Lessons Learned for Large-Scale Testing}

$\triangleright$ Failure Feedback: One of the most difficult aspects of validation was providing useful feedback to participants when failures occurred. The data used in FpVTE was sequestered operational data that could not be shared with participants. Some progress was made in FpVTE by allowing participants to write text-only logs, from their submission executed at NIST, that could then be returned for analysis. NIST reviewed all logs to ensure the logs did not include information related to the imagery or the NIST internal computing environment. Enhancements could be made to the FpVTE API to allow the FpVTE test driver to toggle logging on and off, preventing participants from submitting a separate logging build while maintaining the speed of not logging under normal use.

$\triangleright$ Two-Stage Matching Data Transfer: The FpVTE API specifies a 4 GB RAM disk to allow submissions to write data during the first stage of identification that could be referred to during the second stage. The intent was for all processes running on a compute node to share the same 4 GB RAM disk. As NIST did not say how many processes would be run in parallel, it created problems for submissions that wrote a large amount of data per process during the first stage of identification. Without increasing the RAM disk size, NIST was forced to run fewer processes on each compute node to avoid overfilling the RAM disk, which increased the evaluation time and wasted compute node resources. For instance, a compute node that would typically run twenty stage one identification processes might be limited to running just two or three. Future evaluations should better define the expected RAM disk usage, a minimum number of concurrent processes, and other requirements needed to safely and effectively run multiple searches in parallel.

$\triangleright$ Shared Memory: A key feature of the FpVTE API was that a large enrollment set could be loaded in memory and shared among multiple processes in parallel. An important aspect in allowing this shared memory useage was that the memory must remain static after initialization (see Section 5 for details). This caused problems for some participants and took several validation iterations to correct.

$\triangleright$ Additional Computational Statistics: The FpVTE test driver recorded the resident enrollment set size of identification stage one processes after returning from the identification stage one initialization method. It was expected that participants would use this method to load the entire enrollment set partition into RAM. While this was a fairly good indicator of RAM requirements, some submissions allocated significantly more memory during the core identification stage one method, which in many cases required re-partitioning the enrollment set with an additional compute node. Should FpVTE be repeated, it would be more fair to record additional computational statistics, such as peak RAM consumption over the execution time of the submission, since the RAM usage after initialization did not completely represent the RAM resources required for some submissions to run.

$\triangleright$ Timing Submissions: Keeping timing fair is a difficult task. The baseline of performing a timing test with only a single FpVTE process running proved most successful at keeping timing fair for all participants. The timing test was run against the full enrollment set with the assumption that using a smaller enrollment set would cause the search times to decrease. In cases where any unusual results were noticed, the timing tests were repeated to verify that the results remained consistent.

$\triangleright$ Enrollment and Re-enrollment: Enrolling the full datasets $(\approx 11.4$ million total subjects across all three classes) was not a trivial task. The original assumption was that this enrollment would only be performed for the first submission and not need to be repeated with later submissions. This was not the case and greatly increased the overall time required to complete the evaluation. Any future evaluations of this magnitude should explore performing a "maximum size" template extraction up front, then allowing for adjustments during the finalization stage to only use the minimum amount of information needed by the submissions. This could greatly reduce the need for re-enrollments. Care would need to be taken when reporting the "size" of the enrollment templates for each submission.

$\triangleright$ Enrollment Size - Disk vs. RAM: Another failed assumption was that reporting the size of the template at extraction would be a good indicator of how much RAM the enrollment set required (i.e., the Actual RAM and Reported $R A M$ columns from the tables Section 9 should be relatively close). For most submissions, this was true. Other submissions either compressed the templates and required more memory than it appeared they would need (at least

\begin{tabular}{l|l|l|l|ll|l}
$\mathrm{C}=$ afis team & $\mathrm{D}=3 \mathrm{M}$ Cogent & $\mathrm{E}=$ Neurotechnology & $\mathrm{F}=$ Papillon & $\mathrm{G}=$ Dermalog & $\mathrm{H}=$ Hisign Bio-Info Institute \\
$\mathrm{I}=$ NEC & $\mathrm{J}=$ Sonda & $\mathrm{K}=$ Tiger IT & $\mathrm{L}=$ Innovatrics & $\mathrm{M}=$ SPEX & $\mathrm{O}=$ ID Solutions \\
$\mathrm{P}=\mathrm{id} 3$ & $\mathrm{Q}=$ Morpho & $\mathrm{S}=$ Decatur Industries & $\mathrm{T}=$ BIO-key & $\mathrm{U}=$ Aware & $\mathrm{V}=$ AA Technology
\end{tabular}


one participant warned NIST that this would be the case) or they required less storage than the template sizes indicated they would need (again, at least one participant sent a warning about this issue). While the FpVTE API tried to prevent this by asking for both memory usage in RAM and on disk, there was confusion among the participants on what values, if any, to return. For example, many participants were confused on how to report memory usage of slap images-the participant would segment the fingers and return a single template, but report back four separate RAM usage values. Future evaluations will need to provide better guidance on this issue.

$\triangleright$ Consolidations of Nonmate Searches: A large amount of unexpected time was spent performing consolidations on the "back-end" of the searches. There proved to be a lot more consolidations to examine than originally expected. It took two to three months to clean these up before meaningful results could be produced. A significant improvement to this issue was the decision to flip nonmate search images, as discussed in Subsection 6.5.

$\triangleright$ Operational Sequestered Data: Participants were able to learn things about their specific submissions even though they may not have been one of the top performers. Some participants shared with NIST that they were grateful to test on the large sample of sequestered operational data to which they might not otherwise have access. They may not have had the best performance, but they were looking to learn about limitations with their submissions and make improvements, which was one of stated goals of FpVTE. This should continue to help advance fingerprint matching technologies and support the NIST mission of, "promoting U.S. innovation and industrial competitiveness".

\begin{tabular}{l|l|l|l|l|l|l}
$\mathrm{C}=$ afis team & $\mathrm{D}=3 \mathrm{M}$ Cogent & $\mathrm{E}=$ Neurotechnology & $\mathrm{F}=$ Papillon & $\mathrm{G}=$ Dermalog & $\mathrm{H}=$ Hisign Bio-Info Institute \\
$\mathrm{I}=$ NEC & $\mathrm{J}=$ Sonda & $\mathrm{K}=$ Tiger IT & $\mathrm{L}=$ Innovatrics & $\mathrm{M}=$ SPEX & $\mathrm{O}=$ ID Solutions \\
$\mathrm{P}=$ id3 & $\mathrm{Q}=$ Morpho & $\mathrm{S}=$ Decatur Industries & $\mathrm{T}=$ BIO-key & $\mathrm{U}=$ Aware & $\mathrm{V}=$ AA Technology
\end{tabular}




\section{Way Forward}

NIST plans to publish further research and analysis in addition to this initial results report, including:

$\triangleright$ running larger search sets (300 000 nonmates and 50000 mates) so that DET curves can show accuracy with FPIR rates below $10^{-3}$;

$\triangleright$ performing failure analysis in an attempt to determine if there are common failures among submissions and what causes those failures. Some things to examine during failure analysis include image quality, segmentation errors, gender differences, and consolidation errors;

$\triangleright$ performing analysis of results based on NFIQ values for the datasets;

$\triangleright$ looking at accuracy of subgroups of metadata, such as male versus female.

$\triangleright$ performing further analysis and testing to determine possible causes for four-finger IDFlat slap images being less accurate than two-index finger single-capture images;

If FpVTE were to be repeated, it might be useful to concentrate more on throughput versus accuracy. For instance, rather than set a single maximum search time of 90 seconds, the evaluation could have several search time maximums in an effort to see how different search times impact matching accuracy. It was clear during this evaluation that some participants have finer-grained control over the speed in which searches were performed. A speed-vs-accuracy track/competition would be useful.

\subsection{Related Testing}

\subsubsection{Forensic Palmprint}

As data becomes available, the protocols from this evaluation could be applied to perform an evaluation for latent palmprint matching.

\subsubsection{Mobile Data}

NIST has performed some testing with simulated mobile data, but future evaluations should look at using operational mobile data in the search sets to see how it impacts performance of matching algorithms.

\subsubsection{Cross-Comparison of Modalities}

Additional testing will compare the performance of fingerprint, face, and iris matching algorithms, in which all use a search set of 1.6 million subjects. While the datasets will be captured from different sources and subjects, this will be one of the first steps in comparing different modalities on similar sample sizes.

\begin{tabular}{l|l|l|l|ll|l}
$\mathrm{C}=$ afis team & $\mathrm{D}=3 \mathrm{M}$ Cogent & $\mathrm{E}=$ Neurotechnology & $\mathrm{F}=$ Papillon & $\mathrm{G}=$ Dermalog & $\mathrm{H}=$ Hisign Bio-Info Institute \\
$\mathrm{I}=$ NEC & $\mathrm{J}=$ Sonda & $\mathrm{K}=$ Tiger IT & $\mathrm{L}=$ Innovatrics & $\mathrm{M}=$ SPEX & $\mathrm{O}=$ ID Solutions \\
$\mathrm{P}=\mathrm{id} 3$ & $\mathrm{Q}=$ Morpho & $\mathrm{S}=$ Decatur Industries & $\mathrm{T}=$ BIO-key & $\mathrm{U}=$ Aware & $\mathrm{V}=$ AA Technology
\end{tabular}




\section{References}

[1] Chambers, J., Cleveland, W., Kleiner, B., And Tukey, P. Graphical Methods for Data Analysis. Wadsworth Brooks/Cole, 1983. 198

[2] Criminal Justice information Services Division - Federal Bureau of Investigation. IAFiS-IC-0110 (V3.1) WSQ Gray-scale Fingerprint Image Compression Specification, 2010. 6

[3] Grother, P., Michaels, R., And Phillips, P. J. Face Recognition Vendor Test 2002 Performance Metrics. In Audioand Video-Based Biometric Person Authentication: 4th International Conference, AVBPA 2003, Guildford, UK, June 9-11, 2003 Proceedings (June 2003), J. Kittler and M. S. Nixon, Eds., vol. 2688, Springer Berlin Heidelberg, pp. 937-945. 1

[4] Grother, P., Quinn, G., Matey, J., NGan, M., Salamon, W., Fiumara, G., And Watson, C. Iris Exchange III. NIST Interagency Report 7836 (2012). 18

[5] ImAge Group - NIST. NIST Biometric Evaluations Website. http://www.nist.gov/itl/iad/ig/biometric_ evaluations.cfm. 3

[6] International Committee for Information Technology Standards. American National Standard for Information Technology - Finger Minutiae Format for Data Interchange, ANSI/INCITS 378-2004, 2004. 3

[7] ISO/IEC JTC 1 SC 37. Information technology - Vocabulary - Part 37: Biometrics. http://standards. iso.org/ ittf/PubliclyAvailableStandards/c055194_ISOIEC_2382-37_2012.zip, 2012. 1

[8] KOMARINSKI, P. Automated Fingerprint Identification Systems (AFIS), 1st ed. Elsevier, 2004. ISBN 9780080475981. 19

[9] Martin, A. F., Doddington, G. R., Terri Kamm, M. O., And Przybocki, M. A. The Det curve in assessment of detection task performance, 1997. 18

[10] Open MPI Project. Open MPI: Open Source High Performance Computing. http://www.open-mpi.org/, 2014. [Online; accessed 22 October 2014]. 11

[11] SAlAmON, W., AND FiUmARA, G. Biometric Evaluation Framework. http://www.nist.gov/itl/iad/ig/framework. cfm, 2014. [Online; accessed 28 August 2014]. 11

[12] TABASSI, E., AND Wilson, C. L. A novel approach to fingerprint image quality. In Image Processing, 2005. ICIP 2005. IEEE International Conference on (Sept 2005), vol. 2, pp. II-37-40. 6

[13] TABAssi, E., Wilson, C. L., AND WATSON, C. I. Fingerprint Image Quality. NIST Interagency Report 7151 (2004). 6

[14] Watson, C., Garris, M. D., Tabassi, E., Wilson, C. L., McCabe, R. M., Janet, S., And Ko, K. User's Guide to Export Controlled Distribution of NIST Biometric Image Software (NBIS-EC). NIST Interagency Report 7391 (2007). 6

[15] Watson, C., SAlamon, W., AND Fiumara, G. Fingerprint Vendor Technology Evaluation Implementer's Guide. National Institute of Standards and Technology, http://nigos.nist.gov: 8080/evaluations/fpvte2012/FPVTE2012_API_ Plan_full_060712.pdf, July 2012. xvi, 1, 61

[16] Wayman, J., Jain, A., Maltoni, D., And Mai, D. Biometric Systems: Technology, Design and Performance Evaluation, 2005. ISBN 1852335963. 19

[17] Wilson, C., Hicklin, R. A., Bone, M., Korves, H., Grother, P., Ulery, B., Micheals, R., Zoepfl, M., Otto, S., AND WATSON, C. Fingerprint Vendor Technology Evaluation 2003. NIST Interagency Report 7123 (2004). 82, 83, 84,85

\begin{tabular}{l|l|l|l|l|l|l}
$\mathrm{C}=$ afis team & $\mathrm{D}=$ = 3M Cogent & $\mathrm{E}=$ Neurotechnology & $\mathrm{F}=$ Papillon & $\mathrm{G}=$ Dermalog & $\mathrm{H}=$ Hisign Bio-Info Institute \\
$\mathrm{I}=$ NEC & $\mathrm{J}=$ Sonda & $\mathrm{K}=$ Tiger IT & $\mathrm{L}=$ Innovatrics & $\mathrm{M}=$ SPEX & $\mathrm{O}=$ ID Solutions \\
$\mathrm{P}=\mathrm{id} 3$ & $\mathrm{Q}=$ Morpho & $\mathrm{S}=$ Decatur Industries & $\mathrm{T}=$ BIO-key & $\mathrm{U}=$ Aware & $\mathrm{V}=$ AA Technology
\end{tabular}




\section{A Individual Participant FNIR Plots}

This appendix contains a full size DET curve for every participant in FpVTE. The reader is reminded that enrollment set sizes were 100000 subjects for single index fingers, 1.6 million subjects for two index fingers, 3 million subjects for IDFlats, and 5 million for ten-finger rolled and plain impressions.

\begin{tabular}{l|l|l|l|ll|l}
$\mathrm{C}=$ afis team & $\mathrm{D}=3 \mathrm{M}$ Cogent & $\mathrm{E}=$ Neurotechnology & $\mathrm{F}=$ Papillon & $\mathrm{G}=$ Dermalog & $\mathrm{H}=$ Hisign Bio-Info Institute \\
$\mathrm{I}=$ NEC & $\mathrm{J}=$ Sonda & $\mathrm{K}=$ Tiger IT & $\mathrm{L}=$ Innovatrics & $\mathrm{M}=$ SPEX & $\mathrm{O}=$ ID Solutions \\
$\mathrm{P}=\mathrm{id} 3$ & $\mathrm{Q}=$ Morpho & $\mathrm{S}=$ Decatur Industries & $\mathrm{T}=$ BIO-key & $\mathrm{U}=$ Aware & $\mathrm{V}=$ AA Technology
\end{tabular}




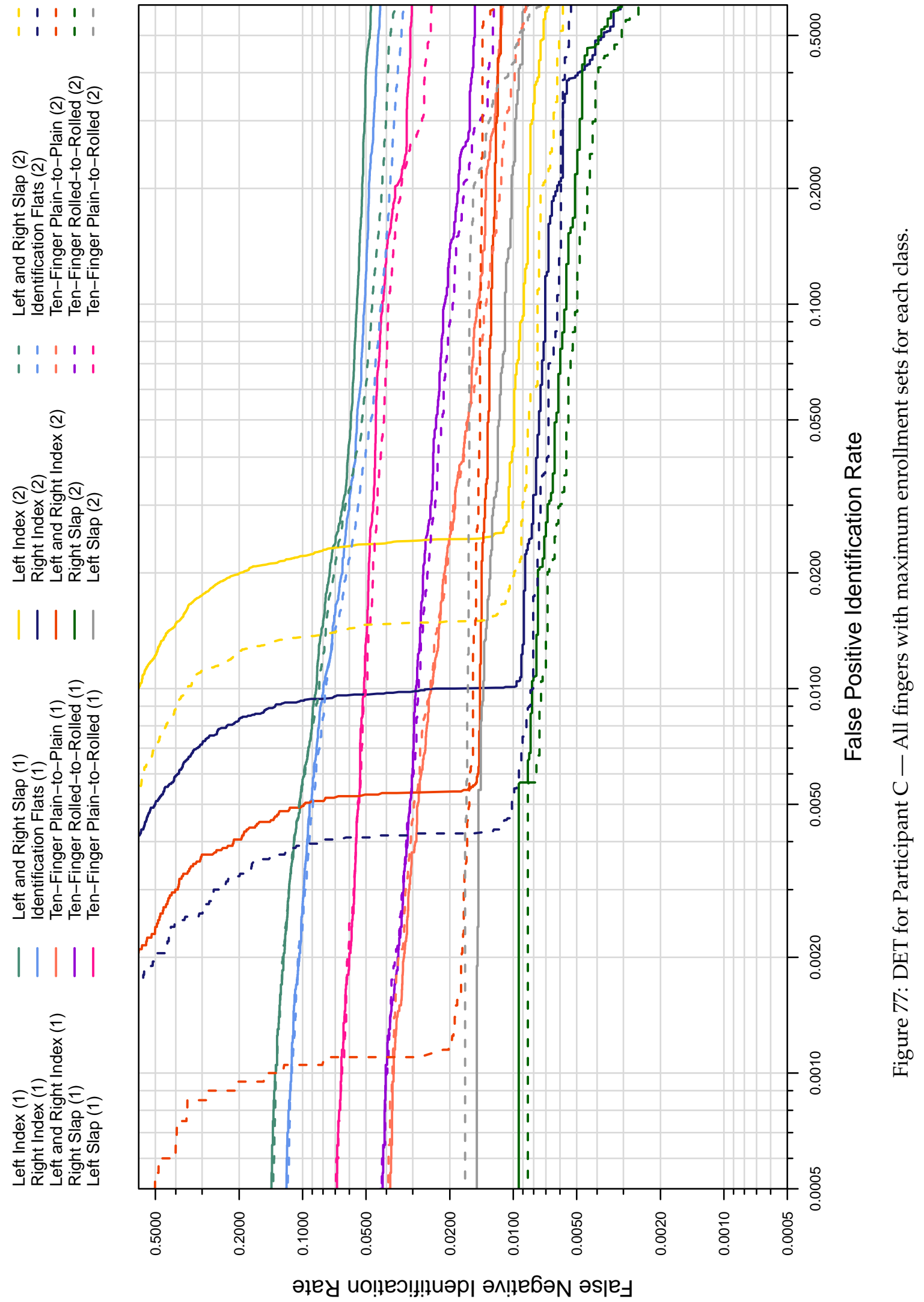


False Negative Identification Rate

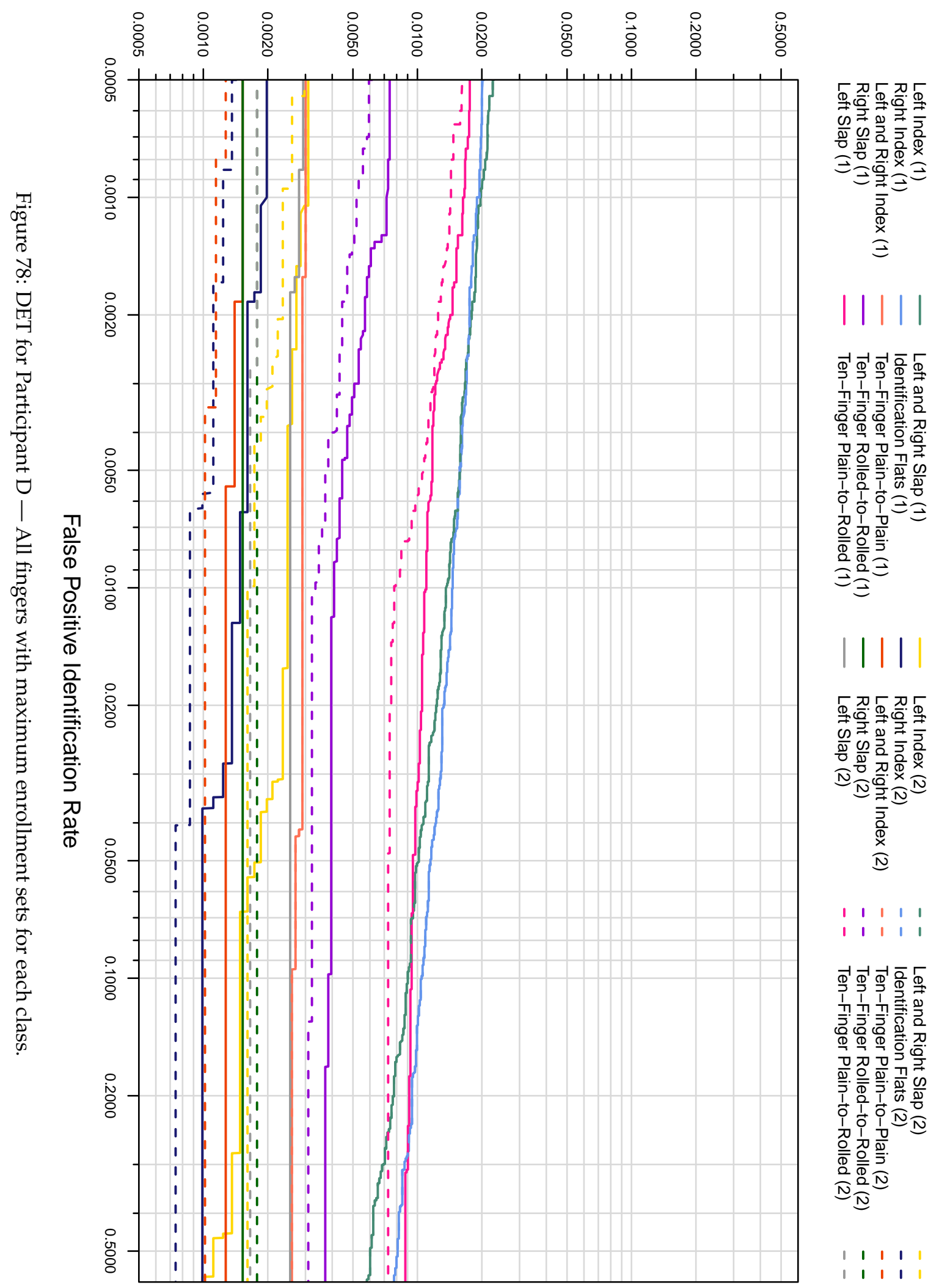

\footnotetext{
$\mathrm{C}=$ afis team $\mathrm{D}=3 \mathrm{M}$ Cogent $\mid \mathrm{E}=$ Neurotechnology $\mid \mathrm{F}=$ Papillon $\quad \mathrm{G}=$ Dermalog $\mathrm{H}=$ Hisign Bio-Info Institute

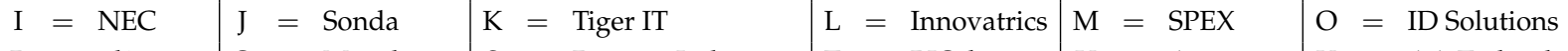

$\mathrm{P}=\mathrm{id} 3 \quad \mathrm{Q}=$ Morpho $\quad \mathrm{S}=$ Decatur Industries $\mid \mathrm{T}=$ BIO-key $\mid \mathrm{U}=$ Aware $\quad \mathrm{V}=$ AA Technology
} 


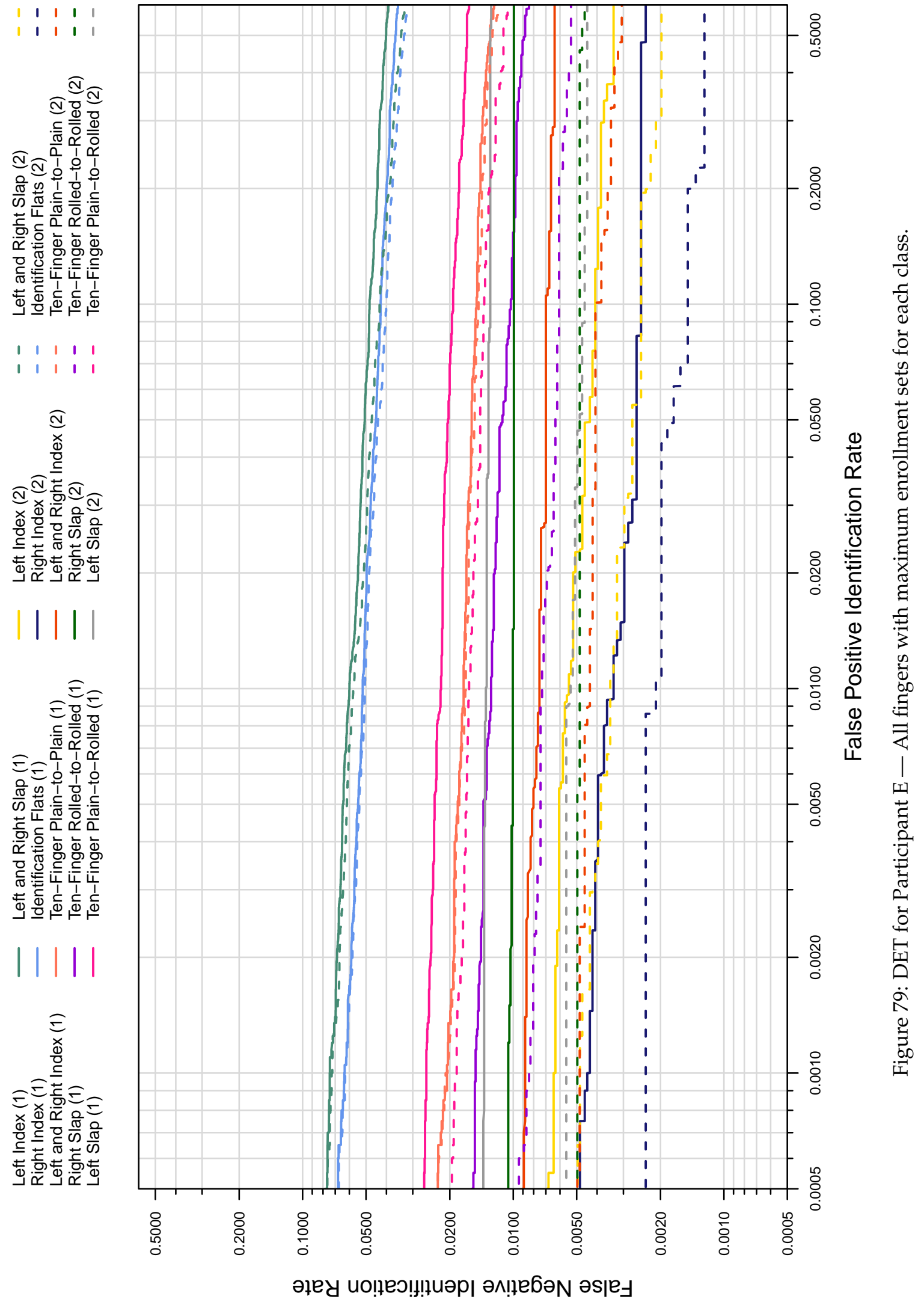


False Negative Identification Rate

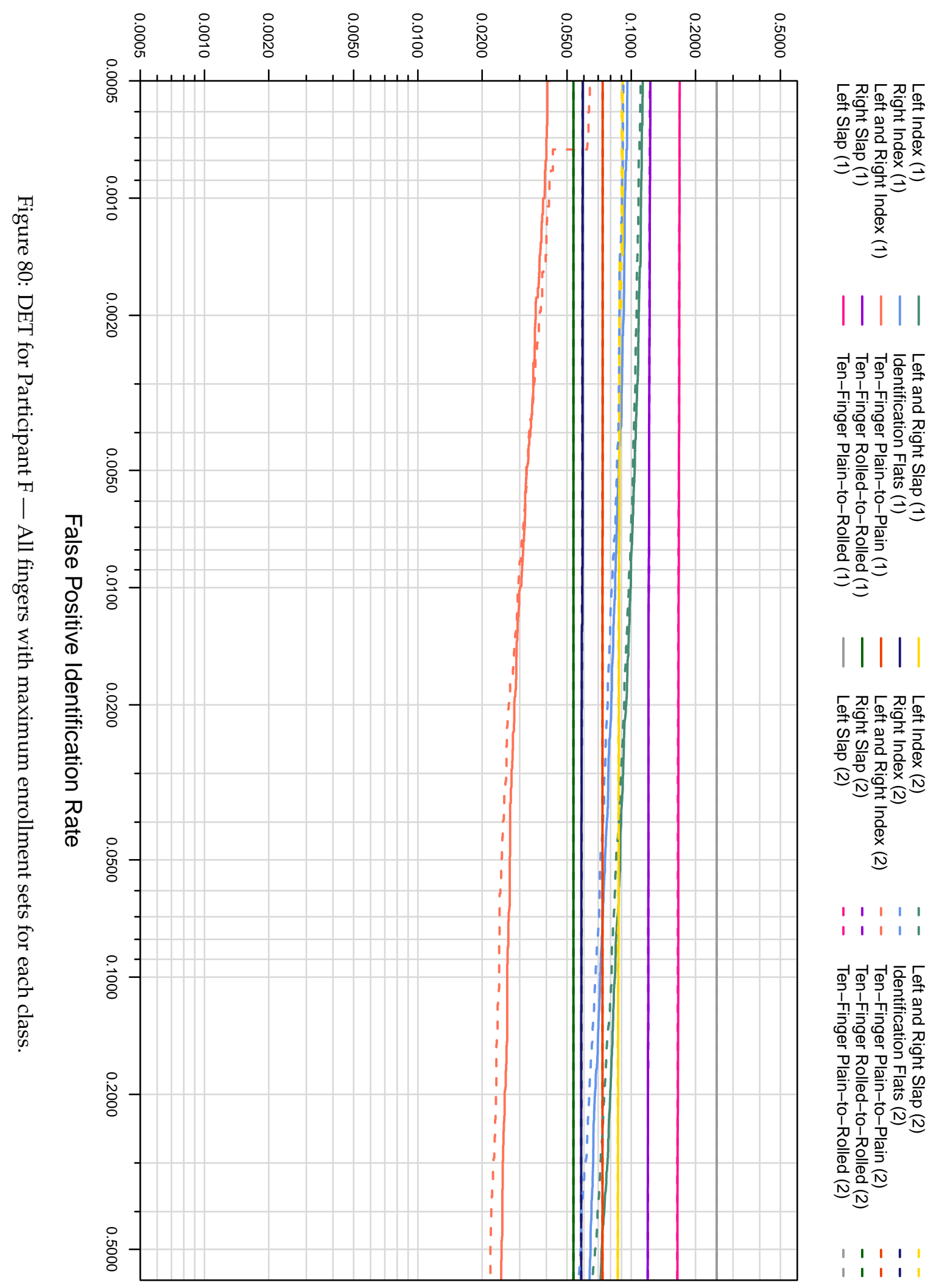

\footnotetext{
$\mathrm{C}=$ afis team $\mathrm{D}=3 \mathrm{M}$ Cogent $\mathrm{E}=$ Neurotechnology $\mid \mathrm{F}=$ Papillon $\quad \mathrm{G}=$ Dermalog $\mathrm{H}=$ Hisign Bio-Info Institute

\begin{tabular}{l|l|l|l|l}
$\mathrm{I}=$ NEC & $\mathrm{J}=$ Sonda & $\mathrm{K}=$ Tiger IT & $\mathrm{L}=$ Innovatrics $\mathrm{M}=$ SPEX & $\mathrm{O}=$ ID Solutions
\end{tabular}

$\mathrm{P}=\mathrm{id} 3 \quad \mathrm{Q}=$ Morpho $\quad \mathrm{S}=$ Decatur Industries $\mid \mathrm{T}=$ BIO-key $\mid \mathrm{U}=$ Aware $\quad \mathrm{V}=$ AA Technology
} 


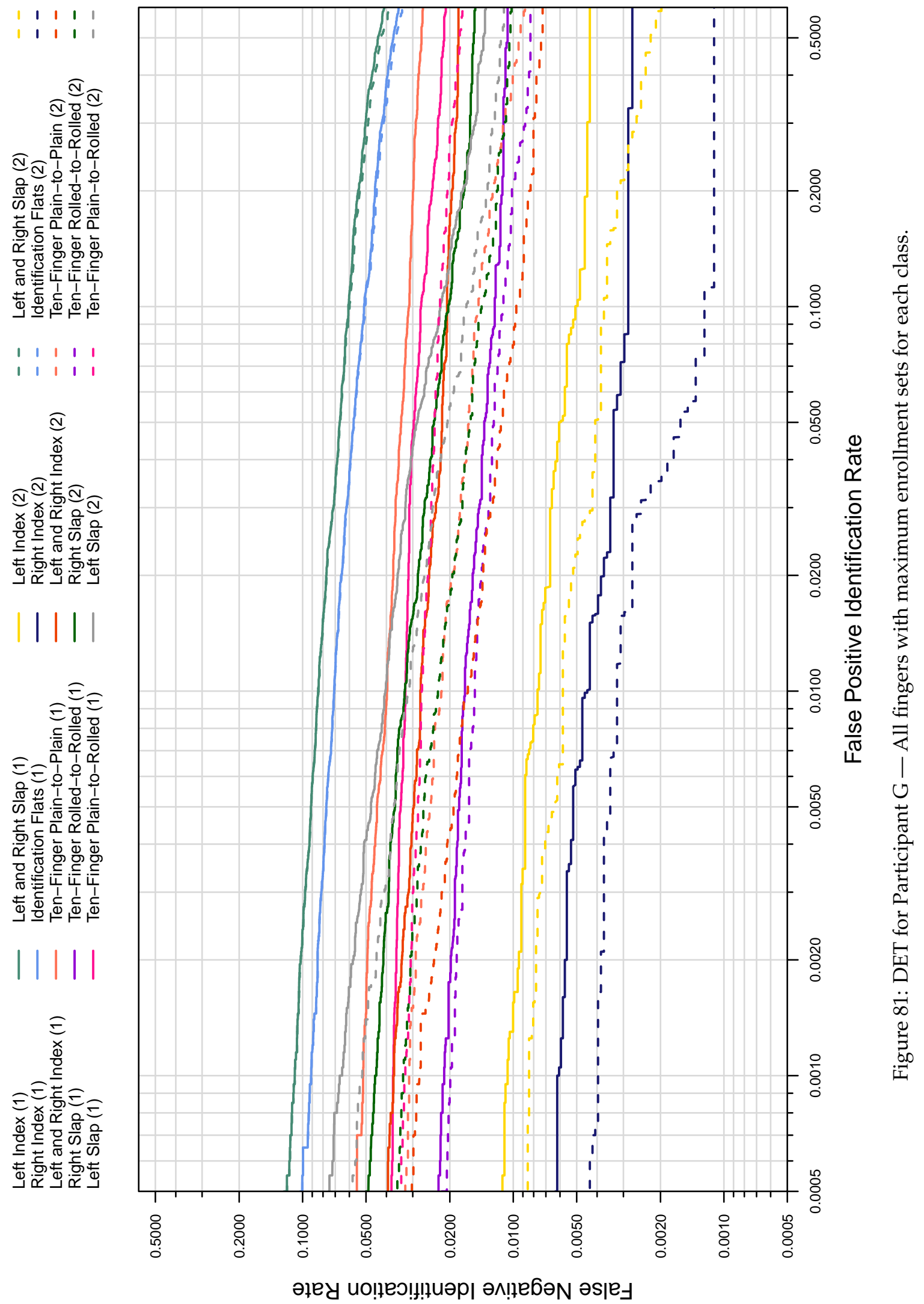


False Negative Identification Rate

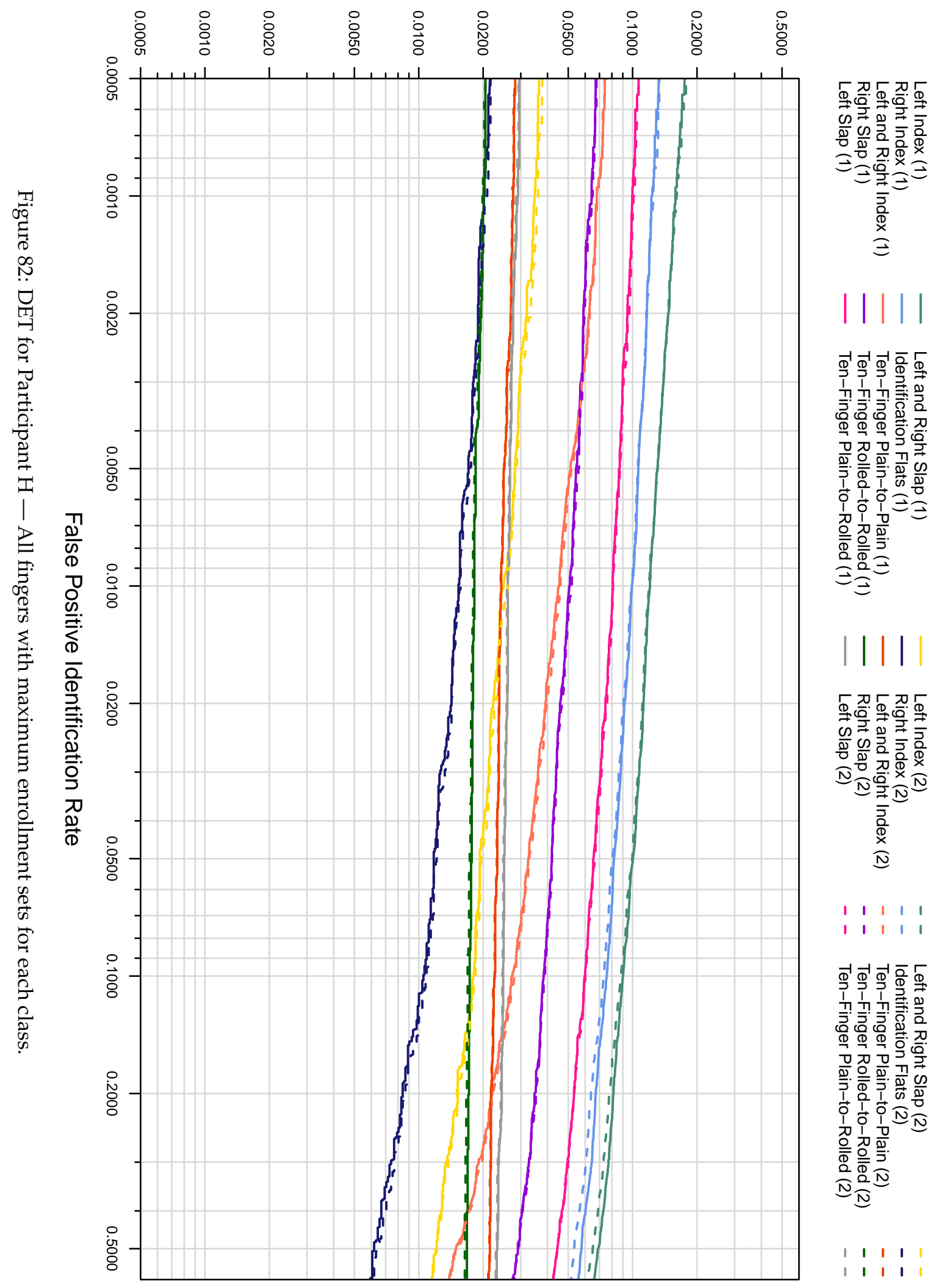

\footnotetext{
$\mathrm{C}=$ afis team $\mathrm{D}=3 \mathrm{M}$ Cogent $\mathrm{E}=$ Neurotechnology $\mid \mathrm{F}=$ Papillon $\quad \mathrm{G}=$ Dermalog $\mathrm{H}=$ Hisign Bio-Info Institute

\begin{tabular}{l|l|l|l|l}
$\mathrm{I}=$ NEC & $\mathrm{J}=$ Sonda & $\mathrm{K}=$ Tiger IT & $\mathrm{L}=$ Innovatrics $\mathrm{M}=$ SPEX & $\mathrm{O}=$ ID Solutions
\end{tabular}

$\mathrm{P}=\mathrm{id} 3 \quad \mathrm{Q}=$ Morpho $\quad \mathrm{S}=$ Decatur Industries $\mathrm{T}=$ BIO-key $\mid \mathrm{U}=$ Aware $\quad \mathrm{V}=$ AA Technology
} 


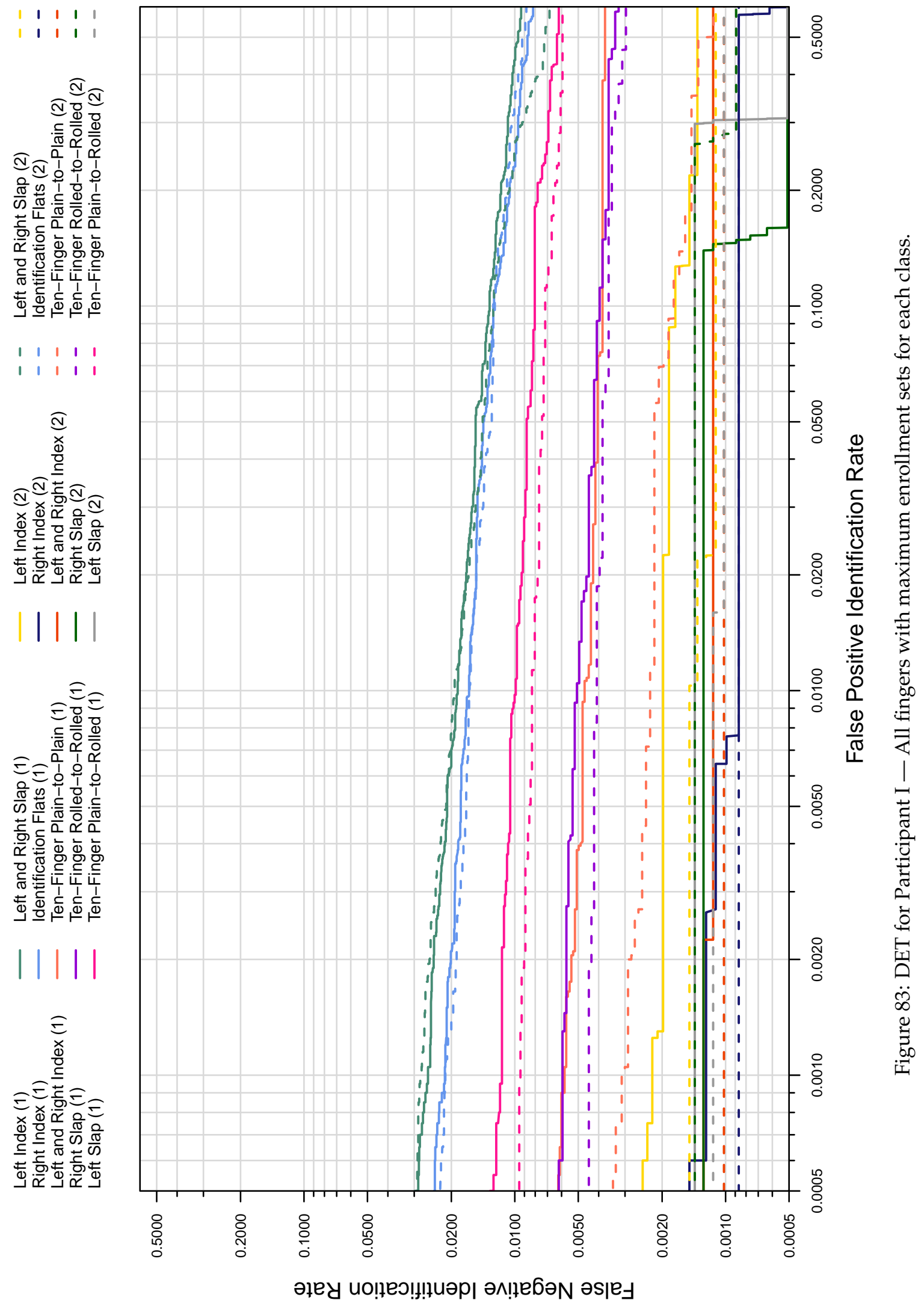


False Negative Identification Rate

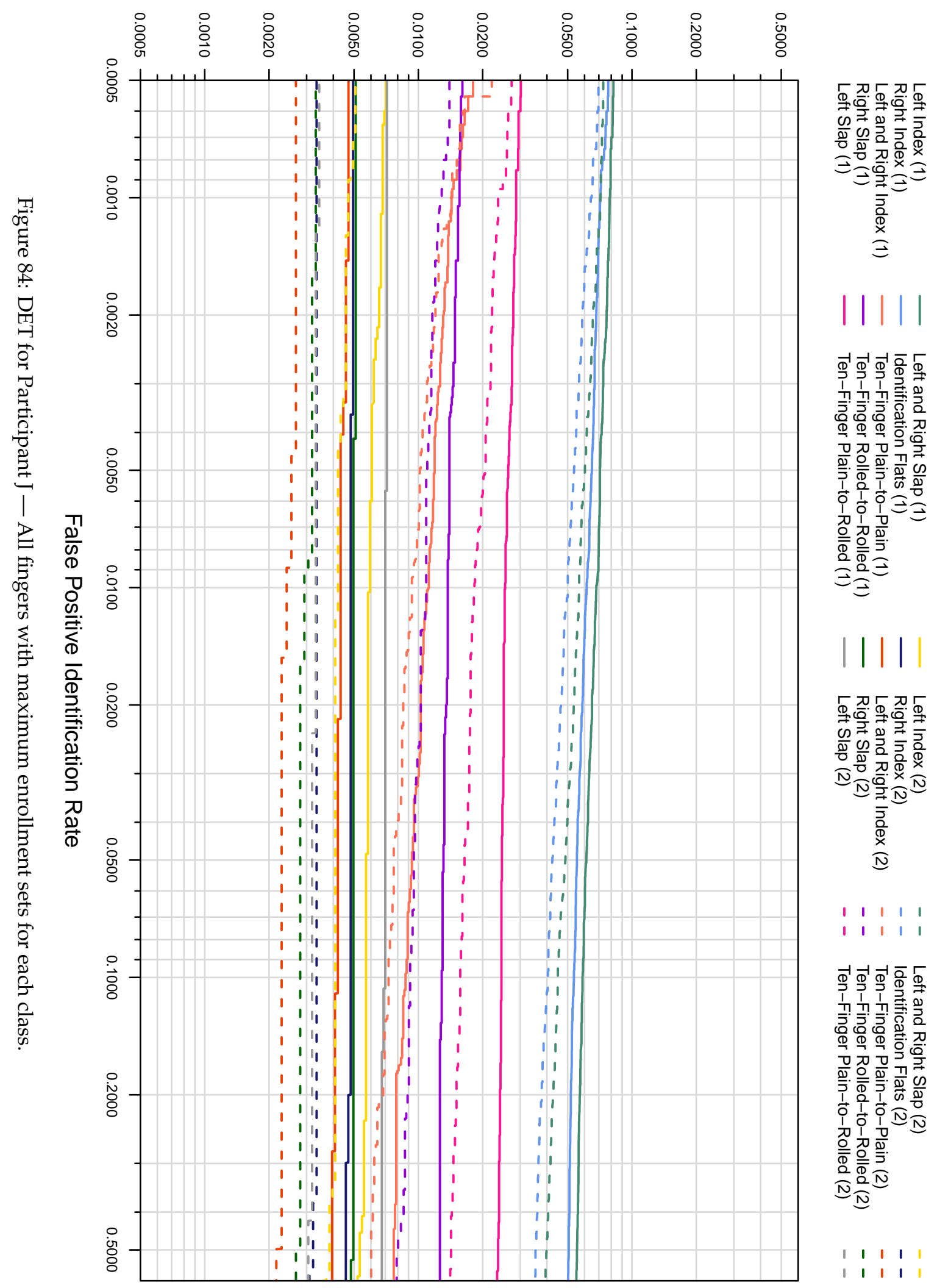

\footnotetext{
$\mathrm{C}=$ afis team $\mathrm{D}=3 \mathrm{M}$ Cogent $\mid \mathrm{E}=$ Neurotechnology $\mid \mathrm{F}=$ Papillon $\quad \mathrm{G}=$ Dermalog $\mathrm{H}=$ Hisign Bio-Info Institute

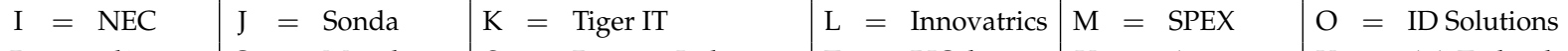

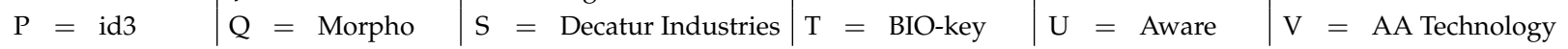




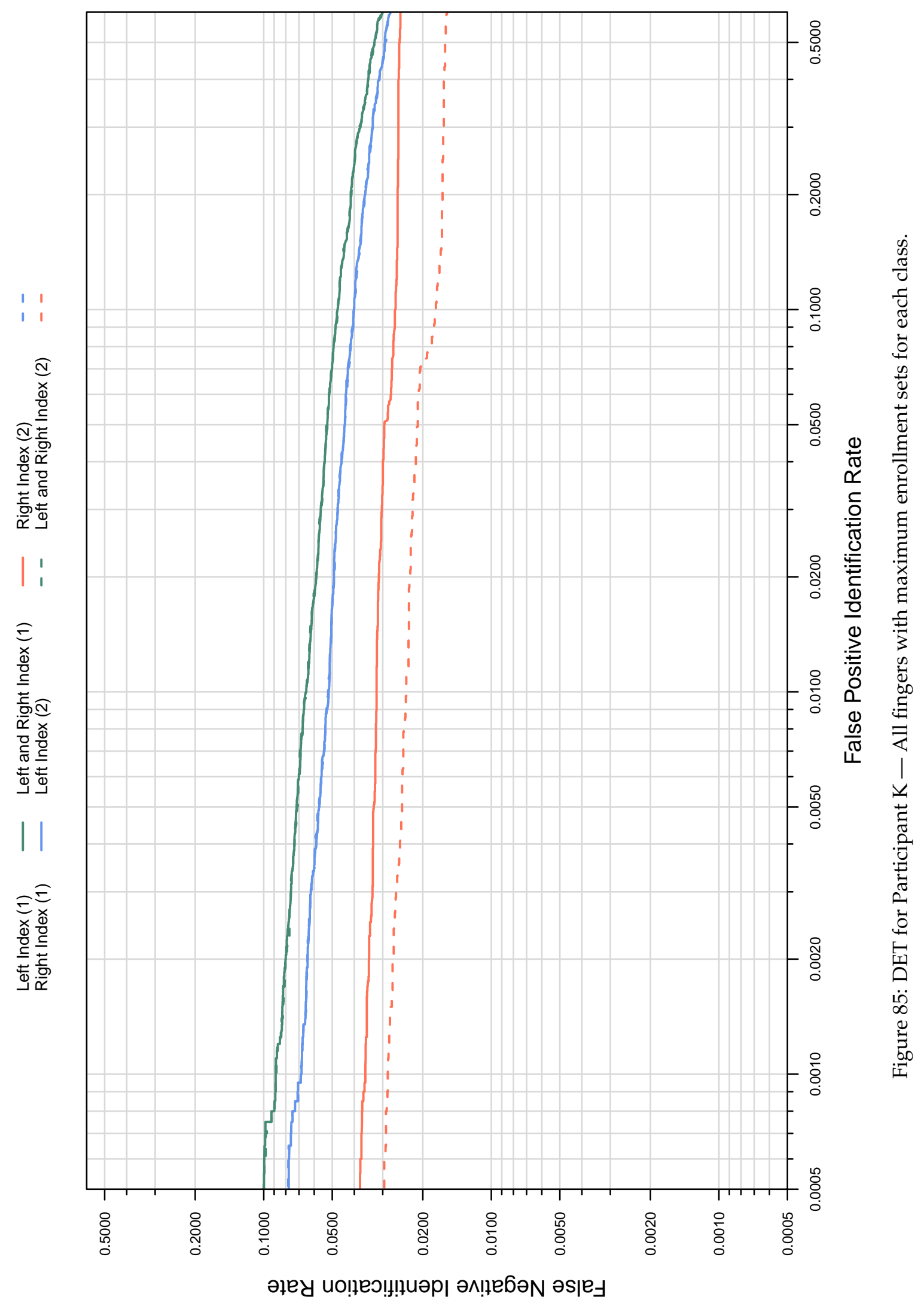


False Negative Identification Rate

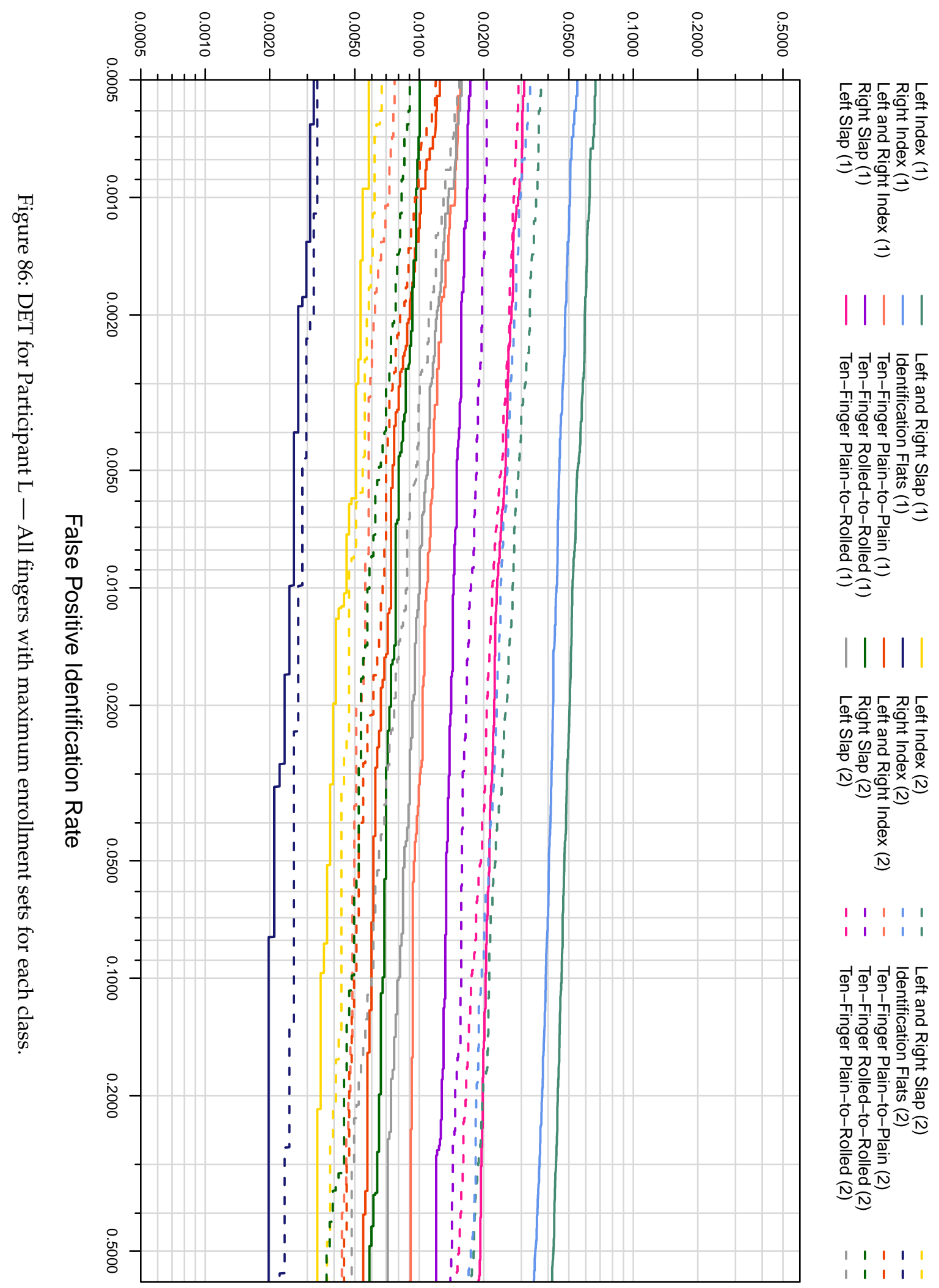

\footnotetext{
$\mathrm{C}=$ afis team $\mathrm{D}=3 \mathrm{M}$ Cogent $\mid \mathrm{E}=$ Neurotechnology $\mid \mathrm{F}=$ Papillon $\quad \mathrm{G}=$ Dermalog $\mathrm{H}=$ Hisign Bio-Info Institute

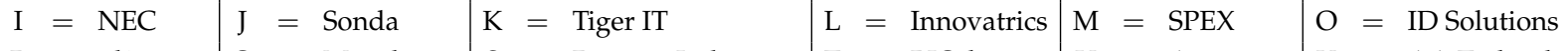

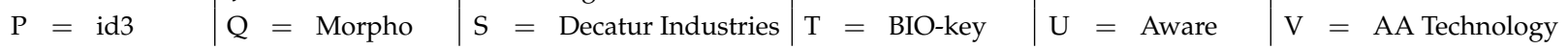




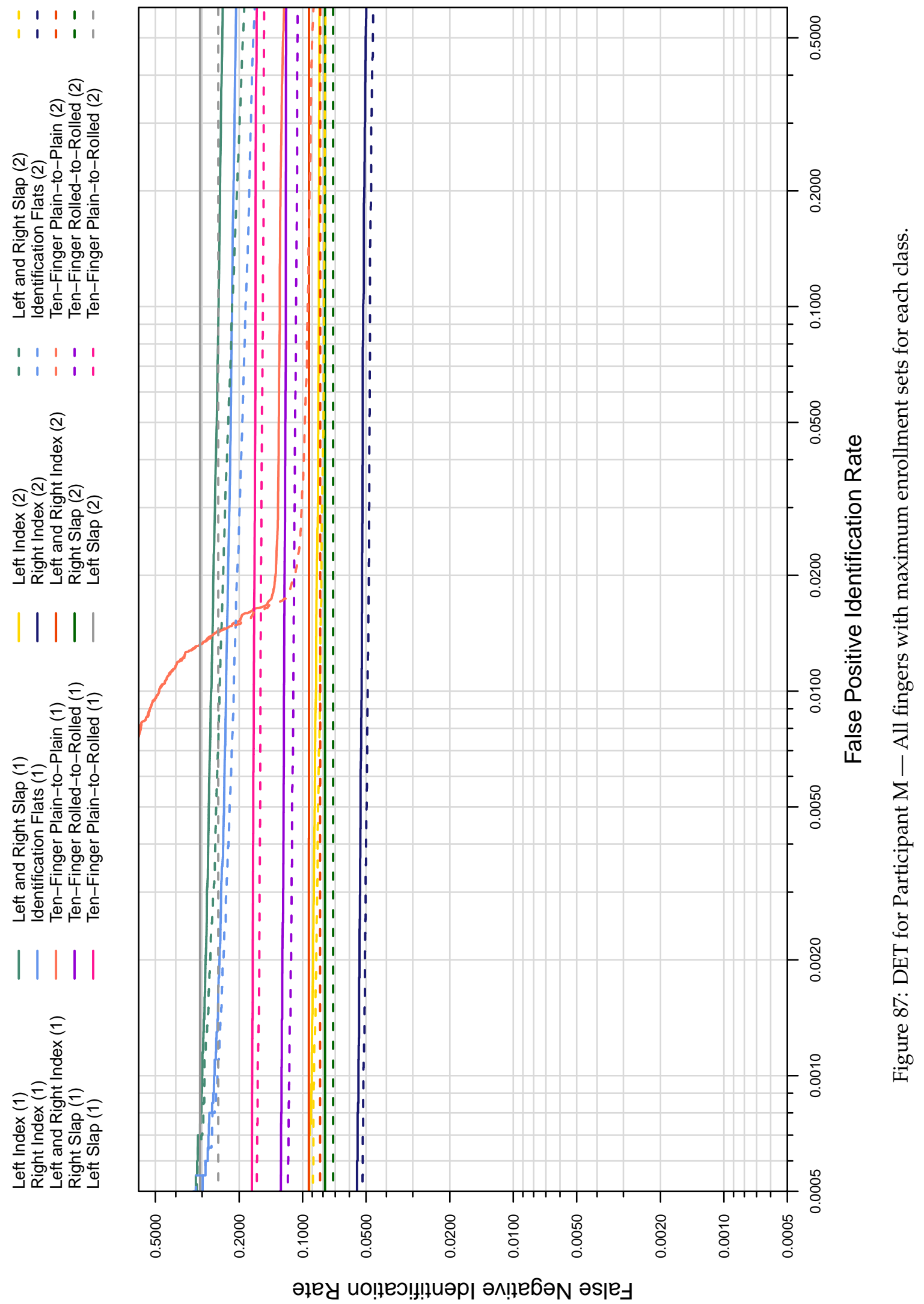


False Negative Identification Rate

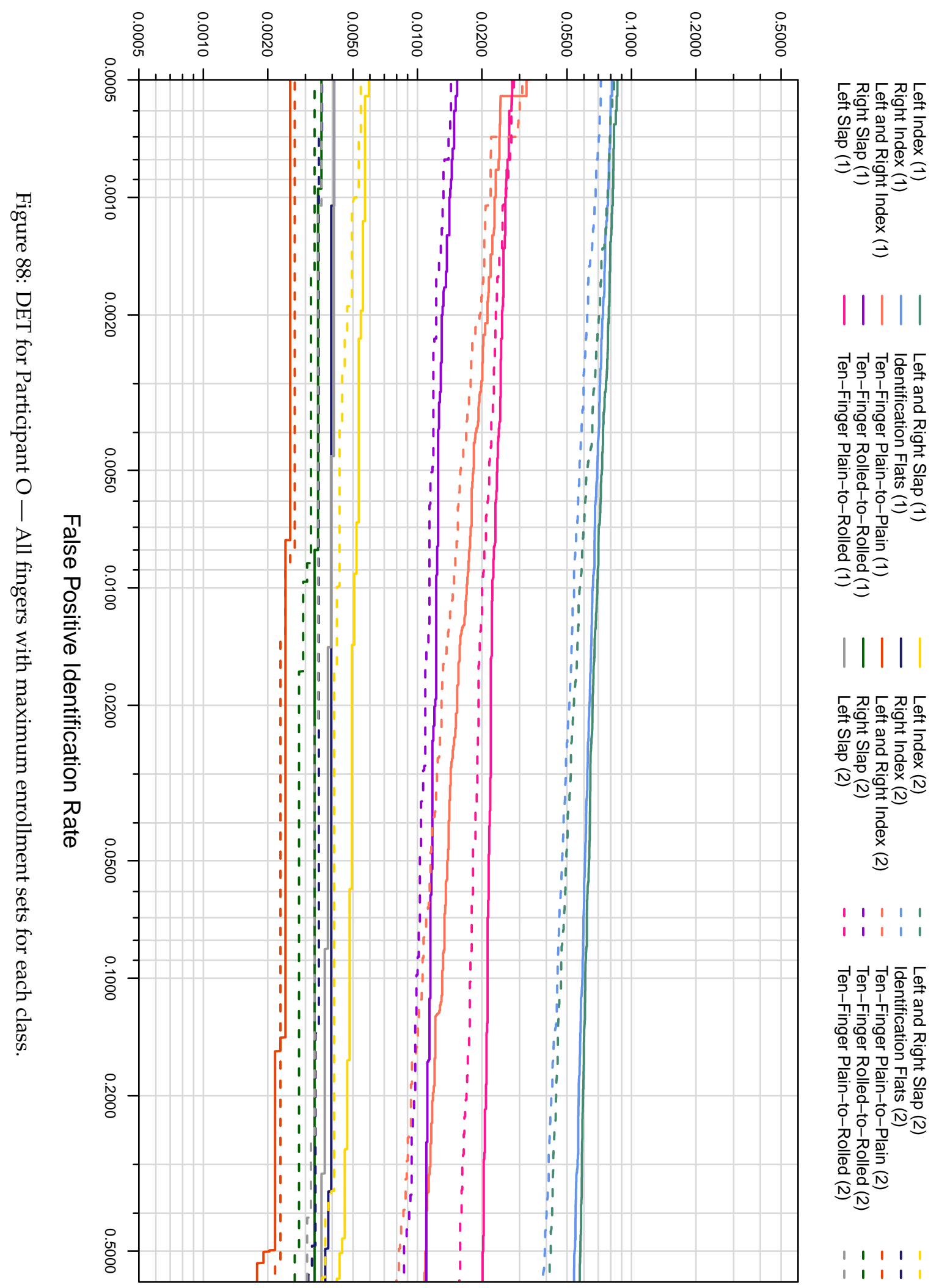

\footnotetext{
$\mathrm{C}=$ afis team $\mathrm{D}=3 \mathrm{M}$ Cogent $\mid \mathrm{E}=$ Neurotechnology $\mid \mathrm{F}=$ Papillon $\quad \mathrm{G}=$ Dermalog $\mathrm{H}=$ Hisign Bio-Info Institute

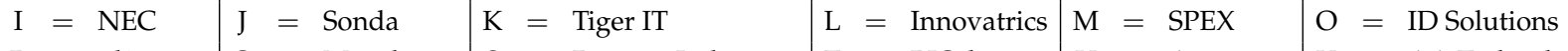

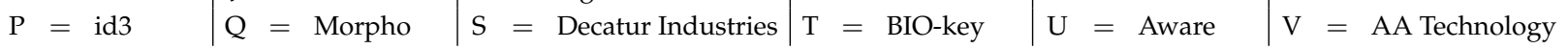




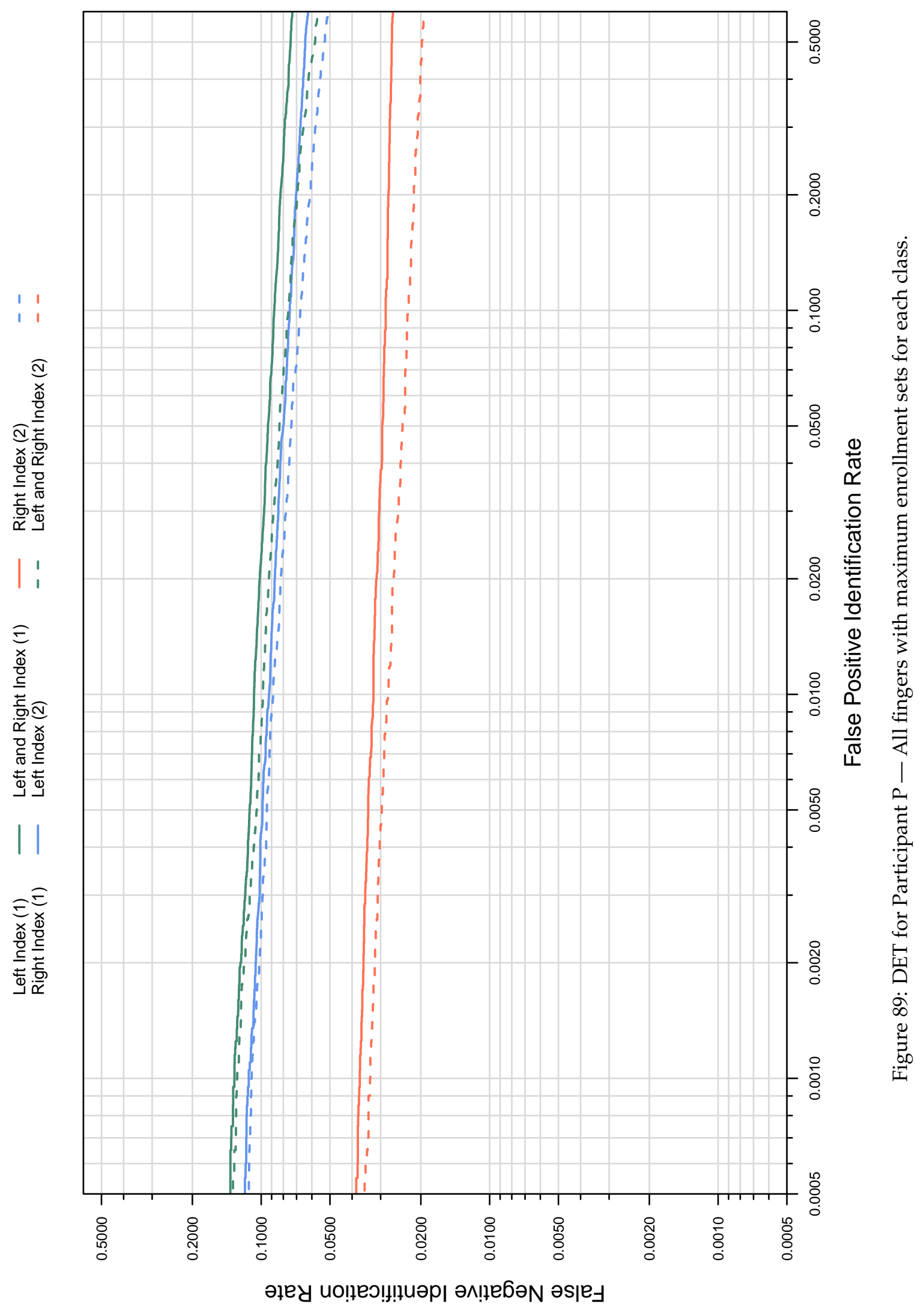


False Negative Identification Rate

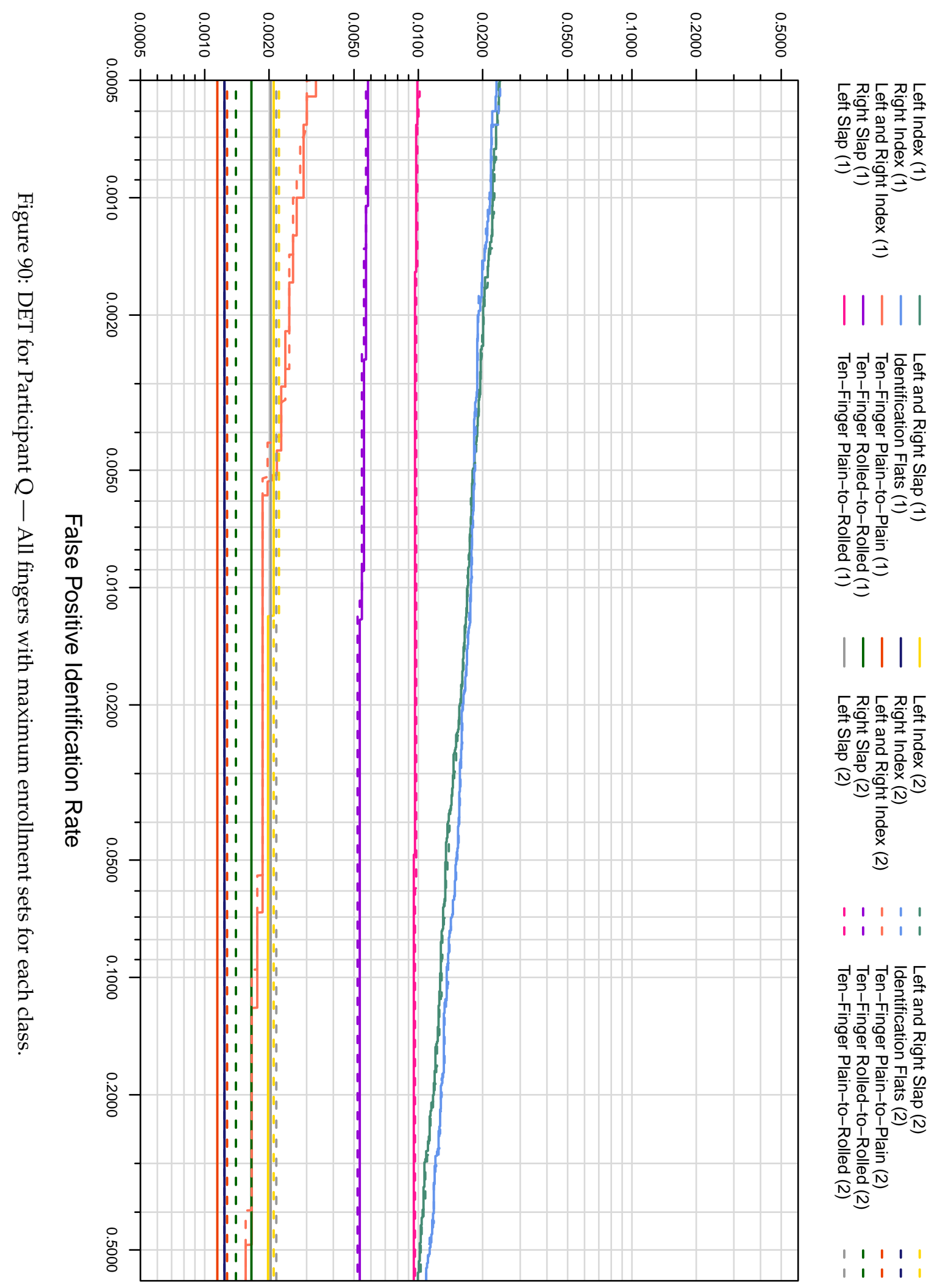

\footnotetext{
$\mathrm{C}=$ afis team $\mathrm{D}=3 \mathrm{M}$ Cogent $\mathrm{E}=$ Neurotechnology $\mid \mathrm{F}=$ Papillon $\quad \mathrm{G}=$ Dermalog $\mathrm{H}=$ Hisign Bio-Info Institute

\begin{tabular}{l|l|l|l|l}
$\mathrm{I}=$ NEC & $\mathrm{J}=$ Sonda & $\mathrm{K}=$ Tiger IT & $\mathrm{L}=$ Innovatrics $\mathrm{M}=$ SPEX & $\mathrm{O}=$ ID Solutions
\end{tabular}

$\mathrm{P}=\mathrm{id} 3 \quad \mathrm{Q}=$ Morpho $\quad \mathrm{S}=$ Decatur Industries $\mid \mathrm{T}=$ BIO-key $\mid \mathrm{U}=$ Aware $\quad \mathrm{V}=$ AA Technology
} 


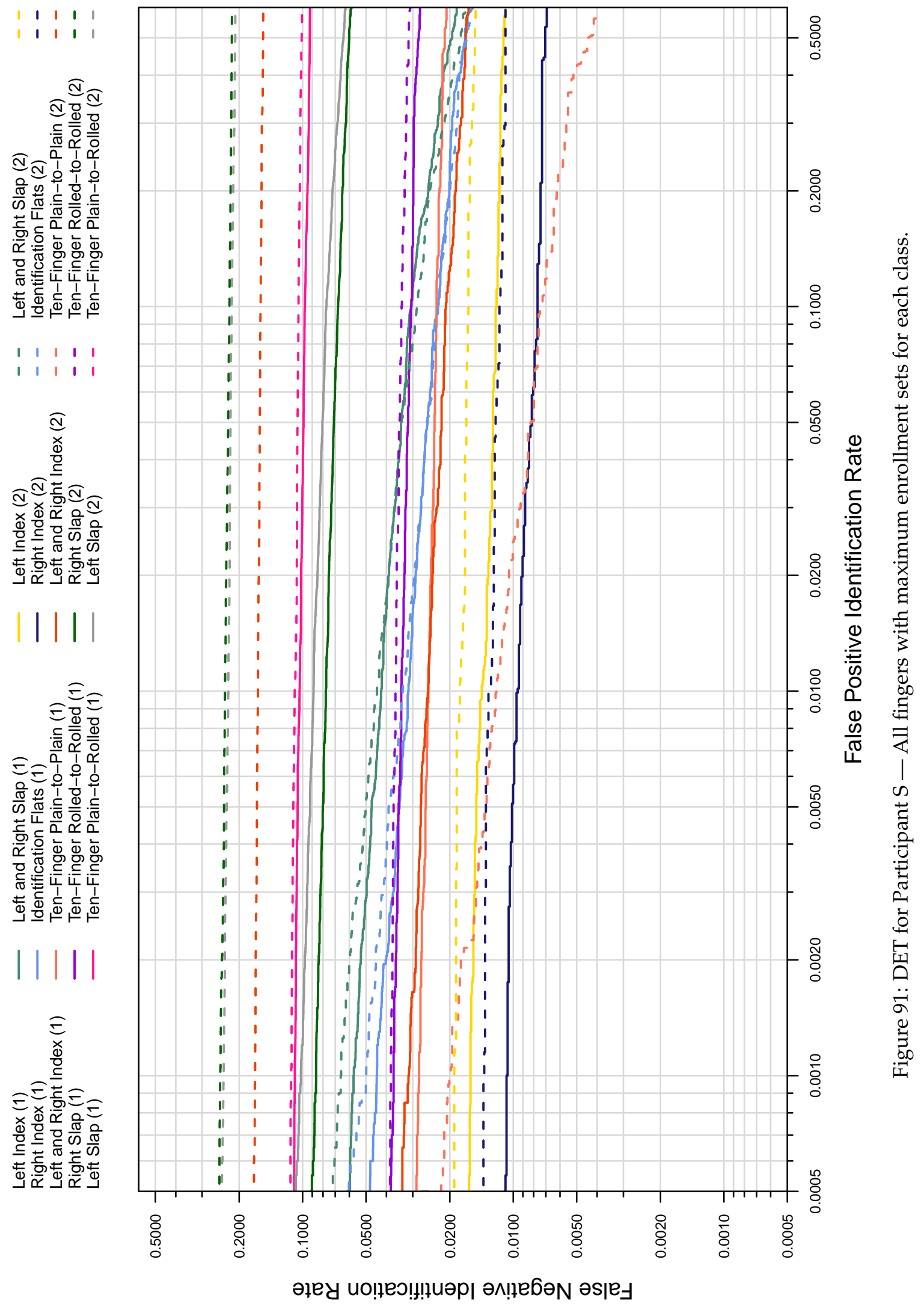


False Negative Identification Rate

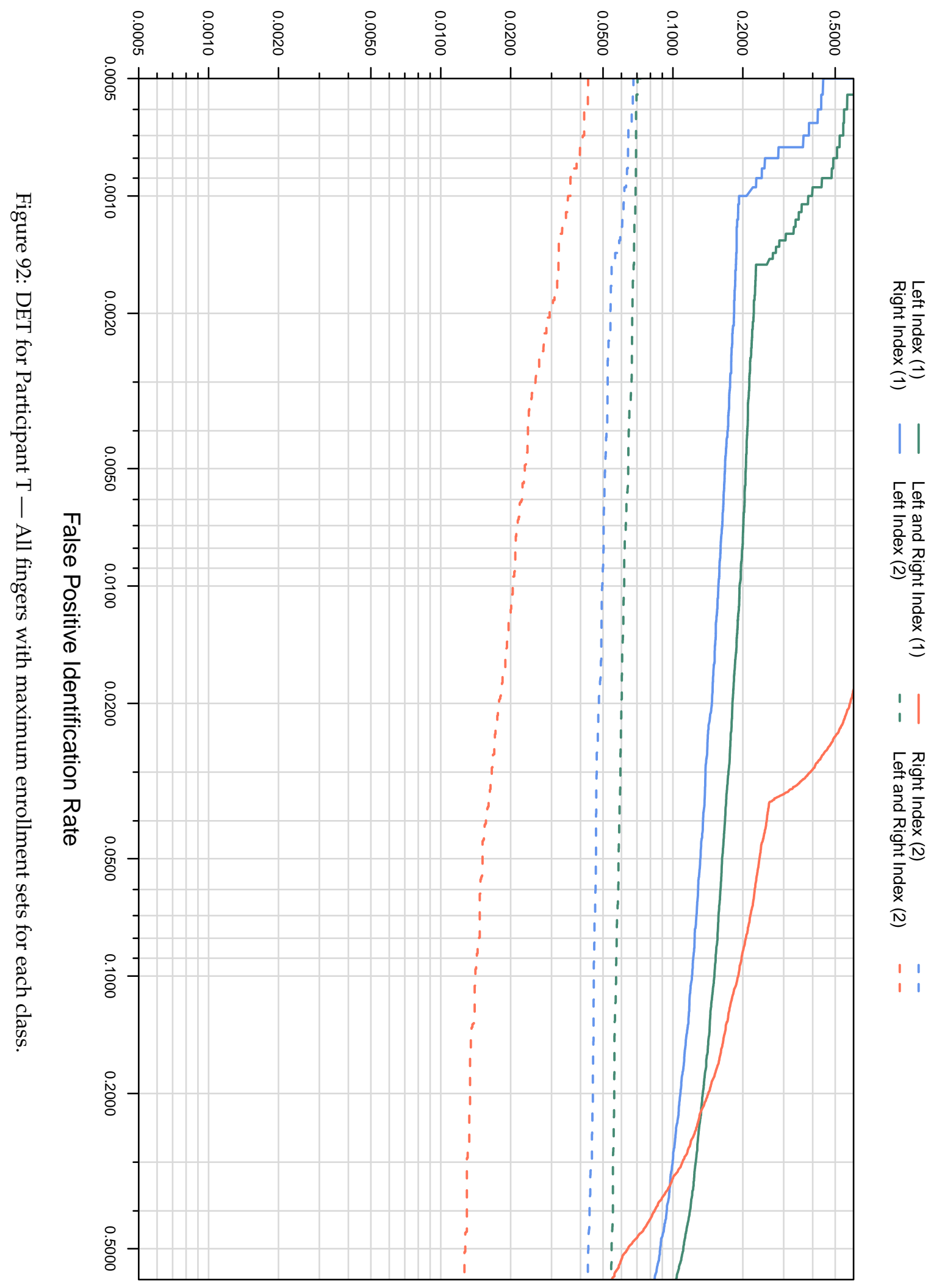

\footnotetext{
$\mathrm{C}=$ afis team $\mid \mathrm{D}=3 \mathrm{M}$ Cogent $\mid \mathrm{E}=$ Neurotechnology $\mid \mathrm{F}=$ Papillon $\mid \mathrm{G}=$ Dermalog $\mathrm{H}=$ Hisign Bio-Info Institute

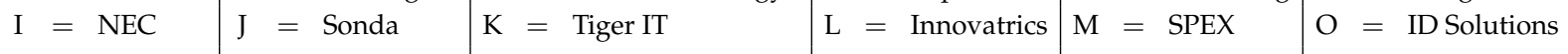

$\mathrm{P}=\mathrm{id} 3 \quad \mathrm{Q}=$ Morpho $\quad \mathrm{S}=$ Decatur Industries $\mathrm{T}=$ BIO-key $\quad \mathrm{U}=$ Aware $\quad \mathrm{V}=$ AA Technology
} 


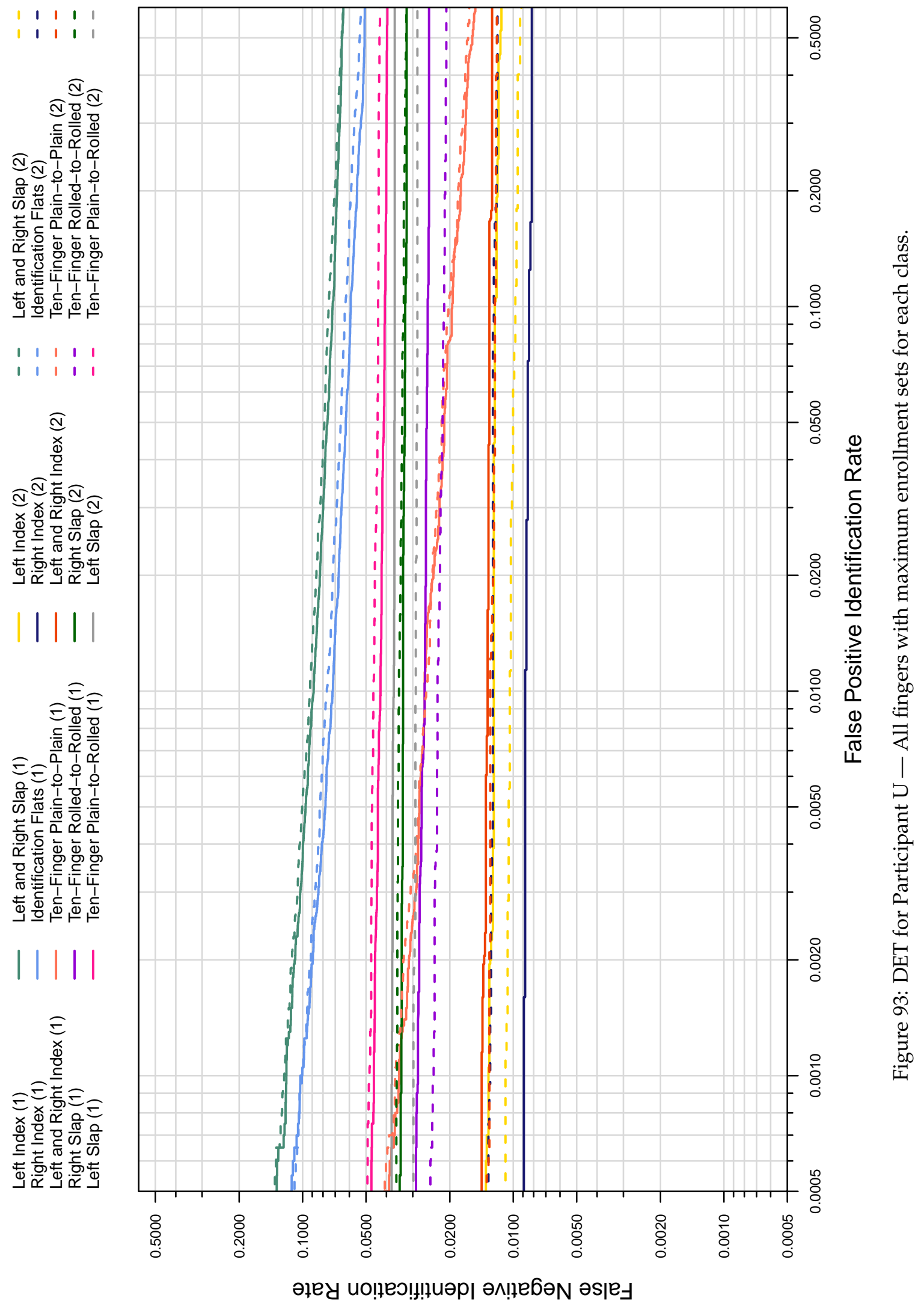


False Negative Identification Rate

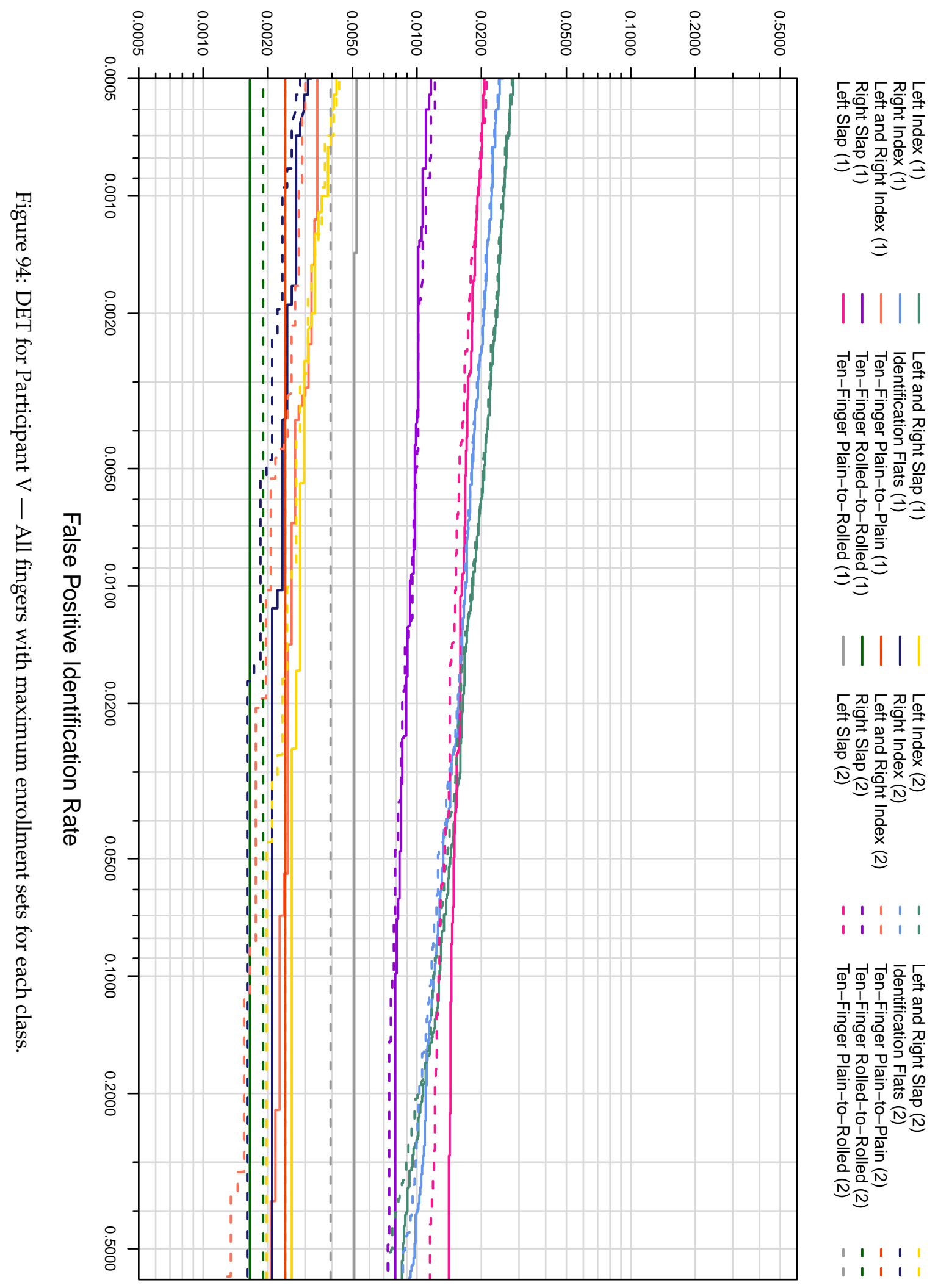

\footnotetext{
$\mathrm{C}=$ afis team $\mathrm{D}=3 \mathrm{M}$ Cogent $\mathrm{E}=$ Neurotechnology $\mid \mathrm{F}=$ Papillon $\quad \mathrm{G}=$ Dermalog $\mathrm{H}=$ Hisign Bio-Info Institute

\begin{tabular}{l|l|l|l|l}
$\mathrm{I}=$ NEC & $\mathrm{J}=$ Sonda & $\mathrm{K}=$ Tiger IT & $\mathrm{L}=$ Innovatrics $\mathrm{M}=$ SPEX & $\mathrm{O}=$ ID Solutions
\end{tabular}

$\mathrm{P}=\mathrm{id} 3 \quad \mathrm{Q}=$ Morpho $\quad \mathrm{S}=$ Decatur Industries $\mathrm{T}=$ BIO-key $\mid \mathrm{U}=$ Aware $\quad \mathrm{V}=$ AA Technology
} 


\section{B Combined Class DETs and CMCs}

This appendix contains DET and CMC curves for all classes and participants grouped together. There is one grouping for the participant's first submission and another for their second submission. The submissions are split for visibility only"first" and "second" submissions do not imply any sort of logical grouping. The reader is reminded that enrollment set sizes were 100000 subjects for single index fingers, 1.6 million subjects for two index fingers, 3 million subjects for IDFlats, and 5 million for ten-finger rolled and plain impressions.

\begin{tabular}{l|l|l|l|ll|l}
$\mathrm{C}=$ afis team & $\mathrm{D}=3 \mathrm{M}$ Cogent & $\mathrm{E}=$ Neurotechnology & $\mathrm{F}=$ Papillon & $\mathrm{G}=$ Dermalog & $\mathrm{H}=$ Hisign Bio-Info Institute \\
$\mathrm{I}=$ NEC & $\mathrm{J}=$ Sonda & $\mathrm{K}=$ Tiger IT & $\mathrm{L}=$ Innovatrics & $\mathrm{M}=$ SPEX & $\mathrm{O}=$ ID Solutions \\
$\mathrm{P}=\mathrm{id} 3$ & $\mathrm{Q}=$ Morpho & $\mathrm{S}=$ Decatur Industries & $\mathrm{T}=$ BIO-key & $\mathrm{U}=$ Aware & $\mathrm{V}=$ AA Technology
\end{tabular}


False Negative Identification Rate

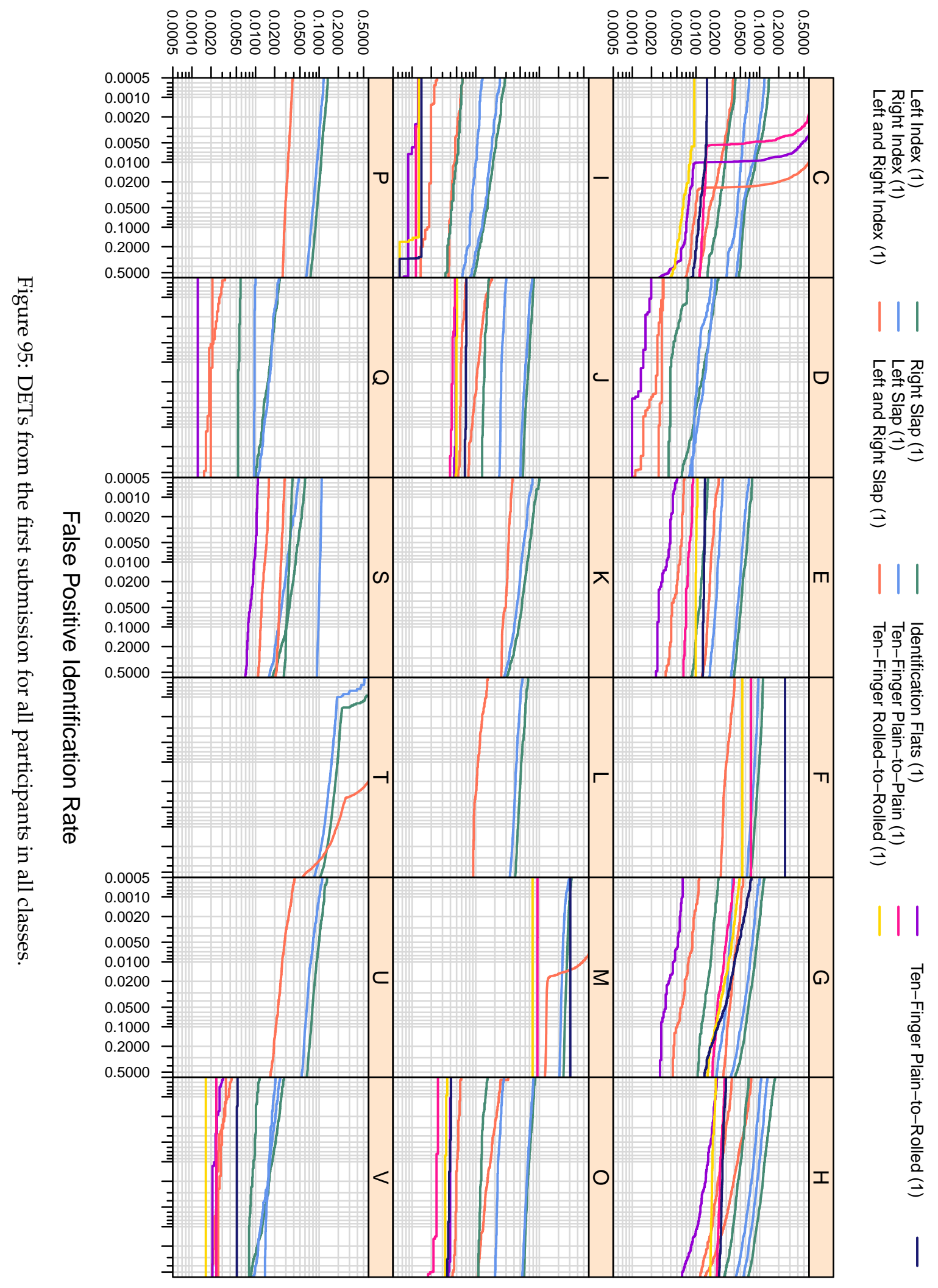

\footnotetext{
$\mathrm{C}=$ afis team $\mathrm{D}=3 \mathrm{M}$ Cogent $\mid \mathrm{E}=$ Neurotechnology $\mid \mathrm{F}=$ Papillon $\quad \mathrm{G}=$ Dermalog $\mathrm{H}=$ Hisign Bio-Info Institute

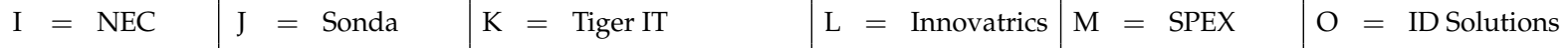

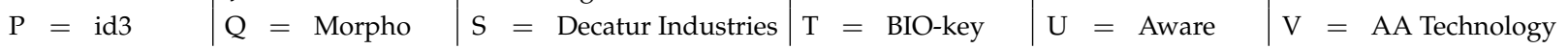




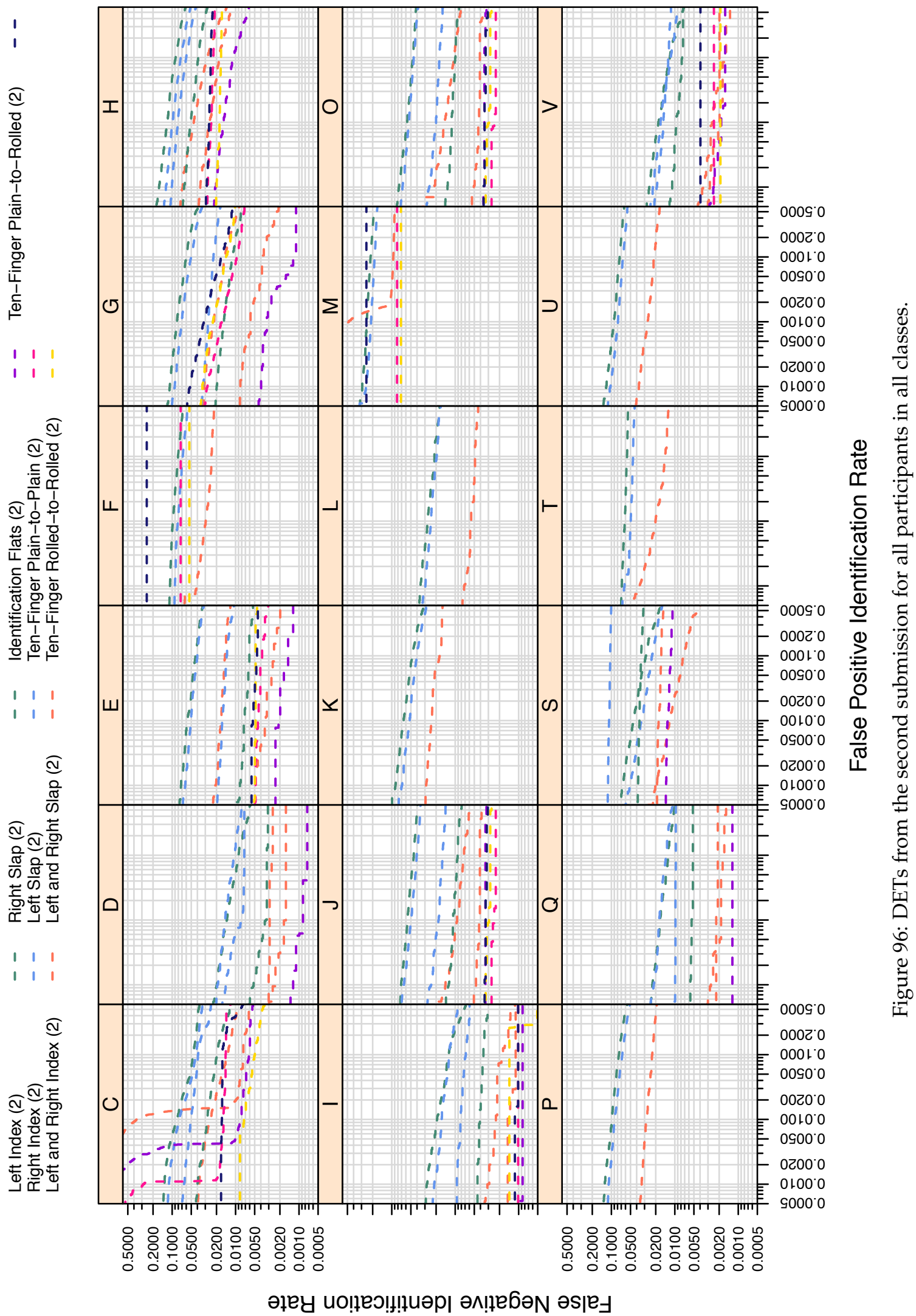

\footnotetext{
$\mathrm{C}=$ afis team $\mathrm{D}=3 \mathrm{M}$ Cogent $\mid \mathrm{E}=$ Neurotechnology $\mid \mathrm{F}=$ Papillon $\mid \mathrm{G}=$ Dermalog $\mid \mathrm{H}=$ Hisign Bio-Info Institute

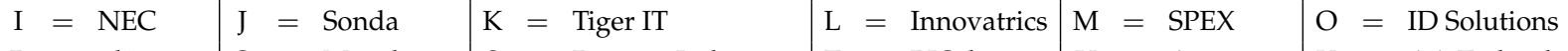

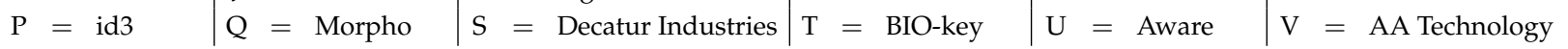




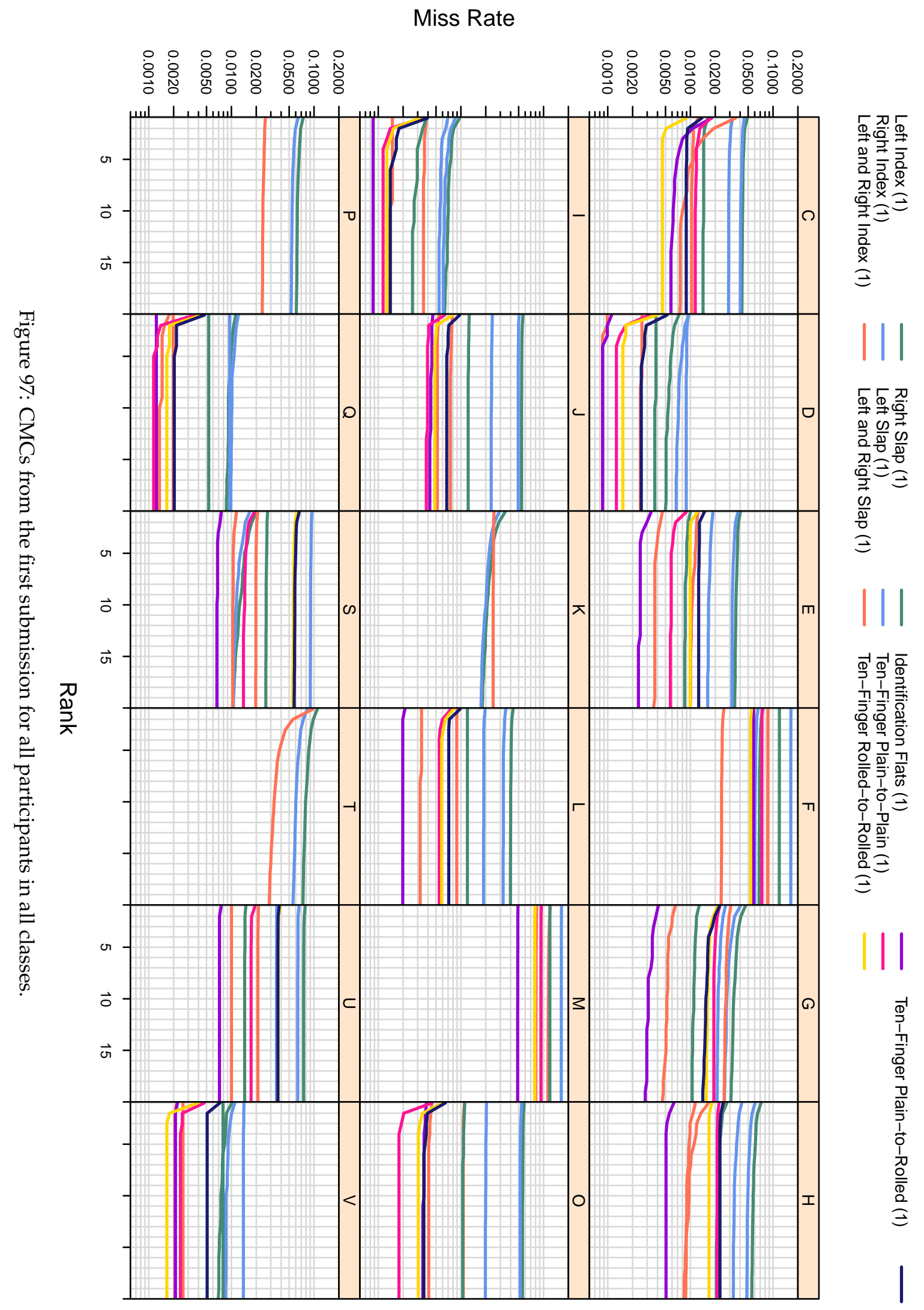

\footnotetext{
$\mathrm{C}=$ afis team $\mathrm{D}=3 \mathrm{M}$ Cogent $\mathrm{E}=$ Neurotechnology $\mid \mathrm{F}=$ Papillon $\mid \mathrm{G}=$ Dermalog $\mathrm{H}=$ Hisign Bio-Info Institute

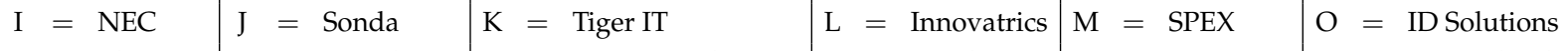

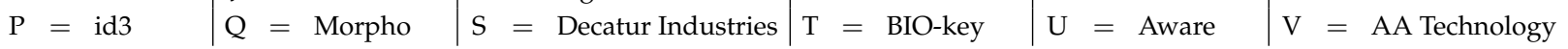




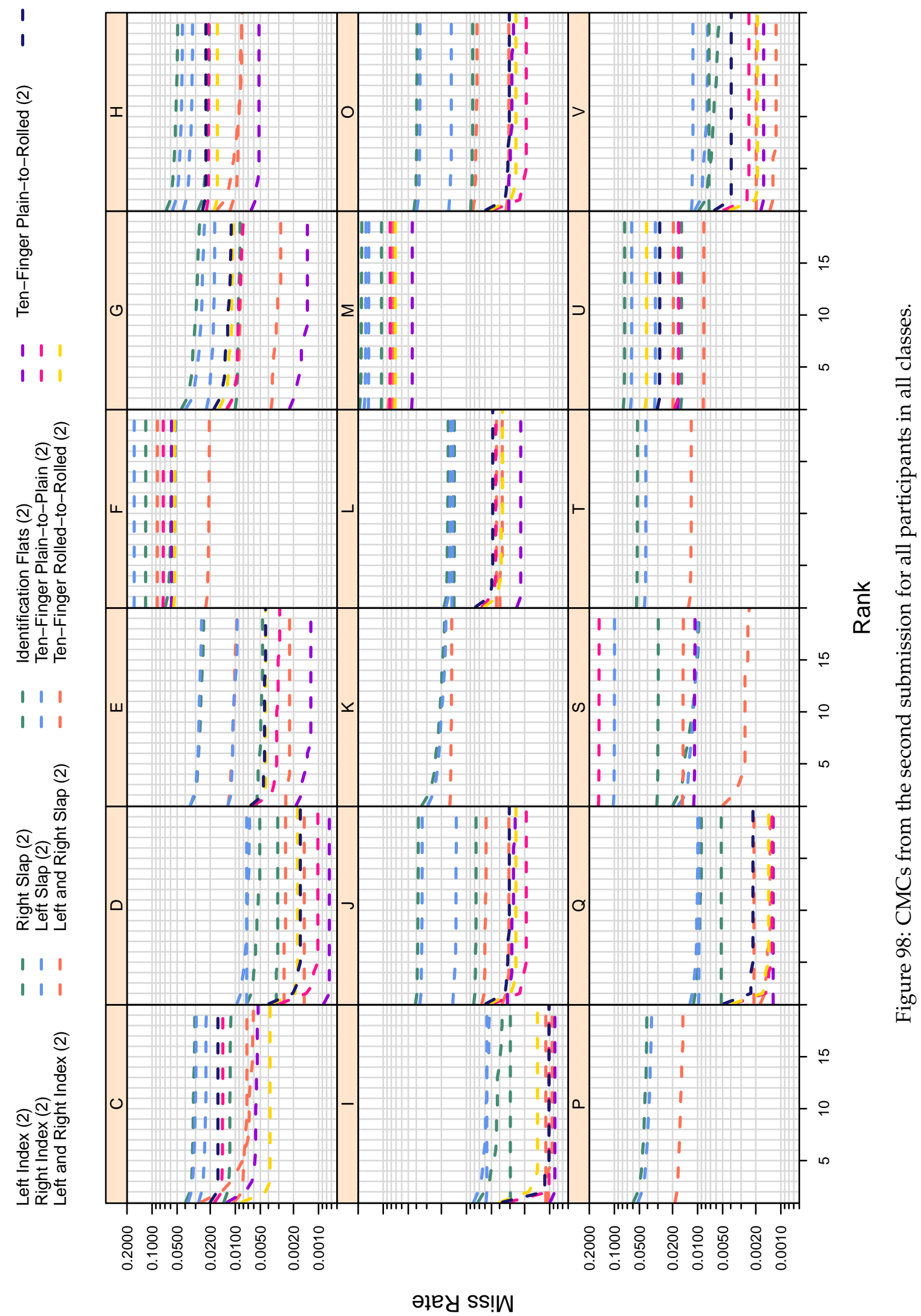

\footnotetext{
$\mathrm{C}=$ afis team $\mathrm{D}=3 \mathrm{M}$ Cogent $\mid \mathrm{E}=$ Neurotechnology $\mid \mathrm{F}=$ Papillon $\quad \mathrm{G}=$ Dermalog $\mathrm{H}=$ Hisign Bio-Info Institute

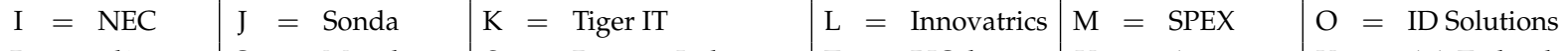

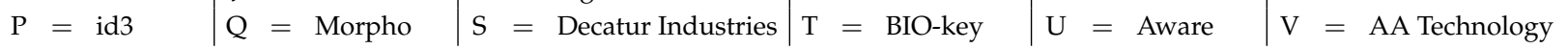




\section{Accuracy Time Tradeoff Detailed Tables with Median Values}

In order to reduce the number of tables in the main body of the report (Section 8), this appendix contains tables that show the search times for each stage of identification, for both a single process and ten processes.

The tables in this appendix report median times. For readers interested in mean times, please refer to Appendix D.

Class A results are in Tables 24 through 29, Class B results are in Tables 30 through 33, and Class C results are in Tables 34 through 36. 


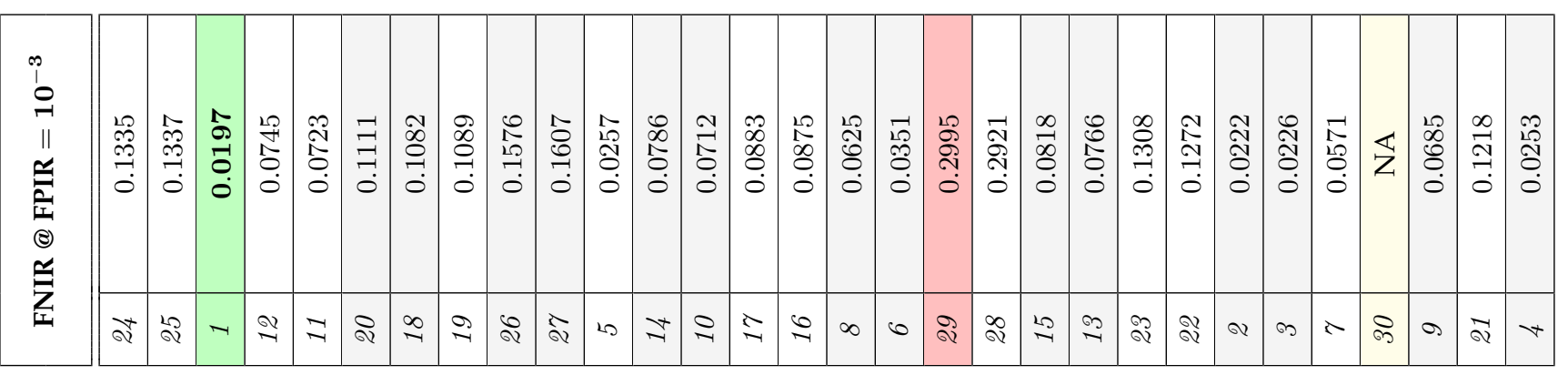

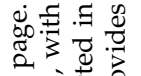

का

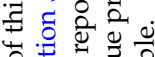

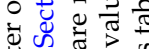

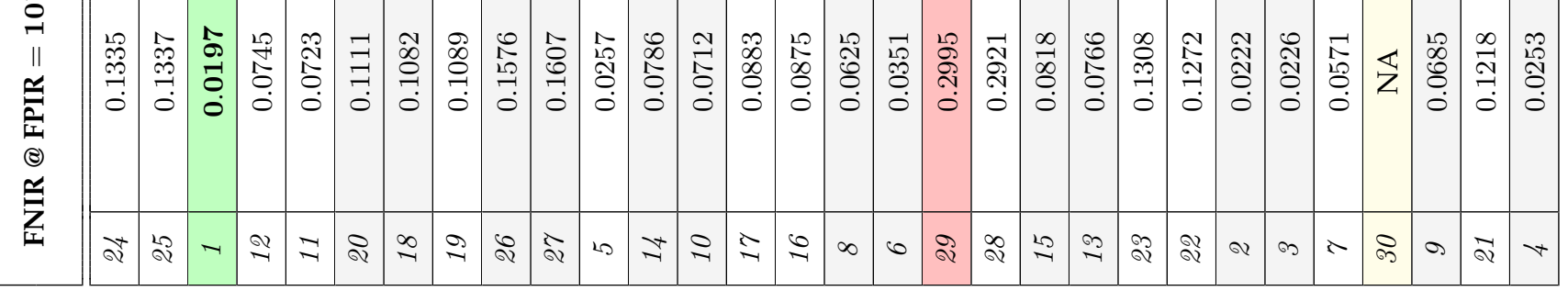

ఖ

政

둥

도.워요

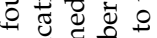

ठㄹㄹ

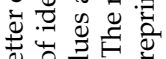

का एक

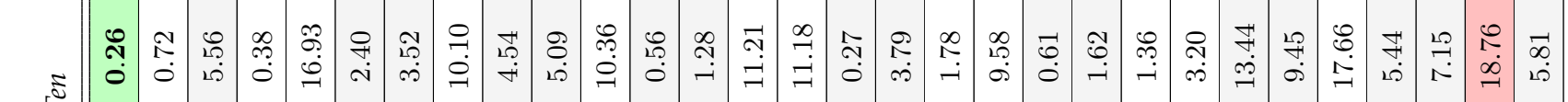

$\stackrel{\widetilde{\pi}}{\stackrel{\varpi}{0}}$

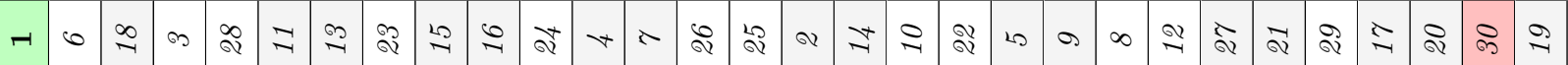

\&

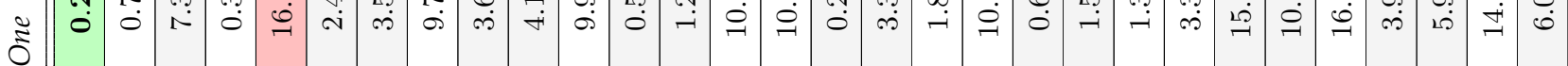

-10 จ

ะ

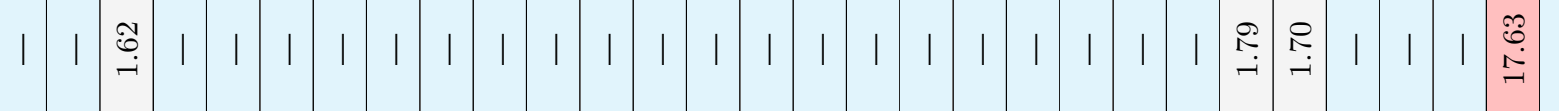

旁

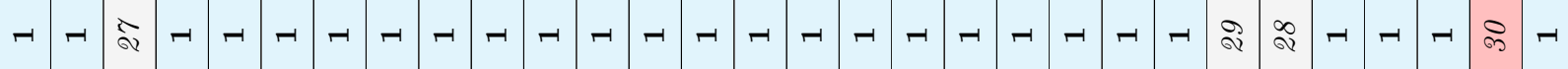

की

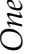

l | | \begin{tabular}{c}
$\underset{1}{1}$ \\
\hdashline
\end{tabular}

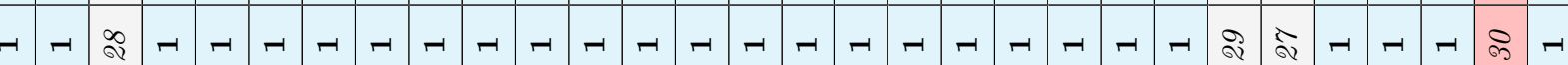

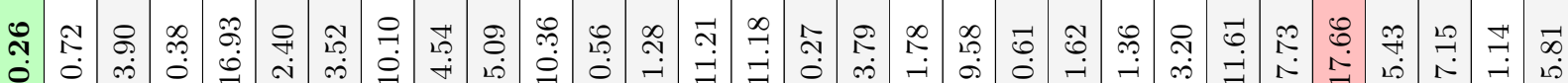

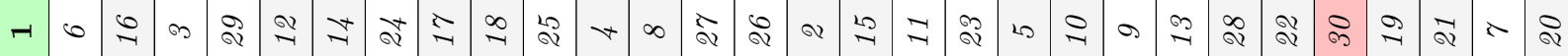

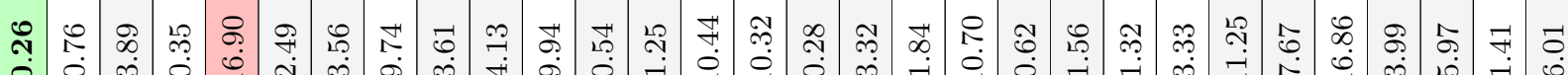
§ั

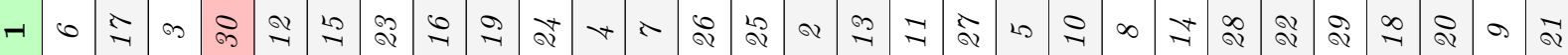

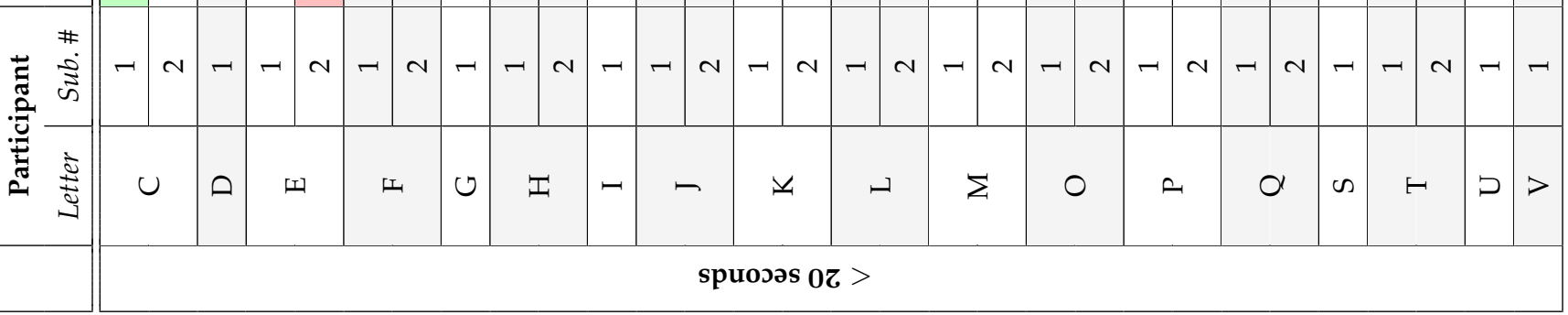

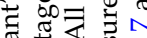

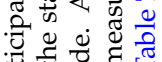

更

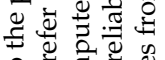

०ै द्व

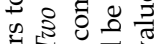

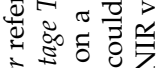

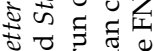

궁ㄷㅀ

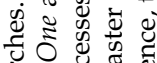

娄

के की

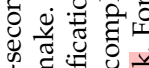

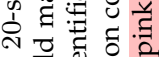

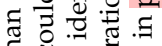

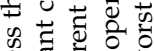

융

×.

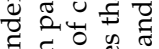

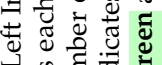

।

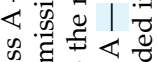

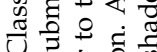

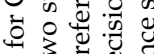

उ.

जै

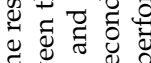

药

工 0 ठ

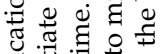

记

क्षे

:

원 월

ष्

0 을 8

.

ब

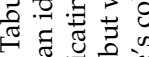

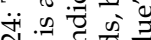

계

जि ज्ञ

$\mathrm{C}=$ afis team $\mathrm{D}=3 \mathrm{M}$ Cogent $\mid \mathrm{E}=$ Neurotechnology $\mid \mathrm{F}=$ Papillon $\mid \mathrm{G}=$ Dermalog $\mid \mathrm{H}=$ Hisign Bio-Info Institute

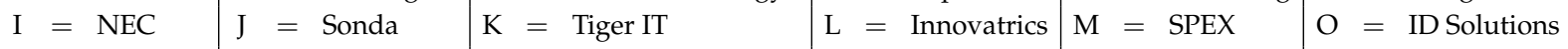

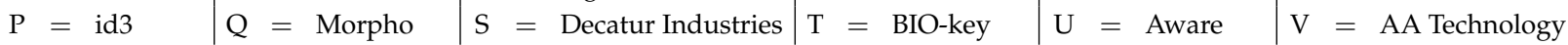




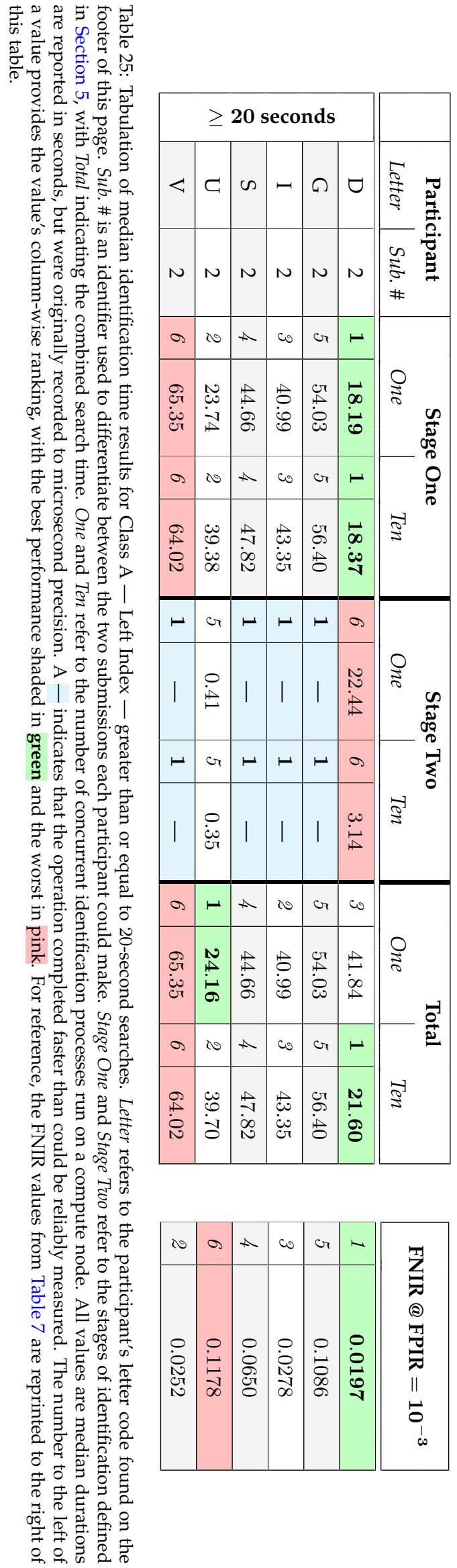

\begin{tabular}{l|l|l|l|l|l|l}
$\mathrm{C}=$ afis team & $\mathrm{D}=3 \mathrm{M}$ Cogent & $\mathrm{E}=$ Neurotechnology & $\mathrm{F}=$ Papillon & $\mathrm{G}=$ Dermalog & $\mathrm{H}=$ Hisign Bio-Info Institute \\
$\mathrm{I}=\mathrm{NEC}$ & $\mathrm{J}=$ Sonda & $\mathrm{K}=$ Tiger IT & $\mathrm{L}=$ Innovatrics & $\mathrm{M}=$ SPEX & $\mathrm{O}=$ ID Solutions \\
$\mathrm{P}=\mathrm{id} 3$ & $\mathrm{Q}=$ Morpho & $\mathrm{S}=$ Decatur Industries & $\mathrm{T}=$ BIO-key & $\mathrm{U}=$ Aware & $\mathrm{V}=$ AA Technology
\end{tabular}




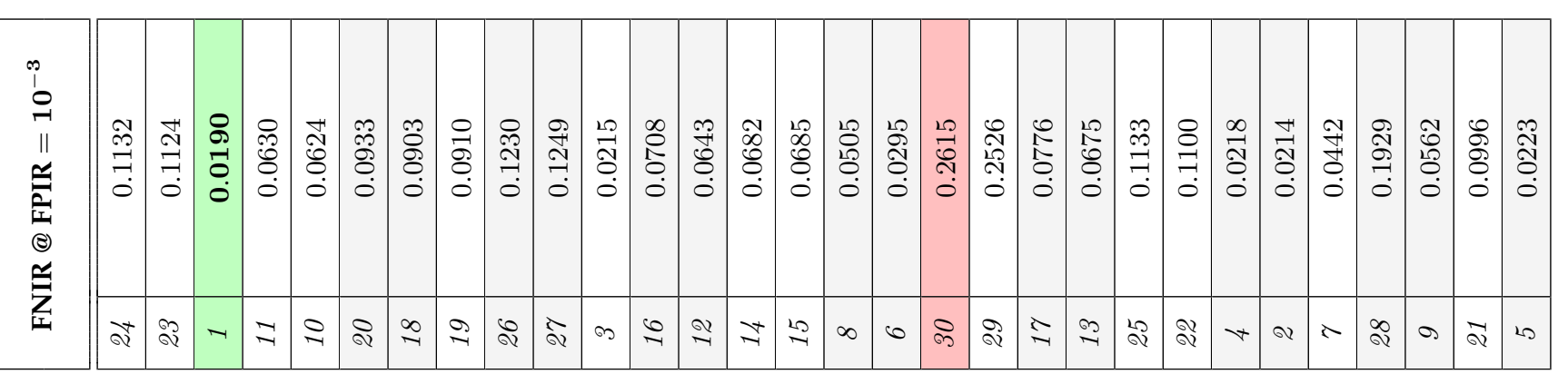

韋 $10.9 \frac{8}{0}$

पे

ปै

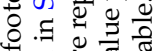

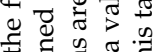

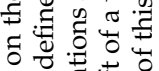

它

D.

粈

ठ덩

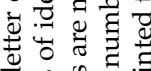

ज

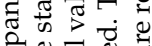

월류

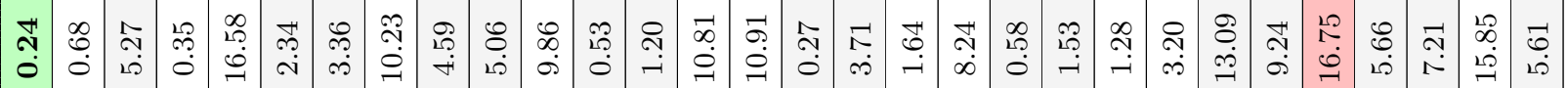

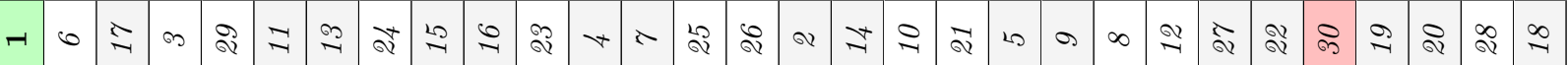

퐁

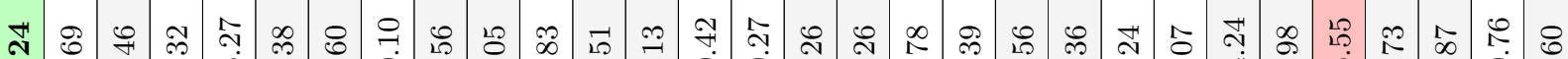

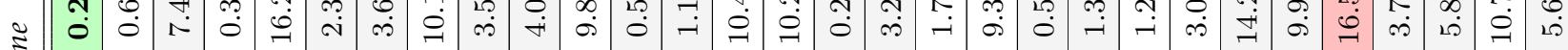
- 0 จ क จे

疍

E

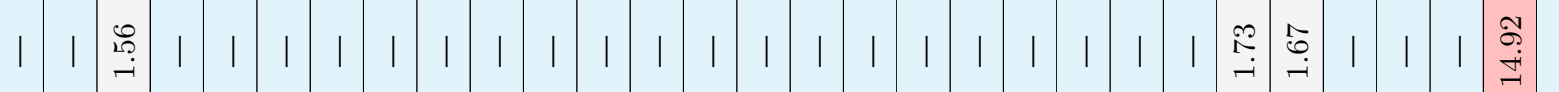

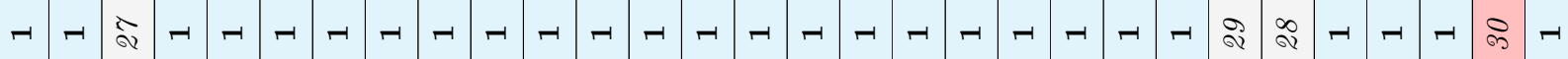

के

$\Xi$

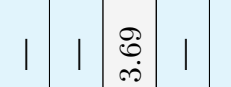

I

I

ָู

훙요 웜

$\pm$

온

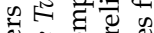

造

के

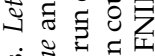

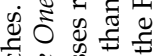

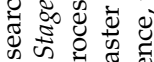

ธี

क है है है

흔.

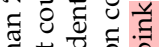

$\nsubseteq+\vec{c}$.

ญ.

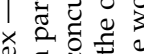

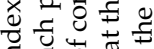

द्व.

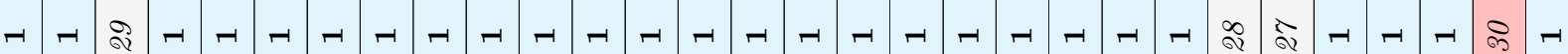

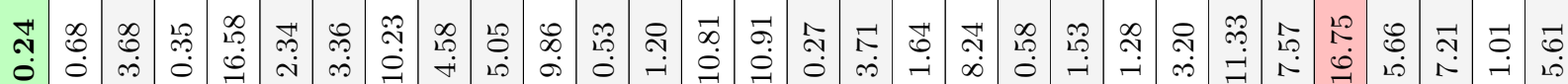

ڤ

$\check{\sigma}$

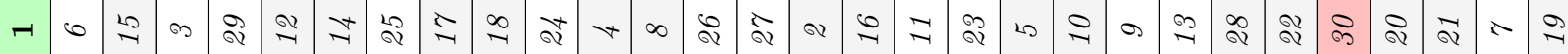

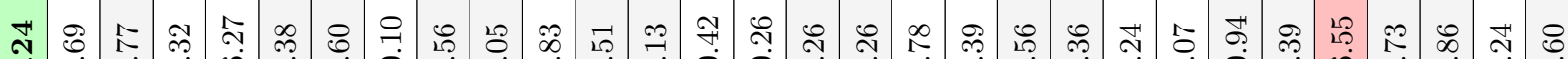

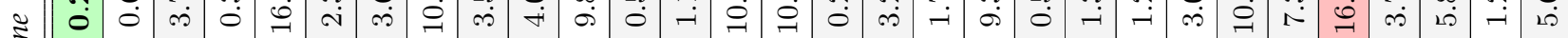

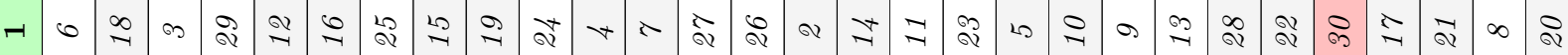

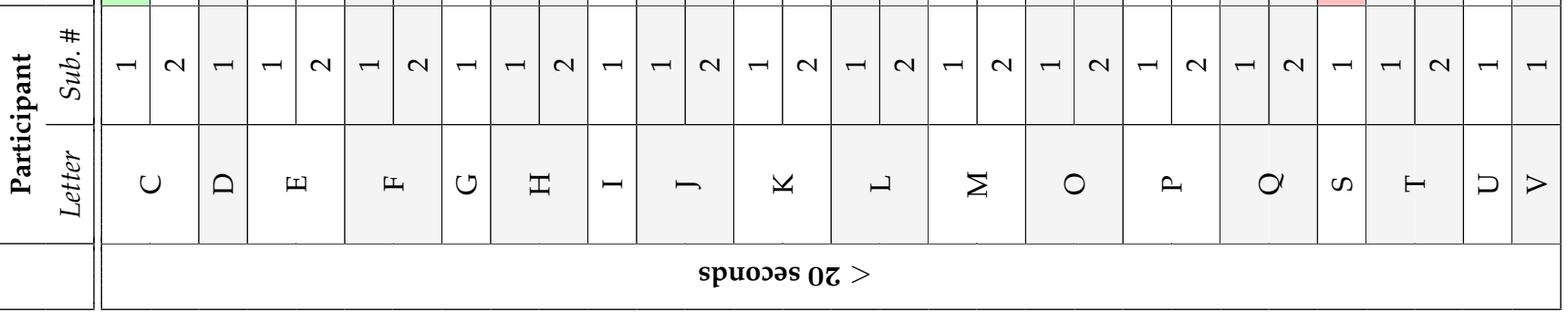

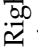

$\varangle$

क के

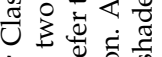

苛

s

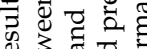

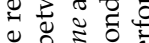

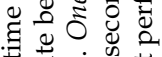

ठี

ฮั

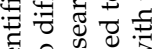

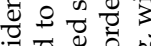

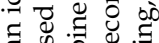

굴 로

द.

范焉

도유. 0005

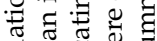

ㄱ. 오 3

元 \#

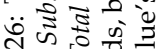

요요요

ส

$\mathrm{C}=$ afis team $\mathrm{D}=3 \mathrm{M}$ Cogent $\mid \mathrm{E}=$ Neurotechnology $\mid \mathrm{F}=$ Papillon $\mid \mathrm{G}=$ Dermalog $\mid \mathrm{H}=$ Hisign Bio-Info Institute

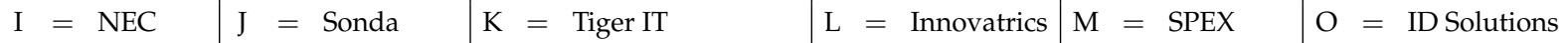

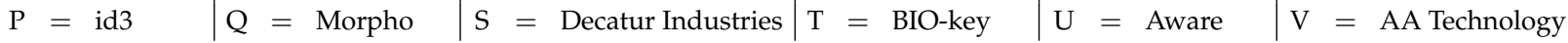




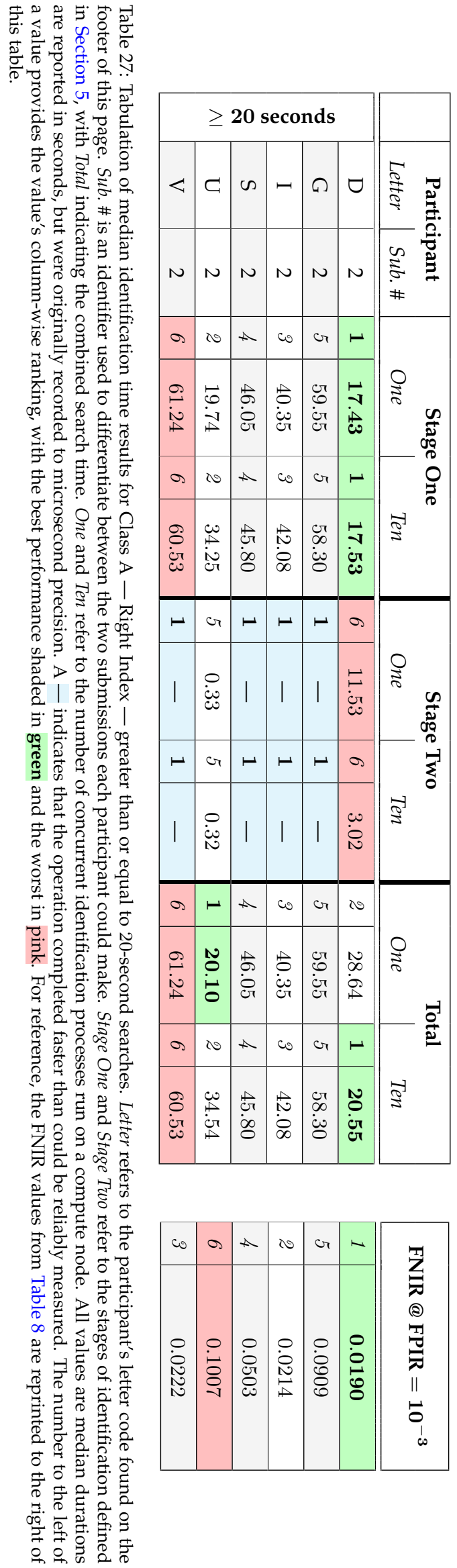

\begin{tabular}{l|l|l|l|ll|l}
$\mathrm{C}=$ afis team & $\mathrm{D}=3 \mathrm{M}$ Cogent & $\mathrm{E}=$ Neurotechnology & $\mathrm{F}=$ Papillon & $\mathrm{G}=$ Dermalog & $\mathrm{H}=$ Hisign Bio-Info Institute \\
$\mathrm{I}=$ NEC & $\mathrm{J}=$ Sonda & $\mathrm{K}=$ Tiger IT & $\mathrm{L}=$ Innovatrics & $\mathrm{M}=$ SPEX & $\mathrm{O}=$ ID Solutions \\
$\mathrm{P}=\mathrm{id} 3$ & $\mathrm{Q}=$ Morpho & $\mathrm{S}=$ Decatur Industries & $\mathrm{T}=$ BIO-key & $\mathrm{U}=$ Aware & $\mathrm{V}=$ AA Technology
\end{tabular}



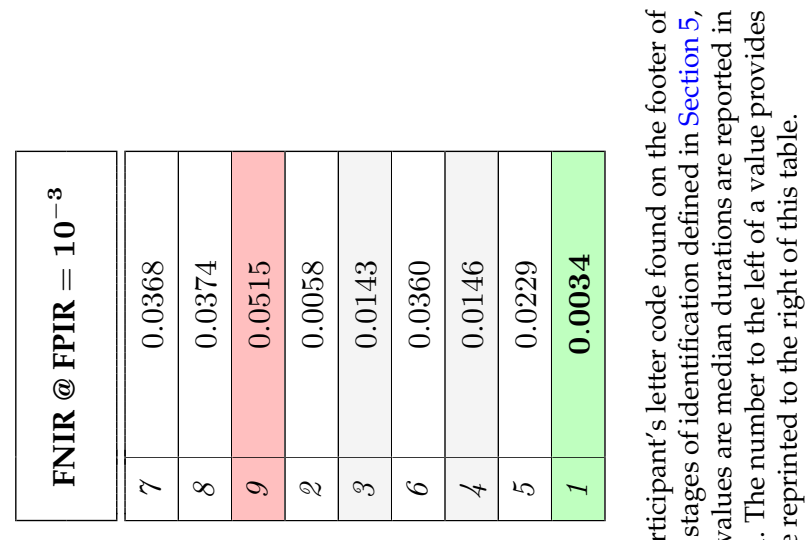

跣卷

记

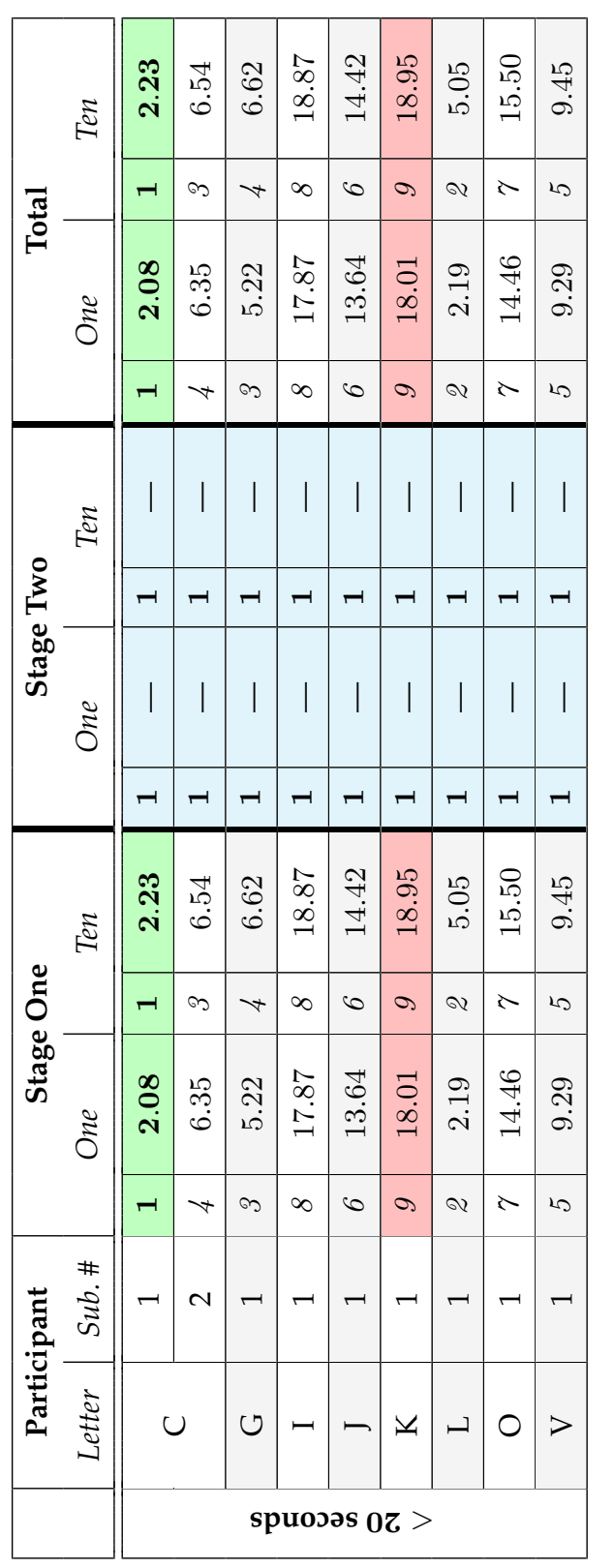

₹

की

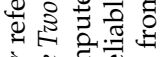

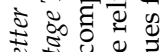

ज部

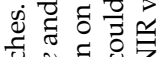

ฮँ ๊

के

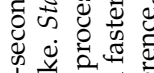

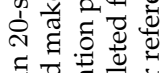

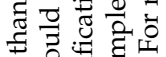

\&

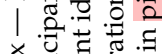

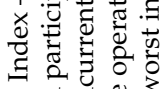

동듀 है छ

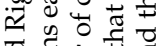

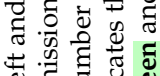

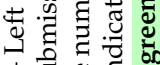

1 实导. .5

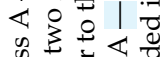

Uึ

ప艹

考

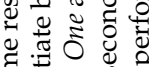

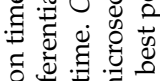

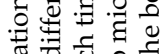

象。

ए 0

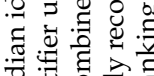

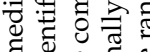

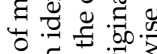

ธี क 50.50

毒. एँّ

สำ ज.

iे

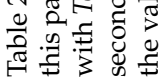

\begin{tabular}{l|l|l|l|l|l|l}
$\mathrm{C}=$ afis team & $\mathrm{D}=3 \mathrm{M}$ Cogent & $\mathrm{E}=$ Neurotechnology & $\mathrm{F}=$ Papillon & $\mathrm{G}=$ Dermalog & $\mathrm{H}=$ Hisign Bio-Info Institute \\
$\mathrm{I}=\mathrm{NEC}$ & $\mathrm{J}=$ Sonda & $\mathrm{K}=$ Tiger IT & $\mathrm{L}=$ Innovatrics & $\mathrm{M}=$ SPEX & $\mathrm{O}=$ ID Solutions \\
$\mathrm{P}=\mathrm{id} 3$ & $\mathrm{Q}=$ Morpho & $\mathrm{S}=$ Decatur Industries & $\mathrm{T}=$ BIO-key & $\mathrm{U}=$ Aware & $\mathrm{V}=$ AA Technology
\end{tabular}




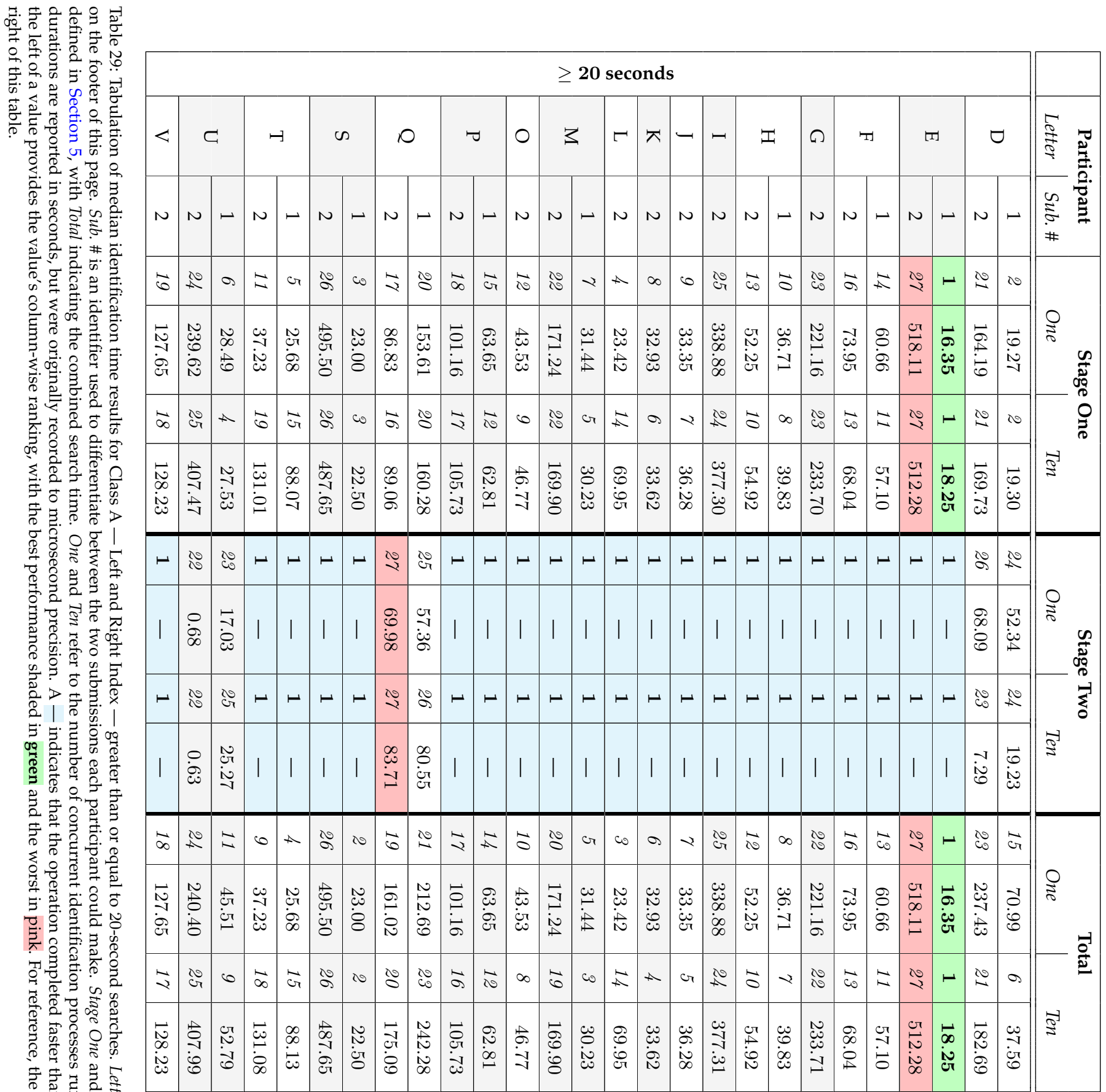

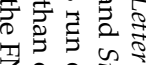

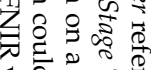

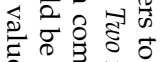

क्ष.

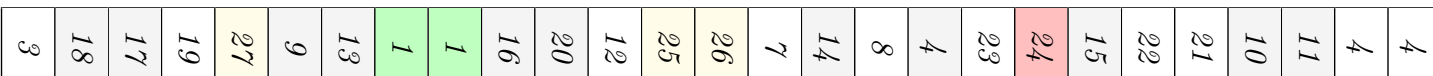

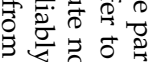

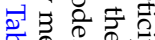

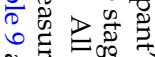

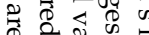

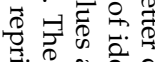

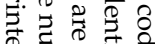

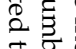

象

\begin{tabular}{|c|c|c|c|c|c|c|c|c|c|c|c|c|c|c|c|c|c|c|c|c|c|c|c|c|c|c|c|}
\hline$<$ & $c$ & & & & & & 1 & & & & 0 & 5 & & $\tau$ & $\pi$ & 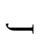 & - & $F$ & & $\Omega$ & $T$ & & $\mathrm{~T}$ & & 5 & & $\stackrel{0}{\$}$ \\
\hline$N$ & $N$ & 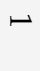 & $N$ & $\leftarrow$ & $N$ & 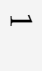 & $N$ & $\leftarrow$ & $N$ & $\bullet$ & N & $N$ & 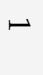 & $N$ & $N$ & N & N & N & 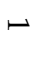 & $N$ & $N$ & $\bullet$ & $N$ & $\vdash$ & $N$ & 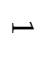 & $\begin{array}{l}\vec{\sigma} \\
\nexists \\
\#\end{array}$ \\
\hline
\end{tabular}

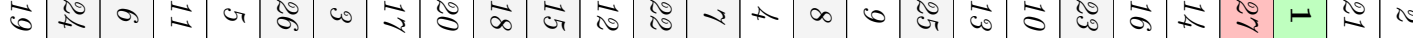

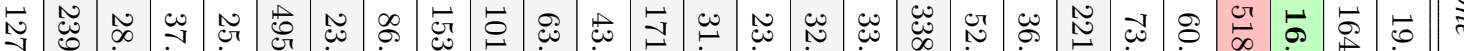

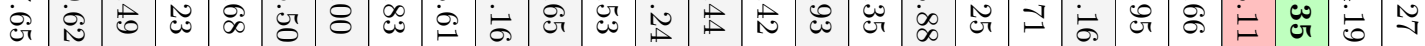

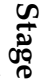

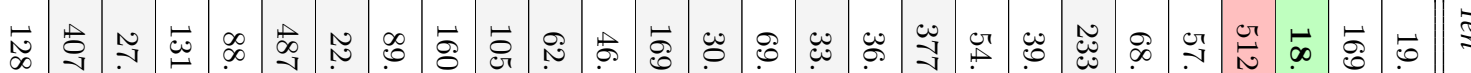

山

\begin{tabular}{|c|c|c|c|c|c|c|c|c|c|c|c|c|c|c|c|c|c|c|c|c|c|c|c|c|c|c|c|}
\hline & ي心 & \begin{tabular}{|l}
0 \\
$\omega_{0}$
\end{tabular} & - & - & - & $r$ & $\mathbb{Y}$ & 心 & 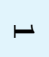 & - & - & $r$ & $r$ & $\sim$ & 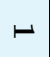 & $\sim$ & $\sim$ & 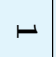 & $r$ & $\sim$ & 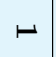 & 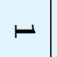 & - & $\sim$ & $\infty$ & $\underset{\$}{\infty}$ & \\
\hline | & $\begin{array}{l}\dot{D} \\
\dot{\infty}\end{array}$ & $\begin{array}{l}\vec{v} \\
\dot{\omega}\end{array}$ & | & | & | & | & $\begin{array}{l}0 \\
0 \\
0 \\
\infty\end{array}$ & $\begin{array}{l}\tilde{c} \\
\dot{y} \\
\dot{\omega}\end{array} \mid$ & 1 & | & | & | & 1 & $\mid$ & I & | & | & $\mid$ & $\mid$ & | & $\mid$ & | & | & | & $\begin{array}{l}\dot{0} \\
\dot{0} \\
\dot{0}\end{array}$ & $\begin{array}{l}\tilde{N} \\
\dot{v} \\
i\end{array}$ & $\underset{\substack{* \\
0}}{\infty}$ \\
\hline- & 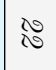 & $\stackrel{心}{c}$ & $\vdash$ & $\leftarrow$ & $\leftarrow$ & $r$ & $\stackrel{N}{\sim}$ & $\stackrel{\infty}{0}$ & $\mapsto$ & 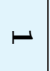 & $\vdash$ & $\leftarrow$ & $\mapsto$ & - & $\vdash$ & $\leftarrow$ & 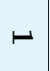 & 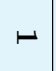 & $\mapsto$ & - & 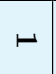 & - & 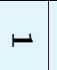 & $\sim$ & $\mathbb{E}$ & 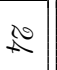 & $\bar{\Sigma}$ \\
\hline | & 赵 & 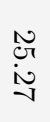 & $\mid$ & | & | & | & 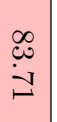 & 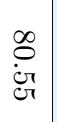 & 1 & | & | & | & 1 & 1 & 1 & | & | & 1 & 1 & 1 & $\mid$ & | & | & | & 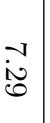 & $\begin{array}{l}\overrightarrow{0} \\
\dot{ن} \\
\dot{\omega}\end{array}$ & $\vec{g}$ \\
\hline$\vec{\infty}$ & ז̊ & $\Xi$ & 0 & $A$ & 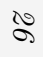 & ه & $\vec{\bullet}$ & 心 & 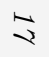 & $\widehat{A}$ & $\vec{\sigma}$ & તి & $G$ & $c_{0}$ & $a$ & $\checkmark$ & $\stackrel{心}{G}$ & $\vec{o}$ & $\infty$ & 心 & $\vec{a}$ & $\vec{\omega}$ & $\stackrel{\$}{\lessgtr}$ & - & ৫્ఠ & $\vec{G}$ & \\
\hline כ. & 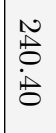 & 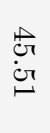 & 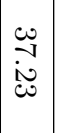 & 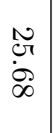 & $\begin{array}{l}\vec{t} \\
\stackrel{\leftrightarrow}{i} \\
\text { in }\end{array}$ & هُ & $\begin{array}{l}\vec{\rho} \\
\dot{\rho} \\
\dot{0}\end{array}$ & 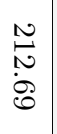 & $\begin{array}{l}\overrightarrow{0} \\
\dot{\sigma}\end{array}$ & 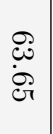 & $\begin{array}{l}\vec{c} \\
\dot{c} \\
\dot{c}\end{array}$ & $\begin{array}{l}\vec{J} \\
i \\
i\end{array}$ & $\begin{array}{l}\stackrel{\omega}{\doteq} \\
\stackrel{\perp}{\perp}\end{array}$ & 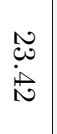 & 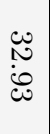 & 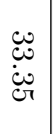 & $\begin{array}{l}\dot{\omega} \\
\infty \\
\infty \\
\infty \\
\infty\end{array}$ & $\begin{array}{l}c \\
N \\
心 \\
心 \\
c\end{array}$ & $\mid \begin{array}{c}\infty \\
\stackrel{\leftrightarrow}{-} \\
\vec{\nu}\end{array}$ & 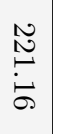 & $\left|\begin{array}{c}-\vec{u} \\
\dot{0} \\
\dot{c}\end{array}\right|$ & $\begin{array}{l}\dot{8} \\
\dot{8}\end{array}$ & $\begin{array}{l}c \\
\infty \\
\sqcup \\
ت\end{array}$ & & 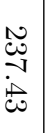 & $\begin{array}{l}\overrightarrow{1} \\
8 \\
0\end{array}$ & \\
\hline v & (ָ) & 0 & $\vec{\infty}_{0}$ & $\vec{c}$ & $\approx$ & ๙ & $\mathbb{8}$ & $\begin{array}{l}\infty \\
\infty \\
\infty\end{array}$ & $\vec{a}$ & $\vec{v}$ & $\infty$ & $\vec{\sigma}$ & $\infty$ & 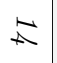 & $A$ & $G$ & న & $\vec{a}$ & $\checkmark$ & $\mathbb{\infty}$ & $\overrightarrow{\omega_{0}}$ & $\Xi$ & 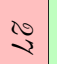 & $\sim$ & $\approx$ & $a$ & \\
\hline $\begin{array}{l}\vec{N} \\
\infty \\
0\end{array}$ & $\begin{array}{l}\stackrel{0}{0} \\
\text { - } \\
0 \\
0\end{array}$ & $\begin{array}{l}\stackrel{c}{N} \\
\stackrel{1}{0}\end{array}$ & $\mid$\begin{tabular}{c|}
$\vec{\omega}$ \\
$\stackrel{\dot{\omega}}{\infty}$ \\
$\dot{\infty}$
\end{tabular} & $\begin{array}{l}\infty \\
\infty \\
\dot{\omega} \omega\end{array}$ & $\begin{array}{l}\Delta \\
\infty \\
-1 \\
\dot{i} \\
\dot{c}\end{array}$ & $\begin{array}{l}\text { Non } \\
\text { iñ }\end{array}$ & $\begin{array}{l}\overrightarrow{\mathrm{v}} \\
\dot{0} \\
\dot{0}\end{array}$ & 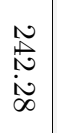 & $\begin{array}{l}\vec{c} \\
\stackrel{c}{c} \\
\stackrel{\omega}{\omega}\end{array}$ & $\begin{array}{l}0 \\
\dot{0} \\
\infty \\
\bullet\end{array}$ & $\begin{array}{l}\stackrel{\Delta}{o} \\
\text { y } \\
\text { v }\end{array}$ & $\begin{array}{l}\overrightarrow{8} \\
8 \\
8\end{array}$ & $\left|\begin{array}{l}\dot{\omega} \\
\dot{0} \\
\dot{\omega} \\
0\end{array}\right|$ & $\begin{array}{l}\mathscr{8} \\
\dot{8} \\
\dot{\mathrm{r}}\end{array}$ & 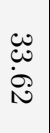 & $\begin{array}{l}\stackrel{\omega}{.} \\
\stackrel{\sim}{\infty} \\
\infty\end{array}$ & $\begin{array}{l}\stackrel{\omega}{u} \\
\vec{\omega} \\
\dot{\omega}\end{array}$ & $\mid \begin{array}{l}c \\
\stackrel{c}{1} \\
0 \\
0\end{array}$ & $\left|\begin{array}{l}0 \\
0 \\
\dot{0} \\
\dot{c}\end{array}\right|$ & 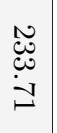 & $\mid \begin{array}{l}\infty \\
\infty \\
\dot{1} \\
1\end{array}$ & 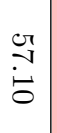 & 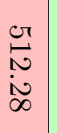 & & $\begin{array}{l}\vec{\infty} \\
0 \\
0 \\
0 \\
0\end{array}$ & 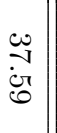 & $\overrightarrow{3}$ \\
\hline
\end{tabular}

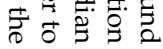




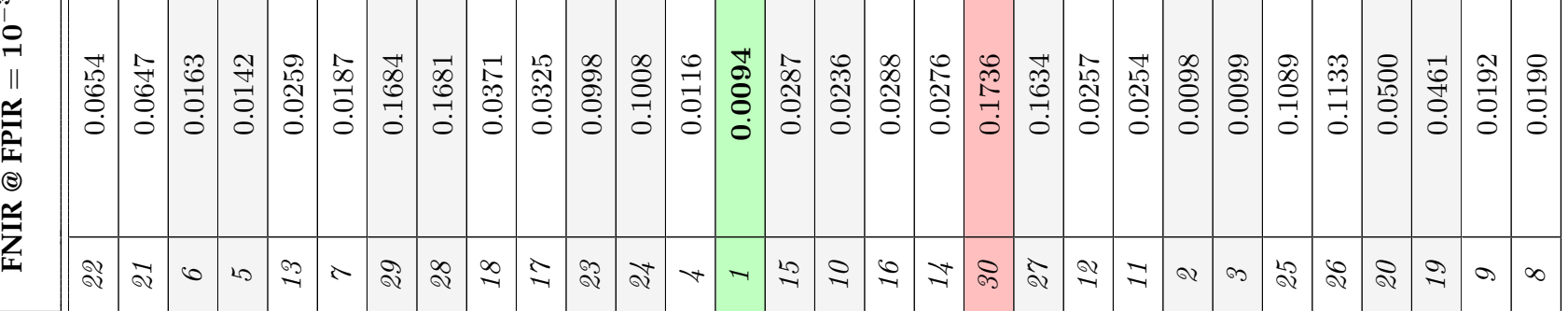

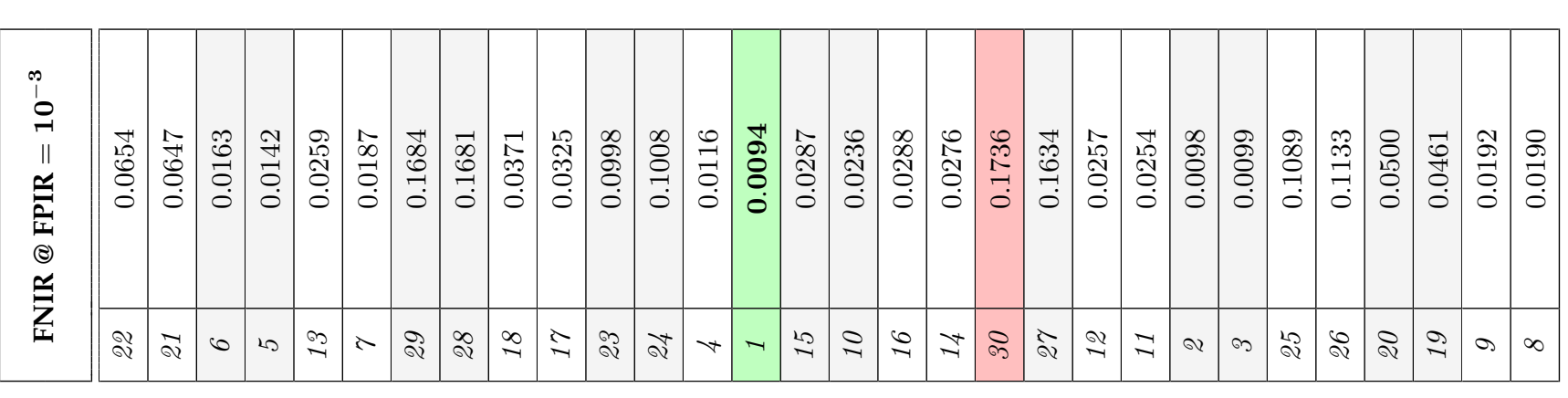

\begin{tabular}{|c|c|c|c|c|c|c|c|c|c|c|c|c|c|c|c|c|c|c|c|c|c|c|c|c|c|c|c|c|c|c|}
\hline \multirow{4}{*}{ స్ } & $\begin{array}{l}\infty \\
\infty \\
\infty \\
\infty\end{array}$ & 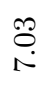 & $\begin{array}{l}\vec{b} \\
\dot{0} \\
i\end{array}$ & $\begin{array}{l}\vec{D} \\
\dot{0} \\
\infty\end{array} \mid$ & $\begin{array}{l}\stackrel{\theta}{\vec{H}} \\
\dot{\infty}\end{array}$ & 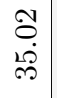 & $\begin{array}{l}10 \\
\infty \\
\text { id } \\
\sigma\end{array}$ & 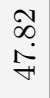 & 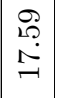 & $\begin{array}{l}8 \\
\dot{0} \\
0\end{array}$ & $\begin{array}{l}\stackrel{20}{\circ} \\
\dot{\theta}\end{array}$ & 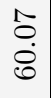 & $\begin{array}{l}\overrightarrow{1} \\
\dot{\theta}\end{array}$ & $\begin{array}{l}\stackrel{8}{S} \\
\stackrel{H}{H}\end{array}$ & $\begin{array}{l}\stackrel{2}{0} \\
\stackrel{N}{N}\end{array}$ & $\begin{array}{l}20 \\
1 \\
\infty \\
1\end{array}$ & \begin{tabular}{l}
$\vec{D}$ \\
$\dot{0}$ \\
\hdashline
\end{tabular} & 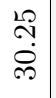 & $\begin{array}{l}\vec{H} \\
\stackrel{\leftrightarrow}{\infty}\end{array}$ & 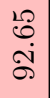 & $\begin{array}{l}\vec{m} \\
\ddot{m} \\
\dot{m}\end{array}$ & 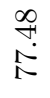 & $\begin{array}{l}\vec{N} \\
\infty \\
b \\
b\end{array}$ & 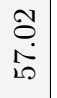 & $\begin{array}{l}\hat{0} \\
\stackrel{+}{1} \\
\stackrel{1}{1}\end{array}$ & $\begin{array}{l}\vec{\infty} \\
\stackrel{+}{1} \\
\stackrel{1}{*}\end{array}$ & 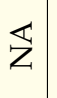 & 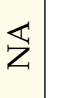 & 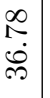 & 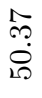 \\
\hline & 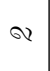 & $\infty$ & $\sigma^{*}$ & $\hat{\otimes}$ & -1 & 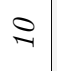 & $\infty$ & \pm & 20 & $\stackrel{\infty}{-\infty}$ & ฉి & $\stackrel{\sim}{-1}$ & $\vec{Q}$ & $\stackrel{\infty}{\sim}$ & 0 & ڤి & $\psi$ & $\therefore$ & $\theta$ & $\begin{array}{l}\infty \\
\stackrel{\infty}{\vee}\end{array}$ & $\stackrel{\sim}{\sim}$ & $\stackrel{20}{\otimes}$ & $\approx$ & $\stackrel{\bullet}{\sim}$ & ชै & $\stackrel{9}{\otimes}$ & 1 & 1 & $\exists$ & $\stackrel{20}{2}$ \\
\hline & $\begin{array}{l}\vec{T} \\
\dot{s}\end{array}$ & $\stackrel{+}{\stackrel{+}{0}}$ & 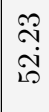 & $\begin{array}{c}0 \\
\infty \\
\infty \\
10\end{array}$ & $\begin{array}{l}\mathcal{N} \\
\infty \\
\end{array}$ & $\begin{array}{l}\hat{\infty} \\
\stackrel{\infty}{\infty} \\
\dot{m}\end{array}$ & $\begin{array}{l}\dot{8} \\
\dot{0} \\
\dot{8}\end{array}$ & 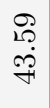 & 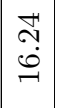 & $\begin{array}{l}\stackrel{9}{+} \\
\stackrel{8}{B}\end{array}$ & $\begin{array}{l}\stackrel{2}{1} \\
\stackrel{9}{F}\end{array}$ & $\begin{array}{l}\stackrel{ }{1} \\
\dot{B}\end{array}$ & $\begin{array}{l}\mathscr{8} \\
10 \\
10\end{array}$ & $\begin{array}{l}0 \\
\stackrel{1}{ } \\
\infty \\
\infty\end{array}$ & $\begin{array}{l}\overrightarrow{0} \\
\stackrel{\leftrightarrow}{\mathrm{d}}\end{array}$ & $\begin{array}{l}\infty \\
\stackrel{\infty}{\sim} \\
\stackrel{+}{N}\end{array}$ & 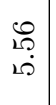 & 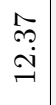 & $\begin{array}{l}\stackrel{-}{二} \\
\overrightarrow{\dot{m}}\end{array}$ & 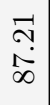 & $\begin{array}{l}\overrightarrow{0} \\
\text { s. } \\
\text {. }\end{array}$ & $\stackrel{10}{\stackrel{10}{P}}$ & $\begin{array}{l}\vec{N} \\
\stackrel{D}{0} \\
\text { is }\end{array}$ & 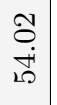 & $\begin{array}{l}\stackrel{g}{\dot{0}} \\
\stackrel{i}{i}\end{array}$ & $\begin{array}{l}\stackrel{5}{1} \\
\dot{\mathrm{N}}\end{array}$ & $\begin{array}{l}\stackrel{0}{0} \\
\dot{\infty}\end{array}$ & $\begin{array}{l}\hat{0} \\
\stackrel{0}{\infty}\end{array}$ & $\begin{array}{l}\hat{D} \\
\dot{0} \\
\dot{\oplus}\end{array}$ & 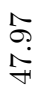 \\
\hline & $\curvearrowright$ & $\psi$ & $\stackrel{\infty}{=}$ & & -1 & $\therefore$ & $\infty$ & \pm & 0 & $\stackrel{2}{\otimes}$ & $\stackrel{0}{\sim}$ & $\approx$ & $\vec{Q}$ & $\stackrel{\oplus}{\sim}$ & $\lambda$ & $\widehat{\hat{Q}}$ & $\infty$ & 20 & $a$ & Qे & $\stackrel{\sim}{\sim}$ & $\mathscr{Q}$ & 2 & 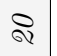 & खे & $\stackrel{2}{2}$ & $\stackrel{\infty}{\otimes}$ & $\stackrel{\circ}{\circ}$ & $\exists$ & $\stackrel{20}{-1}$ \\
\hline
\end{tabular}

\begin{tabular}{|c|c|c|c|c|c|c|c|c|c|c|c|c|c|c|c|c|c|c|c|c|c|c|c|c|c|c|c|c|c|c|}
\hline & 1 & | & $\begin{array}{l}\infty \\
0 \\
0\end{array}$ & $\begin{array}{l}\overleftrightarrow{0} \\
\stackrel{\dot{0}}{0}\end{array}$ & I & 1 & | & | & | & | & | & 1 & 1 & 1 & 1 & | & | & 1 & 1 & 1 & 1 & 1 & $\begin{array}{l}\stackrel{\curvearrowright}{~} \\
\sim\end{array}$ & 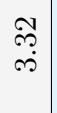 & 1 & | & $\overleftrightarrow{Z}$ & $\overleftrightarrow{Z}$ & 1 & \\
\hline & -1 & $-r$ & $\hat{\widehat{Q}}$ & $\stackrel{\infty}{\otimes}$ & $-r$ & -1 & -1 & $-r$ & -1 & -1 & -1 & - & -1 & -1 & $-r$ & -1 & -1 & -1 & -1 & -1 & -1 & - & $\stackrel{2}{2}$ & $\stackrel{2}{\otimes}$ & -1 & -1 & 1 & I & -1 & - \\
\hline ธี & 1 & | & $\begin{array}{l}\stackrel{\Im}{2} \\
\text { is }\end{array}$ & $\begin{array}{l}\qquad 8 \\
\stackrel{2}{+} \\
+\end{array}$ & | & 1 & 1 & | & 1 & 1 & 1 & 1 & 1 & 1 & 1 & 1 & 1 & 1 & 1 & 1 & 1 & 1 & $\begin{array}{l}\stackrel{\leftrightarrow}{\rightarrow} \\
\stackrel{\infty}{\circ}\end{array}$ & $\begin{array}{l}20 \\
20 \\
03 \\
\infty\end{array}$ & 1 & | & \begin{tabular}{l}
\multirow{0}{0}{} \\
\multirow{r}{*}{}
\end{tabular} & 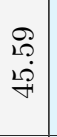 & 1 & | \\
\hline & -1 & $-r$ & $\stackrel{2}{\otimes}$ & $\stackrel{\infty}{\otimes}$ & $-r$ & -1 & -1 & $-r$ & -1 & -1 & -1 & -1 & -1 & -1 & -1 & -1 & -1 & -1 & -1 & -1 & -1 & -1 & $\stackrel{2}{\circ}$ & $\widehat{\widehat{Q}}$ & -1 & -1 & คి & ฉे & -1 & -1 \\
\hline
\end{tabular}

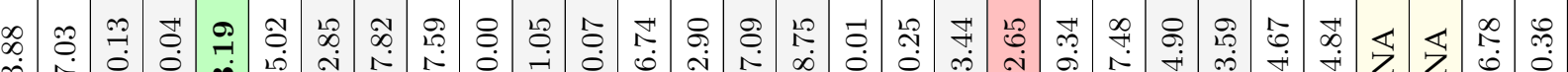
‡

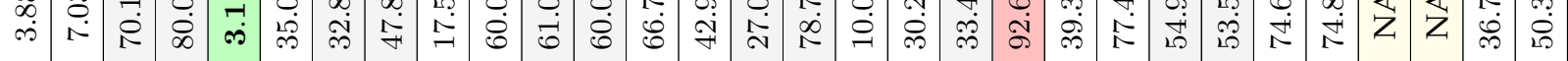

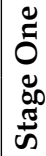
๘ ๙ ๓ ชิ ลิ 芒过

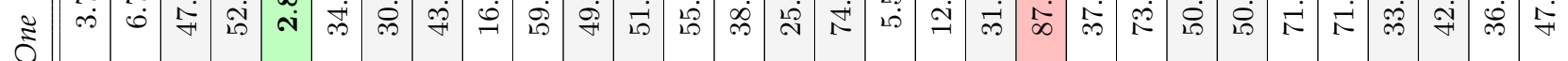

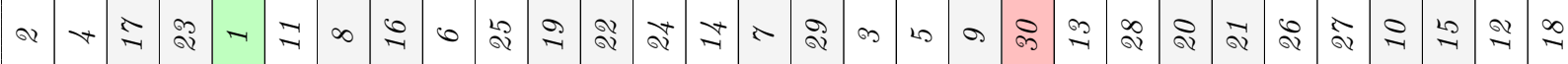

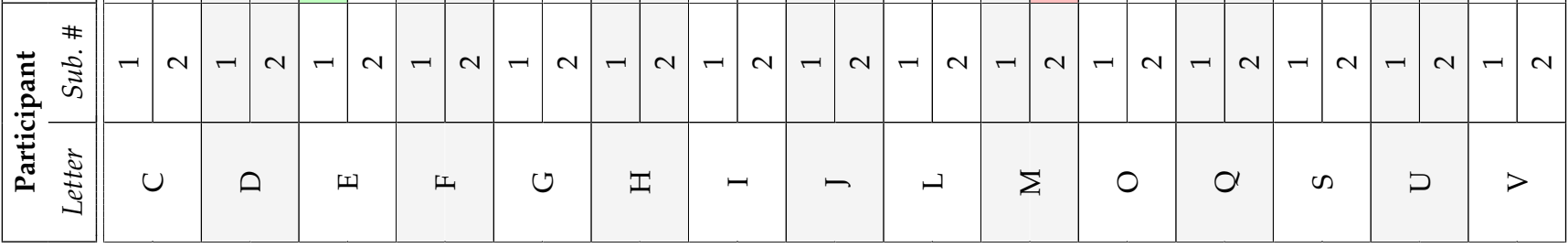




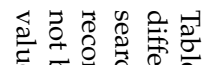

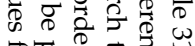

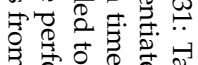

उ象㑒 ?

눙유.

$\checkmark \rightarrow$

궁

을

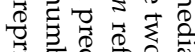

. F.

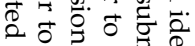

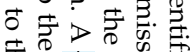

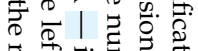

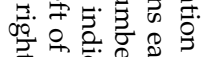

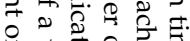

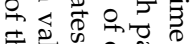

है.

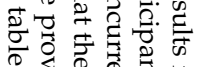

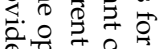

表苛.

क.

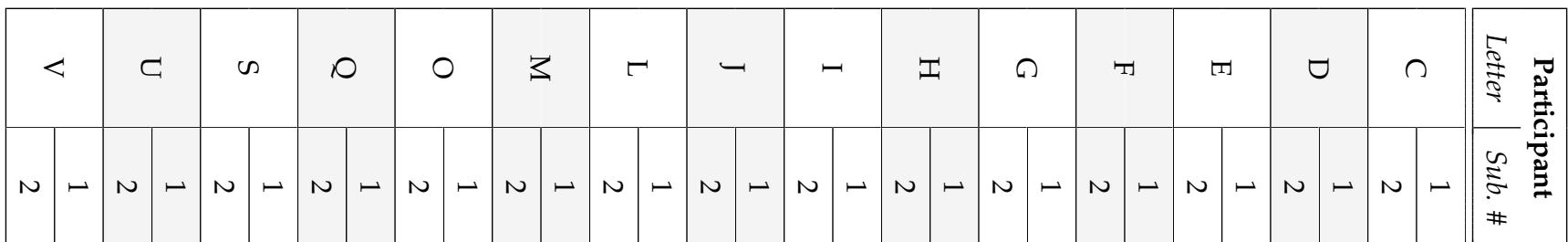

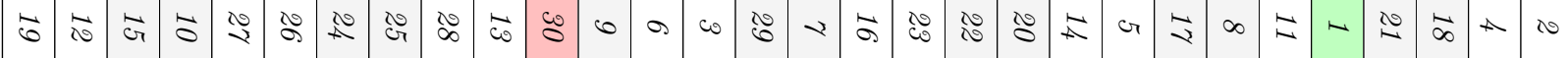

D

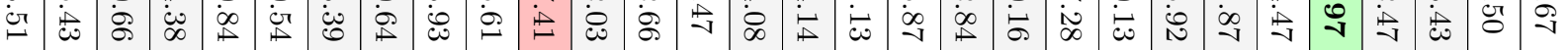

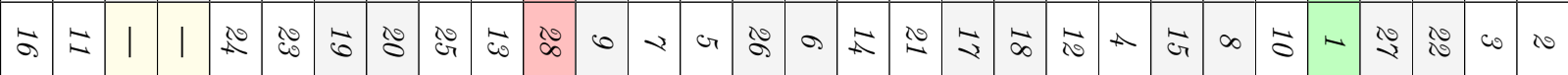

开

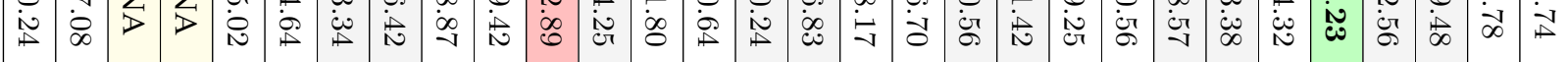

율

s.

\begin{tabular}{|c|c|c|c|c|c|c|c|c|c|c|c|c|c|c|c|c|c|c|c|c|c|c|c|c|c|c|c|c|c|c|}
\hline$\sim$ & $\sim$ & 0 & 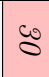 & $\sim$ & $\sim$ & 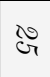 & 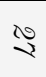 & $\sim$ & $\sim$ & $\sim$ & $\sim$ & $\sim$ & $\sim$ & $\sim$ & $\sim$ & - & $\sim$ & $\sim$ & $\sim$ & $\sim$ & - & - & $\downarrow$ & $\sim$ & $\sim$ & $\infty$ & ఠ) & $\sim$ & $\sim$ & \\
\hline | & 1 & $\begin{array}{l}\bullet \\
\stackrel{0}{0} \\
\dot{\partial}\end{array}$ & 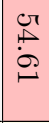 & 1 & $\mid$ & $\begin{array}{l}\dot{c} \\
\dot{c} \\
\stackrel{\perp}{n}\end{array}$ & $\begin{array}{l}\dot{\omega} \\
\dot{G}\end{array}$ & I & | & 1 & 1 & | & | & | & 1 & 1 & 1 & | & | & | & | & 1 & | & | & | & $\stackrel{\stackrel{\bullet}{ \pm}}{\stackrel{*}{*}}$ & $\begin{array}{l}\dot{c} \\
\dot{\tilde{g}}\end{array}$ & | & 1 & $\widetilde{0}$ \\
\hline$\sim$ & - & 1 & 1 & $\sim$ & $\sim$ & $\infty$ & $\stackrel{c}{c}$ & $\sim$ & $\sim$ & - & - & $\sim$ & - & $\sim$ & - & $\sim$ & $\sim$ & $\sim$ & - & $\sim$ & $\sim$ & $\sim$ & $\sim$ & - & $\sim$ & $\infty$ & $\underset{\sim}{\mathbb{V}}$ & $\checkmark$ & $\sim$ & 0 \\
\hline | & 1 & $\underset{>}{Z}$ & $\underset{>}{Z}$ & 1 & $\mid$ & $\begin{array}{l}\dot{\omega} \\
\dot{\dot{\theta}}\end{array}$ & 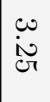 & I & | & I & 1 & | & | & | & 1 & I & | & | & | & | & I & 1 & 1 & I & 1 & $\begin{array}{l}\infty \\
\dot{\vec{D}} \\
\vec{\perp}\end{array}$ & $\begin{array}{l}c \pi \\
\ddot{0}\end{array}$ & I & 1 & \\
\hline
\end{tabular}

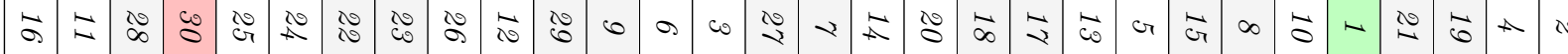

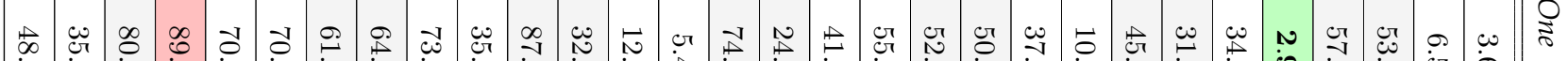

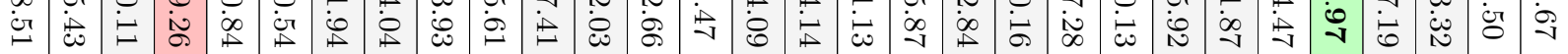

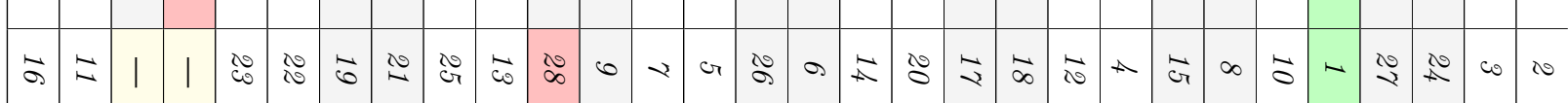

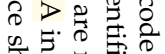

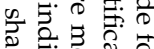

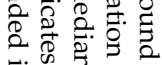

$5 \cdot$

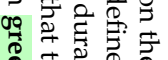

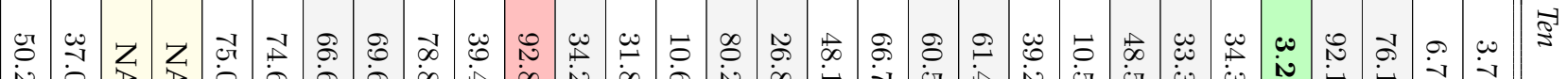

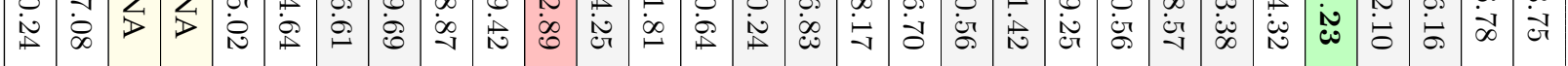

年

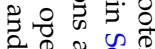

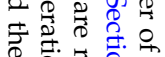

席要

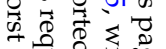

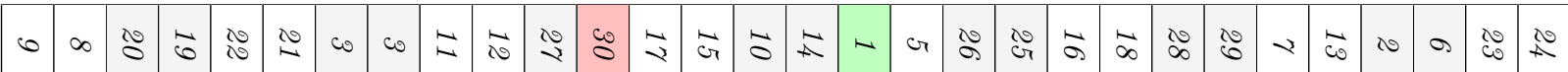

施.

강.

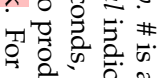

范

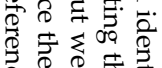

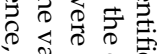

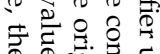




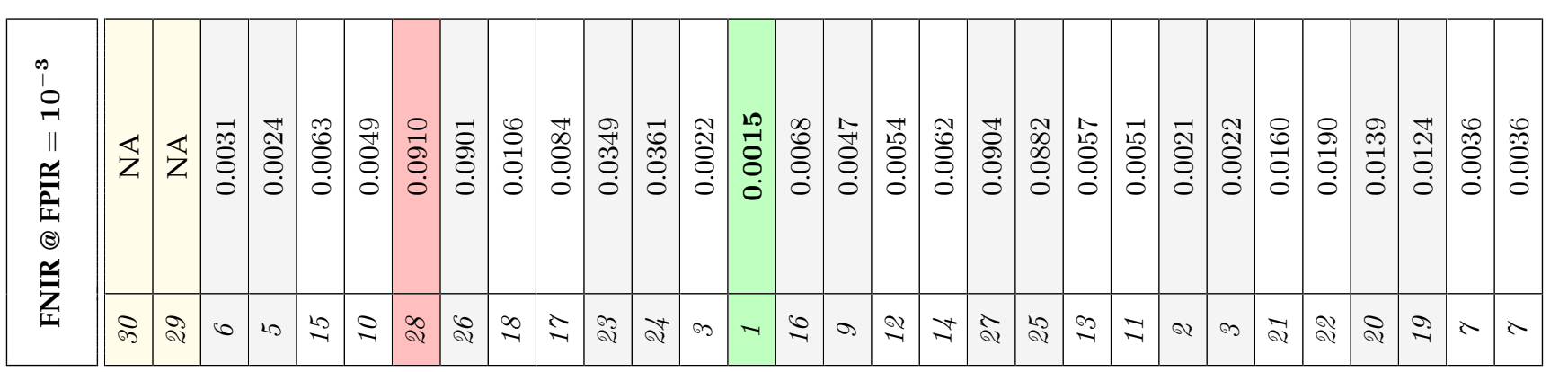

\begin{tabular}{|c|c|c|c|c|c|c|c|c|c|c|c|c|c|c|c|c|c|c|c|c|c|c|c|c|c|c|c|c|c|c|}
\hline \multirow{4}{*}{ 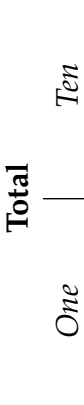 } & $\begin{array}{l}\mathscr{O} \\
0 \\
0\end{array}$ & $\stackrel{ }{\stackrel{ }{二}}$ & $\begin{array}{l}\overrightarrow{0} \\
\dot{0} \\
0 \\
0\end{array}$ & $\begin{array}{l}\stackrel{2}{\rho} \\
\stackrel{-}{=}\end{array}$ & $\stackrel{8}{\circ}$ & 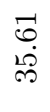 & 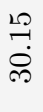 & $\begin{array}{l}m \\
\stackrel{\leftrightarrow}{+} \\
\stackrel{+}{H}\end{array}$ & $\stackrel{\sim}{\stackrel{-}{*}}$ & $\begin{array}{l}\stackrel{\odot}{\circ} \\
\dot{\infty}\end{array}$ & $\begin{array}{l}\infty \\
\overrightarrow{0} \\
\infty \\
\infty\end{array}$ & $\begin{array}{c}0 \\
-1 \\
\infty \\
\infty\end{array}$ & $\begin{array}{l}\infty \\
\stackrel{\infty}{\rightarrow} \\
\stackrel{\leftrightarrow}{*}\end{array}$ & 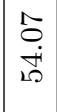 & 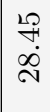 & $\begin{array}{l}\underset{F}{N} \\
\underset{I}{*}\end{array}$ & 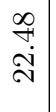 & $\begin{array}{l}\tilde{\infty} \\
\dot{\theta} \\
\dot{0}\end{array}$ & $\begin{array}{l}\overrightarrow{0} \\
\dot{\infty} \\
\dot{\infty}\end{array}$ & $\begin{array}{l}\stackrel{1}{0} \\
\dot{10}\end{array}$ & $\begin{array}{l}\vec{b} \\
\vec{\forall} \\
\vec{F}\end{array}$ & $\begin{array}{l}\stackrel{-}{0} \\
\stackrel{p}{p}\end{array}$ & $\begin{array}{l}\stackrel{R}{R} \\
\stackrel{R}{R}\end{array}$ & $\begin{array}{l}10 \\
10 \\
0 \\
10\end{array}$ & 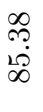 & $\begin{array}{l}7 \\
2 \\
\infty \\
\infty\end{array}$ & $\overleftrightarrow{Z}$ & $\overleftrightarrow{Z}$ & $\begin{array}{l}\stackrel{g}{0} \\
\dot{\forall}\end{array}$ & $\begin{array}{l}\text { จे } \\
\text { in } \\
\text { LD }\end{array}$ \\
\hline & $\infty$ & 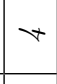 & $\hat{\otimes}$ & $\begin{array}{l}\infty \\
\otimes\end{array}$ & $\checkmark$ & $\approx$ & $\infty$ & $\exists$ & -1 & $\theta$ & $\stackrel{0}{\circ}$ & ๙े & \pm & $\stackrel{0}{1}$ & 0 & ๙ै & 20 & $\stackrel{-1}{-1}$ & $\lambda$ & $\stackrel{20}{-1}$ & $\rightarrow$ & $\vec{Q}$ & จे & $\stackrel{\infty}{\sim}$ & $\stackrel{2}{Q}$ & $\begin{array}{l}\stackrel{Q}{Q} \\
\otimes\end{array}$ & 1 & 1 & $\stackrel{\leftrightarrow}{\sim}$ & $\lesssim$ \\
\hline & $\begin{array}{l}\stackrel{9}{4} \\
0\end{array}$ & $\begin{array}{l}? \\
? \\
0 \\
0\end{array}$ & 竎 & $\begin{array}{l}28 \\
\dot{0} \\
80\end{array}$ & $\stackrel{\mathcal{T}}{6}$ & $\begin{array}{l}\stackrel{8}{\circ} \\
\stackrel{\leftrightarrow}{\circ}\end{array}$ & $\begin{array}{l}\stackrel{N}{N} \\
\stackrel{N}{N}\end{array}$ & 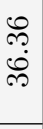 & $\begin{array}{l}\infty \\
\infty \\
\infty \\
\dot{\infty}\end{array}$ & 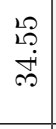 & 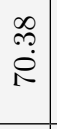 & 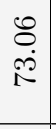 & 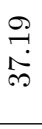 & 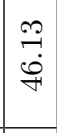 & $\begin{array}{l}10 \\
\rightarrow \\
\stackrel{1}{*}\end{array}$ & $\begin{array}{l}\infty \\
\stackrel{\infty}{1} \\
\stackrel{0}{0}\end{array}$ & 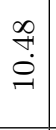 & 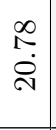 & 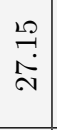 & $\begin{array}{l}\vec{\sigma} \\
\text { is }\end{array}$ & $\begin{array}{ll}\vec{m} \\
\infty \\
\infty \\
\infty \\
\infty\end{array}$ & $\begin{array}{l}0 \\
\mathscr{1} \\
0 \\
0\end{array}$ & $\begin{array}{l}\hat{D} \\
\dot{d} \\
\dot{\delta}\end{array}$ & $\begin{array}{l}\stackrel{?}{1} \\
\text { : } \\
\text { in }\end{array}$ & $\begin{array}{l}\mathscr{m} \\
\ddot{\infty} \\
\ddot{\infty}\end{array}$ & $\mid \begin{array}{c}m \\
\stackrel{\vec{i}}{\sim} \\
\infty\end{array}$ & $\begin{array}{c}\mathcal{O} \\
\dot{0} \\
\infty \\
\infty\end{array}$ & $\begin{array}{c}\stackrel{P}{1} \\
\text { i } \\
\infty\end{array}$ & $\begin{array}{l}\infty \\
0 \\
\infty \\
\infty \\
\infty\end{array}$ & $\begin{array}{l}\infty \\
\infty \\
\text { ì } \\
\text { Dे }\end{array}$ \\
\hline & の & 20 & 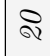 & $\vec{\otimes}$ & 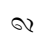 & $\stackrel{2}{\sim}$ & $\sigma$ & $\stackrel{\text { }}{\sim}$ & -1 & 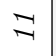 & $\stackrel{20}{\leftrightarrow}$ & $\stackrel{\text { ஜे }}{ }$ & $\stackrel{2}{-2}$ & $\equiv$ & $\lambda$ & ৯े & $*$ & 0 & $\infty$ & $\stackrel{0}{-1}$ & \pm & ๑2 & ๙ै & $\stackrel{2}{\sim}$ & \&े & ลे & ๓े & $\begin{array}{l}\infty \\
\stackrel{\otimes}{*}\end{array}$ & $\stackrel{20}{\longrightarrow}$ & $\stackrel{\infty}{-1}$ \\
\hline
\end{tabular}

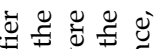

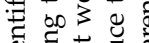

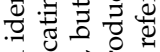

a :

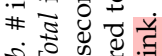

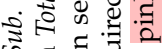

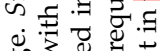

足们

急

पू

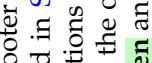

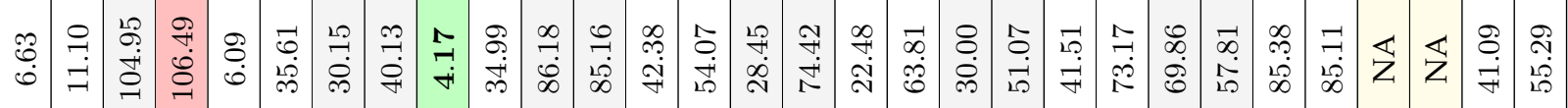

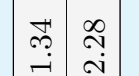

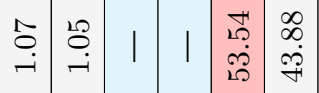

- -1 ลิ

$\stackrel{2}{\circ}$

\section{\begin{tabular}{|l|}
-1 \\
\hline \\
$\infty$ \\
$\infty$ \\
$\infty$ \\
$\infty$ \\
$\infty$ \\
$\infty$ \\
$\infty$
\end{tabular}}

œ

की

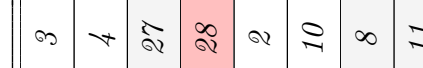

$-\infty$

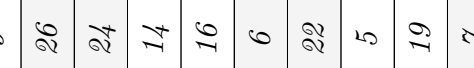

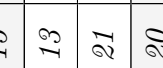

$\infty$

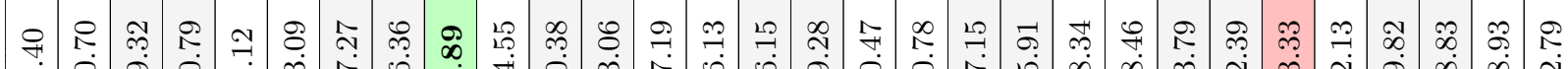

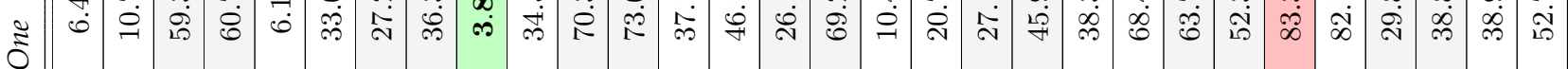

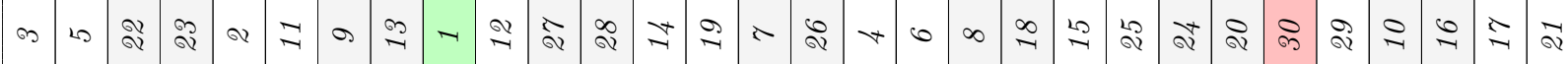

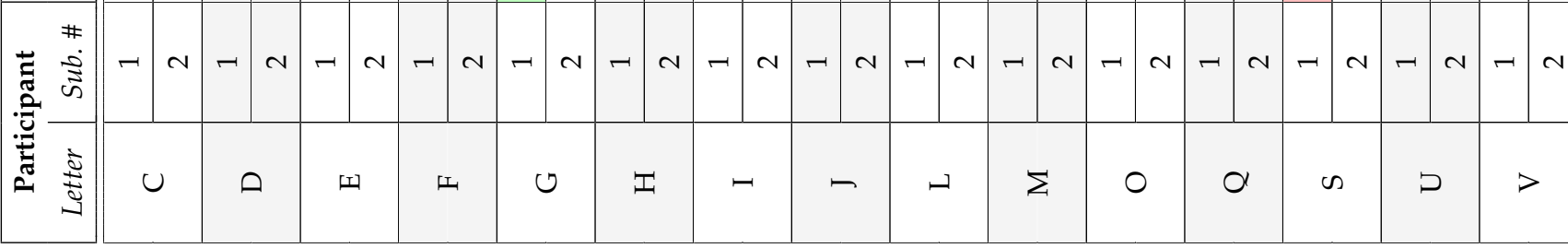

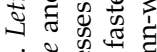

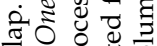
के

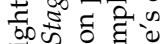

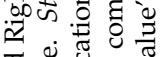
药 击 $\frac{\pi}{3} \cdot \frac{0}{3} \frac{0}{0}$ 


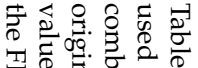

至

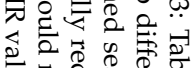

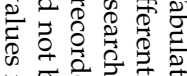

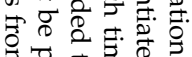

3 它

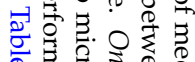

to

.

की

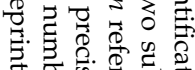

象象

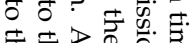

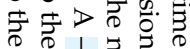

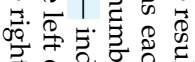

定家 융

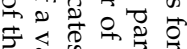

志.

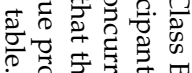

\begin{tabular}{|c|c|c|c|c|c|c|c|c|c|c|c|c|c|c|c|c|c|c|c|c|c|c|c|c|c|c|c|c|c|}
\hline & $\xi$ & $c$ & & & is & 10 & & $c$ & & 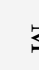 & & 5 & & $=$ & & - & & $I$ & & $\Omega$ & & T & $t$ & $\mathrm{~T}$ & $c$ & & 0 & & $\frac{5}{\frac{5}{9}}$ \\
\hline$\sim$ & $\bullet$ & $N$ & 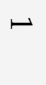 & $N$ & 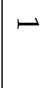 & $N$ & 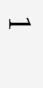 & $N$ & $\bullet$ & $N$ & $\leftarrow$ & $N$ & $\bullet$ & $N$ & $\bullet$ & $N$ & $\bullet$ & $N$ & - & N & $\vec{N}$ & 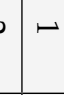 & N & $\bullet$ & $N$ & $\bullet$ & $N$ & 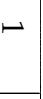 & 5 \\
\hline
\end{tabular}

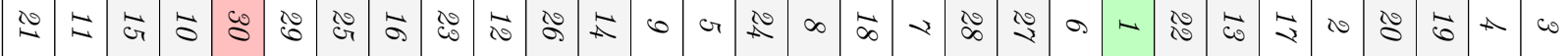

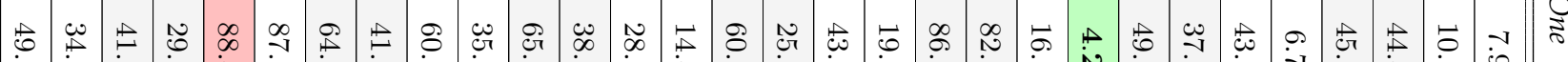

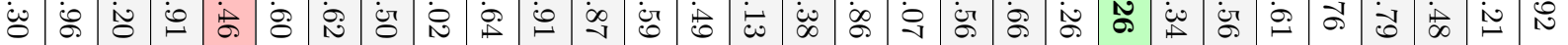

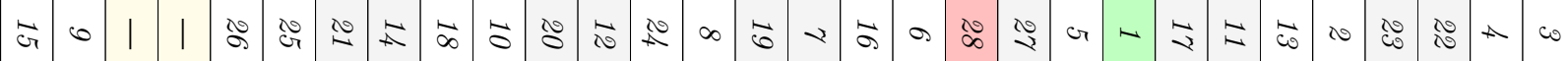

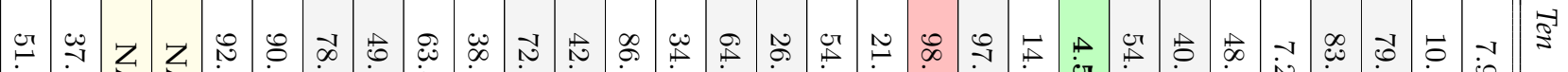

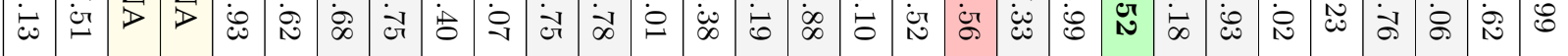

ㄴ.

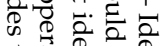

\begin{tabular}{|c|c|c|c|c|c|c|c|c|c|c|c|c|c|c|c|c|c|c|c|c|c|c|c|c|c|c|c|c|c|}
\hline$\sim$ & 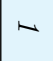 & : & $\approx$ & 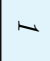 & $\sim$ & $\stackrel{心}{\mathbb{N}}$ & $\infty$ & $\sim$ & $\sim$ & $\sim$ & $\sim$ & $\sim$ & $\sim$ & $\sim$ & 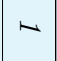 & - & $\sim$ & $\sim$ & $\omega$ & $\sim$ & $\sim$ & -1 & -1 & -1 & -1 & 8 & o- & $\sim$ & \\
\hline | & 1 & 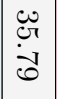 & $\begin{array}{l}c \\
\tilde{y} \\
\dot{8}\end{array}$ & 1 & 1 & $\overrightarrow{\dot{\infty}}$ & $\vec{\infty}$ & 1 & 1 & 1 & 1 & | & 1 & | & 1 & 1 & $\mid$ & | & | & | & | & | & | & | & & & \begin{tabular}{l|l}
0 \\
0 \\
0 & 1
\end{tabular} & 1 & \\
\hline- & - & 1 & 1 & $\sim$ & - & ๙ & cir & 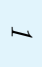 & - & -1 & - & $\sim$ & $\sim$ & $\sim$ & - & - & - & - & $\sim$ & $\sim$ & - & 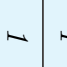 & -1 & -1 & $-1 \lesssim$ & $\infty$ & $\begin{array}{lll}0 & -1\end{array}$ & - & \\
\hline | & 1 & $\underset{>}{Z}$ & Z & 1 & 1 & 卢 & $\dot{8}$ & | & 1 & 1 & I & $\mid$ & 1 & | & 1 & 1 & 1 & I & | & | & I & | & | & | & & \begin{tabular}{c|c}
$\dot{u}$ \\
$\dot{\nu}$
\end{tabular} & $\begin{array}{ll}\overrightarrow{0} & \mid\end{array}$ & | & \\
\hline$\vec{\sigma}$ & $\vec{a}$ & $心$ & $\infty$ & $\theta$ & ৫ి & న & A & 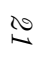 & $\Xi$ & $\aleph_{0}$ & $\overrightarrow{\omega_{0}}$ & 0 & $G$ & $\stackrel{心}{心}$ & $\infty$ & $\vec{a}$ & $\checkmark$ & 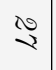 & $\infty$ & $a$ & $\sim$ & 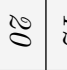 & ๗) & $\vec{G}$ & $\bowtie$ & $=$ & ऐ। & . & \\
\hline $\begin{array}{l}\vec{b} \\
\dot{c}\end{array}$ & 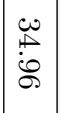 & $\begin{array}{l}\vec{y} \\
\dot{\omega} \\
\infty \\
\infty\end{array}$ & $\mid \begin{array}{l}\infty \\
0 \\
\dot{0} \\
\dot{0}\end{array}$ & $\begin{array}{l}\infty \\
\infty \\
\stackrel{0}{0} \\
\text { Aे }\end{array}$ & $\mid \begin{array}{c}\infty \\
\stackrel{-}{1} \\
\dot{\theta}\end{array}$ & $\begin{array}{l}\mathscr{O} \\
\dot{\ominus} \\
\dot{\ominus}\end{array}$ & $\begin{array}{l}\text { N } \\
0 \\
0 \\
N\end{array}$ & 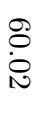 & 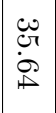 & $\begin{array}{c}0 \\
\dot{i} \\
\dot{0}\end{array}$ & $\begin{array}{l}\dot{c} \\
\infty \\
\infty \\
0 \\
v\end{array}$ & $\begin{array}{l}0 \\
\infty \\
i j \\
0 \\
0\end{array}$ & 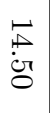 & $\begin{array}{l}\stackrel{g}{0} \\
\dot{\vec{\omega}}\end{array}$ & $\mid \begin{array}{c}0 \\
\dot{c} \\
\dot{\omega} \\
\infty \\
\infty\end{array}$ & \begin{tabular}{|l|l}
$\vec{c}$ \\
$\dot{0}$ \\
$\dot{g}$ \\
$o$
\end{tabular} & $\begin{array}{l}\overrightarrow{0} \\
\dot{0} \\
- \\
-1\end{array}$ & $\begin{array}{l}\infty \\
\infty \\
\dot{c} \\
\dot{c} \\
\sigma\end{array}$ & 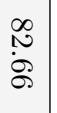 & & $\begin{array}{l}\dot{N} \\
\dot{N}\end{array}$ & $\begin{array}{l}\vec{b} \\
\dot{\omega} \\
\dot{c}\end{array}$ & 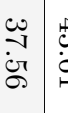 & 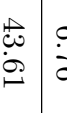 & & & 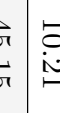 & $\mid \begin{array}{l}-1 \\
0 \\
1\end{array}$ & \\
\hline
\end{tabular}

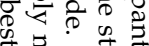

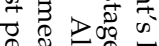

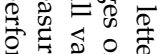

$\vec{G} 0||$ |

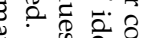

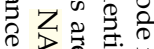

罂:

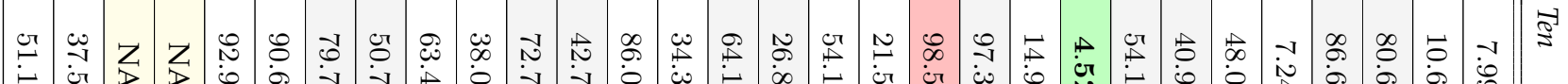

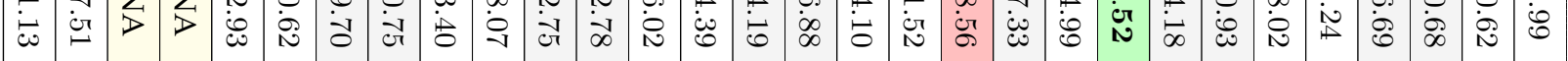

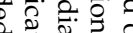

.

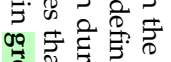

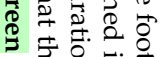

\% हैं 5 .

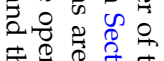

ث융

$\sum \neq$.

का का

․ㅠ

政.

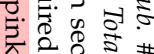

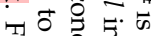

我记

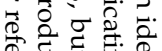

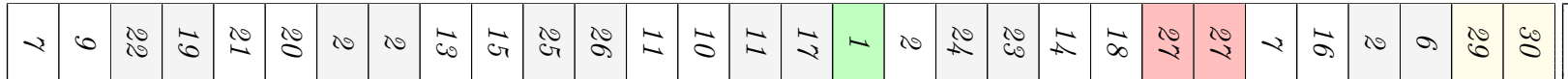

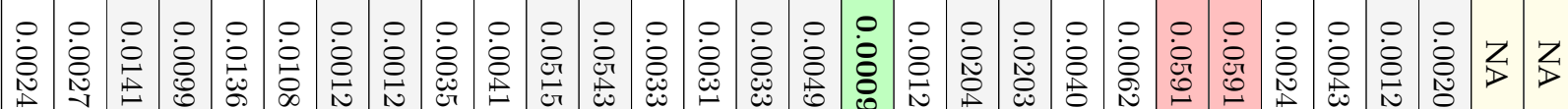


5 .

.

:

के क्षे

ن류

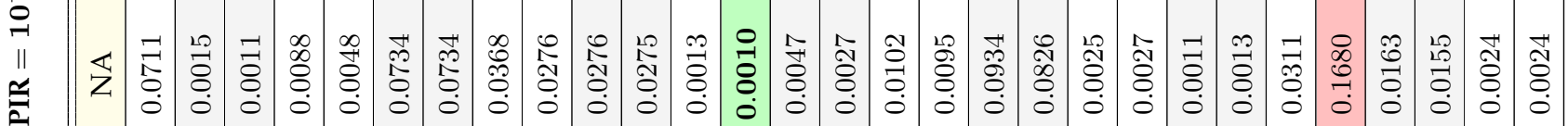

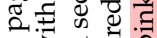

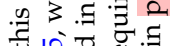

पै

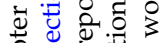

$\circ$ 品

艺

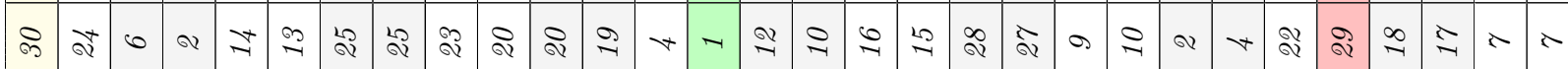

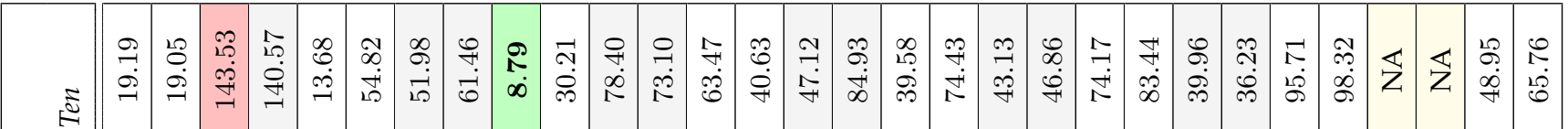

సี

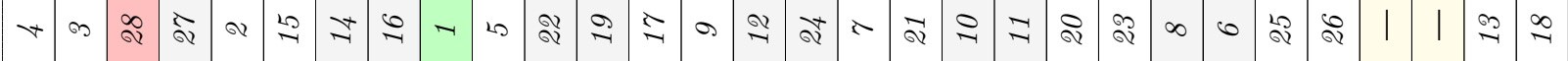

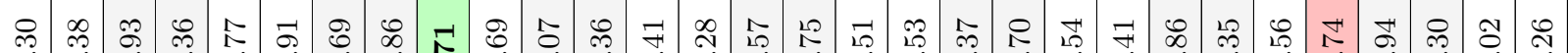

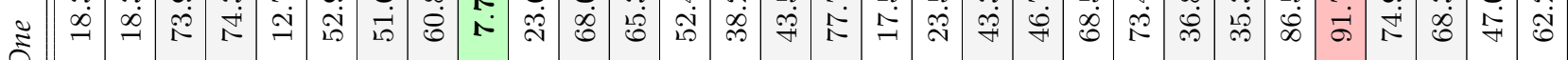

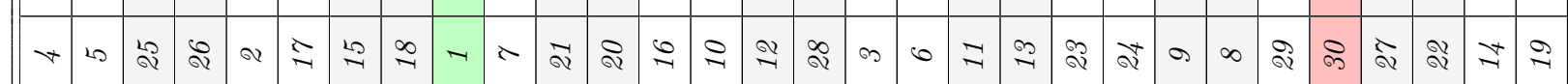

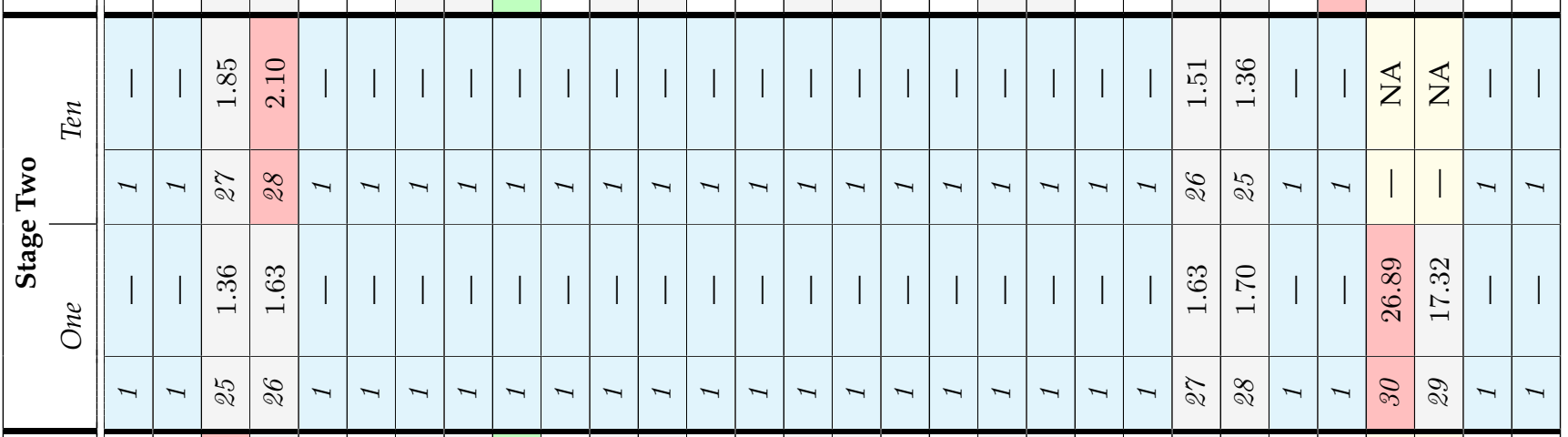

๑

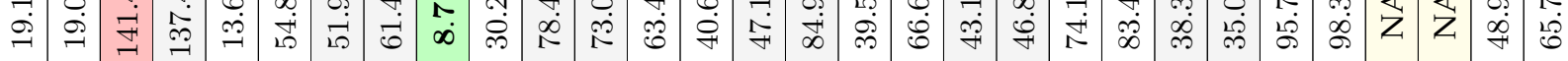

o

$* \infty \infty$

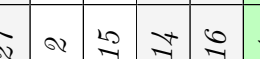

๙ จ ミ

$\stackrel{\infty}{\rightarrow-\infty}$

离

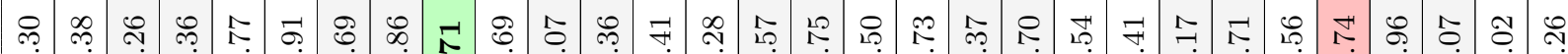

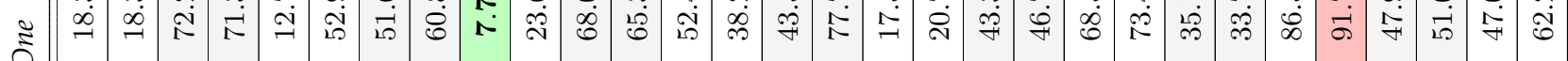

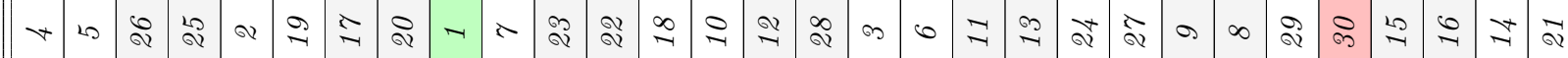

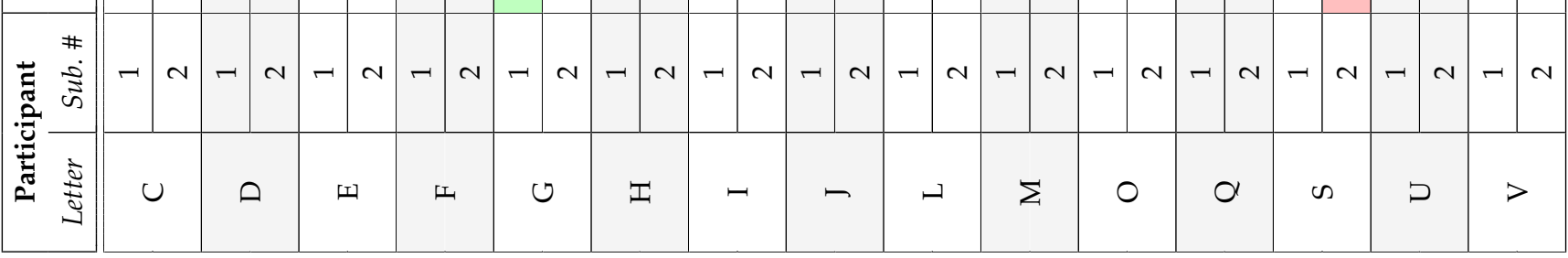




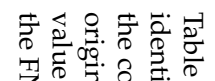

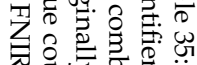

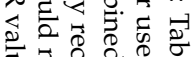
कृ

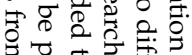
3 잉

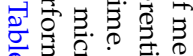

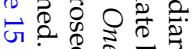

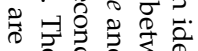

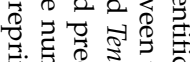

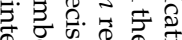
$2 \stackrel{4}{9} \stackrel{9}{9}$ 아의 0 o

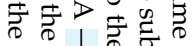
둥. 뭉.

\begin{tabular}{|c|c|c|c|c|c|c|c|c|c|c|c|c|c|c|c|c|c|c|c|c|c|c|c|c|c|c|c|c|c|c|}
\hline$<$ & 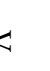 & $c$ & & & s & 10 & & & D & & & $r$ & & - & & r & & 7 & & $a$ & & $\mathrm{~T}$ & & $\mathrm{~T}$ & & C & & & ) & $\frac{5}{\frac{5}{5}}$ \\
\hline N & $\bullet$ & $N$ & $\bullet$ & $N$ & 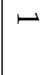 & $N$ & $\bullet$ & $N$ & 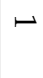 & $N$ & 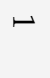 & $N$ & $\bullet$ & $N$ & $\bullet$ & $N$ & $\bullet$ & $N$ & $\bullet$ & $N$ & 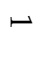 & $N$ & - & $N$ & 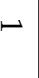 & $N$ & - & $N$ & 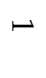 & \\
\hline
\end{tabular}

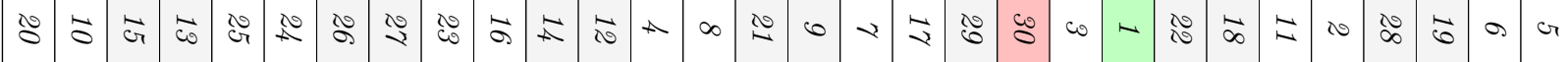

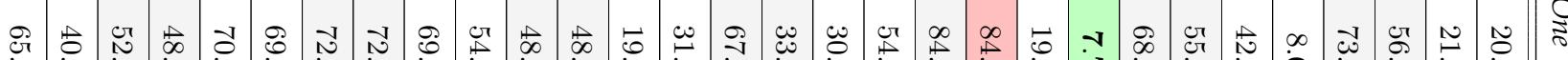

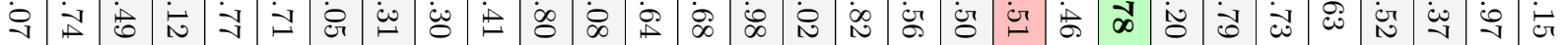

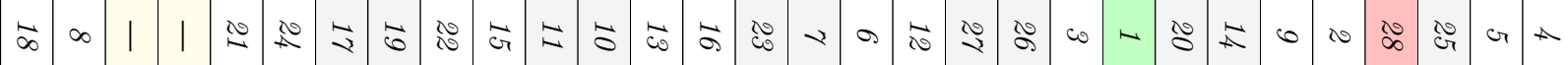

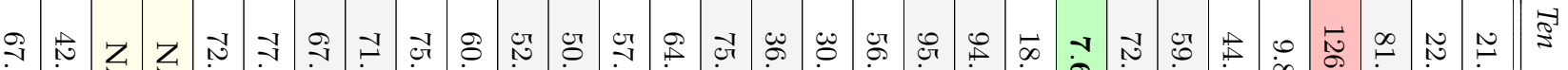

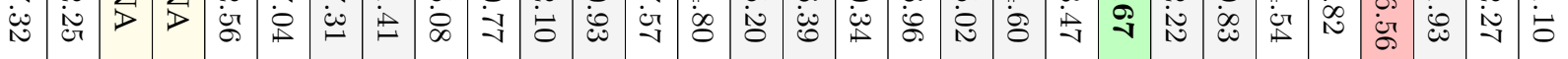

\begin{tabular}{|c|c|c|c|c|c|c|c|c|c|c|c|c|c|c|c|c|c|c|c|c|c|c|c|c|c|c|c|c|c|c|}
\hline- & $\sim$ & $\mathbb{0}$ & 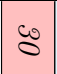 & $\sim$ & $\sim$ & $\stackrel{N}{\checkmark}$ & $\infty$ & $\sim$ & 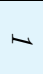 & $\sim$ & $\sim$ & $\sim$ & - & $\sim$ & 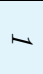 & - & $\sim$ & $\sim$ & $\sim$ & $\sim$ & $\sim$ & $\sim$ & $\sim$ & - & $\sim$ & $\mathbb{G}$ & $\infty$ & $\sim$ & $\downarrow$ & \\
\hline | & $\mid$ & 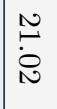 & 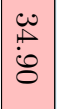 & 1 & 1 & $\begin{array}{l}\stackrel{N}{N} \\
\stackrel{v}{v}\end{array}$ & $\begin{array}{l}N \\
\text { N } \\
\text { N }\end{array}$ & 1 & | & 1 & 1 & 1 & 1 & 1 & | & 1 & 1 & 1 & | & 1 & | & | & 1 & | & | & $\stackrel{\bullet}{\infty}$ & $\begin{array}{l}\stackrel{N}{N} \\
\text { N }\end{array}$ & I & | & $\mathscr{2}$ \\
\hline- & $\downarrow$ & 1 & 1 & - & $\sim$ & $\infty$ & $\underset{\sim}{*}$ & $\sim$ & $\sim$ & $\sim$ & $\sim$ & $\sim$ & $\omega$ & $\sim$ & $\sim$ & - & $\sim$ & $\sim$ & $\sim$ & $\sim$ & $\sim$ & $\sim$ & $\sim$ & - & $\sim$ & 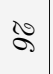 & 心 & - & . & \\
\hline $\mid$ & $\mid$ & Z & $\underset{>}{Z}$ & I & 1 & $\begin{array}{l}\sim \\
\stackrel{v}{c}\end{array}$ & $\begin{array}{l}N \\
\dot{0} \\
0\end{array}$ & 1 & | & 1 & 1 & | & I & | & | & 1 & 1 & I & I & | & | & | & $\mid$ & | & | & $\stackrel{\vec{c}}{\overrightarrow{0}}$ & $\stackrel{\vec{\Delta}}{\vec{\theta}}$ & 1 & | & \\
\hline$\omega_{\infty}$ & $\vec{a}$ & 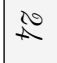 & $\infty$ & $\infty$ & 心্心ে & $\stackrel{心}{心}$ & $\approx$ & 心 & 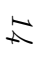 & $\vec{\omega}$ & $\vec{\nabla}$ & $G$ & $\infty$ & $\vec{\theta}$ & 0 & $\checkmark$ & $\vec{c}$ & $\mathbb{0}$ & 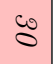 & $c_{0}$ & - & 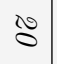 & $\vec{a}$ & $\approx$ & $\infty$ & 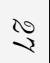 & $\vec{v}$ & $\sigma$ & $A$ & \\
\hline $\begin{array}{c}\stackrel{g}{i} \\
\stackrel{i}{i}\end{array}$ & $\begin{array}{l}\stackrel{B}{O} \\
\stackrel{-}{ \pm}\end{array}$ & $\begin{array}{l}-1 \\
N \\
\dot{D} \\
\infty \\
\infty\end{array}$ & $\begin{array}{l}\infty \\
1 \\
0 \\
0\end{array}$ & $\begin{array}{l}\stackrel{c}{0} \\
\stackrel{-}{-}\end{array}$ & $\mid \begin{array}{l}\dot{\theta} \\
\dot{v} \\
\vec{v}\end{array}$ & $\stackrel{+N}{\stackrel{N}{N}}$ & 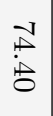 & $\begin{array}{l}\text { சે } \\
\dot{0}\end{array}$ & $\begin{array}{l}\stackrel{c}{c} \\
\stackrel{N}{N}\end{array}$ & $\begin{array}{l}\vec{\infty} \\
\infty \\
\dot{0}\end{array}$ & $\mid \begin{array}{l}\vec{\infty} \\
\dot{\infty} \\
\dot{\infty}\end{array}$ & 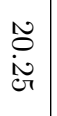 & $\begin{array}{l}\stackrel{\omega}{\bullet} \\
\dot{\infty}\end{array}$ & $\begin{array}{l}9 \\
-1 \\
0 \\
\infty\end{array}$ & 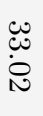 & \begin{tabular}{|l}
$\dot{D}$ \\
$\stackrel{0}{0}$ \\
$\dot{N}$ \\
$N$
\end{tabular} & 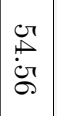 & $\begin{array}{l}\infty \\
\stackrel{\infty}{\dot{c}} \\
\dot{c}\end{array}$ & $\begin{array}{l}\infty \\
\stackrel{\infty}{+} \\
\stackrel{c}{\sim}\end{array}$ & $\begin{array}{l}\vec{b} \\
\dot{\sigma}\end{array}$ & $\begin{array}{l}\vec{y} \\
-1 \\
\infty\end{array}$ & $\begin{array}{l}\infty \\
\dot{0} \\
\dot{\tilde{o}}\end{array}$ & 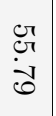 & 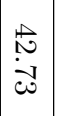 & $\begin{array}{l}\infty \\
\dot{\mathcal{U}}\end{array}$ & $\begin{array}{l}\vec{p} \\
\stackrel{p}{0} \\
\vec{v}\end{array}$ & $\begin{array}{l}\mathbb{C} \\
0 \\
0 \\
0\end{array}$ & 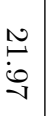 & $\begin{array}{l}\stackrel{N}{\mathscr{C}} \\
\stackrel{\vec{c}}{1}\end{array}$ & \\
\hline 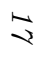 & $\infty$ & I & 1 & ¿ & io & $\vec{\infty}_{0}$ & $心$ & 心్心 & $\AA$ & $\approx$ & $\vec{a}$ & $\vec{a}$ & $\vec{G}$ & ¿্. & $\checkmark$ & 0 & $\vec{\infty}$ & 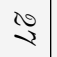 & ๙ూ & $c_{0}$ & $\sim$ & $\overrightarrow{0}$ & $\overrightarrow{\omega_{0}}$ & 0 & $\infty$ & $\infty$ & 心 & $G$ & 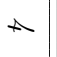 & \\
\hline $\begin{array}{l}9 \\
i \\
i v\end{array}$ & $\begin{array}{l}\text { N } \\
\text { iv } \\
\text { iv }\end{array}$ & Z & $\underset{>}{Z}$ & 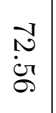 & $\mid \begin{array}{l}1 \\
\dot{1} \\
\dot{1}\end{array}$ & 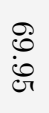 & $\begin{array}{l}-\vec{u} \\
\dot{\omega} \\
\dot{\omega} \\
\omega\end{array}$ & गै & $\stackrel{8}{\partial}$ & $\begin{array}{l}\stackrel{f}{N} \\
\stackrel{0}{0}\end{array}$ & $\left|\begin{array}{l}0 \\
0 \\
0 \\
0 \\
\text { co }\end{array}\right|$ & $\begin{array}{l}\mathscr{D} \\
\dot{\dot{d}} \\
\dot{\mathscr{c}}\end{array}$ & $\stackrel{+}{\infty}$ & चै & $\begin{array}{l}\omega \\
\dot{\omega} \\
\dot{\omega}\end{array}$ & $\begin{array}{l}\dot{\omega} \\
\dot{\omega} \\
\dot{\omega} \\
ث\end{array}$ & $\mid \begin{array}{l}\tilde{E} \\
\mathscr{\mathscr { H }} \\
\mathscr{O}\end{array}$ & 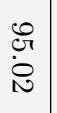 & $\begin{array}{l}\stackrel{\varphi}{+} \\
\dot{8}\end{array}$ & $\begin{array}{l}\vec{\infty} \\
\dot{\vec{v}}\end{array}$ & & $\begin{array}{l}\text { N } \\
\stackrel{N}{N} \\
\text { n. }\end{array}$ & $\mid \begin{array}{c}\tilde{c} \\
0 \\
\infty \\
\omega\end{array}$ & 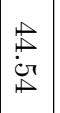 & $\begin{array}{l}0 \\
\infty \\
1\end{array}$ & & & 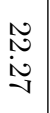 & $\stackrel{\stackrel{N}{\rightleftarrows}}{\sigma}$ & \\
\hline
\end{tabular}

更.

贾

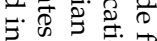

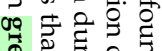

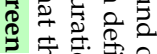
\% है요

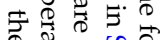

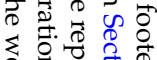

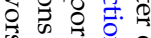
. E. 을. ㄹ. ㄹ. 둥

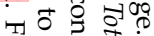
둥 क्ष 2 i 중

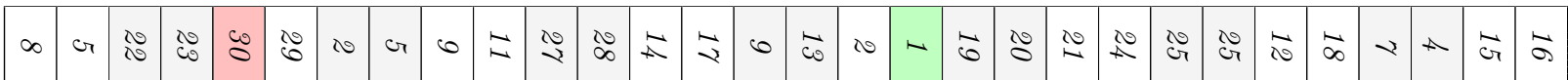

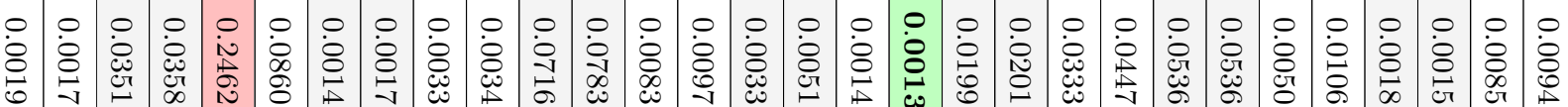




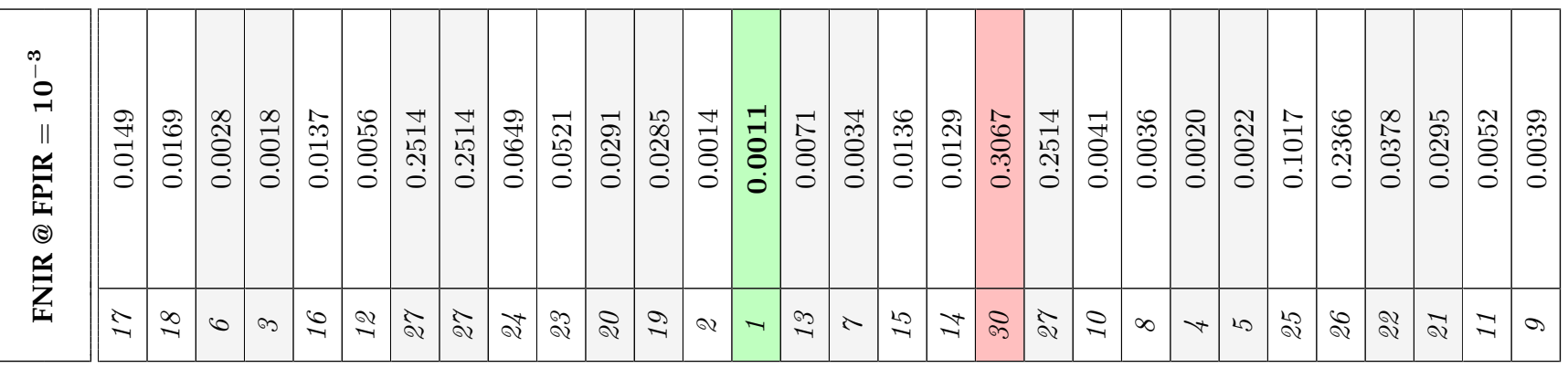

ฮ

曲 3 है

๑

के है

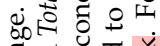

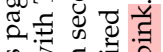

足 3

पै

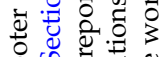

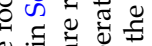

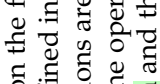

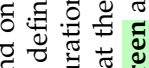

उٓ

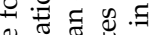

: ‡

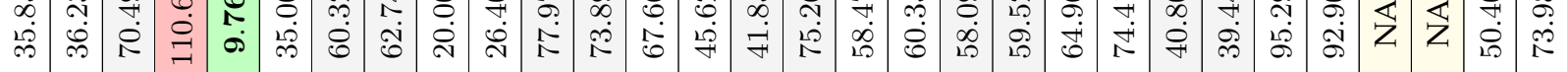

సٓำ

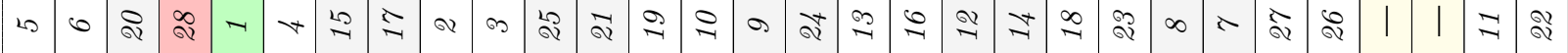

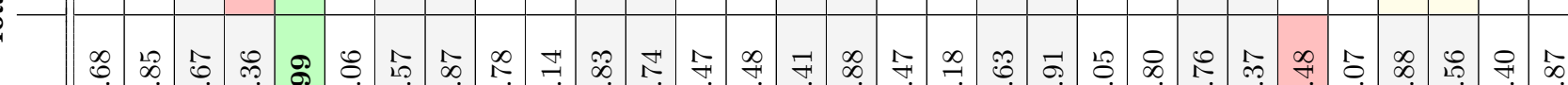

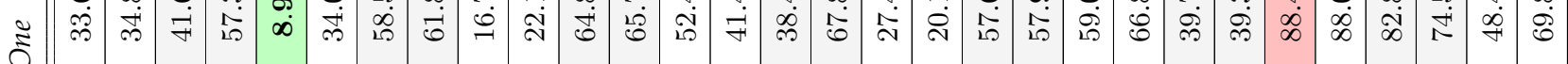
○ $\infty$ ๑

\begin{tabular}{|c|c|c|c|c|c|c|c|c|c|c|c|c|c|c|c|c|c|c|c|c|c|c|c|c|c|c|c|c|c|c|}
\hline \multirow{4}{*}{ 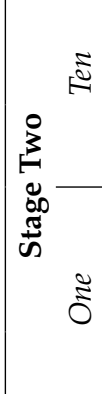 } & 1 & 1 & 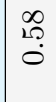 & $\stackrel{0}{\stackrel{0}{-}}$ & 1 & | & 1 & 1 & I & | & 1 & 1 & I & | & I & 1 & 1 & 1 & 1 & 1 & I & 1 & $\begin{array}{l}R \\
\stackrel{R}{-}\end{array}$ & $\begin{array}{l}0 \\
\stackrel{\oplus}{N} \\
i\end{array}$ & I & 1 & $\overleftrightarrow{Z}$ & $\overleftrightarrow{Z}$ & 1 & \\
\hline & -1 & -1 & $\stackrel{2}{Q}$ & $\stackrel{2}{\otimes}$ & -1 & -1 & -1 & -1 & -1 & -1 & -1 & -1 & - & -1 & -1 & -1 & -1 & -1 & -1 & -1 & -1 & -1 & ลิ & 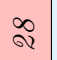 & - & -1 & | & I & -1 & - \\
\hline & | & 1 & $\begin{array}{l}8 \\
\stackrel{0}{0}\end{array}$ & $\underset{\sim}{\stackrel{H}{O}}$ & 1 & I & 1 & 1 & 1 & 1 & 1 & 1 & I & I & I & 1 & 1 & I & 1 & 1 & I & 1 & $\overrightarrow{\sigma_{-}}$ & $\underset{\infty}{\stackrel{\infty}{-}}$ & I & | & $\begin{array}{l}0 \\
\rightarrow \\
\infty \\
\infty\end{array}$ & $\begin{array}{l}\stackrel{8}{\circ} \\
\stackrel{\leftrightarrow}{\sim}\end{array}$ & | & \\
\hline & 7 & 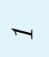 & $\stackrel{2}{Q}$ & $\stackrel{\bullet}{*}$ & -1 & -1 & -1 & -1 & -1 & -1 & -1 & -1 & -1 & -1 & -1 & -1 & -1 & -1 & -1 & -1 & -1 & -1 & $\infty$ & $\hat{\otimes}$ & -1 & -1 & ळे & Qे & -1 & - \\
\hline
\end{tabular}

L ह

它

o

م

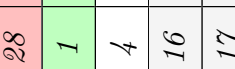

จ

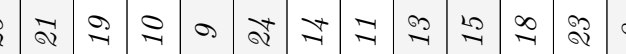

का

崔 ई

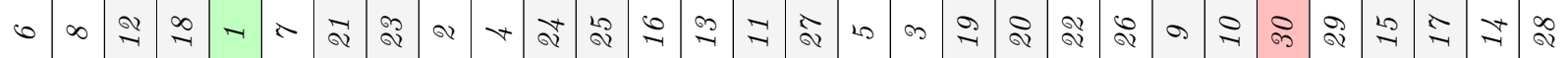

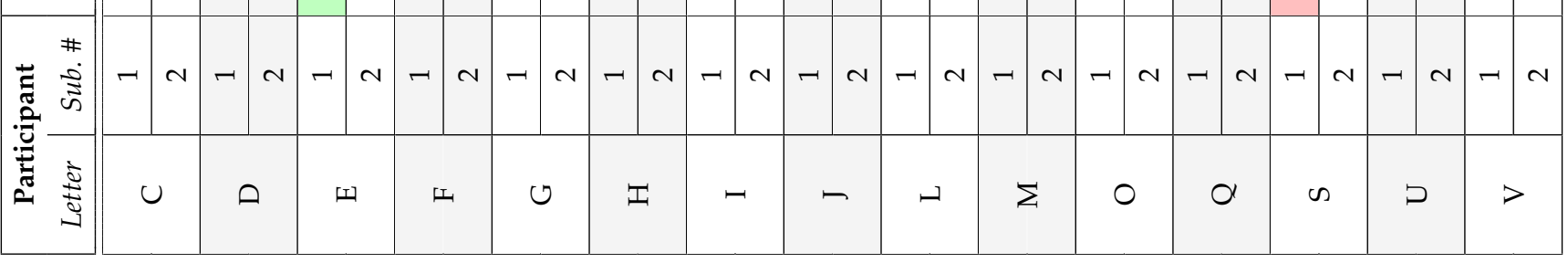

8 :

过

过语范

表品

원

在艺苋

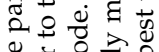

东远:

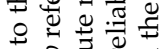

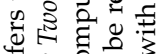

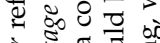

究

ఫृ

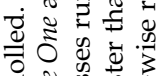

\% \&

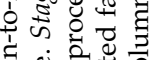

:

중

幽 든

陆

它

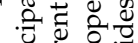

U

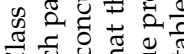

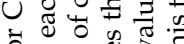

它

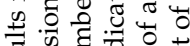

ज.

है है द्व

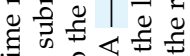

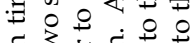

동

苋导

象远造

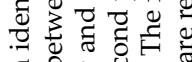

둬워

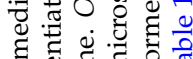

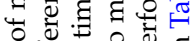

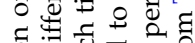

.

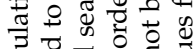

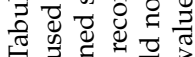

论 


\section{Accuracy Time Tradeoff Detailed Tables with Mean Values}

In order to reduce the number of tables in the main body of the report (Section 8), this appendix contains tables that show the search times for each stage of identification, for both a single process and ten processes.

The tables in this appendix report mean times. For readers interested in median times, please refer to Appendix C.

Class A results are in Tables 37 through 42, Class B results are in Tables 43 through 46, and Class C results are in Tables 47 through 49.

\begin{tabular}{l|l|l|l|ll|l}
$\mathrm{C}=$ afis team & $\mathrm{D}=3 \mathrm{M}$ Cogent & $\mathrm{E}=$ Neurotechnology & $\mathrm{F}=$ Papillon & $\mathrm{G}=$ Dermalog & $\mathrm{H}=$ Hisign Bio-Info Institute \\
$\mathrm{I}=$ NEC & $\mathrm{J}=$ Sonda & $\mathrm{K}=$ Tiger IT & $\mathrm{L}=$ Innovatrics & $\mathrm{M}=$ SPEX & $\mathrm{O}=$ ID Solutions \\
$\mathrm{P}=\mathrm{id} 3$ & $\mathrm{Q}=$ Morpho & $\mathrm{S}=$ Decatur Industries & $\mathrm{T}=$ BIO-key & $\mathrm{U}=$ Aware & $\mathrm{V}=$ AA Technology
\end{tabular}




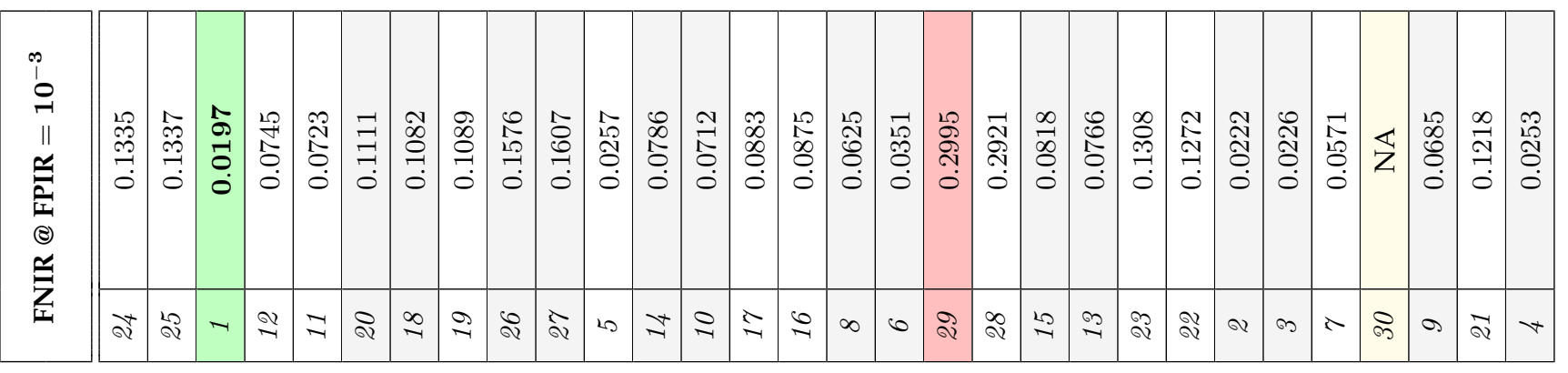

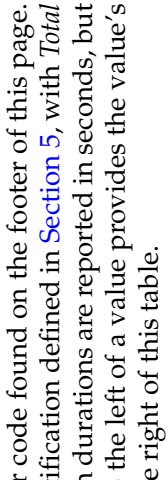

穿

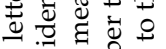

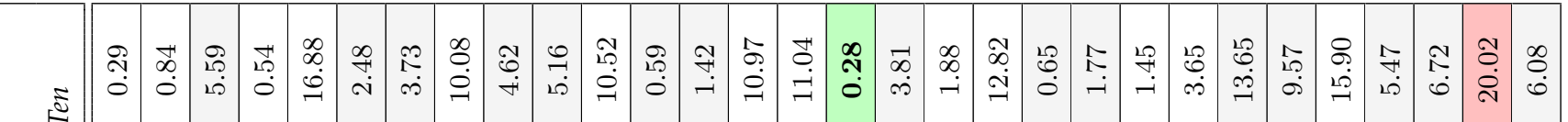

胥

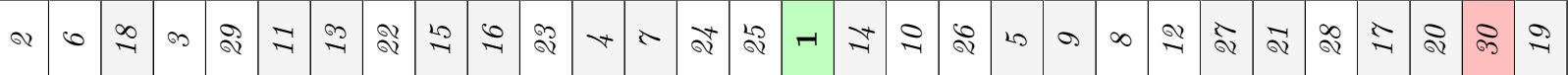

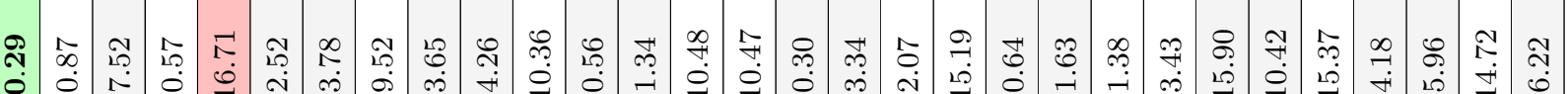

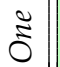

-

\begin{tabular}{|c|c|c|c|c|c|c|c|c|c|c|c|c|c|c|c|c|c|c|c|c|c|c|c|c|c|c|c|c|c|c|}
\hline \multirow{4}{*}{ 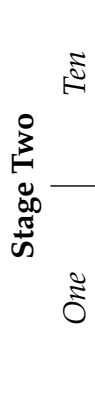 } & 1 & I & $\underset{-}{\stackrel{\theta}{0}}$ & 1 & 1 & | & 1 & 1 & 1 & 1 & 1 & | & 1 & | & 11 & 1 & 1 & | & | & | & | & | & 19 & & ti & 1 & | & 1 & $\begin{array}{l}\vec{\infty} \\
\infty \\
\infty \\
-\infty\end{array}$ & 1 \\
\hline & $r$ & -1 & $\widehat{\hat{Q}}$ & $-r$ & -1 & $r$ & $r$ & -1 & -1 & -1 & -1 & -1 & -1 & -1 & -1 & $-r$ & -1 & -1 & -1 & -1 & -1 & -1 & -1 & Qे & \begin{tabular}{l|l}
$\infty$ & 7 \\
$*$ & 7
\end{tabular} & -1 & -1 & $-1 \delta$ & ळे & -1 \\
\hline & 1 & 1 & $\begin{array}{l}18 \\
\text { iी } \\
\text { is }\end{array}$ & 1 & 1 & | & 1 & 1 & 1 & 1 & 1 & 1 & 1 & 1 & 1 & 1 & 1 & 1 & 1 & I & I & | & 1 & $\begin{array}{l}\mathscr{C} \\
+ \\
+\end{array}$ & is & 1 & I & 1 & 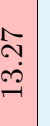 & 1 \\
\hline & $\neg$ & -1 & $\stackrel{\infty}{\infty}$ & -1 & -1 & $r$ & $\neg$ & -1 & $\rightarrow$ & $\neg$ & -1 & $\rightarrow$ & -1 & -1 & -1 & -1 & -1 & -1 & $\neg$ & -1 & $\dashv$ & 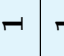 & $\rightarrow$ I & के & $\hat{\alpha}$ & -1 & $\dashv$ & $-1 \delta$ & के & -1 \\
\hline
\end{tabular}

穴

ڤี

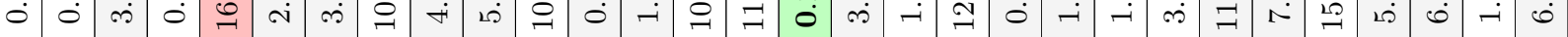

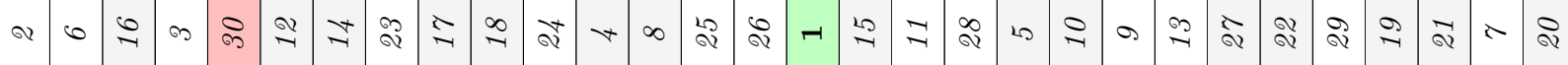

का

ค่

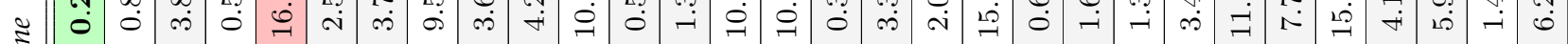

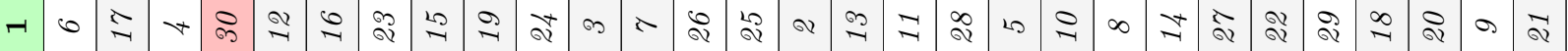

\begin{tabular}{|c|c|c|c|c|c|c|c|c|c|c|c|c|c|c|c|c|c|c|c|c|}
\hline 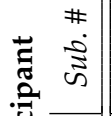 & - & $N$ & - & $-r$ & \begin{tabular}{l|l|}
$N$ & -1
\end{tabular} & $N$ & -1 & $\rightarrow N$ & $\rightarrow$ & $\neg N$ & $\rightarrow N$ & $\neg N$ & $\neg N$ & $\rightarrow N$ & $-\sim N$ & $\neg N$ & -1 & $\rightarrow N$ & - & - \\
\hline 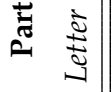 & $u$ & 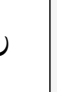 & $\theta$ & 四 & & 工 & 0 & I & - & - & צ & 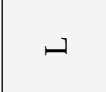 & $\Sigma$ & 0 & a & a & $\infty$ & H & 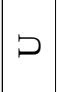 & $>$ \\
\hline
\end{tabular}

$\stackrel{0}{\circ}$

$\stackrel{0}{0.1}$

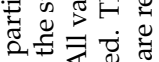

娄艺

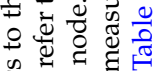

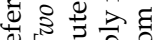

년

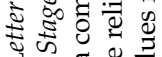

प्र.

क्ष त्

ป气

此

है

造造焉

ลे

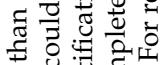

ญ 䒕

| $:=$

๔

द्व

屯 छ

1.0

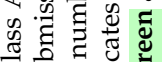

บे के

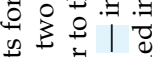

它密《

घี

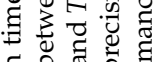

ธี ฮ

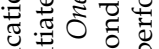

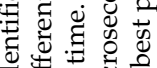

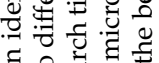

푱요요

\& 0 क

पे

:

득 음

유웛

등 $00 \%$.

승. 믐

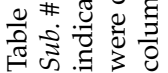

$\mathrm{C}=$ afis team $\mathrm{D}=3 \mathrm{M}$ Cogent $\mid \mathrm{E}=$ Neurotechnology $\mid \mathrm{F}=$ Papillon $\mid \mathrm{G}=$ Dermalog $\mid \mathrm{H}=$ Hisign Bio-Info Institute

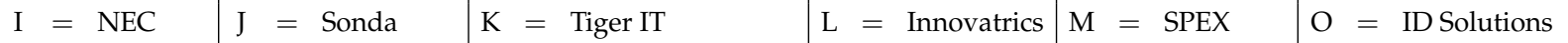

\begin{tabular}{l|l|l|l|l}
$\mathrm{P}=\mathrm{id} 3$ & $\mathrm{Q}=$ Morpho & $\mathrm{S}=$ Decatur Industries $\mathrm{T}=$ BIO-key & $\mathrm{U}=$ Aware & $\mathrm{V}=$ AA Technology
\end{tabular} 


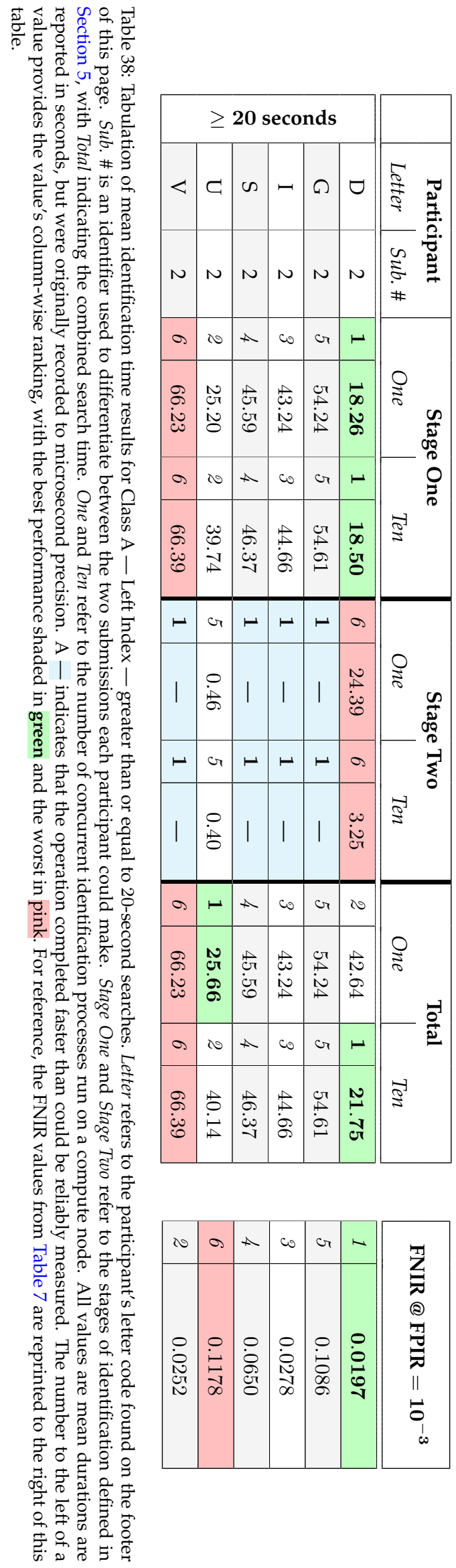

\begin{tabular}{l|l|l|l|ll|l}
$\mathrm{C}=$ afis team & $\mathrm{D}=3 \mathrm{M}$ Cogent & $\mathrm{E}=$ Neurotechnology & $\mathrm{F}=$ Papillon & $\mathrm{G}=$ Dermalog & $\mathrm{H}=$ Hisign Bio-Info Institute \\
$\mathrm{I}=$ NEC & $\mathrm{J}=$ Sonda & $\mathrm{K}=$ Tiger IT & $\mathrm{L}=$ Innovatrics & $\mathrm{M}=$ SPEX & $\mathrm{O}=$ ID Solutions \\
$\mathrm{P}=\mathrm{id} 3$ & $\mathrm{Q}=$ Morpho & $\mathrm{S}=$ Decatur Industries & $\mathrm{T}=$ BIO-key & $\mathrm{U}=$ Aware & $\mathrm{V}=$ AA Technology
\end{tabular}




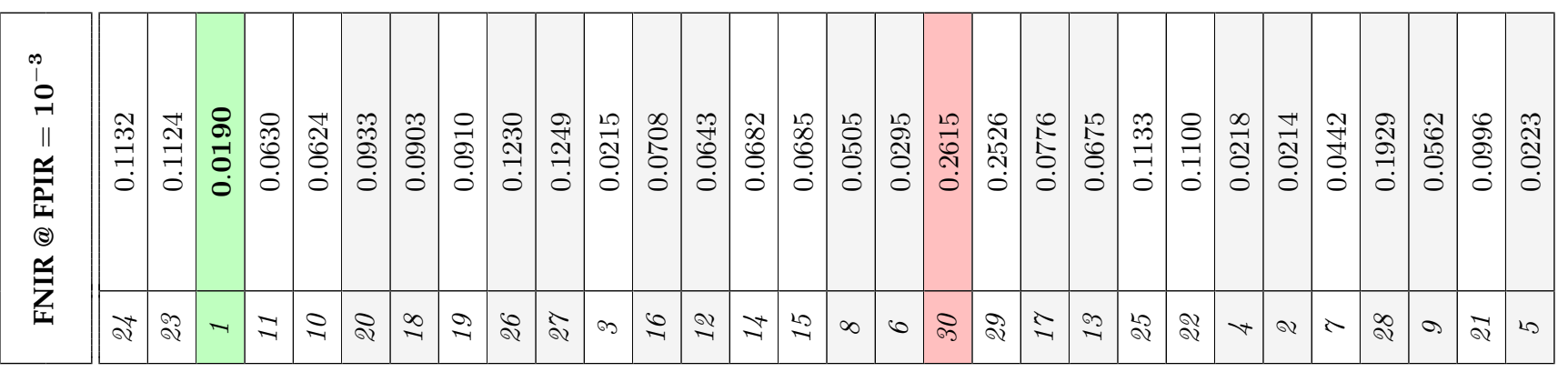

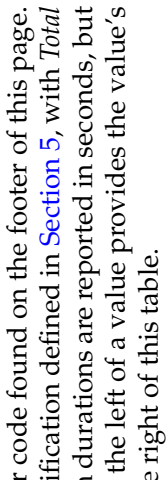

穿

远

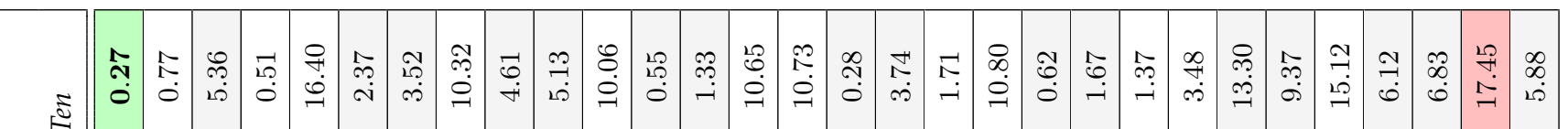

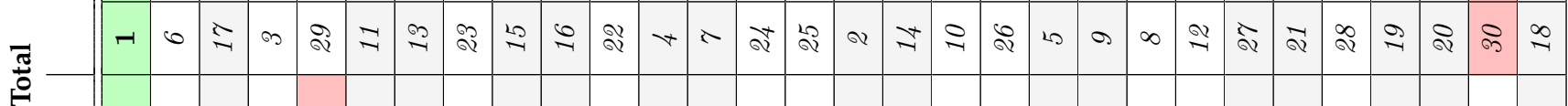

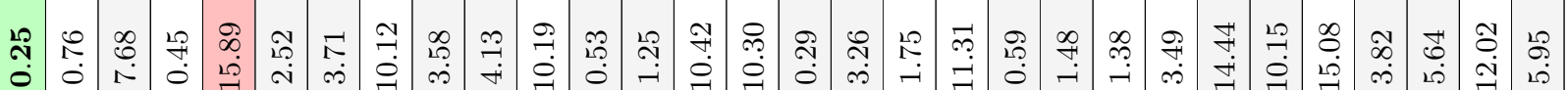
Ð - 0 จ

\begin{tabular}{|c|c|c|c|c|c|c|c|c|c|c|c|c|c|c|c|c|c|c|c|c|c|c|c|c|c|c|}
\hline \multirow{4}{*}{ 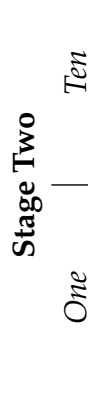 } & 1 & I & 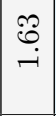 & 1 & I & I & I & | & 1 & | & | & | & 1 & | & | & | & I & 1 & | & 1 & $1 \stackrel{0}{\stackrel{0}{7}}$ & 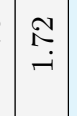 & 1 & $1 \mid 1$ & $\begin{array}{c}\hat{2} \\
\tilde{r} \\
\dot{0} \\
-1\end{array}$ & $\mid$ \\
\hline & -1 & -1 & $\hat{\mathrm{Q}}$ & -1 & -1 & -1 & -1 & -1. & -1 & -17 & -17 & $-r$ & -1 & -1 & -17 & $-1-$ & -1 & -17 & -17 & -1 & -12 & $\begin{array}{l}\infty \\
\propto\end{array}$ & -1 & \begin{tabular}{r|r}
- & -
\end{tabular} & ๓ & $r$ \\
\hline & 1 & | & 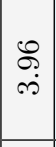 & 1 & | & | & I & I & | & | & | & | & I & | & | & | & I & 1 & 1 & I & | & $\begin{array}{l}\vec{\Gamma} \\
\stackrel{i}{*}\end{array}$ & 1 & $1 \mid 1$ & $\begin{array}{l}\mathcal{N} \\
\hat{N} \\
\stackrel{9}{9}\end{array}$ & 1 \\
\hline & -1 & -1 & 2 & -1 & -1 & -1 & -1 & -1 & -1 & $-1-$ & $-1-$ & $-1-1$ & -1 & -1 & $-r$ & $-1-$ & $-1-1$ & -1. & -1 & $-r$ & 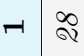 & बे & -1 & -1 & ๓ & $r$ \\
\hline \multirow{4}{*}{ 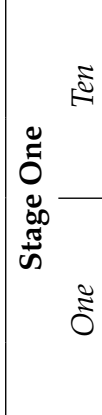 } & స̂̀ & 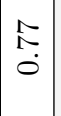 & 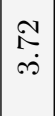 & $\mid \begin{array}{c}\overrightarrow{0} \\
0 \\
0\end{array}$ & $\begin{array}{l}\stackrel{9}{+} \\
\dot{\bullet}\end{array}$ & ๙ֶ. & $\begin{array}{l}\stackrel{T}{i s} \\
\stackrel{s}{0}\end{array}$ & 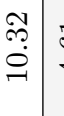 & 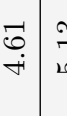 & 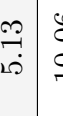 & 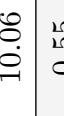 & 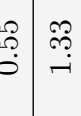 & \begin{tabular}{l}
$: 0$ \\
$\stackrel{0}{0}$ \\
\hdashline
\end{tabular} & $\left|\begin{array}{l}\Re \\
\stackrel{1}{0} \\
0 \\
-1\end{array}\right|$ & 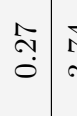 & 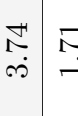 & $=\begin{array}{l}\infty \\
\infty \\
\stackrel{0}{\oplus}\end{array}$ & 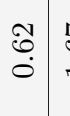 & $\begin{array}{lll}\hat{0} & 1 \\
- & 0\end{array}$ & \begin{tabular}{c|c}
$\stackrel{\sim}{\infty}$ & $g$ \\
- & $\rho$
\end{tabular} & $\begin{array}{ll}\infty \\
\stackrel{\infty}{+} \\
\stackrel{\sim}{+}\end{array}$ & $\begin{array}{l}\stackrel{\bullet}{0} \\
\stackrel{\sim}{\sim}\end{array}$ & $\stackrel{\mathcal{7}}{7}$ & \begin{tabular}{c|c}
$\mathcal{T}$ & $\mathscr{7}$ \\
\hdashline & $\infty$ \\
0 & 0
\end{tabular} & 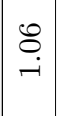 & $\begin{array}{l}\infty \\
\infty \\
i j \\
10\end{array}$ \\
\hline & -1 & 0 & 20 & $\infty$ & ळे & ำ & \pm & बें : & $\approx 9$ & $\stackrel{\infty}{\sim} \approx$ & 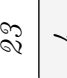 & $+\infty$ & $\stackrel{2}{Q}$ & ๙ & $\diamond$ & $\stackrel{0}{*}=$ & $\overline{\widehat{Q}}$ & 20 & $\therefore$ & $\sigma:$ & 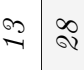 & ช & शे & จ & 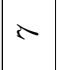 & 2 \\
\hline & $\begin{array}{c}\stackrel{L}{\text { N }} \\
\stackrel{0}{0}\end{array}$ & $\begin{array}{l}0 \\
0 \\
0\end{array}$ & $\begin{array}{l}\mathcal{N} \\
\sim \\
\infty\end{array}$ & $\stackrel{2}{\stackrel{2}{9}}$ & $\begin{array}{l}\infty \\
\infty \\
\stackrel{1}{-1} \\
-1\end{array}$ & $\begin{array}{l}\text { î } \\
\text { งิ }\end{array}$ & $\begin{array}{l}\Gamma \\
\infty \\
\infty\end{array}$ & 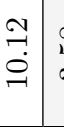 & 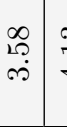 & $\stackrel{\mathscr{m}}{\rightarrow}$ & 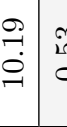 & 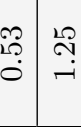 & 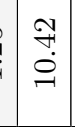 & 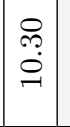 & 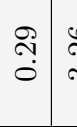 & 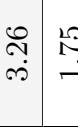 & 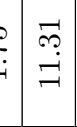 & $\begin{array}{c}0 \\
0 \\
0 \\
0 \\
0\end{array}$ & \begin{tabular}{l|l}
$\stackrel{\infty}{+}$ \\
$\stackrel{-}{-}$
\end{tabular} & \begin{tabular}{c|c}
$\stackrel{\infty}{\leftrightarrow}$ & $\wp$ \\
- & $\wp$
\end{tabular} & \begin{tabular}{c|c}
$\stackrel{\leftrightarrow}{+}$ & $\stackrel{\overbrace{}}{\leftrightarrows}$ \\
\hdashline & $=$
\end{tabular} & 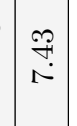 & 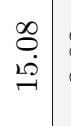 & 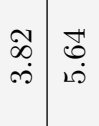 & 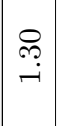 & $\begin{array}{l}28 \\
20 \\
10\end{array}$ \\
\hline & -1 & 0 & $\equiv$ & $\infty$ & คे & $\stackrel{\vartheta}{\sim}$ & $\stackrel{2}{1}$ & $\stackrel{2}{Q}$ & 29 & 2 & Qैं & $+\lambda$ & จ & $\begin{array}{ll}20 \\
\otimes\end{array}$ & $\diamond$ & $\rightarrow=$ & $\begin{array}{l}\infty \\
\otimes \\
\otimes\end{array}$ & 20 & $\therefore$ & $0>$ & $\approx$ จ & Q & 2 & \begin{tabular}{l|l}
$\infty$ & 8 \\
-1
\end{tabular} & $\infty$ & बे \\
\hline \multirow{2}{*}{ 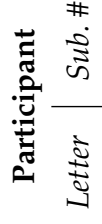 } & - & $N$ & -1 & - & $N$ & - & $N$ & -1 & $\rightarrow c$ & $\begin{array}{lll}N & 7\end{array}$ & -7 & $\rightarrow N$ & $1-7$ & $N$ & $\rightarrow 0$ & N & $\begin{aligned} n \\
-1\end{aligned}$ & -1 & $\begin{array}{lll}N & -\end{array}$ & -0 & $N-r$ & $N$ & - & $r \mid N$ & - & - \\
\hline & $c$ & J & $\theta$ & II & 니 & 工 & & 0 & I & & - & $\curvearrowleft$ & $\forall$ & & - & & $\Sigma$ & 0 & & a & & a & $\infty$ & 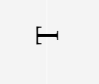 & $\supset$ & $>$ \\
\hline & \multicolumn{26}{|c|}{ puojas $0 z>$} \\
\hline
\end{tabular}

की

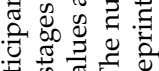
的范 号考它 요요 원

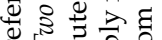
$\rightarrow+\frac{0}{\sigma}$ 害安 $\rightarrow$ i $\pi: \frac{\pi}{0}$ 串

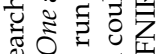
क

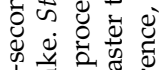
के ลิ

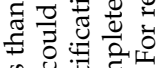
记 泀 1. ช. 들 2 ज.

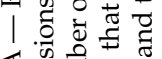
«. U ज्ञ क्षे

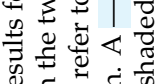
ष

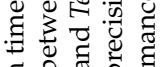
. ฮ ّ

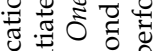

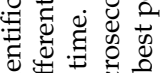

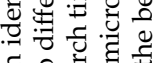
도웛요

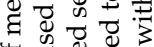
Oे . 可

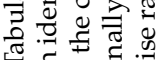

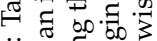
oิ. क्ष

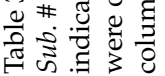




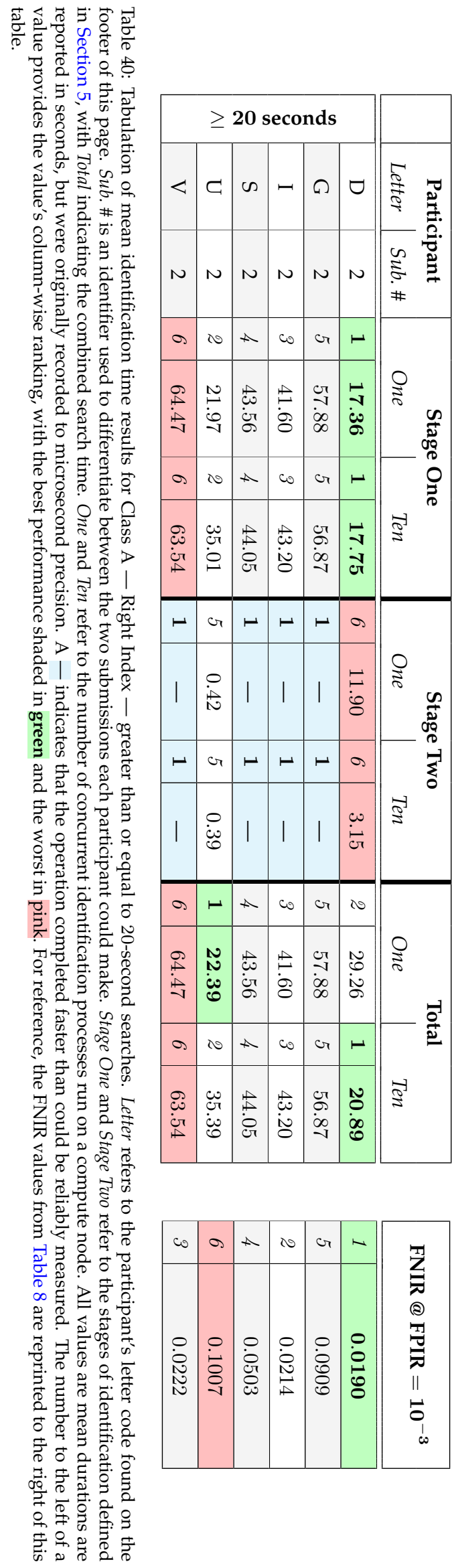

\begin{tabular}{l|l|l|l|l|l|l}
$\mathrm{C}=$ afis team & $\mathrm{D}=3 \mathrm{M}$ Cogent & $\mathrm{E}=$ Neurotechnology & $\mathrm{F}=$ Papillon & $\mathrm{G}=$ Dermalog & $\mathrm{H}=$ Hisign Bio-Info Institute \\
$\mathrm{I}=\mathrm{NEC}$ & $\mathrm{J}=$ Sonda & $\mathrm{K}=$ Tiger IT & $\mathrm{L}=$ Innovatrics & $\mathrm{M}=$ SPEX & $\mathrm{O}=$ ID Solutions \\
$\mathrm{P}=\mathrm{id} 3$ & $\mathrm{Q}=$ Morpho & $\mathrm{S}=$ Decatur Industries & $\mathrm{T}=$ BIO-key & $\mathrm{U}=$ Aware & $\mathrm{V}=$ AA Technology
\end{tabular}



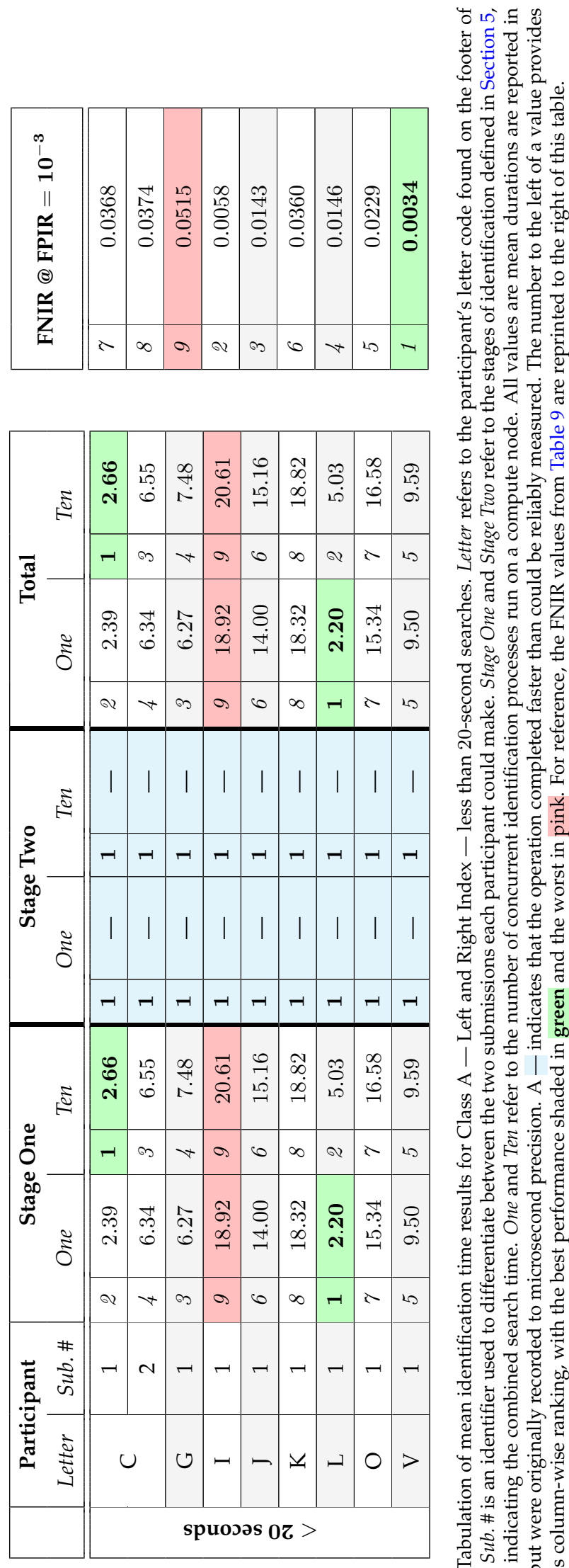

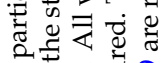

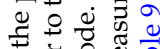

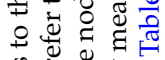

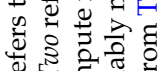

늠

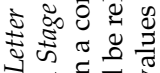

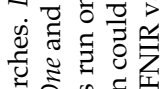

ปี

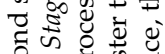

过选

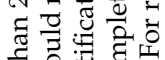

के

$\because$ 車

ช.

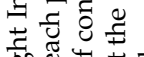

类 00

ट.

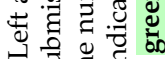

के 5.9

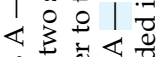

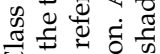

पै एँ

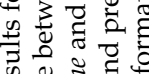

ڤ્屯

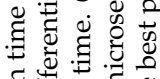

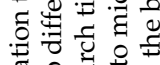

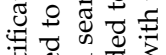

记

늠

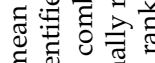

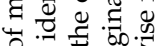

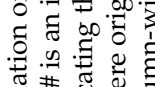

\#

चี ज.

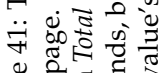

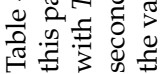

\begin{tabular}{l|l|l|l|ll|l|l}
$\mathrm{C}=$ afis team & $\mathrm{D}=3 \mathrm{M}$ Cogent & $\mathrm{E}=$ Neurotechnology & $\mathrm{F}=$ Papillon & $\mathrm{G}=$ Dermalog & $\mathrm{H}=$ Hisign Bio-Info Institute \\
$\mathrm{I}=$ NEC & $\mathrm{J}=$ Sonda & $\mathrm{K}=$ Tiger IT & $\mathrm{L}=$ Innovatrics & $\mathrm{M}=$ SPEX & $\mathrm{O}=$ ID Solutions \\
$\mathrm{P}=\mathrm{id} 3$ & $\mathrm{Q}=$ Morpho & $\mathrm{S}=$ Decatur Industries & $\mathrm{T}=$ BIO-key & $\mathrm{U}=$ Aware & $\mathrm{V}=$ AA Technology
\end{tabular}




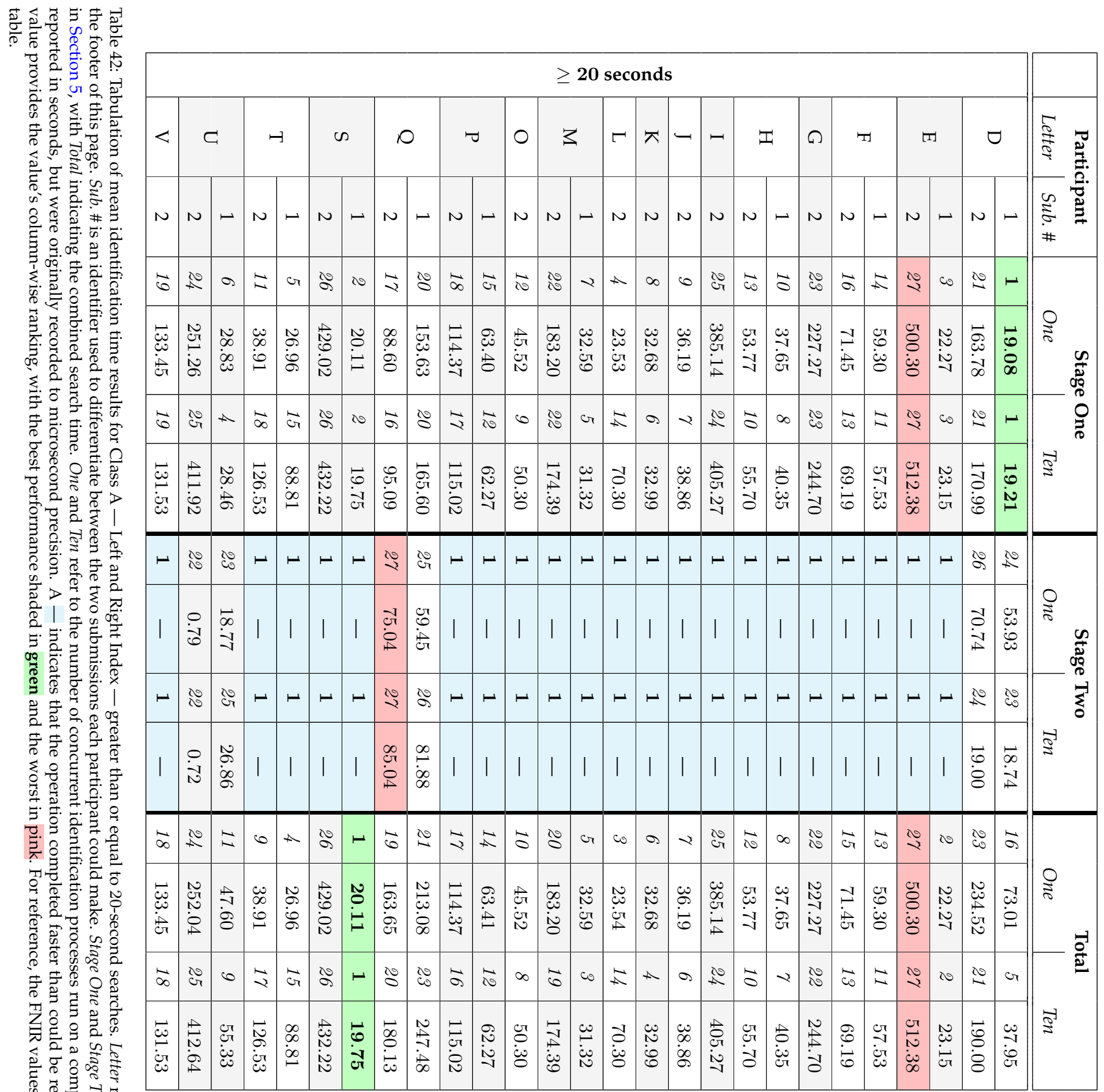

象:

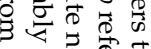

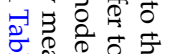

o

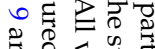

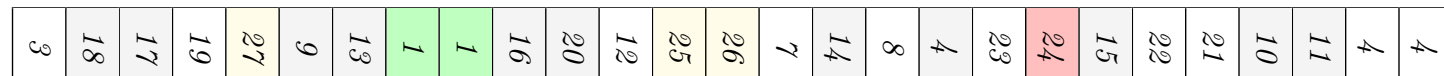

要

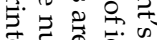

व.

o

के

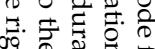

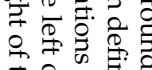

空: 


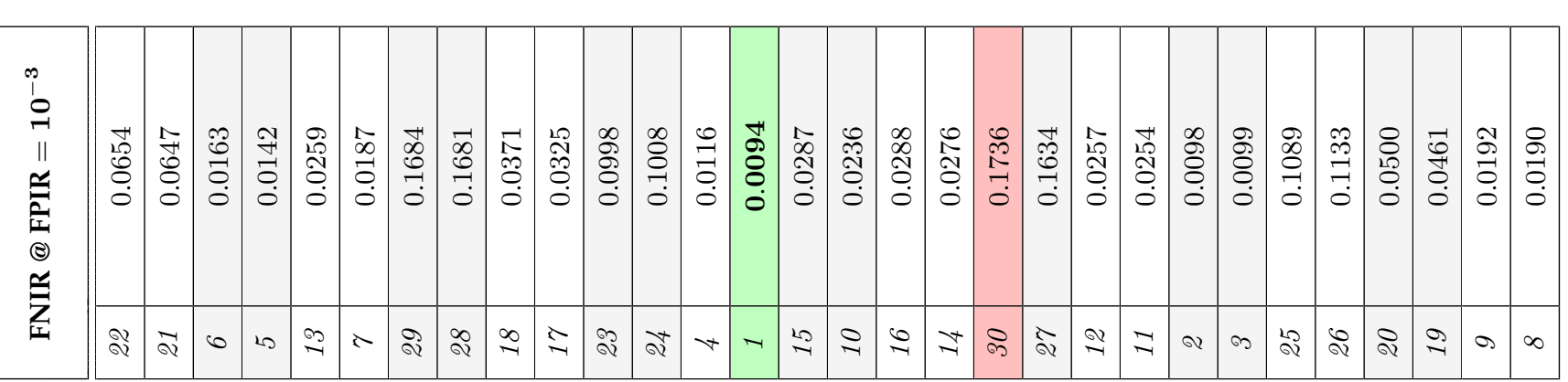

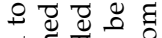

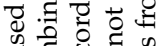

द्व

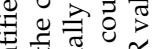

일.

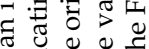

.

ن.

制造焉

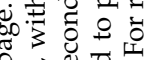

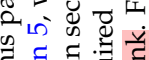

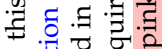

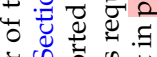

品.

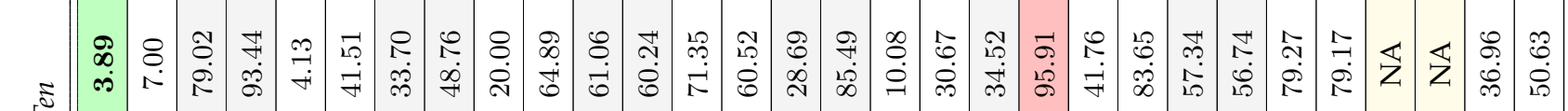

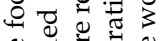

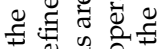

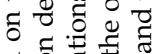

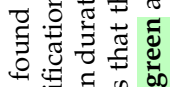

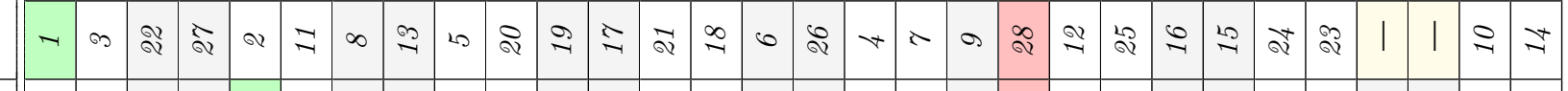

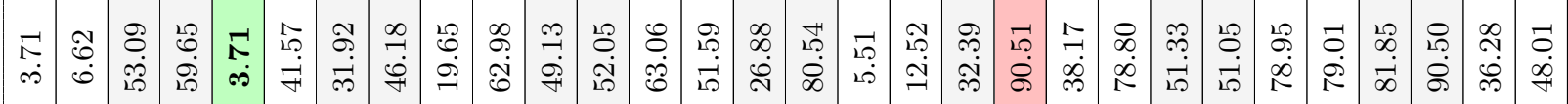
$\stackrel{5}{5}$

8 ष

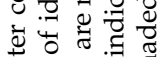

过 \& 8

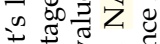

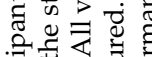

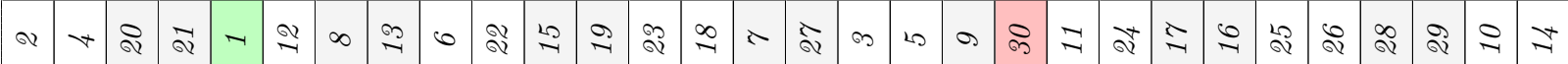

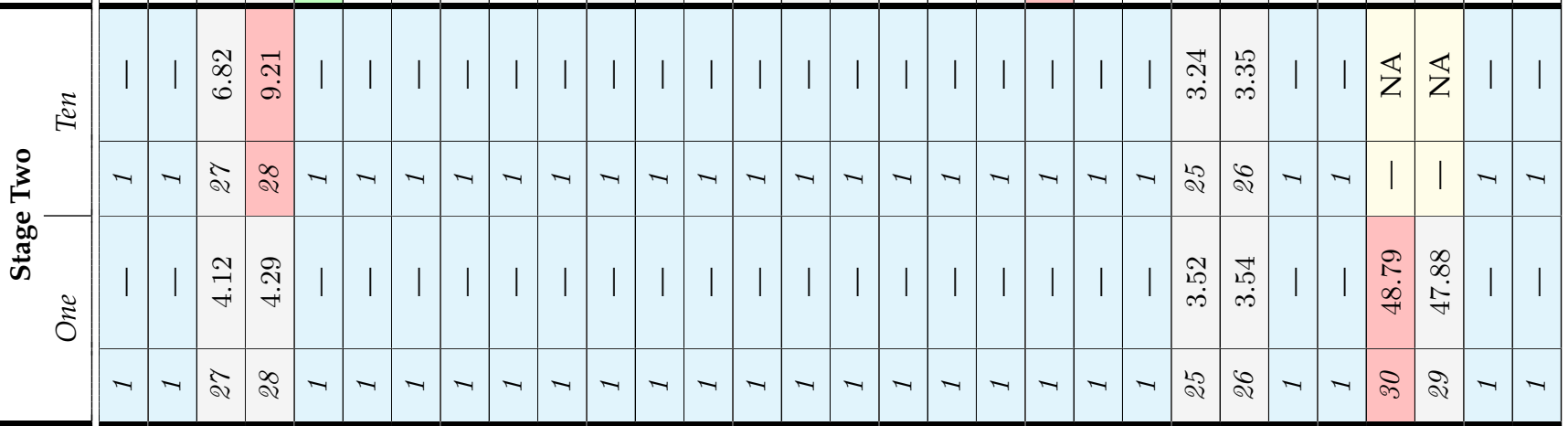

Eㅇ 웜웜

正

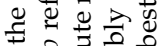

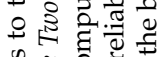

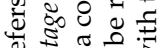

का

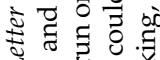

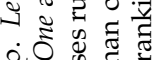

की

का

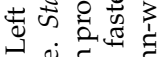

1 递

$\infty$ ह च

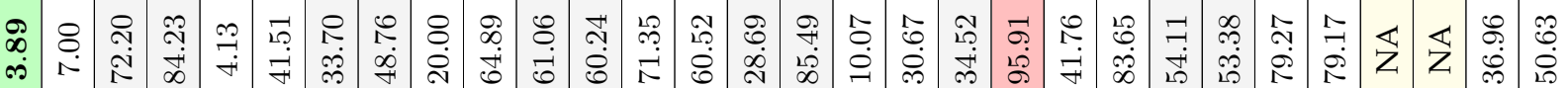

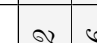

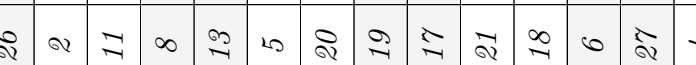

$+a$

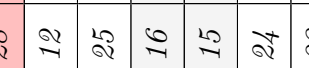

|

के

구 §

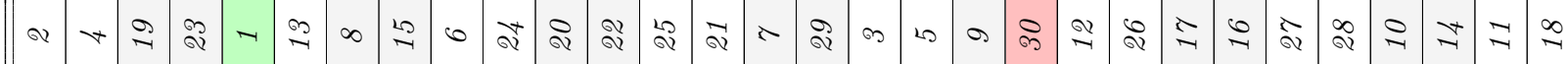

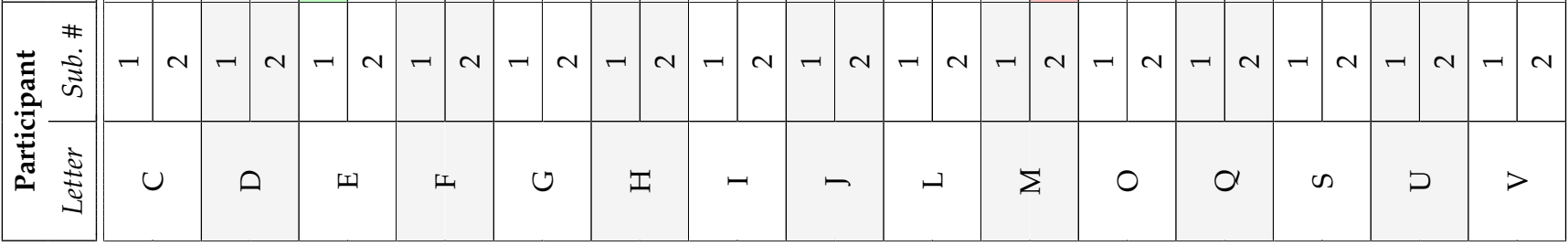

पै

进 $\overrightarrow{0}$.

s. 문

क्ष

年它

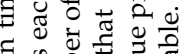

ธ

है क्षे है

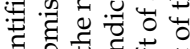

들

की

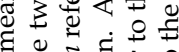

당

द वे की है

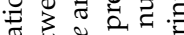

䨔

元 


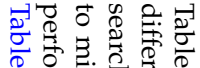

思

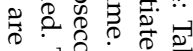

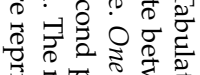

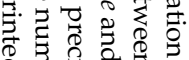

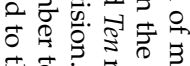

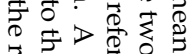

oa

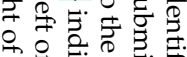

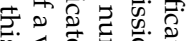

政政

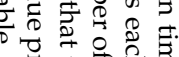

후웅

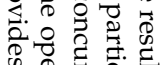

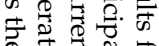

을. 롱

है 0

is

0 .

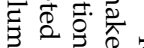

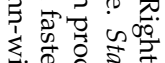

c.

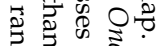

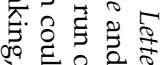

కद

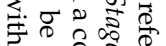

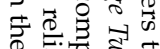

हैं है

ब

ते

赵赵范

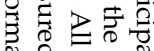

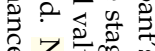

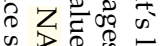

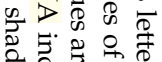

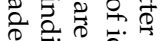

今.

\begin{tabular}{|c|c|c|c|c|c|c|c|c|c|c|c|c|c|c|c|c|c|c|c|c|c|c|c|c|c|c|c|c|c|c|}
\hline & $\xi$ & $c$ & $\Rightarrow$ & & s & 10 & & & & 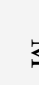 & & 5 & & - & & - & & $I$ & & O & & $T$ & & T & & $\nabla$ & & $\Omega$ & & $\frac{5}{\frac{5}{5}}$ \\
\hline$N$ & 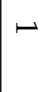 & $N$ & - & $N$ & 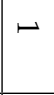 & $N$ & $\bullet$ & $N$ & - & $N$ & 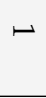 & $N$ & 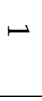 & $N$ & $\bullet$ & $N$ & $\bullet$ & $N$ & - & $N$ & $\bullet$ & $N$ & $\bullet$ & $N$ & - & $N$ & - & $N$ & - & $\begin{array}{l}\text { E } \\
\text { \# }\end{array}$ \\
\hline
\end{tabular}

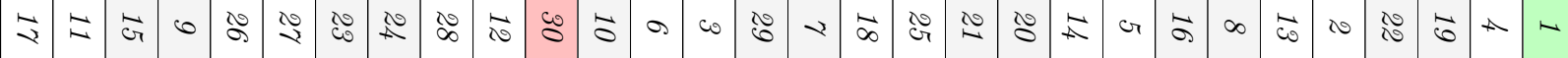

D

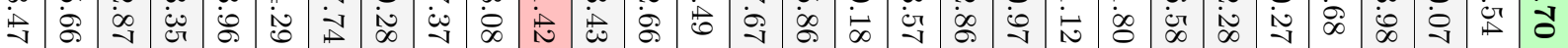

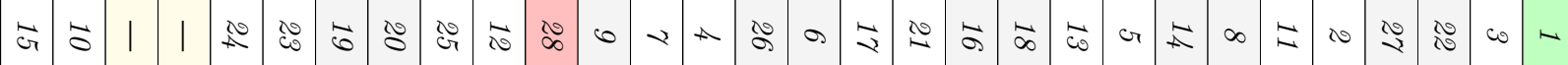

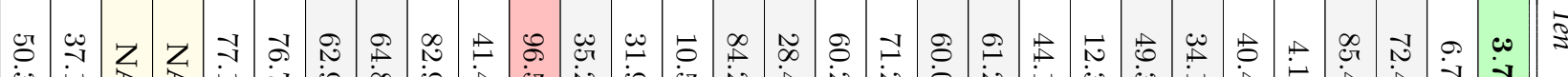

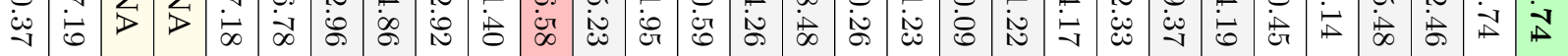

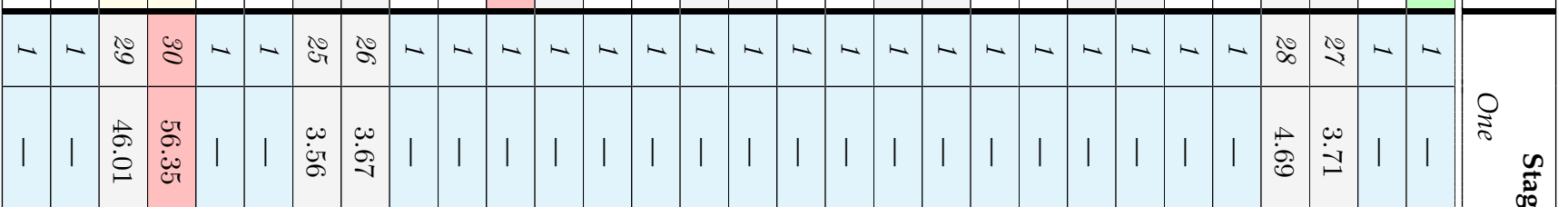

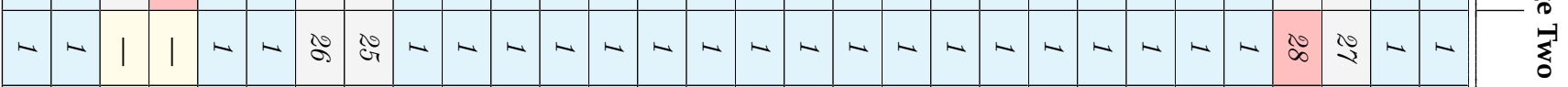

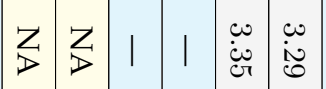
$\begin{array}{lll}0 & 0 \\ \dot{0} & \dot{\theta} \\ ن\end{array}$

$\vec{G} \vec{b}$ d

幽

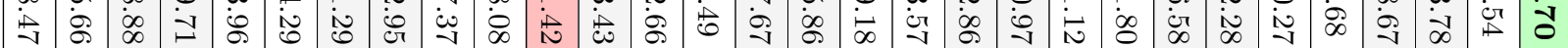
$\vec{c} \vec{\sigma}|\quad|$ d

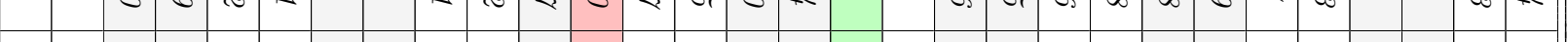

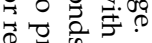

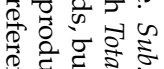

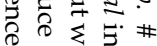

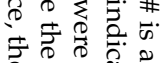
节

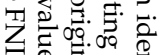
范

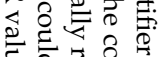

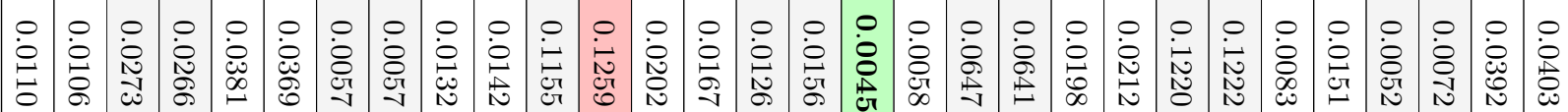




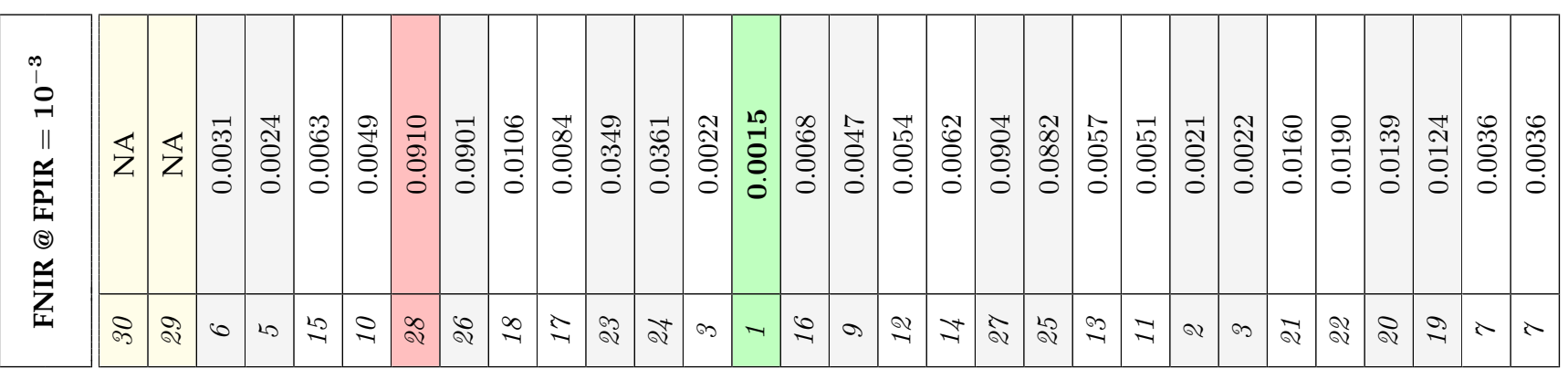

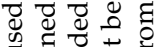

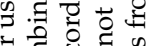

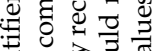

可

ㄴ.0.0

.

\# .

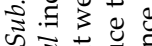

की

논

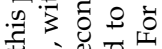

吾

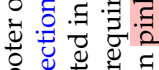

o d :

Ð $\cong 0.0$

ㅎำ

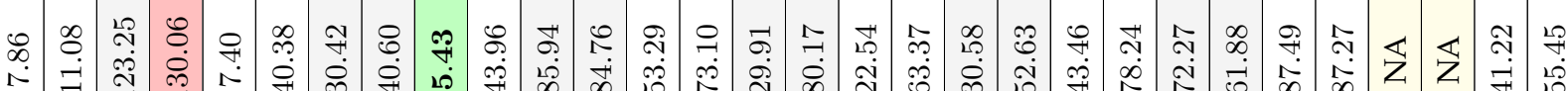

సٓํำ

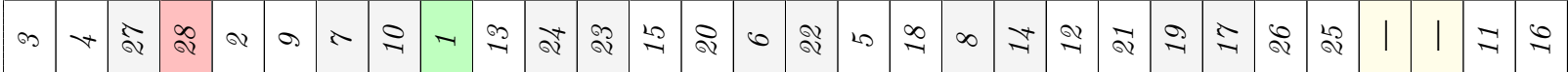

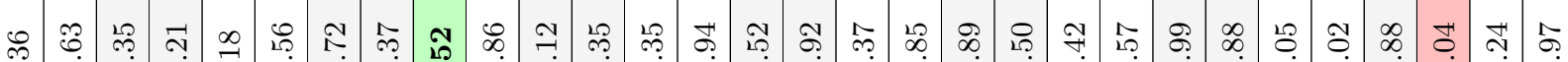

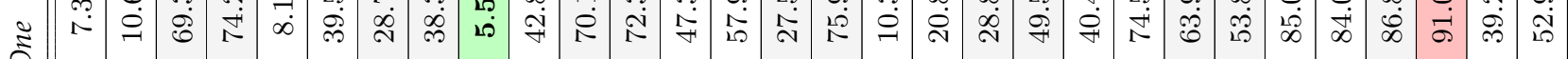

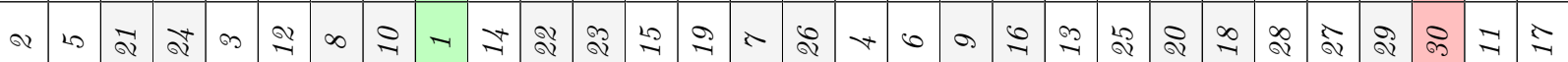

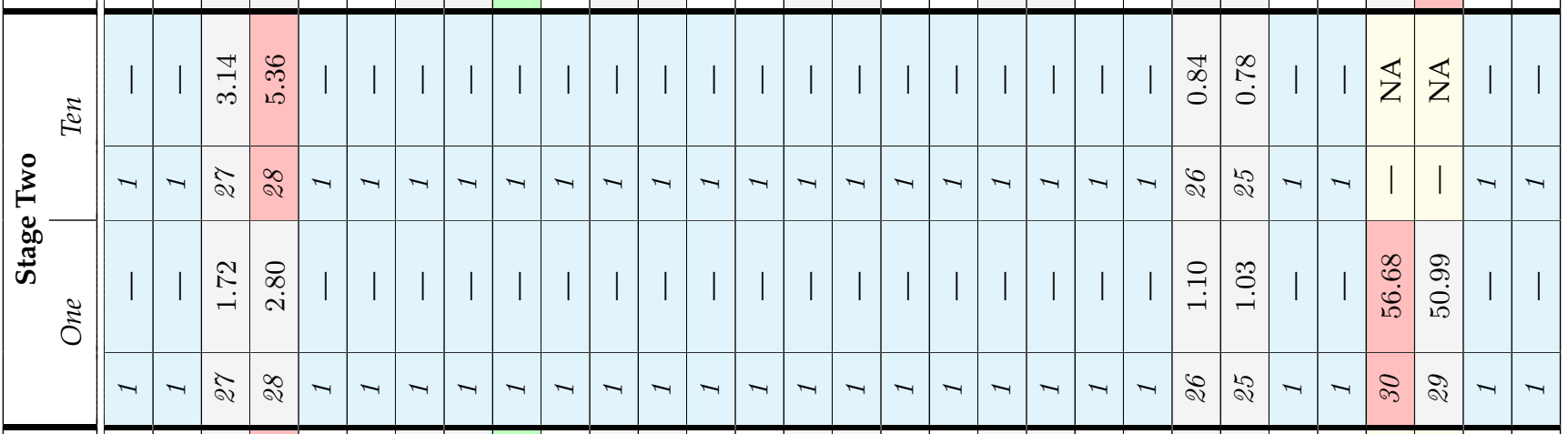

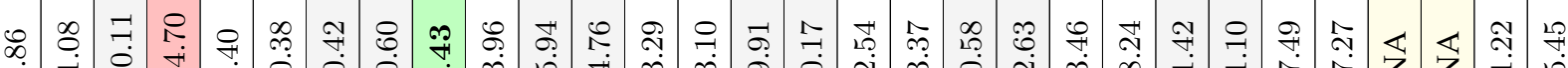
ะ

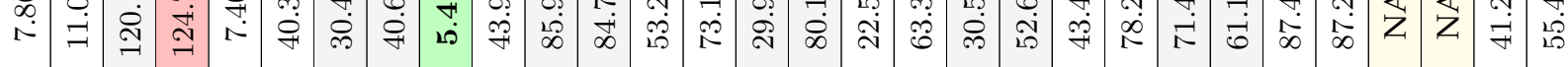

$\tilde{\sigma}$

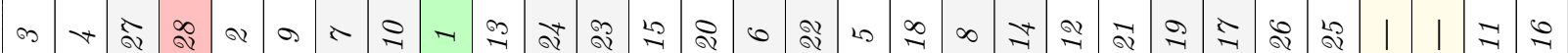

के

舟

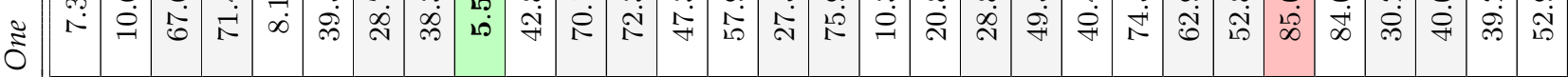
๙ ⿻

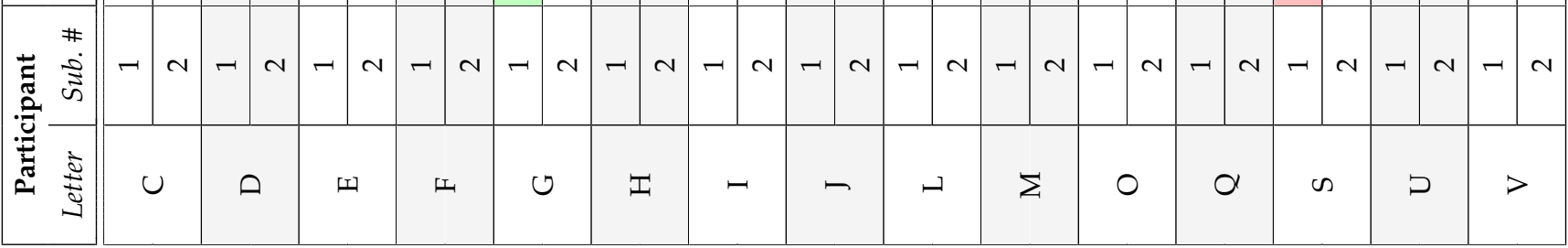

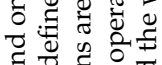

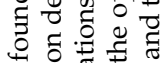

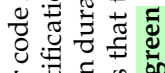

पै

웜

ज诺

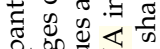

:

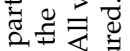

요요

원 छ

닌

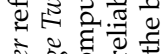

₹

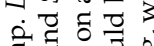

कँ 5 ह

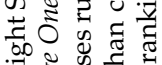

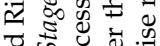

政 8

过茛

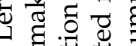

1 중

o

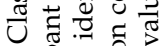

농 :

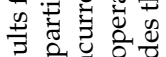

क्ष

踏

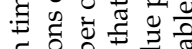

.

๘

굴 웡

क्षे की

눈

ฮ

면 5 .

पे

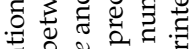

c 0 웜

总 0 . 


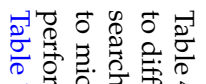

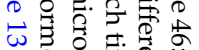

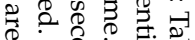

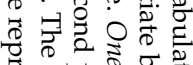

.

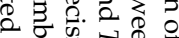

웜 9

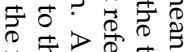

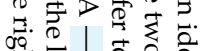

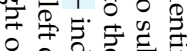

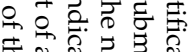

E. 2 通局

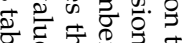

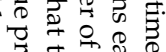

훙

क⿺辶

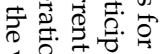

记

○ 0

क 5 .

웅

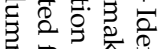

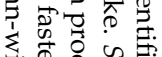

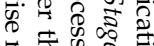

ज्ञ

창 8 를 풀

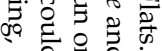

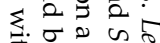

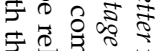

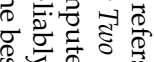

争

遂

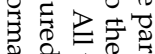

年

n.

हैं हैं क

ㄱ. 훙

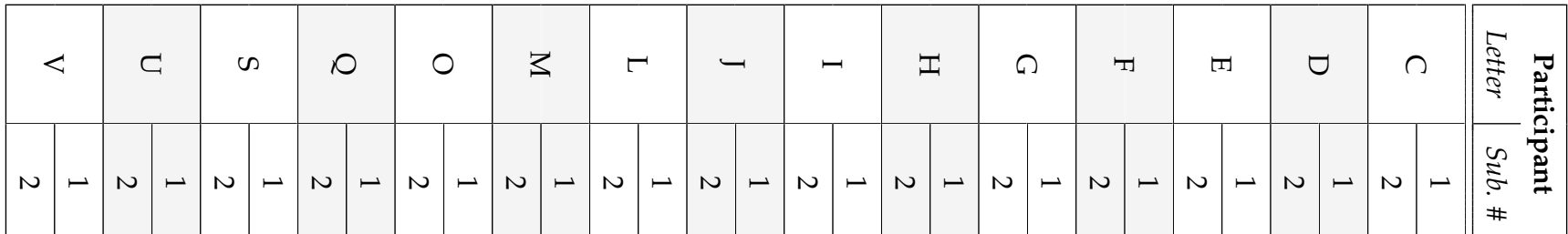

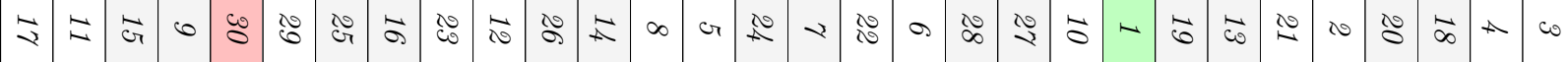

岂

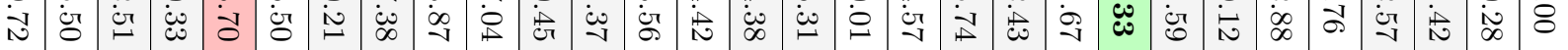

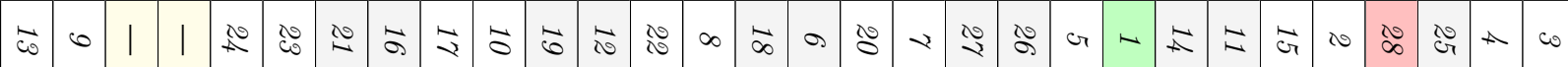

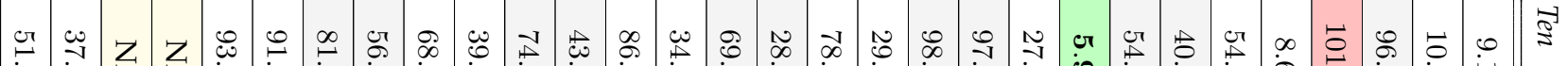

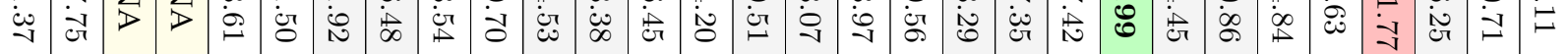

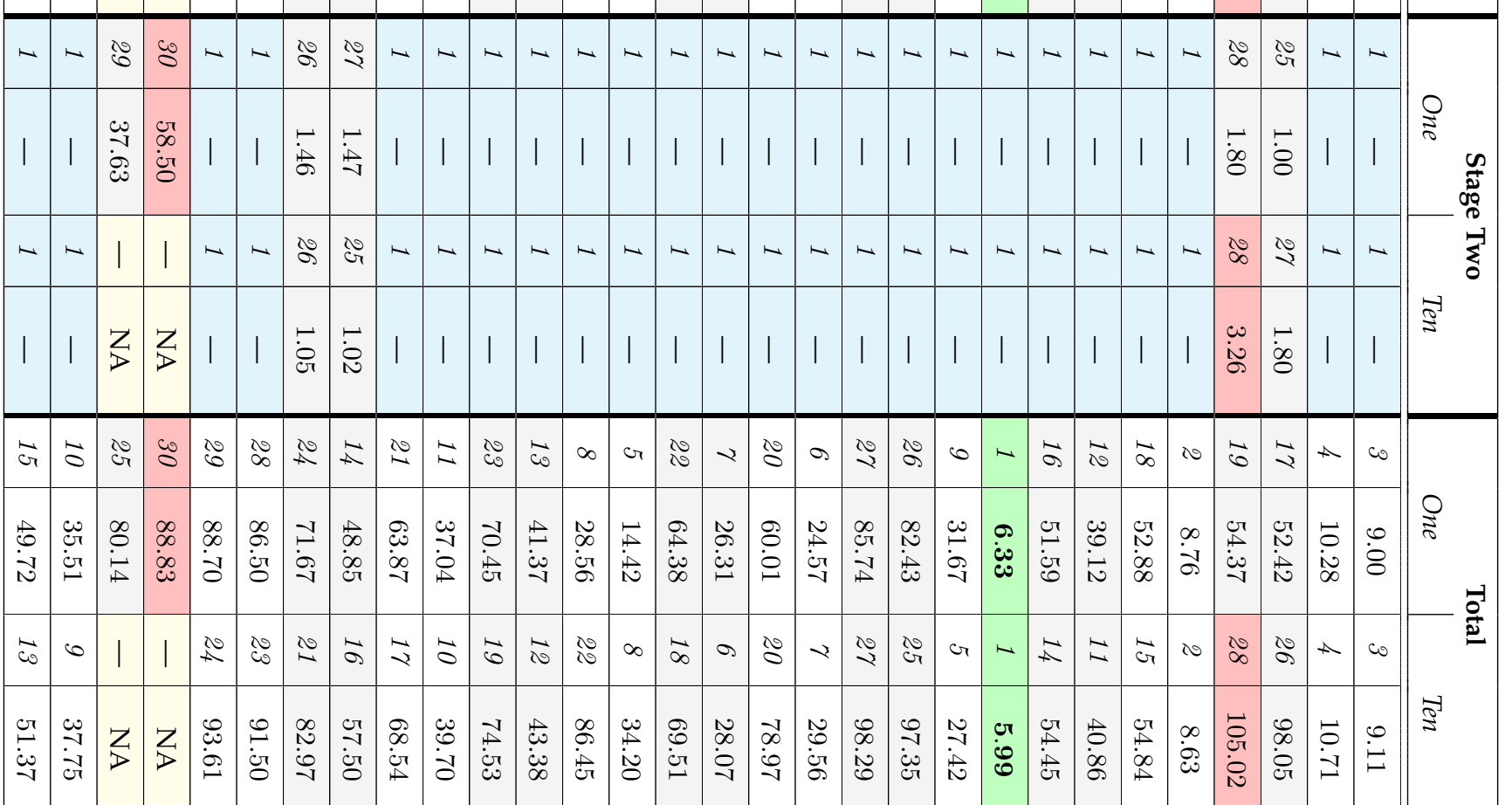

$\sum \stackrel{2}{2} \stackrel{0}{2}$

象

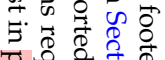

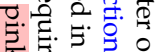

율

T일.

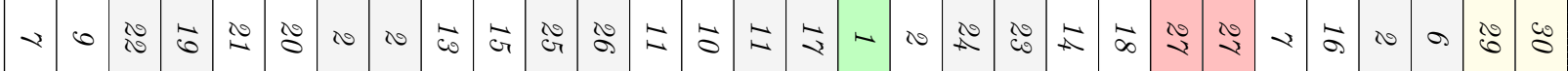

范

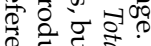

तु

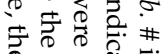

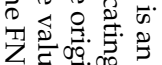

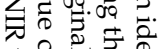

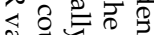

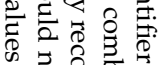

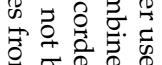

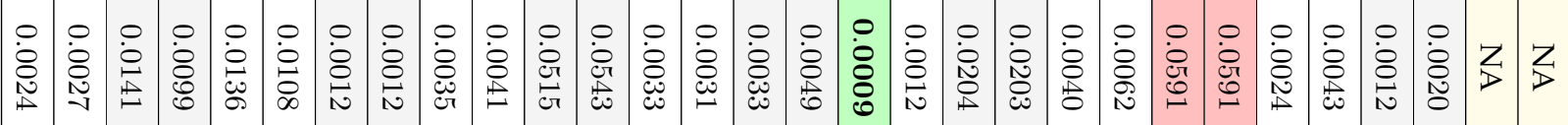

$\mathrm{C}=$ afis team $\mathrm{D}=3 \mathrm{M}$ Cogent $\mid \mathrm{E}=$ Neurotechnology $\mid \mathrm{F}=$ Papillon $\mid \mathrm{G}=$ Dermalog $\mid \mathrm{H}=$ Hisign Bio-Info Institute

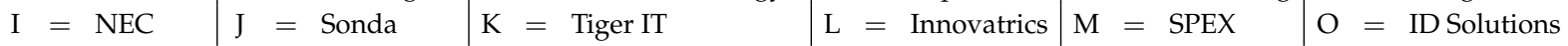

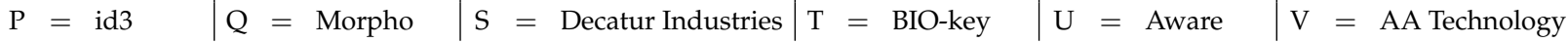




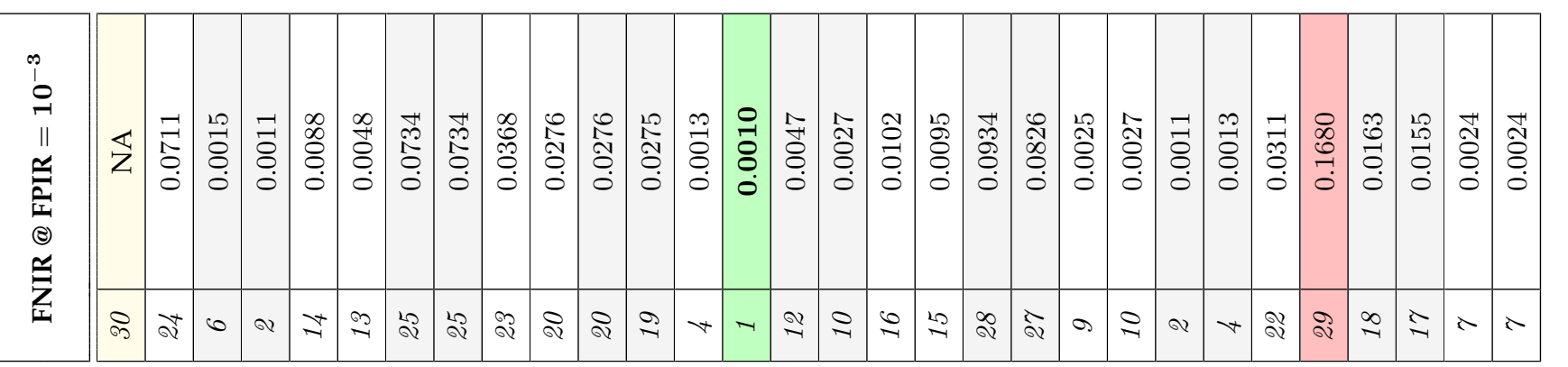

ฮ

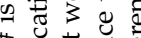

\#.:

के

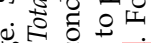

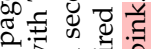

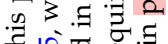

₹

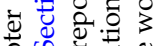

.

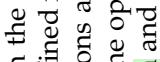

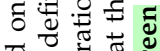

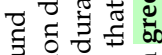

D

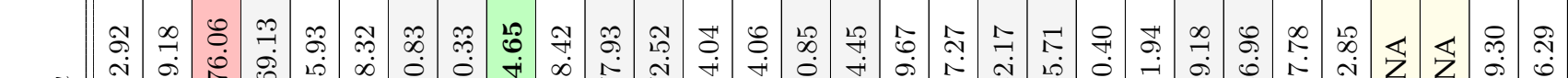

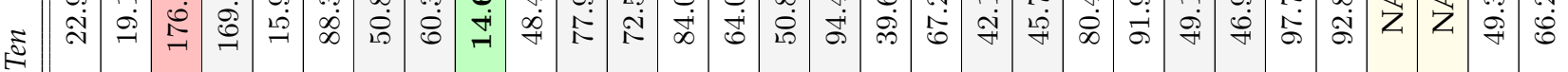

$\frac{\pi}{0}$

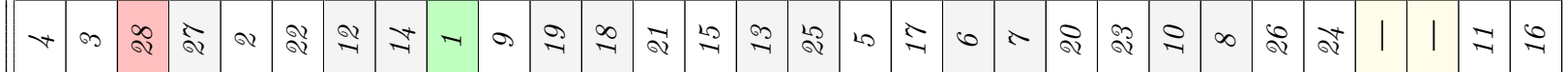

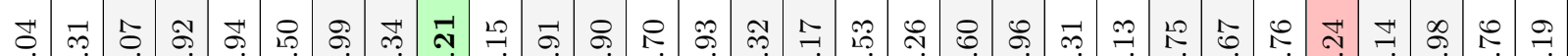
ई

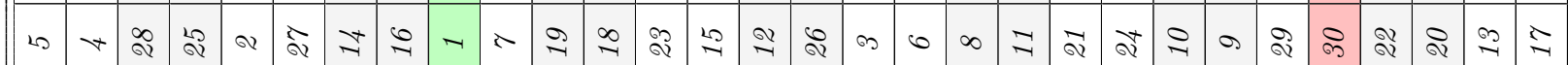

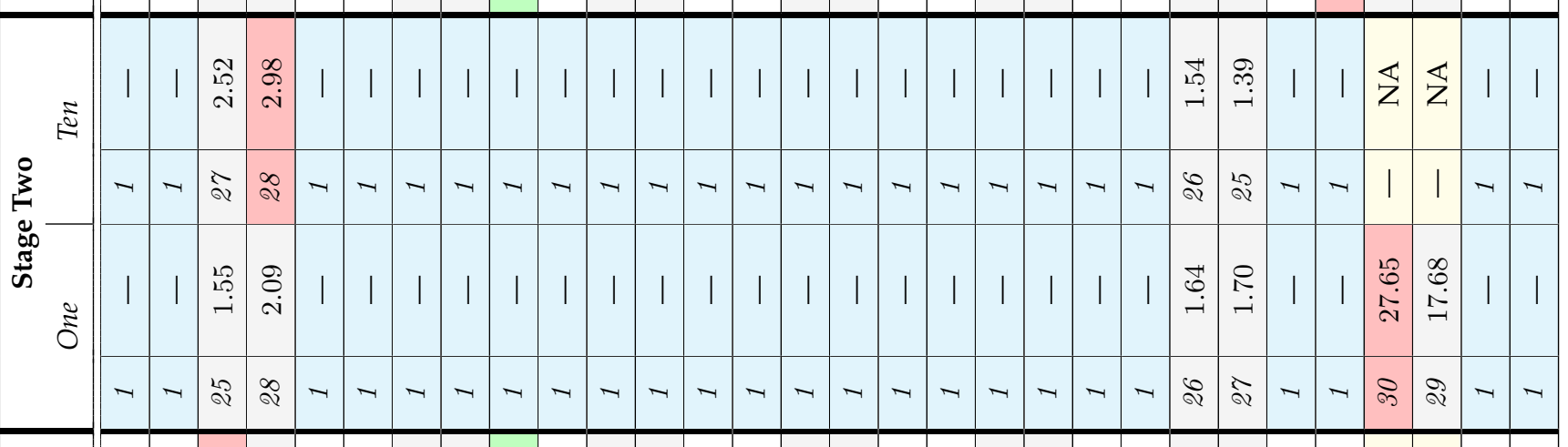

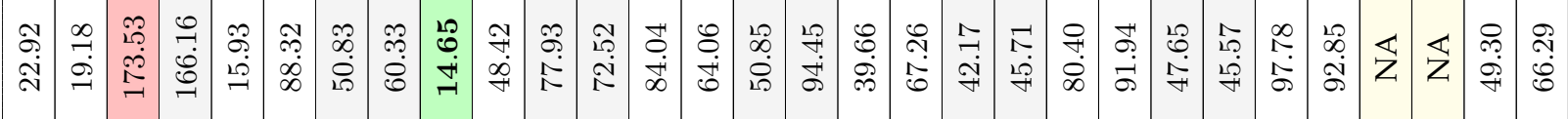

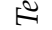

O

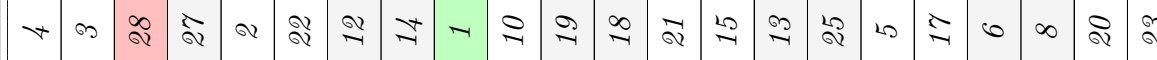

$\exists \stackrel{2}{2}$

कृ

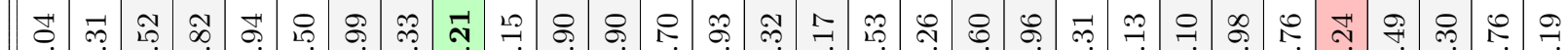

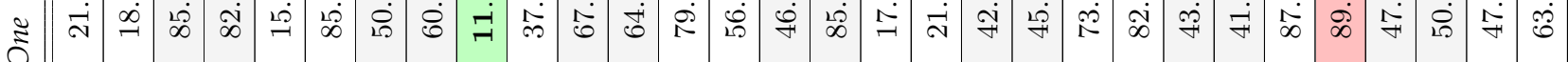

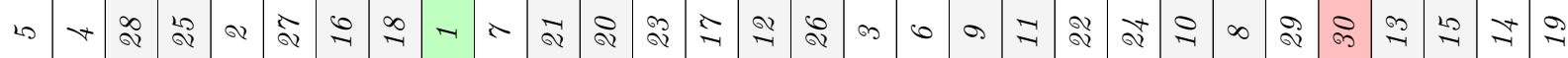

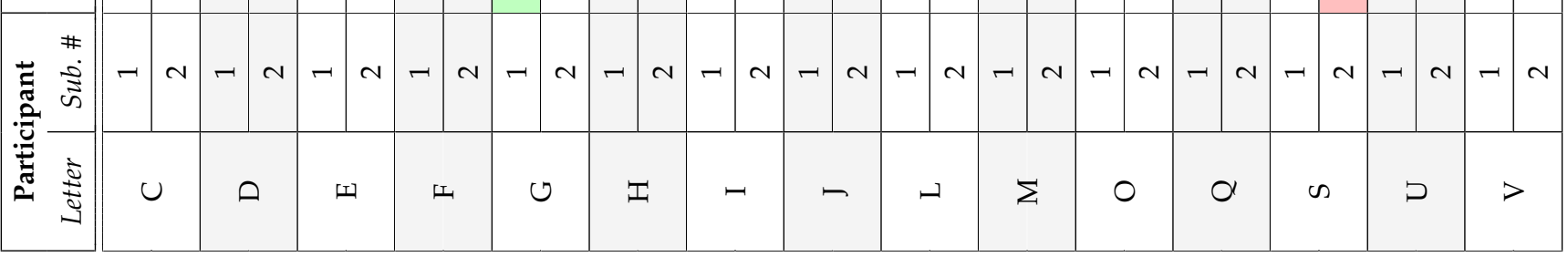
8 远

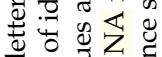
क

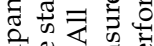
: 훙요

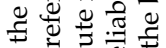

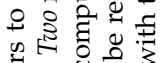

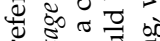




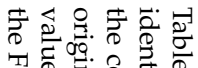

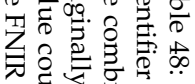

可

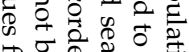

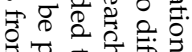

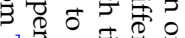

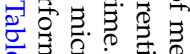
$\rightarrow \stackrel{0}{\circ} \circ$ $\because \rightarrow 0$ ० $\overrightarrow{0}$.

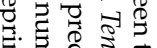

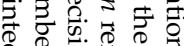
20 更 के

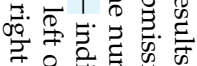

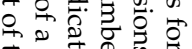
F 7 क्ष

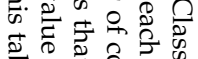

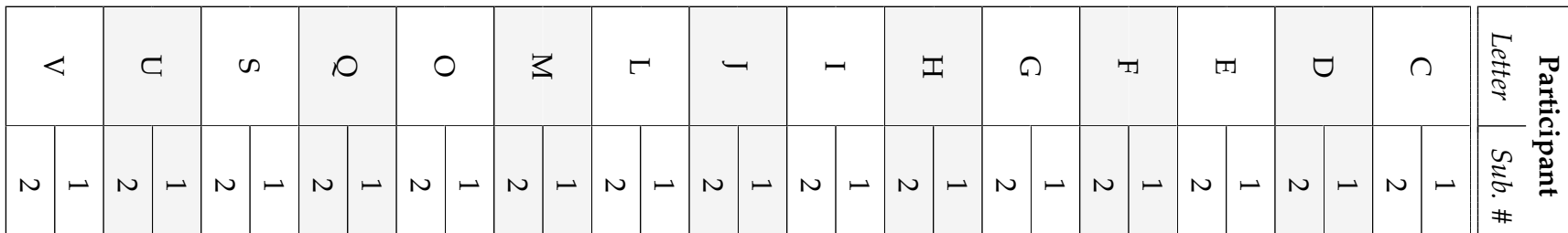

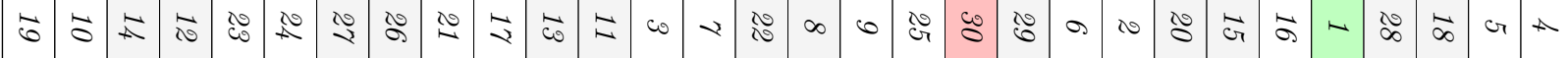

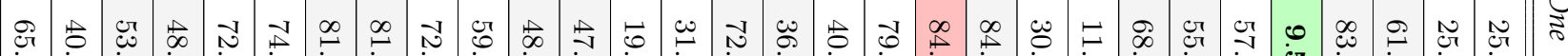

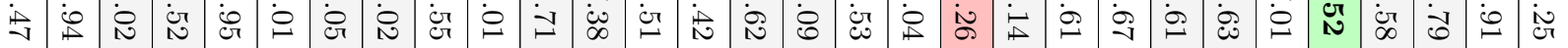

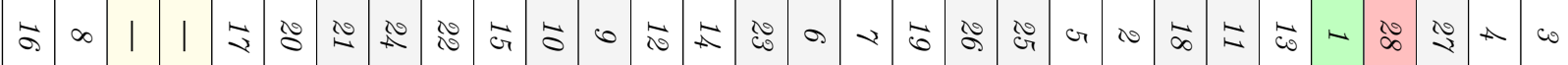

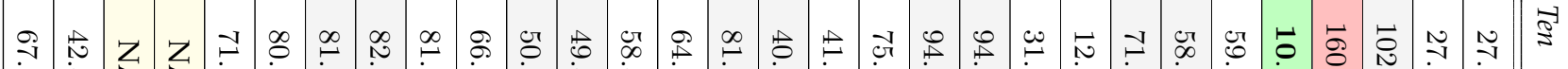

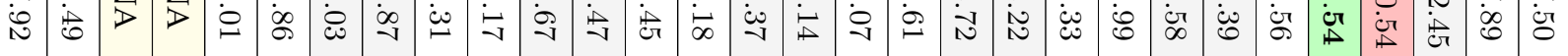

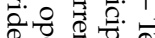

क.

के 류.

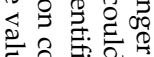

\&

s.

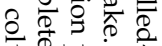

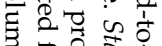

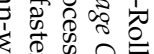

实.

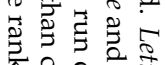

당. 8 क

वa

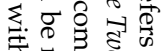

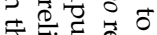

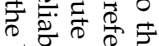

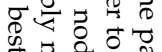

유유.

웅

范范

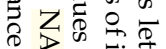

告专

造.

\begin{tabular}{|c|c|c|c|c|c|c|c|c|c|c|c|c|c|c|c|c|c|c|c|c|c|c|c|c|c|c|c|c|c|c|}
\hline- & - & $\mathbb{0}$ & 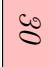 & - & - & $\infty$ & 心 & - & - & - & $\sim$ & - & - & - & - & - & - & $\sim$ & $\sim$ & $\sim$ & $\sim$ & - & - & $\sim$ & - & $\underset{\sim}{*}$ & $\infty$ & - & - & \\
\hline | & 1 & $\mid \begin{array}{l}0 \\
\dot{\omega} \\
\sigma \\
\sigma\end{array}$ & 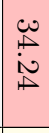 & I & $\mid$ & $\mid \begin{array}{l}0 \\
\dot{\dot{\theta}}\end{array}$ & 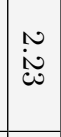 & 1 & 1 & 1 & | & $\mid$ & 1 & 1 & 1 & 1 & 1 & | & | & | & | & 1 & $\mid$ & | & 1 & & $\stackrel{\vec{t}}{\vec{v}}$ & 1 & I & 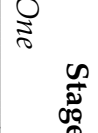 \\
\hline- & $\sim$ & 1 & 1 & - & - & $\stackrel{N}{-1}$ & $\infty$ & $\sim$ & $\sim$ & - & $\sim$ & - & - & - & - & - & - & - & - & $\sim$ & $\sim$ & - & - & - & - & ঝ & 心 & - & $\sim$ & \\
\hline | & 1 & $\underset{>}{Z}$ & $\underset{>}{Z}$ & I & | & $\mid \begin{array}{l}0 \\
\dot{c} \\
\dot{c}\end{array}$ & \begin{tabular}{|l|l}
$N$ \\
$\dot{c} \tilde{\omega}$
\end{tabular} & 1 & 1 & | & | & 1 & 1 & 1 & 1 & | & 1 & | & | & | & | & 1 & 1 & 1 & | & ب̂̀ & $\overrightarrow{\dot{\theta}}$ & 1 & I & \\
\hline$\vec{a}$ & $\vec{\sigma}$ & ¿ & 心 & No & $\frac{10}{d x}$ & $\stackrel{N}{v}$ & $\infty$ & $\overrightarrow{0}$ & $\vec{c}$ & $\vec{\diamond}$ & 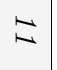 & $\omega$ & $\checkmark$ & ৫ & $\infty$ & 0 & నo & రి & $\infty$ & $a$ & ৯ & $\vec{\infty}$ & $\vec{\omega}$ & 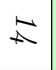 & - & $\infty$ & 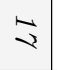 & $G$ & $A$ & \\
\hline 突 & $\begin{array}{l}\qquad \\
0 \\
0 \\
\qquad\end{array}$ & $\mid \begin{array}{c}\vec{T} \\
\dot{d} \\
\dot{c}\end{array}$ & $\begin{array}{l}\infty \\
\stackrel{\infty}{\sim} \\
\stackrel{\sigma}{\sigma}\end{array}$ & 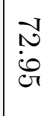 & $\stackrel{\vec{P}}{\stackrel{P}{0}}$ & 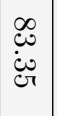 & 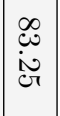 & $\mid \begin{array}{l}N \\
N \\
\dot{c} \\
ن\end{array}$ & $\mid \begin{array}{l}\mathcal{J} \\
0 \\
0 \\
0\end{array}$ & $\begin{array}{l}\infty \\
\infty \\
\dot{v} \\
\forall\end{array}$ & $\begin{array}{l}\vec{v} \\
\dot{i} \\
\infty\end{array}$ & 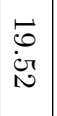 & $\begin{array}{l}\stackrel{\omega}{*} \\
\stackrel{\vec{N}}{N}\end{array}$ & $\begin{array}{c}-1 \\
\cdots \\
0 \\
N \\
N\end{array}$ & $\begin{array}{l}\dot{\omega} \\
\stackrel{0}{0} \\
\dot{0}\end{array}$ & $\begin{array}{l}\theta \\
0 \\
\dot{d} \\
\dot{c}\end{array}$ & $\mid \begin{array}{l}-1 \\
\vdots \\
\dot{1}\end{array}$ & 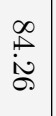 & 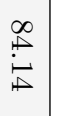 & 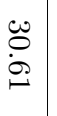 & $\begin{array}{l}\rightleftarrows \\
\dot{v} \\
\vec{v}\end{array}$ & $\mid \begin{array}{l}\ddot{2} \\
\dot{0} \\
\dot{\ominus}\end{array}$ & 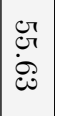 & $\begin{array}{l}\tilde{c} \\
-1 \\
0 \\
0 \\
\text { v }\end{array}$ & $\begin{array}{l}0 \\
\dot{j} \\
\text { j }\end{array}$ & $\begin{array}{l}\infty \\
0 \\
\dot{0} \\
0 \\
0\end{array}$ & 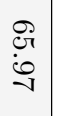 & 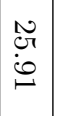 & 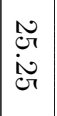 & \\
\hline$\vec{\sigma}$ & $\infty$ & 1 & | & $\vec{\nabla}$ & 8 & D & 必 & : & $\vec{G}$ & $\vec{\sigma}$ & 0 & $\vec{\infty}$ & $\hat{A}$ & 心্ঠি & $\infty$ & 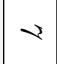 & $\overrightarrow{0}$ & ఠం & $\mathbb{G}$ & $G$ & $\diamond$ & $\vec{\infty}_{0}$ & $\vec{\beth}$ & $\overrightarrow{\omega_{0}}$ & $\leftarrow$ & $\infty$ & $\underset{\sim}{*}$ & $\lambda$ & $\infty$ & \\
\hline$\sim$ & $\begin{array}{l}\vec{N} \\
\overrightarrow{0} \\
\overrightarrow{0}\end{array}$ & $Z_{>}$ & $\underset{>}{Z}$ & $\overrightarrow{2}$ & $\begin{array}{l}\infty \\
0 \\
0\end{array}$ & $\mid$ & $\left|\begin{array}{l}\infty \\
0 \\
\dot{d} \\
0 \\
0\end{array}\right|$ & $\mid \begin{array}{l}\infty \\
\stackrel{\infty}{\omega}\end{array}$ & $\mid \begin{array}{l}\mathscr{g} \\
\stackrel{g}{v}\end{array}$ & 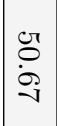 & $\overrightarrow{0}$ & $\left|\begin{array}{l}c \\
\infty \\
\infty \\
\mathbb{1}\end{array}\right|$ & $\mid \begin{array}{l}\stackrel{P}{+} \\
\stackrel{\overrightarrow{0}}{0}\end{array}$ & $\mid \begin{array}{l}\infty \\
\stackrel{\infty}{\dot{\omega}} \\
-v\end{array}$ & $\begin{array}{l}\stackrel{\theta}{0} \\
\dot{\vec{t}}\end{array}$ & $\begin{array}{l}\stackrel{\bullet}{\ominus} \\
\dot{\rho} \\
\mathcal{v}\end{array}$ & $\begin{array}{l}\vec{\jmath} \\
\dot{\rho} \\
\bullet\end{array}$ & $\begin{array}{l}\stackrel{\bullet}{\stackrel{N}{N}} \\
\stackrel{N}{2}\end{array}$ & 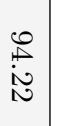 & 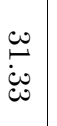 & $\vec{N}$ & $\mid \begin{array}{l}\vec{y} \\
\dot{d} \\
\infty\end{array}$ & $\mid \begin{array}{l}\tilde{r} \\
\infty \\
\dot{j} \\
0\end{array}$ & $\begin{array}{l}\mathcal{J} \\
0 \\
\dot{y} \\
0\end{array}$ & $\begin{array}{l}\overrightarrow{0} \\
\dot{\sigma} \pi \\
\vec{\Delta}\end{array}$ & & $\begin{array}{l}\overrightarrow{0} \\
\stackrel{+}{\dot{\omega}} \\
\dot{\sigma}\end{array}$ & $\begin{array}{l}N \\
-1 \\
0 \\
0\end{array}$ & $\left|\begin{array}{l}0 \\
-1 \\
\dot{c} \\
\dot{c}\end{array}\right|$ & \\
\hline
\end{tabular}

与.

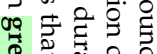

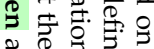

है क 8 क

車 5

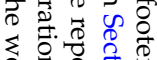

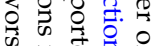

.

둘. 을

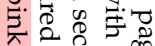

웅

记

象

\begin{tabular}{|c|c|c|c|c|c|c|c|c|c|c|c|c|c|c|c|c|c|c|c|c|c|c|c|c|c|c|c|c|}
\hline$\infty$ & $G$ & ه্ & i & 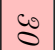 & 8 & $\infty$ & Gr & 0 & $\Xi$ & $\mathbb{N}$ & $\infty$ & 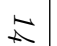 & $\vec{v}$ & 0 & $\overrightarrow{c_{0}}$ & $\infty$ & L & $\overrightarrow{0}$ & 8 온 & 0 is & d & cis & $\vec{\phi}$ & $\infty_{\infty} \mid z$ & 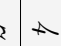 & $\vec{c}$ & $\vec{a}$ & \\
\hline$\stackrel{0}{?}$ & 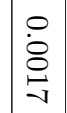 & 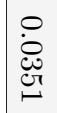 & $\begin{array}{l}0 \\
\dot{0} \\
\dot{d} \\
\tilde{d} \\
\infty\end{array}$ & 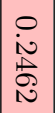 & \begin{tabular}{|l|}
0 \\
$\dot{8}$ \\
$\&$ \\
8 \\
8
\end{tabular} & $\begin{array}{l}0 \\
\dot{8} \\
\stackrel{8}{1} \\
\end{array}$ & $\begin{array}{l}\circ \\
\dot{8}\end{array}$ & $\begin{array}{l}\stackrel{0}{8} \\
\dot{8}\end{array}$ & 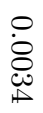 & 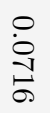 & $\begin{array}{l}\stackrel{0}{0} \\
\dot{0}\end{array}$ & $\begin{array}{l}0 \\
\dot{0} \\
\dot{0} \\
\infty \\
0 \\
ن\end{array}$ & $\begin{array}{l}\circ \\
\dot{8} \\
\stackrel{0}{0} \\
-1\end{array}$ & 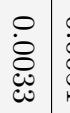 & $\begin{array}{l}\stackrel{0}{0} \\
\dot{8} \\
\stackrel{c}{\oplus}\end{array}$ & & 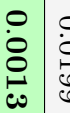 & 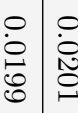 & 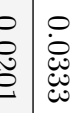 & 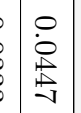 & 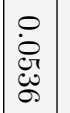 & 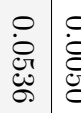 & 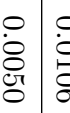 & 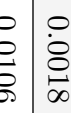 & 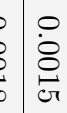 & 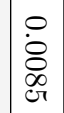 & 영 & \\
\hline
\end{tabular}




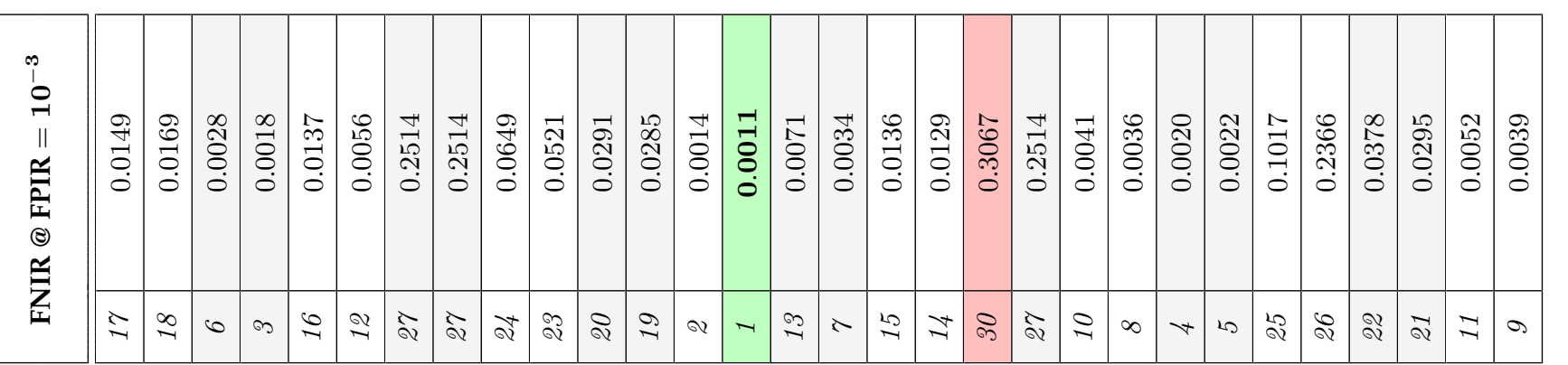

ฮี

. 3 है

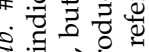

का के

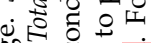

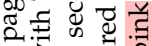

. 3.5

₹ 10 ठ

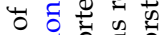

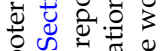

象

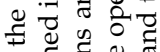

ธ。

चี

요묘

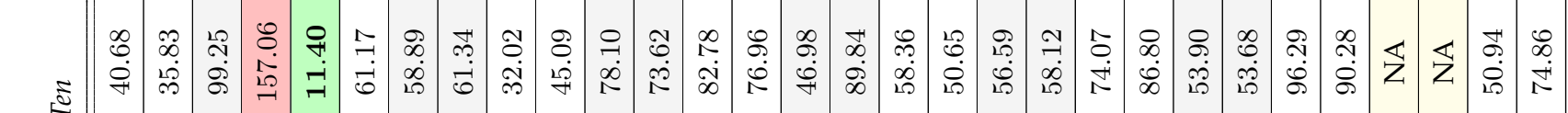

คี

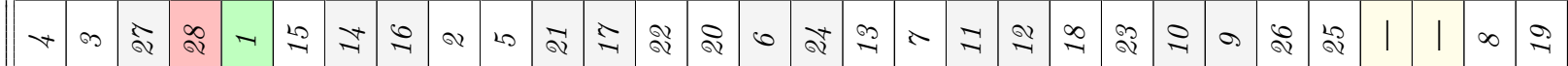

L そ

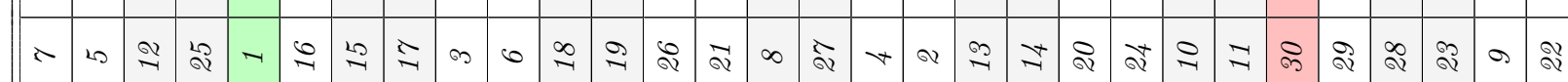

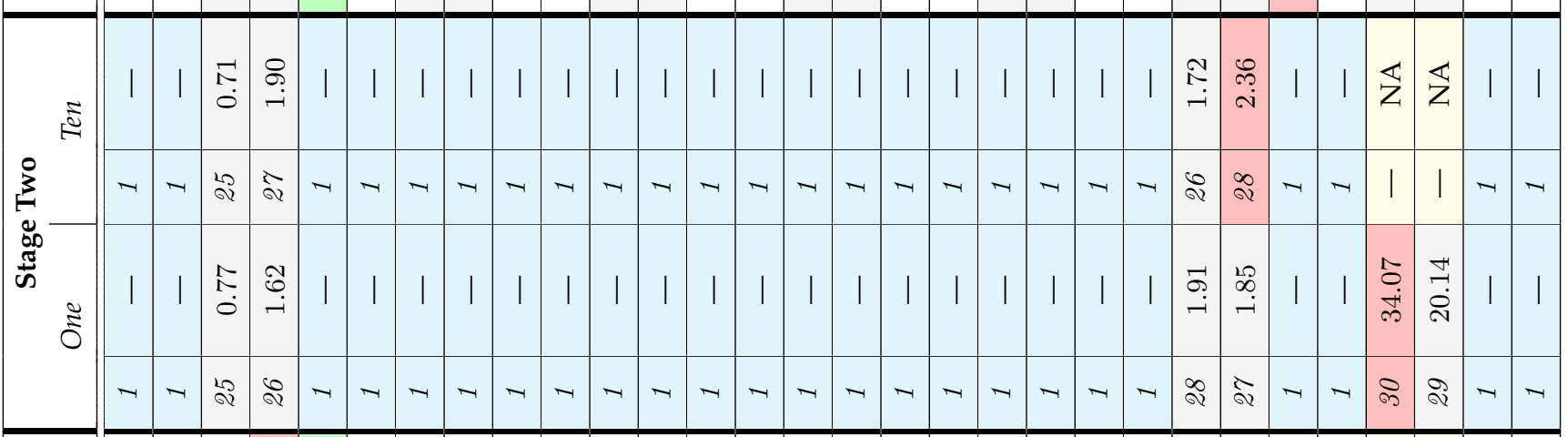

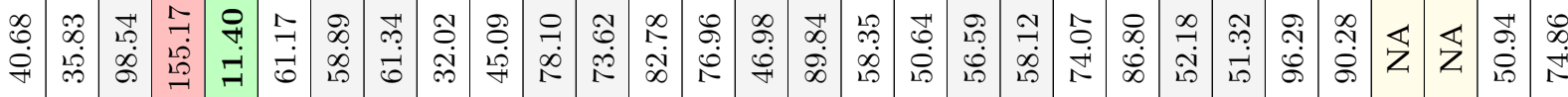

$-\infty$

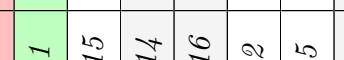

จิ

จ 6 สं ชั

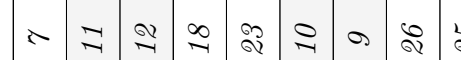

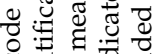

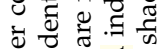

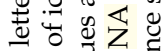

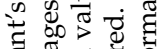

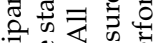

प्ञ

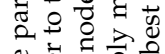

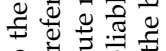

울

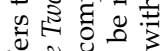

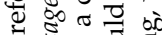

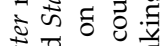

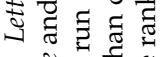

ठ்

空

के

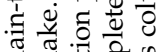

ब ब है है क्ष

就 등

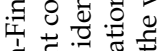

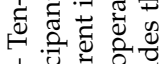

U

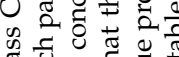

造记

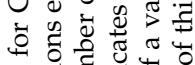

क क्ष

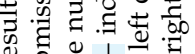

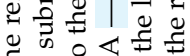

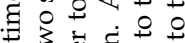

L

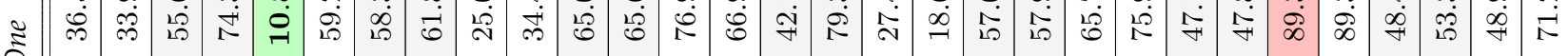

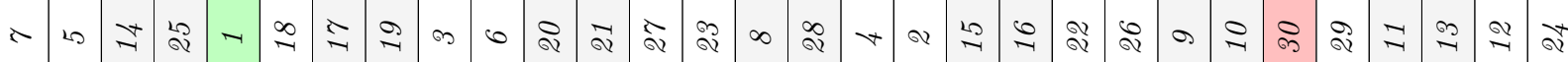

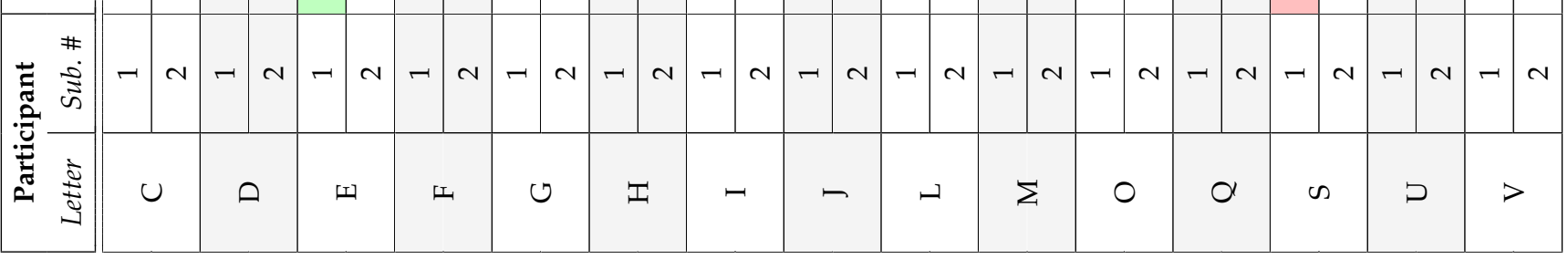

王

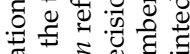

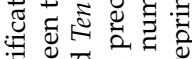

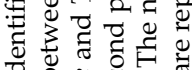

궁

⿷

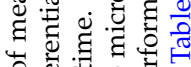

웜

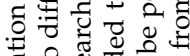

준 志岛 


\section{E Progression for Last Two Submissions}

This appendix provides additional information in reference to comments from Section 8 that looked at accuracy versus search time. The tables in this appendix show search times for the last two of the three submission periods during the evaluation. Generally, participants attempt to improve accuracy with potential tradeoffs in speed and template size. The tables in this section show the search times and FNIR @ FPIR $=10^{-3}$ for the these two submissions.

Class A results are in Tables 50 through 52, Class B results are in Tables 53 through 56, and Class C results are in Tables 57 through 59.

\begin{tabular}{l|l|l|l|ll|l}
$\mathrm{C}=$ afis team & $\mathrm{D}=3 \mathrm{M}$ Cogent & $\mathrm{E}=$ Neurotechnology & $\mathrm{F}=$ Papillon & $\mathrm{G}=$ Dermalog & $\mathrm{H}=$ Hisign Bio-Info Institute \\
$\mathrm{I}=$ NEC & $\mathrm{J}=$ Sonda & $\mathrm{K}=$ Tiger IT & $\mathrm{L}=$ Innovatrics & $\mathrm{M}=$ SPEX & $\mathrm{O}=$ ID Solutions \\
$\mathrm{P}=\mathrm{id} 3$ & $\mathrm{Q}=$ Morpho & $\mathrm{S}=$ Decatur Industries & $\mathrm{T}=$ BIO-key & $\mathrm{U}=$ Aware & $\mathrm{V}=$ AA Technology
\end{tabular}




\begin{tabular}{|c|c|c|c|c|c|c|c|c|c|c|}
\hline & \multicolumn{2}{|c|}{ Participant } & \multicolumn{4}{|c|}{ Second } & \multicolumn{4}{|c|}{ Third } \\
\hline & Letter & Sub.\# & \multicolumn{2}{|c|}{ Time } & \multicolumn{2}{|c|}{ FNIR } & \multicolumn{2}{|c|}{ Time } & \multicolumn{2}{|c|}{ FNIR } \\
\hline \multirow{30}{*}{ 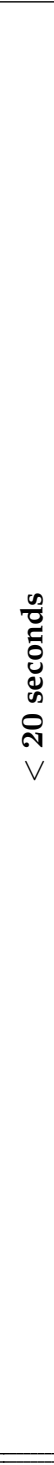 } & \multirow{2}{*}{$\mathrm{C}$} & 1 & 1 & 0.12 & 16 & 0.1077 & 1 & 0.29 & 24 & 0.1335 \\
\hline & & 2 & 6 & 0.75 & 15 & 0.0984 & 6 & 0.87 & 25 & 0.1337 \\
\hline & $\mathrm{D}$ & 1 & 9 & 1.79 & 2 & 0.0376 & 20 & 7.52 & 1 & 0.0197 \\
\hline & \multirow{2}{*}{$\mathrm{E}$} & 1 & 3 & 0.42 & 10 & 0.0794 & 4 & 0.57 & 12 & 0.0745 \\
\hline & & 2 & 22 & 15.40 & 8 & 0.0757 & 30 & 16.71 & 11 & 0.0723 \\
\hline & \multirow{2}{*}{$\mathrm{F}$} & 1 & 11 & 2.50 & 18 & 0.1122 & 11 & 2.52 & 20 & 0.1111 \\
\hline & & 2 & 15 & 3.64 & 17 & 0.1093 & 15 & 3.78 & 18 & 0.1082 \\
\hline & G & 1 & 16 & 5.30 & 23 & 0.1221 & 21 & 9.52 & 19 & 0.1089 \\
\hline & \multirow{2}{*}{$\mathrm{H}$} & 1 & 5 & 0.71 & 20 & 0.1168 & 14 & 3.65 & 26 & 0.1576 \\
\hline & & 2 & 7 & 0.94 & 19 & 0.1160 & 17 & 4.26 & 27 & 0.1607 \\
\hline & I & 1 & 18 & 6.19 & 1 & 0.0306 & 22 & 10.36 & 5 & 0.0257 \\
\hline & \multirow{2}{*}{$\mathrm{J}$} & 1 & 2 & 0.35 & 13 & 0.0900 & 3 & 0.56 & 14 & 0.0786 \\
\hline & & 2 & 4 & 0.60 & 9 & 0.0773 & 7 & 1.34 & 10 & 0.0712 \\
\hline & \multirow{2}{*}{$\mathrm{K}$} & 1 & - & - & - & - & 25 & 10.48 & 17 & 0.0883 \\
\hline & & 2 & - & - & - & - & 24 & 10.47 & 16 & 0.0875 \\
\hline & \multirow{2}{*}{$\mathrm{L}$} & 1 & 8 & 1.76 & 14 & 0.0913 & 2 & 0.30 & 8 & 0.0625 \\
\hline & & 2 & 14 & 3.04 & 12 & 0.0881 & 12 & 3.34 & 6 & 0.0351 \\
\hline & \multirow{2}{*}{ M } & 1 & - & - & - & - & 10 & 2.07 & 29 & 0.2995 \\
\hline & & 2 & - & - & - & - & 27 & 15.19 & 28 & 0.2921 \\
\hline & \multirow{2}{*}{$\mathrm{O}$} & 1 & 10 & 1.96 & 7 & 0.0751 & 5 & 0.64 & 15 & 0.0818 \\
\hline & & 2 & 13 & 2.95 & 6 & 0.0735 & 9 & 1.63 & 13 & 0.0766 \\
\hline & \multirow{2}{*}{$\mathrm{P}$} & 1 & - & - & - & - & 8 & 1.38 & 23 & 0.1308 \\
\hline & & 2 & 12 & 2.83 & 24 & 0.1343 & 13 & 3.43 & 22 & 0.1272 \\
\hline & \multirow{2}{*}{ Q } & 1 & 20 & 6.97 & 4 & 0.0507 & 29 & 15.90 & 2 & 0.0222 \\
\hline & & 2 & 19 & 6.42 & 5 & 0.0511 & 23 & 10.42 & 3 & 0.0226 \\
\hline & $S$ & 1 & 17 & 5.99 & 11 & 0.0811 & 28 & 15.37 & 7 & 0.0571 \\
\hline & \multirow{2}{*}{$\mathrm{T}$} & 1 & 23 & 16.49 & 21 & 0.1181 & 16 & 4.18 & 30 & NA \\
\hline & & 2 & - & - & - & - & 18 & 5.96 & 9 & 0.0685 \\
\hline & $\mathrm{U}$ & 1 & 24 & 18.62 & 22 & 0.1209 & 26 & 14.72 & 21 & 0.1218 \\
\hline & $\mathrm{V}$ & 1 & 21 & 10.34 & 3 & 0.0402 & 19 & 6.22 & 4 & 0.0253 \\
\hline \multirow{6}{*}{ 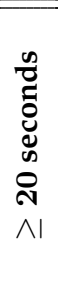 } & $\mathrm{D}$ & 2 & 1 & 3.99 & 1 & 0.0269 & 2 & 42.64 & 1 & 0.0197 \\
\hline & G & 2 & 4 & 27.35 & 5 & 0.1210 & 5 & 54.24 & 5 & 0.1086 \\
\hline & $\mathrm{I}$ & 2 & 3 & 9.04 & 2 & 0.0274 & 3 & 43.24 & 3 & 0.0278 \\
\hline & $S$ & 2 & 2 & 5.94 & 4 & 0.0811 & 4 & 45.59 & 4 & 0.0650 \\
\hline & $\mathrm{U}$ & 2 & - & - & - & - & 1 & 25.66 & 6 & 0.1178 \\
\hline & $\mathrm{V}$ & 2 & 5 & 61.50 & 3 & 0.0395 & 6 & 66.23 & 2 & 0.0252 \\
\hline
\end{tabular}

Table 50: Tabulation of the progression of identification timing and accuracy for Class A - Left Index. Submissions were split into two groups. The first group includes submissions that, in Third, performed searches on average in less than 20 seconds, and the second includes those that took, on average, 20 seconds or longer. Letter refers to the participant's letter code found on the footer of this page. Sub. \# is an identifier used to differentiate between the two submissions each participant could make. The Time column shows the time used to perform a search over an enrollment set of 100000 in seconds, but was originally recorded to microsecond precision. The FNIR column shows FNIR for each submission at FPIR $=10^{-3}$. NA indicates that the operations required to produce the value could not be performed. - indicates that there was not a validated submission during that submission period. The number to the left of a value provides the value's column-wise ranking.

\begin{tabular}{l|l|l|l|ll|l}
$\mathrm{C}=$ afis team & $\mathrm{D}=3 \mathrm{M}$ Cogent & $\mathrm{E}=$ Neurotechnology & $\mathrm{F}=$ Papillon & $\mathrm{G}=$ Dermalog & $\mathrm{H}=$ Hisign Bio-Info Institute \\
$\mathrm{I}=$ NEC & $\mathrm{J}=$ Sonda & $\mathrm{K}=$ Tiger IT & $\mathrm{L}=$ Innovatrics & $\mathrm{M}=$ SPEX & $\mathrm{O}=$ ID Solutions \\
$\mathrm{P}=\mathrm{id} 3$ & $\mathrm{Q}=$ Morpho & $\mathrm{S}=$ Decatur Industries & $\mathrm{T}=$ BIO-key & $\mathrm{U}=$ Aware & $\mathrm{V}=$ AA Technology
\end{tabular}




\begin{tabular}{|c|c|c|c|c|c|c|c|c|c|c|}
\hline & \multicolumn{2}{|c|}{ Participant } & \multicolumn{4}{|c|}{ Second } & \multicolumn{4}{|c|}{ Third } \\
\hline & \multirow{3}{*}{$\frac{\text { Letter }}{\mathrm{C}}$} & \multirow{2}{*}{$\frac{\text { Sub. \# }}{2}$} & \multicolumn{2}{|c|}{ Time } & \multicolumn{2}{|c|}{ FNIR } & \multicolumn{2}{|c|}{ Time } & \multicolumn{2}{|c|}{ FNIR } \\
\hline \multirow{30}{*}{ 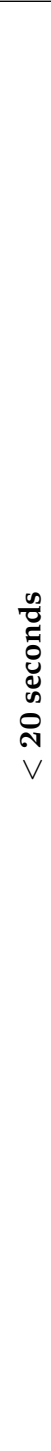 } & & & 1 & 0.11 & 20 & 0.1022 & 1 & 0.25 & 24 & 0.1132 \\
\hline & & 2 & 6 & 0.67 & 15 & 0.0899 & 6 & 0.76 & 23 & 0.1124 \\
\hline & D & 1 & 9 & 1.77 & 5 & 0.0337 & 20 & 7.68 & 1 & 0.0190 \\
\hline & \multirow{2}{*}{$\mathrm{E}$} & 1 & 2 & 0.37 & 12 & 0.0733 & 3 & 0.45 & 11 & 0.0630 \\
\hline & & 2 & 23 & 14.66 & 10 & 0.0709 & 30 & 15.89 & 10 & 0.0624 \\
\hline & \multirow{2}{*}{$\mathrm{F}$} & 1 & 11 & 2.26 & 18 & 0.0945 & 11 & 2.52 & 20 & 0.0933 \\
\hline & & 2 & 15 & 3.30 & 17 & 0.0928 & 15 & 3.71 & 18 & 0.0903 \\
\hline & G & 1 & 16 & 5.39 & 19 & 0.1018 & 21 & 10.12 & 19 & 0.0910 \\
\hline & \multirow{2}{*}{$\mathrm{H}$} & 1 & 5 & 0.65 & 22 & 0.1035 & 14 & 3.58 & 26 & 0.1230 \\
\hline & & 2 & 7 & 0.88 & 21 & 0.1025 & 17 & 4.13 & 27 & 0.1249 \\
\hline & I & 1 & 17 & 5.91 & 1 & 0.0232 & 23 & 10.19 & 3 & 0.0215 \\
\hline & \multirow{2}{*}{$\mathrm{J}$} & 1 & 3 & 0.37 & 14 & 0.0800 & 4 & 0.53 & 16 & 0.0708 \\
\hline & & 2 & 4 & 0.55 & 9 & 0.0700 & 7 & 1.25 & 12 & 0.0643 \\
\hline & \multirow{2}{*}{$\mathrm{K}$} & 1 & - & - & - & - & 25 & 10.42 & 14 & 0.0682 \\
\hline & & 2 & - & - & - & - & 24 & 10.30 & 15 & 0.0685 \\
\hline & \multirow{2}{*}{$\mathrm{L}$} & 1 & 8 & 1.54 & 13 & 0.0739 & 2 & 0.29 & 8 & 0.0505 \\
\hline & & 2 & 14 & 2.94 & 11 & 0.0721 & 12 & 3.26 & 6 & 0.0295 \\
\hline & \multirow{2}{*}{ M } & 1 & - & - & - & - & 10 & 1.75 & 30 & 0.2615 \\
\hline & & 2 & - & - & - & - & 26 & 11.31 & 29 & 0.2526 \\
\hline & \multirow{2}{*}{$\mathrm{O}$} & 1 & 10 & 1.89 & 7 & 0.0672 & 5 & 0.59 & 17 & 0.0776 \\
\hline & & 2 & 13 & 2.79 & 8 & 0.0673 & 9 & 1.48 & 13 & 0.0675 \\
\hline & \multirow{2}{*}{$\mathrm{P}$} & 1 & - & - & - & - & 8 & 1.38 & 25 & 0.1133 \\
\hline & & 2 & 12 & 2.62 & 24 & 0.1120 & 13 & 3.49 & 22 & 0.1100 \\
\hline & \multirow{2}{*}{$\mathrm{Q}$} & 1 & 22 & 12.96 & 3 & 0.0248 & 28 & 14.44 & 4 & 0.0218 \\
\hline & & 2 & 19 & 9.23 & 2 & 0.0242 & 22 & 10.15 & 2 & 0.0214 \\
\hline & $S$ & 1 & 18 & 6.18 & 6 & 0.0640 & 29 & 15.08 & 7 & 0.0442 \\
\hline & \multirow{2}{*}{$\mathrm{T}$} & 1 & 21 & 12.30 & 16 & 0.0924 & 16 & 3.82 & 28 & 0.1929 \\
\hline & & 2 & - & - & - & - & 18 & 5.64 & 9 & 0.0562 \\
\hline & $\mathrm{U}$ & 1 & 24 & 15.97 & 23 & 0.1048 & 27 & 12.02 & 21 & 0.0996 \\
\hline & V & 1 & 20 & 9.96 & 4 & 0.0331 & 19 & 5.95 & 5 & 0.0223 \\
\hline \multirow{6}{*}{ 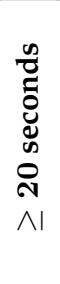 } & D & 2 & 2 & 6.25 & 2 & 0.0248 & 2 & 29.26 & 1 & 0.0190 \\
\hline & G & 2 & 4 & 26.76 & 5 & 0.1007 & 5 & 57.88 & 5 & 0.0909 \\
\hline & I & 2 & 3 & 9.31 & 1 & 0.0217 & 3 & 41.60 & 2 & 0.0214 \\
\hline & $S$ & 2 & 1 & 6.20 & 4 & 0.0641 & 4 & 43.56 & 4 & 0.0503 \\
\hline & $\mathrm{U}$ & 2 & - & - & - & - & 1 & 22.39 & 6 & 0.1007 \\
\hline & V & 2 & 5 & 60.50 & 3 & 0.0330 & 6 & 64.47 & 3 & 0.0222 \\
\hline
\end{tabular}

Table 51: Tabulation of the progression of identification timing and accuracy for Class A - Right Index. Submissions were split into two groups. The first group includes submissions that, in Third, performed searches on average in less than 20 seconds, and the second includes those that took, on average, 20 seconds or longer. Letter refers to the participant's letter code found on the footer of this page. Sub. \# is an identifier used to differentiate between the two submissions each participant could make. The Time column shows the time used to perform a search over an enrollment set of 100000 in seconds, but was originally recorded to microsecond precision. The FNIR column shows FNIR for each submission at FPIR $=10^{-3}$. - indicates that there was not a validated submission during that submission period. The number to the left of a value provides the value's column-wise ranking.

\begin{tabular}{l|l|l|l|ll|l}
$\mathrm{C}=$ afis team & $\mathrm{D}=3 \mathrm{M}$ Cogent & $\mathrm{E}=$ Neurotechnology & $\mathrm{F}=$ Papillon & $\mathrm{G}=$ Dermalog & $\mathrm{H}=$ Hisign Bio-Info Institute \\
$\mathrm{I}=$ NEC & $\mathrm{J}=$ Sonda & $\mathrm{K}=$ Tiger IT & $\mathrm{L}=$ Innovatrics & $\mathrm{M}=$ SPEX & $\mathrm{O}=$ ID Solutions \\
$\mathrm{P}=\mathrm{id} 3$ & $\mathrm{Q}=$ Morpho & $\mathrm{S}=$ Decatur Industries & $\mathrm{T}=$ BIO-key & $\mathrm{U}=$ Aware & $\mathrm{V}=$ AA Technology
\end{tabular}




\begin{tabular}{|c|c|c|c|c|c|c|c|c|c|c|}
\hline & \multicolumn{2}{|c|}{ Participant } & \multicolumn{4}{|c|}{ Second } & \multicolumn{4}{|c|}{ Third } \\
\hline & Letter & Sub.\# & \multicolumn{2}{|c|}{ Time } & \multicolumn{2}{|c|}{ FNIR } & \multicolumn{2}{|c|}{ Time } & \multicolumn{2}{|c|}{ FNIR } \\
\hline \multirow{9}{*}{ 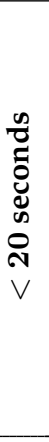 } & \multirow{2}{*}{$\mathrm{C}$} & 1 & 1 & 1.48 & 6 & 0.0313 & 2 & 2.39 & 7 & 0.0368 \\
\hline & & 2 & 2 & 6.30 & 7 & 0.0354 & 4 & 6.34 & 8 & 0.0374 \\
\hline & G & 1 & 3 & 7.38 & 8 & 0.1426 & 3 & 6.27 & 9 & 0.0515 \\
\hline & $\mathrm{I}$ & 1 & 7 & 19.73 & 2 & 0.0073 & 9 & 18.92 & 2 & 0.0058 \\
\hline & $\mathrm{J}$ & 1 & 5 & 9.20 & 4 & 0.0161 & 6 & 14.00 & 3 & 0.0143 \\
\hline & $\mathrm{K}$ & 1 & - & - & - & - & 8 & 18.32 & 6 & 0.0360 \\
\hline & $\mathrm{L}$ & 1 & 6 & 17.81 & 5 & 0.0306 & 1 & 2.20 & 4 & 0.0146 \\
\hline & $\mathrm{O}$ & 1 & 8 & 57.36 & 3 & 0.0132 & 7 & 15.34 & 5 & 0.0229 \\
\hline & $\mathrm{V}$ & 1 & 4 & 8.56 & 1 & 0.0056 & 5 & 9.50 & 1 & 0.0034 \\
\hline \multirow{27}{*}{ 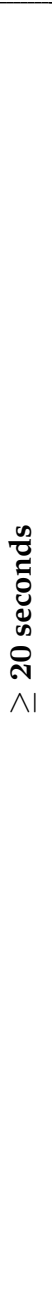 } & \multirow{2}{*}{$\mathrm{D}$} & 1 & 2 & 14.06 & 5 & 0.0046 & 16 & 73.01 & 4 & 0.0030 \\
\hline & & 2 & 9 & 46.57 & 1 & 0.0033 & 23 & 234.52 & 4 & 0.0030 \\
\hline & \multirow{2}{*}{$\mathrm{E}$} & 1 & 4 & 17.36 & 10 & 0.0216 & 2 & 22.27 & 11 & 0.0207 \\
\hline & & 2 & 22 & 463.95 & 9 & 0.0208 & 27 & 500.30 & 10 & 0.0202 \\
\hline & \multirow{2}{*}{$\mathrm{F}$} & 1 & 12 & 56.49 & 15 & 0.0392 & 13 & 59.30 & 21 & 0.0386 \\
\hline & & 2 & 13 & 67.36 & 16 & 0.0407 & 15 & 71.45 & 22 & 0.0412 \\
\hline & G & 2 & 19 & 166.37 & 21 & 0.0558 & 22 & 227.27 & 15 & 0.0311 \\
\hline & \multirow{2}{*}{$\mathrm{H}$} & 1 & 5 & 18.42 & 20 & 0.0538 & 8 & 37.65 & 24 & 0.0686 \\
\hline & & 2 & 6 & 25.38 & 19 & 0.0537 & 12 & 53.77 & 23 & 0.0684 \\
\hline & I & 2 & 18 & 162.34 & 4 & 0.0038 & 25 & 385.14 & 4 & 0.0030 \\
\hline & $\mathrm{J}$ & 2 & 3 & 16.23 & 8 & 0.0137 & 7 & 36.19 & 8 & 0.0143 \\
\hline & $\mathrm{K}$ & 2 & - & - & - & - & 6 & 32.68 & 14 & 0.0286 \\
\hline & $\mathrm{L}$ & 2 & 7 & 34.31 & 13 & 0.0365 & 3 & 23.54 & 7 & 0.0072 \\
\hline & \multirow{2}{*}{ M } & 1 & - & - & - & - & 5 & 32.59 & 26 & NA \\
\hline & & 2 & - & - & - & - & 20 & 183.20 & 25 & NA \\
\hline & $\mathrm{O}$ & 2 & 15 & 85.37 & 7 & 0.0134 & 10 & 45.52 & 12 & 0.0214 \\
\hline & \multirow{2}{*}{$\mathrm{P}$} & 1 & - & - & - & - & 14 & 63.41 & 20 & 0.0370 \\
\hline & & 2 & 16 & 89.78 & 17 & 0.0408 & 17 & 114.37 & 16 & 0.0333 \\
\hline & \multirow{2}{*}{ Q } & 1 & 14 & 85.29 & 3 & 0.0035 & 21 & 213.08 & 1 & 0.0027 \\
\hline & & 2 & 10 & 48.40 & 2 & 0.0034 & 19 & 163.65 & 1 & 0.0027 \\
\hline & \multirow{2}{*}{$S$} & 1 & 1 & 13.37 & 14 & 0.0379 & 1 & 20.11 & 13 & 0.0281 \\
\hline & & 2 & 11 & 54.11 & 11 & 0.0316 & 26 & 429.02 & 9 & 0.0195 \\
\hline & \multirow{2}{*}{$\mathrm{T}$} & 1 & 17 & 138.43 & 12 & 0.0318 & 4 & 26.96 & 27 & NA \\
\hline & & 2 & - & - & - & - & 9 & 38.91 & 19 & 0.0366 \\
\hline & \multirow{2}{*}{$\mathrm{U}$} & 1 & 8 & 38.87 & 18 & 0.0532 & 11 & 47.60 & 17 & 0.0336 \\
\hline & & 2 & 21 & 288.82 & 22 & 0.2620 & 24 & 252.04 & 18 & 0.0358 \\
\hline & $\mathrm{V}$ & 2 & 20 & 197.77 & 6 & 0.0048 & 18 & 133.45 & 3 & 0.0028 \\
\hline
\end{tabular}

Table 52: Tabulation of the progression of identification timing and accuracy for Class A — Left and Right Index. Submissions were split into two groups. The first group includes submissions that, in Third, performed searches on average in less than 20 seconds, and the second includes those that took, on average, 20 seconds or longer. Letter refers to the participant's letter code found on the footer of this page. Sub. \# is an identifier used to differentiate between the two submissions each participant could make. The Time column shows the time used to perform a search over an enrollment set of 1600000 in seconds, but was originally recorded to microsecond precision. The FNIR column shows FNIR for each submission at FPIR $=10^{-3}$. NA indicates that the operations required to produce the value could not be performed. — indicates that there was not a validated submission during that submission period. The number to the left of a value provides the value's column-wise ranking.

\begin{tabular}{l|l|l|l|ll|l}
$\mathrm{C}=$ afis team & $\mathrm{D}=3 \mathrm{M}$ Cogent & $\mathrm{E}=$ Neurotechnology & $\mathrm{F}=$ Papillon & $\mathrm{G}=$ Dermalog & $\mathrm{H}=$ Hisign Bio-Info Institute \\
$\mathrm{I}=$ NEC & $\mathrm{J}=$ Sonda & $\mathrm{K}=$ Tiger IT & $\mathrm{L}=$ Innovatrics & $\mathrm{M}=$ SPEX & $\mathrm{O}=$ ID Solutions \\
$\mathrm{P}=\mathrm{id} 3$ & $\mathrm{Q}=$ Morpho & $\mathrm{S}=$ Decatur Industries & $\mathrm{T}=$ BIO-key & $\mathrm{U}=$ Aware & $\mathrm{V}=$ AA Technology
\end{tabular}




\begin{tabular}{|c|c|c|c|c|c|c|c|c|c|}
\hline \multicolumn{2}{|c|}{ Participant } & \multicolumn{4}{|c|}{ Second } & \multicolumn{4}{|c|}{ Third } \\
\hline Letter & Sub.\# & \multicolumn{2}{|c|}{ Time } & \multicolumn{2}{|c|}{ FNIR } & \multicolumn{2}{|c|}{ Time } & \multicolumn{2}{|c|}{ FNIR } \\
\hline \multirow{2}{*}{$\mathrm{C}$} & 1 & 1 & 3.20 & 14 & 0.0667 & 2 & 3.71 & 22 & 0.0654 \\
\hline & 2 & 3 & 6.18 & 15 & 0.0669 & 4 & 6.62 & 21 & 0.0647 \\
\hline \multirow{2}{*}{$\mathrm{D}$} & 1 & 14 & 39.90 & 5 & 0.0178 & 20 & 53.09 & 6 & 0.0163 \\
\hline & 2 & 18 & 55.83 & 7 & 0.0242 & 21 & 59.65 & 5 & 0.0142 \\
\hline \multirow{2}{*}{$\mathrm{E}$} & 1 & 2 & 3.37 & 12 & 0.0397 & 1 & 3.71 & 13 & 0.0259 \\
\hline & 2 & 7 & 19.68 & 8 & 0.0277 & 12 & 41.57 & 7 & 0.0187 \\
\hline \multirow{2}{*}{$\mathrm{F}$} & 1 & - & - & - & - & 8 & 31.92 & 29 & 0.1684 \\
\hline & 2 & - & - & - & - & 13 & 46.18 & 28 & 0.1681 \\
\hline \multirow{2}{*}{ G } & 1 & 12 & 33.42 & 17 & 0.0704 & 6 & 19.65 & 18 & 0.0371 \\
\hline & 2 & 19 & 61.44 & 16 & 0.0677 & 22 & 62.98 & 17 & 0.0325 \\
\hline \multirow{2}{*}{$\mathrm{H}$} & 1 & 13 & 39.16 & 18 & 0.0798 & 15 & 49.13 & 23 & 0.0998 \\
\hline & 2 & 17 & 46.21 & 20 & 0.0813 & 19 & 52.05 & 24 & 0.1008 \\
\hline \multirow{2}{*}{ I } & 1 & 21 & 77.87 & 4 & 0.0161 & 23 & 63.06 & 4 & 0.0116 \\
\hline & 2 & 20 & 67.21 & 3 & 0.0127 & 18 & 51.59 & 1 & 0.0094 \\
\hline \multirow{2}{*}{$\mathrm{J}$} & 1 & 5 & 11.15 & 13 & 0.0491 & 7 & 26.88 & 15 & 0.0287 \\
\hline & 2 & 11 & 31.32 & 9 & 0.0340 & 27 & 80.54 & 10 & 0.0236 \\
\hline \multirow{2}{*}{$\mathrm{L}$} & 1 & 4 & 10.66 & 19 & 0.0809 & 3 & 5.51 & 16 & 0.0288 \\
\hline & 2 & 8 & 23.68 & 20 & 0.0813 & 5 & 12.52 & 14 & 0.0276 \\
\hline \multirow{2}{*}{ M } & 1 & - & - & - & - & 9 & 32.39 & 30 & 0.1736 \\
\hline & 2 & 22 & 89.64 & 22 & 0.1634 & 30 & 90.51 & 27 & 0.1634 \\
\hline \multirow{2}{*}{$\mathrm{O}$} & 1 & 6 & 19.29 & 11 & 0.0366 & 11 & 38.17 & 12 & 0.0257 \\
\hline & 2 & 9 & 30.71 & 10 & 0.0349 & 24 & 78.80 & 11 & 0.0254 \\
\hline \multirow{2}{*}{ Q } & 1 & 16 & 45.67 & 1 & 0.0104 & 17 & 51.33 & 2 & 0.0098 \\
\hline & 2 & 10 & 31.26 & 2 & 0.0116 & 16 & 51.05 & 3 & 0.0099 \\
\hline \multirow{2}{*}{ S } & 1 & - & - & - & - & 25 & 78.95 & 25 & 0.1089 \\
\hline & 2 & - & - & - & - & 26 & 79.01 & 26 & 0.1133 \\
\hline \multirow{2}{*}{$\mathrm{U}$} & 1 & - & - & - & - & 28 & 81.85 & 20 & 0.0500 \\
\hline & 2 & - & - & - & - & 29 & 90.50 & 19 & 0.0461 \\
\hline \multirow{2}{*}{$\mathrm{V}$} & 1 & 15 & 42.01 & 6 & 0.0239 & 10 & 36.28 & 9 & 0.0192 \\
\hline & 2 & - & - & - & - & 14 & 48.01 & 8 & 0.0190 \\
\hline
\end{tabular}

Table 53: Tabulation of the progression of identification timing and accuracy for Class B — Left Slap. Letter refers to the participant's letter code found on the footer of this page. Sub. \# is an identifier used to differentiate between the two submissions each participant could make. The Time column shows the time used to perform a search over an enrollment set of 3000000 in seconds, but was originally recorded to microsecond precision. The FNIR column shows FNIR for each submission at FPIR $=10^{-3}$. - indicates that there was not a validated submission during that submission period. The number to the left of a value provides the value's column-wise ranking.

\begin{tabular}{l|l|l|l|ll|l}
$\mathrm{C}=$ afis team & $\mathrm{D}=3 \mathrm{M}$ Cogent & $\mathrm{E}=$ Neurotechnology & $\mathrm{F}=$ Papillon & $\mathrm{G}=$ Dermalog & $\mathrm{H}=$ Hisign Bio-Info Institute \\
$\mathrm{I}=$ NEC & $\mathrm{J}=$ Sonda & $\mathrm{K}=$ Tiger IT & $\mathrm{L}=$ Innovatrics & $\mathrm{M}=$ SPEX & $\mathrm{O}=$ ID Solutions \\
$\mathrm{P}=\mathrm{id} 3$ & $\mathrm{Q}=$ Morpho & $\mathrm{S}=$ Decatur Industries & $\mathrm{T}=$ BIO-key & $\mathrm{U}=$ Aware & $\mathrm{V}=$ AA Technology
\end{tabular}




\begin{tabular}{|c|c|c|c|c|c|c|c|c|c|}
\hline \multicolumn{2}{|c|}{ Participant } & \multicolumn{4}{|c|}{ Second } & \multicolumn{4}{|c|}{ Third } \\
\hline Letter & Sub.\# & \multicolumn{2}{|c|}{ Time } & \multicolumn{2}{|c|}{ FNIR } & \multicolumn{2}{|c|}{ Time } & \multicolumn{2}{|c|}{ FNIR } \\
\hline \multirow{2}{*}{$\mathrm{C}$} & 1 & 1 & 3.16 & 16 & 0.0391 & 1 & 3.70 & 24 & 0.0403 \\
\hline & 2 & 3 & 6.10 & 14 & 0.0386 & 4 & 6.54 & 23 & 0.0392 \\
\hline \multirow{2}{*}{$\mathrm{D}$} & 1 & 13 & 37.74 & 5 & 0.0082 & 19 & 53.78 & 6 & 0.0072 \\
\hline & 2 & 19 & 56.55 & 6 & 0.0100 & 20 & 58.67 & 2 & 0.0052 \\
\hline \multirow{2}{*}{$\mathrm{E}$} & 1 & 2 & 3.71 & 11 & 0.0220 & 2 & 4.68 & 13 & 0.0151 \\
\hline & 2 & 9 & 25.48 & 7 & 0.0134 & 12 & 40.27 & 7 & 0.0083 \\
\hline \multirow{2}{*}{$\mathrm{F}$} & 1 & - & - & - & - & 8 & 32.28 & 29 & 0.1222 \\
\hline & 2 & - & - & - & - & 14 & 46.58 & 28 & 0.1220 \\
\hline \multirow{2}{*}{ G } & 1 & 6 & 19.93 & 17 & 0.0423 & 5 & 11.80 & 18 & 0.0212 \\
\hline & 2 & 15 & 41.42 & 15 & 0.0388 & 13 & 41.12 & 16 & 0.0198 \\
\hline \multirow{2}{*}{$\mathrm{H}$} & 1 & 14 & 39.40 & 20 & 0.0554 & 17 & 50.97 & 25 & 0.0641 \\
\hline & 2 & 17 & 46.02 & 21 & 0.0563 & 18 & 52.86 & 26 & 0.0647 \\
\hline \multirow{2}{*}{ I } & 1 & 21 & 79.29 & 4 & 0.0074 & 23 & 63.57 & 5 & 0.0058 \\
\hline & 2 & 20 & 77.42 & 1 & 0.0057 & 16 & 49.18 & 1 & 0.0045 \\
\hline \multirow{2}{*}{$\mathrm{J}$} & 1 & 5 & 11.62 & 13 & 0.0313 & 7 & 25.86 & 14 & 0.0156 \\
\hline & 2 & 11 & 32.12 & 9 & 0.0210 & 27 & 77.67 & 10 & 0.0126 \\
\hline \multirow{2}{*}{$\mathrm{L}$} & 1 & 4 & 11.09 & 18 & 0.0526 & 3 & 5.49 & 15 & 0.0167 \\
\hline & 2 & 8 & 23.42 & 19 & 0.0536 & 6 & 12.66 & 17 & 0.0202 \\
\hline \multirow{2}{*}{ M } & 1 & - & - & - & - & 9 & 33.43 & 30 & 0.1259 \\
\hline & 2 & 22 & 90.28 & 22 & 0.1157 & 30 & 91.42 & 27 & 0.1155 \\
\hline \multirow{2}{*}{$\mathrm{O}$} & 1 & 7 & 20.12 & 12 & 0.0231 & 11 & 38.08 & 12 & 0.0142 \\
\hline & 2 & 10 & 32.04 & 10 & 0.0212 & 26 & 77.37 & 11 & 0.0132 \\
\hline \multirow{2}{*}{ Q } & 1 & 18 & 53.98 & 2 & 0.0064 & 22 & 62.95 & 3 & 0.0057 \\
\hline & 2 & 12 & 33.57 & 3 & 0.0069 & 21 & 61.29 & 3 & 0.0057 \\
\hline \multirow{2}{*}{ S } & 1 & - & - & - & - & 25 & 74.29 & 21 & 0.0369 \\
\hline & 2 & - & - & - & - & 24 & 73.96 & 22 & 0.0381 \\
\hline \multirow{2}{*}{$\mathrm{U}$} & 1 & - & - & - & - & 29 & 89.71 & 19 & 0.0266 \\
\hline & 2 & - & - & - & - & 28 & 88.88 & 20 & 0.0273 \\
\hline \multirow{2}{*}{$\mathrm{V}$} & 1 & 16 & 42.11 & 8 & 0.0148 & 10 & 35.66 & 8 & 0.0106 \\
\hline & 2 & - & - & - & - & 15 & 48.47 & 9 & 0.0110 \\
\hline
\end{tabular}

Table 54: Tabulation of the progression of identification timing and accuracy for Class B - Right Slap. Letter refers to the participant's letter code found on the footer of this page. Sub. \# is an identifier used to differentiate between the two submissions each participant could make. The Time column shows the time used to perform a search over an enrollment set of 3000000 in seconds, but was originally recorded to microsecond precision. The FNIR column shows FNIR for each submission at FPIR $=10^{-3}$. - indicates that there was not a validated submission during that submission period. The number to the left of a value provides the value's column-wise ranking. 


\begin{tabular}{|c|c|c|c|c|c|c|c|c|c|}
\hline \multicolumn{2}{|c|}{ Participant } & \multicolumn{4}{|c|}{ Second } & \multicolumn{4}{|c|}{ Third } \\
\hline Letter & Sub.\# & \multicolumn{2}{|c|}{ Time } & \multicolumn{2}{|c|}{ FNIR } & \multicolumn{2}{|c|}{ Time } & \multicolumn{2}{|c|}{ FNIR } \\
\hline \multirow{2}{*}{ C } & 1 & 1 & 3.44 & 21 & 0.0365 & 2 & 7.36 & 30 & NA \\
\hline & 2 & 3 & 4.33 & 20 & 0.0356 & 5 & 10.63 & 29 & NA \\
\hline \multirow{2}{*}{$\mathrm{D}$} & 1 & 13 & 38.51 & 5 & 0.0040 & 21 & 69.35 & 6 & 0.0031 \\
\hline & 2 & 17 & 48.86 & 6 & 0.0045 & 24 & 74.21 & 5 & 0.0024 \\
\hline \multirow{2}{*}{$\mathrm{E}$} & 1 & 4 & 6.39 & 11 & 0.0098 & 3 & 8.18 & 15 & 0.0063 \\
\hline & 2 & 18 & 52.30 & 8 & 0.0074 & 12 & 39.56 & 10 & 0.0049 \\
\hline \multirow{2}{*}{$\mathrm{F}$} & 1 & - & - & - & - & 8 & 28.72 & 28 & 0.0910 \\
\hline & 2 & - & - & - & - & 10 & 38.37 & 26 & 0.0901 \\
\hline \multirow{2}{*}{ G } & 1 & 2 & 4.27 & 13 & 0.0132 & 1 & 5.52 & 18 & 0.0106 \\
\hline & 2 & 7 & 22.15 & 16 & 0.0235 & 14 & 42.86 & 17 & 0.0084 \\
\hline \multirow{2}{*}{$\mathrm{H}$} & 1 & 19 & 52.34 & 18 & 0.0325 & 22 & 70.12 & 23 & 0.0349 \\
\hline & 2 & 20 & 61.14 & 19 & 0.0340 & 23 & 72.35 & 24 & 0.0361 \\
\hline \multirow{2}{*}{ I } & 1 & 22 & 79.56 & 4 & 0.0031 & 15 & 47.35 & 3 & 0.0022 \\
\hline & 2 & 21 & 69.84 & 3 & 0.0027 & 19 & 57.94 & 1 & 0.0015 \\
\hline \multirow{2}{*}{$\mathrm{J}$} & 1 & 5 & 11.06 & 15 & 0.0228 & 7 & 27.52 & 16 & 0.0068 \\
\hline & 2 & 12 & 32.23 & 10 & 0.0090 & 26 & 75.92 & 9 & 0.0047 \\
\hline \multirow{2}{*}{$\mathrm{L}$} & 1 & 8 & 25.03 & 17 & 0.0312 & 4 & 10.37 & 12 & 0.0054 \\
\hline & 2 & 10 & 26.13 & 14 & 0.0216 & 6 & 20.85 & 14 & 0.0062 \\
\hline \multirow{2}{*}{ M } & 1 & - & - & - & - & 9 & 28.89 & 27 & 0.0904 \\
\hline & 2 & 16 & 48.74 & 22 & 0.0882 & 16 & 49.50 & 25 & 0.0882 \\
\hline \multirow{2}{*}{$\mathrm{O}$} & 1 & 6 & 19.15 & 12 & 0.0106 & 13 & 40.42 & 13 & 0.0057 \\
\hline & 2 & 11 & 31.55 & 9 & 0.0088 & 25 & 74.57 & 11 & 0.0051 \\
\hline \multirow{2}{*}{$\mathrm{Q}$} & 1 & 15 & 47.54 & 1 & 0.0019 & 20 & 63.99 & 2 & 0.0021 \\
\hline & 2 & 9 & 25.99 & 2 & 0.0020 & 18 & 53.88 & 3 & 0.0022 \\
\hline \multirow{2}{*}{$S$} & 1 & - & - & - & - & 28 & 85.05 & 21 & 0.0160 \\
\hline & 2 & - & - & - & - & 27 & 84.02 & 22 & 0.0190 \\
\hline \multirow{2}{*}{$\mathrm{U}$} & 1 & - & - & - & - & 29 & 86.88 & 20 & 0.0139 \\
\hline & 2 & - & - & - & - & 30 & 91.04 & 19 & 0.0124 \\
\hline \multirow{2}{*}{ V } & 1 & 14 & 46.90 & 7 & 0.0051 & 11 & 39.24 & 7 & 0.0036 \\
\hline & 2 & - & - & - & - & 17 & 52.97 & 7 & 0.0036 \\
\hline
\end{tabular}

Table 55: Tabulation of the progression of identification timing and accuracy for Class B - Left and Right Slap. Letter refers to the participant's letter code found on the footer of this page. Sub. \# is an identifier used to differentiate between the two submissions each participant could make. The Time column shows the time used to perform a search over an enrollment set of 3000000 in seconds, but was originally recorded to microsecond precision. The FNIR column shows FNIR for each submission at FPIR $=10^{-3}$. NA indicates that the operations required to produce the value could not be performed. indicates that there was not a validated submission during that submission period. The number to the left of a value provides the value's column-wise ranking.

\begin{tabular}{l|l|l|l|ll|l}
$\mathrm{C}=$ afis team & $\mathrm{D}=3 \mathrm{M}$ Cogent & $\mathrm{E}=$ Neurotechnology & $\mathrm{F}=$ Papillon & $\mathrm{G}=$ Dermalog & $\mathrm{H}=$ Hisign Bio-Info Institute \\
$\mathrm{I}=$ NEC & $\mathrm{J}=$ Sonda & $\mathrm{K}=$ Tiger IT & $\mathrm{L}=$ Innovatrics & $\mathrm{M}=$ SPEX & $\mathrm{O}=$ ID Solutions \\
$\mathrm{P}=\mathrm{id} 3$ & $\mathrm{Q}=$ Morpho & $\mathrm{S}=$ Decatur Industries & $\mathrm{T}=$ BIO-key & $\mathrm{U}=$ Aware & $\mathrm{V}=$ AA Technology
\end{tabular}




\begin{tabular}{|c|c|c|c|c|c|c|c|c|c|}
\hline \multicolumn{2}{|c|}{ Participant } & \multicolumn{4}{|c|}{ Second } & \multicolumn{4}{|c|}{ Third } \\
\hline Letter & Sub.\# & \multicolumn{2}{|c|}{ Time } & \multicolumn{2}{|c|}{ FNIR } & \multicolumn{2}{|c|}{ Time } & \multicolumn{2}{|c|}{ FNIR } \\
\hline \multirow{2}{*}{$\mathrm{C}$} & 1 & 2 & 4.67 & 20 & 0.0276 & 3 & 9.00 & 30 & NA \\
\hline & 2 & 3 & 5.01 & 19 & 0.0268 & 4 & 10.28 & 29 & NA \\
\hline \multirow{2}{*}{ D } & 1 & 9 & 26.83 & 6 & 0.0028 & 17 & 52.42 & 6 & 0.0020 \\
\hline & 2 & 13 & 33.98 & 5 & 0.0027 & 19 & 54.37 & 2 & 0.0012 \\
\hline \multirow{2}{*}{$\mathrm{E}$} & 1 & 4 & 7.71 & 11 & 0.0071 & 2 & 8.76 & 16 & 0.0043 \\
\hline & 2 & 20 & 70.09 & 8 & 0.0043 & 18 & 52.88 & 7 & 0.0024 \\
\hline \multirow{2}{*}{$\mathrm{F}$} & 1 & - & - & - & - & 12 & 39.12 & 27 & 0.0591 \\
\hline & 2 & - & - & - & - & 16 & 51.59 & 27 & 0.0591 \\
\hline \multirow{2}{*}{ G } & 1 & 1 & 2.50 & 12 & 0.0101 & 1 & 6.33 & 18 & 0.0062 \\
\hline & 2 & 6 & 12.62 & 14 & 0.0157 & 9 & 31.67 & 14 & 0.0040 \\
\hline \multirow{2}{*}{$\mathrm{H}$} & 1 & 18 & 56.70 & 16 & 0.0220 & 26 & 82.43 & 23 & 0.0203 \\
\hline & 2 & 19 & 67.68 & 17 & 0.0249 & 27 & 85.74 & 24 & 0.0204 \\
\hline \multirow{2}{*}{ I } & 1 & 22 & 75.61 & 4 & 0.0021 & 6 & 24.57 & 2 & 0.0012 \\
\hline & 2 & 17 & 50.74 & 2 & 0.0012 & 20 & 60.01 & 1 & 0.0009 \\
\hline \multirow{2}{*}{$\mathrm{J}$} & 1 & 5 & 8.13 & 22 & 0.0614 & 7 & 26.31 & 17 & 0.0049 \\
\hline & 2 & 11 & 31.60 & 9 & 0.0054 & 22 & 64.38 & 11 & 0.0033 \\
\hline \multirow{2}{*}{$\mathrm{L}$} & 1 & 14 & 36.59 & 18 & 0.0250 & 5 & 14.42 & 10 & 0.0031 \\
\hline & 2 & 12 & 33.50 & 13 & 0.0147 & 8 & 28.56 & 11 & 0.0033 \\
\hline \multirow{2}{*}{$\mathrm{M}$} & 1 & - & - & - & - & 13 & 41.37 & 26 & 0.0543 \\
\hline & 2 & 21 & 70.37 & 21 & 0.0515 & 23 & 70.45 & 25 & 0.0515 \\
\hline \multirow{2}{*}{$\mathrm{O}$} & 1 & 7 & 16.34 & 15 & 0.0173 & 11 & 37.04 & 15 & 0.0041 \\
\hline & 2 & 10 & 30.74 & 10 & 0.0056 & 21 & 63.87 & 13 & 0.0035 \\
\hline \multirow{2}{*}{ Q } & 1 & 16 & 48.59 & 1 & 0.0010 & 14 & 48.85 & 2 & 0.0012 \\
\hline & 2 & 8 & 26.38 & 2 & 0.0012 & 24 & 71.67 & 2 & 0.0012 \\
\hline \multirow{2}{*}{$S$} & 1 & - & - & - & - & 28 & 86.50 & 20 & 0.0108 \\
\hline & 2 & - & - & - & - & 29 & 88.70 & 21 & 0.0136 \\
\hline \multirow{2}{*}{$\mathrm{U}$} & 1 & - & - & - & - & 30 & 88.83 & 19 & 0.0099 \\
\hline & 2 & - & - & - & - & 25 & 80.14 & 22 & 0.0141 \\
\hline \multirow{2}{*}{ V } & 1 & 15 & 42.75 & 7 & 0.0041 & 10 & 35.51 & 9 & 0.0027 \\
\hline & 2 & - & - & - & - & 15 & 49.72 & 7 & 0.0024 \\
\hline
\end{tabular}

Table 56: Tabulation of the progression of identification timing and accuracy for Class B - Identification Flats. Letter refers to the participant's letter code found on the footer of this page. Sub. \# is an identifier used to differentiate between the two submissions each participant could make. The Time column shows the time used to perform a search over an enrollment set of 3000000 in seconds, but was originally recorded to microsecond precision. The FNIR column shows FNIR for each submission at FPIR $=10^{-3}$. NA indicates that the operations required to produce the value could not be performed. indicates that there was not a validated submission during that submission period. The number to the left of a value provides the value's column-wise ranking.

\begin{tabular}{l|l|l|l|ll|l}
$\mathrm{C}=$ afis team & $\mathrm{D}=3 \mathrm{M}$ Cogent & $\mathrm{E}=$ Neurotechnology & $\mathrm{F}=$ Papillon & $\mathrm{G}=$ Dermalog & $\mathrm{H}=$ Hisign Bio-Info Institute \\
$\mathrm{I}=$ NEC & $\mathrm{J}=$ Sonda & $\mathrm{K}=$ Tiger IT & $\mathrm{L}=$ Innovatrics & $\mathrm{M}=$ SPEX & $\mathrm{O}=$ ID Solutions \\
$\mathrm{P}=\mathrm{id} 3$ & $\mathrm{Q}=$ Morpho & $\mathrm{S}=$ Decatur Industries & $\mathrm{T}=$ BIO-key & $\mathrm{U}=$ Aware & $\mathrm{V}=$ AA Technology
\end{tabular}




\begin{tabular}{|c|c|c|c|c|c|c|c|c|c|}
\hline \multicolumn{2}{|c|}{ Participant } & \multicolumn{4}{|c|}{ Second } & \multicolumn{4}{|c|}{ Third } \\
\hline Letter & Sub.\# & \multicolumn{2}{|c|}{ Time } & \multicolumn{2}{|c|}{ FNIR } & \multicolumn{2}{|c|}{ Time } & \multicolumn{2}{|c|}{ FNIR } \\
\hline \multirow{2}{*}{$\mathrm{C}$} & 1 & 3 & 10.27 & 16 & 0.0574 & 5 & 21.04 & 30 & NA \\
\hline & 2 & 2 & 10.04 & 17 & 0.0584 & 4 & 18.31 & 24 & 0.0711 \\
\hline \multirow{2}{*}{$\mathrm{D}$} & 1 & 10 & 44.21 & 4 & 0.0024 & 28 & 87.07 & 6 & 0.0015 \\
\hline & 2 & 20 & 74.46 & 3 & 0.0018 & 25 & 84.92 & 2 & 0.0011 \\
\hline \multirow{2}{*}{$\mathrm{E}$} & 1 & 6 & 27.61 & 11 & 0.0143 & 2 & 15.94 & 14 & 0.0088 \\
\hline & 2 & 21 & 79.95 & 9 & 0.0083 & 27 & 85.50 & 13 & 0.0048 \\
\hline \multirow{2}{*}{$\mathrm{F}$} & 1 & 12 & 50.99 & 18 & 0.0734 & 14 & 50.99 & 25 & 0.0734 \\
\hline & 2 & 17 & 60.34 & 18 & 0.0734 & 16 & 60.34 & 25 & 0.0734 \\
\hline \multirow{2}{*}{ G } & 1 & 1 & 8.43 & 21 & 0.1923 & 1 & 11.21 & 23 & 0.0368 \\
\hline & 2 & 5 & 21.36 & 20 & 0.0780 & 7 & 37.15 & 20 & 0.0276 \\
\hline \multirow{2}{*}{$\mathrm{H}$} & 1 & 18 & 68.30 & 15 & 0.0377 & 19 & 67.91 & 20 & 0.0276 \\
\hline & 2 & 15 & 59.28 & 14 & 0.0363 & 18 & 64.90 & 19 & 0.0275 \\
\hline \multirow{2}{*}{ I } & 1 & 14 & 56.55 & 5 & 0.0033 & 23 & 79.70 & 4 & 0.0013 \\
\hline & 2 & 9 & 40.52 & 7 & 0.0050 & 15 & 56.93 & 1 & 0.0010 \\
\hline \multirow{2}{*}{$\mathrm{J}$} & 1 & 7 & 35.33 & 10 & 0.0104 & 12 & 46.32 & 12 & 0.0047 \\
\hline & 2 & 19 & 69.82 & 7 & 0.0050 & 26 & 85.17 & 10 & 0.0027 \\
\hline \multirow{2}{*}{$\mathrm{L}$} & 1 & 13 & 54.67 & 12 & 0.0159 & 3 & 17.53 & 16 & 0.0102 \\
\hline & 2 & 11 & 47.51 & 13 & 0.0221 & 6 & 21.26 & 15 & 0.0095 \\
\hline \multirow{2}{*}{ M } & 1 & - & - & - & - & 8 & 42.60 & 28 & 0.0934 \\
\hline & 2 & - & - & - & - & 11 & 45.96 & 27 & 0.0826 \\
\hline \multirow{2}{*}{$\mathrm{O}$} & 1 & - & - & - & - & 21 & 73.31 & 9 & 0.0025 \\
\hline & 2 & - & - & - & - & 24 & 82.13 & 10 & 0.0027 \\
\hline \multirow{2}{*}{$\mathrm{Q}$} & 1 & 8 & 38.03 & 1 & 0.0014 & 10 & 44.75 & 2 & 0.0011 \\
\hline & 2 & 4 & 20.02 & 2 & 0.0017 & 9 & 43.67 & 4 & 0.0013 \\
\hline \multirow{2}{*}{$S$} & 1 & - & - & - & - & 29 & 87.76 & 22 & 0.0311 \\
\hline & 2 & - & - & - & - & 30 & 89.24 & 29 & 0.1680 \\
\hline \multirow{2}{*}{$\mathrm{U}$} & 1 & - & - & - & - & 22 & 75.14 & 18 & 0.0163 \\
\hline & 2 & - & - & - & - & 20 & 67.98 & 17 & 0.0155 \\
\hline \multirow{2}{*}{ V } & 1 & 16 & 59.38 & 6 & 0.0034 & 13 & 47.76 & 7 & 0.0024 \\
\hline & 2 & - & - & - & - & 17 & 63.19 & 7 & 0.0024 \\
\hline
\end{tabular}

Table 57: Tabulation of the progression of identification timing and accuracy for Class C - Ten-Finger Plain-to-Plain. Letter refers to the participant's letter code found on the footer of this page. Sub. \# is an identifier used to differentiate between the two submissions each participant could make. The Time column shows the time used to perform a search over an enrollment set of 5000000 in seconds, but was originally recorded to microsecond precision. The FNIR column shows FNIR for each submission at FPIR $=10^{-3}$. NA indicates that the operations required to produce the value could not be performed. - indicates that there was not a validated submission during that submission period. The number to the left of a value provides the value's column-wise ranking.

\begin{tabular}{l|l|l|l|ll|l}
$\mathrm{C}=$ afis team & $\mathrm{D}=3 \mathrm{M}$ Cogent & $\mathrm{E}=$ Neurotechnology & $\mathrm{F}=$ Papillon & $\mathrm{G}=$ Dermalog & $\mathrm{H}=$ Hisign Bio-Info Institute \\
$\mathrm{I}=$ NEC & $\mathrm{J}=$ Sonda & $\mathrm{K}=$ Tiger IT & $\mathrm{L}=$ Innovatrics & $\mathrm{M}=$ SPEX & $\mathrm{O}=$ ID Solutions \\
$\mathrm{P}=\mathrm{id} 3$ & $\mathrm{Q}=$ Morpho & $\mathrm{S}=$ Decatur Industries & $\mathrm{T}=$ BIO-key & $\mathrm{U}=$ Aware & $\mathrm{V}=$ AA Technology
\end{tabular}




\begin{tabular}{|c|c|c|c|c|c|c|c|c|c|}
\hline \multicolumn{2}{|c|}{ Participant } & \multicolumn{4}{|c|}{ Second } & \multicolumn{4}{|c|}{ Third } \\
\hline Letter & Sub.\# & \multicolumn{2}{|c|}{ Time } & \multicolumn{2}{|c|}{ FNIR } & \multicolumn{2}{|c|}{ Time } & \multicolumn{2}{|c|}{ FNIR } \\
\hline \multirow{2}{*}{$\mathrm{C}$} & 1 & 2 & 19.26 & 21 & 0.0826 & 4 & 25.25 & 16 & 0.0094 \\
\hline & 2 & 7 & 28.17 & 20 & 0.0822 & 5 & 25.91 & 15 & 0.0085 \\
\hline \multirow{2}{*}{$\mathrm{D}$} & 1 & 11 & 46.42 & 6 & 0.0028 & 17 & 65.97 & 4 & 0.0015 \\
\hline & 2 & 16 & 70.86 & 5 & 0.0023 & 30 & 86.39 & 7 & 0.0018 \\
\hline \multirow{2}{*}{$\mathrm{E}$} & 1 & 1 & 12.49 & 17 & 0.0285 & 1 & 9.52 & 18 & 0.0106 \\
\hline & 2 & 8 & 29.59 & 9 & 0.0121 & 14 & 57.02 & 12 & 0.0050 \\
\hline \multirow{2}{*}{$\mathrm{F}$} & 1 & 13 & 55.63 & 18 & 0.0536 & 13 & 55.63 & 25 & 0.0536 \\
\hline & 2 & 15 & 68.61 & 18 & 0.0536 & 18 & 68.61 & 25 & 0.0536 \\
\hline \multirow{2}{*}{ G } & 1 & 3 & 20.17 & 15 & 0.0239 & 2 & 11.67 & 24 & 0.0447 \\
\hline & 2 & 5 & 23.05 & 13 & 0.0195 & 6 & 30.61 & 21 & 0.0333 \\
\hline \multirow{2}{*}{$\mathrm{H}$} & 1 & 19 & 77.49 & 15 & 0.0239 & 28 & 84.14 & 20 & 0.0201 \\
\hline & 2 & 17 & 71.01 & 14 & 0.0237 & 29 & 84.26 & 19 & 0.0199 \\
\hline \multirow{2}{*}{ I } & 1 & 10 & 40.06 & 1 & 0.0015 & 24 & 79.04 & 1 & 0.0013 \\
\hline & 2 & 4 & 20.92 & 7 & 0.0032 & 9 & 40.53 & 2 & 0.0014 \\
\hline \multirow{2}{*}{$\mathrm{J}$} & 1 & 6 & 26.55 & 12 & 0.0168 & 8 & 36.09 & 13 & 0.0051 \\
\hline & 2 & 12 & 55.60 & 8 & 0.0045 & 20 & 72.62 & 9 & 0.0033 \\
\hline \multirow{2}{*}{$\mathrm{L}$} & 1 & 18 & 75.36 & 10 & 0.0123 & 7 & 31.42 & 17 & 0.0097 \\
\hline & 2 & 20 & 80.46 & 11 & 0.0160 & 3 & 19.52 & 14 & 0.0083 \\
\hline \multirow{2}{*}{ M } & 1 & - & - & - & - & 11 & 47.38 & 28 & 0.0783 \\
\hline & 2 & - & - & - & - & 12 & 48.71 & 27 & 0.0716 \\
\hline \multirow{2}{*}{$\mathrm{O}$} & 1 & - & - & - & - & 15 & 59.01 & 11 & 0.0034 \\
\hline & 2 & - & - & - & - & 19 & 72.55 & 9 & 0.0033 \\
\hline \multirow{2}{*}{$\mathrm{Q}$} & 1 & 14 & 67.74 & 1 & 0.0015 & 26 & 83.25 & 5 & 0.0017 \\
\hline & 2 & 9 & 37.87 & 3 & 0.0019 & 27 & 83.35 & 2 & 0.0014 \\
\hline \multirow{2}{*}{$S$} & 1 & - & - & - & - & 22 & 74.01 & 29 & 0.0860 \\
\hline & 2 & - & - & - & - & 21 & 72.95 & 30 & 0.2462 \\
\hline \multirow{2}{*}{$\mathrm{U}$} & 1 & - & - & - & - & 25 & 82.76 & 23 & 0.0358 \\
\hline & 2 & - & - & - & - & 23 & 74.39 & 22 & 0.0351 \\
\hline \multirow{2}{*}{$\mathrm{V}$} & 1 & 21 & 91.48 & 4 & 0.0022 & 10 & 40.94 & 5 & 0.0017 \\
\hline & 2 & - & - & - & - & 16 & 65.47 & 8 & 0.0019 \\
\hline
\end{tabular}

Table 58: Tabulation of the progression of identification timing and accuracy for Class C - Ten-Finger Rolled-to-Rolled. Letter refers to the participant's letter code found on the footer of this page. Sub. \# is an identifier used to differentiate between the two submissions each participant could make. The Time column shows the time used to perform a search over an enrollment set of 5000000 in seconds, but was originally recorded to microsecond precision. The FNIR column shows FNIR for each submission at FPIR $=10^{-3}$. - indicates that there was not a validated submission during that submission period. The number to the left of a value provides the value's column-wise ranking.

\begin{tabular}{l|l|l|l|ll|l}
$\mathrm{C}=$ afis team & $\mathrm{D}=3 \mathrm{M}$ Cogent & $\mathrm{E}=$ Neurotechnology & $\mathrm{F}=$ Papillon & $\mathrm{G}=$ Dermalog & $\mathrm{H}=$ Hisign Bio-Info Institute \\
$\mathrm{I}=$ NEC & $\mathrm{J}=$ Sonda & $\mathrm{K}=$ Tiger IT & $\mathrm{L}=$ Innovatrics & $\mathrm{M}=$ SPEX & $\mathrm{O}=$ ID Solutions \\
$\mathrm{P}=\mathrm{id} 3$ & $\mathrm{Q}=$ Morpho & $\mathrm{S}=$ Decatur Industries & $\mathrm{T}=$ BIO-key & $\mathrm{U}=$ Aware & $\mathrm{V}=$ AA Technology
\end{tabular}




\begin{tabular}{|c|c|c|c|c|c|c|c|c|c|}
\hline \multicolumn{2}{|c|}{ Participant } & \multicolumn{4}{|c|}{ Second } & \multicolumn{4}{|c|}{ Third } \\
\hline Letter & Sub.\# & \multicolumn{2}{|c|}{ Time } & \multicolumn{2}{|c|}{ FNIR } & \multicolumn{2}{|c|}{ Time } & \multicolumn{2}{|c|}{ FNIR } \\
\hline \multirow{2}{*}{$\mathrm{C}$} & 1 & 3 & 18.23 & 16 & 0.0979 & 7 & 36.55 & 17 & 0.0149 \\
\hline & 2 & 4 & 19.43 & 17 & 0.1124 & 5 & 33.93 & 18 & 0.0169 \\
\hline \multirow{2}{*}{$\mathrm{D}$} & 1 & 8 & 39.02 & 6 & 0.0048 & 12 & 56.38 & 6 & 0.0028 \\
\hline & 2 & 14 & 58.60 & 2 & 0.0034 & 25 & 76.00 & 3 & 0.0018 \\
\hline \multirow{2}{*}{$\mathrm{E}$} & 1 & 5 & 20.29 & 13 & 0.0383 & 1 & 10.81 & 16 & 0.0137 \\
\hline & 2 & 10 & 55.20 & 9 & 0.0164 & 16 & 59.20 & 12 & 0.0056 \\
\hline \multirow{2}{*}{$\mathrm{F}$} & 1 & 13 & 58.39 & 20 & 0.2514 & 15 & 58.39 & 27 & 0.2514 \\
\hline & 2 & 16 & 61.82 & 20 & 0.2514 & 17 & 61.82 & 27 & 0.2514 \\
\hline \multirow{2}{*}{ G } & 1 & 1 & 11.46 & 19 & 0.2393 & 3 & 25.05 & 24 & 0.0649 \\
\hline & 2 & 2 & 11.62 & 18 & 0.1455 & 6 & 34.47 & 23 & 0.0521 \\
\hline \multirow{2}{*}{$\mathrm{H}$} & 1 & 20 & 70.66 & 15 & 0.0429 & 18 & 65.03 & 20 & 0.0291 \\
\hline & 2 & 18 & 62.51 & 14 & 0.0411 & 19 & 65.69 & 19 & 0.0285 \\
\hline \multirow{2}{*}{ I } & 1 & 11 & 56.80 & 5 & 0.0039 & 26 & 76.98 & 2 & 0.0014 \\
\hline & 2 & 12 & 58.34 & 4 & 0.0037 & 21 & 66.90 & 1 & 0.0011 \\
\hline \multirow{2}{*}{$\mathrm{J}$} & 1 & 7 & 30.56 & 10 & 0.0174 & 8 & 42.13 & 13 & 0.0071 \\
\hline & 2 & 17 & 62.05 & 8 & 0.0053 & 27 & 79.37 & 7 & 0.0034 \\
\hline \multirow{2}{*}{$\mathrm{L}$} & 1 & 15 & 60.76 & 11 & 0.0251 & 4 & 27.43 & 15 & 0.0136 \\
\hline & 2 & 21 & 72.40 & 12 & 0.0358 & 2 & 18.62 & 14 & 0.0129 \\
\hline \multirow{2}{*}{ M } & 1 & - & - & - & - & 13 & 57.06 & 30 & 0.3067 \\
\hline & 2 & - & - & - & - & 14 & 57.90 & 27 & 0.2514 \\
\hline \multirow{2}{*}{$\mathrm{O}$} & 1 & - & - & - & - & 20 & 65.71 & 10 & 0.0041 \\
\hline & 2 & - & - & - & - & 24 & 75.98 & 8 & 0.0036 \\
\hline \multirow{2}{*}{ Q } & 1 & 9 & 42.45 & 1 & 0.0028 & 10 & 49.07 & 4 & 0.0020 \\
\hline & 2 & 6 & 22.94 & 2 & 0.0034 & 11 & 49.72 & 5 & 0.0022 \\
\hline \multirow{2}{*}{$S$} & 1 & - & - & - & - & 30 & 89.37 & 25 & 0.1017 \\
\hline & 2 & - & - & - & - & 29 & 89.34 & 26 & 0.2366 \\
\hline \multirow{2}{*}{$\mathrm{U}$} & 1 & - & - & - & - & 28 & 82.50 & 22 & 0.0378 \\
\hline & 2 & - & - & - & - & 23 & 73.46 & 21 & 0.0295 \\
\hline \multirow{2}{*}{$\mathrm{V}$} & 1 & 19 & 66.49 & 7 & 0.0051 & 9 & 48.91 & 11 & 0.0052 \\
\hline & 2 & - & - & - & - & 22 & 71.28 & 9 & 0.0039 \\
\hline
\end{tabular}

Table 59: Tabulation of the progression of identification timing and accuracy for Class C - Ten-Finger Plain-to-Rolled. Letter refers to the participant's letter code found on the footer of this page. Sub. \# is an identifier used to differentiate between the two submissions each participant could make. The Time column shows the time used to perform a search over an enrollment set of 5000000 in seconds, but was originally recorded to microsecond precision. The FNIR column shows FNIR for each submission at FPIR $=10^{-3}$. - indicates that there was not a validated submission during that submission period. The number to the left of a value provides the value's column-wise ranking.

\begin{tabular}{l|l|l|l|ll|l}
$\mathrm{C}=$ afis team & $\mathrm{D}=3 \mathrm{M}$ Cogent & $\mathrm{E}=$ Neurotechnology & $\mathrm{F}=$ Papillon & $\mathrm{G}=$ Dermalog & $\mathrm{H}=$ Hisign Bio-Info Institute \\
$\mathrm{I}=$ NEC & $\mathrm{J}=$ Sonda & $\mathrm{K}=$ Tiger IT & $\mathrm{L}=$ Innovatrics & $\mathrm{M}=$ SPEX & $\mathrm{O}=$ ID Solutions \\
$\mathrm{P}=\mathrm{id} 3$ & $\mathrm{Q}=$ Morpho & $\mathrm{S}=$ Decatur Industries & $\mathrm{T}=$ BIO-key & $\mathrm{U}=$ Aware & $\mathrm{V}=$ AA Technology
\end{tabular}




\section{F Enrollment Size}

\begin{tabular}{l|l|l|l|l|l|l}
$\mathrm{C}=$ afis team & $\mathrm{D}=3 \mathrm{M}$ Cogent & $\mathrm{E}=$ Neurotechnology & $\mathrm{F}=$ Papillon & $\mathrm{G}=$ Dermalog & $\mathrm{H}=$ Hisign Bio-Info Institute \\
$\mathrm{I}=\mathrm{NEC}$ & $\mathrm{J}=$ Sonda & $\mathrm{K}=$ Tiger IT & $\mathrm{L}=$ Innovatrics & $\mathrm{M}=$ SPEX & $\mathrm{O}=$ ID Solutions \\
$\mathrm{P}=\mathrm{id} 3$ & $\mathrm{Q}=$ Morpho & $\mathrm{S}=$ Decatur Industries & $\mathrm{T}=$ BIO-key & $\mathrm{U}=$ Aware & $\mathrm{V}=$ AA Technology
\end{tabular}




\begin{tabular}{|c|c|c|c|c|c|c|c|c|c|c|c|}
\hline \multicolumn{2}{|c|}{ Participant } & \multicolumn{4}{|c|}{ RAM } & \multicolumn{4}{|c|}{ On Disk } & \multirow{2}{*}{\multicolumn{2}{|c|}{ FNIR $@$ FPIR $=10^{-3}$}} \\
\hline Letter & Sub.\# & \multicolumn{2}{|c|}{ Actual } & \multicolumn{2}{|c|}{ Reported } & \multicolumn{2}{|c|}{ Finalized } & \multicolumn{2}{|c|}{ Stored } & & \\
\hline \multirow{2}{*}{ C } & 1 & 7 & 0.18 & 10 & 0.16 & 8 & 0.17 & 8 & 0.16 & 30 & 0.1335 \\
\hline & 2 & 8 & 0.18 & 10 & 0.16 & 8 & 0.17 & 8 & 0.16 & 31 & 0.1337 \\
\hline \multirow{2}{*}{$\mathrm{D}$} & 1 & 21 & 0.61 & 18 & 0.33 & 29 & 1.25 & 18 & 0.34 & 1 & 0.0197 \\
\hline & 2 & 21 & 0.61 & 18 & 0.33 & 30 & 1.40 & 18 & 0.34 & 1 & 0.0197 \\
\hline \multirow{2}{*}{$\mathrm{E}$} & 1 & 30 & 1.12 & 27 & 0.50 & 23 & 0.50 & 27 & 0.50 & 16 & 0.0745 \\
\hline & 2 & 29 & 1.11 & 27 & 0.50 & 23 & 0.50 & 27 & 0.50 & 15 & 0.0723 \\
\hline \multirow{2}{*}{$\mathrm{F}$} & 1 & 6 & 0.18 & 7 & 0.11 & 10 & 0.17 & 10 & 0.17 & 25 & 0.1111 \\
\hline & 2 & 5 & 0.18 & 7 & 0.11 & 10 & 0.17 & 10 & 0.17 & 22 & 0.1082 \\
\hline \multirow{2}{*}{ G } & 1 & 20 & 0.57 & 29 & 0.50 & 5 & 0.15 & 5 & 0.15 & 24 & 0.1089 \\
\hline & 2 & 19 & 0.57 & 29 & 0.50 & 5 & 0.15 & 5 & 0.15 & 23 & 0.1086 \\
\hline \multirow{2}{*}{$\mathrm{H}$} & 1 & 11 & 0.33 & 14 & 0.29 & 12 & 0.29 & 14 & 0.29 & 32 & 0.1576 \\
\hline & 2 & 11 & 0.33 & 14 & 0.29 & 12 & 0.29 & 14 & 0.29 & 33 & 0.1607 \\
\hline \multirow{2}{*}{ I } & 1 & 23 & 0.70 & 35 & 0.68 & 31 & 1.54 & 35 & 0.68 & 7 & 0.0257 \\
\hline & 2 & 28 & 1.04 & 36 & 1.03 & 32 & 1.55 & 36 & 1.03 & 8 & 0.0278 \\
\hline \multirow{2}{*}{$\mathrm{J}$} & 1 & 9 & 0.32 & 12 & 0.27 & 14 & 0.31 & 12 & 0.28 & 18 & 0.0786 \\
\hline & 2 & 9 & 0.32 & 12 & 0.27 & 14 & 0.31 & 12 & 0.28 & 14 & 0.0712 \\
\hline \multirow{2}{*}{ K } & 1 & 35 & 3.87 & 25 & 0.47 & 35 & 4.60 & 25 & 0.48 & 21 & 0.0883 \\
\hline & 2 & 36 & 3.87 & 25 & 0.47 & 35 & 4.60 & 25 & 0.48 & 20 & 0.0875 \\
\hline \multirow{2}{*}{$\mathrm{L}$} & 1 & 31 & 1.14 & 9 & 0.15 & 7 & 0.16 & 7 & 0.16 & 11 & 0.0625 \\
\hline & 2 & 34 & 2.32 & 22 & 0.46 & 20 & 0.46 & 22 & 0.46 & 9 & 0.0351 \\
\hline \multirow{2}{*}{ M } & 1 & 3 & 0.14 & 5 & 0.11 & 3 & 0.13 & 3 & 0.13 & 35 & 0.2995 \\
\hline & 2 & 4 & 0.14 & 5 & 0.11 & 3 & 0.13 & 3 & 0.13 & 34 & 0.2921 \\
\hline \multirow{2}{*}{$\mathrm{O}$} & 1 & 15 & 0.35 & 16 & 0.30 & 16 & 0.32 & 16 & 0.31 & 19 & 0.0818 \\
\hline & 2 & 15 & 0.35 & 16 & 0.30 & 16 & 0.32 & 16 & 0.31 & 17 & 0.0766 \\
\hline \multirow{2}{*}{$\mathrm{P}$} & 1 & 27 & 0.78 & 3 & 0.03 & 1 & 0.03 & 1 & 0.03 & 29 & 0.1308 \\
\hline & 2 & 26 & 0.78 & 3 & 0.03 & 1 & 0.03 & 1 & 0.03 & 28 & 0.1272 \\
\hline \multirow{2}{*}{ Q } & 1 & 13 & 0.34 & 1 & 0.03 & 27 & 1.07 & 33 & 0.68 & 3 & 0.0222 \\
\hline & 2 & 14 & 0.34 & 1 & 0.03 & 27 & 1.07 & 33 & 0.68 & 4 & 0.0226 \\
\hline \multirow{2}{*}{$S$} & 1 & 24 & 0.72 & 23 & 0.47 & 21 & 0.47 & 23 & 0.47 & 10 & 0.0571 \\
\hline & 2 & 24 & 0.72 & 23 & 0.47 & 21 & 0.47 & 23 & 0.47 & 12 & 0.0650 \\
\hline \multirow{2}{*}{$\mathrm{T}$} & 1 & 2 & 0.01 & 33 & 0.60 & 25 & 0.91 & 31 & 0.60 & 36 & NA \\
\hline & 2 & 1 & 0.01 & 33 & 0.60 & 25 & 0.91 & 31 & 0.60 & 13 & 0.0685 \\
\hline 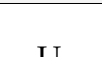 & 1 & 33 & 1.70 & 31 & 0.53 & 33 & 1.96 & 29 & 0.54 & 27 & 0.1218 \\
\hline U & 2 & 32 & 1.42 & 31 & 0.53 & 33 & 1.96 & 29 & 0.54 & 26 & 0.1178 \\
\hline $\mathrm{V}$ & 1 & 18 & 0.39 & 20 & 0.38 & 18 & 0.39 & 20 & 0.38 & 6 & 0.0253 \\
\hline$v$ & 2 & 17 & 0.39 & 20 & 0.38 & 18 & 0.39 & 20 & 0.38 & 5 & 0.0252 \\
\hline
\end{tabular}

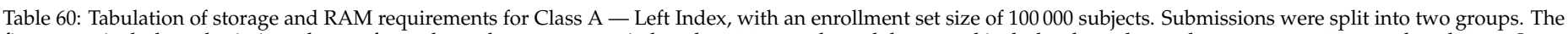

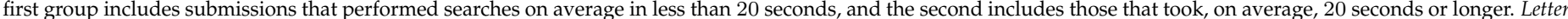

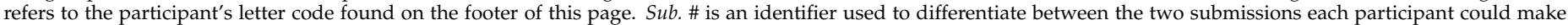

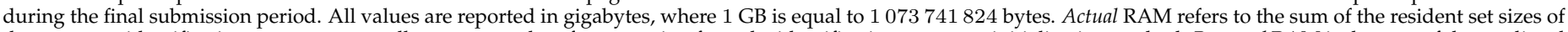

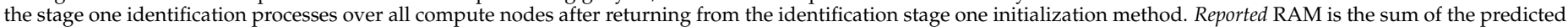

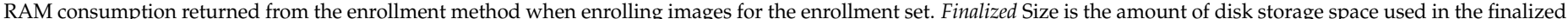

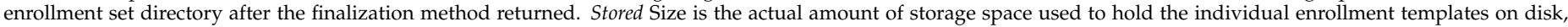

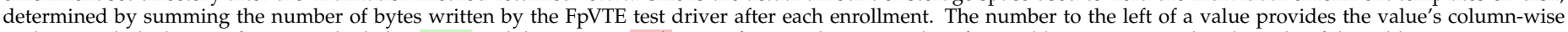
ranking, with the best performance shaded in green and the worst in pink. For reference, the FNIR values from Table 7 are reprinted to the right of this table.

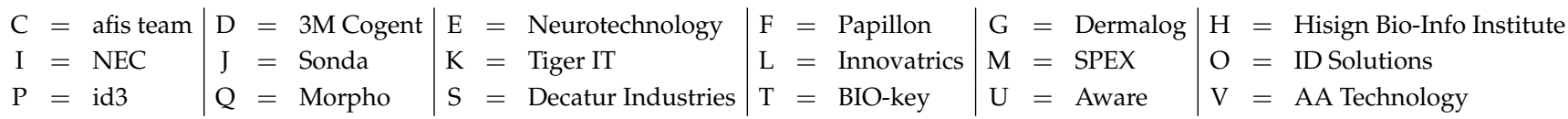




\begin{tabular}{|c|c|c|c|c|c|c|c|c|c|}
\hline \multicolumn{2}{|c|}{ Participant } & \multicolumn{4}{|c|}{ RAM } & \multicolumn{4}{|c|}{ On Disk } \\
\hline Letter & Sub.\# & \multicolumn{2}{|c|}{ Actual } & \multicolumn{2}{|c|}{ Reported } & \multicolumn{2}{|c|}{ Finalized } & \multicolumn{2}{|c|}{ Stored } \\
\hline \multirow{2}{*}{$\mathrm{C}$} & 1 & 7 & 0.17 & 10 & 0.15 & 8 & 0.16 & 8 & 0.16 \\
\hline & 2 & 8 & 0.18 & 10 & 0.15 & 8 & 0.16 & 8 & 0.16 \\
\hline \multirow{2}{*}{$\mathrm{D}$} & 1 & 21 & 0.61 & 18 & 0.33 & 29 & 1.25 & 18 & 0.33 \\
\hline & 2 & 21 & 0.61 & 18 & 0.33 & 30 & 1.40 & 18 & 0.33 \\
\hline \multirow{2}{*}{$\mathrm{E}$} & 1 & 29 & 1.07 & 27 & 0.48 & 23 & 0.48 & 27 & 0.48 \\
\hline & 2 & 30 & 1.07 & 27 & 0.48 & 23 & 0.48 & 27 & 0.48 \\
\hline \multirow{2}{*}{$\mathrm{F}$} & 1 & 6 & 0.17 & 7 & 0.11 & 10 & 0.17 & 10 & 0.16 \\
\hline & 2 & 5 & 0.17 & 7 & 0.11 & 10 & 0.17 & 10 & 0.16 \\
\hline \multirow{2}{*}{ G } & 1 & 19 & 0.57 & 29 & 0.48 & 5 & 0.15 & 5 & 0.15 \\
\hline & 2 & 20 & 0.57 & 29 & 0.48 & 5 & 0.15 & 5 & 0.15 \\
\hline \multirow{2}{*}{$\mathrm{H}$} & 1 & 14 & 0.33 & 14 & 0.29 & 14 & 0.29 & 14 & 0.29 \\
\hline & 2 & 13 & 0.33 & 14 & 0.29 & 14 & 0.29 & 14 & 0.29 \\
\hline \multirow{2}{*}{ I } & 1 & 23 & 0.69 & 35 & 0.67 & 31 & 1.54 & 35 & 0.67 \\
\hline & 2 & 28 & 1.01 & 36 & 0.99 & 32 & 1.55 & 36 & 0.99 \\
\hline \multirow{2}{*}{$\mathrm{J}$} & 1 & 10 & 0.31 & 12 & 0.26 & 12 & 0.29 & 12 & 0.27 \\
\hline & 2 & 9 & 0.31 & 12 & 0.26 & 12 & 0.29 & 12 & 0.27 \\
\hline \multirow{2}{*}{$\mathrm{K}$} & 1 & 36 & 3.87 & 25 & 0.46 & 35 & 4.60 & 25 & 0.47 \\
\hline & 2 & 35 & 3.87 & 25 & 0.46 & 35 & 4.60 & 25 & 0.47 \\
\hline \multirow{2}{*}{$\mathrm{L}$} & 1 & 31 & 1.14 & 9 & 0.15 & 7 & 0.16 & 7 & 0.15 \\
\hline & 2 & 34 & 2.32 & 22 & 0.44 & 20 & 0.45 & 22 & 0.45 \\
\hline \multirow{2}{*}{$\mathrm{M}$} & 1 & 3 & 0.14 & 5 & 0.11 & 3 & 0.13 & 3 & 0.12 \\
\hline & 2 & 3 & 0.14 & 5 & 0.11 & 3 & 0.13 & 3 & 0.12 \\
\hline \multirow{2}{*}{$\mathrm{O}$} & 1 & 15 & 0.34 & 16 & 0.29 & 16 & 0.30 & 16 & 0.30 \\
\hline & 2 & 16 & 0.34 & 16 & 0.29 & 16 & 0.30 & 16 & 0.30 \\
\hline \multirow{2}{*}{$\mathrm{P}$} & 1 & 26 & 0.76 & 3 & 0.03 & 1 & 0.03 & 1 & 0.03 \\
\hline & 2 & 27 & 0.76 & 3 & 0.03 & 1 & 0.03 & 1 & 0.03 \\
\hline \multirow{2}{*}{ Q } & 1 & 11 & 0.33 & 1 & 0.03 & 27 & 1.03 & 33 & 0.66 \\
\hline & 2 & 11 & 0.33 & 1 & 0.03 & 27 & 1.03 & 33 & 0.66 \\
\hline \multirow{2}{*}{$S$} & 1 & 24 & 0.71 & 23 & 0.46 & 21 & 0.46 & 23 & 0.46 \\
\hline & 2 & 25 & 0.71 & 23 & 0.46 & 21 & 0.46 & 23 & 0.46 \\
\hline \multirow{2}{*}{$\mathrm{T}$} & 1 & 1 & 0.01 & 33 & 0.57 & 25 & 0.87 & 31 & 0.57 \\
\hline & 2 & 1 & 0.01 & 33 & 0.57 & 25 & 0.87 & 31 & 0.57 \\
\hline \multirow{2}{*}{$\mathrm{U}$} & 1 & 33 & 1.56 & 31 & 0.52 & 33 & 1.79 & 29 & 0.52 \\
\hline & 2 & 32 & 1.30 & 31 & 0.52 & 33 & 1.79 & 29 & 0.52 \\
\hline \multirow{2}{*}{$\mathrm{V}$} & 1 & 18 & 0.39 & 20 & 0.38 & 18 & 0.38 & 20 & 0.38 \\
\hline & 2 & 17 & 0.39 & 20 & 0.38 & 18 & 0.38 & 20 & 0.38 \\
\hline
\end{tabular}

\begin{tabular}{|c|c|}
\hline \multicolumn{2}{|c|}{ FNIR $@$ FPIR $=10^{-3}$} \\
\hline 30 & 0.1132 \\
\hline 29 & 0.1124 \\
\hline 1 & 0.0190 \\
\hline 1 & 0.0190 \\
\hline 15 & 0.0630 \\
\hline 14 & 0.0624 \\
\hline 25 & 0.0933 \\
\hline 22 & 0.0903 \\
\hline 24 & 0.0910 \\
\hline 23 & 0.0909 \\
\hline 32 & 0.1230 \\
\hline 33 & 0.1249 \\
\hline 5 & 0.0215 \\
\hline 3 & 0.0214 \\
\hline 20 & 0.0708 \\
\hline 16 & 0.0643 \\
\hline 18 & 0.0682 \\
\hline 19 & 0.0685 \\
\hline 12 & 0.0505 \\
\hline 9 & 0.0295 \\
\hline 36 & 0.2615 \\
\hline 35 & 0.2526 \\
\hline 21 & 0.0776 \\
\hline 17 & 0.0675 \\
\hline 31 & 0.1133 \\
\hline 28 & 0.1100 \\
\hline 6 & 0.0218 \\
\hline 3 & 0.0214 \\
\hline 10 & 0.0442 \\
\hline 11 & 0.0503 \\
\hline 34 & 0.1929 \\
\hline 13 & 0.0562 \\
\hline 26 & 0.0996 \\
\hline 27 & 0.1007 \\
\hline 8 & 0.0223 \\
\hline 7 & 0.0222 \\
\hline
\end{tabular}

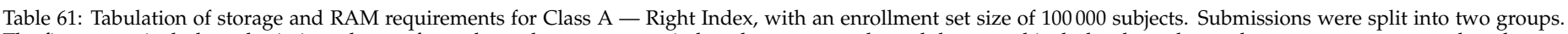

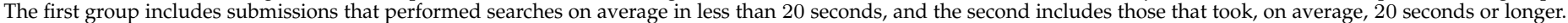

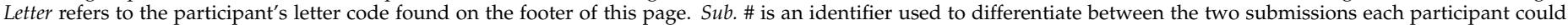

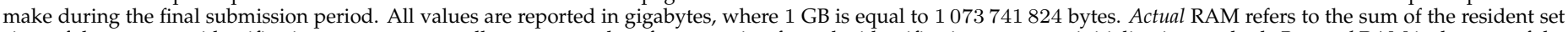

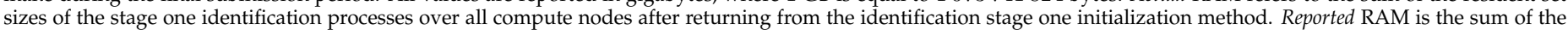

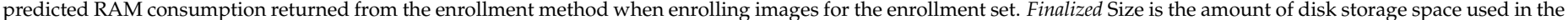

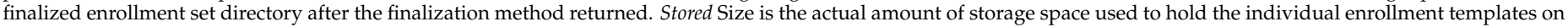

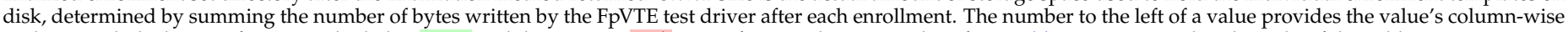
ranking, with the best performance shaded in green and the worst in pink. For reference, the FNIR values from Table 8 are reprinted to the right of this table.

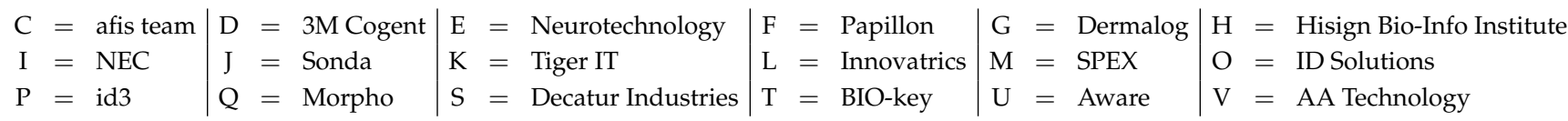




\begin{tabular}{|c|c|c|c|c|c|c|c|c|c|c|c|}
\hline \multicolumn{2}{|c|}{ Participant } & \multicolumn{4}{|c|}{ RAM } & \multicolumn{4}{|c|}{ On Disk } & \multirow{2}{*}{\multicolumn{2}{|c|}{ FNIR @ FPIR $=10^{-3}$}} \\
\hline Letter & Sub.\# & \multicolumn{2}{|c|}{ Actual } & \multicolumn{2}{|c|}{ Reported } & \multicolumn{2}{|c|}{ Finalized } & \multicolumn{2}{|c|}{ Stored } & & \\
\hline \multirow{2}{*}{$\mathrm{C}$} & 1 & 8 & 4.87 & 10 & 4.66 & 8 & 4.78 & 8 & 4.69 & 26 & 0.0368 \\
\hline & 2 & 7 & 4.87 & 10 & 4.66 & 8 & 4.78 & 8 & 4.69 & 28 & 0.0374 \\
\hline \multirow{2}{*}{$\mathrm{D}$} & 1 & 24 & 18.58 & 18 & 10.30 & 30 & 40.04 & 18 & 10.31 & 4 & 0.0030 \\
\hline & 2 & 24 & 18.58 & 18 & 10.30 & 31 & 44.68 & 18 & 10.31 & 4 & 0.0030 \\
\hline \multirow{2}{*}{$\mathrm{E}$} & 1 & 30 & 33.40 & 27 & 14.95 & 23 & 15.02 & 27 & 14.96 & 15 & 0.0207 \\
\hline & 2 & 31 & 33.41 & 27 & 14.95 & 23 & 15.02 & 27 & 14.96 & 14 & 0.0202 \\
\hline \multirow{2}{*}{$\mathrm{F}$} & 1 & 5 & 4.86 & 5 & 3.21 & 10 & 4.86 & 10 & 4.78 & 29 & 0.0386 \\
\hline & 2 & 5 & 4.86 & 5 & 3.21 & 10 & 4.86 & 10 & 4.78 & 30 & 0.0412 \\
\hline \multirow{2}{*}{ G } & 1 & 23 & 16.38 & 31 & 15.63 & 6 & 4.75 & 6 & 4.67 & 31 & 0.0515 \\
\hline & 2 & 22 & 16.38 & 31 & 15.63 & 6 & 4.75 & 6 & 4.67 & 20 & 0.0311 \\
\hline \multirow{2}{*}{$\mathrm{H}$} & 1 & 16 & 9.36 & 16 & 9.19 & 16 & 9.32 & 16 & 9.19 & 33 & 0.0686 \\
\hline & 2 & 15 & 9.36 & 16 & 9.19 & 16 & 9.32 & 16 & 9.19 & 32 & 0.0684 \\
\hline \multirow{2}{*}{ I } & 1 & 21 & 15.83 & 33 & 15.68 & 25 & 24.61 & 31 & 15.68 & 8 & 0.0058 \\
\hline & 2 & 29 & 30.83 & 36 & 30.68 & 32 & 46.83 & 36 & 30.68 & 4 & 0.0030 \\
\hline \multirow{2}{*}{$\mathrm{J}$} & 1 & 9 & 8.30 & 12 & 8.07 & 12 & 8.68 & 12 & 8.15 & 10 & 0.0143 \\
\hline & 2 & 10 & 8.30 & 12 & 8.07 & 12 & 8.68 & 12 & 8.15 & 10 & 0.0143 \\
\hline \multirow{2}{*}{ K } & 1 & 36 & 61.81 & 25 & 14.74 & 35 & 73.67 & 25 & 14.80 & 24 & 0.0360 \\
\hline & 2 & 35 & 61.81 & 25 & 14.74 & 35 & 73.67 & 25 & 14.80 & 19 & 0.0286 \\
\hline \multirow{2}{*}{$\mathrm{L}$} & 1 & 26 & 18.76 & 9 & 4.55 & 5 & 4.68 & 5 & 4.61 & 12 & 0.0146 \\
\hline & 2 & 34 & 53.98 & 22 & 13.64 & 20 & 13.73 & 22 & 13.66 & 9 & 0.0072 \\
\hline \multirow{2}{*}{ M } & 1 & 3 & 3.75 & 5 & 3.21 & 3 & 3.75 & 3 & 3.68 & 35 & NA \\
\hline & 2 & 4 & 3.76 & 5 & 3.21 & 3 & 3.75 & 3 & 3.68 & 34 & NA \\
\hline \multirow{2}{*}{$\mathrm{O}$} & 1 & 12 & 9.05 & 14 & 8.82 & 14 & 8.84 & 14 & 8.90 & 17 & 0.0229 \\
\hline & 2 & 11 & 9.05 & 14 & 8.82 & 14 & 8.84 & 14 & 8.90 & 16 & 0.0214 \\
\hline \multirow{2}{*}{$\mathrm{P}$} & 1 & 28 & 21.42 & 3 & 0.86 & 1 & 0.89 & 1 & 0.83 & 27 & 0.0370 \\
\hline & 2 & 27 & 21.41 & 3 & 0.86 & 1 & 0.89 & 1 & 0.83 & 21 & 0.0333 \\
\hline \multirow{2}{*}{ Q } & 1 & 14 & 9.14 & 1 & 0.82 & 28 & 30.63 & 34 & 20.48 & 1 & 0.0027 \\
\hline & 2 & 13 & 9.14 & 1 & 0.82 & 28 & 30.63 & 34 & 20.48 & 1 & 0.0027 \\
\hline \multirow{2}{*}{$S$} & 1 & 19 & 14.82 & 23 & 14.57 & 21 & 14.63 & 23 & 14.57 & 18 & 0.0281 \\
\hline & 2 & 19 & 14.82 & 23 & 14.57 & 21 & 14.63 & 23 & 14.57 & 13 & 0.0195 \\
\hline \multirow{2}{*}{$\mathrm{T}$} & 1 & 1 & 0.01 & 34 & 17.99 & 26 & 27.40 & 32 & 18.02 & 36 & NA \\
\hline & 2 & 1 & 0.01 & 34 & 17.99 & 26 & 27.40 & 32 & 18.02 & 25 & 0.0366 \\
\hline 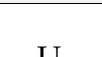 & 1 & 33 & 44.63 & 29 & 15.30 & 33 & 51.84 & 29 & 15.46 & 22 & 0.0336 \\
\hline U & 2 & 32 & 37.35 & 29 & 15.30 & 33 & 51.84 & 29 & 15.46 & 23 & 0.0358 \\
\hline $\mathrm{V}$ & 1 & 18 & 11.77 & 20 & 11.69 & 18 & 11.77 & 20 & 11.71 & 7 & 0.0034 \\
\hline$v$ & 2 & 17 & 11.77 & 20 & 11.69 & 18 & 11.77 & 20 & 11.71 & 3 & 0.0028 \\
\hline
\end{tabular}

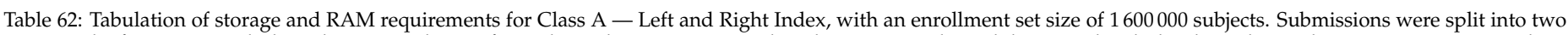

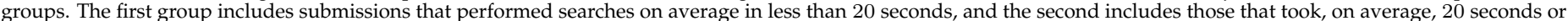

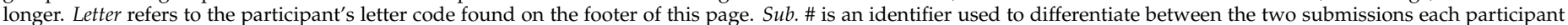

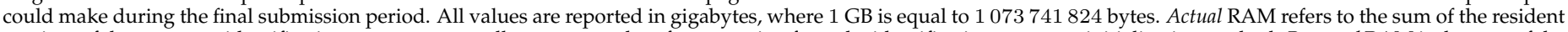

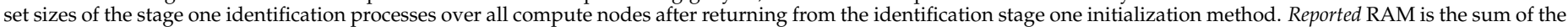

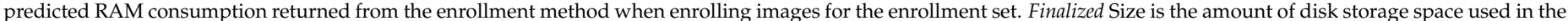

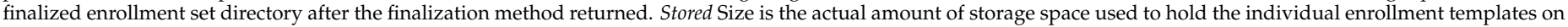

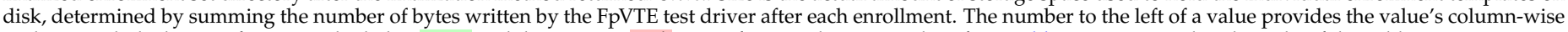
ranking, with the best performance shaded in green and the worst in pink. For reference, the FNIR values from Table 9 are reprinted to the right of this table.

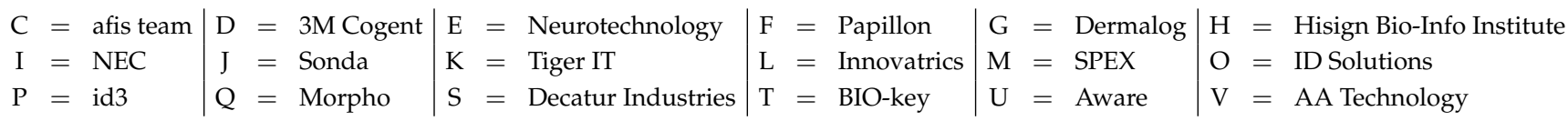




\begin{tabular}{|c|c|c|c|c|c|c|c|c|c|c|c|}
\hline \multicolumn{2}{|c|}{ Participant } & \multicolumn{4}{|c|}{ RAM } & \multicolumn{4}{|c|}{ On Disk } & \multirow{2}{*}{\multicolumn{2}{|c|}{ FNIR $@$ FPIR $=10^{-3}$}} \\
\hline Letter & Sub.\# & \multicolumn{2}{|c|}{ Actual } & \multicolumn{2}{|c|}{ Reported } & \multicolumn{2}{|c|}{ Finalized } & \multicolumn{2}{|c|}{ Stored } & & \\
\hline \multirow{2}{*}{$\mathrm{C}$} & 1 & 3 & 46.49 & 4 & 45.92 & 2 & 46.34 & 2 & 45.98 & 30 & NA \\
\hline & 2 & 4 & 46.49 & 4 & 45.92 & 2 & 46.34 & 2 & 45.98 & 29 & NA \\
\hline \multirow{2}{*}{$\mathrm{D}$} & 1 & 12 & 79.43 & 16 & 84.51 & 18 & 86.78 & 18 & 86.59 & 6 & 0.0020 \\
\hline & 2 & 13 & 79.43 & 15 & 83.67 & 15 & 85.95 & 17 & 85.76 & 2 & 0.0012 \\
\hline \multirow{2}{*}{$\mathrm{E}$} & 1 & 14 & 79.73 & 3 & 29.86 & 1 & 30.00 & 1 & 29.89 & 16 & 0.0043 \\
\hline & 2 & 28 & 317.95 & 30 & 176.65 & 26 & 176.79 & 28 & 176.67 & 7 & 0.0024 \\
\hline \multirow{2}{*}{$\mathrm{F}$} & 1 & 10 & 77.02 & 11 & 53.02 & 12 & 77.01 & 13 & 76.88 & 27 & 0.0591 \\
\hline & 2 & 10 & 77.02 & 11 & 53.02 & 12 & 77.01 & 13 & 76.88 & 27 & 0.0591 \\
\hline \multirow{2}{*}{ G } & 1 & 25 & 156.12 & 26 & 152.16 & 4 & 47.21 & 4 & 47.07 & 18 & 0.0062 \\
\hline & 2 & 26 & 156.12 & 26 & 152.16 & 4 & 47.21 & 4 & 47.07 & 14 & 0.0040 \\
\hline \multirow{2}{*}{$\mathrm{H}$} & 1 & 15 & 86.46 & 17 & 84.85 & 16 & 86.42 & 15 & 84.85 & 23 & 0.0203 \\
\hline & 2 & 16 & 86.46 & 17 & 84.85 & 16 & 86.42 & 15 & 84.85 & 24 & 0.0204 \\
\hline \multirow{2}{*}{ I } & 1 & 5 & 49.68 & 8 & 49.40 & 14 & 82.58 & 8 & 49.40 & 2 & 0.0012 \\
\hline & 2 & 21 & 108.71 & 23 & 108.43 & 23 & 133.80 & 23 & 108.43 & 1 & 0.0009 \\
\hline \multirow{2}{*}{$\mathrm{J}$} & 1 & 18 & 101.00 & 21 & 101.67 & 21 & 100.73 & 21 & 100.75 & 17 & 0.0049 \\
\hline & 2 & 17 & 101.00 & 21 & 101.67 & 21 & 100.73 & 21 & 100.75 & 11 & 0.0033 \\
\hline \multirow{2}{*}{$\mathrm{L}$} & 1 & 22 & 119.79 & 7 & 47.80 & 7 & 47.92 & 7 & 47.77 & 10 & 0.0031 \\
\hline & 2 & 27 & 177.48 & 6 & 46.73 & 6 & 47.56 & 6 & 47.42 & 11 & 0.0033 \\
\hline \multirow{2}{*}{ M } & 1 & 6 & 57.53 & 9 & 53.02 & 8 & 57.52 & 9 & 57.39 & 26 & 0.0543 \\
\hline & 2 & 6 & 57.53 & 9 & 53.02 & 8 & 57.52 & 9 & 57.39 & 25 & 0.0515 \\
\hline \multirow{2}{*}{$\mathrm{O}$} & 1 & 19 & 101.00 & 19 & 101.67 & 19 & 100.73 & 19 & 100.75 & 15 & 0.0041 \\
\hline & 2 & 20 & 101.00 & 19 & 101.67 & 19 & 100.73 & 19 & 100.75 & 13 & 0.0035 \\
\hline \multirow{2}{*}{$\mathrm{Q}$} & 1 & 1 & 7.54 & 1 & 8.87 & 27 & 232.47 & 29 & 222.31 & 2 & 0.0012 \\
\hline & 2 & 2 & 7.54 & 1 & 8.87 & 27 & 232.47 & 29 & 222.31 & 2 & 0.0012 \\
\hline \multirow{2}{*}{$S$} & 1 & 24 & 150.35 & 24 & 147.31 & 24 & 147.42 & 24 & 147.31 & 20 & 0.0108 \\
\hline & 2 & 23 & 150.35 & 24 & 147.31 & 24 & 147.42 & 24 & 147.31 & 21 & 0.0136 \\
\hline \multirow{2}{*}{$\mathrm{U}$} & 1 & 29 & 440.68 & 28 & 155.98 & 29 & 512.65 & 26 & 157.35 & 19 & 0.0099 \\
\hline & 2 & 30 & 540.60 & 29 & 170.16 & 30 & 625.07 & 27 & 171.53 & 22 & 0.0141 \\
\hline \multirow{2}{*}{$\mathrm{V}$} & 1 & 8 & 63.53 & 13 & 63.39 & 10 & 63.52 & 11 & 63.41 & 9 & 0.0027 \\
\hline & 2 & 9 & 63.53 & 13 & 63.39 & 10 & 63.52 & 11 & 63.41 & 7 & 0.0024 \\
\hline
\end{tabular}

Table 63: Tabulation of storage and RAM requirements for Class B - Identification Flats, with an enrollment set size of 3000000 subjects. Letter refers to the participant's letter code found on the footer of this page. Sub. \# is an identifier used to differentiate between the two submissions each participant could make during the final submission period. All values are reported in gigabytes, where 1 GB is equal to 1073741824 bytes. Actual RAM refers to the sum of the resident set sizes of the stage one identification processes over all compute nodes after returning from the identification stage one initialization method. Reported RAM is the sum of the predicted RAM consumption returned from the enrollment method when enrolling images for the enrollment set. Finalized Size is the amount of disk storage space used in the finalized enrollment set directory after the finalization method returned. Stored Size is the actual amount of storage space used to hold the individual enrollment templates on disk, determined by summing the number of bytes written by the FpVTE test driver after each enrollment. The number to the left of a value provides the value's column-wise ranking, with the best performance shaded in green and the worst in pink. For reference, the FNIR values from Table 13 are reprinted to the right of this table.

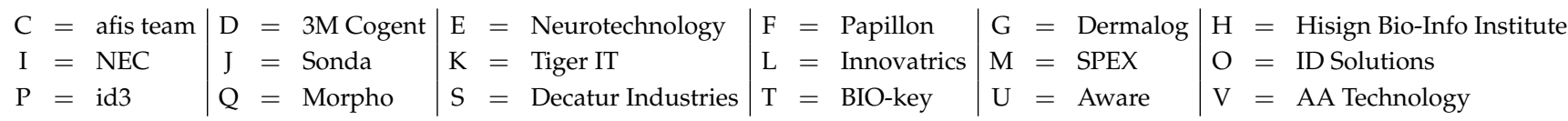




\begin{tabular}{|c|c|c|c|c|c|c|c|c|c|}
\hline \multicolumn{2}{|c|}{ Participant } & \multicolumn{4}{|c|}{ RAM } & \multicolumn{4}{|c|}{ On Disk } \\
\hline Letter & Sub.\# & \multicolumn{2}{|c|}{ Actual } & \multicolumn{2}{|c|}{ Reported } & \multicolumn{2}{|c|}{ Finalized } & \multicolumn{2}{|c|}{ Stored } \\
\hline \multirow{2}{*}{ C } & 1 & 9 & 92.86 & 11 & 96.24 & 10 & 92.63 & 11 & 96.33 \\
\hline & 2 & 10 & 92.86 & 11 & 96.24 & 10 & 92.63 & 11 & 96.33 \\
\hline \multirow{2}{*}{$\mathrm{D}$} & 1 & 16 & 132.37 & 19 & 155.88 & 19 & 159.68 & 19 & 159.37 \\
\hline & 2 & 15 & 132.37 & 18 & 153.13 & 18 & 156.93 & 18 & 156.62 \\
\hline \multirow{2}{*}{$\mathrm{E}$} & 1 & 14 & 127.39 & 3 & 57.47 & 1 & 57.71 & 1 & 57.52 \\
\hline & 2 & 28 & 573.25 & 30 & 318.66 & 26 & 318.89 & 28 & 318.70 \\
\hline \multirow{2}{*}{$\mathrm{F}$} & 1 & 7 & 90.93 & 6 & 82.27 & 6 & 90.92 & 7 & 90.70 \\
\hline & 2 & 6 & 90.93 & 6 & 82.27 & 6 & 90.92 & 7 & 90.70 \\
\hline \multirow{2}{*}{ G } & 1 & 25 & 274.35 & 26 & 267.49 & 4 & 82.40 & 4 & 82.17 \\
\hline & 2 & 26 & 274.35 & 26 & 267.49 & 4 & 82.40 & 4 & 82.17 \\
\hline \multirow{2}{*}{$\mathrm{H}$} & 1 & 17 & 144.02 & 16 & 142.06 & 14 & 143.97 & 16 & 142.06 \\
\hline & 2 & 18 & 144.02 & 16 & 142.06 & 14 & 143.97 & 16 & 142.06 \\
\hline \multirow{2}{*}{ I } & 1 & 4 & 84.42 & 10 & 83.96 & 16 & 144.38 & 6 & 83.96 \\
\hline & 2 & 11 & 110.22 & 13 & 109.76 & 17 & 153.35 & 13 & 109.76 \\
\hline \multirow{2}{*}{$\mathrm{J}$} & 1 & 20 & 181.00 & 22 & 182.11 & 20 & 182.47 & 22 & 180.58 \\
\hline & 2 & 21 & 181.00 & 23 & 182.11 & 23 & 182.47 & 23 & 180.58 \\
\hline \multirow{2}{*}{$\mathrm{L}$} & 1 & 23 & 192.19 & 4 & 72.47 & 2 & 72.67 & 2 & 72.43 \\
\hline & 2 & 27 & 274.95 & 4 & 72.47 & 3 & 72.67 & 2 & 72.43 \\
\hline \multirow{2}{*}{ M } & 1 & 5 & 90.93 & 8 & 82.27 & 8 & 90.92 & 9 & 90.70 \\
\hline & 2 & 8 & 90.93 & 8 & 82.27 & 8 & 90.92 & 9 & 90.70 \\
\hline \multirow{2}{*}{$\mathrm{O}$} & 1 & 19 & 181.00 & 20 & 182.11 & 21 & 182.47 & 20 & 180.58 \\
\hline & 2 & 22 & 181.00 & 20 & 182.11 & 21 & 182.47 & 20 & 180.58 \\
\hline \multirow{2}{*}{ Q } & 1 & 2 & 13.33 & 1 & 15.77 & 27 & 412.86 & 29 & 395.06 \\
\hline & 2 & 1 & 13.33 & 1 & 15.77 & 27 & 412.86 & 29 & 395.06 \\
\hline \multirow{2}{*}{ S } & 1 & 24 & 260.37 & 24 & 257.08 & 25 & 257.27 & 24 & 257.09 \\
\hline & 2 & 3 & 61.04 & 24 & 257.08 & 24 & 257.27 & 24 & 257.09 \\
\hline \multirow{2}{*}{$\mathrm{U}$} & 1 & 29 & 811.45 & 28 & 293.25 & 29 & 941.34 & 26 & 295.54 \\
\hline & 2 & 29 & 811.45 & 28 & 293.25 & 29 & 941.34 & 26 & 295.54 \\
\hline \multirow{2}{*}{$\mathrm{V}$} & 1 & 13 & 121.80 & 14 & 121.56 & 12 & 121.79 & 14 & 121.60 \\
\hline & 2 & 12 & 121.79 & 14 & 121.56 & 12 & 121.79 & 14 & 121.60 \\
\hline
\end{tabular}

\begin{tabular}{|c|c|}
\hline \multicolumn{2}{|c|}{ FNIR $@$ FPIR $=10^{-3}$} \\
\hline 30 & NA \\
\hline 24 & 0.0711 \\
\hline 6 & 0.0015 \\
\hline 2 & 0.0011 \\
\hline 14 & 0.0088 \\
\hline 13 & 0.0048 \\
\hline 25 & 0.0734 \\
\hline 25 & 0.0734 \\
\hline 23 & 0.0368 \\
\hline 20 & 0.0276 \\
\hline 20 & 0.0276 \\
\hline 19 & 0.0275 \\
\hline 4 & 0.0013 \\
\hline 1 & 0.0010 \\
\hline 12 & 0.0047 \\
\hline 10 & 0.0027 \\
\hline 16 & 0.0102 \\
\hline 15 & 0.0095 \\
\hline 28 & 0.0934 \\
\hline 27 & 0.0826 \\
\hline 9 & 0.0025 \\
\hline 10 & 0.0027 \\
\hline 2 & 0.0011 \\
\hline 4 & 0.0013 \\
\hline 22 & 0.0311 \\
\hline 29 & 0.1680 \\
\hline 18 & 0.0163 \\
\hline 17 & 0.0155 \\
\hline 7 & 0.0024 \\
\hline 7 & 0.0024 \\
\hline
\end{tabular}

Table 64: Tabulation of storage and RAM requirements for Class C - Ten-Finger Plain-to-Plain, with an enrollment set size of 5000000 subjects. Letter refers to the participant's letter code found on the footer of this page. Sub. \# is an identifier used to differentiate between the two submissions each participant could make during the final submission period. All values are reported in gigabytes, where 1 GB is equal to 1073741824 bytes. Actual RAM refers to the sum of the resident set sizes of the stage one identification processes over all compute nodes after returning from the identification stage one initialization method. Reported RAM is the sum of the predicted RAM consumption returned from the enrollment method when enrolling images for the enrollment set. Finalized Size is the amount of disk storage space used in the finalized enrollment set directory after the finalization method returned. Stored Size is the actual amount of storage space used to hold the individual enrollment templates on disk, determined by summing the number of bytes written by the FpVTE test driver after each enrollment. The number to the left of a value provides the value's column-wise ranking, with the best performance shaded in green and the worst in pink. For reference, the FNIR values from Table 14 are reprinted to the right of this table.

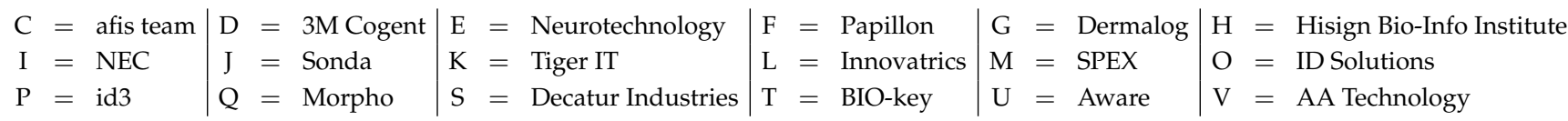




\begin{tabular}{|c|c|c|c|c|c|c|c|c|c|}
\hline \multicolumn{2}{|c|}{ Participant } & \multicolumn{4}{|c|}{ RAM } & \multicolumn{4}{|c|}{ On Disk } \\
\hline Letter & Sub.\# & \multicolumn{2}{|c|}{ Actual } & \multicolumn{2}{|c|}{ Reported } & \multicolumn{2}{|c|}{ Finalized } & \multicolumn{2}{|c|}{ Stored } \\
\hline \multirow{2}{*}{$\mathrm{C}$} & 1 & 14 & 183.36 & 14 & 190.50 & 14 & 183.11 & 14 & 190.59 \\
\hline & 2 & 15 & 183.36 & 14 & 190.50 & 14 & 183.11 & 14 & 190.59 \\
\hline \multirow{2}{*}{$\mathrm{D}$} & 1 & 10 & 132.40 & 22 & 302.53 & 18 & 303.52 & 24 & 303.21 \\
\hline & 2 & 9 & 132.37 & 27 & 309.65 & 19 & 310.65 & 25 & 310.33 \\
\hline \multirow{2}{*}{$\mathrm{E}$} & 1 & 16 & 191.66 & 3 & 95.45 & 1 & 95.69 & 1 & 95.50 \\
\hline & 2 & 30 & 930.48 & 30 & 528.40 & 26 & 528.63 & 28 & 528.45 \\
\hline \multirow{2}{*}{$\mathrm{F}$} & 1 & 7 & 130.29 & 5 & 118.45 & 4 & 130.29 & 5 & 130.07 \\
\hline & 2 & 6 & 130.29 & 5 & 118.45 & 4 & 130.29 & 5 & 130.07 \\
\hline \multirow{2}{*}{ G } & 1 & 20 & 261.29 & 18 & 254.66 & 2 & 97.99 & 2 & 97.76 \\
\hline & 2 & 19 & 261.29 & 18 & 254.66 & 2 & 97.99 & 2 & 97.76 \\
\hline \multirow{2}{*}{$\mathrm{H}$} & 1 & 13 & 144.02 & 10 & 143.46 & 8 & 143.97 & 10 & 143.46 \\
\hline & 2 & 12 & 144.02 & 10 & 143.46 & 8 & 143.97 & 10 & 143.46 \\
\hline \multirow{2}{*}{ I } & 1 & 4 & 113.80 & 4 & 113.34 & 12 & 153.41 & 4 & 113.34 \\
\hline & 2 & 11 & 137.03 & 9 & 136.57 & 13 & 153.69 & 9 & 136.57 \\
\hline \multirow{2}{*}{$\mathrm{J}$} & 1 & 22 & 303.13 & 24 & 304.24 & 21 & 355.15 & 21 & 302.68 \\
\hline & 2 & 24 & 303.13 & 23 & 304.24 & 20 & 355.15 & 20 & 302.68 \\
\hline \multirow{2}{*}{$\mathrm{L}$} & 1 & 21 & 280.49 & 12 & 151.41 & 10 & 151.61 & 12 & 151.37 \\
\hline & 2 & 26 & 367.82 & 12 & 151.41 & 11 & 151.61 & 12 & 151.37 \\
\hline \multirow{2}{*}{ M } & 1 & 8 & 130.29 & 7 & 118.45 & 6 & 130.29 & 7 & 130.07 \\
\hline & 2 & 5 & 130.29 & 7 & 118.45 & 6 & 130.29 & 7 & 130.07 \\
\hline \multirow{2}{*}{$\mathrm{O}$} & 1 & 25 & 303.13 & 25 & 304.24 & 22 & 355.15 & 22 & 302.68 \\
\hline & 2 & 23 & 303.13 & 25 & 304.24 & 22 & 355.15 & 22 & 302.68 \\
\hline \multirow{2}{*}{ Q } & 1 & 1 & 20.22 & 1 & 25.61 & 27 & 666.73 & 29 & 641.13 \\
\hline & 2 & 2 & 20.22 & 1 & 25.61 & 27 & 666.73 & 29 & 641.13 \\
\hline \multirow{2}{*}{$S$} & 1 & 27 & 382.88 & 28 & 381.17 & 25 & 381.36 & 26 & 381.17 \\
\hline & 2 & 3 & 78.28 & 28 & 381.17 & 24 & 381.36 & 26 & 381.17 \\
\hline \multirow{2}{*}{$\mathrm{U}$} & 1 & 28 & 806.17 & 20 & 257.87 & 29 & 937.76 & 18 & 260.17 \\
\hline & 2 & 28 & 806.17 & 20 & 257.87 & 29 & 937.76 & 18 & 260.17 \\
\hline \multirow{2}{*}{$\mathrm{V}$} & 1 & 17 & 234.03 & 16 & 233.80 & 16 & 234.03 & 16 & 233.84 \\
\hline & 2 & 18 & 234.03 & 16 & 233.80 & 16 & 234.03 & 16 & 233.84 \\
\hline
\end{tabular}

\begin{tabular}{|c|c|}
\hline \multicolumn{2}{|c|}{ FNIR $@$ FPIR $=10^{-3}$} \\
\hline 16 & 0.0094 \\
\hline 15 & 0.0085 \\
\hline 4 & 0.0015 \\
\hline 7 & 0.0018 \\
\hline 18 & 0.0106 \\
\hline 12 & 0.0050 \\
\hline 25 & 0.0536 \\
\hline 25 & 0.0536 \\
\hline 24 & 0.0447 \\
\hline 21 & 0.0333 \\
\hline 20 & 0.0201 \\
\hline 19 & 0.0199 \\
\hline 1 & 0.0013 \\
\hline 2 & 0.0014 \\
\hline 13 & 0.0051 \\
\hline 9 & 0.0033 \\
\hline 17 & 0.0097 \\
\hline 14 & 0.0083 \\
\hline 28 & 0.0783 \\
\hline 27 & 0.0716 \\
\hline 11 & 0.0034 \\
\hline 9 & 0.0033 \\
\hline 5 & 0.0017 \\
\hline 2 & 0.0014 \\
\hline 29 & 0.0860 \\
\hline 30 & 0.2462 \\
\hline 23 & 0.0358 \\
\hline 22 & 0.0351 \\
\hline 5 & 0.0017 \\
\hline 8 & 0.0019 \\
\hline
\end{tabular}

Table 65: Tabulation of storage and RAM requirements for Class C - Ten-Finger Rolled-to-Rolled, with an enrollment set size of 5000000 subjects. Letter refers to the participant's letter code found on the footer of this page. Sub. \# is an identifier used to differentiate between the two submissions each participant could make during the final submission period. All values are reported in gigabytes, where 1 GB is equal to 1073741824 bytes. Actual RAM refers to the sum of the resident set sizes of the stage one identification processes over all compute nodes after returning from the identification stage one initialization method. Reported RAM is the sum of the predicted RAM consumption returned from the enrollment method when enrolling images for the enrollment set. Finalized Size is the amount of disk storage space used in the finalized enrollment set directory after the finalization method returned. Stored Size is the actual amount of storage space used to hold the individual enrollment templates on disk, determined by summing the number of bytes written by the FpVTE test driver after each enrollment. The number to the left of a value provides the value's column-wise ranking, with the best performance shaded in green and the worst in pink. For reference, the FNIR values from Table 15 are reprinted to the right of this table.

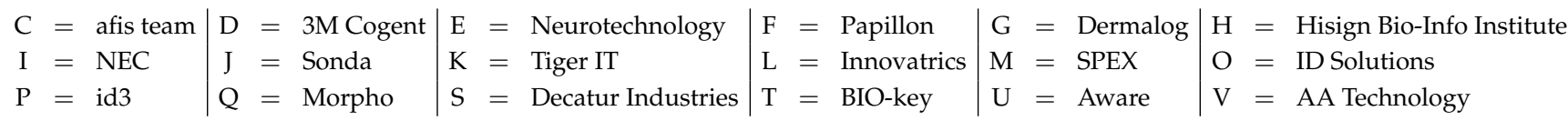




\section{G Search Template Sizes}

\section{G.1 Mean Values}

\begin{tabular}{l|l|l|l|l|l}
$\mathrm{C}=$ afis team & $\mathrm{D}=3 \mathrm{M}$ Cogent & $\mathrm{E}=$ Neurotechnology & $\mathrm{F}=$ Papillon & $\mathrm{G}=$ Dermalog & $\mathrm{H}=$ Hisign Bio-Info Institute \\
$\mathrm{I}=\mathrm{NEC}$ & $\mathrm{J}=$ Sonda & $\mathrm{K}=$ Tiger IT & $\mathrm{L}=$ Innovatrics & $\mathrm{M}=$ SPEX & $\mathrm{O}=$ ID Solutions \\
$\mathrm{P}=\mathrm{id} 3$ & $\mathrm{Q}=$ Morpho & $\mathrm{S}=$ Decatur Industries & $\mathrm{T}=$ BIO-key & $\mathrm{U}=$ Aware & $\mathrm{V}=$ AA Technology
\end{tabular}




\begin{tabular}{|c|c|c|c|c|c|c|c|c|c|c|c|c|c|}
\hline \multicolumn{2}{|c|}{ Participant } & \multicolumn{4}{|c|}{ Left Index } & \multicolumn{4}{|c|}{ Right Index } & \multicolumn{4}{|c|}{ Left and Right Index } \\
\hline Letter & Sub.\# & \multicolumn{2}{|c|}{ Size } & \multicolumn{2}{|c|}{ FNIR } & \multicolumn{2}{|c|}{ Size } & \multicolumn{2}{|c|}{ FNIR } & \multicolumn{2}{|c|}{ Size } & \multicolumn{2}{|c|}{ FNIR } \\
\hline \multirow{2}{*}{$\mathrm{C}$} & 1 & 5 & 1.49 & 30 & 0.1335 & 5 & 1.51 & 30 & 0.1132 & 5 & 2.98 & 26 & 0.0368 \\
\hline & 2 & 5 & 1.49 & 31 & 0.1337 & 5 & 1.51 & 29 & 0.1124 & 5 & 2.98 & 28 & 0.0374 \\
\hline \multirow{2}{*}{$\mathrm{D}$} & 1 & 18 & 3.31 & 1 & 0.0197 & 18 & 3.34 & 1 & 0.0190 & 18 & 6.65 & 4 & 0.0030 \\
\hline & 2 & 18 & 3.31 & 1 & 0.0197 & 18 & 3.34 & 1 & 0.0190 & 18 & 6.65 & 4 & 0.0030 \\
\hline \multirow{2}{*}{$\mathrm{E}$} & 1 & 27 & 4.83 & 16 & 0.0745 & 27 & 4.89 & 15 & 0.0630 & 27 & 9.71 & 15 & 0.0207 \\
\hline & 2 & 27 & 4.83 & 15 & 0.0723 & 27 & 4.89 & 14 & 0.0624 & 27 & 9.71 & 14 & 0.0202 \\
\hline \multirow{2}{*}{$\mathrm{F}$} & 1 & 8 & 1.54 & 25 & 0.1111 & 8 & 1.55 & 25 & 0.0933 & 7 & 3.00 & 29 & 0.0386 \\
\hline & 2 & 8 & 1.54 & 22 & 0.1082 & 8 & 1.55 & 22 & 0.0903 & 7 & 3.00 & 30 & 0.0412 \\
\hline \multirow{2}{*}{ G } & 1 & 10 & 2.51 & 24 & 0.1089 & 10 & 2.52 & 24 & 0.0910 & 10 & 5.02 & 31 & 0.0515 \\
\hline & 2 & 10 & 2.51 & 23 & 0.1086 & 10 & 2.52 & 23 & 0.0909 & 10 & 5.02 & 20 & 0.0311 \\
\hline \multirow{2}{*}{$\mathrm{H}$} & 1 & 16 & 3.00 & 32 & 0.1576 & 16 & 3.00 & 32 & 0.1230 & 16 & 5.99 & 33 & 0.0686 \\
\hline & 2 & 16 & 3.00 & 33 & 0.1607 & 16 & 3.00 & 33 & 0.1249 & 16 & 5.99 & 32 & 0.0684 \\
\hline \multirow{2}{*}{ I } & 1 & 35 & 6.83 & 7 & 0.0257 & 35 & 6.87 & 5 & 0.0215 & 29 & 10.19 & 8 & 0.0058 \\
\hline & 2 & 36 & 10.07 & 8 & 0.0278 & 36 & 10.18 & 3 & 0.0214 & 36 & 20.17 & 4 & 0.0030 \\
\hline \multirow{2}{*}{$\mathrm{J}$} & 1 & 12 & 2.68 & 18 & 0.0786 & 12 & 2.72 & 20 & 0.0708 & 12 & 5.26 & 10 & 0.0143 \\
\hline & 2 & 12 & 2.68 & 14 & 0.0712 & 12 & 2.72 & 16 & 0.0643 & 12 & 5.26 & 10 & 0.0143 \\
\hline \multirow{2}{*}{ K } & 1 & 25 & 4.82 & 21 & 0.0883 & 25 & 4.85 & 18 & 0.0682 & 25 & 9.67 & 24 & 0.0360 \\
\hline & 2 & 25 & 4.82 & 20 & 0.0875 & 25 & 4.85 & 19 & 0.0685 & 25 & 9.67 & 19 & 0.0286 \\
\hline \multirow{2}{*}{$\mathrm{L}$} & 1 & 7 & 1.52 & 11 & 0.0625 & 7 & 1.53 & 12 & 0.0505 & 9 & 3.01 & 12 & 0.0146 \\
\hline & 2 & 22 & 4.47 & 9 & 0.0351 & 22 & 4.51 & 9 & 0.0295 & 22 & 8.93 & 9 & 0.0072 \\
\hline \multirow{2}{*}{ M } & 1 & 3 & 1.21 & 35 & 0.2995 & 3 & 1.18 & 36 & 0.2615 & 3 & 2.31 & 35 & NA \\
\hline & 2 & 3 & 1.21 & 34 & 0.2921 & 3 & 1.18 & 35 & 0.2526 & 3 & 2.31 & 34 & NA \\
\hline \multirow{2}{*}{$\mathrm{O}$} & 1 & 14 & 2.93 & 19 & 0.0818 & 14 & 2.98 & 21 & 0.0776 & 14 & 5.77 & 17 & 0.0229 \\
\hline & 2 & 14 & 2.93 & 17 & 0.0766 & 14 & 2.98 & 17 & 0.0675 & 14 & 5.77 & 16 & 0.0214 \\
\hline \multirow{2}{*}{$\mathrm{P}$} & 1 & 1 & 0.28 & 29 & 0.1308 & 1 & 0.28 & 31 & 0.1133 & 1 & 0.53 & 27 & 0.0370 \\
\hline & 2 & 1 & 0.28 & 28 & 0.1272 & 1 & 0.28 & 28 & 0.1100 & 1 & 0.53 & 21 & 0.0333 \\
\hline$\Omega$ & 1 & 33 & 6.52 & 3 & 0.0222 & 33 & 6.57 & 6 & 0.0218 & 34 & 13.08 & 1 & 0.0027 \\
\hline$Q$ & 2 & 33 & 6.52 & 4 & 0.0226 & 33 & 6.57 & 3 & 0.0214 & 34 & 13.08 & 1 & 0.0027 \\
\hline$S$ & 1 & 23 & 4.74 & 10 & 0.0571 & 23 & 4.77 & 10 & 0.0442 & 23 & 9.52 & 18 & 0.0281 \\
\hline 3 & 2 & 23 & 4.74 & 12 & 0.0650 & 23 & 4.77 & 11 & 0.0503 & 23 & 9.52 & 13 & 0.0195 \\
\hline$T$ & 1 & 31 & 6.00 & 36 & NA & 31 & 6.09 & 34 & 0.1929 & 32 & 12.09 & 36 & NA \\
\hline $\mathrm{T}$ & 2 & 31 & 6.00 & 13 & 0.0685 & 31 & 6.09 & 13 & 0.0562 & 32 & 12.09 & 25 & 0.0366 \\
\hline I & 1 & 29 & 5.17 & 27 & 0.1218 & 29 & 5.20 & 26 & 0.0996 & 30 & 10.36 & 22 & 0.0336 \\
\hline U & 2 & 29 & 5.17 & 26 & 0.1178 & 29 & 5.20 & 27 & 0.1007 & 30 & 10.36 & 23 & 0.0358 \\
\hline $\mathrm{V}$ & 1 & 20 & 3.70 & 6 & 0.0253 & 20 & 3.72 & 8 & 0.0223 & 20 & 7.41 & 7 & 0.0034 \\
\hline$v$ & 2 & 20 & 3.70 & 5 & 0.0252 & 20 & 3.72 & 7 & 0.0222 & 20 & 7.41 & 3 & 0.0028 \\
\hline
\end{tabular}

Table 66: Tabulation of mean search template sizes for Class A. Letter refers to the participant's letter code found on the footer of this page. Sub. \# is an identifier used to differentiate between the two submissions each participant could make. Size values indicate the mean kilobytes used to store a search template on disk for a single subject, where $1 \mathrm{kB}$ is equal to 1024 bytes. The FNIR column shows FNIR for each submission at FPIR $=10^{-3}$. NA indicates that the operations required to produce the value could not be performed. The number to the left of a value provides the value's column-wise ranking, with the best performance shaded in green and the worst in pink.

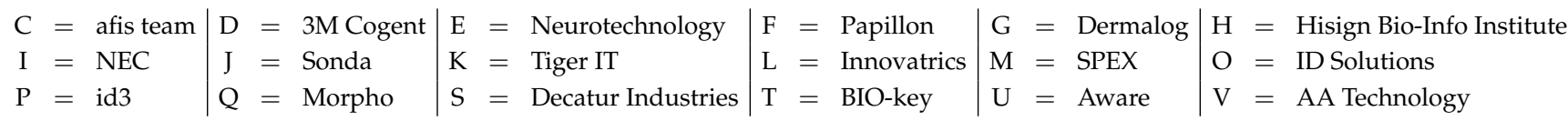




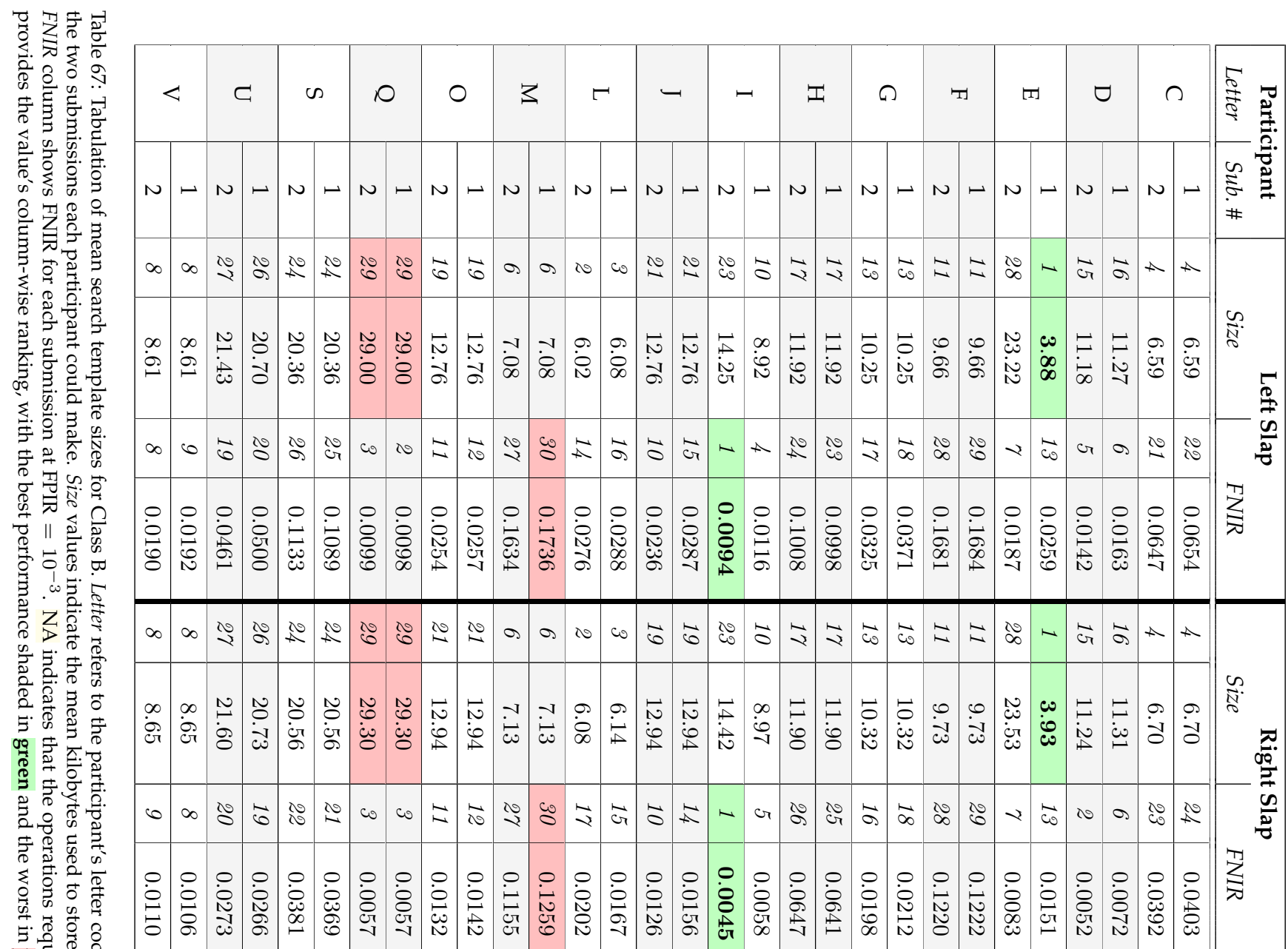

ㄷ. ㄷ. 용. 2 .

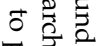

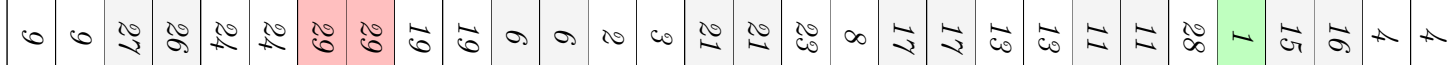

궁

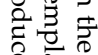

政离

के

눙

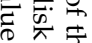

8 के

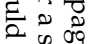

$\exists$.

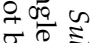

일

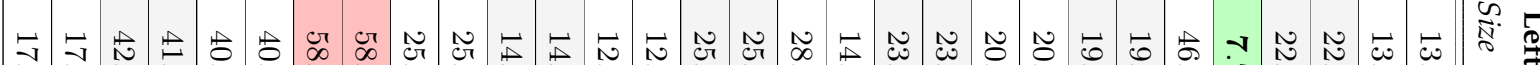

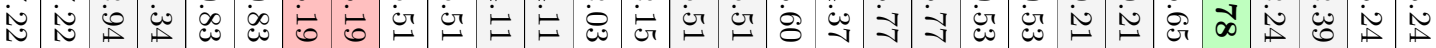

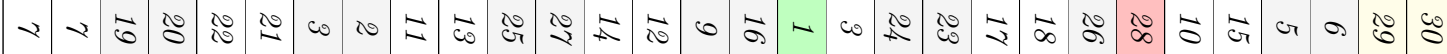

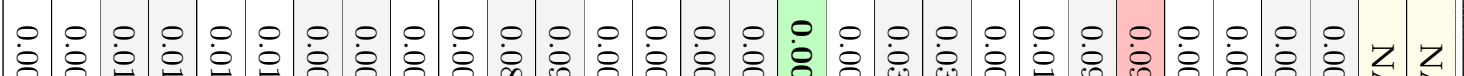

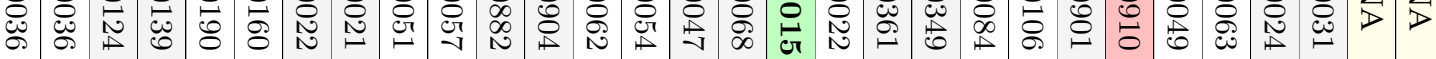
$\underset{3}{\pi}$

证.

贾 $\stackrel{0}{\rightarrow}$

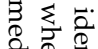

․․․

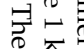

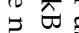

क.

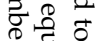

$\stackrel{4}{\circ}$

융

居 $\rightarrow \overrightarrow{0}$

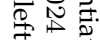

0 过

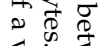

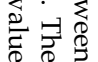

○ 0 传

N N iv iv

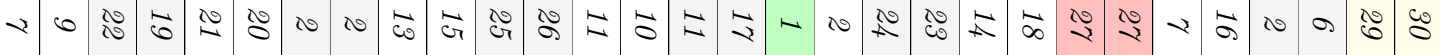

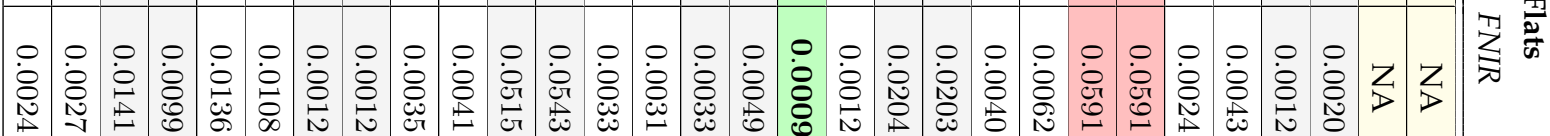

$\mathrm{C}=$ afis team $\mid \mathrm{D}=3 \mathrm{M}$ Cogent $\mid \mathrm{E}=$ Neurotechnology $\mid \mathrm{F}=$ Papillon $\mid \mathrm{G}=$ Dermalog $\mid \mathrm{H}=$ Hisign Bio-Info Institute

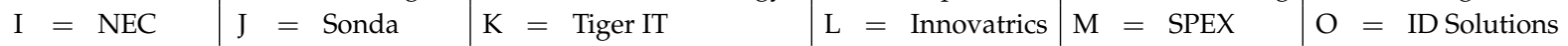

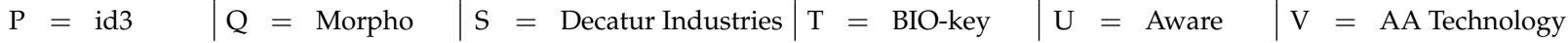




\begin{tabular}{|c|c|c|c|c|c|c|c|c|c|}
\hline \multicolumn{2}{|c|}{ Participant } & \multicolumn{4}{|c|}{ Ten-Finger Plain-to-Plain } & \multicolumn{4}{|c|}{ Ten-Finger Rolled-to-Rolled } \\
\hline Letter & Sub.\# & \multicolumn{2}{|c|}{ Size } & \multicolumn{2}{|c|}{ FNIR } & \multicolumn{2}{|c|}{ Size } & \multicolumn{2}{|c|}{ FNIR } \\
\hline \multirow{2}{*}{$\mathrm{C}$} & 1 & 9 & 20.91 & 30 & NA & 14 & 40.15 & 16 & 0.0094 \\
\hline & 2 & 9 & 20.91 & 24 & 0.0711 & 14 & 40.15 & 15 & 0.0085 \\
\hline \multirow{2}{*}{$\mathrm{D}$} & 1 & 19 & 33.66 & 6 & 0.0015 & 24 & 63.63 & 4 & 0.0015 \\
\hline & 2 & 18 & 33.16 & 2 & 0.0011 & 25 & 65.36 & 7 & 0.0018 \\
\hline \multirow{2}{*}{ E } & 1 & 1 & 12.10 & 14 & 0.0088 & 1 & 19.95 & 18 & 0.0106 \\
\hline & 2 & 28 & 67.07 & 13 & 0.0048 & 28 & 110.22 & 12 & 0.0050 \\
\hline \multirow{2}{*}{$F$} & 1 & 5 & 19.45 & 25 & 0.0734 & 3 & 27.41 & 25 & 0.0536 \\
\hline & 2 & 5 & 19.45 & 25 & 0.0734 & 3 & 27.41 & 25 & 0.0536 \\
\hline \multirow{2}{*}{ G } & 1 & 14 & 27.52 & 23 & 0.0368 & 10 & 30.76 & 24 & 0.0447 \\
\hline & 2 & 14 & 27.52 & 20 & 0.0276 & 10 & 30.76 & 21 & 0.0333 \\
\hline \multirow{2}{*}{$\mathrm{H}$} & 1 & 16 & 29.72 & 20 & 0.0276 & 8 & 29.96 & 20 & 0.0201 \\
\hline & 2 & 16 & 29.72 & 19 & 0.0275 & 8 & 29.96 & 19 & 0.0199 \\
\hline \multirow{2}{*}{ I } & 1 & 4 & 17.76 & 4 & 0.0013 & 2 & 23.76 & 1 & 0.0013 \\
\hline & 2 & 11 & 23.15 & 1 & 0.0010 & 7 & 28.49 & 2 & 0.0014 \\
\hline \multirow{2}{*}{$\mathrm{J}$} & 1 & 23 & 38.56 & 12 & 0.0047 & 23 & 63.60 & 13 & 0.0051 \\
\hline & 2 & 22 & 38.56 & 10 & 0.0027 & 20 & 63.60 & 9 & 0.0033 \\
\hline \multirow{2}{*}{$\mathrm{L}$} & 1 & 2 & 15.74 & 16 & 0.0102 & 12 & 32.38 & 17 & 0.0097 \\
\hline & 2 & 2 & 15.74 & 15 & 0.0095 & 12 & 32.38 & 14 & 0.0083 \\
\hline \multirow{2}{*}{ M } & 1 & 5 & 19.45 & 28 & 0.0934 & 3 & 27.41 & 28 & 0.0783 \\
\hline & 2 & 5 & 19.45 & 27 & 0.0826 & 3 & 27.41 & 27 & 0.0716 \\
\hline \multirow{2}{*}{$\mathrm{O}$} & 1 & 20 & 38.56 & 9 & 0.0025 & 21 & 63.60 & 11 & 0.0034 \\
\hline & 2 & 20 & 38.56 & 10 & 0.0027 & 21 & 63.60 & 9 & 0.0033 \\
\hline \multirow{2}{*}{ Q } & 1 & 29 & 83.85 & 2 & 0.0011 & 29 & 134.42 & 5 & 0.0017 \\
\hline & 2 & 29 & 83.85 & 4 & 0.0013 & 29 & 134.42 & 2 & 0.0014 \\
\hline \multirow{2}{*}{$S$} & 1 & 24 & 54.12 & 22 & 0.0311 & 26 & 80.04 & 29 & 0.0860 \\
\hline & 2 & 24 & 54.12 & 29 & 0.1680 & 26 & 80.04 & 30 & 0.2462 \\
\hline \multirow{2}{*}{$\mathrm{U}$} & 1 & 26 & 62.80 & 18 & 0.0163 & 18 & 54.62 & 23 & 0.0358 \\
\hline & 2 & 26 & 62.80 & 17 & 0.0155 & 18 & 54.62 & 22 & 0.0351 \\
\hline \multirow{2}{*}{ V } & 1 & 12 & 25.78 & 7 & 0.0024 & 16 & 48.74 & 5 & 0.0017 \\
\hline & 2 & 12 & 25.78 & 7 & 0.0024 & 16 & 48.74 & 8 & 0.0019 \\
\hline
\end{tabular}

Table 68: Tabulation of mean search template sizes for Class C. Letter refers to the participant's letter code found on the footer of this page. Sub. \# is an identifier used to differentiate between the two submissions each participant could make. Size values indicate the mean kilobytes used to store a search template on disk for a single subject, where $1 \mathrm{kB}$ is equal to 1024 bytes. The FNIR column shows FNIR for each submission at FPIR $=10^{-3}$. NA indicates that the operations required to produce the value could not be performed. The number to the left of a value provides the value's column-wise ranking, with the best performance shaded in green and the worst in pink.

\begin{tabular}{l|l|l|l|ll|l}
$\mathrm{C}=$ afis team & $\mathrm{D}=3 \mathrm{M}$ Cogent & $\mathrm{E}=$ Neurotechnology & $\mathrm{F}=$ Papillon & $\mathrm{G}=$ Dermalog & $\mathrm{H}=$ Hisign Bio-Info Institute \\
$\mathrm{I}=$ NEC & $\mathrm{J}=$ Sonda & $\mathrm{K}=$ Tiger IT & $\mathrm{L}=$ Innovatrics & $\mathrm{M}=$ SPEX & $\mathrm{O}=$ ID Solutions \\
$\mathrm{P}=\mathrm{id} 3$ & $\mathrm{Q}=$ Morpho & $\mathrm{S}=$ Decatur Industries & $\mathrm{T}=$ BIO-key & $\mathrm{U}=$ Aware & $\mathrm{V}=$ AA Technology
\end{tabular}




\section{G.2 Median Values}

\begin{tabular}{l|l|l|l|l|l}
$\mathrm{C}=$ afis team & $\mathrm{D}=3 \mathrm{M}$ Cogent & $\mathrm{E}=$ Neurotechnology & $\mathrm{F}=$ Papillon & $\mathrm{G}=$ Dermalog & $\mathrm{H}=$ Hisign Bio-Info Institute \\
$\mathrm{I}=\mathrm{NEC}$ & $\mathrm{J}=$ Sonda & $\mathrm{K}=$ Tiger IT & $\mathrm{L}=$ Innovatrics & $\mathrm{M}=$ SPEX & $\mathrm{O}=$ ID Solutions \\
$\mathrm{P}=\mathrm{id} 3$ & $\mathrm{Q}=$ Morpho & $\mathrm{S}=$ Decatur Industries & $\mathrm{T}=$ BIO-key & $\mathrm{U}=$ Aware & $\mathrm{V}=$ AA Technology
\end{tabular}




\begin{tabular}{|c|c|c|c|c|c|c|c|c|c|c|c|c|c|}
\hline \multicolumn{2}{|c|}{ Participant } & \multicolumn{4}{|c|}{ Left Index } & \multicolumn{4}{|c|}{ Right Index } & \multicolumn{4}{|c|}{ Left and Right Index } \\
\hline Letter & Sub.\# & \multicolumn{2}{|c|}{ Size } & \multicolumn{2}{|c|}{ FNIR } & \multicolumn{2}{|c|}{ Size } & \multicolumn{2}{|c|}{ FNIR } & \multicolumn{2}{|c|}{ Size } & \multicolumn{2}{|c|}{ FNIR } \\
\hline \multirow{2}{*}{$\mathrm{C}$} & 1 & 5 & 1.49 & 30 & 0.1335 & 5 & 1.51 & 30 & 0.1132 & 5 & 2.94 & 26 & 0.0368 \\
\hline & 2 & 5 & 1.49 & 31 & 0.1337 & 5 & 1.51 & 29 & 0.1124 & 5 & 2.94 & 28 & 0.0374 \\
\hline \multirow{2}{*}{$\mathrm{D}$} & 1 & 18 & 3.27 & 1 & 0.0197 & 18 & 3.30 & 1 & 0.0190 & 18 & 6.57 & 4 & 0.0030 \\
\hline & 2 & 18 & 3.27 & 1 & 0.0197 & 18 & 3.30 & 1 & 0.0190 & 18 & 6.57 & 4 & 0.0030 \\
\hline \multirow{2}{*}{$\mathrm{E}$} & 1 & 27 & 4.84 & 16 & 0.0745 & 27 & 4.91 & 15 & 0.0630 & 27 & 9.71 & 15 & 0.0207 \\
\hline & 2 & 27 & 4.84 & 15 & 0.0723 & 27 & 4.91 & 14 & 0.0624 & 27 & 9.71 & 14 & 0.0202 \\
\hline \multirow{2}{*}{$\mathrm{F}$} & 1 & 8 & 1.52 & 25 & 0.1111 & 8 & 1.53 & 25 & 0.0933 & 8 & 2.97 & 29 & 0.0386 \\
\hline & 2 & 8 & 1.52 & 22 & 0.1082 & 8 & 1.53 & 22 & 0.0903 & 8 & 2.97 & 30 & 0.0412 \\
\hline \multirow{2}{*}{ G } & 1 & 10 & 2.35 & 24 & 0.1089 & 10 & 2.36 & 24 & 0.0910 & 10 & 4.81 & 31 & 0.0515 \\
\hline & 2 & 10 & 2.35 & 23 & 0.1086 & 10 & 2.36 & 23 & 0.0909 & 10 & 4.81 & 20 & 0.0311 \\
\hline \multirow{2}{*}{$\mathrm{H}$} & 1 & 16 & 3.00 & 32 & 0.1576 & 16 & 3.00 & 32 & 0.1230 & 16 & 6.00 & 33 & 0.0686 \\
\hline & 2 & 16 & 3.00 & 33 & 0.1607 & 16 & 3.00 & 33 & 0.1249 & 16 & 6.00 & 32 & 0.0684 \\
\hline \multirow{2}{*}{ I } & 1 & 35 & 6.84 & 7 & 0.0257 & 35 & 6.88 & 5 & 0.0215 & 31 & 10.19 & 8 & 0.0058 \\
\hline & 2 & 36 & 10.02 & 8 & 0.0278 & 36 & 10.12 & 3 & 0.0214 & 36 & 20.05 & 4 & 0.0030 \\
\hline \multirow{2}{*}{$\mathrm{J}$} & 1 & 12 & 2.65 & 18 & 0.0786 & 12 & 2.68 & 20 & 0.0708 & 12 & 5.19 & 10 & 0.0143 \\
\hline & 2 & 12 & 2.65 & 14 & 0.0712 & 12 & 2.68 & 16 & 0.0643 & 12 & 5.19 & 10 & 0.0143 \\
\hline \multirow{2}{*}{ K } & 1 & 25 & 4.76 & 21 & 0.0883 & 25 & 4.79 & 18 & 0.0682 & 25 & 9.55 & 24 & 0.0360 \\
\hline & 2 & 25 & 4.76 & 20 & 0.0875 & 25 & 4.79 & 19 & 0.0685 & 25 & 9.55 & 19 & 0.0286 \\
\hline \multirow{2}{*}{$\mathrm{L}$} & 1 & 7 & 1.50 & 11 & 0.0625 & 7 & 1.51 & 12 & 0.0505 & 7 & 2.97 & 12 & 0.0146 \\
\hline & 2 & 22 & 4.41 & 9 & 0.0351 & 22 & 4.45 & 9 & 0.0295 & 22 & 8.81 & 9 & 0.0072 \\
\hline \multirow{2}{*}{ M } & 1 & 3 & 1.20 & 35 & 0.2995 & 3 & 1.17 & 36 & 0.2615 & 3 & 2.28 & 35 & NA \\
\hline & 2 & 3 & 1.20 & 34 & 0.2921 & 3 & 1.17 & 35 & 0.2526 & 3 & 2.28 & 34 & NA \\
\hline \multirow{2}{*}{$\mathrm{O}$} & 1 & 14 & 2.90 & 19 & 0.0818 & 14 & 2.94 & 21 & 0.0776 & 14 & 5.69 & 17 & 0.0229 \\
\hline & 2 & 14 & 2.90 & 17 & 0.0766 & 14 & 2.94 & 17 & 0.0675 & 14 & 5.69 & 16 & 0.0214 \\
\hline \multirow{2}{*}{$\mathrm{P}$} & 1 & 1 & 0.28 & 29 & 0.1308 & 1 & 0.28 & 31 & 0.1133 & 1 & 0.53 & 27 & 0.0370 \\
\hline & 2 & 1 & 0.28 & 28 & 0.1272 & 1 & 0.28 & 28 & 0.1100 & 1 & 0.53 & 21 & 0.0333 \\
\hline$\Omega$ & 1 & 33 & 6.46 & 3 & 0.0222 & 33 & 6.51 & 6 & 0.0218 & 34 & 12.94 & 1 & 0.0027 \\
\hline$Q$ & 2 & 33 & 6.46 & 4 & 0.0226 & 33 & 6.51 & 3 & 0.0214 & 34 & 12.94 & 1 & 0.0027 \\
\hline$S$ & 1 & 23 & 4.69 & 10 & 0.0571 & 23 & 4.71 & 10 & 0.0442 & 23 & 9.40 & 18 & 0.0281 \\
\hline $\mathrm{S}$ & 2 & 23 & 4.69 & 12 & 0.0650 & 23 & 4.71 & 11 & 0.0503 & 23 & 9.40 & 13 & 0.0195 \\
\hline$T$ & 1 & 31 & 5.81 & 36 & NA & 31 & 5.89 & 34 & 0.1929 & 32 & 11.73 & 36 & NA \\
\hline $\mathrm{T}$ & 2 & 31 & 5.81 & 13 & 0.0685 & 31 & 5.89 & 13 & 0.0562 & 32 & 11.73 & 25 & 0.0366 \\
\hline I & 1 & 29 & 5.01 & 27 & 0.1218 & 29 & 5.04 & 26 & 0.0996 & 29 & 10.05 & 22 & 0.0336 \\
\hline U & 2 & 29 & 5.01 & 26 & 0.1178 & 29 & 5.04 & 27 & 0.1007 & 29 & 10.05 & 23 & 0.0358 \\
\hline $\mathrm{V}$ & 1 & 20 & 3.63 & 6 & 0.0253 & 20 & 3.65 & 8 & 0.0223 & 20 & 7.27 & 7 & 0.0034 \\
\hline$V$ & 2 & 20 & 3.63 & 5 & 0.0252 & 20 & 3.65 & 7 & 0.0222 & 20 & 7.27 & 3 & 0.0028 \\
\hline
\end{tabular}

Table 69: Tabulation of median search template sizes for Class A. Letter refers to the participant's letter code found on the footer of this page. Sub. \# is an identifier used to differentiate between the two submissions each participant could make. Size values indicate the median kilobytes used to store a search template on disk for a single subject, where $1 \mathrm{kB}$ is equal to 1024 bytes. The FNIR column shows FNIR for each submission at FPIR $=10^{-3}$. NA indicates that the operations required to produce the value could not be performed. The number to the left of a value provides the value's column-wise ranking, with the best performance shaded in green and the worst in pink.

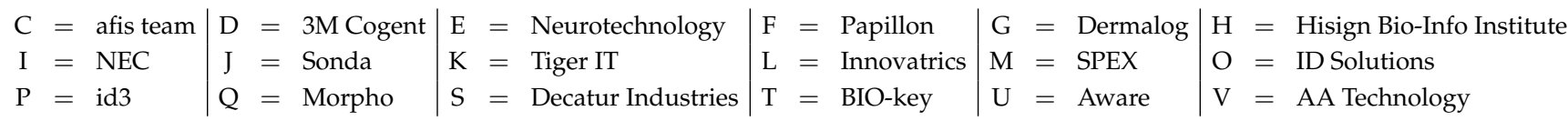




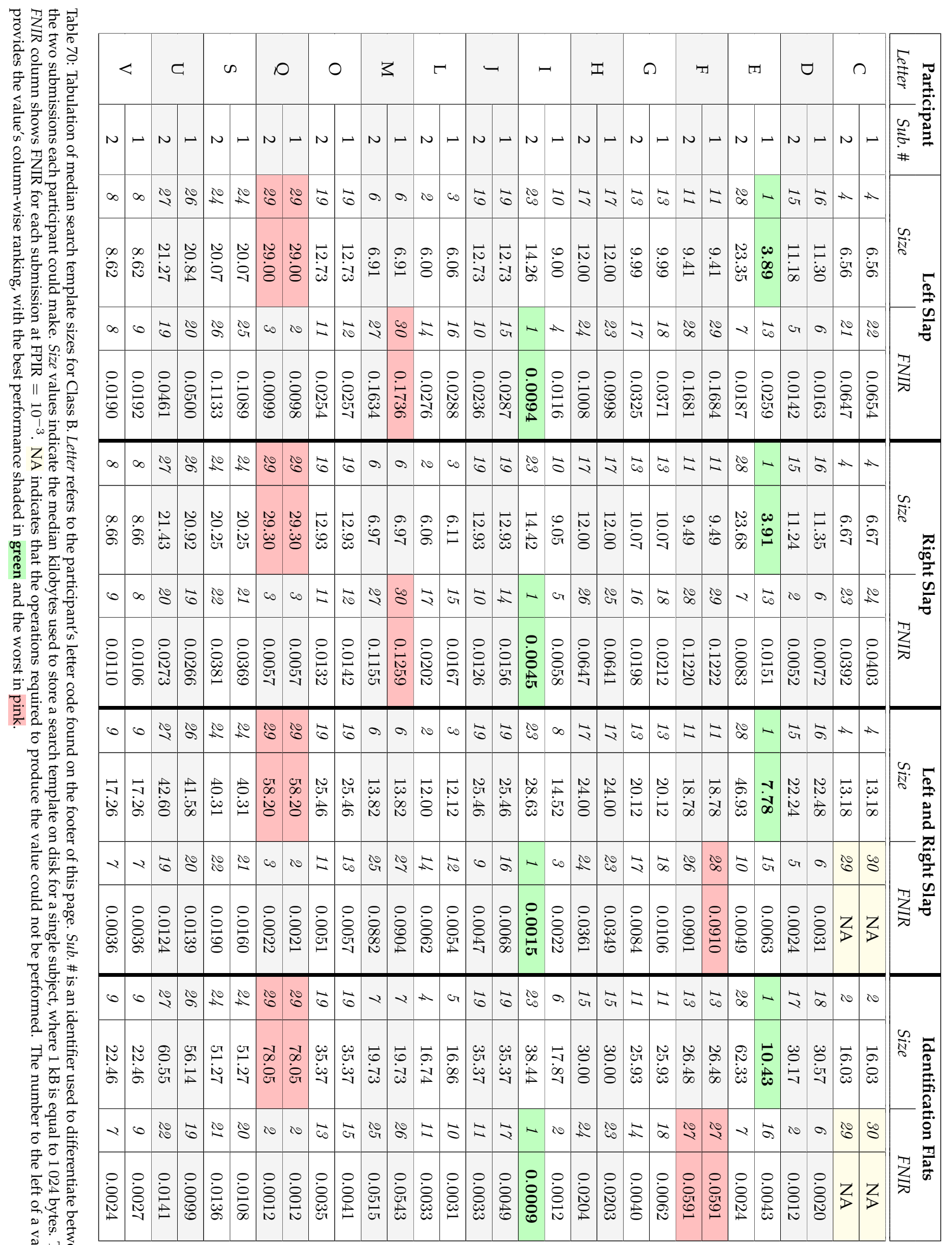

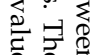

$\mathrm{C}=$ afis team $\mathrm{D}=3 \mathrm{M}$ Cogent $\mid \mathrm{E}=$ Neurotechnology $\mid \mathrm{F}=$ Papillon $\mid \mathrm{G}=$ Dermalog $\mid \mathrm{H}=$ Hisign Bio-Info Institute

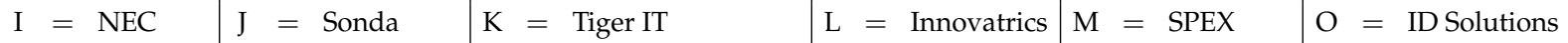

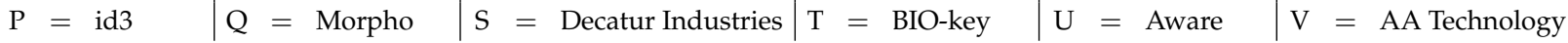




\begin{tabular}{|c|c|c|c|c|c|c|c|c|c|}
\hline \multicolumn{2}{|c|}{ Participant } & \multicolumn{4}{|c|}{ Ten-Finger Plain-to-Plain } & \multicolumn{4}{|c|}{ Ten-Finger Rolled-to-Rolled } \\
\hline \multirow{3}{*}{$\frac{\text { Letter }}{\mathrm{C}}$} & \multirow{2}{*}{$\frac{S u b . \#}{1}$} & \multicolumn{2}{|c|}{ Size } & \multicolumn{2}{|c|}{ FNIR } & \multicolumn{2}{|c|}{ Size } & \multicolumn{2}{|c|}{ FNIR } \\
\hline & & 9 & 20.80 & 30 & NA & 14 & 40.13 & 16 & 0.0094 \\
\hline & 2 & 9 & 20.80 & 24 & 0.0711 & 14 & 40.13 & 15 & 0.0085 \\
\hline \multirow{2}{*}{$\mathrm{D}$} & 1 & 19 & 33.58 & 6 & 0.0015 & 20 & 63.49 & 4 & 0.0015 \\
\hline & 2 & 18 & 33.00 & 2 & 0.0011 & 25 & 65.19 & 7 & 0.0018 \\
\hline \multirow{2}{*}{$\mathrm{E}$} & 1 & 1 & 12.07 & 14 & 0.0088 & 1 & 19.69 & 18 & 0.0106 \\
\hline & 2 & 28 & 66.94 & 13 & 0.0048 & 28 & 108.80 & 12 & 0.0050 \\
\hline \multirow{2}{*}{$\mathrm{F}$} & 1 & 5 & 19.35 & 25 & 0.0734 & 3 & 27.48 & 25 & 0.0536 \\
\hline & 2 & 5 & 19.35 & 25 & 0.0734 & 3 & 27.48 & 25 & 0.0536 \\
\hline \multirow{2}{*}{ G } & 1 & 14 & 26.87 & 23 & 0.0368 & 10 & 30.12 & 24 & 0.0447 \\
\hline & 2 & 14 & 26.87 & 20 & 0.0276 & 10 & 30.12 & 21 & 0.0333 \\
\hline \multirow{2}{*}{$\mathrm{H}$} & 1 & 16 & 30.00 & 20 & 0.0276 & 8 & 30.00 & 20 & 0.0201 \\
\hline & 2 & 16 & 30.00 & 19 & 0.0275 & 8 & 30.00 & 19 & 0.0199 \\
\hline \multirow{2}{*}{ I } & 1 & 4 & 17.86 & 4 & 0.0013 & 2 & 23.92 & 1 & 0.0013 \\
\hline & 2 & 11 & 23.17 & 1 & 0.0010 & 7 & 28.90 & 2 & 0.0014 \\
\hline \multirow{2}{*}{$\mathrm{J}$} & 1 & 20 & 38.52 & 12 & 0.0047 & 21 & 63.55 & 13 & 0.0051 \\
\hline & 2 & 20 & 38.52 & 10 & 0.0027 & 21 & 63.55 & 9 & 0.0033 \\
\hline \multirow{2}{*}{$\mathrm{L}$} & 1 & 2 & 15.77 & 16 & 0.0102 & 12 & 32.65 & 17 & 0.0097 \\
\hline & 2 & 2 & 15.77 & 15 & 0.0095 & 12 & 32.65 & 14 & 0.0083 \\
\hline \multirow{2}{*}{ M } & 1 & 5 & 19.35 & 28 & 0.0934 & 3 & 27.48 & 28 & 0.0783 \\
\hline & 2 & 5 & 19.35 & 27 & 0.0826 & 3 & 27.48 & 27 & 0.0716 \\
\hline \multirow{2}{*}{$\mathrm{O}$} & 1 & 20 & 38.52 & 9 & 0.0025 & 21 & 63.55 & 11 & 0.0034 \\
\hline & 2 & 20 & 38.52 & 10 & 0.0027 & 21 & 63.55 & 9 & 0.0033 \\
\hline \multirow{2}{*}{ Q } & 1 & 29 & 83.49 & 2 & 0.0011 & 29 & 135.01 & 5 & 0.0017 \\
\hline & 2 & 29 & 83.49 & 4 & 0.0013 & 29 & 135.01 & 2 & 0.0014 \\
\hline \multirow{2}{*}{$S$} & 1 & 24 & 53.59 & 22 & 0.0311 & 26 & 78.67 & 29 & 0.0860 \\
\hline & 2 & 24 & 53.59 & 29 & 0.1680 & 26 & 78.67 & 30 & 0.2462 \\
\hline \multirow{2}{*}{$\mathrm{U}$} & 1 & 26 & 63.23 & 18 & 0.0163 & 18 & 54.73 & 23 & 0.0358 \\
\hline & 2 & 26 & 63.23 & 17 & 0.0155 & 18 & 54.73 & 22 & 0.0351 \\
\hline \multirow{2}{*}{ V } & 1 & 12 & 25.68 & 7 & 0.0024 & 16 & 48.85 & 5 & 0.0017 \\
\hline & 2 & 12 & 25.68 & 7 & 0.0024 & 16 & 48.85 & 8 & 0.0019 \\
\hline
\end{tabular}

Table 71: Tabulation of median search template sizes for Class C. Letter refers to the participant's letter code found on the footer of this page. Sub. \# is an identifier used to differentiate between the two submissions each participant could make. Size values indicate the median kilobytes used to store a search template on disk for a single subject, where $1 \mathrm{kB}$ is equal to 1024 bytes. The FNIR column shows FNIR for each submission at FPIR $=10^{-3}$. NA indicates that the operations required to produce the value could not be performed. The number to the left of a value provides the value's column-wise ranking, with the best performance shaded in green and the worst in pink.

\begin{tabular}{l|l|l|l|ll|l}
$\mathrm{C}=$ afis team & $\mathrm{D}=3 \mathrm{M}$ Cogent & $\mathrm{E}=$ Neurotechnology & $\mathrm{F}=$ Papillon & $\mathrm{G}=$ Dermalog & $\mathrm{H}=$ Hisign Bio-Info Institute \\
$\mathrm{I}=$ NEC & $\mathrm{J}=$ Sonda & $\mathrm{K}=$ Tiger IT & $\mathrm{L}=$ Innovatrics & $\mathrm{M}=$ SPEX & $\mathrm{O}=$ ID Solutions \\
$\mathrm{P}=\mathrm{id} 3$ & $\mathrm{Q}=$ Morpho & $\mathrm{S}=$ Decatur Industries & $\mathrm{T}=$ BIO-key & $\mathrm{U}=$ Aware & $\mathrm{V}=$ AA Technology
\end{tabular}




\section{H Template Creation Times}

\begin{tabular}{l|l|l|l|l|l}
$\mathrm{C}=$ afis team & $\mathrm{D}=3 \mathrm{M}$ Cogent & $\mathrm{E}=$ Neurotechnology & $\mathrm{F}=$ Papillon & $\mathrm{G}=$ Dermalog & $\mathrm{H}=$ Hisign Bio-Info Institute \\
$\mathrm{I}=$ NEC & $\mathrm{J}=$ Sonda & $\mathrm{K}=$ Tiger IT & $\mathrm{L}=$ Innovatrics & $\mathrm{M}=$ SPEX & $\mathrm{O}=$ ID Solutions \\
$\mathrm{P}=\mathrm{id} 3$ & $\mathrm{Q}=$ Morpho & $\mathrm{S}=$ Decatur Industries & $\mathrm{T}=$ BIO-key & $\mathrm{U}=$ Aware & $\mathrm{V}=$ AA Technology
\end{tabular}




\begin{tabular}{|c|c|c|c|c|c|c|c|c|c|c|c|}
\hline \multicolumn{2}{|c|}{ Participant } & \multicolumn{4}{|c|}{ Enrollment } & \multicolumn{4}{|c|}{ Search } & \multirow{2}{*}{\multicolumn{2}{|c|}{ FNIR $@$ FPIR $=10^{-3}$}} \\
\hline Letter & Sub.\# & \multicolumn{2}{|c|}{ Mean } & \multicolumn{2}{|c|}{ Median } & \multicolumn{2}{|c|}{ Mean } & \multicolumn{2}{|c|}{ Median } & & \\
\hline \multirow{2}{*}{$\mathrm{C}$} & 1 & 21 & 0.45 & 19 & 0.41 & 19 & 0.41 & 19 & 0.39 & 30 & 0.1335 \\
\hline & 2 & 21 & 0.45 & 19 & 0.41 & 19 & 0.41 & 19 & 0.39 & 31 & 0.1337 \\
\hline \multirow{2}{*}{$\mathrm{D}$} & 1 & 35 & 1.79 & 34 & 1.46 & 35 & 1.57 & 34 & 1.43 & 1 & 0.0197 \\
\hline & 2 & 34 & 1.79 & 33 & 1.46 & 34 & 1.55 & 33 & 1.42 & 1 & 0.0197 \\
\hline \multirow{2}{*}{$\mathrm{E}$} & 1 & 11 & 0.37 & 15 & 0.36 & 15 & 0.35 & 15 & 0.35 & 16 & 0.0745 \\
\hline & 2 & 11 & 0.37 & 15 & 0.36 & 15 & 0.35 & 15 & 0.35 & 15 & 0.0723 \\
\hline \multirow{2}{*}{ F } & 1 & 31 & 1.21 & 31 & 1.13 & 29 & 1.11 & 29 & 1.07 & 25 & 0.1111 \\
\hline & 2 & 31 & 1.21 & 31 & 1.13 & 29 & 1.11 & 29 & 1.07 & 22 & 0.1082 \\
\hline \multirow{2}{*}{ G } & 1 & 19 & 0.43 & 21 & 0.41 & 21 & 0.57 & 23 & 0.55 & 24 & 0.1089 \\
\hline & 2 & 19 & 0.43 & 21 & 0.41 & 21 & 0.57 & 23 & 0.55 & 23 & 0.1086 \\
\hline \multirow{2}{*}{$\mathrm{H}$} & 1 & 13 & 0.37 & 7 & 0.31 & 11 & 0.33 & 7 & 0.30 & 32 & 0.1576 \\
\hline & 2 & 13 & 0.37 & 7 & 0.31 & 11 & 0.33 & 7 & 0.30 & 33 & 0.1607 \\
\hline \multirow{2}{*}{ I } & 1 & 33 & 1.49 & 35 & 1.47 & 33 & 1.48 & 35 & 1.46 & 7 & 0.0257 \\
\hline & 2 & 36 & 2.29 & 36 & 2.20 & 36 & 2.19 & 36 & 2.15 & 8 & 0.0278 \\
\hline \multirow{2}{*}{$\mathrm{J}$} & 1 & 7 & 0.35 & 13 & 0.34 & 9 & 0.33 & 13 & 0.32 & 18 & 0.0786 \\
\hline & 2 & 7 & 0.35 & 13 & 0.34 & 9 & 0.33 & 13 & 0.32 & 14 & 0.0712 \\
\hline \multirow{2}{*}{ K } & 1 & 27 & 1.09 & 27 & 1.07 & 27 & 1.06 & 27 & 1.05 & 21 & 0.0883 \\
\hline & 2 & 27 & 1.09 & 27 & 1.07 & 27 & 1.06 & 27 & 1.05 & 20 & 0.0875 \\
\hline \multirow{2}{*}{$\mathrm{L}$} & 1 & 1 & 0.07 & 1 & 0.06 & 1 & 0.07 & 1 & 0.05 & 11 & 0.0625 \\
\hline & 2 & 4 & 0.19 & 4 & 0.16 & 4 & 0.17 & 4 & 0.15 & 9 & 0.0351 \\
\hline \multirow{2}{*}{ M } & 1 & 29 & 1.21 & 29 & 1.13 & 31 & 1.12 & 31 & 1.07 & 35 & 0.2995 \\
\hline & 2 & 29 & 1.21 & 29 & 1.13 & 31 & 1.12 & 31 & 1.07 & 34 & 0.2921 \\
\hline \multirow{2}{*}{$\mathrm{O}$} & 1 & 5 & 0.29 & 5 & 0.28 & 5 & 0.28 & 5 & 0.27 & 19 & 0.0818 \\
\hline & 2 & 5 & 0.29 & 5 & 0.28 & 5 & 0.28 & 5 & 0.27 & 17 & 0.0766 \\
\hline \multirow{2}{*}{$\mathrm{P}$} & 1 & 17 & 0.42 & 17 & 0.38 & 17 & 0.38 & 17 & 0.36 & 29 & 0.1308 \\
\hline & 2 & 17 & 0.42 & 17 & 0.38 & 17 & 0.38 & 17 & 0.36 & 28 & 0.1272 \\
\hline \multirow{2}{*}{ Q } & 1 & 23 & 0.64 & 23 & 0.56 & 23 & 0.57 & 21 & 0.54 & 3 & 0.0222 \\
\hline & 2 & 23 & 0.64 & 23 & 0.56 & 23 & 0.57 & 21 & 0.54 & 4 & 0.0226 \\
\hline \multirow{2}{*}{$S$} & 1 & 25 & 0.91 & 25 & 0.89 & 25 & 0.90 & 25 & 0.88 & 10 & 0.0571 \\
\hline & 2 & 25 & 0.91 & 25 & 0.89 & 25 & 0.90 & 25 & 0.88 & 12 & 0.0650 \\
\hline \multirow{2}{*}{$\mathrm{T}$} & 1 & 9 & 0.35 & 9 & 0.32 & 7 & 0.33 & 9 & 0.31 & 36 & NA \\
\hline & 2 & 9 & 0.35 & 9 & 0.32 & 7 & 0.33 & 9 & 0.31 & 13 & 0.0685 \\
\hline I & 1 & 2 & 0.17 & 2 & 0.16 & 2 & 0.16 & 2 & 0.15 & 27 & 0.1218 \\
\hline U & 2 & 2 & 0.17 & 2 & 0.16 & 2 & 0.16 & 2 & 0.15 & 26 & 0.1178 \\
\hline $\mathrm{V}$ & 1 & 15 & 0.37 & 11 & 0.33 & 13 & 0.34 & 11 & 0.32 & 6 & 0.0253 \\
\hline$V$ & 2 & 15 & 0.37 & 11 & 0.33 & 13 & 0.34 & 11 & 0.32 & 5 & 0.0252 \\
\hline
\end{tabular}

Table 72: Tabulation of enrollment time results for Class A — Left Index. Letter refers to the participant's letter code found on the footer of this page. Sub. $\#$ is an identifier used to differentiate between the two submissions each participant could make. Enrollment shows the time used to create a fingerprint template to be used in an enrollment set. Search shows the time used to create a search template to be used for a query. All values are reported in seconds, but were originally recorded to microsecond precision. The number to the left of a value provides the value's column-wise ranking, with the best performance shaded in green and the worst in pink. For reference, the FNIR values from Table 7 are reprinted to the right of this table.

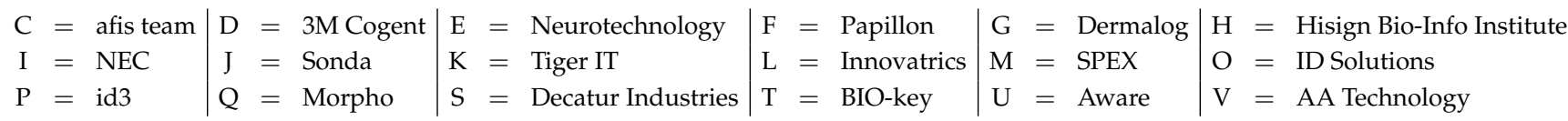




\begin{tabular}{|c|c|c|c|c|c|c|c|c|c|c|c|}
\hline \multicolumn{2}{|c|}{ Participant } & \multicolumn{4}{|c|}{ Enrollment } & \multicolumn{4}{|c|}{ Search } & \multirow{2}{*}{\multicolumn{2}{|c|}{ FNIR $@$ FPIR $=10^{-3}$}} \\
\hline Letter & Sub.\# & \multicolumn{2}{|c|}{ Mean } & \multicolumn{2}{|c|}{ Median } & \multicolumn{2}{|c|}{ Mean } & \multicolumn{2}{|c|}{ Median } & & \\
\hline \multirow{2}{*}{$\mathrm{C}$} & 1 & 21 & 0.45 & 19 & 0.41 & 19 & 0.41 & 19 & 0.39 & 30 & 0.1132 \\
\hline & 2 & 21 & 0.45 & 19 & 0.41 & 19 & 0.41 & 19 & 0.39 & 29 & 0.1124 \\
\hline \multirow{2}{*}{$\mathrm{D}$} & 1 & 34 & 1.78 & 33 & 1.45 & 35 & 1.58 & 34 & 1.43 & 1 & 0.0190 \\
\hline & 2 & 35 & 1.80 & 34 & 1.46 & 34 & 1.57 & 33 & 1.43 & 1 & 0.0190 \\
\hline \multirow{2}{*}{$\mathrm{E}$} & 1 & 13 & 0.37 & 15 & 0.37 & 15 & 0.37 & 15 & 0.36 & 15 & 0.0630 \\
\hline & 2 & 13 & 0.37 & 15 & 0.37 & 15 & 0.37 & 15 & 0.36 & 14 & 0.0624 \\
\hline \multirow{2}{*}{$\mathrm{F}$} & 1 & 29 & 1.23 & 29 & 1.15 & 31 & 1.14 & 29 & 1.09 & 25 & 0.0933 \\
\hline & 2 & 29 & 1.23 & 29 & 1.15 & 31 & 1.14 & 29 & 1.09 & 22 & 0.0903 \\
\hline \multirow{2}{*}{ G } & 1 & 19 & 0.43 & 21 & 0.41 & 21 & 0.57 & 23 & 0.55 & 24 & 0.0910 \\
\hline & 2 & 19 & 0.43 & 21 & 0.41 & 21 & 0.57 & 23 & 0.55 & 23 & 0.0909 \\
\hline \multirow{2}{*}{$\mathrm{H}$} & 1 & 15 & 0.38 & 7 & 0.31 & 9 & 0.34 & 7 & 0.30 & 32 & 0.1230 \\
\hline & 2 & 15 & 0.38 & 7 & 0.31 & 9 & 0.34 & 7 & 0.30 & 33 & 0.1249 \\
\hline \multirow{2}{*}{ I } & 1 & 33 & 1.51 & 35 & 1.48 & 33 & 1.49 & 35 & 1.46 & 5 & 0.0215 \\
\hline & 2 & 36 & 2.29 & 36 & 2.20 & 36 & 2.19 & 36 & 2.15 & 3 & 0.0214 \\
\hline \multirow{2}{*}{$\mathrm{J}$} & 1 & 7 & 0.35 & 13 & 0.33 & 11 & 0.34 & 13 & 0.33 & 20 & 0.0708 \\
\hline & 2 & 7 & 0.35 & 13 & 0.33 & 11 & 0.34 & 13 & 0.33 & 16 & 0.0643 \\
\hline \multirow{2}{*}{ K } & 1 & 27 & 1.09 & 27 & 1.06 & 27 & 1.06 & 27 & 1.05 & 18 & 0.0682 \\
\hline & 2 & 27 & 1.09 & 27 & 1.06 & 27 & 1.06 & 27 & 1.05 & 19 & 0.0685 \\
\hline \multirow{2}{*}{$\mathrm{L}$} & 1 & 1 & 0.07 & 1 & 0.06 & 1 & 0.07 & 1 & 0.05 & 12 & 0.0505 \\
\hline & 2 & 4 & 0.19 & 4 & 0.16 & 4 & 0.17 & 4 & 0.16 & 9 & 0.0295 \\
\hline \multirow{2}{*}{ M } & 1 & 31 & 1.24 & 31 & 1.16 & 29 & 1.14 & 31 & 1.09 & 36 & 0.2615 \\
\hline & 2 & 31 & 1.24 & 31 & 1.16 & 29 & 1.14 & 31 & 1.09 & 35 & 0.2526 \\
\hline \multirow{2}{*}{$\mathrm{O}$} & 1 & 5 & 0.29 & 5 & 0.28 & 5 & 0.28 & 5 & 0.27 & 21 & 0.0776 \\
\hline & 2 & 5 & 0.29 & 5 & 0.28 & 5 & 0.28 & 5 & 0.27 & 17 & 0.0675 \\
\hline \multirow{2}{*}{$\mathrm{P}$} & 1 & 17 & 0.42 & 17 & 0.38 & 17 & 0.39 & 17 & 0.37 & 31 & 0.1133 \\
\hline & 2 & 17 & 0.42 & 17 & 0.38 & 17 & 0.39 & 17 & 0.37 & 28 & 0.1100 \\
\hline \multirow{2}{*}{ Q } & 1 & 23 & 0.64 & 23 & 0.56 & 23 & 0.58 & 21 & 0.54 & 6 & 0.0218 \\
\hline & 2 & 23 & 0.64 & 23 & 0.56 & 23 & 0.58 & 21 & 0.54 & 3 & 0.0214 \\
\hline \multirow{2}{*}{ S } & 1 & 25 & 0.91 & 25 & 0.89 & 25 & 0.89 & 25 & 0.88 & 10 & 0.0442 \\
\hline & 2 & 25 & 0.91 & 25 & 0.89 & 25 & 0.89 & 25 & 0.88 & 11 & 0.0503 \\
\hline \multirow{2}{*}{$\mathrm{T}$} & 1 & 9 & 0.35 & 9 & 0.32 & 7 & 0.33 & 9 & 0.31 & 34 & 0.1929 \\
\hline & 2 & 9 & 0.35 & 9 & 0.32 & 7 & 0.33 & 9 & 0.31 & 13 & 0.0562 \\
\hline $\mathrm{J}$ & 1 & 2 & 0.17 & 2 & 0.16 & 2 & 0.16 & 2 & 0.15 & 26 & 0.0996 \\
\hline U & 2 & 2 & 0.17 & 2 & 0.16 & 2 & 0.16 & 2 & 0.15 & 27 & 0.1007 \\
\hline $\mathrm{V}$ & 1 & 11 & 0.37 & 11 & 0.33 & 13 & 0.34 & 11 & 0.32 & 8 & 0.0223 \\
\hline$v$ & 2 & 11 & 0.37 & 11 & 0.33 & 13 & 0.34 & 11 & 0.32 & 7 & 0.0222 \\
\hline
\end{tabular}

Table 73: Tabulation of enrollment time results for Class A - Right Index. Letter refers to the participant's letter code found on the footer of this page. Sub. $\#$ is an identifier used to differentiate between the two submissions each participant could make. Enrollment shows the time used to create a fingerprint template to be used in an enrollment set. Search shows the time used to create a search template to be used for a query. All values are reported in seconds, but were originally recorded to microsecond precision. The number to the left of a value provides the value's column-wise ranking, with the best performance shaded in green and the worst in pink. For reference, the FNIR values from Table 8 are reprinted to the right of this table.

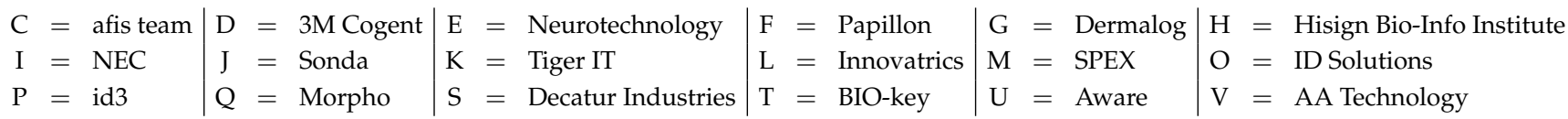




\begin{tabular}{|c|c|c|c|c|c|c|c|c|c|c|c|}
\hline \multicolumn{2}{|c|}{ Participant } & \multicolumn{4}{|c|}{ Enrollment } & \multicolumn{4}{|c|}{ Search } & \multirow{2}{*}{\multicolumn{2}{|c|}{ FNIR $@$ FPIR $=10^{-3}$}} \\
\hline Letter & Sub.\# & \multicolumn{2}{|c|}{ Mean } & \multicolumn{2}{|c|}{ Median } & \multicolumn{2}{|c|}{ Mean } & \multicolumn{2}{|c|}{ Median } & & \\
\hline \multirow{2}{*}{ C } & 1 & 21 & 0.88 & 21 & 0.80 & 19 & 0.80 & 19 & 0.78 & 26 & 0.0368 \\
\hline & 2 & 21 & 0.88 & 21 & 0.80 & 19 & 0.80 & 19 & 0.78 & 28 & 0.0374 \\
\hline \multirow{2}{*}{$\mathrm{D}$} & 1 & 35 & 3.57 & 34 & 2.91 & 34 & 3.10 & 33 & 2.84 & 4 & 0.0030 \\
\hline & 2 & 34 & 3.55 & 33 & 2.89 & 35 & 3.12 & 34 & 2.84 & 4 & 0.0030 \\
\hline \multirow{2}{*}{$\mathrm{E}$} & 1 & 15 & 0.74 & 15 & 0.73 & 15 & 0.70 & 15 & 0.70 & 15 & 0.0207 \\
\hline & 2 & 15 & 0.74 & 15 & 0.73 & 15 & 0.70 & 15 & 0.70 & 14 & 0.0202 \\
\hline \multirow{2}{*}{$\mathrm{F}$} & 1 & 29 & 2.42 & 29 & 2.26 & 29 & 2.23 & 29 & 2.15 & 29 & 0.0386 \\
\hline & 2 & 29 & 2.42 & 29 & 2.26 & 29 & 2.23 & 29 & 2.15 & 30 & 0.0412 \\
\hline \multirow{2}{*}{ G } & 1 & 19 & 0.84 & 19 & 0.80 & 21 & 1.13 & 23 & 1.09 & 31 & 0.0515 \\
\hline & 2 & 19 & 0.84 & 19 & 0.80 & 21 & 1.13 & 23 & 1.09 & 20 & 0.0311 \\
\hline \multirow{2}{*}{$\mathrm{H}$} & 1 & 13 & 0.74 & 7 & 0.62 & 9 & 0.66 & 7 & 0.60 & 33 & 0.0686 \\
\hline & 2 & 13 & 0.74 & 7 & 0.62 & 9 & 0.66 & 7 & 0.60 & 32 & 0.0684 \\
\hline \multirow{2}{*}{ I } & 1 & 33 & 2.98 & 35 & 2.96 & 33 & 2.96 & 35 & 2.93 & 8 & 0.0058 \\
\hline & 2 & 36 & 4.59 & 36 & 4.40 & 36 & 4.37 & 36 & 4.30 & 4 & 0.0030 \\
\hline \multirow{2}{*}{$\mathrm{J}$} & 1 & 7 & 0.69 & 13 & 0.67 & 11 & 0.66 & 13 & 0.65 & 10 & 0.0143 \\
\hline & 2 & 7 & 0.69 & 13 & 0.67 & 11 & 0.66 & 13 & 0.65 & 10 & 0.0143 \\
\hline \multirow{2}{*}{ K } & 1 & 27 & 2.17 & 27 & 2.13 & 27 & 2.11 & 27 & 2.09 & 24 & 0.0360 \\
\hline & 2 & 27 & 2.17 & 27 & 2.13 & 27 & 2.11 & 27 & 2.09 & 19 & 0.0286 \\
\hline \multirow{2}{*}{$\mathrm{L}$} & 1 & 1 & 0.12 & 1 & 0.11 & 1 & 0.11 & 1 & 0.11 & 12 & 0.0146 \\
\hline & 2 & 4 & 0.36 & 4 & 0.32 & 4 & 0.33 & 4 & 0.31 & 9 & 0.0072 \\
\hline \multirow{2}{*}{ M } & 1 & 31 & 2.42 & 31 & 2.27 & 31 & 2.25 & 31 & 2.16 & 35 & NA \\
\hline & 2 & 31 & 2.42 & 31 & 2.27 & 31 & 2.25 & 31 & 2.16 & 34 & NA \\
\hline \multirow{2}{*}{$\mathrm{O}$} & 1 & 5 & 0.57 & 5 & 0.55 & 5 & 0.55 & 5 & 0.53 & 17 & 0.0229 \\
\hline & 2 & 5 & 0.57 & 5 & 0.55 & 5 & 0.55 & 5 & 0.53 & 16 & 0.0214 \\
\hline \multirow{2}{*}{$\mathrm{P}$} & 1 & 17 & 0.83 & 17 & 0.76 & 17 & 0.77 & 17 & 0.72 & 27 & 0.0370 \\
\hline & 2 & 17 & 0.83 & 17 & 0.76 & 17 & 0.77 & 17 & 0.72 & 21 & 0.0333 \\
\hline \multirow{2}{*}{ Q } & 1 & 23 & 1.27 & 23 & 1.10 & 23 & 1.13 & 21 & 1.08 & 1 & 0.0027 \\
\hline & 2 & 23 & 1.27 & 23 & 1.10 & 23 & 1.13 & 21 & 1.08 & 1 & 0.0027 \\
\hline \multirow{2}{*}{$S$} & 1 & 25 & 1.81 & 25 & 1.78 & 25 & 1.77 & 25 & 1.75 & 18 & 0.0281 \\
\hline & 2 & 25 & 1.81 & 25 & 1.78 & 25 & 1.77 & 25 & 1.75 & 13 & 0.0195 \\
\hline \multirow{2}{*}{$\mathrm{T}$} & 1 & 9 & 0.70 & 9 & 0.64 & 7 & 0.65 & 9 & 0.62 & 36 & NA \\
\hline & 2 & 9 & 0.70 & 9 & 0.64 & 7 & 0.65 & 9 & 0.62 & 25 & 0.0366 \\
\hline 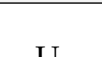 & 1 & 2 & 0.32 & 2 & 0.31 & 2 & 0.30 & 2 & 0.30 & 22 & 0.0336 \\
\hline $\mathrm{U}$ & 2 & 2 & 0.32 & 2 & 0.31 & 2 & 0.30 & 2 & 0.30 & 23 & 0.0358 \\
\hline & 1 & 11 & 0.73 & 11 & 0.65 & 13 & 0.68 & 11 & 0.64 & 7 & 0.0034 \\
\hline$V$ & 2 & 11 & 0.73 & 11 & 0.65 & 13 & 0.68 & 11 & 0.64 & 3 & 0.0028 \\
\hline
\end{tabular}

Table 74: Tabulation of enrollment time results for Class A — Left and Right Index. Letter refers to the participant's letter code found on the footer of this page. Sub. \# is an identifier used to differentiate between the two submissions each participant could make. Enrollment shows the time used to create a fingerprint template to be used in an enrollment set. Search shows the time used to create a search template to be used for a query. All values are reported in seconds, but were originally recorded to microsecond precision. The number to the left of a value provides the value's column-wise ranking, with the best performance shaded in green and the worst in pink. For reference, the FNIR values from Table 9 are reprinted to the right of this table.

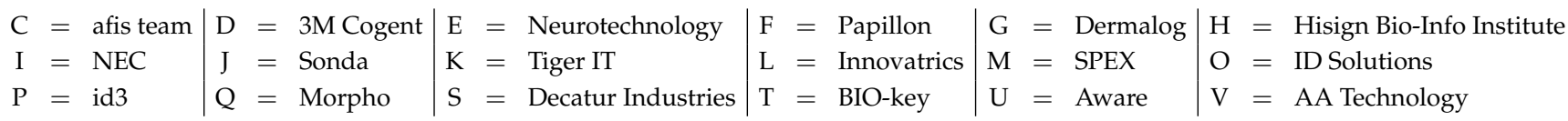




\begin{tabular}{|c|c|c|c|c|c|c|c|}
\hline \multicolumn{2}{|c|}{ Participant } & \multicolumn{4}{|c|}{ Search } & \multirow{2}{*}{\multicolumn{2}{|c|}{ FNIR @ FPIR $=10^{-3}$}} \\
\hline Letter & Sub.\# & \multicolumn{2}{|c|}{ Mean } & \multicolumn{2}{|c|}{ Median } & & \\
\hline \multirow{2}{*}{$\mathrm{C}$} & 1 & 12 & 2.13 & 12 & 2.12 & 22 & 0.0654 \\
\hline & 2 & 12 & 2.13 & 12 & 2.12 & 21 & 0.0647 \\
\hline \multirow{2}{*}{$\mathrm{D}$} & 1 & 14 & 2.16 & 14 & 2.15 & 6 & 0.0163 \\
\hline & 2 & 24 & 4.18 & 24 & 4.17 & 5 & 0.0142 \\
\hline \multirow{2}{*}{$\mathrm{E}$} & 1 & 11 & 1.95 & 11 & 1.95 & 13 & 0.0259 \\
\hline & 2 & 15 & 2.21 & 15 & 2.21 & 7 & 0.0187 \\
\hline \multirow{2}{*}{$\mathrm{F}$} & 1 & 28 & 6.94 & 26 & 6.73 & 29 & 0.1684 \\
\hline & 2 & 28 & 6.94 & 26 & 6.73 & 28 & 0.1681 \\
\hline \multirow{2}{*}{ G } & 1 & 18 & 2.81 & 18 & 2.83 & 18 & 0.0371 \\
\hline & 2 & 18 & 2.81 & 18 & 2.83 & 17 & 0.0325 \\
\hline \multirow{2}{*}{$\mathrm{H}$} & 1 & 9 & 1.68 & 9 & 1.67 & 23 & 0.0998 \\
\hline & 2 & 9 & 1.68 & 9 & 1.67 & 24 & 0.1008 \\
\hline \multirow{2}{*}{ I } & 1 & 25 & 6.50 & 25 & 6.50 & 4 & 0.0116 \\
\hline & 2 & 30 & 7.68 & 30 & 7.67 & 1 & 0.0094 \\
\hline \multirow{2}{*}{$\mathrm{J}$} & 1 & 7 & 1.22 & 3 & 1.21 & 15 & 0.0287 \\
\hline & 2 & 7 & 1.22 & 3 & 1.21 & 10 & 0.0236 \\
\hline \multirow{2}{*}{$\mathrm{L}$} & 1 & 2 & 1.13 & 2 & 1.11 & 16 & 0.0288 \\
\hline & 2 & 1 & 0.33 & 1 & 0.33 & 14 & 0.0276 \\
\hline \multirow{2}{*}{ M } & 1 & 26 & 6.94 & 28 & 6.73 & 30 & 0.1736 \\
\hline & 2 & 26 & 6.94 & 28 & 6.73 & 27 & 0.1634 \\
\hline \multirow{2}{*}{$\mathrm{O}$} & 1 & 5 & 1.22 & 5 & 1.21 & 12 & 0.0257 \\
\hline & 2 & 5 & 1.22 & 5 & 1.21 & 11 & 0.0254 \\
\hline \multirow{2}{*}{ Q } & 1 & 20 & 3.41 & 20 & 3.41 & 2 & 0.0098 \\
\hline & 2 & 20 & 3.41 & 20 & 3.41 & 3 & 0.0099 \\
\hline \multirow{2}{*}{$S$} & 1 & 22 & 3.97 & 22 & 3.96 & 25 & 0.1089 \\
\hline & 2 & 22 & 3.97 & 22 & 3.96 & 26 & 0.1133 \\
\hline \multirow{2}{*}{$\mathrm{U}$} & 1 & 17 & 2.46 & 17 & 2.42 & 20 & 0.0500 \\
\hline & 2 & 16 & 2.45 & 16 & 2.41 & 19 & 0.0461 \\
\hline \multirow{2}{*}{ V } & 1 & 3 & 1.21 & 7 & 1.22 & 9 & 0.0192 \\
\hline & 2 & 3 & 1.21 & 7 & 1.22 & 8 & 0.0190 \\
\hline
\end{tabular}

Table 75: Tabulation of enrollment time results for Class B - Left Slap. Letter refers to the participant's letter code found on the footer of this page. Sub. \# is an identifier used to differentiate between the two submissions each participant could make. Search shows the time used to create a search template to be used for a query. All values are reported in seconds, but were originally recorded to microsecond precision. The number to the left of a value provides the value's column-wise ranking, with the best performance shaded in green and the worst in pink. For reference, the FNIR values from Table 10 are reprinted to the right of this table.

\begin{tabular}{l|l|l|l|ll|l}
$\mathrm{C}=$ afis team & $\mathrm{D}=3 \mathrm{M}$ Cogent & $\mathrm{E}=$ Neurotechnology & $\mathrm{F}=$ Papillon & $\mathrm{G}=$ Dermalog & $\mathrm{H}=$ Hisign Bio-Info Institute \\
$\mathrm{I}=$ NEC & $\mathrm{J}=$ Sonda & $\mathrm{K}=$ Tiger IT & $\mathrm{L}=$ Innovatrics & $\mathrm{M}=$ SPEX & $\mathrm{O}=$ ID Solutions \\
$\mathrm{P}=\mathrm{id} 3$ & $\mathrm{Q}=$ Morpho & $\mathrm{S}=$ Decatur Industries & $\mathrm{T}=$ BIO-key & $\mathrm{U}=$ Aware & $\mathrm{V}=$ AA Technology
\end{tabular}




\begin{tabular}{|c|c|c|c|c|c|c|c|}
\hline \multicolumn{2}{|c|}{ Participant } & \multicolumn{4}{|c|}{ Search } & \multirow{2}{*}{\multicolumn{2}{|c|}{ FNIR $@$ FPIR $=10^{-3}$}} \\
\hline Letter & Sub.\# & \multicolumn{2}{|c|}{ Mean } & \multicolumn{2}{|c|}{ Median } & & \\
\hline \multirow{2}{*}{$\mathrm{C}$} & 1 & 12 & 2.10 & 12 & 2.09 & 24 & 0.0403 \\
\hline & 2 & 12 & 2.10 & 12 & 2.09 & 23 & 0.0392 \\
\hline \multirow{2}{*}{$\mathrm{D}$} & 1 & 14 & 2.15 & 14 & 2.14 & 6 & 0.0072 \\
\hline & 2 & 24 & 4.15 & 24 & 4.13 & 2 & 0.0052 \\
\hline \multirow{2}{*}{ E } & 1 & 11 & 1.92 & 11 & 1.91 & 13 & 0.0151 \\
\hline & 2 & 15 & 2.18 & 15 & 2.18 & 7 & 0.0083 \\
\hline \multirow{2}{*}{$\mathrm{F}$} & 1 & 26 & 6.86 & 26 & 6.68 & 29 & 0.1222 \\
\hline & 2 & 26 & 6.86 & 26 & 6.68 & 28 & 0.1220 \\
\hline \multirow{2}{*}{ G } & 1 & 18 & 2.76 & 18 & 2.78 & 18 & 0.0212 \\
\hline & 2 & 18 & 2.76 & 18 & 2.78 & 16 & 0.0198 \\
\hline \multirow{2}{*}{$\mathrm{H}$} & 1 & 9 & 1.64 & 9 & 1.63 & 25 & 0.0641 \\
\hline & 2 & 9 & 1.64 & 9 & 1.63 & 26 & 0.0647 \\
\hline \multirow{2}{*}{ I } & 1 & 25 & 6.47 & 25 & 6.47 & 5 & 0.0058 \\
\hline & 2 & 30 & 7.65 & 30 & 7.65 & 1 & 0.0045 \\
\hline \multirow{2}{*}{$\mathrm{J}$} & 1 & 5 & 1.21 & 5 & 1.20 & 14 & 0.0156 \\
\hline & 2 & 5 & 1.21 & 5 & 1.20 & 10 & 0.0126 \\
\hline \multirow{2}{*}{$\mathrm{L}$} & 1 & 2 & 1.12 & 2 & 1.11 & 15 & 0.0167 \\
\hline & 2 & 1 & 0.33 & 1 & 0.33 & 17 & 0.0202 \\
\hline \multirow{2}{*}{ M } & 1 & 28 & 6.88 & 28 & 6.69 & 30 & 0.1259 \\
\hline & 2 & 28 & 6.88 & 28 & 6.69 & 27 & 0.1155 \\
\hline \multirow{2}{*}{$\mathrm{O}$} & 1 & 7 & 1.21 & 7 & 1.20 & 12 & 0.0142 \\
\hline & 2 & 7 & 1.21 & 7 & 1.20 & 11 & 0.0132 \\
\hline \multirow{2}{*}{ Q } & 1 & 20 & 3.41 & 20 & 3.41 & 3 & 0.0057 \\
\hline & 2 & 20 & 3.41 & 20 & 3.41 & 3 & 0.0057 \\
\hline \multirow{2}{*}{$S$} & 1 & 22 & 3.89 & 22 & 3.88 & 21 & 0.0369 \\
\hline & 2 & 22 & 3.89 & 22 & 3.88 & 22 & 0.0381 \\
\hline \multirow{2}{*}{$\mathrm{U}$} & 1 & 16 & 2.43 & 16 & 2.39 & 19 & 0.0266 \\
\hline & 2 & 17 & 2.43 & 17 & 2.39 & 20 & 0.0273 \\
\hline \multirow{2}{*}{$\mathrm{V}$} & 1 & 3 & 1.19 & 3 & 1.19 & 8 & 0.0106 \\
\hline & 2 & 3 & 1.19 & 3 & 1.19 & 9 & 0.0110 \\
\hline
\end{tabular}

Table 76: Tabulation of enrollment time results for Class B - Right Slap. Letter refers to the participant's letter code found on the footer of this page. $S u b$. \# is an identifier used to differentiate between the two submissions each participant could make. Search shows the time used to create a search template to be used for a query. All values are reported in seconds, but were originally recorded to microsecond precision. The number to the left of a value provides the value's column-wise ranking, with the best performance shaded in green and the worst in pink. For reference, the FNIR values from Table 11 are reprinted to the right of this table.

\begin{tabular}{l|l|l|l|ll|l}
$\mathrm{C}=$ afis team & $\mathrm{D}=3 \mathrm{M}$ Cogent & $\mathrm{E}=$ Neurotechnology & $\mathrm{F}=$ Papillon & $\mathrm{G}=$ Dermalog & $\mathrm{H}=$ Hisign Bio-Info Institute \\
$\mathrm{I}=$ NEC & $\mathrm{J}=$ Sonda & $\mathrm{K}=$ Tiger IT & $\mathrm{L}=$ Innovatrics & $\mathrm{M}=$ SPEX & $\mathrm{O}=$ ID Solutions \\
$\mathrm{P}=\mathrm{id} 3$ & $\mathrm{Q}=$ Morpho & $\mathrm{S}=$ Decatur Industries & $\mathrm{T}=$ BIO-key & $\mathrm{U}=$ Aware & $\mathrm{V}=$ AA Technology
\end{tabular}




\begin{tabular}{|c|c|c|c|c|c|c|c|}
\hline \multicolumn{2}{|c|}{ Participant } & \multicolumn{4}{|c|}{ Search } & \multirow{2}{*}{\multicolumn{2}{|c|}{ FNIR $@$ FPIR $=10^{-3}$}} \\
\hline Letter & Sub.\# & \multicolumn{2}{|c|}{ Mean } & \multicolumn{2}{|c|}{ Median } & & \\
\hline \multirow{2}{*}{$\mathrm{C}$} & 1 & 12 & 4.22 & 12 & 4.20 & 30 & NA \\
\hline & 2 & 12 & 4.22 & 12 & 4.20 & 29 & NA \\
\hline \multirow{2}{*}{$\mathrm{D}$} & 1 & 14 & 4.29 & 14 & 4.28 & 6 & 0.0031 \\
\hline & 2 & 24 & 8.31 & 24 & 8.28 & 5 & 0.0024 \\
\hline \multirow{2}{*}{$\mathrm{E}$} & 1 & 11 & 3.86 & 11 & 3.85 & 15 & 0.0063 \\
\hline & 2 & 15 & 4.39 & 15 & 4.38 & 10 & 0.0049 \\
\hline \multirow{2}{*}{$\mathrm{F}$} & 1 & 26 & 13.78 & 26 & 13.49 & 28 & 0.0910 \\
\hline & 2 & 26 & 13.78 & 26 & 13.49 & 26 & 0.0901 \\
\hline \multirow{2}{*}{ G } & 1 & 18 & 5.59 & 18 & 5.62 & 18 & 0.0106 \\
\hline & 2 & 18 & 5.59 & 18 & 5.62 & 17 & 0.0084 \\
\hline \multirow{2}{*}{$\mathrm{H}$} & 1 & 9 & 3.31 & 9 & 3.31 & 23 & 0.0349 \\
\hline & 2 & 9 & 3.31 & 9 & 3.31 & 24 & 0.0361 \\
\hline \multirow{2}{*}{ I } & 1 & 25 & 12.94 & 25 & 12.96 & 3 & 0.0022 \\
\hline & 2 & 30 & 15.30 & 30 & 15.33 & 1 & 0.0015 \\
\hline \multirow{2}{*}{$\mathrm{J}$} & 1 & 7 & 2.42 & 5 & 2.40 & 16 & 0.0068 \\
\hline & 2 & 7 & 2.42 & 5 & 2.40 & 9 & 0.0047 \\
\hline \multirow{2}{*}{$\mathrm{L}$} & 1 & 2 & 2.24 & 2 & 2.23 & 12 & 0.0054 \\
\hline & 2 & 1 & 0.66 & 1 & 0.66 & 14 & 0.0062 \\
\hline \multirow{2}{*}{ M } & 1 & 28 & 13.79 & 28 & 13.53 & 27 & 0.0904 \\
\hline & 2 & 28 & 13.79 & 28 & 13.53 & 25 & 0.0882 \\
\hline \multirow{2}{*}{$\mathrm{O}$} & 1 & 5 & 2.42 & 3 & 2.40 & 13 & 0.0057 \\
\hline & 2 & 5 & 2.42 & 3 & 2.40 & 11 & 0.0051 \\
\hline \multirow{2}{*}{ Q } & 1 & 20 & 6.84 & 20 & 6.84 & 2 & 0.0021 \\
\hline & 2 & 20 & 6.84 & 20 & 6.84 & 3 & 0.0022 \\
\hline \multirow{2}{*}{$S$} & 1 & 22 & 7.82 & 22 & 7.83 & 21 & 0.0160 \\
\hline & 2 & 22 & 7.82 & 22 & 7.83 & 22 & 0.0190 \\
\hline \multirow{2}{*}{$\mathrm{U}$} & 1 & 17 & 4.91 & 16 & 4.82 & 20 & 0.0139 \\
\hline & 2 & 16 & 4.89 & 17 & 4.83 & 19 & 0.0124 \\
\hline \multirow{2}{*}{ V } & 1 & 3 & 2.40 & 7 & 2.40 & 7 & 0.0036 \\
\hline & 2 & 3 & 2.40 & 7 & 2.40 & 7 & 0.0036 \\
\hline
\end{tabular}

Table 77: Tabulation of enrollment time results for Class B — Left and Right Slap. Letter refers to the participant's letter code found on the footer of this page. Sub. \# is an identifier used to differentiate between the two submissions each participant could make. Search shows the time used to create a search template to be used for a query. All values are reported in seconds, but were originally recorded to microsecond precision. The number to the left of a value provides the value's column-wise ranking, with the best performance shaded in green and the worst in pink. For reference, the FNIR values from Table 12 are reprinted to the right of this table.

\begin{tabular}{l|l|l|l|ll|l}
$\mathrm{C}=$ afis team & $\mathrm{D}=3 \mathrm{M}$ Cogent & $\mathrm{E}=$ Neurotechnology & $\mathrm{F}=$ Papillon & $\mathrm{G}=$ Dermalog & $\mathrm{H}=$ Hisign Bio-Info Institute \\
$\mathrm{I}=$ NEC & $\mathrm{J}=$ Sonda & $\mathrm{K}=$ Tiger IT & $\mathrm{L}=$ Innovatrics & $\mathrm{M}=$ SPEX & $\mathrm{O}=$ ID Solutions \\
$\mathrm{P}=\mathrm{id} 3$ & $\mathrm{Q}=$ Morpho & $\mathrm{S}=$ Decatur Industries & $\mathrm{T}=$ BIO-key & $\mathrm{U}=$ Aware & $\mathrm{V}=$ AA Technology
\end{tabular}




\begin{tabular}{|c|c|c|c|c|c|c|c|c|c|c|c|}
\hline \multicolumn{2}{|c|}{ Participant } & \multicolumn{4}{|c|}{ Enrollment } & \multicolumn{4}{|c|}{ Search } & \multirow{2}{*}{\multicolumn{2}{|c|}{ FNIR $@$ FPIR $=10^{-3}$}} \\
\hline Letter & Sub.\# & & lean & & dian & & ean & & dian & & \\
\hline \multirow{2}{*}{ C } & 1 & 11 & 4.92 & 11 & 5.06 & 11 & 5.16 & 11 & 5.13 & 30 & NA \\
\hline & 2 & 11 & 4.92 & 11 & 5.06 & 11 & 5.16 & 11 & 5.13 & 29 & NA \\
\hline \multirow{2}{*}{$\mathrm{D}$} & 1 & 19 & 5.82 & 19 & 5.97 & 17 & 6.19 & 17 & 6.16 & 6 & 0.0020 \\
\hline & 2 & 24 & 11.20 & 24 & 11.47 & 24 & 11.90 & 24 & 11.86 & 2 & 0.0012 \\
\hline \multirow{2}{*}{$\mathrm{E}$} & 1 & 13 & 5.11 & 13 & 5.28 & 13 & 5.35 & 13 & 5.34 & 16 & 0.0043 \\
\hline & 2 & 18 & 5.69 & 18 & 5.89 & 16 & 5.95 & 16 & 5.93 & 7 & 0.0024 \\
\hline \multirow{2}{*}{$\mathrm{F}$} & 1 & 28 & 17.69 & 28 & 17.86 & 28 & 18.36 & 26 & 18.07 & 27 & 0.0591 \\
\hline & 2 & 28 & 17.69 & 28 & 17.86 & 28 & 18.36 & 26 & 18.07 & 27 & 0.0591 \\
\hline \multirow{2}{*}{ G } & 1 & 14 & 5.41 & 14 & 5.60 & 18 & 7.89 & 18 & 7.90 & 18 & 0.0062 \\
\hline & 2 & 14 & 5.41 & 14 & 5.60 & 18 & 7.89 & 18 & 7.90 & 14 & 0.0040 \\
\hline \multirow{2}{*}{$\mathrm{H}$} & 1 & 9 & 4.15 & 9 & 4.28 & 9 & 4.37 & 9 & 4.37 & 23 & 0.0203 \\
\hline & 2 & 9 & 4.15 & 9 & 4.28 & 9 & 4.37 & 9 & 4.37 & 24 & 0.0204 \\
\hline \multirow{2}{*}{ I } & 1 & 25 & 15.80 & 25 & 16.47 & 25 & 16.50 & 25 & 16.52 & 2 & 0.0012 \\
\hline & 2 & 30 & 18.56 & 30 & 19.35 & 30 & 19.36 & 30 & 19.36 & 1 & 0.0009 \\
\hline \multirow{2}{*}{$\mathrm{J}$} & 1 & 3 & 3.20 & 3 & 3.27 & 7 & 3.38 & 3 & 3.35 & 17 & 0.0049 \\
\hline & 2 & 3 & 3.20 & 3 & 3.27 & 7 & 3.38 & 3 & 3.35 & 11 & 0.0033 \\
\hline \multirow{2}{*}{$\mathrm{L}$} & 1 & 2 & 2.94 & 2 & 3.02 & 2 & 3.05 & 2 & 3.05 & 10 & 0.0031 \\
\hline & 2 & 1 & 0.84 & 1 & 0.87 & 1 & 0.88 & 1 & 0.88 & 11 & 0.0033 \\
\hline \multirow{2}{*}{ M } & 1 & 26 & 17.66 & 26 & 17.85 & 26 & 18.36 & 28 & 18.11 & 26 & 0.0543 \\
\hline & 2 & 26 & 17.66 & 26 & 17.85 & 26 & 18.36 & 28 & 18.11 & 25 & 0.0515 \\
\hline \multirow{2}{*}{$\mathrm{O}$} & 1 & 7 & 3.20 & 5 & 3.27 & 5 & 3.38 & 5 & 3.36 & 15 & 0.0041 \\
\hline & 2 & 7 & 3.20 & 5 & 3.27 & 5 & 3.38 & 5 & 3.36 & 13 & 0.0035 \\
\hline \multirow{2}{*}{ Q } & 1 & 20 & 8.50 & 20 & 8.85 & 20 & 8.89 & 20 & 8.92 & 2 & 0.0012 \\
\hline & 2 & 20 & 8.50 & 20 & 8.85 & 20 & 8.89 & 20 & 8.92 & 2 & 0.0012 \\
\hline \multirow{2}{*}{$S$} & 1 & 22 & 9.80 & 22 & 10.19 & 22 & 10.24 & 22 & 10.31 & 20 & 0.0108 \\
\hline & 2 & 22 & 9.80 & 22 & 10.19 & 22 & 10.24 & 22 & 10.31 & 21 & 0.0136 \\
\hline \multirow{2}{*}{$\mathrm{U}$} & 1 & 17 & 5.66 & 17 & 5.73 & 15 & 5.90 & 15 & 5.82 & 19 & 0.0099 \\
\hline & 2 & 16 & 5.56 & 16 & 5.66 & 14 & 5.80 & 14 & 5.73 & 22 & 0.0141 \\
\hline \multirow{2}{*}{$\mathrm{V}$} & 1 & 5 & 3.20 & 7 & 3.30 & 3 & 3.35 & 7 & 3.36 & 9 & 0.0027 \\
\hline & 2 & 5 & 3.20 & 7 & 3.30 & 3 & 3.35 & 7 & 3.36 & 7 & 0.0024 \\
\hline
\end{tabular}

Table 78: Tabulation of enrollment time results for Class B - Identification Flats. Letter refers to the participant's letter code found on the footer of this page. Sub. \# is an identifier used to differentiate between the two submissions each participant could make. Enrollment shows the time used to create a fingerprint template to be used in an enrollment set. Search shows the time used to create a search template to be used for a query. All values are reported in seconds, but were originally recorded to microsecond precision. The number to the left of a value provides the value's column-wise ranking, with the best performance shaded in green and the worst in pink. For reference, the FNIR values from Table 13 are reprinted to the right of this table. 


\begin{tabular}{|c|c|c|c|c|c|c|c|c|c|c|c|}
\hline \multicolumn{2}{|c|}{ Participant } & \multicolumn{4}{|c|}{ Enrollment } & \multicolumn{4}{|c|}{ Search } & \multirow{2}{*}{\multicolumn{2}{|c|}{ FNIR $@$ FPIR $=10^{-3}$}} \\
\hline Letter & Sub.\# & & lean & & dian & & ean & & dian & & \\
\hline \multirow{2}{*}{ C } & 1 & 15 & 5.93 & 13 & 5.89 & 13 & 5.84 & 13 & 5.80 & 30 & NA \\
\hline & 2 & 15 & 5.93 & 13 & 5.89 & 13 & 5.84 & 13 & 5.80 & 24 & 0.0711 \\
\hline \multirow{2}{*}{$\mathrm{D}$} & 1 & 19 & 7.10 & 19 & 7.09 & 17 & 7.12 & 17 & 7.10 & 6 & 0.0015 \\
\hline & 2 & 24 & 13.55 & 24 & 13.52 & 24 & 13.69 & 24 & 13.66 & 2 & 0.0011 \\
\hline \multirow{2}{*}{$\mathrm{E}$} & 1 & 11 & 5.19 & 11 & 5.21 & 11 & 5.20 & 11 & 5.20 & 14 & 0.0088 \\
\hline & 2 & 12 & 5.20 & 12 & 5.22 & 12 & 5.21 & 12 & 5.20 & 13 & 0.0048 \\
\hline \multirow{2}{*}{$\mathrm{F}$} & 1 & 27 & 15.96 & 25 & 15.88 & 27 & 15.96 & 27 & 15.87 & 25 & 0.0734 \\
\hline & 2 & 27 & 15.96 & 25 & 15.88 & 27 & 15.96 & 27 & 15.87 & 25 & 0.0734 \\
\hline \multirow{2}{*}{ G } & 1 & 13 & 5.93 & 15 & 5.91 & 18 & 8.35 & 18 & 8.32 & 23 & 0.0368 \\
\hline & 2 & 13 & 5.93 & 15 & 5.91 & 18 & 8.35 & 18 & 8.32 & 20 & 0.0276 \\
\hline \multirow{2}{*}{$\mathrm{H}$} & 1 & 9 & 5.18 & 9 & 5.21 & 9 & 5.16 & 9 & 5.16 & 20 & 0.0276 \\
\hline & 2 & 9 & 5.18 & 9 & 5.21 & 9 & 5.16 & 9 & 5.16 & 19 & 0.0275 \\
\hline \multirow{2}{*}{ I } & 1 & 29 & 16.65 & 29 & 16.67 & 29 & 16.71 & 29 & 16.67 & 4 & 0.0013 \\
\hline & 2 & 30 & 17.15 & 30 & 17.07 & 30 & 17.04 & 30 & 16.99 & 1 & 0.0010 \\
\hline \multirow{2}{*}{$\mathrm{J}$} & 1 & 3 & 3.61 & 3 & 3.58 & 3 & 3.62 & 5 & 3.62 & 12 & 0.0047 \\
\hline & 2 & 6 & 3.63 & 6 & 3.60 & 6 & 3.64 & 6 & 3.62 & 10 & 0.0027 \\
\hline \multirow{2}{*}{$\mathrm{L}$} & 1 & 1 & 3.56 & 1 & 3.52 & 1 & 3.51 & 1 & 3.48 & 16 & 0.0102 \\
\hline & 2 & 1 & 3.56 & 1 & 3.52 & 1 & 3.51 & 1 & 3.48 & 15 & 0.0095 \\
\hline \multirow{2}{*}{ M } & 1 & 25 & 15.95 & 27 & 15.89 & 25 & 15.91 & 25 & 15.83 & 28 & 0.0934 \\
\hline & 2 & 25 & 15.95 & 27 & 15.89 & 25 & 15.91 & 25 & 15.83 & 27 & 0.0826 \\
\hline \multirow{2}{*}{$\mathrm{O}$} & 1 & 4 & 3.63 & 4 & 3.60 & 4 & 3.63 & 3 & 3.61 & 9 & 0.0025 \\
\hline & 2 & 4 & 3.63 & 4 & 3.60 & 4 & 3.63 & 3 & 3.61 & 10 & 0.0027 \\
\hline \multirow{2}{*}{ Q } & 1 & 20 & 9.10 & 20 & 9.08 & 20 & 9.14 & 20 & 9.11 & 2 & 0.0011 \\
\hline & 2 & 20 & 9.10 & 20 & 9.08 & 20 & 9.14 & 20 & 9.11 & 4 & 0.0013 \\
\hline \multirow{2}{*}{$S$} & 1 & 22 & 10.86 & 22 & 10.87 & 22 & 10.91 & 22 & 10.87 & 22 & 0.0311 \\
\hline & 2 & 22 & 10.86 & 22 & 10.87 & 22 & 10.91 & 22 & 10.87 & 29 & 0.1680 \\
\hline \multirow{2}{*}{$\mathrm{U}$} & 1 & 17 & 6.34 & 17 & 6.09 & 15 & 6.13 & 15 & 5.91 & 18 & 0.0163 \\
\hline & 2 & 17 & 6.34 & 17 & 6.09 & 15 & 6.13 & 15 & 5.91 & 17 & 0.0155 \\
\hline \multirow{2}{*}{ V } & 1 & 7 & 3.68 & 7 & 3.67 & 7 & 3.69 & 7 & 3.66 & 7 & 0.0024 \\
\hline & 2 & 7 & 3.68 & 7 & 3.67 & 7 & 3.69 & 7 & 3.66 & 7 & 0.0024 \\
\hline
\end{tabular}

Table 79: Tabulation of enrollment time results for Class C - Ten-Finger Plain-to-Plain. Letter refers to the participant's letter code found on the footer of this page. Sub. \# is an identifier used to differentiate between the two submissions each participant could make. Enrollment shows the time used to create a fingerprint template to be used in an enrollment set. Search shows the time used to create a search template to be used for a query. All values are reported in seconds, but were originally recorded to microsecond precision. The number to the left of a value provides the value's column-wise ranking, with the best performance shaded in green and the worst in pink. For reference, the FNIR values from Table 14 are reprinted to the right of this table.

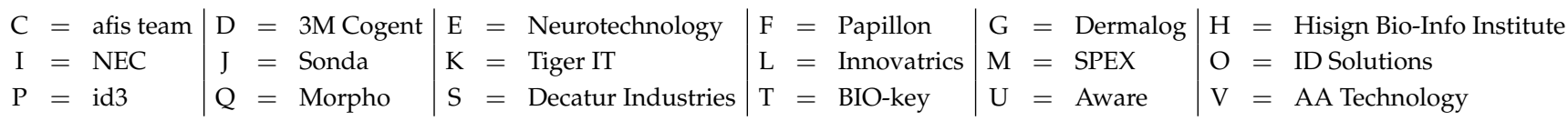




\begin{tabular}{|c|c|c|c|c|c|c|c|c|c|c|c|}
\hline \multicolumn{2}{|c|}{ Participant } & \multicolumn{4}{|c|}{ Enrollment } & \multicolumn{4}{|c|}{ Search } & \multirow{2}{*}{\multicolumn{2}{|c|}{ FNIR $@$ FPIR $=10^{-3}$}} \\
\hline Letter & Sub.\# & & lean & & dian & & Iean & & dian & & \\
\hline \multirow{2}{*}{$\mathrm{C}$} & 1 & 13 & 11.18 & 13 & 11.00 & 13 & 10.79 & 13 & 10.69 & 16 & 0.0094 \\
\hline & 2 & 13 & 11.18 & 13 & 11.00 & 13 & 10.79 & 13 & 10.69 & 15 & 0.0085 \\
\hline \multirow{2}{*}{$\mathrm{D}$} & 1 & 21 & 17.37 & 21 & 17.27 & 23 & 17.44 & 21 & 17.39 & 4 & 0.0015 \\
\hline & 2 & 30 & 30.78 & 30 & 30.70 & 30 & 30.43 & 30 & 30.47 & 7 & 0.0018 \\
\hline \multirow{2}{*}{$\mathrm{E}$} & 1 & 12 & 9.93 & 12 & 9.94 & 12 & 9.90 & 12 & 9.92 & 18 & 0.0106 \\
\hline & 2 & 11 & 9.92 & 11 & 9.94 & 11 & 9.89 & 11 & 9.91 & 12 & 0.0050 \\
\hline \multirow{2}{*}{$\mathrm{F}$} & 1 & 26 & 21.06 & 28 & 20.95 & 28 & 21.07 & 28 & 20.92 & 25 & 0.0536 \\
\hline & 2 & 26 & 21.06 & 28 & 20.95 & 28 & 21.07 & 28 & 20.92 & 25 & 0.0536 \\
\hline \multirow{2}{*}{ G } & 1 & 15 & 11.21 & 15 & 11.19 & 19 & 15.55 & 19 & 15.50 & 24 & 0.0447 \\
\hline & 2 & 15 & 11.21 & 15 & 11.19 & 19 & 15.55 & 19 & 15.50 & 21 & 0.0333 \\
\hline \multirow{2}{*}{$\mathrm{H}$} & 1 & 17 & 12.36 & 17 & 12.36 & 15 & 12.08 & 15 & 12.17 & 20 & 0.0201 \\
\hline & 2 & 17 & 12.36 & 17 & 12.36 & 15 & 12.08 & 15 & 12.17 & 19 & 0.0199 \\
\hline \multirow{2}{*}{ I } & 1 & 25 & 20.25 & 25 & 20.25 & 25 & 20.21 & 25 & 20.23 & 1 & 0.0013 \\
\hline & 2 & 24 & 18.75 & 24 & 18.76 & 24 & 18.72 & 24 & 18.73 & 2 & 0.0014 \\
\hline \multirow{2}{*}{$\mathrm{J}$} & 1 & 7 & 6.78 & 5 & 6.67 & 5 & 6.78 & 5 & 6.69 & 13 & 0.0051 \\
\hline & 2 & 8 & 6.80 & 8 & 6.71 & 8 & 6.82 & 8 & 6.74 & 9 & 0.0033 \\
\hline \multirow{2}{*}{$\mathrm{L}$} & 1 & 3 & 4.43 & 3 & 4.44 & 3 & 4.36 & 3 & 4.36 & 17 & 0.0097 \\
\hline & 2 & 3 & 4.43 & 3 & 4.44 & 3 & 4.36 & 3 & 4.36 & 14 & 0.0083 \\
\hline \multirow{2}{*}{ M } & 1 & 28 & 21.08 & 26 & 20.92 & 26 & 21.02 & 26 & 20.89 & 28 & 0.0783 \\
\hline & 2 & 28 & 21.08 & 26 & 20.92 & 26 & 21.02 & 26 & 20.89 & 27 & 0.0716 \\
\hline \multirow{2}{*}{$\mathrm{O}$} & 1 & 5 & 6.77 & 6 & 6.67 & 6 & 6.80 & 6 & 6.71 & 11 & 0.0034 \\
\hline & 2 & 5 & 6.77 & 6 & 6.67 & 6 & 6.80 & 6 & 6.71 & 9 & 0.0033 \\
\hline \multirow{2}{*}{$\mathrm{Q}$} & 1 & 19 & 12.94 & 19 & 13.03 & 17 & 12.95 & 17 & 13.06 & 5 & 0.0017 \\
\hline & 2 & 19 & 12.94 & 19 & 13.03 & 17 & 12.95 & 17 & 13.06 & 2 & 0.0014 \\
\hline \multirow{2}{*}{$S$} & 1 & 22 & 17.54 & 22 & 17.67 & 21 & 17.43 & 22 & 17.57 & 29 & 0.0860 \\
\hline & 2 & 22 & 17.54 & 22 & 17.67 & 21 & 17.43 & 22 & 17.57 & 30 & 0.2462 \\
\hline \multirow{2}{*}{$\mathrm{U}$} & 1 & 1 & 2.95 & 1 & 2.88 & 1 & 2.94 & 1 & 2.87 & 23 & 0.0358 \\
\hline & 2 & 1 & 2.95 & 1 & 2.88 & 1 & 2.94 & 1 & 2.87 & 22 & 0.0351 \\
\hline \multirow{2}{*}{ V } & 1 & 9 & 8.60 & 9 & 8.62 & 9 & 8.48 & 9 & 8.50 & 5 & 0.0017 \\
\hline & 2 & 9 & 8.60 & 9 & 8.62 & 9 & 8.48 & 9 & 8.50 & 8 & 0.0019 \\
\hline
\end{tabular}

Table 80: Tabulation of enrollment time results for Class C - Ten-Finger Rolled-to-Rolled. Letter refers to the participant's letter code found on the footer of this page. Sub. \# is an identifier used to differentiate between the two submissions each participant could make. Enrollment shows the time used to create a fingerprint template to be used in an enrollment set. Search shows the time used to create a search template to be used for a query. All values are reported in seconds, but were originally recorded to microsecond precision. The number to the left of a value provides the value's column-wise ranking, with the best performance shaded in green and the worst in pink. For reference, the FNIR values from Table 15 are reprinted to the right of this table. 


\section{Ranked Results}

In order to reduce the number of tables in Section 10 of the main body of the report, this appendix contains the tables showing ranked results for all three classes. The tables from the main body of the document are repeated in this appendix so there is a complete set of tables here for the reader to analyze.

Class A results are in Tables 81 through 83, Class B results are in Tables 84 through 87, and Class C results are in Tables 88 through 89.

\begin{tabular}{l|l|l|l|ll|l}
$\mathrm{C}=$ afis team & $\mathrm{D}=3 \mathrm{M}$ Cogent & $\mathrm{E}=$ Neurotechnology & $\mathrm{F}=$ Papillon & $\mathrm{G}=$ Dermalog & $\mathrm{H}=$ Hisign Bio-Info Institute \\
$\mathrm{I}=$ NEC & $\mathrm{J}=$ Sonda & $\mathrm{K}=$ Tiger IT & $\mathrm{L}=$ Innovatrics & $\mathrm{M}=$ SPEX & $\mathrm{O}=$ ID Solutions \\
$\mathrm{P}=\mathrm{id} 3$ & $\mathrm{Q}=$ Morpho & $\mathrm{S}=$ Decatur Industries & $\mathrm{T}=$ BIO-key & $\mathrm{U}=$ Aware & $\mathrm{V}=$ AA Technology
\end{tabular}




\begin{tabular}{|c|c|c|c|c|c|c|c|c|c|c|c|c|c|c|}
\hline & \multicolumn{2}{|c|}{ Participant } & \multirow{2}{*}{\multicolumn{2}{|c|}{ FNIR }} & \multicolumn{4}{|c|}{ Identification } & \multicolumn{4}{|c|}{ Search Enrollment } & \multirow{2}{*}{\multicolumn{2}{|c|}{ RAM }} \\
\hline & Letter & Sub.\# & & & \multicolumn{2}{|c|}{ Mean } & \multicolumn{2}{|c|}{ Median } & \multicolumn{2}{|c|}{ Mean } & \multicolumn{2}{|c|}{ Median } & & \\
\hline \multirow{30}{*}{ 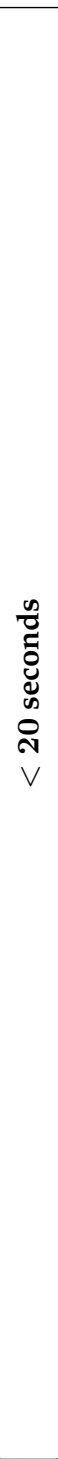 } & $\mathrm{D}$ & 1 & 1 & 0.0197 & 20 & 7.52 & 20 & 7.32 & 30 & 1.57 & 29 & 1.43 & 19 & 0.61 \\
\hline & $Q$ & 1 & 2 & 0.0222 & 29 & 15.90 & 28 & 15.70 & 20 & 0.57 & 19 & 0.54 & 13 & 0.34 \\
\hline & $\mathrm{Q}$ & 2 & 3 & 0.0226 & 23 & 10.42 & 24 & 10.43 & 20 & 0.57 & 19 & 0.54 & 14 & 0.34 \\
\hline & $\mathrm{V}$ & 1 & 4 & 0.0253 & 19 & 6.22 & 19 & 6.01 & 12 & 0.34 & 10 & 0.32 & 17 & 0.39 \\
\hline & I & 1 & 5 & 0.0257 & 22 & 10.36 & 22 & 9.94 & 29 & 1.48 & 30 & 1.46 & 20 & 0.70 \\
\hline & $\mathrm{L}$ & 2 & 6 & 0.0351 & 12 & 3.34 & 12 & 3.32 & 3 & 0.17 & 3 & 0.15 & 28 & 2.32 \\
\hline & $S$ & 1 & 7 & 0.0571 & 28 & 15.37 & 29 & 16.86 & 22 & 0.90 & 22 & 0.88 & 21 & 0.72 \\
\hline & $\mathrm{L}$ & 1 & 8 & 0.0625 & 2 & 0.30 & 2 & 0.29 & 1 & 0.07 & 1 & 0.05 & 26 & 1.14 \\
\hline & $\mathrm{T}$ & 2 & 9 & 0.0685 & 18 & 5.96 & 18 & 5.97 & 6 & 0.33 & 8 & 0.31 & 1 & 0.01 \\
\hline & $\mathrm{J}$ & 2 & 10 & 0.0712 & 7 & 1.34 & 7 & 1.25 & 8 & 0.33 & 11 & 0.32 & 9 & 0.32 \\
\hline & $\mathrm{E}$ & 2 & 11 & 0.0723 & 30 & 16.71 & 30 & 16.90 & 13 & 0.35 & 13 & 0.35 & 24 & 1.11 \\
\hline & $\mathrm{E}$ & 1 & 12 & 0.0745 & 4 & 0.57 & 3 & 0.35 & 13 & 0.35 & 13 & 0.35 & 25 & 1.12 \\
\hline & $\mathrm{O}$ & 2 & 13 & 0.0766 & 9 & 1.63 & 9 & 1.56 & 4 & 0.28 & 4 & 0.27 & 15 & 0.35 \\
\hline & $\mathrm{J}$ & 1 & 14 & 0.0786 & 3 & 0.56 & 4 & 0.54 & 8 & 0.33 & 11 & 0.32 & 9 & 0.32 \\
\hline & $\mathrm{O}$ & 1 & 15 & 0.0818 & 5 & 0.64 & 5 & 0.62 & 4 & 0.28 & 4 & 0.27 & 15 & 0.35 \\
\hline & $\mathrm{K}$ & 2 & 16 & 0.0875 & 24 & 10.47 & 23 & 10.32 & 23 & 1.06 & 23 & 1.05 & 30 & 3.87 \\
\hline & $\mathrm{K}$ & 1 & 17 & 0.0883 & 25 & 10.48 & 25 & 10.44 & 23 & 1.06 & 23 & 1.05 & 29 & 3.87 \\
\hline & $\mathrm{F}$ & 2 & 18 & 0.1082 & 15 & 3.78 & 14 & 3.56 & 25 & 1.11 & 25 & 1.07 & 5 & 0.18 \\
\hline & G & 1 & 19 & 0.1089 & 21 & 9.52 & 21 & 9.74 & 19 & 0.57 & 21 & 0.55 & 18 & 0.57 \\
\hline & $\mathrm{F}$ & 1 & 20 & 0.1111 & 11 & 2.52 & 11 & 2.49 & 25 & 1.11 & 25 & 1.07 & 6 & 0.18 \\
\hline & $\mathrm{U}$ & 1 & 21 & 0.1218 & 26 & 14.72 & 27 & 14.42 & 2 & 0.16 & 2 & 0.15 & 27 & 1.70 \\
\hline & $\mathrm{P}$ & 2 & 22 & 0.1272 & 13 & 3.43 & 13 & 3.33 & 15 & 0.38 & 15 & 0.36 & 22 & 0.78 \\
\hline & $\mathrm{P}$ & 1 & 23 & 0.1308 & 8 & 1.38 & 8 & 1.32 & 15 & 0.38 & 15 & 0.36 & 23 & 0.78 \\
\hline & C & 1 & 24 & 0.1335 & 1 & 0.29 & 1 & 0.26 & 17 & 0.41 & 17 & 0.39 & 7 & 0.18 \\
\hline & $\mathrm{C}$ & 2 & 25 & 0.1337 & 6 & 0.87 & 6 & 0.76 & 17 & 0.41 & 17 & 0.39 & 8 & 0.18 \\
\hline & $\mathrm{H}$ & 1 & 26 & 0.1576 & 14 & 3.65 & 15 & 3.61 & 10 & 0.33 & 6 & 0.30 & 11 & 0.33 \\
\hline & $\mathrm{H}$ & 2 & 27 & 0.1607 & 17 & 4.26 & 17 & 4.13 & 10 & 0.33 & 6 & 0.30 & 11 & 0.33 \\
\hline & $\mathrm{M}$ & 2 & 28 & 0.2921 & 27 & 15.19 & 26 & 10.70 & 27 & 1.12 & 27 & 1.07 & 4 & 0.14 \\
\hline & $\mathrm{M}$ & 1 & 29 & 0.2995 & 10 & 2.07 & 10 & 1.84 & 27 & 1.12 & 27 & 1.07 & 3 & 0.14 \\
\hline & $\mathrm{T}$ & 1 & 30 & NA & 16 & 4.18 & 16 & 3.99 & 6 & 0.33 & 8 & 0.31 & 2 & 0.01 \\
\hline \multirow{6}{*}{ 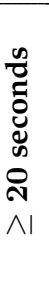 } & $\mathrm{D}$ & 2 & 1 & 0.0197 & 2 & 42.64 & 3 & 41.84 & 5 & 1.55 & 5 & 1.42 & 3 & 0.61 \\
\hline & $\mathrm{V}$ & 2 & 2 & 0.0252 & 6 & 66.23 & 6 & 65.35 & 2 & 0.34 & 2 & 0.32 & 1 & 0.39 \\
\hline & I & 2 & 3 & 0.0278 & 3 & 43.24 & 2 & 40.99 & 6 & 2.19 & 6 & 2.15 & 5 & 1.04 \\
\hline & $S$ & 2 & 4 & 0.0650 & 4 & 45.59 & 4 & 44.66 & 4 & 0.90 & 4 & 0.88 & 4 & 0.72 \\
\hline & $G$ & 2 & 5 & 0.1086 & 5 & 54.24 & 5 & 54.03 & 3 & 0.57 & 3 & 0.55 & 2 & 0.57 \\
\hline & $\mathrm{U}$ & 2 & 6 & 0.1178 & 1 & 25.66 & 1 & 24.16 & 1 & 0.16 & 1 & 0.15 & 6 & 1.42 \\
\hline
\end{tabular}

Table 81: Tabulation of ranked results for Class A — Left Index. Submissions were split into two groups. The first group includes submissions that performed searches on average in less than 20 seconds, and the second includes those that took, on average, 20 seconds or longer. Letter refers to the participant's letter code found on the footer of this page. Sub. \# is an identifier used to differentiate between the two submissions each participant could make. The FNIR column was computed at the score threshold that gave FPIR $=10^{-3}$. NA indicates that the operations required to produce the value could not be performed. The Identification column shows the time used to perform a search over an enrollment set of 100000 , as seen in Table 17 . The Search Enrollment column shows the time used to create a search template to be used for a query, as seen in Table 72. Identification and Search Enrollment durations are reported in seconds, but were originally recorded to microsecond precision. RAM refers to the sum of the resident set sizes of the stage one identification processes over all compute nodes after returning from the identification stage one initialization method, as seen in Table 60. $R A M$ is reported in gigabytes, where 1 GB is equal to 1073741824 bytes. The number to the left of a value provides the value's column-wise ranking, with the best performance shaded in green and the worst in pink. The table is sorted on the FNIR column-wise ranking.

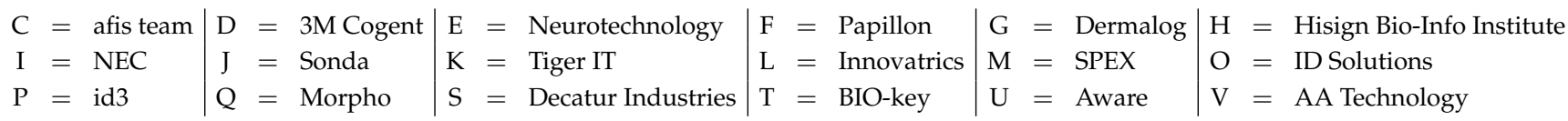




\begin{tabular}{|c|c|c|c|c|c|c|c|c|c|c|c|c|c|c|}
\hline & \multicolumn{2}{|c|}{ Participant } & \multirow{2}{*}{\multicolumn{2}{|c|}{ FNIR }} & \multicolumn{4}{|c|}{ Identification } & \multicolumn{4}{|c|}{ Search Enrollment } & \multirow{2}{*}{\multicolumn{2}{|c|}{ RAM }} \\
\hline & Letter & Sub.\# & & & \multicolumn{2}{|c|}{ Mean } & \multicolumn{2}{|c|}{ Median } & \multicolumn{2}{|c|}{ Mean } & \multicolumn{2}{|c|}{ Median } & & \\
\hline \multirow{30}{*}{ 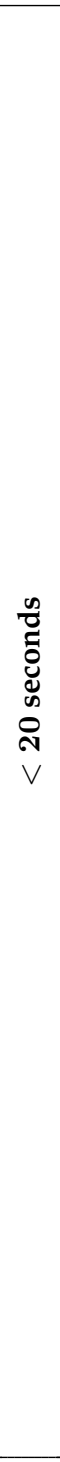 } & $\mathrm{D}$ & 1 & 1 & 0.0190 & 20 & 7.68 & 20 & 7.46 & 30 & 1.58 & 29 & 1.43 & 19 & 0.61 \\
\hline & $Q$ & 2 & 2 & 0.0214 & 22 & 10.15 & 23 & 9.98 & 20 & 0.58 & 19 & 0.54 & 11 & 0.33 \\
\hline & I & 1 & 3 & 0.0215 & 23 & 10.19 & 22 & 9.83 & 29 & 1.49 & 30 & 1.46 & 20 & 0.69 \\
\hline & Q & 1 & 4 & 0.0218 & 28 & 14.44 & 28 & 14.24 & 20 & 0.58 & 19 & 0.54 & 11 & 0.33 \\
\hline & $\mathrm{V}$ & 1 & 5 & 0.0223 & 19 & 5.95 & 18 & 5.60 & 12 & 0.34 & 10 & 0.32 & 17 & 0.39 \\
\hline & $\mathrm{L}$ & 2 & 6 & 0.0295 & 12 & 3.26 & 13 & 3.26 & 3 & 0.17 & 3 & 0.16 & 28 & 2.32 \\
\hline & $\mathrm{S}$ & 1 & 7 & 0.0442 & 29 & 15.08 & 30 & 16.55 & 22 & 0.89 & 22 & 0.88 & 21 & 0.71 \\
\hline & $\mathrm{L}$ & 1 & 8 & 0.0505 & 2 & 0.29 & 2 & 0.26 & 1 & 0.07 & 1 & 0.05 & 26 & 1.14 \\
\hline & $\mathrm{T}$ & 2 & 9 & 0.0562 & 18 & 5.64 & 19 & 5.87 & 6 & 0.33 & 8 & 0.31 & 1 & 0.01 \\
\hline & $\mathrm{E}$ & 2 & 10 & 0.0624 & 30 & 15.89 & 29 & 16.27 & 13 & 0.37 & 13 & 0.36 & 25 & 1.07 \\
\hline & $E$ & 1 & 11 & 0.0630 & 3 & 0.45 & 3 & 0.32 & 13 & 0.37 & 13 & 0.36 & 24 & 1.07 \\
\hline & $\mathrm{J}$ & 2 & 12 & 0.0643 & 7 & 1.25 & 7 & 1.13 & 10 & 0.34 & 11 & 0.33 & 9 & 0.31 \\
\hline & $\mathrm{O}$ & 2 & 13 & 0.0675 & 9 & 1.48 & 9 & 1.36 & 4 & 0.28 & 4 & 0.27 & 16 & 0.34 \\
\hline & $\mathrm{K}$ & 1 & 14 & 0.0682 & 25 & 10.42 & 26 & 10.42 & 23 & 1.06 & 23 & 1.05 & 30 & 3.87 \\
\hline & $\mathrm{K}$ & 2 & 15 & 0.0685 & 24 & 10.30 & 25 & 10.27 & 23 & 1.06 & 23 & 1.05 & 29 & 3.87 \\
\hline & $\mathrm{J}$ & 1 & 16 & 0.0708 & 4 & 0.53 & 4 & 0.51 & 10 & 0.34 & 11 & 0.33 & 10 & 0.31 \\
\hline & $\mathrm{O}$ & 1 & 17 & 0.0776 & 5 & 0.59 & 5 & 0.56 & 4 & 0.28 & 4 & 0.27 & 15 & 0.34 \\
\hline & $\mathrm{F}$ & 2 & 18 & 0.0903 & 15 & 3.71 & 15 & 3.60 & 27 & 1.14 & 25 & 1.09 & 5 & 0.17 \\
\hline & G & 1 & 19 & 0.0910 & 21 & 10.12 & 24 & 10.10 & 19 & 0.57 & 21 & 0.55 & 18 & 0.57 \\
\hline & $\mathrm{F}$ & 1 & 20 & 0.0933 & 11 & 2.52 & 11 & 2.38 & 27 & 1.14 & 25 & 1.09 & 6 & 0.17 \\
\hline & $\mathrm{U}$ & 1 & 21 & 0.0996 & 27 & 12.02 & 27 & 10.76 & 2 & 0.16 & 2 & 0.15 & 27 & 1.56 \\
\hline & $\mathrm{P}$ & 2 & 22 & 0.1100 & 13 & 3.49 & 12 & 3.07 & 15 & 0.39 & 15 & 0.37 & 23 & 0.76 \\
\hline & $\mathrm{C}$ & 2 & 23 & 0.1124 & 6 & 0.76 & 6 & 0.69 & 17 & 0.41 & 17 & 0.39 & 8 & 0.18 \\
\hline & C & 1 & 24 & 0.1132 & 1 & 0.25 & 1 & 0.24 & 17 & 0.41 & 17 & 0.39 & 7 & 0.17 \\
\hline & $\mathrm{P}$ & 1 & 25 & 0.1133 & 8 & 1.38 & 8 & 1.24 & 15 & 0.39 & 15 & 0.37 & 22 & 0.76 \\
\hline & $\mathrm{H}$ & 1 & 26 & 0.1230 & 14 & 3.58 & 14 & 3.56 & 8 & 0.34 & 6 & 0.30 & 14 & 0.33 \\
\hline & $\mathrm{H}$ & 2 & 27 & 0.1249 & 17 & 4.13 & 17 & 4.05 & 8 & 0.34 & 6 & 0.30 & 13 & 0.33 \\
\hline & $\mathrm{T}$ & 1 & 28 & 0.1929 & 16 & 3.82 & 16 & 3.73 & 6 & 0.33 & 8 & 0.31 & 1 & 0.01 \\
\hline & $\mathrm{M}$ & 2 & 29 & 0.2526 & 26 & 11.31 & 21 & 9.39 & 25 & 1.14 & 27 & 1.09 & 3 & 0.14 \\
\hline & $\mathrm{M}$ & 1 & 30 & 0.2615 & 10 & 1.75 & 10 & 1.78 & 25 & 1.14 & 27 & 1.09 & 3 & 0.14 \\
\hline \multirow{6}{*}{ 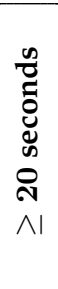 } & D & 2 & 1 & 0.0190 & 2 & 29.26 & 2 & 28.64 & 5 & 1.57 & 5 & 1.43 & 3 & 0.61 \\
\hline & I & 2 & 2 & 0.0214 & 3 & 41.60 & 3 & 40.35 & 6 & 2.19 & 6 & 2.15 & 5 & 1.01 \\
\hline & $\mathrm{V}$ & 2 & 3 & 0.0222 & 6 & 64.47 & 6 & 61.24 & 2 & 0.34 & 2 & 0.32 & 1 & 0.39 \\
\hline & $S$ & 2 & 4 & 0.0503 & 4 & 43.56 & 4 & 46.05 & 4 & 0.89 & 4 & 0.88 & 4 & 0.71 \\
\hline & G & 2 & 5 & 0.0909 & 5 & 57.88 & 5 & 59.55 & 3 & 0.57 & 3 & 0.55 & 2 & 0.57 \\
\hline & $\mathrm{U}$ & 2 & 6 & 0.1007 & 1 & 22.39 & 1 & 20.10 & 1 & 0.16 & 1 & 0.15 & 6 & 1.30 \\
\hline
\end{tabular}

Table 82: Tabulation of ranked results for Class A - Right Index. Submissions were split into two groups. The first group includes submissions that performed searches on average in less than 20 seconds, and the second includes those that took, on average, 20 seconds or longer. Letter refers to the participant's letter code found on the footer of this page. Sub. \# is an identifier used to differentiate between the two submissions each participant could make. The FNIR column was computed at the score threshold that gave FPIR $=10^{-3}$. The Identification column shows the time used to perform a search over an enrollment set of 100000 , as seen in Table 17. The Search Enrollment column shows the time used to create a search template to be used for a query, as seen in Table 73. Identification and Search Enrollment durations are reported in seconds, but were originally recorded to microsecond precision. $R A M$ refers to the sum of the resident set sizes of the stage one identification processes over all compute nodes after returning from the identification stage one initialization method, as seen in Table 61. RAM is reported in gigabytes, where 1 GB is equal to 1073741824 bytes. The number to the left of a value provides the value's column-wise ranking, with the best performance shaded in green and the worst in pink. The table is sorted on the FNIR column-wise ranking. 


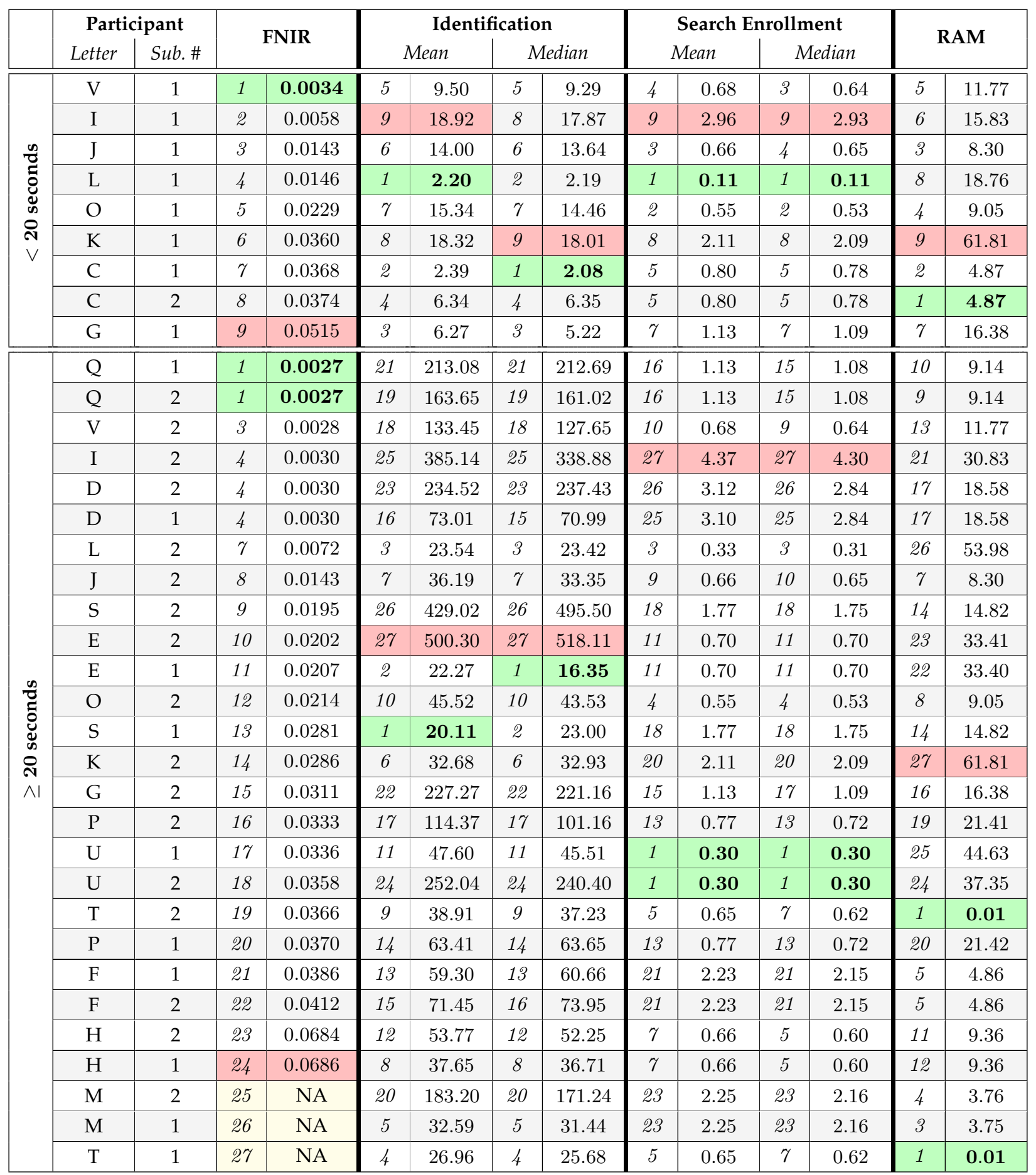

Table 83: Tabulation of ranked results for Class A - Left and Right Index. Submissions were split into two groups. The first group includes submissions that performed searches on average in less than 20 seconds, and the second includes those that took, on average, 20 seconds or longer. Letter refers to the participant's letter code found on the footer of this page. Sub. \# is an identifier used to differentiate between the two submissions each participant could make. The FNIR column was computed at the score threshold that gave FPIR $=10^{-3}$. NA indicates that the operations required to produce the value could not be performed. The Identification column shows the time used to perform a search over an enrollment set of 1600000 , as seen in Table 18 . The Search Enrollment column shows the time used to create a search template to be used for a query, as seen in Table 74. Identification and Search Enrollment durations are reported in seconds, but were originally recorded to microsecond precision. RAM refers to the sum of the resident set sizes of the stage one identification processes over all compute nodes after returning from the identification stage one initialization method, as seen in Table 62. $R A M$ is reported in gigabytes, where 1 GB is equal to 1073741824 bytes. The number to the left of a value provides the value's column-wise ranking, with the best performance shaded in green and the worst in pink. The table is sorted on the FNIR column-wise ranking. 


\begin{tabular}{|c|c|c|c|c|c|c|c|c|c|c|c|c|c|}
\hline \multicolumn{2}{|c|}{ Participant } & \multirow{2}{*}{\multicolumn{2}{|c|}{ FNIR }} & \multicolumn{4}{|c|}{ Identification } & \multicolumn{4}{|c|}{ Search Enrollment } & \multirow{2}{*}{\multicolumn{2}{|c|}{ RAM }} \\
\hline Letter & Sub.\# & & & \multicolumn{2}{|c|}{ Mean } & \multicolumn{2}{|c|}{ Median } & \multicolumn{2}{|c|}{ Mean } & \multicolumn{2}{|c|}{ Median } & & \\
\hline I & 2 & 1 & 0.0094 & 18 & 51.59 & 13 & 38.26 & 30 & 7.68 & 30 & 7.67 & 21 & 108.71 \\
\hline $\mathrm{Q}$ & 1 & 2 & 0.0098 & 17 & 51.33 & 19 & 53.24 & 20 & 3.41 & 20 & 3.41 & 1 & 7.54 \\
\hline Q & 2 & 3 & 0.0099 & 16 & 51.05 & 20 & 54.02 & 20 & 3.41 & 20 & 3.41 & 2 & 7.54 \\
\hline $\mathrm{I}$ & 1 & 4 & 0.0116 & 23 & 63.06 & 21 & 55.96 & 25 & 6.50 & 25 & 6.50 & 5 & 49.68 \\
\hline $\mathrm{D}$ & 2 & 5 & 0.0142 & 21 & 59.65 & 22 & 58.10 & 24 & 4.18 & 24 & 4.17 & 12 & 79.43 \\
\hline $\mathrm{D}$ & 1 & 6 & 0.0163 & 20 & 53.09 & 18 & 52.23 & 14 & 2.16 & 14 & 2.15 & 13 & 79.43 \\
\hline $\mathrm{E}$ & 2 & 7 & 0.0187 & 12 & 41.57 & 10 & 34.37 & 15 & 2.21 & 15 & 2.21 & 28 & 318.03 \\
\hline $\mathrm{V}$ & 2 & 8 & 0.0190 & 14 & 48.01 & 15 & 47.97 & 3 & 1.21 & 7 & 1.22 & 9 & 63.53 \\
\hline $\mathrm{V}$ & 1 & 9 & 0.0192 & 10 & 36.28 & 11 & 36.07 & 3 & 1.21 & 7 & 1.22 & 8 & 63.53 \\
\hline $\mathrm{J}$ & 2 & 10 & 0.0236 & 27 & 80.54 & 27 & 74.38 & 7 & 1.22 & 3 & 1.21 & 19 & 101.00 \\
\hline $\mathrm{O}$ & 2 & 11 & 0.0254 & 24 & 78.80 & 26 & 73.15 & 5 & 1.22 & 5 & 1.21 & 18 & 101.00 \\
\hline $\mathrm{O}$ & 1 & 12 & 0.0257 & 11 & 38.17 & 12 & 37.01 & 5 & 1.22 & 5 & 1.21 & 17 & 101.00 \\
\hline $\mathrm{E}$ & 1 & 13 & 0.0259 & 1 & 3.71 & 1 & 2.82 & 11 & 1.95 & 11 & 1.95 & 14 & 79.72 \\
\hline $\mathrm{L}$ & 2 & 14 & 0.0276 & 5 & 12.52 & 5 & 12.37 & 1 & 0.33 & 1 & 0.33 & 27 & 177.49 \\
\hline $\mathrm{J}$ & 1 & 15 & 0.0287 & 7 & 26.88 & 7 & 25.61 & 7 & 1.22 & 3 & 1.21 & 20 & 101.00 \\
\hline $\mathrm{L}$ & 1 & 16 & 0.0288 & 3 & 5.51 & 3 & 5.56 & 2 & 1.13 & 2 & 1.11 & 22 & 119.79 \\
\hline $\mathrm{G}$ & 2 & 17 & 0.0325 & 22 & 62.98 & 23 & 59.49 & 18 & 2.81 & 18 & 2.83 & 25 & 156.12 \\
\hline G & 1 & 18 & 0.0371 & 6 & 19.65 & 6 & 16.24 & 18 & 2.81 & 18 & 2.83 & 26 & 156.12 \\
\hline $\mathrm{U}$ & 2 & 19 & 0.0461 & 29 & 90.50 & 30 & 89.07 & 16 & 2.45 & 16 & 2.41 & 30 & 540.60 \\
\hline $\mathrm{U}$ & 1 & 20 & 0.0500 & 28 & 81.85 & 28 & 80.03 & 17 & 2.46 & 17 & 2.42 & 29 & 440.67 \\
\hline C & 2 & 21 & 0.0647 & 4 & 6.62 & 4 & 6.74 & 12 & 2.13 & 12 & 2.12 & 4 & 46.49 \\
\hline $\mathrm{C}$ & 1 & 22 & 0.0654 & 2 & 3.71 & 2 & 3.74 & 12 & 2.13 & 12 & 2.12 & 3 & 46.49 \\
\hline $\mathrm{H}$ & 1 & 23 & 0.0998 & 15 & 49.13 & 16 & 49.75 & 9 & 1.68 & 9 & 1.67 & 16 & 86.46 \\
\hline $\mathrm{H}$ & 2 & 24 & 0.1008 & 19 & 52.05 & 17 & 51.77 & 9 & 1.68 & 9 & 1.67 & 15 & 86.46 \\
\hline S & 1 & 25 & 0.1089 & 25 & 78.95 & 24 & 71.69 & 22 & 3.97 & 22 & 3.96 & 24 & 150.35 \\
\hline$S$ & 2 & 26 & 0.1133 & 26 & 79.01 & 25 & 71.77 & 22 & 3.97 & 22 & 3.96 & 23 & 150.35 \\
\hline $\mathrm{M}$ & 2 & 27 & 0.1634 & 30 & 90.51 & 29 & 87.21 & 26 & 6.94 & 28 & 6.73 & 7 & 57.53 \\
\hline $\mathrm{F}$ & 2 & 28 & 0.1681 & 13 & 46.18 & 14 & 43.59 & 28 & 6.94 & 26 & 6.73 & 10 & 77.02 \\
\hline $\mathrm{F}$ & 1 & 29 & 0.1684 & 8 & 31.92 & 8 & 30.09 & 28 & 6.94 & 26 & 6.73 & 11 & 77.02 \\
\hline $\mathrm{M}$ & 1 & 30 & 0.1736 & 9 & 32.39 & 9 & 31.17 & 26 & 6.94 & 28 & 6.73 & 6 & 57.53 \\
\hline
\end{tabular}

Table 84: Tabulation of ranked results for Class B - Left Slap. Letter refers to the participant's letter code found on the footer of this page. Sub. \# is an identifier used to differentiate between the two submissions each participant could make. The FNIR column was computed at the score threshold that gave FPIR $=10^{-3}$. The Identification column shows the time used to perform a search over an enrollment set of 3000000 , as seen in Table 19. The Search Enrollment column shows the time used to create a search template to be used for a query, as seen in Table 75. Identification and Search Enrollment durations are reported in seconds, but were originally recorded to microsecond precision. $R A M$ refers to the sum of the resident set sizes of the stage one identification processes over all compute nodes after returning from the identification stage one initialization method, as seen in Table 63 . RAM is reported in gigabytes, where 1 GB is equal to 1073741824 bytes. The number to the left of a value provides the value's column-wise ranking, with the best performance shaded in green and the worst in pink. The table is sorted on the FNIR column-wise ranking. 


\begin{tabular}{|c|c|c|c|c|c|c|c|c|c|c|c|c|c|}
\hline \multicolumn{2}{|c|}{ Participant } & \multirow{2}{*}{\multicolumn{2}{|c|}{ FNIR }} & \multicolumn{4}{|c|}{ Identification } & \multicolumn{4}{|c|}{ Search Enrollment } & \multirow{2}{*}{\multicolumn{2}{|c|}{ RAM }} \\
\hline Letter & Sub.\# & & & \multicolumn{2}{|c|}{ Mean } & \multicolumn{2}{|c|}{ Median } & \multicolumn{2}{|c|}{ Mean } & \multicolumn{2}{|c|}{ Median } & & \\
\hline I & 2 & 1 & 0.0045 & 16 & 49.18 & 14 & 41.13 & 30 & 7.65 & 30 & 7.65 & 21 & 108.71 \\
\hline $\mathrm{D}$ & 2 & 2 & 0.0052 & 20 & 58.67 & 21 & 57.19 & 24 & 4.15 & 24 & 4.13 & 12 & 79.43 \\
\hline Q & 1 & 3 & 0.0057 & 22 & 62.95 & 23 & 64.04 & 20 & 3.41 & 20 & 3.41 & 1 & 7.54 \\
\hline $\mathrm{Q}$ & 2 & 3 & 0.0057 & 21 & 61.29 & 22 & 61.94 & 20 & 3.41 & 20 & 3.41 & 2 & 7.54 \\
\hline $\mathrm{I}$ & 1 & 5 & 0.0058 & 23 & 63.57 & 20 & 55.87 & 25 & 6.47 & 25 & 6.47 & 5 & 49.68 \\
\hline $\mathrm{D}$ & 1 & 6 & 0.0072 & 19 & 53.78 & 19 & 53.32 & 14 & 2.15 & 14 & 2.14 & 13 & 79.43 \\
\hline $\mathrm{E}$ & 2 & 7 & 0.0083 & 12 & 40.27 & 10 & 34.47 & 15 & 2.18 & 15 & 2.18 & 28 & 318.00 \\
\hline V & 1 & 8 & 0.0106 & 10 & 35.66 & 11 & 35.43 & 3 & 1.19 & 3 & 1.19 & 8 & 63.53 \\
\hline $\mathrm{V}$ & 2 & 9 & 0.0110 & 15 & 48.47 & 16 & 48.51 & 3 & 1.19 & 3 & 1.19 & 9 & 63.53 \\
\hline $\mathrm{J}$ & 2 & 10 & 0.0126 & 27 & 77.67 & 27 & 74.09 & 5 & 1.21 & 5 & 1.20 & 19 & 101.00 \\
\hline $\mathrm{O}$ & 2 & 11 & 0.0132 & 26 & 77.37 & 26 & 73.93 & 7 & 1.21 & 7 & 1.20 & 20 & 101.00 \\
\hline $\mathrm{O}$ & 1 & 12 & 0.0142 & 11 & 38.08 & 12 & 35.61 & 7 & 1.21 & 7 & 1.20 & 17 & 101.00 \\
\hline $\mathrm{E}$ & 1 & 13 & 0.0151 & 2 & 4.68 & 1 & 2.97 & 11 & 1.92 & 11 & 1.91 & 14 & 79.72 \\
\hline $\mathrm{J}$ & 1 & 14 & 0.0156 & 7 & 25.86 & 7 & 24.14 & 5 & 1.21 & 5 & 1.20 & 18 & 101.00 \\
\hline $\mathrm{L}$ & 1 & 15 & 0.0167 & 3 & 5.49 & 3 & 5.47 & 2 & 1.12 & 2 & 1.11 & 22 & 119.78 \\
\hline G & 2 & 16 & 0.0198 & 13 & 41.12 & 13 & 37.28 & 18 & 2.76 & 18 & 2.78 & 25 & 156.12 \\
\hline $\mathrm{L}$ & 2 & 17 & 0.0202 & 6 & 12.66 & 6 & 12.66 & 1 & 0.33 & 1 & 0.33 & 27 & 177.49 \\
\hline G & 1 & 18 & 0.0212 & 5 & 11.80 & 5 & 10.13 & 18 & 2.76 & 18 & 2.78 & 26 & 156.12 \\
\hline $\mathrm{U}$ & 1 & 19 & 0.0266 & 29 & 89.71 & 30 & 89.26 & 16 & 2.43 & 16 & 2.39 & 29 & 440.67 \\
\hline $\mathrm{U}$ & 2 & 20 & 0.0273 & 28 & 88.88 & 28 & 80.11 & 17 & 2.43 & 17 & 2.39 & 30 & 540.59 \\
\hline$S$ & 1 & 21 & 0.0369 & 25 & 74.29 & 24 & 70.54 & 22 & 3.89 & 22 & 3.88 & 23 & 150.35 \\
\hline $\mathrm{S}$ & 2 & 22 & 0.0381 & 24 & 73.96 & 25 & 70.84 & 22 & 3.89 & 22 & 3.88 & 24 & 150.35 \\
\hline $\mathrm{C}$ & 2 & 23 & 0.0392 & 4 & 6.54 & 4 & 6.50 & 12 & 2.10 & 12 & 2.09 & 3 & 46.49 \\
\hline $\mathrm{C}$ & 1 & 24 & 0.0403 & 1 & 3.70 & 2 & 3.67 & 12 & 2.10 & 12 & 2.09 & 4 & 46.49 \\
\hline $\mathrm{H}$ & 1 & 25 & 0.0641 & 17 & 50.97 & 17 & 50.16 & 9 & 1.64 & 9 & 1.63 & 16 & 86.46 \\
\hline $\mathrm{H}$ & 2 & 26 & 0.0647 & 18 & 52.86 & 18 & 52.84 & 9 & 1.64 & 9 & 1.63 & 15 & 86.46 \\
\hline $\mathrm{M}$ & 2 & 27 & 0.1155 & 30 & 91.42 & 29 & 87.41 & 28 & 6.88 & 28 & 6.69 & 6 & 57.53 \\
\hline $\mathrm{F}$ & 2 & 28 & 0.1220 & 14 & 46.58 & 15 & 45.92 & 26 & 6.86 & 26 & 6.68 & 10 & 77.02 \\
\hline $\mathrm{F}$ & 1 & 29 & 0.1222 & 8 & 32.28 & 8 & 31.87 & 26 & 6.86 & 26 & 6.68 & 11 & 77.02 \\
\hline $\mathrm{M}$ & 1 & 30 & 0.1259 & 9 & 33.43 & 9 & 32.03 & 28 & 6.88 & 28 & 6.69 & 7 & 57.53 \\
\hline
\end{tabular}

Table 85: Tabulation of ranked results for Class B - Right Slap. Letter refers to the participant's letter code found on the footer of this page. Sub. \# is an identifier used to differentiate between the two submissions each participant could make. The FNIR column was computed at the score threshold that gave FPIR $=10^{-3}$. The Identification column shows the time used to perform a search over an enrollment set of 3000000 , as seen in Table 19. The Search Enrollment column shows the time used to create a search template to be used for a query, as seen in Table 76. Identification and Search Enrollment durations are reported in seconds, but were originally recorded to microsecond precision. $R A M$ refers to the sum of the resident set sizes of the stage one identification processes over all compute nodes after returning from the identification stage one initialization method, as seen in Table 63 . $R A M$ is reported in gigabytes, where 1 GB is equal to 1073741824 bytes. The number to the left of a value provides the value's column-wise ranking, with the best performance shaded in green and the worst in pink. The table is sorted on the FNIR column-wise ranking. 


\begin{tabular}{|c|c|c|c|c|c|c|c|c|c|c|c|c|c|}
\hline \multicolumn{2}{|c|}{ Participant } & \multirow{2}{*}{\multicolumn{2}{|c|}{ FNIR }} & \multicolumn{4}{|c|}{ Identification } & \multicolumn{4}{|c|}{ Search Enrollment } & \multirow{2}{*}{\multicolumn{2}{|c|}{ RAM }} \\
\hline Letter & Sub.\# & & & \multicolumn{2}{|c|}{ Mean } & \multicolumn{2}{|c|}{ Median } & \multicolumn{2}{|c|}{ Mean } & \multicolumn{2}{|c|}{ Median } & & \\
\hline I & 2 & 1 & 0.0015 & 19 & 57.94 & 17 & 46.13 & 30 & 15.30 & 30 & 15.33 & 21 & 108.71 \\
\hline$Q$ & 1 & 2 & 0.0021 & 20 & 63.99 & 22 & 65.02 & 20 & 6.84 & 20 & 6.84 & 2 & 7.54 \\
\hline $\mathrm{Q}$ & 2 & 3 & 0.0022 & 18 & 53.88 & 19 & 53.40 & 20 & 6.84 & 20 & 6.84 & 1 & 7.54 \\
\hline I & 1 & 3 & 0.0022 & 15 & 47.35 & 13 & 37.19 & 25 & 12.94 & 25 & 12.96 & 5 & 49.68 \\
\hline $\mathrm{D}$ & 2 & 5 & 0.0024 & 24 & 74.21 & 21 & 63.05 & 24 & 8.31 & 24 & 8.28 & 13 & 79.43 \\
\hline $\mathrm{D}$ & 1 & 6 & 0.0031 & 21 & 69.35 & 20 & 61.24 & 14 & 4.29 & 14 & 4.28 & 12 & 79.43 \\
\hline $\mathrm{V}$ & 1 & 7 & 0.0036 & 11 & 39.24 & 15 & 38.93 & 3 & 2.40 & 7 & 2.40 & 9 & 63.53 \\
\hline $\mathrm{V}$ & 2 & 7 & 0.0036 & 17 & 52.97 & 18 & 52.80 & 3 & 2.40 & 7 & 2.40 & 8 & 63.53 \\
\hline $\mathrm{J}$ & 2 & 9 & 0.0047 & 26 & 75.92 & 24 & 69.28 & 7 & 2.42 & 5 & 2.40 & 17 & 101.00 \\
\hline$E$ & 2 & 10 & 0.0049 & 12 & 39.56 & 10 & 33.09 & 15 & 4.39 & 15 & 4.38 & 28 & 317.99 \\
\hline $\mathrm{O}$ & 2 & 11 & 0.0051 & 25 & 74.57 & 23 & 68.46 & 5 & 2.42 & 3 & 2.40 & 19 & 101.00 \\
\hline $\mathrm{L}$ & 1 & 12 & 0.0054 & 4 & 10.37 & 4 & 10.48 & 2 & 2.24 & 2 & 2.23 & 22 & 119.77 \\
\hline $\mathrm{O}$ & 1 & 13 & 0.0057 & 13 & 40.42 & 14 & 38.34 & 5 & 2.42 & 3 & 2.40 & 20 & 101.00 \\
\hline $\mathrm{L}$ & 2 & 14 & 0.0062 & 6 & 20.85 & 6 & 20.78 & 1 & 0.66 & 1 & 0.66 & 27 & 177.49 \\
\hline $\mathrm{E}$ & 1 & 15 & 0.0063 & 3 & 8.18 & 2 & 6.12 & 11 & 3.86 & 11 & 3.85 & 14 & 79.72 \\
\hline $\mathrm{J}$ & 1 & 16 & 0.0068 & 7 & 27.52 & 7 & 26.15 & 7 & 2.42 & 5 & 2.40 & 18 & 101.00 \\
\hline G & 2 & 17 & 0.0084 & 14 & 42.86 & 11 & 34.55 & 18 & 5.59 & 18 & 5.62 & 25 & 156.12 \\
\hline $\mathrm{G}$ & 1 & 18 & 0.0106 & 1 & 5.52 & 1 & 3.89 & 18 & 5.59 & 18 & 5.62 & 26 & 156.12 \\
\hline $\mathrm{U}$ & 2 & 19 & 0.0124 & 30 & 91.04 & 28 & 82.40 & 16 & 4.89 & 17 & 4.83 & 30 & 540.59 \\
\hline $\mathrm{U}$ & 1 & 20 & 0.0139 & 29 & 86.88 & 30 & 83.62 & 17 & 4.91 & 16 & 4.82 & 29 & 440.67 \\
\hline$S$ & 1 & 21 & 0.0160 & 28 & 85.05 & 29 & 83.33 & 22 & 7.82 & 22 & 7.83 & 23 & 150.35 \\
\hline$S$ & 2 & 22 & 0.0190 & 27 & 84.02 & 27 & 82.13 & 22 & 7.82 & 22 & 7.83 & 24 & 150.35 \\
\hline $\mathrm{H}$ & 1 & 23 & 0.0349 & 22 & 70.12 & 25 & 70.38 & 9 & 3.31 & 9 & 3.31 & 16 & 86.46 \\
\hline $\mathrm{H}$ & 2 & 24 & 0.0361 & 23 & 72.35 & 26 & 73.06 & 9 & 3.31 & 9 & 3.31 & 15 & 86.46 \\
\hline $\mathrm{M}$ & 2 & 25 & 0.0882 & 16 & 49.50 & 16 & 45.91 & 28 & 13.79 & 28 & 13.53 & 6 & 57.53 \\
\hline $\mathrm{F}$ & 2 & 26 & 0.0901 & 10 & 38.37 & 12 & 36.36 & 26 & 13.78 & 26 & 13.49 & 11 & 77.02 \\
\hline M & 1 & 27 & 0.0904 & 9 & 28.89 & 8 & 27.15 & 28 & 13.79 & 28 & 13.53 & 7 & 57.53 \\
\hline $\mathrm{F}$ & 1 & 28 & 0.0910 & 8 & 28.72 & 9 & 27.27 & 26 & 13.78 & 26 & 13.49 & 10 & 77.02 \\
\hline$C$ & 2 & 29 & NA & 5 & 10.63 & 5 & 10.70 & 12 & 4.22 & 12 & 4.20 & 4 & 46.49 \\
\hline C & 1 & 30 & NA & 2 & 7.36 & 3 & 6.40 & 12 & 4.22 & 12 & 4.20 & 3 & 46.49 \\
\hline
\end{tabular}

Table 86: Tabulation of ranked results for Class B - Left and Right Slap. Letter refers to the participant's letter code found on the footer of this page. Sub. \# is an identifier used to differentiate between the two submissions each participant could make. The FNIR column was computed at the score threshold that gave FPIR $=10^{-3}$. NA indicates that the operations required to produce the value could not be performed. The Identification column shows the time used to perform a search over an enrollment set of 3000000 , as seen in Table 19. The Search Enrollment column shows the time used to create a search template to be used for a query, as seen in Table 77. Identification and Search Enrollment durations are reported in seconds, but were originally recorded to microsecond precision. RAM refers to the sum of the resident set sizes of the stage one identification processes over all compute nodes after returning from the identification stage one initialization method, as seen in Table 63. RAM is reported in gigabytes, where 1 GB is equal to 1073741824 bytes. The number to the left of a value provides the value's column-wise ranking, with the best performance shaded in green and the worst in pink. The table is sorted on the FNIR column-wise ranking. 


\begin{tabular}{|c|c|c|c|c|c|c|c|c|c|c|c|c|c|}
\hline \multicolumn{2}{|c|}{ Participant } & \multirow{2}{*}{\multicolumn{2}{|c|}{ FNIR }} & \multicolumn{4}{|c|}{ Identification } & \multicolumn{4}{|c|}{ Search Enrollment } & \multirow{2}{*}{\multicolumn{2}{|c|}{ RAM }} \\
\hline Letter & Sub.\# & & & \multicolumn{2}{|c|}{ Mean } & \multicolumn{2}{|c|}{ Median } & \multicolumn{2}{|c|}{ Mean } & \multicolumn{2}{|c|}{ Median } & & \\
\hline I & 2 & 1 & 0.0009 & 20 & 60.01 & 16 & 43.86 & 30 & 19.36 & 30 & 19.36 & 21 & 108.71 \\
\hline$Q$ & 1 & 2 & 0.0012 & 14 & 48.85 & 14 & 42.92 & 20 & 8.89 & 20 & 8.92 & 1 & 7.54 \\
\hline $\mathrm{Q}$ & 2 & 2 & 0.0012 & 24 & 71.67 & 24 & 66.01 & 20 & 8.89 & 20 & 8.92 & 2 & 7.54 \\
\hline I & 1 & 2 & 0.0012 & 6 & 24.57 & 7 & 19.07 & 25 & 16.50 & 25 & 16.52 & 5 & 49.68 \\
\hline $\mathrm{D}$ & 2 & 2 & 0.0012 & 19 & 54.37 & 18 & 46.70 & 24 & 11.90 & 24 & 11.86 & 13 & 79.43 \\
\hline $\mathrm{D}$ & 1 & 6 & 0.0020 & 17 & 52.42 & 17 & 45.15 & 17 & 6.19 & 17 & 6.16 & 12 & 79.43 \\
\hline $\mathrm{V}$ & 2 & 7 & 0.0024 & 15 & 49.72 & 19 & 49.30 & 3 & 3.35 & 7 & 3.36 & 9 & 63.53 \\
\hline $\mathrm{E}$ & 2 & 7 & 0.0024 & 18 & 52.88 & 15 & 43.61 & 16 & 5.95 & 16 & 5.93 & 28 & 317.95 \\
\hline $\mathrm{V}$ & 1 & 9 & 0.0027 & 10 & 35.51 & 10 & 34.96 & 3 & 3.35 & 7 & 3.36 & 8 & 63.53 \\
\hline $\mathrm{L}$ & 1 & 10 & 0.0031 & 5 & 14.42 & 5 & 14.50 & 2 & 3.05 & 2 & 3.05 & 22 & 119.79 \\
\hline $\mathrm{L}$ & 2 & 11 & 0.0033 & 8 & 28.56 & 9 & 28.59 & 1 & 0.88 & 1 & 0.88 & 27 & 177.48 \\
\hline $\mathrm{J}$ & 2 & 11 & 0.0033 & 22 & 64.38 & 22 & 60.13 & 7 & 3.38 & 3 & 3.35 & 17 & 101.00 \\
\hline $\mathrm{O}$ & 2 & 13 & 0.0035 & 21 & 63.87 & 21 & 60.02 & 5 & 3.38 & 5 & 3.36 & 20 & 101.00 \\
\hline G & 2 & 14 & 0.0040 & 9 & 31.67 & 6 & 16.26 & 18 & 7.89 & 18 & 7.90 & 26 & 156.12 \\
\hline $\mathrm{O}$ & 1 & 15 & 0.0041 & 11 & 37.04 & 11 & 35.64 & 5 & 3.38 & 5 & 3.36 & 19 & 101.00 \\
\hline $\mathrm{E}$ & 1 & 16 & 0.0043 & 2 & 8.76 & 2 & 6.76 & 13 & 5.35 & 13 & 5.34 & 14 & 79.73 \\
\hline $\mathrm{J}$ & 1 & 17 & 0.0049 & 7 & 26.31 & 8 & 25.38 & 7 & 3.38 & 3 & 3.35 & 18 & 101.00 \\
\hline $\mathrm{G}$ & 1 & 18 & 0.0062 & 1 & 6.33 & 1 & 4.26 & 18 & 7.89 & 18 & 7.90 & 25 & 156.12 \\
\hline $\mathrm{U}$ & 1 & 19 & 0.0099 & 30 & 88.83 & 28 & 86.60 & 15 & 5.90 & 15 & 5.82 & 29 & 440.68 \\
\hline$S$ & 1 & 20 & 0.0108 & 28 & 86.50 & 29 & 87.60 & 22 & 10.24 & 22 & 10.31 & 24 & 150.35 \\
\hline$S$ & 2 & 21 & 0.0136 & 29 & 88.70 & 30 & 88.46 & 22 & 10.24 & 22 & 10.31 & 23 & 150.35 \\
\hline $\mathrm{U}$ & 2 & 22 & 0.0141 & 25 & 80.14 & 25 & 75.28 & 14 & 5.80 & 14 & 5.73 & 30 & 540.60 \\
\hline $\mathrm{H}$ & 1 & 23 & 0.0203 & 26 & 82.43 & 26 & 82.66 & 9 & 4.37 & 9 & 4.37 & 15 & 86.46 \\
\hline $\mathrm{H}$ & 2 & 24 & 0.0204 & 27 & 85.74 & 27 & 86.56 & 9 & 4.37 & 9 & 4.37 & 16 & 86.46 \\
\hline $\mathrm{M}$ & 2 & 25 & 0.0515 & 23 & 70.45 & 23 & 65.91 & 26 & 18.36 & 28 & 18.11 & 6 & 57.53 \\
\hline $\mathrm{M}$ & 1 & 26 & 0.0543 & 13 & 41.37 & 13 & 38.87 & 26 & 18.36 & 28 & 18.11 & 6 & 57.53 \\
\hline $\mathrm{F}$ & 1 & 27 & 0.0591 & 12 & 39.12 & 12 & 37.56 & 28 & 18.36 & 26 & 18.07 & 10 & 77.02 \\
\hline $\mathrm{F}$ & 2 & 27 & 0.0591 & 16 & 51.59 & 20 & 49.34 & 28 & 18.36 & 26 & 18.07 & 10 & 77.02 \\
\hline$C$ & 2 & 29 & NA & 4 & 10.28 & 4 & 10.21 & 11 & 5.16 & 11 & 5.13 & 4 & 46.49 \\
\hline C & 1 & 30 & NA & 3 & 9.00 & 3 & 7.92 & 11 & 5.16 & 11 & 5.13 & 3 & 46.49 \\
\hline
\end{tabular}

Table 87: Tabulation of ranked results for Class B - Identification Flats. Letter refers to the participant's letter code found on the footer of this page. Sub. \# is an identifier used to differentiate between the two submissions each participant could make. The FNIR column was computed at the score threshold that gave FPIR $=10^{-3}$. NA indicates that the operations required to produce the value could not be performed. The Identification column shows the time used to perform a search over an enrollment set of 3000000 , as seen in Table 19. The Search Enrollment column shows the time used to create a search template to be used for a query, as seen in Table 78. Identification and Search Enrollment durations are reported in seconds, but were originally recorded to microsecond precision. RAM refers to the sum of the resident set sizes of the stage one identification processes over all compute nodes after returning from the identification stage one initialization method, as seen in Table 63. RAM is reported in gigabytes, where 1 GB is equal to 1073741824 bytes. The number to the left of a value provides the value's column-wise ranking, with the best performance shaded in green and the worst in pink. The table is sorted on the FNIR column-wise ranking.

\footnotetext{
$\mathrm{C}=$ afis team $\mid \mathrm{D}=3 \mathrm{M}$ Cogent $\mid \mathrm{E}=$ Neurotechnology $\mid \mathrm{F}=$ Papillon $\mid \mathrm{G}=$ Dermalog $\mathrm{H}=$ Hisign Bio-Info Institute

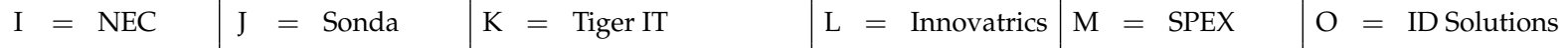

$\mathrm{P}=\mathrm{id} 3 \quad \mathrm{Q}=$ Morpho $\quad \mathrm{S}=$ Decatur Industries $\mathrm{T}=$ BIO-key $\quad \mathrm{U}=$ Aware $\quad \mathrm{V}=$ AA Technology
} 


\begin{tabular}{|c|c|c|c|c|c|c|c|c|c|c|c|c|c|}
\hline \multicolumn{2}{|c|}{ Participant } & \multirow{2}{*}{\multicolumn{2}{|c|}{ FNIR }} & \multicolumn{4}{|c|}{ Identification } & \multicolumn{4}{|c|}{ Search Enrollment } & \multirow{2}{*}{\multicolumn{2}{|c|}{ RAM }} \\
\hline Letter & Sub.\# & & & \multicolumn{2}{|c|}{ Mean } & \multicolumn{2}{|c|}{ Median } & \multicolumn{2}{|c|}{ Mean } & \multicolumn{2}{|c|}{ Median } & & \\
\hline I & 2 & 1 & 0.0010 & 15 & 56.93 & 10 & 38.28 & 30 & 17.04 & 30 & 16.99 & 11 & 110.22 \\
\hline Q & 1 & 2 & 0.0011 & 10 & 44.75 & 9 & 36.86 & 20 & 9.14 & 20 & 9.11 & 2 & 13.33 \\
\hline $\mathrm{D}$ & 2 & 2 & 0.0011 & 25 & 84.92 & 26 & 74.36 & 24 & 13.69 & 24 & 13.66 & 15 & 132.37 \\
\hline$Q$ & 2 & 4 & 0.0013 & 9 & 43.67 & 8 & 35.35 & 20 & 9.14 & 20 & 9.11 & 1 & 13.33 \\
\hline I & 1 & 4 & 0.0013 & 23 & 79.70 & 16 & 52.41 & 29 & 16.71 & 29 & 16.67 & 4 & 84.42 \\
\hline $\mathrm{D}$ & 1 & 6 & 0.0015 & 28 & 87.07 & 25 & 73.93 & 17 & 7.12 & 17 & 7.10 & 16 & 132.37 \\
\hline $\mathrm{V}$ & 1 & 7 & 0.0024 & 13 & 47.76 & 14 & 47.02 & 7 & 3.69 & 7 & 3.66 & 13 & 121.80 \\
\hline $\mathrm{V}$ & 2 & 7 & 0.0024 & 17 & 63.19 & 19 & 62.26 & 7 & 3.69 & 7 & 3.66 & 12 & 121.79 \\
\hline $\mathrm{O}$ & 1 & 9 & 0.0025 & 21 & 73.31 & 23 & 68.54 & 4 & 3.63 & 3 & 3.61 & 19 & 181.00 \\
\hline $\mathrm{O}$ & 2 & 10 & 0.0027 & 24 & 82.13 & 24 & 73.41 & 4 & 3.63 & 3 & 3.61 & 22 & 181.00 \\
\hline $\mathrm{J}$ & 2 & 10 & 0.0027 & 26 & 85.17 & 28 & 77.75 & 6 & 3.64 & 6 & 3.62 & 21 & 181.00 \\
\hline $\mathrm{J}$ & 1 & 12 & 0.0047 & 12 & 46.32 & 12 & 43.57 & 3 & 3.62 & 5 & 3.62 & 20 & 181.00 \\
\hline$E$ & 2 & 13 & 0.0048 & 27 & 85.50 & 17 & 52.91 & 12 & 5.21 & 12 & 5.20 & 28 & 573.25 \\
\hline $\mathrm{E}$ & 1 & 14 & 0.0088 & 2 & 15.94 & 2 & 12.77 & 11 & 5.20 & 11 & 5.20 & 14 & 127.39 \\
\hline $\mathrm{L}$ & 2 & 15 & 0.0095 & 6 & 21.26 & 6 & 23.53 & 1 & 3.51 & 1 & 3.48 & 27 & 274.95 \\
\hline $\mathrm{L}$ & 1 & 16 & 0.0102 & 3 & 17.53 & 3 & 17.51 & 1 & 3.51 & 1 & 3.48 & 23 & 192.19 \\
\hline $\mathrm{U}$ & 2 & 17 & 0.0155 & 20 & 67.98 & 22 & 68.30 & 15 & 6.13 & 15 & 5.91 & 29 & 811.45 \\
\hline $\mathrm{U}$ & 1 & 18 & 0.0163 & 22 & 75.14 & 27 & 74.94 & 15 & 6.13 & 15 & 5.91 & 29 & 811.45 \\
\hline $\mathrm{H}$ & 2 & 19 & 0.0275 & 18 & 64.90 & 20 & 65.36 & 9 & 5.16 & 9 & 5.16 & 18 & 144.02 \\
\hline G & 2 & 20 & 0.0276 & 7 & 37.15 & 7 & 23.69 & 18 & 8.35 & 18 & 8.32 & 26 & 274.35 \\
\hline $\mathrm{H}$ & 1 & 20 & 0.0276 & 19 & 67.91 & 21 & 68.07 & 9 & 5.16 & 9 & 5.16 & 17 & 144.02 \\
\hline$S$ & 1 & 22 & 0.0311 & 29 & 87.76 & 29 & 86.56 & 22 & 10.91 & 22 & 10.87 & 24 & 260.37 \\
\hline$G$ & 1 & 23 & 0.0368 & 1 & 11.21 & 1 & 7.71 & 18 & 8.35 & 18 & 8.32 & 25 & 274.35 \\
\hline C & 2 & 24 & 0.0711 & 4 & 18.31 & 5 & 18.38 & 13 & 5.84 & 13 & 5.80 & 10 & 92.86 \\
\hline $\mathrm{F}$ & 1 & 25 & 0.0734 & 14 & 50.99 & 15 & 51.69 & 27 & 15.96 & 27 & 15.87 & 7 & 90.93 \\
\hline $\mathrm{F}$ & 2 & 25 & 0.0734 & 16 & 60.34 & 18 & 60.86 & 27 & 15.96 & 27 & 15.87 & 6 & 90.93 \\
\hline $\mathrm{M}$ & 2 & 27 & 0.0826 & 11 & 45.96 & 13 & 46.70 & 25 & 15.91 & 25 & 15.83 & 8 & 90.93 \\
\hline $\mathrm{M}$ & 1 & 28 & 0.0934 & 8 & 42.60 & 11 & 43.37 & 25 & 15.91 & 25 & 15.83 & 5 & 90.93 \\
\hline$S$ & 2 & 29 & 0.1680 & 30 & 89.24 & 30 & 91.74 & 22 & 10.91 & 22 & 10.87 & 3 & 61.04 \\
\hline $\mathrm{C}$ & 1 & 30 & NA & 5 & 21.04 & 4 & 18.30 & 13 & 5.84 & 13 & 5.80 & 9 & 92.86 \\
\hline
\end{tabular}

Table 88: Tabulation of ranked results for Class C - Ten-Finger Plain-to-Plain. Letter refers to the participant's letter code found on the footer of this page. Sub. \# is an identifier used to differentiate between the two submissions each participant could make. The FNIR column was computed at the score threshold that gave FPIR $=10^{-3}$. NA indicates that the operations required to produce the value could not be performed. The Identification column shows the time used to perform a search over an enrollment set of 5000000 , as seen in Table 20. The Search Enrollment column shows the time used to create a search template to be used for a query, as seen in Table 79. Identification and Search Enrollment durations are reported in seconds, but were originally recorded to microsecond precision. RAM refers to the sum of the resident set sizes of the stage one identification processes over all compute nodes after returning from the identification stage one initialization method, as seen in Table 64 . RAM is reported in gigabytes, where 1 GB is equal to 1073741824 bytes. The number to the left of a value provides the value's column-wise ranking, with the best performance shaded in green and the worst in pink. The table is sorted on the FNIR column-wise ranking.

\footnotetext{
$\mathrm{C}=$ afis team $\mid \mathrm{D}=3 \mathrm{M}$ Cogent $\mid \mathrm{E}=$ Neurotechnology $\mid \mathrm{F}=$ Papillon $\mid \mathrm{G}=$ Dermalog $\mid \mathrm{H}=$ Hisign Bio-Info Institute

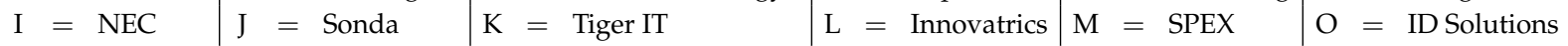

$\mathrm{P}=\mathrm{id} 3 \quad \mathrm{Q}=$ Morpho $\quad \mathrm{S}=$ Decatur Industries $\mathrm{T}=$ BIO-key $\quad \mathrm{U}=$ Aware $\quad \mathrm{V}=$ AA Technology
} 


\begin{tabular}{|c|c|c|c|c|c|c|c|c|c|c|c|c|c|}
\hline \multicolumn{2}{|c|}{ Participant } & \multirow{2}{*}{\multicolumn{2}{|c|}{ FNIR }} & \multicolumn{4}{|c|}{ Identification } & \multicolumn{4}{|c|}{ Search Enrollment } & \multirow{2}{*}{\multicolumn{2}{|c|}{ RAM }} \\
\hline Letter & Sub.\# & & & \multicolumn{2}{|c|}{ Mean } & \multicolumn{2}{|c|}{ Median } & \multicolumn{2}{|c|}{ Mean } & \multicolumn{2}{|c|}{ Median } & & \\
\hline I & 1 & 1 & 0.0013 & 24 & 79.04 & 15 & 54.56 & 25 & 20.21 & 25 & 20.23 & 4 & 113.80 \\
\hline$Q$ & 2 & 2 & 0.0014 & 27 & 83.35 & 25 & 74.22 & 17 & 12.95 & 17 & 13.06 & 2 & 20.22 \\
\hline I & 2 & 2 & 0.0014 & 9 & 40.53 & 7 & 30.82 & 24 & 18.72 & 24 & 18.73 & 11 & 137.03 \\
\hline $\mathrm{D}$ & 1 & 4 & 0.0015 & 17 & 65.97 & 17 & 58.92 & 23 & 17.44 & 21 & 17.39 & 10 & 132.40 \\
\hline Q & 1 & 5 & 0.0017 & 26 & 83.25 & 26 & 74.40 & 17 & 12.95 & 17 & 13.06 & 1 & 20.22 \\
\hline $\mathrm{V}$ & 1 & 5 & 0.0017 & 10 & 40.94 & 10 & 40.74 & 9 & 8.48 & 9 & 8.50 & 17 & 234.03 \\
\hline $\mathrm{D}$ & 2 & 7 & 0.0018 & 30 & 86.39 & 27 & 74.97 & 30 & 30.43 & 30 & 30.47 & 9 & 132.37 \\
\hline V & 2 & 8 & 0.0019 & 16 & 65.47 & 18 & 65.07 & 9 & 8.48 & 9 & 8.50 & 18 & 234.03 \\
\hline $\mathrm{O}$ & 2 & 9 & 0.0033 & 19 & 72.55 & 21 & 69.30 & 6 & 6.80 & 6 & 6.71 & 23 & 303.13 \\
\hline $\mathrm{J}$ & 2 & 9 & 0.0033 & 20 & 72.62 & 19 & 67.98 & 8 & 6.82 & 8 & 6.74 & 24 & 303.13 \\
\hline $\mathrm{O}$ & 1 & 11 & 0.0034 & 15 & 59.01 & 14 & 54.42 & 6 & 6.80 & 6 & 6.71 & 25 & 303.13 \\
\hline $\mathrm{E}$ & 2 & 12 & 0.0050 & 14 & 57.02 & 11 & 42.73 & 11 & 9.89 & 11 & 9.91 & 30 & 930.48 \\
\hline $\mathrm{J}$ & 1 & 13 & 0.0051 & 8 & 36.09 & 9 & 33.02 & 5 & 6.78 & 5 & 6.69 & 22 & 303.13 \\
\hline $\mathrm{L}$ & 2 & 14 & 0.0083 & 3 & 19.52 & 5 & 20.25 & 3 & 4.36 & 3 & 4.36 & 26 & 367.82 \\
\hline $\mathrm{C}$ & 2 & 15 & 0.0085 & 5 & 25.91 & 6 & 21.97 & 13 & 10.79 & 13 & 10.69 & 15 & 183.36 \\
\hline C & 1 & 16 & 0.0094 & 4 & 25.25 & 4 & 20.15 & 13 & 10.79 & 13 & 10.69 & 14 & 183.36 \\
\hline $\mathrm{L}$ & 1 & 17 & 0.0097 & 7 & 31.42 & 8 & 31.68 & 3 & 4.36 & 3 & 4.36 & 21 & 280.49 \\
\hline $\mathrm{E}$ & 1 & 18 & 0.0106 & 1 & 9.52 & 2 & 8.63 & 12 & 9.90 & 12 & 9.92 & 16 & 191.66 \\
\hline $\mathrm{H}$ & 2 & 19 & 0.0199 & 29 & 84.26 & 29 & 84.50 & 15 & 12.08 & 15 & 12.17 & 12 & 144.02 \\
\hline $\mathrm{H}$ & 1 & 20 & 0.0201 & 28 & 84.14 & 30 & 84.51 & 15 & 12.08 & 15 & 12.17 & 13 & 144.02 \\
\hline G & 2 & 21 & 0.0333 & 6 & 30.61 & 3 & 19.46 & 19 & 15.55 & 19 & 15.50 & 19 & 261.29 \\
\hline $\mathrm{U}$ & 2 & 22 & 0.0351 & 23 & 74.39 & 24 & 72.48 & 1 & 2.94 & 1 & 2.87 & 28 & 806.17 \\
\hline $\mathrm{U}$ & 1 & 23 & 0.0358 & 25 & 82.76 & 28 & 82.61 & 1 & 2.94 & 1 & 2.87 & 28 & 806.17 \\
\hline$G$ & 1 & 24 & 0.0447 & 2 & 11.67 & 1 & 7.78 & 19 & 15.55 & 19 & 15.50 & 20 & 261.29 \\
\hline $\mathrm{F}$ & 1 & 25 & 0.0536 & 13 & 55.63 & 16 & 55.79 & 28 & 21.07 & 28 & 20.92 & 7 & 130.29 \\
\hline $\mathrm{F}$ & 2 & 25 & 0.0536 & 18 & 68.61 & 20 & 68.20 & 28 & 21.07 & 28 & 20.92 & 6 & 130.29 \\
\hline$M$ & 2 & 27 & 0.0716 & 12 & 48.71 & 13 & 48.80 & 26 & 21.02 & 26 & 20.89 & 5 & 130.29 \\
\hline $\mathrm{M}$ & 1 & 28 & 0.0783 & 11 & 47.38 & 12 & 48.08 & 26 & 21.02 & 26 & 20.89 & 8 & 130.29 \\
\hline $\mathrm{S}$ & 1 & 29 & 0.0860 & 22 & 74.01 & 22 & 69.71 & 21 & 17.43 & 22 & 17.57 & 27 & 382.88 \\
\hline$S$ & 2 & 30 & 0.2462 & 21 & 72.95 & 23 & 70.77 & 21 & 17.43 & 22 & 17.57 & 3 & 78.28 \\
\hline
\end{tabular}

Table 89: Tabulation of ranked results for Class C - Ten-Finger Rolled-to-Rolled. Letter refers to the participant's letter code found on the footer of this page. Sub. \# is an identifier used to differentiate between the two submissions each participant could make. The FNIR column was computed at the score threshold that gave FPIR $=10^{-3}$. The Identification column shows the time used to perform a search over an enrollment set of 5000000 , as seen in Table 20. The Search Enrollment column shows the time used to create a search template to be used for a query, as seen in Table 80. Identification and Search Enrollment durations are reported in seconds, but were originally recorded to microsecond precision. RAM refers to the sum of the resident set sizes of the stage one identification processes over all compute nodes after returning from the identification stage one initialization method, as seen in Table 65. RAM is reported in gigabytes, where $1 \mathrm{~GB}$ is equal to 1073741824 bytes. The number to the left of a value provides the value's column-wise ranking, with the best performance shaded in green and the worst in pink. The table is sorted on the FNIR column-wise ranking.

\begin{tabular}{l|l|l|l|ll|l}
$\mathrm{C}=$ afis team & $\mathrm{D}=3 \mathrm{M}$ Cogent & $\mathrm{E}=$ Neurotechnology & $\mathrm{F}=$ Papillon & $\mathrm{G}=$ Dermalog & $\mathrm{H}=$ Hisign Bio-Info Institute \\
$\mathrm{I}=$ NEC & $\mathrm{J}=$ Sonda & $\mathrm{K}=$ Tiger IT & $\mathrm{L}=$ Innovatrics & $\mathrm{M}=$ SPEX & $\mathrm{O}=$ ID Solutions \\
$\mathrm{P}=\mathrm{id} 3$ & $\mathrm{Q}=$ Morpho & $\mathrm{S}=$ Decatur Industries & $\mathrm{T}=$ BIO-key & $\mathrm{U}=$ Aware & $\mathrm{V}=$ AA Technology
\end{tabular}




\section{J Relative Combined Results}

The tables in Appendix I show the detailed values for FNIR at a fixed FPIR of $10^{-3}$, identification time, search template enrollment time, and RAM consumption. While the column-wise ranking for each variable is given, it can be difficult to visualize relative comparisons among submissions for each of these values. Use of star plots can help with this visualization. For more information on how to read star plots, please refer to the explanation in Appendix K.

Class A plots are in Figures 99 through 101, Class B plots are in Figures 102 through 105, and Class C plots are in Figures 106 through 107.

Some notable observations based on the plotted shape include that:

$\triangleright$ For almost all finger combinations, I uses the longest enrollment times while providing the highest accuracy.

$\triangleright$ D's submission strike a balance between identification time and enrollment time in order to provide high accuracy.

$\triangleright$ Class A results are nearly identical, regardless of which index finger combinations are used.

$\triangleright \mathbf{J 1}, \mathbf{L 1}, \mathbf{Q}, \mathbf{0 1}$, and V1 provide high accuracy while limiting time and computational resources for most finger combinations.

$\triangleright$ Shapes formed by participants appear to remain similar regardless of finger combination.

$\triangleright$ Tradeoffs between a participant's two submissions are very easily seen. For instance, in class Class A, V1 and S1 use significantly shorter identification times than $\mathbf{V} 2$ and $\mathbf{S} 2$ respectively, but result in similar relative accuracies. 

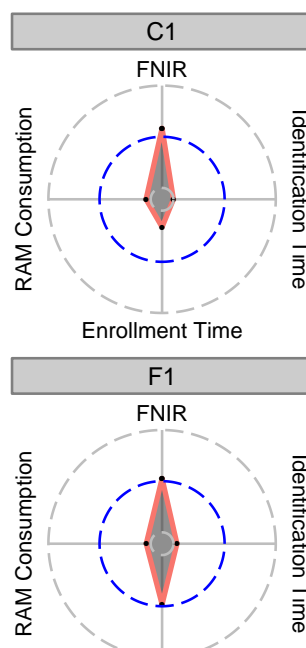

Enrollment Time

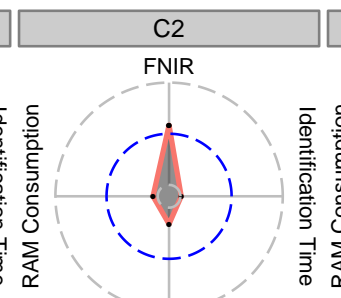

Enrollment Time

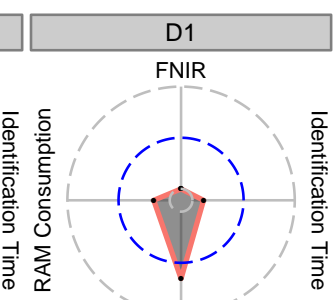

Enrollment Time

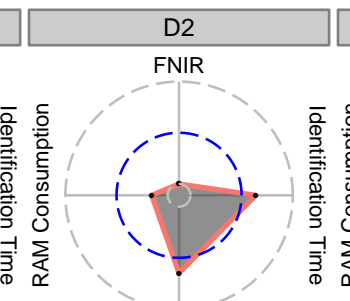

Enrollment Time

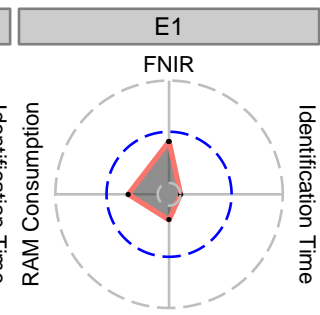

Enrollment Time

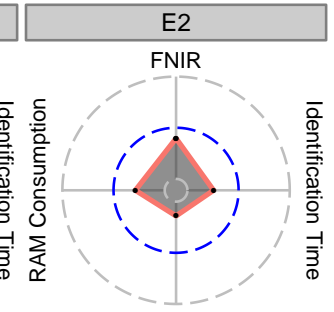

Enrollment Time
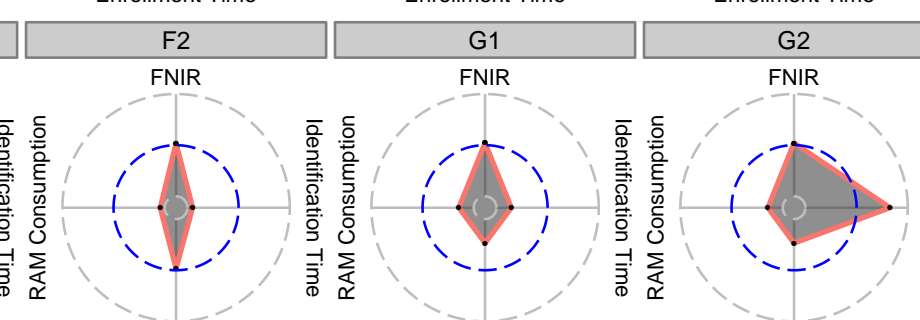

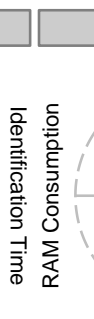

$\mathrm{H} 1$

\begin{tabular}{|c|}
\hline $\mathrm{H} 2$ \\
\hline FNIR
\end{tabular}

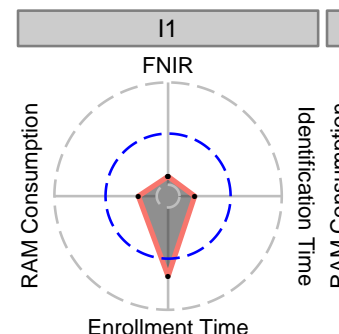

Enrollment Time

Enrollment Time

Enrollment Time

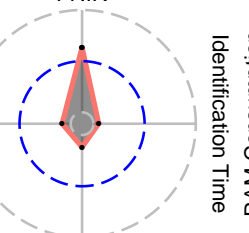

Enrollment Time
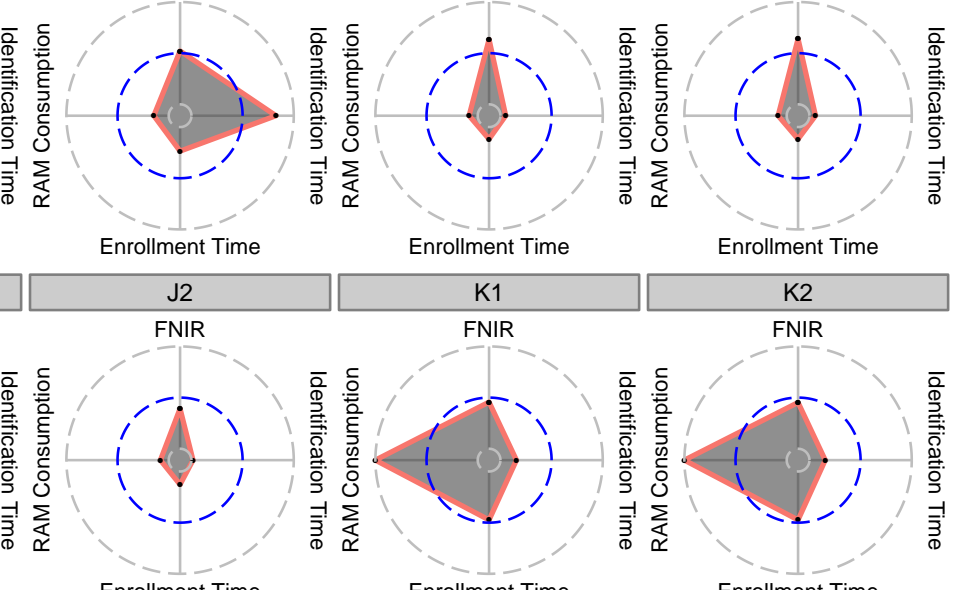

Enrollment Time

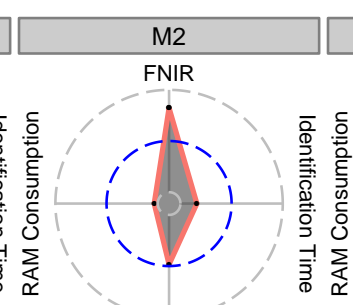

Enrollment Time

Enrollment Time
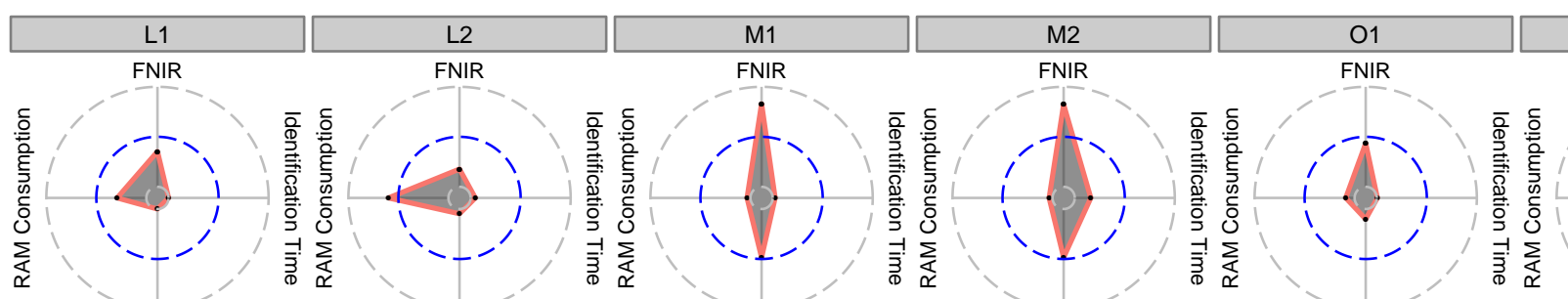

$\mathrm{O} 2$

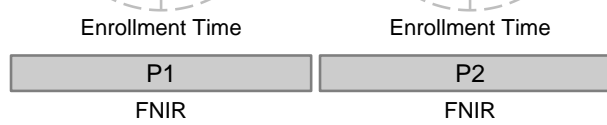

Q1
FNIR

\begin{tabular}{c} 
Enrollment Time \\
\hline Q2 \\
FNIR
\end{tabular}

\begin{tabular}{|c|} 
Enrollment Time \\
\hline S1 \\
FNIR
\end{tabular}
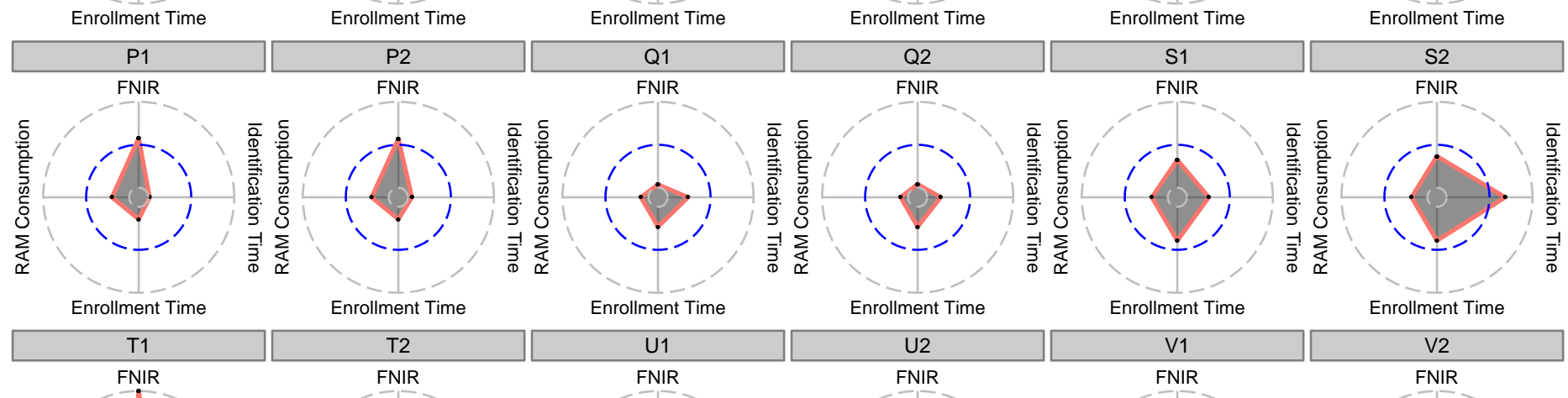

$\begin{array}{r}\text { U2 } \\ \text { FNIR } \\ \hline \text { FII }\end{array}$
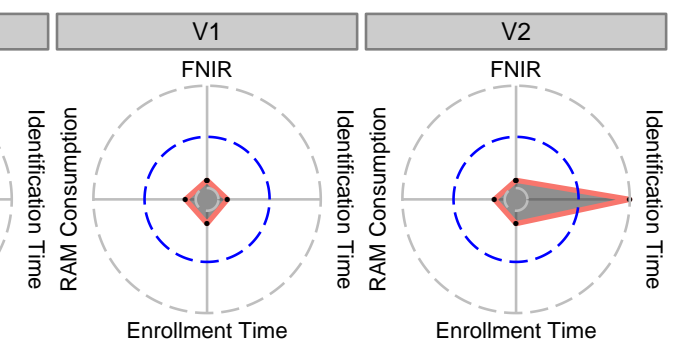

Enrollment Time
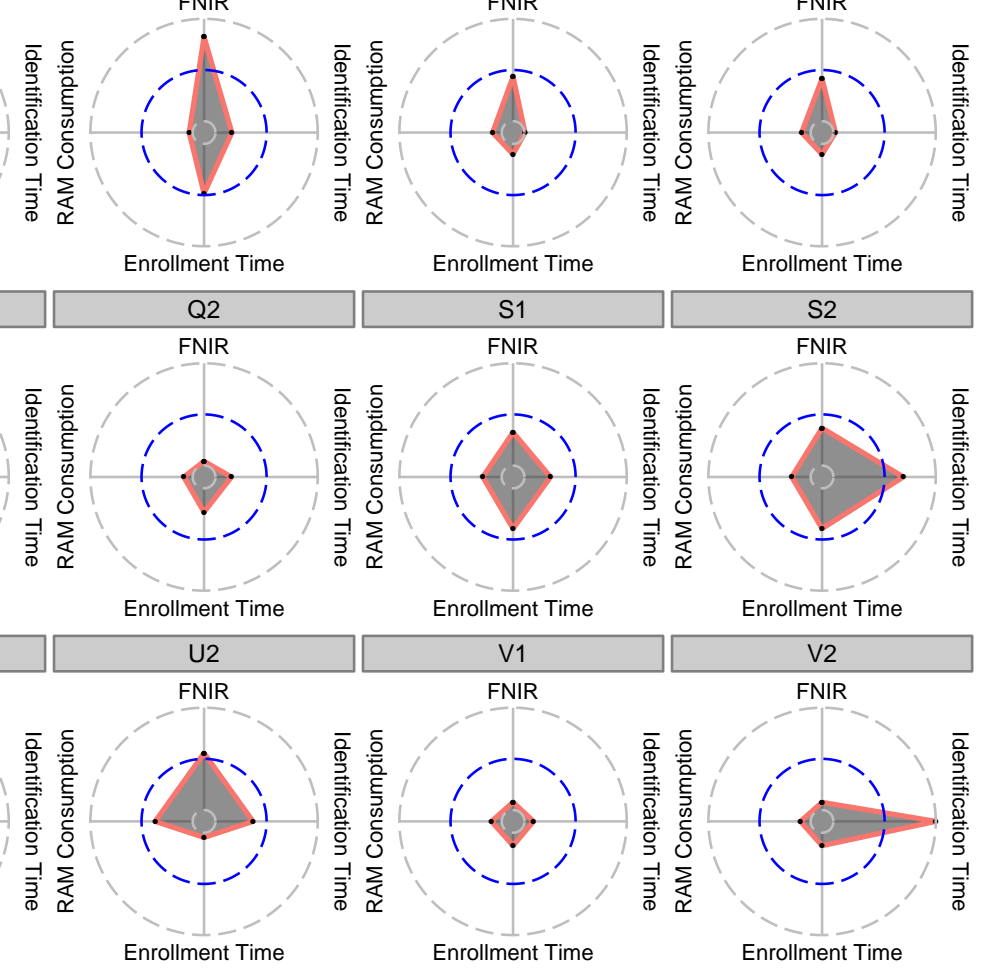

Enrollment Time

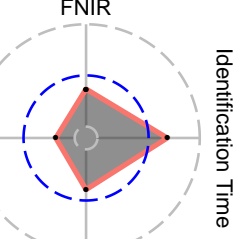

Enrollment Time

Figure 99: Star plot of combined results for Class A — Left Index. The values in this plot have been independently scaled from 0 to 1 from the values printed in Table 81, with the exception of FNIR, whose $\log _{10}$ values were scaled. Any values printed as NA were set to 1 before scaling. The intersection point of a radius and the circumference of a circle indicate the scaled values $0,0.5$, and 1 , with 1 resting on the outer dashed-gray circle. The title above each plot represents the participant's letter code found on the footer of this page and an identifier used to differentiate between the two submissions each participant could make.

\begin{tabular}{l|l|l|l|l|l}
$\mathrm{C}=$ afis team & $\mathrm{D}=3 \mathrm{M}$ Cogent & $\mathrm{E}=$ Neurotechnology & $\mathrm{F}=$ Papillon & $\mathrm{G}=$ Dermalog & $\mathrm{H}=$ Hisign Bio-Info Institute \\
$\mathrm{I}=\mathrm{NEC}$ & $\mathrm{J}=$ Sonda & $\mathrm{K}=$ Tiger IT & $\mathrm{L}=$ Innovatrics & $\mathrm{M}=$ SPEX & $\mathrm{O}=$ ID Solutions \\
$\mathrm{P}=\mathrm{id} 3$ & $\mathrm{Q}=$ Morpho & $\mathrm{S}=$ Decatur Industries & $\mathrm{T}=$ BIO-key & $\mathrm{U}=$ Aware & $\mathrm{V}=$ AA Technology
\end{tabular}




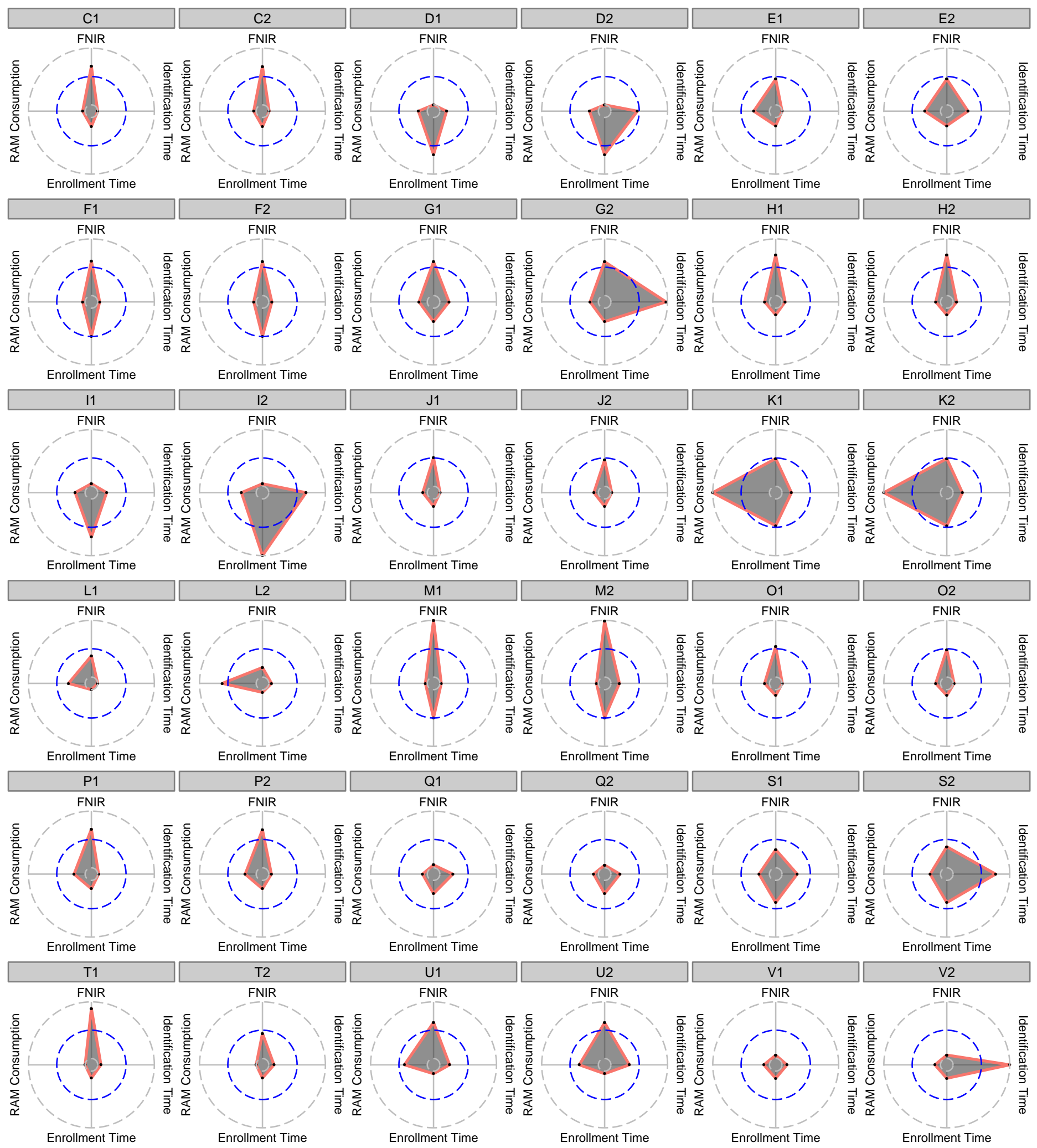

Figure 100: Star plot of combined results for Class A - Right Index. The values in this plot have been independently scaled from 0 to 1 from the values printed in Table 82, with the exception of FNIR, whose $\log _{10}$ values were scaled. The intersection point of a radius and the circumference of a circle indicate the scaled values $0,0.5$, and 1 , with 1 resting on the outer dashed-gray circle. The title above each plot represents the participant's letter code found on the footer of this page and an identifier used to differentiate between the two submissions each participant could make.

\begin{tabular}{l|l|l|l|l|l|l}
$\mathrm{C}=$ afis team & $\mathrm{D}=3 \mathrm{M}$ Cogent & $\mathrm{E}=$ Neurotechnology & $\mathrm{F}=$ Papillon & $\mathrm{G}=$ Dermalog & $\mathrm{H}=$ Hisign Bio-Info Institute \\
$\mathrm{I}=$ NEC & $\mathrm{J}=$ Sonda & $\mathrm{K}=$ Tiger IT & $\mathrm{L}=$ Innovatrics & $\mathrm{M}=$ SPEX & $\mathrm{O}=$ ID Solutions \\
$\mathrm{P}=$ id3 & $\mathrm{Q}=$ Morpho & $\mathrm{S}=$ Decatur Industries & $\mathrm{T}=$ BIO-key & $\mathrm{U}=$ Aware & $\mathrm{V}=$ AA Technology
\end{tabular}



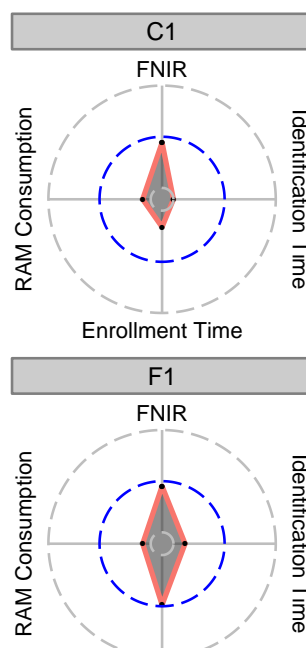

Enrollment Time

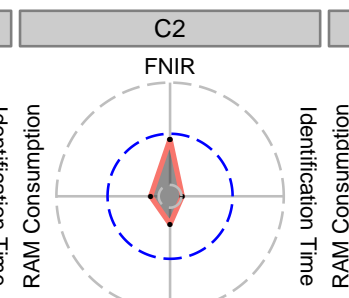

Enrollment Time

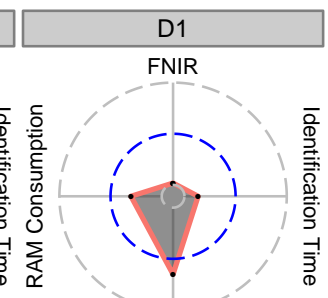

Enrollment Time

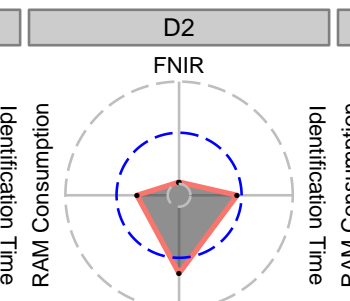

Enrollment Time

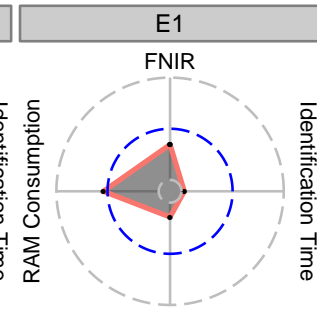

Enrollment Time

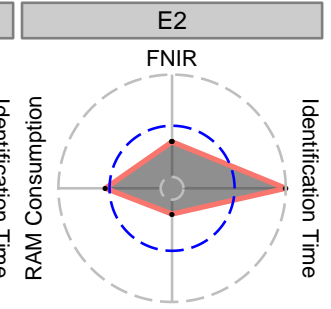

Enrollment Time
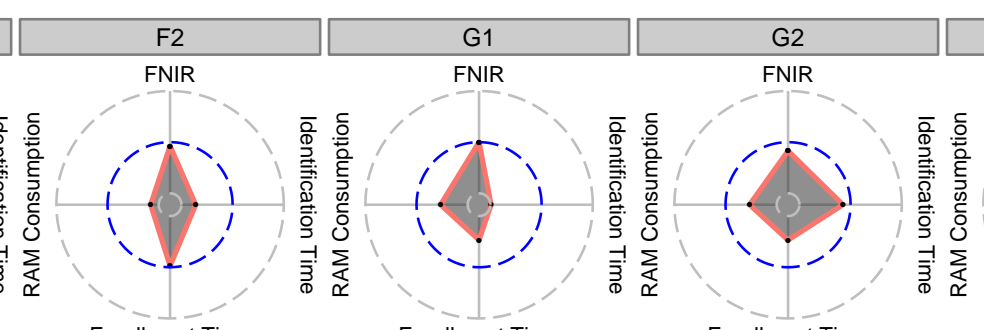

$\mathrm{H} 1$
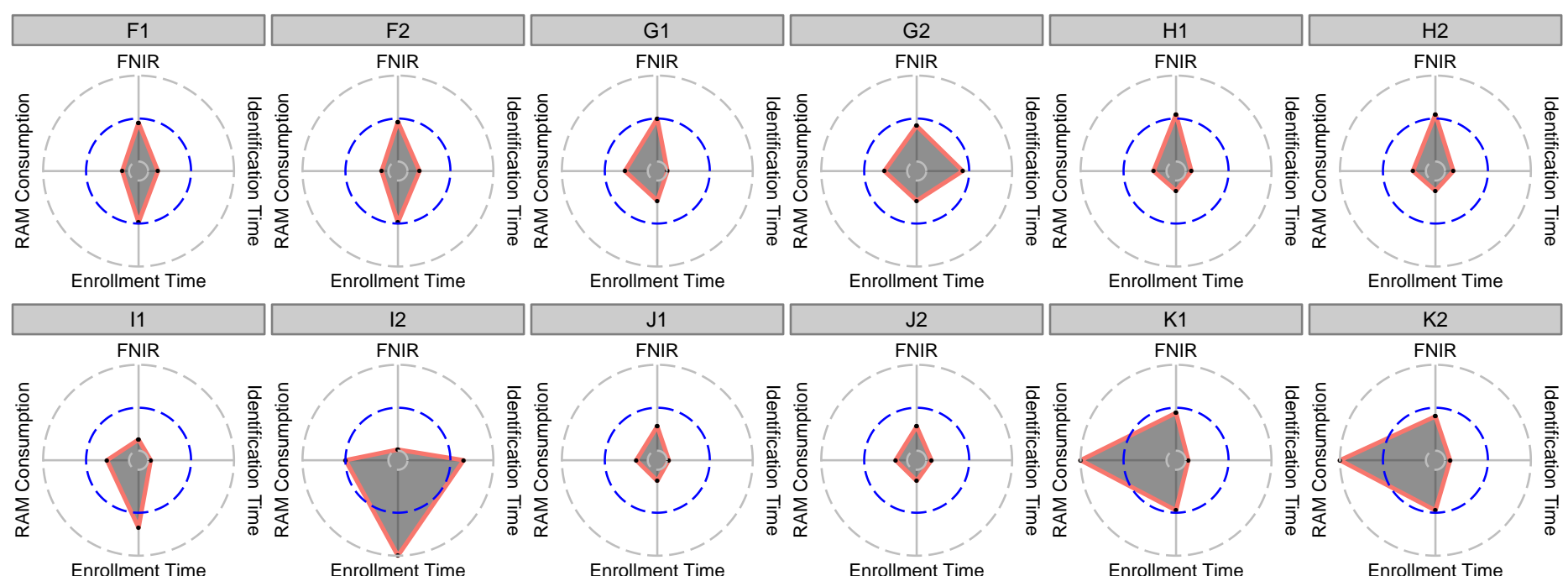

Enrollment Time

Enrollment Time

Enrollment Time

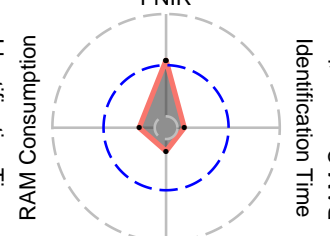

Enrollment Time
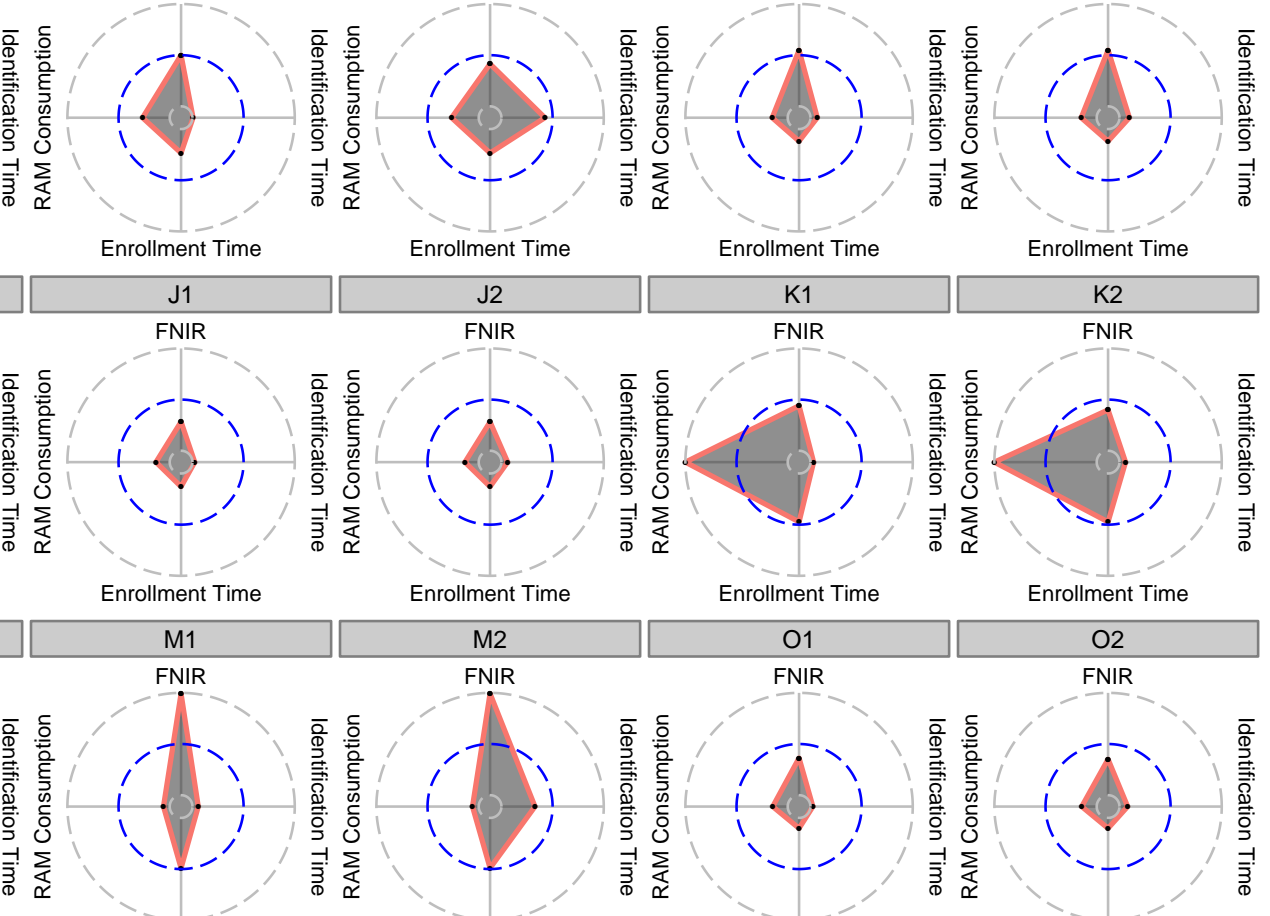

Enrollment Time

Enrollment Time

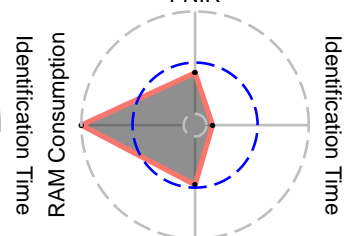

Enrollment Time
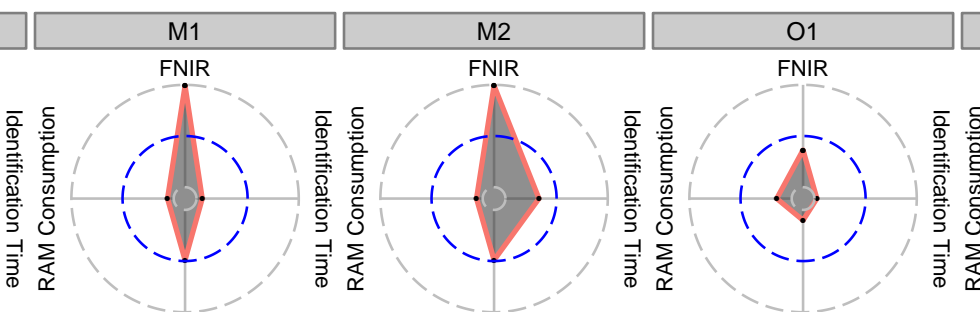

Enrollment Time
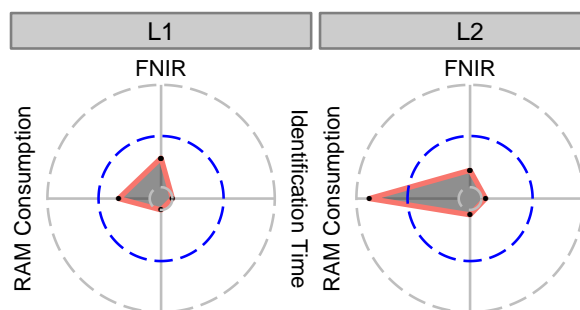

Enrollment Time
Q2
FNIR

\begin{tabular}{|c|} 
Enrollment Time \\
\hline S1 \\
FNIR
\end{tabular}

\begin{tabular}{|c|}
\hline O2 \\
\hline FNIR \\
\hline
\end{tabular}
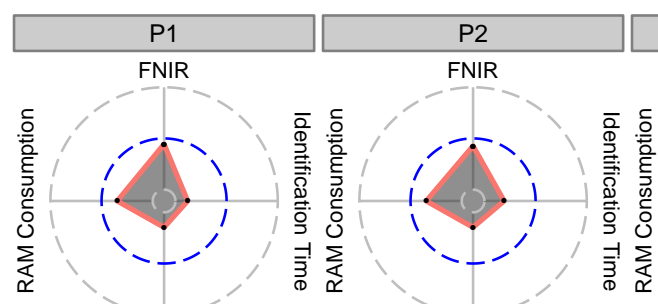

Enrollment Time
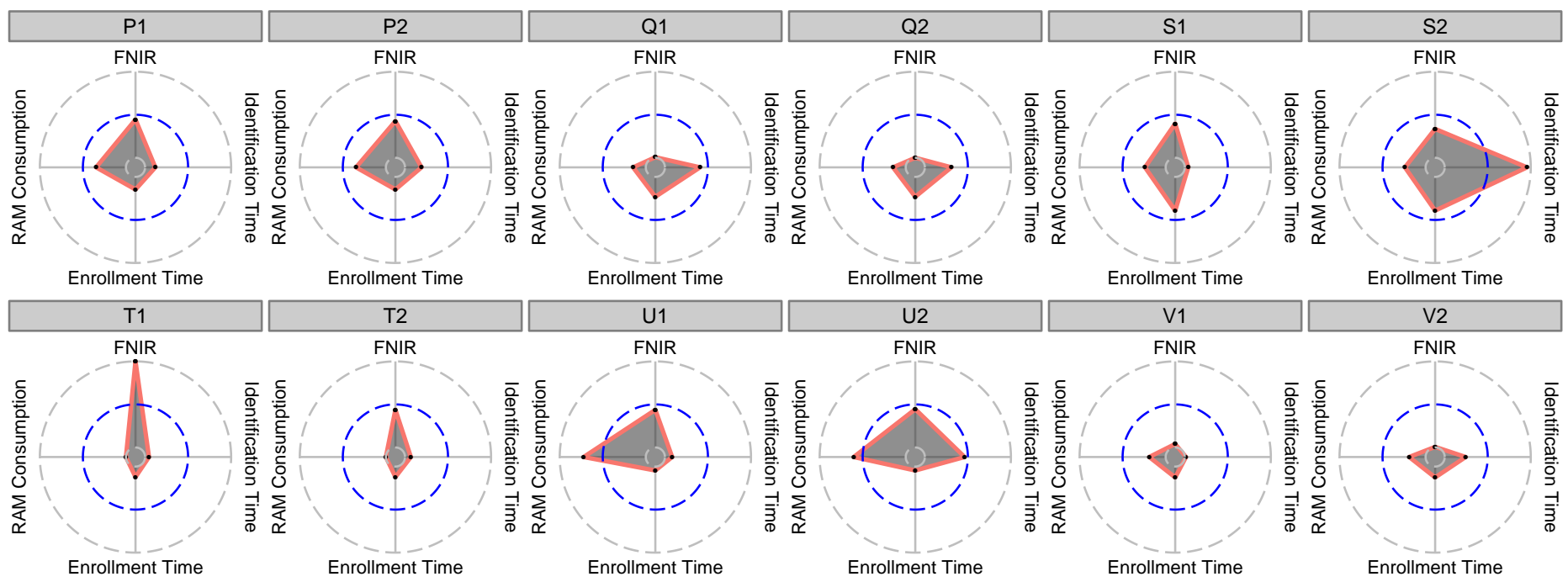

Figure 101: Star plot of combined results for Class A - Left and Right Index. The values in this plot have been independently scaled from 0 to 1 from the values printed in Table 21, with the exception of FNIR, whose $\log _{10}$ values were scaled. Any values printed as NA were set to 1 before scaling. The intersection point of a radius and the circumference of a circle indicate the scaled values $0,0.5$, and 1 , with 1 resting on the outer dashed-gray circle. The title above each plot represents the participant's letter code found on the footer of this page and an identifier used to differentiate between the two submissions each participant could make.

\begin{tabular}{l|l|l|l|l|l}
$\mathrm{C}=$ afis team & $\mathrm{D}=3 \mathrm{M}$ Cogent & $\mathrm{E}=$ Neurotechnology & $\mathrm{F}=$ Papillon & $\mathrm{G}=$ Dermalog & $\mathrm{H}=$ Hisign Bio-Info Institute \\
$\mathrm{I}=\mathrm{NEC}$ & $\mathrm{J}=$ Sonda & $\mathrm{K}=$ Tiger IT & $\mathrm{L}=$ Innovatrics & $\mathrm{M}=$ SPEX & $\mathrm{O}=$ ID Solutions \\
$\mathrm{P}=\mathrm{id} 3$ & $\mathrm{Q}=$ Morpho & $\mathrm{S}=$ Decatur Industries & $\mathrm{T}=$ BIO-key & $\mathrm{U}=$ Aware & $\mathrm{V}=$ AA Technology
\end{tabular}




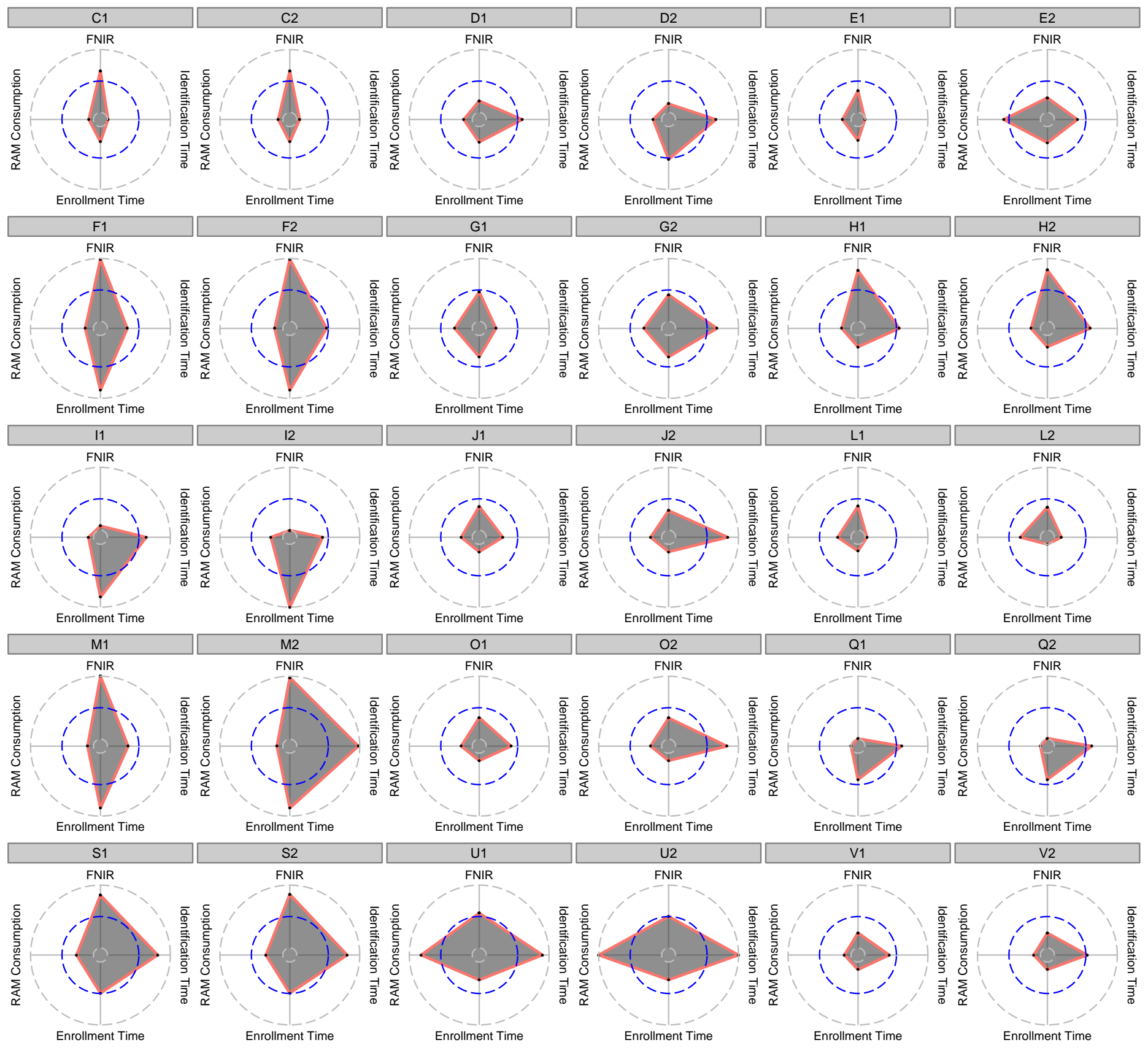

Figure 102: Star plot of combined results for Class B — Left Slap. The values in this plot have been independently scaled from 0 to 1 from the values printed in Table 84, with the exception of FNIR, whose $\log _{10}$ values were scaled. The intersection point of a radius and the circumference of a circle indicate the scaled values $0,0.5$, and 1 , with 1 resting on the outer dashed-gray circle. The title above each plot represents the participant's letter code found on the footer of this page and an identifier used to differentiate between the two submissions each participant could make.

\begin{tabular}{l|l|l|l|ll|l}
$\mathrm{C}=$ afis team & $\mathrm{D}=3 \mathrm{M}$ Cogent & $\mathrm{E}=$ Neurotechnology & $\mathrm{F}=$ Papillon & $\mathrm{G}=$ Dermalog & $\mathrm{H}=$ Hisign Bio-Info Institute \\
$\mathrm{I}=$ NEC & $\mathrm{J}=$ Sonda & $\mathrm{K}=$ Tiger IT & $\mathrm{L}=$ Innovatrics & $\mathrm{M}=$ SPEX & $\mathrm{O}=$ ID Solutions \\
$\mathrm{P}=\mathrm{id} 3$ & $\mathrm{Q}=$ Morpho & $\mathrm{S}=$ Decatur Industries & $\mathrm{T}=$ BIO-key & $\mathrm{U}=$ Aware & $\mathrm{V}=$ AA Technology
\end{tabular}




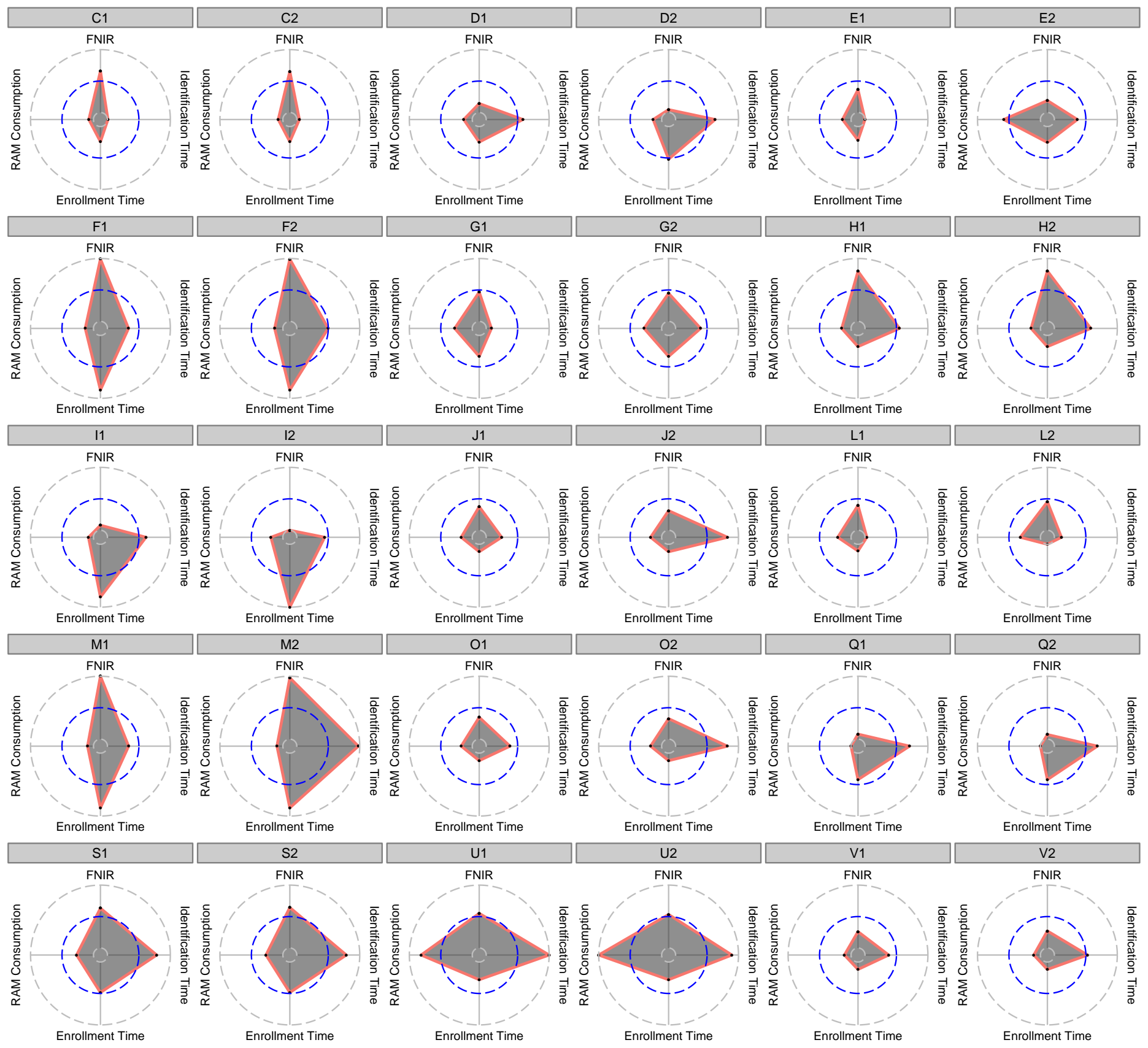

Figure 103: Star plot of combined results for Class B - Right Slap. The values in this plot have been independently scaled from 0 to 1 from the values printed in Table 85, with the exception of FNIR, whose $\log _{10}$ values were scaled. The intersection point of a radius and the circumference of a circle indicate the scaled values $0,0.5$, and 1 , with 1 resting on the outer dashed-gray circle. The title above each plot represents the participant's letter code found on the footer of this page and an identifier used to differentiate between the two submissions each participant could make.

\begin{tabular}{l|l|l|l|ll|l}
$\mathrm{C}=$ afis team & $\mathrm{D}=$ = 3M Cogent & $\mathrm{E}=$ Neurotechnology & $\mathrm{F}=$ Papillon & $\mathrm{G}=$ Dermalog & $\mathrm{H}=$ Hisign Bio-Info Institute \\
$\mathrm{I}=$ NEC & $\mathrm{J}=$ Sonda & $\mathrm{K}=$ Tiger IT & $\mathrm{L}=$ Innovatrics & $\mathrm{M}=$ SPEX & $\mathrm{O}=$ ID Solutions \\
$\mathrm{P}=\mathrm{id} 3$ & $\mathrm{Q}=$ Morpho & $\mathrm{S}=$ Decatur Industries & $\mathrm{T}=$ BIO-key & $\mathrm{U}=$ Aware & $\mathrm{V}=$ AA Technology
\end{tabular}




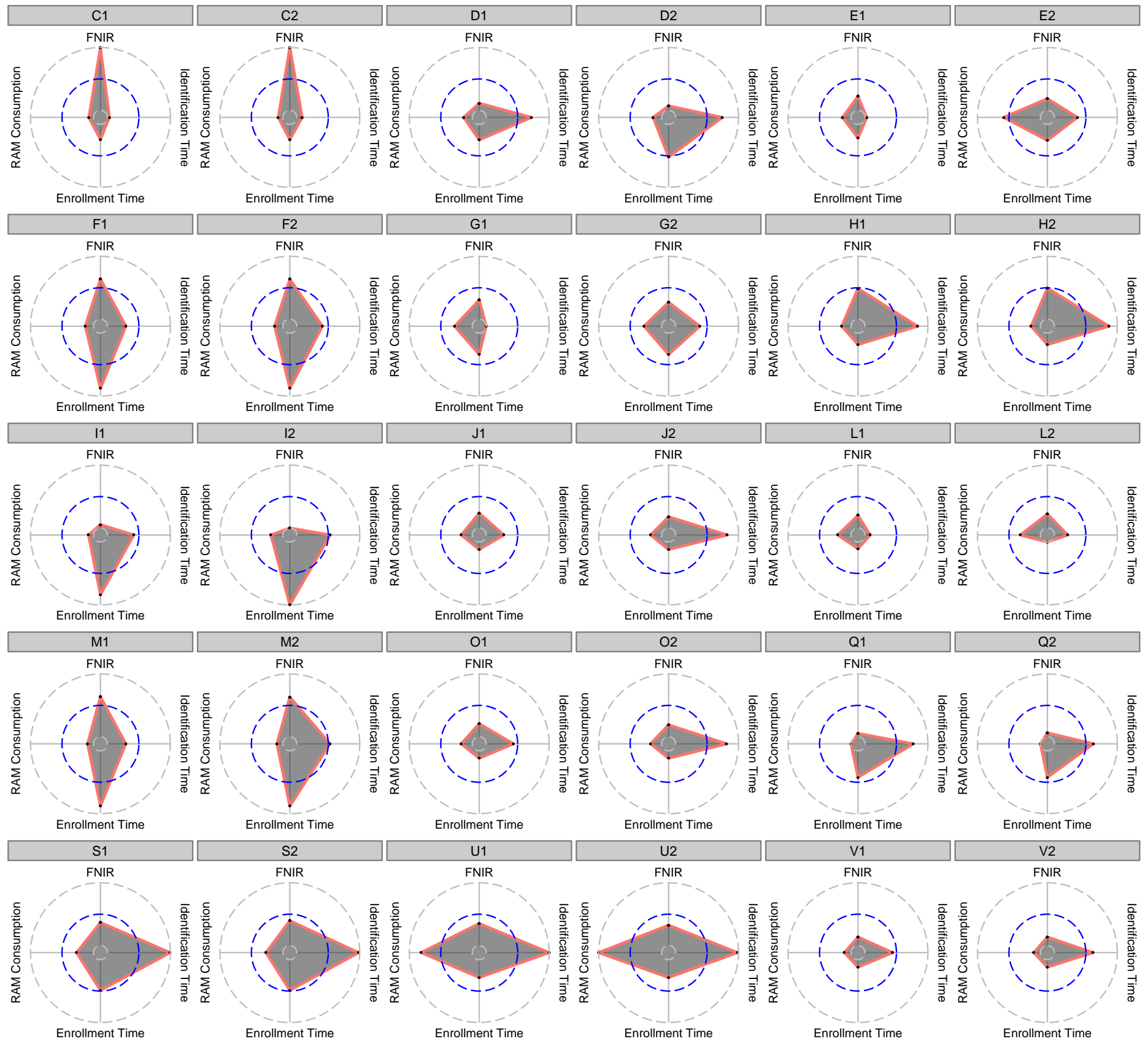

Figure 104: Star plot of combined results for Class B - Left and Right Slap. The values in this plot have been independently scaled from 0 to 1 from the values printed in Table 86, with the exception of FNIR, whose $\log _{10}$ values were scaled. Any values printed as NA were set to 1 before scaling. The intersection point of a radius and the circumference of a circle indicate the scaled values $0,0.5$, and 1 , with 1 resting on the outer dashed-gray circle. The title above each plot represents the participant's letter code found on the footer of this page and an identifier used to differentiate between the two submissions each participant could make.

\begin{tabular}{l|l|l|l|ll|l}
$\mathrm{C}=$ afis team & $\mathrm{D}=3 \mathrm{M}$ Cogent & $\mathrm{E}=$ Neurotechnology & $\mathrm{F}=$ Papillon & $\mathrm{G}=$ Dermalog & $\mathrm{H}=$ Hisign Bio-Info Institute \\
$\mathrm{I}=$ NEC & $\mathrm{J}=$ Sonda & $\mathrm{K}=$ Tiger IT & $\mathrm{L}=$ Innovatrics & $\mathrm{M}=$ SPEX & $\mathrm{O}=$ ID Solutions \\
$\mathrm{P}=\mathrm{id} 3$ & $\mathrm{Q}=$ Morpho & $\mathrm{S}=$ Decatur Industries & $\mathrm{T}=$ BIO-key & $\mathrm{U}=$ Aware & $\mathrm{V}=$ AA Technology
\end{tabular}




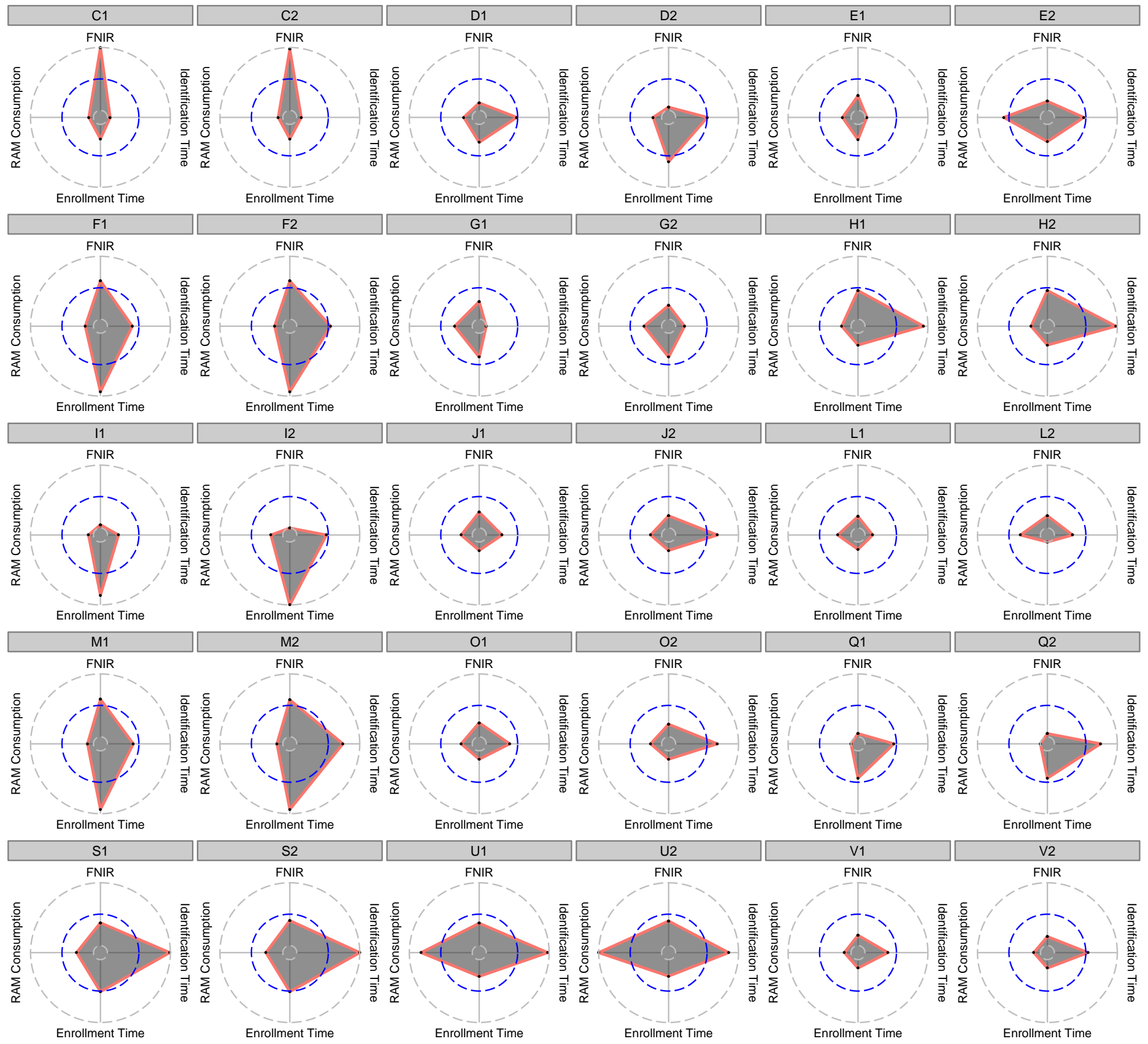

Figure 105: Star plot of combined results for Class B - Identification Flats. The values in this plot have been independently scaled from 0 to 1 from the values printed in Table 22, with the exception of FNIR, whose $\log _{10}$ values were scaled. Any values printed as NA were set to 1 before scaling. The intersection point of a radius and the circumference of a circle indicate the scaled values $0,0.5$, and 1 , with 1 resting on the outer dashed-gray circle. The title above each plot represents the participant's letter code found on the footer of this page and an identifier used to differentiate between the two submissions each participant could make.

\begin{tabular}{l|l|l|l|l|l|l}
$\mathrm{C}=$ afis team & $\mathrm{D}=$ = 3M Cogent & $\mathrm{E}=$ Neurotechnology & $\mathrm{F}=$ Papillon & $\mathrm{G}=$ Dermalog & $\mathrm{H}=$ Hisign Bio-Info Institute \\
$\mathrm{I}=$ NEC & $\mathrm{J}=$ Sonda & $\mathrm{K}=$ Tiger IT & $\mathrm{L}=$ Innovatrics & $\mathrm{M}=$ SPEX & $\mathrm{O}=$ ID Solutions \\
$\mathrm{P}=\mathrm{id} 3$ & $\mathrm{Q}=$ Morpho & $\mathrm{S}=$ Decatur Industries & $\mathrm{T}=$ BIO-key & $\mathrm{U}=$ Aware & $\mathrm{V}=$ AA Technology
\end{tabular}




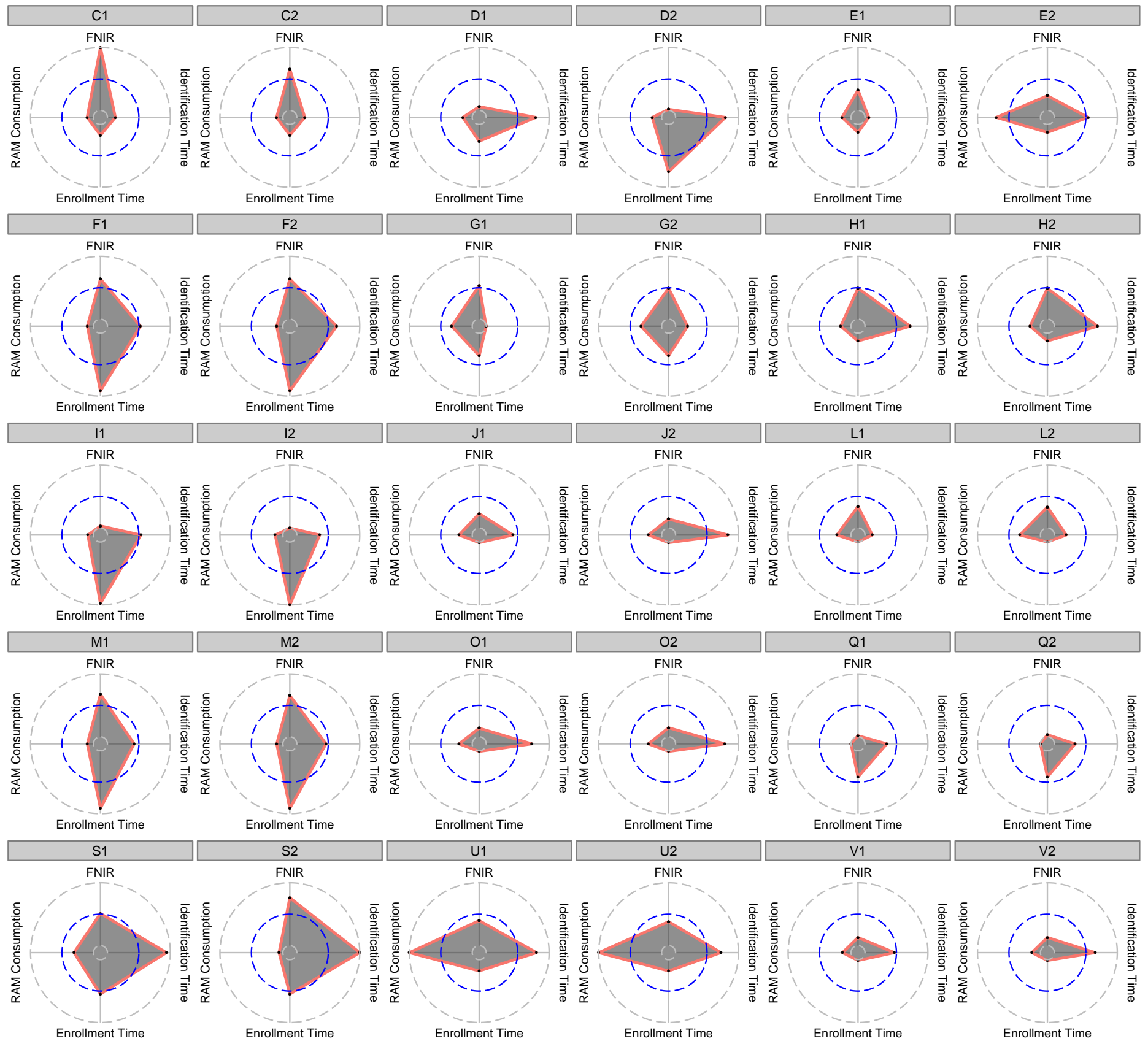

Figure 106: Star plot of combined results for Class C - Ten-Finger Plain-to-Plain. The values in this plot have been independently scaled from 0 to 1 from the values printed in Table 88, with the exception of FNIR, whose $\log _{10}$ values were scaled. Any values printed as NA were set to 1 before scaling. The intersection point of a radius and the circumference of a circle indicate the scaled values $0,0.5$, and 1 , with 1 resting on the outer dashed-gray circle. The title above each plot represents the participant's letter code found on the footer of this page and an identifier used to differentiate between the two submissions each participant could make.

\begin{tabular}{l|l|l|l|ll|l}
$\mathrm{C}=$ afis team & $\mathrm{D}=3 \mathrm{M}$ Cogent & $\mathrm{E}=$ Neurotechnology & $\mathrm{F}=$ Papillon & $\mathrm{G}=$ Dermalog & $\mathrm{H}=$ Hisign Bio-Info Institute \\
$\mathrm{I}=$ NEC & $\mathrm{J}=$ Sonda & $\mathrm{K}=$ Tiger IT & $\mathrm{L}=$ Innovatrics & $\mathrm{M}=$ SPEX & $\mathrm{O}=$ ID Solutions \\
$\mathrm{P}=\mathrm{id} 3$ & $\mathrm{Q}=$ Morpho & $\mathrm{S}=$ Decatur Industries & $\mathrm{T}=$ BIO-key & $\mathrm{U}=$ Aware & $\mathrm{V}=$ AA Technology
\end{tabular}




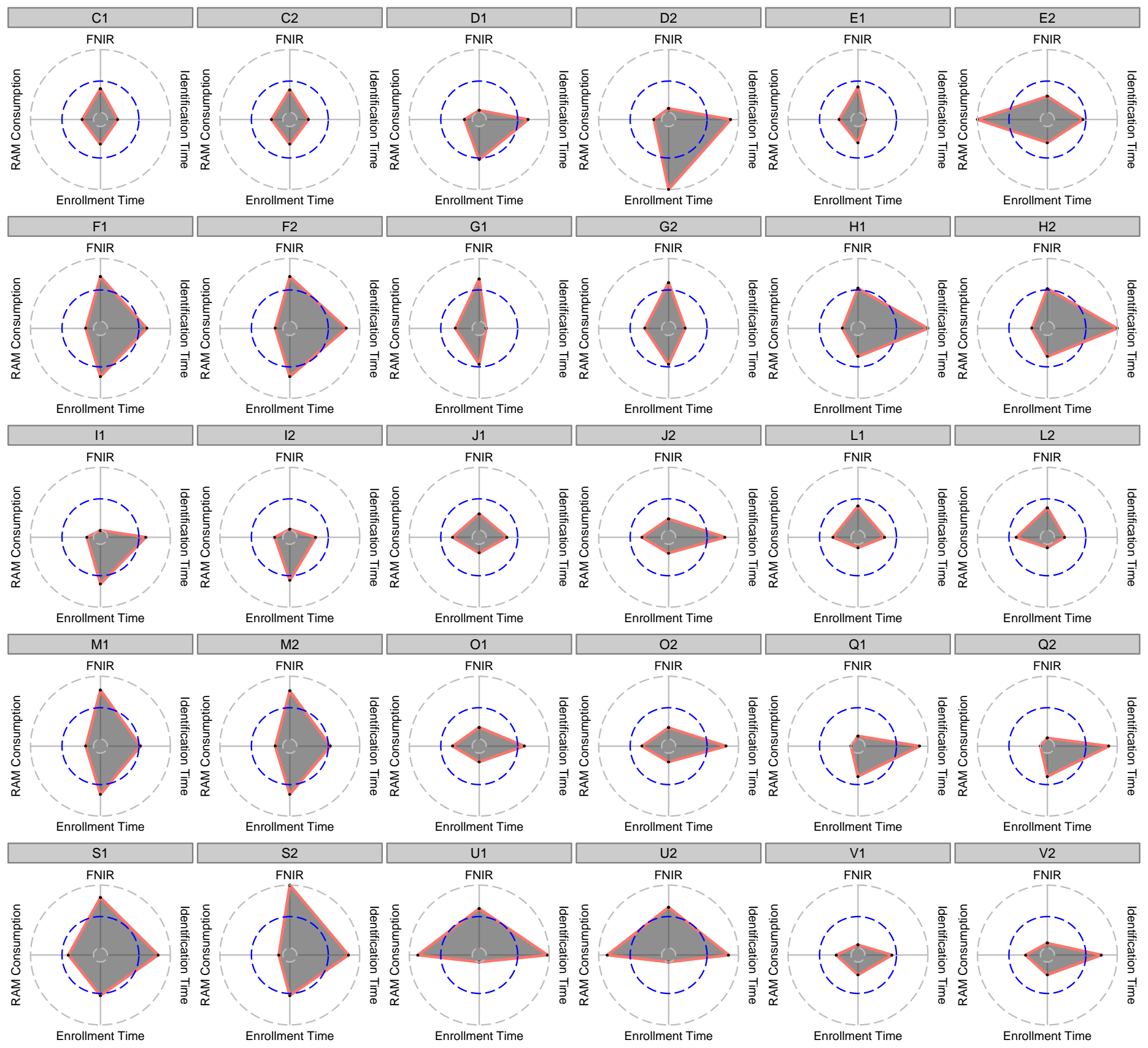

Figure 107: Star plot of combined results for Class C - Ten-Finger Rolled-to-Rolled. The values in this plot have been independently scaled from 0 to 1 from the values printed in Table 23, with the exception of $F N I R$, whose $\log _{10}$ values were scaled. The intersection point of a radius and the circumference of a circle indicate the scaled values $0,0.5$, and 1 , with 1 resting on the outer dashed-gray circle. The title above each plot represents the participant's letter code found on the footer of this page and an identifier used to differentiate between the two submissions each participant could make.

\begin{tabular}{l|l|l|l|ll|l}
$\mathrm{C}=$ afis team & $\mathrm{D}=$ = 3M Cogent & $\mathrm{E}=$ Neurotechnology & $\mathrm{F}=$ Papillon & $\mathrm{G}=$ Dermalog & $\mathrm{H}=$ Hisign Bio-Info Institute \\
$\mathrm{I}=$ NEC & $\mathrm{J}=$ Sonda & $\mathrm{K}=$ Tiger IT & $\mathrm{L}=$ Innovatrics & $\mathrm{M}=$ SPEX & $\mathrm{O}=$ ID Solutions \\
$\mathrm{P}=\mathrm{id} 3$ & $\mathrm{Q}=$ Morpho & $\mathrm{S}=$ Decatur Industries & $\mathrm{T}=$ BIO-key & $\mathrm{U}=$ Aware & $\mathrm{V}=$ AA Technology
\end{tabular}




\section{K Relative Accuracy and Number of Fingers}

In Section 7, DETs, tables, and other data visualization methods were used to show exact FNIR values at FPIR $=10^{-3}$. While this data is important for exact comparisons, it is difficult to quickly compare relative accuracy among submissions, or to see how a submission fares across finger combinations. To facilitate these comparisons, star plots [1] (also called spider or radar plots) are included in this section. These plots are used to quickly examine relative values among multiple variables.

In each star plot, values are plotted along multiple radii, where each radius represents a single variable. The plot area is delineated with three circles. The points on the circumference of these circles indicate different values where they intersect the radii. The intersection with the smallest circle represents 0 , the next-largest circle (dashed-blue) represents 0.5 , and the largest circle (dashed-gray) represents 1 . All values plotted have been scaled between 0 and 1 against other values for the same variable in order to fit within the largest circle. These plotted values were then connected to adjacent plotted values, creating a polygon. Many traits may be quickly inferred by the shape of these polygons:

$\triangleright$ Regular polygons indicate submissions that have similar accuracy for all plotted variables.

$\triangleright$ A submission that is the most accurate in terms of every variable would inscribe the center circle and the least accurate would inscribe the largest circle.

$\triangleright$ The smaller the polygon, the more accurate the submission.

\begin{tabular}{l|l|l|l|l|l|l}
$\mathrm{C}=$ afis team & $\mathrm{D}=3 \mathrm{M}$ Cogent & $\mathrm{E}=$ Neurotechnology & $\mathrm{F}=$ Papillon & $\mathrm{G}=$ Dermalog & $\mathrm{H}=$ Hisign Bio-Info Institute \\
$\mathrm{I}=$ NEC & $\mathrm{J}=$ Sonda & $\mathrm{K}=$ Tiger IT & $\mathrm{L}=$ Innovatrics & $\mathrm{M}=$ SPEX & $\mathrm{O}=$ ID Solutions \\
$\mathrm{P}=$ id3 & $\mathrm{Q}=$ Morpho & $\mathrm{S}=$ Decatur Industries & $\mathrm{T}=$ BIO-key & $\mathrm{U}=$ Aware & $\mathrm{V}=$ AA Technology
\end{tabular}




\section{K.1 Class A}

Relative FNIR results for left index, right index, and left and right index searches are shown in Figure 108. Note that the enrollment set sizes differed between single index and two-index searches.

Some notable observations include:

$\triangleright$ The most accurate submissions $(\mathbf{D}, \mathbf{I}, \mathbf{Q}, \mathbf{V})$ are instantly recognizable by their small shape.

$\triangleright$ Most submissions have near-identical accuracy, regardless of being provided one or two index fingers, as indicated by the equilateral triangle drawn. This may have impacts on data collection and storage. Extreme examples include $\mathbf{D}, \mathbf{K}, \mathbf{M}$, and $\mathbf{V}$.

$\triangleright$ No participants appear to have wildly-differing accuracy among any of the index finger combinations.

\begin{tabular}{l|l|l|l|l|l|l}
$\mathrm{C}=$ afis team & $\mathrm{D}=3 \mathrm{M}$ Cogent & $\mathrm{E}=$ Neurotechnology & $\mathrm{F}=$ Papillon & $\mathrm{G}=$ Dermalog & $\mathrm{H}=$ Hisign Bio-Info Institute \\
$\mathrm{I}=\mathrm{NEC}$ & $\mathrm{J}=$ Sonda & $\mathrm{K}=$ Tiger IT & $\mathrm{L}=$ Innovatrics & $\mathrm{M}=$ SPEX & $\mathrm{O}=$ ID Solutions \\
$\mathrm{P}=$ id3 & $\mathrm{Q}=$ Morpho & $\mathrm{S}=$ Decatur Industries & $\mathrm{T}=$ BIO-key & $\mathrm{U}=$ Aware & $\mathrm{V}=$ AA Technology
\end{tabular}



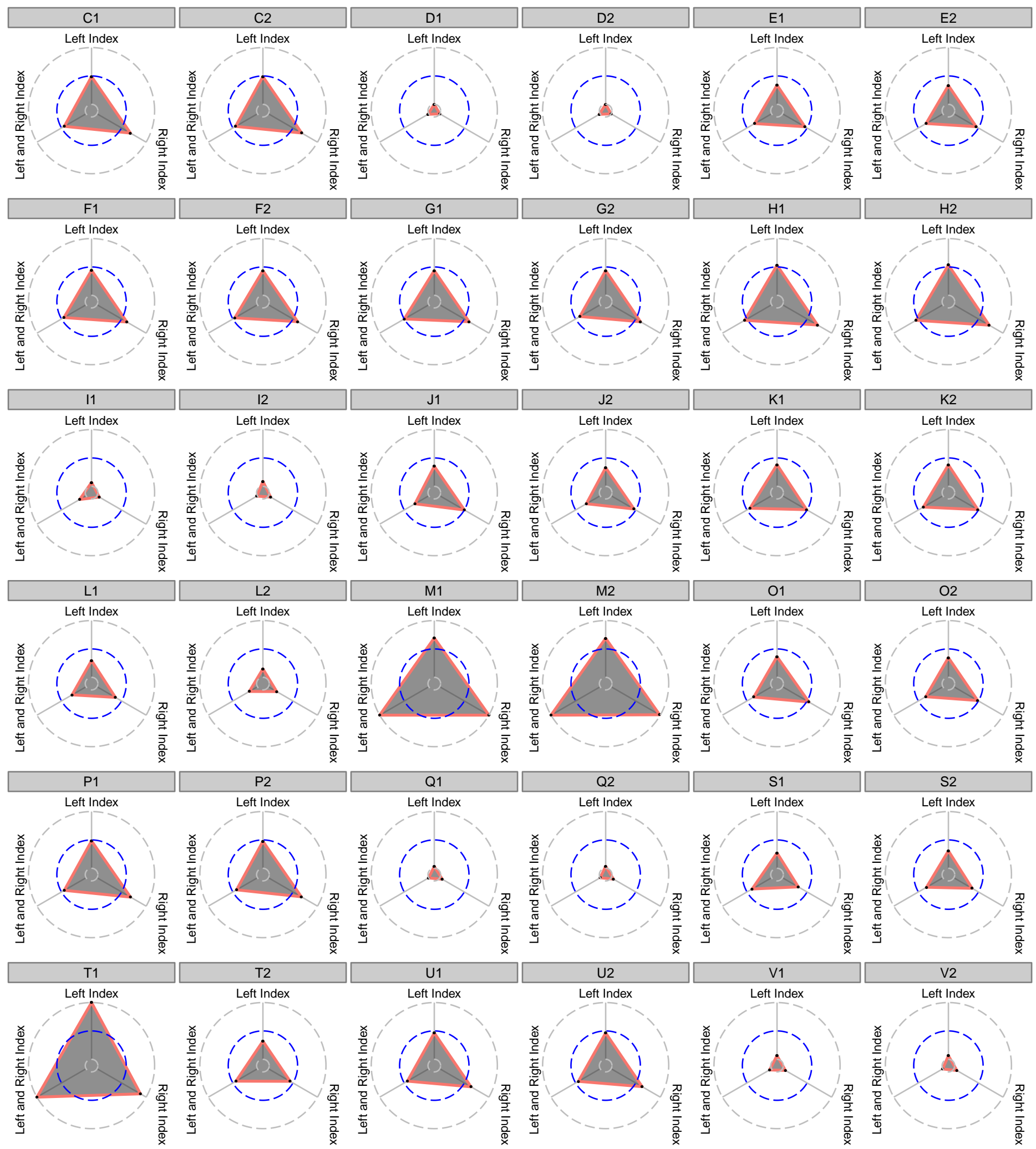

Figure 108: Star plots of relative accuracy for Class A. Values plotted along each radius are $\log _{10}$ of values from Tables 7 through 9 , scaled independently from 0 to 1 . Values printed as NA in those tables were set to 1 prior to scaling. 30000 searches were run against an enrollment set of 100000 subjects for left index and right index, and 1600000 subjects for left and right index. The intersection point of a radius and the circumference of a circle indicate the scaled values $0,0.5$, and 1 , with 1 resting on the outer dashed-gray circle. The title above each plot represents the participant's letter code found on the footer of this page and an identifier used to differentiate between the two submissions each participant could make.

$$
\begin{array}{l|l|l|l|l|l|l}
\mathrm{C}=\text { afis team } & \mathrm{D}=3 \mathrm{M} \text { Cogent } & \mathrm{E}=\text { Neurotechnology } & \mathrm{F}=\text { Papillon } & \mathrm{G}=\text { Dermalog } & \mathrm{H}=\text { Hisign Bio-Info Institute } \\
\mathrm{I}=\mathrm{NEC} & \mathrm{J}=\text { Sonda } & \mathrm{K}=\text { Tiger IT } & \mathrm{L}=\text { Innovatrics } & \mathrm{M}=\text { SPEX } & \mathrm{O}=\text { ID Solutions } \\
\mathrm{P}=\mathrm{id} 3 & \mathrm{Q}=\text { Morpho } & \mathrm{S}=\text { Decatur Industries } & \mathrm{T}=\text { BIO-key } & \mathrm{U}=\text { Aware } & \mathrm{V}=\text { AA Technology }
\end{array}
$$




\section{K.2 Class B}

Relative FNIR results for left slap, right slap, left and right slap, and IDFlat searches are shown in Figure 109. Some notable observations include:

$\triangleright$ The most accurate submissions (I and $\mathbf{Q}$ ) are instantly recognizable by their small shape.

$\triangleright$ The most accurate submissions are also square, meaning they perform equally well for all finger combinations.

$\triangleright$ Most submissions appear to skew towards slightly worse accuracy on left slap and right slap searches, as indicated by the obtuse angles at the vertices for IDFlats and left and right slap.

$\triangleright$ Some submissions, like C, F, H, M, and S, vary significantly as the number of fingers at their disposal changes.

\begin{tabular}{l|l|l|l|l|l|l}
$\mathrm{C}=$ afis team & $\mathrm{D}=3 \mathrm{M}$ Cogent & $\mathrm{E}=$ Neurotechnology & $\mathrm{F}=$ Papillon & $\mathrm{G}=$ Dermalog & $\mathrm{H}=$ Hisign Bio-Info Institute \\
$\mathrm{I}=$ NEC & $\mathrm{J}=$ Sonda & $\mathrm{K}=$ Tiger IT & $\mathrm{L}=$ Innovatrics & $\mathrm{M}=$ SPEX & $\mathrm{O}=$ ID Solutions \\
$\mathrm{P}=$ id3 & $\mathrm{Q}=$ Morpho & $\mathrm{S}=$ Decatur Industries & $\mathrm{T}=$ BIO-key & $\mathrm{U}=$ Aware & $\mathrm{V}=$ AA Technology
\end{tabular}




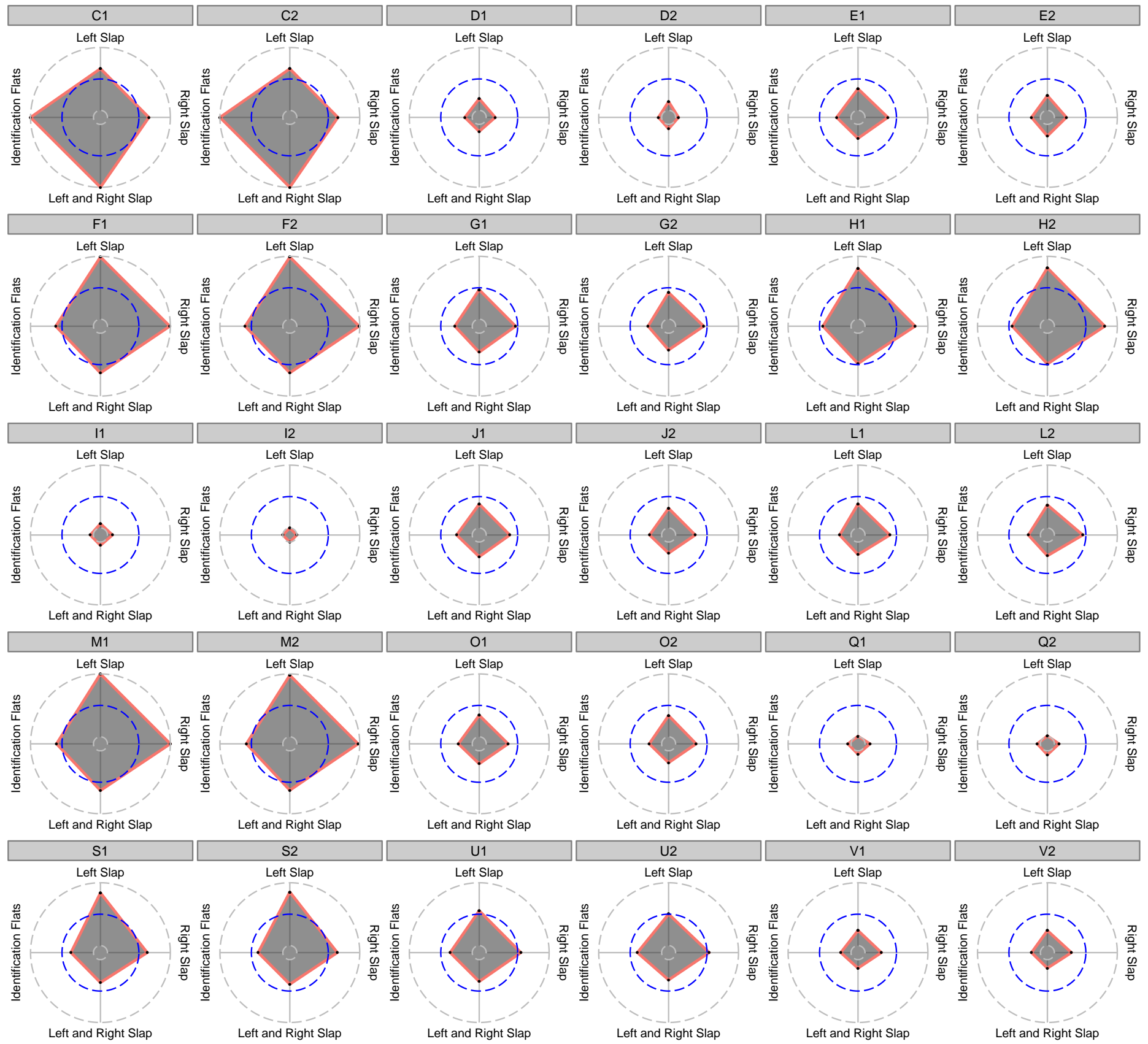

Figure 109: Star plots of relative accuracy for Class B. Values plotted along each radius are $\log _{10}$ of values from Tables 10 through 13 , scaled independently from 0 to 1 . Values printed as NA in those tables were set to 1 prior to scaling. 30000 searches were run against an enrollment set of 3000000 subjects. The intersection point of a radius and the circumference of a circle indicate the scaled values $0,0.5$, and 1 , with 1 resting on the outer dashed-gray circle. The title above each plot represents the participant's letter code found on the footer of this page and an identifier used to differentiate between the two submissions each participant could make.

\begin{tabular}{l|l|l|l|ll|l}
$\mathrm{C}=$ afis team & $\mathrm{D}=3 \mathrm{M}$ Cogent & $\mathrm{E}=$ Neurotechnology & $\mathrm{F}=$ Papillon & $\mathrm{G}=$ Dermalog & $\mathrm{H}=$ Hisign Bio-Info Institute \\
$\mathrm{I}=$ NEC & $\mathrm{J}=$ Sonda & $\mathrm{K}=$ Tiger IT & $\mathrm{L}=$ Innovatrics & $\mathrm{M}=$ SPEX & $\mathrm{O}=$ ID Solutions \\
$\mathrm{P}=\mathrm{id} 3$ & $\mathrm{Q}=$ Morpho & $\mathrm{S}=$ Decatur Industries & $\mathrm{T}=$ BIO-key & $\mathrm{U}=$ Aware & $\mathrm{V}=$ AA Technology
\end{tabular}




\section{K.3 Class C}

Relative FNIR results for ten-finger plain-to-plain, ten-finger rolled-to-rolled, and ten-finger plain-to-rolled searches are shown in Figure 110. Some notable observations include:

$\triangleright$ The most accurate submissions (D, I, and $\mathbf{Q})$ are instantly recognizable by their small shape and are seemingly equilateral, indicating that they perform equally well for all finger combinations.

$\triangleright$ Many submissions had lower accuracy with plain-to-rolled searching over searching homogeneous impressions, including $\mathbf{F}, \mathbf{G}$, and $\mathbf{M}$. 


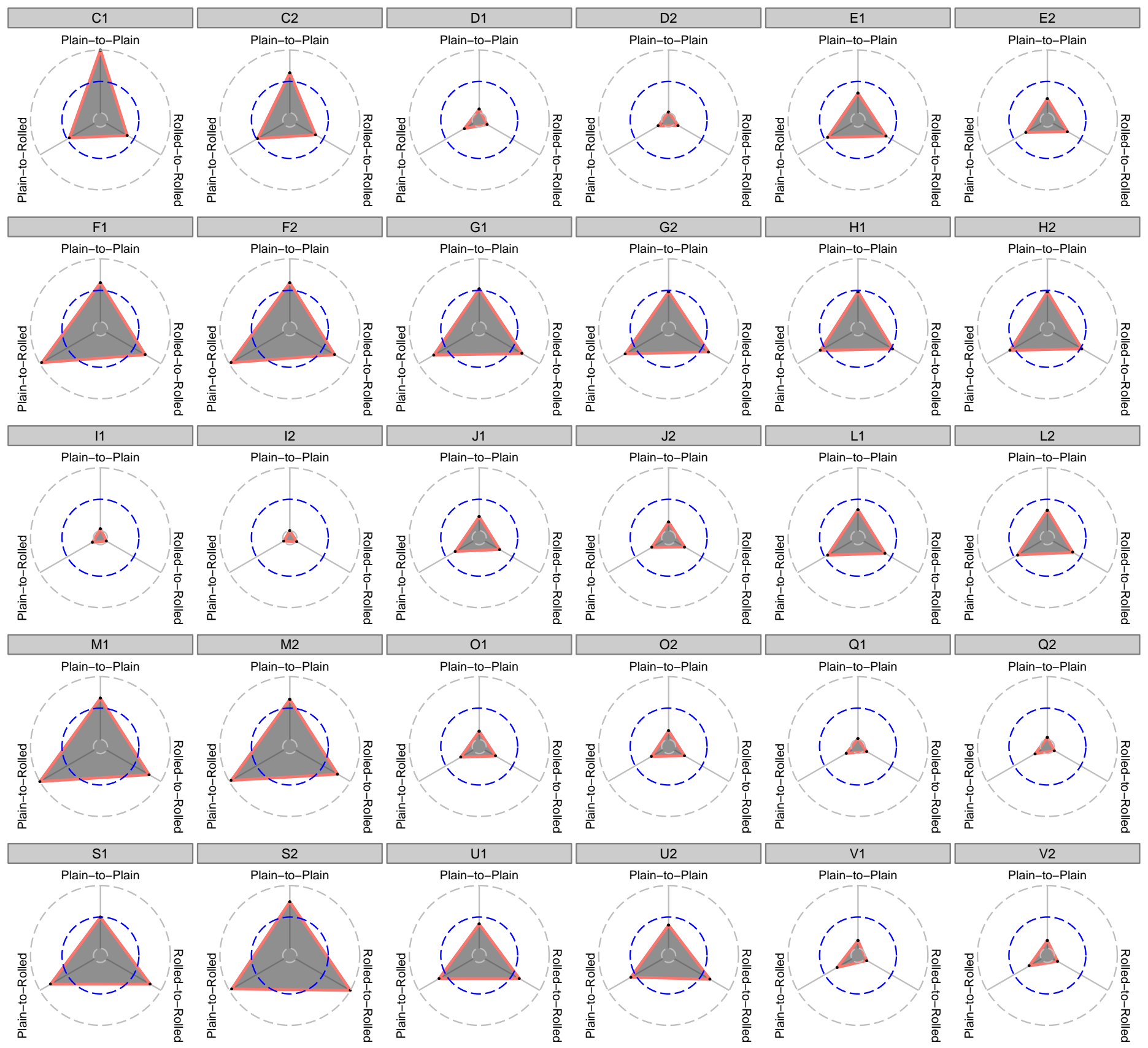

Figure 110: Star plots of relative accuracy for Class C. Values plotted along each radius are $\log _{10}$ of values from Tables 14 through 16 , scaled independently from 0 to 1 . Values printed as NA in those tables were set to 1 prior to scaling. 30000 searches were run against an enrollment set of 5000000 subjects. The intersection point of a radius and the circumference of a circle indicate the scaled values $0,0.5$, and 1 , with 1 resting on the outer dashedgray circle. The title above each plot represents the participant's letter code found on the footer of this page and an identifier used to differentiate between the two submissions each participant could make.

\begin{tabular}{l|l|l|l|ll|l}
$\mathrm{C}=$ afis team & $\mathrm{D}=3 \mathrm{M}$ Cogent & $\mathrm{E}=$ Neurotechnology & $\mathrm{F}=$ Papillon & $\mathrm{G}=$ Dermalog & $\mathrm{H}=$ Hisign Bio-Info Institute \\
$\mathrm{I}=$ NEC & $\mathrm{J}=$ Sonda & $\mathrm{K}=$ Tiger IT & $\mathrm{L}=$ Innovatrics & $\mathrm{M}=$ SPEX & $\mathrm{O}=$ ID Solutions \\
$\mathrm{P}=\mathrm{id} 3$ & $\mathrm{Q}=$ Morpho & $\mathrm{S}=$ Decatur Industries & $\mathrm{T}=$ BIO-key & $\mathrm{U}=$ Aware & $\mathrm{V}=$ AA Technology
\end{tabular}




\section{K.4 All Classes}

Relative FNIR results for left and right index, IDFlat, and ten-finger rolled-to-rolled searches are shown in Figure 111. Note that the enrollment set sizes differed between all three finger searching combinations. Some notable observations include:

$\triangleright$ The top-performing submissions from Class A, B, and C (D, I, Q, and V) are instantly recognizable by their small shape.

$\triangleright$ Many of the most accurate submissions from all three classes appear equally as accurate in each individual class.

$\triangleright$ If a submission had trouble with a particular class in FpVTE, it appears to be Class C, as seen by the long line segments extending from the ten-finger rolled-to-rolled vertex in most plots that do not contain a mostly equilateral triangle.

\begin{tabular}{l|l|l|l|ll|l}
$\mathrm{C}=$ afis team & $\mathrm{D}=3 \mathrm{M}$ Cogent & $\mathrm{E}=$ Neurotechnology & $\mathrm{F}=$ Papillon & $\mathrm{G}=$ Dermalog & $\mathrm{H}=$ Hisign Bio-Info Institute \\
$\mathrm{I}=$ NEC & $\mathrm{J}=$ Sonda & $\mathrm{K}=$ Tiger IT & $\mathrm{L}=$ Innovatrics & $\mathrm{M}=$ SPEX & $\mathrm{O}=$ ID Solutions \\
$\mathrm{P}=\mathrm{id} 3$ & $\mathrm{Q}=$ Morpho & $\mathrm{S}=$ Decatur Industries & $\mathrm{T}=$ BIO-key & $\mathrm{U}=$ Aware & $\mathrm{V}=$ AA Technology
\end{tabular}




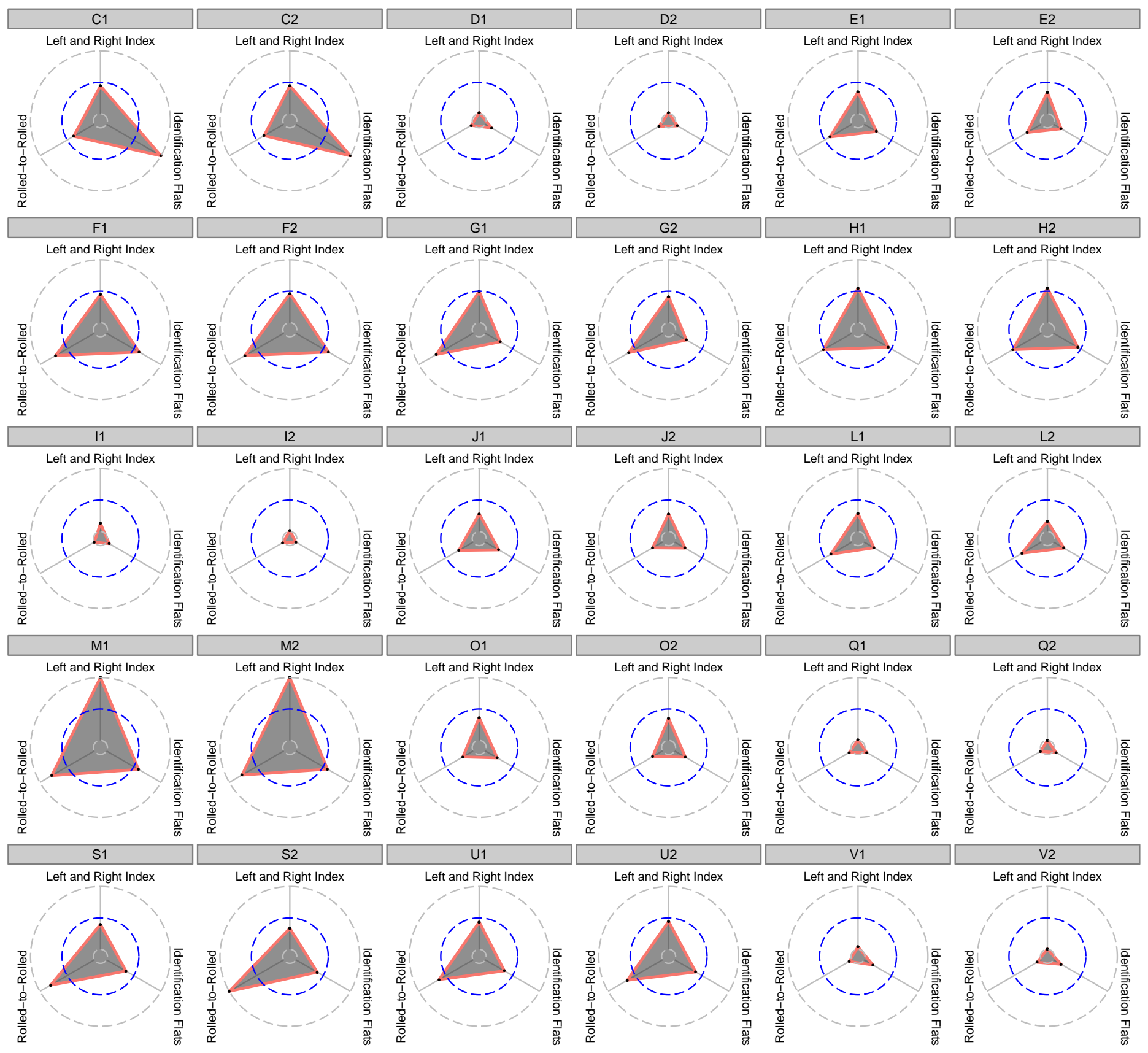

Figure 111: Star plots of relative accuracy for two-index finger, IDFlat, and ten-finger rolled-to-rolled comparisons. Values plotted along each radius are $\log _{10}$ of values from Tables 9, 13, and 15, scaled independently from 0 to 1 . Values printed as NA in those tables were set to 1 prior to scaling. 30000 searches were run against an enrollment set of 1600000 subjects for left and right index, 3000000 subjects for IDFlats, and 5000000 subjects for ten-finger rolled-to-rolled. The intersection point of a radius and the circumference of a circle indicate the scaled values $0,0.5$, and 1 , with 1 resting on the outer dashed-gray circle. The title above each plot represents the participant's letter code found on the footer of this page and an identifier used to differentiate between the two submissions each participant could make.

\begin{tabular}{l|l|l|l|l|l|l}
$\mathrm{C}=$ afis team & $\mathrm{D}=3 \mathrm{M}$ Cogent & $\mathrm{E}=$ Neurotechnology & $\mathrm{F}=$ Papillon & $\mathrm{G}=$ Dermalog & $\mathrm{H}=$ Hisign Bio-Info Institute \\
$\mathrm{I}=$ NEC & $\mathrm{J}=$ Sonda & $\mathrm{K}=$ Tiger IT & $\mathrm{L}=$ Innovatrics & $\mathrm{M}=$ SPEX & $\mathrm{O}=$ ID Solutions \\
$\mathrm{P}=$ id3 & $\mathrm{Q}=$ Morpho & $\mathrm{S}=$ Decatur Industries & $\mathrm{T}=$ BIO-key & $\mathrm{U}=$ Aware & $\mathrm{V}=$ AA Technology
\end{tabular}




\section{Combined Sorted Rankings for a Theoretical Use Case}

In prior sections, numerous tables and plots were presented showcasing individual statistics of participant submissions. Tables 91 through 98 attempt to coalesce all the presented identification statistics and categorize the overall performance of the submissions. Note that these rankings are theoretical and do not imply quality or endorsement of any kind. Please read the disclaimer for more information.

For each statistic, the values and overall ranks from Sections 7 through 9 are displayed. The Score column is computed by weighting percentiles of the results per class. The weights and percentiles were chosen based on values that provided reasonable and consistent results across all enrollment sets for all classes.

$\triangleright$ Search Template Enrollment Time

$$
\begin{aligned}
& +2 B \Longleftrightarrow V_{f} \leq P_{10} \text { or } \\
& +b \Longleftrightarrow V_{f} \leq P_{15} \text { or } \\
& -b \Longleftrightarrow V_{f} \geq P_{85} \\
& +\left(N-R_{\mathrm{FNIR}}\right) \\
& +5 B \Longleftrightarrow V_{f} \leq P_{5} \quad \text { or } \\
& +3 B \Longleftrightarrow V_{f} \leq P_{15} \text { or } \\
& +B \Longleftrightarrow V_{f} \leq P_{5} \quad \text { or } \\
& -5 B \Longleftrightarrow V_{f} \geq P_{95} \text { or } \\
& -3 B \Longleftrightarrow V_{f} \geq P_{90} \text { or } \\
& -B \Longleftrightarrow V_{f} \geq P_{75}
\end{aligned}
$$$$
\triangle \text { FNIR }
$$

$\triangleright$ RAM Consumption

$$
\begin{aligned}
+2 b & \Longleftrightarrow V_{f} \leq P_{10} \quad \text { or } \\
+b & \Longleftrightarrow V_{f} \leq P_{15} \quad \text { or } \\
-2 B & \Longleftrightarrow V_{f} \geq P_{85}
\end{aligned}
$$

$\triangleright$ Identification Time

$$
\begin{aligned}
+\left(N-R_{\text {Time }}\right) & \\
+2 B & \Longleftrightarrow V_{f} \leq P_{10} \quad \text { or } \\
+B & \Longleftrightarrow V_{f} \leq P_{15} \quad \text { or } \\
-2 B & \Longleftrightarrow V_{f} \geq P_{90} \quad \text { or } \\
-B & \Longleftrightarrow V_{f} \geq P_{85}
\end{aligned}
$$

$$
\text { where } \begin{aligned}
N & =\text { number of participants for finger } \\
B & =\max \left({ }^{N} / 2.0,15\right) \\
b & =\max \left({ }^{N} / 6.0,3\right) \\
R_{f} & =\text { submission's rank for factor } f \\
V_{f} & =\text { submission's value for factor } f \\
P_{x} & =x^{t h} \text { percentile of } V_{f}
\end{aligned}
$$

As shown, the primary factors used in scoring were accuracy and identification speed. While enrollment speed is important, the FpVTE API limited the time that could be used, and most participants completed enrollment in a relatively similar timeframe. Using a very high amount of RAM was penalized heavily, as greater amounts of RAM require additional compute nodes, while less RAM could allow additional identification processes to be run, decreasing search time. High accuracy was rewarded the most of any factor-regardless of how fast or resource-friendly a submission is, it doesn't matter if the results are incorrect.

The color bands are indicative of the $80^{\text {th }}$ percentile, $55^{\text {th }}$ percentile, and below. The best performance in each category is shaded in green and the worst in pink. Time values in the Identification and Enroll columns are reported in seconds, but were originally recorded to microsecond precision. $R A M$ is the sum of the resident set sizes of the stage one identification processes over all compute nodes after returning from the identification stage one initialization method.

Some notable observations from Tables 90 through 98 include:

$\triangleright$ Some of the most accurate submissions used such a small amount of RAM that only a single compute node was necessary.

$\triangleright$ The most accurate submissions did not use the entire 90 seconds to perform identifications. This dramatically reduced the score given to submissions that used the maximum amount of time but were not with the top accuracy range.

\begin{tabular}{l|l|l|l|ll|l}
$\mathrm{C}=$ afis team & $\mathrm{D}=$ = 3M Cogent & $\mathrm{E}=$ Neurotechnology & $\mathrm{F}=$ Papillon & $\mathrm{G}=$ Dermalog & $\mathrm{H}=$ Hisign Bio-Info Institute \\
$\mathrm{I}=$ NEC & $\mathrm{J}=$ Sonda & $\mathrm{K}=$ Tiger IT & $\mathrm{L}=$ Innovatrics & $\mathrm{M}=$ SPEX & $\mathrm{O}=$ ID Solutions \\
$\mathrm{P}=\mathrm{id} 3$ & $\mathrm{Q}=$ Morpho & $\mathrm{S}=$ Decatur Industries & $\mathrm{T}=$ BIO-key & $\mathrm{U}=$ Aware & $\mathrm{V}=$ AA Technology
\end{tabular}


$\triangleright$ In Class C, there are a number of submissions that achieve an "acceptable" level of accuracy in much less time than the most accurate submissions.

\begin{tabular}{l|l|l|l|l|l|l|l}
$\mathrm{C}=$ afis team & $\mathrm{D}=3 \mathrm{M}$ Cogent & $\mathrm{E}=$ Neurotechnology & $\mathrm{F}=$ Papillon & $\mathrm{G}=$ Dermalog & $\mathrm{H}=$ Hisign Bio-Info Institute \\
$\mathrm{I}=$ NEC & $\mathrm{J}=$ Sonda & $\mathrm{K}=$ Tiger IT & $\mathrm{L}=$ Innovatrics & $\mathrm{M}=$ SPEX & $\mathrm{O}=$ ID Solutions \\
$\mathrm{P}=\mathrm{id} 3$ & $\mathrm{Q}=$ Morpho & $\mathrm{S}=$ Decatur Industries & $\mathrm{T}=$ BIO-key & $\mathrm{U}=$ Aware & $\mathrm{V}=$ AA Technology
\end{tabular}




\begin{tabular}{|c|c|c|c|c|c|c|c|c|c|c|c|}
\hline & \multicolumn{2}{|c|}{ Participant } & \multirow{2}{*}{ Score } & \multirow{2}{*}{\multicolumn{2}{|c|}{ FNIR }} & \multirow{2}{*}{\multicolumn{2}{|c|}{ Identification }} & \multirow{2}{*}{\multicolumn{2}{|c|}{ Enrollment }} & \multirow{2}{*}{\multicolumn{2}{|c|}{ RAM }} \\
\hline & Letter & Sub.\# & & & & & & & & & \\
\hline \multirow{30}{*}{ 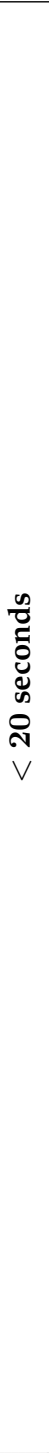 } & $\mathrm{D}$ & 1 & 109 & 1 & 0.0197 & 20 & 7.32 & 29 & 1.43 & 19 & 0.61 \\
\hline & $\mathrm{L}$ & 1 & 95 & 8 & 0.0625 & 2 & 0.29 & 1 & 0.05 & 26 & 1.14 \\
\hline & $\mathrm{V}$ & 1 & 82 & 4 & 0.0253 & 19 & 6.01 & 10 & 0.32 & 17 & 0.39 \\
\hline & $\mathrm{Q}$ & 2 & 79 & 3 & 0.0226 & 24 & 10.43 & 19 & 0.54 & 14 & 0.34 \\
\hline & $Q$ & 1 & 74 & 2 & 0.0222 & 28 & 15.70 & 19 & 0.54 & 13 & 0.34 \\
\hline & $\mathrm{I}$ & 1 & 73 & 5 & 0.0257 & 22 & 9.94 & 30 & 1.46 & 20 & 0.70 \\
\hline & $\overline{\mathrm{J}}$ & 1 & 73 & 14 & 0.0786 & 4 & 0.54 & 11 & 0.32 & 9 & 0.32 \\
\hline & $\mathrm{O}$ & 1 & 60 & 15 & 0.0818 & 5 & 0.62 & 4 & 0.27 & 15 & 0.35 \\
\hline & $\bar{E}$ & 1 & 59 & 12 & 0.0745 & 3 & 0.35 & 13 & 0.35 & 25 & 1.12 \\
\hline & $\mathrm{L}$ & 2 & 57 & 6 & 0.0351 & 12 & 3.32 & 3 & 0.15 & 28 & 2.32 \\
\hline & C & 1 & 50 & 24 & 0.1335 & 1 & 0.26 & 17 & 0.39 & 7 & 0.18 \\
\hline & $\mathrm{O}$ & 2 & 43 & 13 & 0.0766 & 9 & 1.56 & 4 & 0.27 & 15 & 0.35 \\
\hline & $\mathrm{T}$ & 2 & 43 & 9 & 0.0685 & 18 & 5.97 & 8 & 0.31 & 1 & 0.01 \\
\hline & $\bar{J}$ & 2 & 43 & 10 & 0.0712 & 7 & 1.25 & 11 & 0.32 & 9 & 0.32 \\
\hline & $F$ & 2 & 27 & 18 & 0.1082 & 14 & 3.56 & 25 & 1.07 & 5 & 0.18 \\
\hline & $\mathrm{P}$ & 2 & 25 & 22 & 0.1272 & 13 & 3.33 & 15 & 0.36 & 22 & 0.78 \\
\hline & $F$ & 1 & 24 & 20 & 0.1111 & 11 & 2.49 & 25 & 1.07 & 6 & 0.18 \\
\hline & G & 1 & 20 & 19 & 0.1089 & 21 & 9.74 & 21 & 0.55 & 18 & 0.57 \\
\hline & $\mathrm{C}$ & 2 & 14 & 25 & 0.1337 & 6 & 0.76 & 17 & 0.39 & 8 & 0.18 \\
\hline & $\mathrm{P}$ & 1 & 14 & 23 & 0.1308 & 8 & 1.32 & 15 & 0.36 & 23 & 0.78 \\
\hline & $S$ & 1 & 10 & 7 & 0.0571 & 29 & 16.86 & 22 & 0.88 & 21 & 0.72 \\
\hline & $\mathrm{H}$ & 1 & 5 & 26 & 0.1576 & 15 & 3.61 & 6 & 0.30 & 11 & 0.33 \\
\hline & $\mathrm{H}$ & 2 & 1 & 27 & 0.1607 & 17 & 4.13 & 6 & 0.30 & 11 & 0.33 \\
\hline & $\mathrm{U}$ & 1 & -2 & 21 & 0.1218 & 27 & 14.42 & 2 & 0.15 & 27 & 1.70 \\
\hline & $\mathrm{K}$ & 2 & -10 & 16 & 0.0875 & 23 & 10.32 & 23 & 1.05 & 30 & 3.87 \\
\hline & $\mathrm{E}$ & 2 & -11 & 11 & 0.0723 & 30 & 16.90 & 13 & 0.35 & 24 & 1.11 \\
\hline & $\mathrm{K}$ & 1 & -12 & 17 & 0.0883 & 25 & 10.44 & 23 & 1.05 & 29 & 3.87 \\
\hline & $\mathrm{M}$ & 1 & -49 & 29 & 0.2995 & 10 & 1.84 & 27 & 1.07 & 3 & 0.14 \\
\hline & $\mathrm{T}$ & 1 & -51 & 30 & NA & 16 & 3.99 & 8 & 0.31 & 2 & 0.01 \\
\hline & $\mathrm{M}$ & 2 & -55 & 28 & 0.2921 & 26 & 10.70 & 27 & 1.07 & 4 & 0.14 \\
\hline \multirow{6}{*}{ 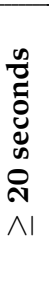 } & $\mathrm{D}$ & 2 & 84 & 1 & 0.0197 & 3 & 41.84 & 5 & 1.42 & 3 & 0.61 \\
\hline & $\mathrm{S}$ & 2 & 4 & 4 & 0.0650 & 4 & 44.66 & 4 & 0.88 & 4 & 0.72 \\
\hline & I & 2 & 3 & 3 & 0.0278 & 2 & 40.99 & 6 & 2.15 & 5 & 1.04 \\
\hline & $\mathrm{V}$ & 2 & -5 & 2 & 0.0252 & 6 & 65.35 & 2 & 0.32 & 1 & 0.39 \\
\hline & G & 2 & -13 & 5 & 0.1086 & 5 & 54.03 & 3 & 0.55 & 2 & 0.57 \\
\hline & $\mathrm{U}$ & 2 & -40 & 6 & 0.1178 & 1 & 24.16 & 1 & 0.15 & 6 & 1.42 \\
\hline
\end{tabular}

Table 90: Tabulation of operationally-ranked results for Class A - Left Index. Submissions were split into two groups. The first group includes submissions that performed searches on average in less than 20 seconds, and the second includes those that took, on average, 20 seconds or longer. Letter refers to the participant's letter code found on the footer of this page. Sub. \# is an identifier used to differentiate between the two submissions each participant could make. Score refers to the sum of the scoring equations shown in Appendix L. The color bands are indicative of the $80^{t h}$ percentile, $55^{\text {th }}$ percentile, and below of the Score column. The FNIR column was computed at the score threshold that gave FPIR $=10^{-3}$. NA indicates that the operations required to produce the value could not be performed. The Identification column shows the time used to perform a search over an enrollment set of 100000 , as seen in Table 17. The Enrollment column shows the time used to create a search template to be used for a query, as seen in Table 72. Identification and Enrollment durations are reported in seconds, but were originally recorded to microsecond precision. RAM refers to the sum of the resident set sizes of the stage one identification processes over all compute nodes after returning from the identification stage one initialization method, as seen in Table 60. RAM is reported in gigabytes, where 1 GB is equal to 1073741824 bytes. The number to the left of a value provides the value's column-wise ranking, with the best performance shaded in green and the worst in pink. The table is sorted on descending Score.

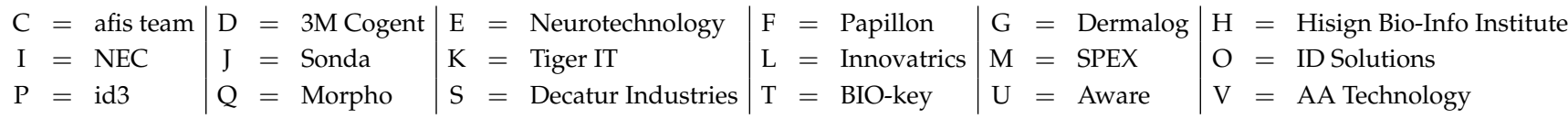




\begin{tabular}{|c|c|c|c|c|c|c|c|c|c|c|c|}
\hline & \multicolumn{2}{|c|}{ Participant } & \multirow{2}{*}{ Score } & \multirow{2}{*}{\multicolumn{2}{|c|}{ FNIR }} & \multirow{2}{*}{\multicolumn{2}{|c|}{ Identification }} & \multirow{2}{*}{\multicolumn{2}{|c|}{ Enrollment }} & \multirow{2}{*}{\multicolumn{2}{|c|}{ RAM }} \\
\hline & Letter & Sub.\# & & & & & & & & & \\
\hline \multirow{30}{*}{ 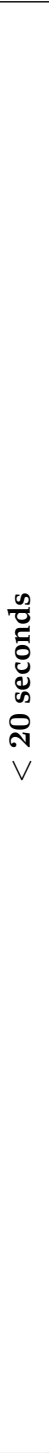 } & $\mathrm{Q}$ & 2 & 111 & 2 & 0.0214 & 23 & 9.98 & 19 & 0.54 & 11 & 0.33 \\
\hline & $\mathrm{D}$ & 1 & 109 & 1 & 0.0190 & 20 & 7.46 & 29 & 1.43 & 19 & 0.61 \\
\hline & $\mathrm{L}$ & 1 & 95 & 8 & 0.0505 & 2 & 0.26 & 1 & 0.05 & 26 & 1.14 \\
\hline & $\mathrm{V}$ & 1 & 81 & 5 & 0.0223 & 18 & 5.60 & 10 & 0.32 & 17 & 0.39 \\
\hline & $\mathrm{E}$ & 1 & 76 & 11 & 0.0630 & 3 & 0.32 & 13 & 0.36 & 24 & 1.07 \\
\hline & I & 1 & 74 & 3 & 0.0215 & 22 & 9.83 & 30 & 1.46 & 20 & 0.69 \\
\hline & $\mathrm{O}$ & 1 & 58 & 17 & 0.0776 & 5 & 0.56 & 4 & 0.27 & 15 & 0.34 \\
\hline & $\mathrm{L}$ & 2 & 57 & 6 & 0.0295 & 13 & 3.26 & 3 & 0.16 & 28 & 2.32 \\
\hline & $\mathrm{J}$ & 1 & 55 & 16 & 0.0708 & 4 & 0.51 & 11 & 0.33 & 10 & 0.31 \\
\hline & $\mathrm{C}$ & 1 & 50 & 24 & 0.1132 & 1 & 0.24 & 17 & 0.39 & 7 & 0.17 \\
\hline & $\mathrm{Q}$ & 1 & 43 & 4 & 0.0218 & 28 & 14.24 & 19 & 0.54 & 11 & 0.33 \\
\hline & $\mathrm{O}$ & 2 & 43 & 13 & 0.0675 & 9 & 1.36 & 4 & 0.27 & 16 & 0.34 \\
\hline & $\mathrm{T}$ & 2 & 43 & 9 & 0.0562 & 19 & 5.87 & 8 & 0.31 & 1 & 0.01 \\
\hline & $\bar{J}$ & 2 & 41 & 12 & 0.0643 & 7 & 1.13 & 11 & 0.33 & 9 & 0.31 \\
\hline & F & 2 & 27 & 18 & 0.0903 & 15 & 3.60 & 25 & 1.09 & 5 & 0.17 \\
\hline & $\mathrm{P}$ & 2 & 25 & 22 & 0.1100 & 12 & 3.07 & 15 & 0.37 & 23 & 0.76 \\
\hline & $\mathrm{F}$ & 1 & 24 & 20 & 0.0933 & 11 & 2.38 & 25 & 1.09 & 6 & 0.17 \\
\hline & G & 1 & 20 & 19 & 0.0910 & 24 & 10.10 & 21 & 0.55 & 18 & 0.57 \\
\hline & $\mathrm{C}$ & 2 & 16 & 23 & 0.1124 & 6 & 0.69 & 17 & 0.39 & 8 & 0.18 \\
\hline & $\mathrm{P}$ & 1 & 12 & 25 & 0.1133 & 8 & 1.24 & 15 & 0.37 & 22 & 0.76 \\
\hline & $\mathrm{S}$ & 1 & 9 & 7 & 0.0442 & 30 & 16.55 & 22 & 0.88 & 21 & 0.71 \\
\hline & $\mathrm{H}$ & 1 & 5 & 26 & 0.1230 & 14 & 3.56 & 6 & 0.30 & 14 & 0.33 \\
\hline & $\mathrm{H}$ & 2 & 1 & 27 & 0.1249 & 17 & 4.05 & 6 & 0.30 & 13 & 0.33 \\
\hline & $\mathrm{U}$ & 1 & -3 & 21 & 0.0996 & 27 & 10.76 & 2 & 0.15 & 27 & 1.56 \\
\hline & $\mathrm{K}$ & 2 & -9 & 15 & 0.0685 & 25 & 10.27 & 23 & 1.05 & 29 & 3.87 \\
\hline & K & 1 & -9 & 14 & 0.0682 & 26 & 10.42 & 23 & 1.05 & 30 & 3.87 \\
\hline & $\mathrm{E}$ & 2 & -10 & 10 & 0.0624 & 29 & 16.27 & 13 & 0.36 & 25 & 1.07 \\
\hline & $\mathrm{T}$ & 1 & -19 & 28 & 0.1929 & 16 & 3.73 & 8 & 0.31 & 1 & 0.01 \\
\hline & $\mathrm{M}$ & 1 & -50 & 30 & 0.2615 & 10 & 1.78 & 27 & 1.09 & 3 & 0.14 \\
\hline & M & 2 & -80 & 29 & 0.2526 & 21 & 9.39 & 27 & 1.09 & 3 & 0.14 \\
\hline \multirow{6}{*}{ 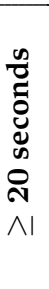 } & $\bar{D}$ & 2 & 84 & 1 & 0.0190 & 2 & 28.64 & 5 & 1.43 & 3 & 0.61 \\
\hline & I & 2 & 19 & 2 & 0.0214 & 3 & 40.35 & 6 & 2.15 & 5 & 1.01 \\
\hline & $\mathrm{S}$ & 2 & 4 & 4 & 0.0503 & 4 & 46.05 & 4 & 0.88 & 4 & 0.71 \\
\hline & G & 2 & -13 & 5 & 0.0909 & 5 & 59.55 & 3 & 0.55 & 2 & 0.57 \\
\hline & V & 2 & -21 & 3 & 0.0222 & 6 & 61.24 & 2 & 0.32 & 1 & 0.39 \\
\hline & $\mathrm{U}$ & 2 & -40 & 6 & 0.1007 & 1 & 20.10 & 1 & 0.15 & 6 & 1.30 \\
\hline
\end{tabular}

Table 91: Tabulation of operationally-ranked results for Class A - Right Index. Submissions were split into two groups. The first group includes submissions that performed searches on average in less than 20 seconds, and the second includes those that took, on average, 20 seconds or longer. Letter refers to the participant's letter code found on the footer of this page. Sub. \# is an identifier used to differentiate between the two submissions each participant could make. Score refers to the sum of the scoring equations shown in Appendix L. The color bands are indicative of the $80^{t h}$ percentile, $55^{\text {th }}$ percentile, and below of the Score column. The FNIR column was computed at the score threshold that gave FPIR $=10^{-3}$. The Identification column shows the time used to perform a search over an enrollment set of 100000 , as seen in Table 17. The Enrollment column shows the time used to create a search template to be used for a query, as seen in Table 73. Identification and Enrollment durations are reported in seconds, but were originally recorded to microsecond precision. RAM refers to the sum of the resident set sizes of the stage one identification processes over all compute nodes after returning from the identification stage one initialization method, as seen in Table 61. RAM is reported in gigabytes, where 1 GB is equal to 1073741824 bytes. The number to the left of a value provides the value's column-wise ranking, with the best performance shaded in green and the worst in pink. The table is sorted on descending Score.

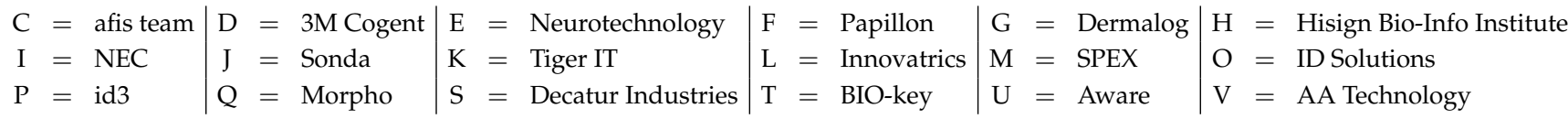




\begin{tabular}{|c|c|c|c|c|c|c|c|c|c|c|c|}
\hline & \multicolumn{2}{|c|}{ Participant } & \multirow{2}{*}{ Score } & \multirow{2}{*}{\multicolumn{2}{|c|}{ FNIR }} & \multirow{2}{*}{\multicolumn{2}{|c|}{ Identification }} & \multirow{2}{*}{\multicolumn{2}{|c|}{ Enrollment }} & \multirow{2}{*}{\multicolumn{2}{|c|}{ RAM }} \\
\hline & Letter & Sub. \# & & & & & & & & & \\
\hline \multirow{9}{*}{ 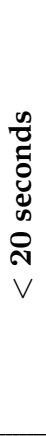 } & $\mathrm{V}$ & 1 & 87 & 1 & 0.0034 & 5 & 9.29 & 3 & 0.64 & 5 & 11.77 \\
\hline & $\mathrm{L}$ & 1 & 43 & 4 & 0.0146 & 2 & 2.19 & 1 & 0.11 & 8 & 18.76 \\
\hline & $\overline{\mathrm{J}}$ & 1 & 24 & 3 & 0.0143 & 6 & 13.64 & 4 & 0.65 & 3 & 8.30 \\
\hline & I & 1 & 19 & 2 & 0.0058 & 8 & 17.87 & 9 & 2.93 & 6 & 15.83 \\
\hline & $\mathrm{C}$ & 1 & 12 & 7 & 0.0368 & 1 & 2.08 & 5 & 0.78 & 2 & 4.87 \\
\hline & $\mathrm{O}$ & 1 & 9 & 5 & 0.0229 & 7 & 14.46 & 2 & 0.53 & 4 & 9.05 \\
\hline & C & 2 & -3 & 8 & 0.0374 & 4 & 6.35 & 5 & 0.78 & 1 & 4.87 \\
\hline & $\mathrm{K}$ & 1 & -44 & 6 & 0.0360 & 9 & 18.01 & 8 & 2.09 & 9 & 61.81 \\
\hline & G & 1 & -69 & 9 & 0.0515 & 3 & 5.22 & 7 & 1.09 & 7 & 16.38 \\
\hline \multirow{27}{*}{ 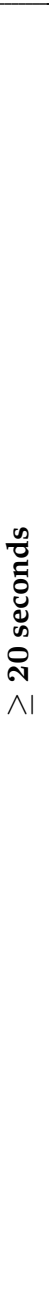 } & $\bar{Q}$ & 2 & $\overline{109}$ & 1 & 0.0027 & 19 & 161.02 & 15 & 1.08 & 9 & 9.14 \\
\hline & $\mathrm{Q}$ & 1 & 107 & 1 & 0.0027 & 21 & 212.69 & 15 & 1.08 & 10 & 9.14 \\
\hline & $\mathrm{L}$ & 2 & 89 & 7 & 0.0072 & 3 & 23.42 & 3 & 0.31 & 26 & 53.98 \\
\hline & $\mathrm{V}$ & 2 & 78 & 3 & 0.0028 & 18 & 127.65 & 9 & 0.64 & 13 & 11.77 \\
\hline & $\mathrm{D}$ & 1 & 74 & 4 & 0.0030 & 15 & 70.99 & 25 & 2.84 & 17 & 18.58 \\
\hline & $\mathrm{E}$ & 1 & 71 & 11 & 0.0207 & 1 & 16.35 & 11 & 0.70 & 22 & 33.40 \\
\hline & $S$ & 1 & 70 & 13 & 0.0281 & 2 & 23.00 & 18 & 1.75 & 14 & 14.82 \\
\hline & $\mathrm{D}$ & 2 & 68 & 4 & 0.0030 & 23 & 237.43 & 26 & 2.84 & 17 & 18.58 \\
\hline & $\bar{J}$ & 2 & 39 & 8 & 0.0143 & 7 & 33.35 & 10 & 0.65 & 7 & 8.30 \\
\hline & $\mathrm{O}$ & 2 & 36 & 12 & 0.0214 & 10 & 43.53 & 4 & 0.53 & 8 & 9.05 \\
\hline & $\mathrm{I}$ & 2 & 36 & 4 & 0.0030 & 25 & 338.88 & 27 & 4.30 & 21 & 30.83 \\
\hline & $\mathrm{T}$ & 2 & 35 & 19 & 0.0366 & 9 & 37.23 & 7 & 0.62 & 1 & 0.01 \\
\hline & $\mathrm{U}$ & 1 & 26 & 17 & 0.0336 & 11 & 45.51 & 1 & 0.30 & 25 & 44.63 \\
\hline & $\mathrm{P}$ & 2 & 21 & 16 & 0.0333 & 17 & 101.16 & 13 & 0.72 & 19 & 21.41 \\
\hline & $\mathrm{P}$ & 1 & 20 & 20 & 0.0370 & 14 & 63.65 & 13 & 0.72 & 20 & 21.42 \\
\hline & $G$ & 2 & 17 & 15 & 0.0311 & 22 & 221.16 & 17 & 1.09 & 16 & 16.38 \\
\hline & $\mathrm{H}$ & 1 & 7 & 24 & 0.0686 & 8 & 36.71 & 5 & 0.60 & 12 & 9.36 \\
\hline & $\mathrm{F}$ & 1 & 5 & 21 & 0.0386 & 13 & 60.66 & 21 & 2.15 & 5 & 4.86 \\
\hline & $\mathrm{K}$ & 2 & 4 & 14 & 0.0286 & 6 & 32.93 & 20 & 2.09 & 27 & 61.81 \\
\hline & $\mathrm{H}$ & 2 & 4 & 23 & 0.0684 & 12 & 52.25 & 5 & 0.60 & 11 & 9.36 \\
\hline & $\bar{F}$ & 2 & 2 & 22 & 0.0412 & 16 & 73.95 & 21 & 2.15 & 5 & 4.86 \\
\hline & $\mathrm{U}$ & 2 & -3 & 18 & 0.0358 & 24 & 240.40 & 1 & 0.30 & 24 & 37.35 \\
\hline & $\mathrm{S}$ & 2 & -11 & 9 & 0.0195 & 26 & 495.50 & 18 & 1.75 & 14 & 14.82 \\
\hline & $E$ & 2 & -13 & 10 & 0.0202 & 27 & 518.11 & 11 & 0.70 & 23 & 33.41 \\
\hline & $\mathrm{T}$ & 1 & -28 & 27 & NA & 4 & 25.68 & 7 & 0.62 & 1 & 0.01 \\
\hline & $\mathrm{M}$ & 2 & -36 & 25 & NA & 20 & 171.24 & 23 & 2.16 & 4 & 3.76 \\
\hline & M & 1 & -48 & 26 & NA & 5 & 31.44 & 23 & 2.16 & 3 & 3.75 \\
\hline
\end{tabular}

Table 92: Tabulation of operationally-ranked results for Class A - Left and Right Index. Submissions were split into two groups. The first group includes submissions that performed searches on average in less than 20 seconds, and the second includes those that took, on average, 20 seconds or longer. Letter refers to the participant's letter code found on the footer of this page. Sub. \# is an identifier used to differentiate between the two submissions each participant could make. Score refers to the sum of the scoring equations shown in Appendix L. The color bands are indicative of the $80^{\text {th }}$ percentile, $55^{\text {th }}$ percentile, and below of the Score column. The FNIR column was computed at the score threshold that gave FPIR $=10^{-3}$. NA indicates that the operations required to produce the value could not be performed. The Identification column shows the time used to perform a search over an enrollment set of 1600000 , as seen in Table 18. The Enrollment column shows the time used to create a search template to be used for a query, as seen in Table 74. Identification and Enrollment durations are reported in seconds, but were originally recorded to microsecond precision. RAM refers to the sum of the resident set sizes of the stage one identification processes over all compute nodes after returning from the identification stage one initialization method, as seen in Table 62. RAM is reported in gigabytes, where 1 GB is equal to 1073741824 bytes. The number to the left of a value provides the value's column-wise ranking, with the best performance shaded in green and the worst in pink. The table is sorted on descending Score.

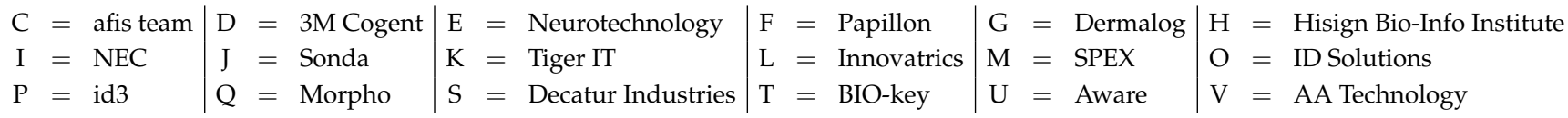




\begin{tabular}{|c|c|c|c|c|c|c|c|c|c|c|}
\hline \multicolumn{2}{|c|}{ Participant } & \multirow{3}{*}{$\begin{array}{c}\text { Score } \\
126 \\
\end{array}$} & \multirow{2}{*}{\multicolumn{2}{|c|}{ FNIR }} & \multirow{2}{*}{\multicolumn{2}{|c|}{ Identification }} & \multirow{2}{*}{\multicolumn{2}{|c|}{ Enrollment }} & \multirow{2}{*}{\multicolumn{2}{|c|}{ RAM }} \\
\hline \multirow{2}{*}{$\frac{\text { Letter }}{\mathrm{Q}}$} & \multirow{2}{*}{$\frac{\text { Sub. \# }}{1}$} & & & & & & & & & \\
\hline & & & 2 & 0.0098 & 19 & 53.24 & 20 & 3.41 & 1 & 7.54 \\
\hline I & 2 & 111 & 1 & 0.0094 & 13 & 38.26 & 30 & 7.67 & 21 & 108.71 \\
\hline $\mathrm{L}$ & 1 & 101 & 16 & 0.0288 & 3 & 5.56 & 2 & 1.11 & 22 & 119.79 \\
\hline Q & 2 & 96 & 3 & 0.0099 & 20 & 54.02 & 20 & 3.41 & 2 & 7.54 \\
\hline I & 1 & 83 & 4 & 0.0116 & 21 & 55.96 & 25 & 6.50 & 5 & 49.68 \\
\hline V & 2 & 83 & 8 & 0.0190 & 15 & 47.97 & 7 & 1.22 & 9 & 63.53 \\
\hline $\mathrm{D}$ & 2 & 79 & 5 & 0.0142 & 22 & 58.10 & 24 & 4.17 & 12 & 79.43 \\
\hline C & 1 & 76 & 22 & 0.0654 & 2 & 3.74 & 12 & 2.12 & 3 & 46.49 \\
\hline $\mathrm{E}$ & 1 & 76 & 13 & 0.0259 & 1 & 2.82 & 11 & 1.95 & 14 & 79.72 \\
\hline $\mathrm{V}$ & 1 & 71 & 9 & 0.0192 & 11 & 36.07 & 7 & 1.22 & 8 & 63.53 \\
\hline $\mathrm{L}$ & 2 & 56 & 14 & 0.0276 & 5 & 12.37 & 1 & 0.33 & 27 & 177.49 \\
\hline $\mathrm{C}$ & 2 & 55 & 21 & 0.0647 & 4 & 6.74 & 12 & 2.12 & 4 & 46.49 \\
\hline $\mathrm{D}$ & 1 & 49 & 6 & 0.0163 & 18 & 52.23 & 14 & 2.15 & 13 & 79.43 \\
\hline $\mathrm{O}$ & 1 & 42 & 12 & 0.0257 & 12 & 37.01 & 5 & 1.21 & 17 & 101.00 \\
\hline $\mathrm{J}$ & 1 & 38 & 15 & 0.0287 & 7 & 25.61 & 3 & 1.21 & 20 & 101.00 \\
\hline $\mathrm{O}$ & 2 & 30 & 11 & 0.0254 & 26 & 73.15 & 5 & 1.21 & 18 & 101.00 \\
\hline $\mathrm{E}$ & 2 & 26 & 7 & 0.0187 & 10 & 34.37 & 15 & 2.21 & 28 & 318.03 \\
\hline G & 2 & 21 & 17 & 0.0325 & 23 & 59.49 & 18 & 2.83 & 25 & 156.12 \\
\hline $\mathrm{J}$ & 2 & 8 & 10 & 0.0236 & 27 & 74.38 & 3 & 1.21 & 19 & 101.00 \\
\hline $\mathrm{H}$ & 1 & 7 & 23 & 0.0998 & 16 & 49.75 & 9 & 1.67 & 16 & 86.46 \\
\hline G & 1 & 6 & 18 & 0.0371 & 6 & 16.24 & 18 & 2.83 & 26 & 156.12 \\
\hline $\mathrm{H}$ & 2 & 2 & 24 & 0.1008 & 17 & 51.77 & 9 & 1.67 & 15 & 86.46 \\
\hline$S$ & 1 & -5 & 25 & 0.1089 & 24 & 71.69 & 22 & 3.96 & 24 & 150.35 \\
\hline$S$ & 2 & -22 & 26 & 0.1133 & 25 & 71.77 & 22 & 3.96 & 23 & 150.35 \\
\hline$F$ & 2 & -31 & 28 & 0.1681 & 14 & 43.59 & 26 & 6.73 & 10 & 77.02 \\
\hline $\mathrm{M}$ & 2 & -47 & 27 & 0.1634 & 29 & 87.21 & 28 & 6.73 & 7 & 57.53 \\
\hline $\mathrm{U}$ & 1 & -48 & 20 & 0.0500 & 28 & 80.03 & 17 & 2.42 & 29 & 440.67 \\
\hline $\mathrm{U}$ & 2 & -48 & 19 & 0.0461 & 30 & 89.07 & 16 & 2.41 & 30 & 540.60 \\
\hline $\mathrm{F}$ & 1 & -57 & 29 & 0.1684 & 8 & 30.09 & 26 & 6.73 & 11 & 77.02 \\
\hline $\mathrm{M}$ & 1 & -59 & 30 & 0.1736 & 9 & 31.17 & 28 & 6.73 & 6 & 57.53 \\
\hline
\end{tabular}

Table 93: Tabulation of operationally-ranked results for Class B - Left Slap. Letter refers to the participant's letter code found on the footer of this page. Sub. \# is an identifier used to differentiate between the two submissions each participant could make. Score refers to the sum of the scoring equations shown in Appendix L. The color bands are indicative of the $80^{\text {th }}$ percentile, $55^{\text {th }}$ percentile, and below of the Score column. The FNIR column was computed at the score threshold that gave FPIR $=10^{-3}$. The Identification column shows the time used to perform a search over an enrollment set of 3000000 , as seen in Table 19. The Enrollment column shows the time used to create a search template to be used for a query, as seen in Table 75 . Identification and Enrollment durations are reported in seconds, but were originally recorded to microsecond precision. RAM refers to the sum of the resident set sizes of the stage one identification processes over all compute nodes after returning from the identification stage one initialization method, as seen in Table 63. RAM is reported in gigabytes, where 1 GB is equal to 1073741824 bytes. The number to the left of a value provides the value's column-wise ranking, with the best performance shaded in green and the worst in pink. The table is sorted on descending Score.

\begin{tabular}{l|l|l|l|ll|l}
$\mathrm{C}=$ afis team & $\mathrm{D}=3 \mathrm{M}$ Cogent & $\mathrm{E}=$ Neurotechnology & $\mathrm{F}=$ Papillon & $\mathrm{G}=$ Dermalog & $\mathrm{H}=$ Hisign Bio-Info Institute \\
$\mathrm{I}=$ NEC & $\mathrm{J}=$ Sonda & $\mathrm{K}=$ Tiger IT & $\mathrm{L}=$ Innovatrics & $\mathrm{M}=$ SPEX & $\mathrm{O}=$ ID Solutions \\
$\mathrm{P}=\mathrm{id} 3$ & $\mathrm{Q}=$ Morpho & $\mathrm{S}=$ Decatur Industries & $\mathrm{T}=$ BIO-key & $\mathrm{U}=$ Aware & $\mathrm{V}=$ AA Technology
\end{tabular}




\begin{tabular}{|c|c|c|c|c|c|c|c|c|c|c|}
\hline \multicolumn{2}{|c|}{ Participant } & \multirow{3}{*}{$\begin{array}{c}\text { Score } \\
113\end{array}$} & \multirow{2}{*}{\multicolumn{2}{|c|}{ FNIR }} & \multirow{2}{*}{\multicolumn{2}{|c|}{ Identification }} & \multirow{2}{*}{\multicolumn{2}{|c|}{ Enrollment }} & \multirow{2}{*}{\multicolumn{2}{|c|}{ RAM }} \\
\hline \multirow{2}{*}{$\frac{\text { Letter }}{\mathrm{I}}$} & \multirow{2}{*}{$\frac{\text { Sub. \# }}{2}$} & & & & & & & & & \\
\hline & & & 1 & 0.0045 & 14 & 41.13 & 30 & 7.65 & 21 & 108.71 \\
\hline $\mathrm{D}$ & 2 & 113 & 2 & 0.0052 & 21 & 57.19 & 24 & 4.13 & 12 & 79.43 \\
\hline $\mathrm{L}$ & 1 & 102 & 15 & 0.0167 & 3 & 5.47 & 2 & 1.11 & 22 & 119.78 \\
\hline $\mathrm{Q}$ & 2 & 91 & 3 & 0.0057 & 22 & 61.94 & 20 & 3.41 & 2 & 7.54 \\
\hline$Q$ & 1 & 90 & 3 & 0.0057 & 23 & 64.04 & 20 & 3.41 & 1 & 7.54 \\
\hline V & 1 & 87 & 8 & 0.0106 & 11 & 35.43 & 3 & 1.19 & 8 & 63.53 \\
\hline $\bar{I}$ & 1 & 82 & 5 & 0.0058 & 20 & 55.87 & 25 & 6.47 & 5 & 49.68 \\
\hline $\mathrm{E}$ & 1 & 75 & 13 & 0.0151 & 1 & 2.97 & 11 & 1.91 & 14 & 79.72 \\
\hline V & 2 & 66 & 9 & 0.0110 & 16 & 48.51 & 3 & 1.19 & 9 & 63.53 \\
\hline $\mathrm{C}$ & 1 & 55 & 24 & 0.0403 & 2 & 3.67 & 12 & 2.09 & 4 & 46.49 \\
\hline $\mathrm{D}$ & 1 & 50 & 6 & 0.0072 & 19 & 53.32 & 14 & 2.14 & 13 & 79.43 \\
\hline $\mathrm{J}$ & 1 & 44 & 14 & 0.0156 & 7 & 24.14 & 5 & 1.20 & 18 & 101.00 \\
\hline C & 2 & 43 & 23 & 0.0392 & 4 & 6.50 & 12 & 2.09 & 3 & 46.49 \\
\hline $\mathrm{O}$ & 1 & 37 & 12 & 0.0142 & 12 & 35.61 & 7 & 1.20 & 17 & 101.00 \\
\hline $\mathrm{L}$ & 2 & 37 & 17 & 0.0202 & 6 & 12.66 & 1 & 0.33 & 27 & 177.49 \\
\hline G & 2 & 31 & 16 & 0.0198 & 13 & 37.28 & 18 & 2.78 & 25 & 156.12 \\
\hline $\mathrm{E}$ & 2 & 26 & 7 & 0.0083 & 10 & 34.47 & 15 & 2.18 & 28 & 318.00 \\
\hline G & 1 & 22 & 18 & 0.0212 & 5 & 10.13 & 18 & 2.78 & 26 & 156.12 \\
\hline $\mathrm{S}$ & 2 & 14 & 22 & 0.0381 & 25 & 70.84 & 22 & 3.88 & 24 & 150.35 \\
\hline S & 1 & 14 & 21 & 0.0369 & 24 & 70.54 & 22 & 3.88 & 23 & 150.35 \\
\hline $\mathrm{J}$ & 2 & 13 & 10 & 0.0126 & 27 & 74.09 & 5 & 1.20 & 19 & 101.00 \\
\hline $\mathrm{O}$ & 2 & 8 & 11 & 0.0132 & 26 & 73.93 & 7 & 1.20 & 20 & 101.00 \\
\hline $\mathrm{H}$ & 1 & 3 & 25 & 0.0641 & 17 & 50.16 & 9 & 1.63 & 16 & 86.46 \\
\hline $\mathrm{H}$ & 2 & 1 & 26 & 0.0647 & 18 & 52.84 & 9 & 1.63 & 15 & 86.46 \\
\hline $\mathrm{F}$ & 2 & -32 & 28 & 0.1220 & 15 & 45.92 & 26 & 6.68 & 10 & 77.02 \\
\hline M & 2 & -47 & 27 & 0.1155 & 29 & 87.41 & 28 & 6.69 & 6 & 57.53 \\
\hline $\mathrm{U}$ & 1 & -48 & 19 & 0.0266 & 30 & 89.26 & 16 & 2.39 & 29 & 440.67 \\
\hline $\mathrm{U}$ & 2 & -48 & 20 & 0.0273 & 28 & 80.11 & 17 & 2.39 & 30 & 540.59 \\
\hline $\mathrm{F}$ & 1 & -57 & 29 & 0.1222 & 8 & 31.87 & 26 & 6.68 & 11 & 77.02 \\
\hline $\mathrm{M}$ & 1 & -59 & 30 & 0.1259 & 9 & 32.03 & 28 & 6.69 & 7 & 57.53 \\
\hline
\end{tabular}

Table 94: Tabulation of operationally-ranked results for Class B - Right Slap. Letter refers to the participant's letter code found on the footer of this page. Sub. \# is an identifier used to differentiate between the two submissions each participant could make. Score refers to the sum of the scoring equations shown in Appendix L. The color bands are indicative of the $80^{\text {th }}$ percentile, $55^{\text {th }}$ percentile, and below of the Score column. The FNIR column was computed at the score threshold that gave FPIR $=10^{-3}$. The Identification column shows the time used to perform a search over an enrollment set of 3000000 , as seen in Table 19. The Enrollment column shows the time used to create a search template to be used for a query, as seen in Table 76. Identification and Enrollment durations are reported in seconds, but were originally recorded to microsecond precision. RAM refers to the sum of the resident set sizes of the stage one identification processes over all compute nodes after returning from the identification stage one initialization method, as seen in Table 63. RAM is reported in gigabytes, where 1 GB is equal to 1073741824 bytes. The number to the left of a value provides the value's column-wise ranking, with the best performance shaded in green and the worst in pink. The table is sorted on descending Score.

\begin{tabular}{l|l|l|l|ll|l}
$\mathrm{C}=$ afis team & $\mathrm{D}=3 \mathrm{M}$ Cogent & $\mathrm{E}=$ Neurotechnology & $\mathrm{F}=$ Papillon & $\mathrm{G}=$ Dermalog & $\mathrm{H}=$ Hisign Bio-Info Institute \\
$\mathrm{I}=$ NEC & $\mathrm{J}=$ Sonda & $\mathrm{K}=$ Tiger IT & $\mathrm{L}=$ Innovatrics & $\mathrm{M}=$ SPEX & $\mathrm{O}=$ ID Solutions \\
$\mathrm{P}=\mathrm{id} 3$ & $\mathrm{Q}=$ Morpho & $\mathrm{S}=$ Decatur Industries & $\mathrm{T}=$ BIO-key & $\mathrm{U}=$ Aware & $\mathrm{V}=$ AA Technology
\end{tabular}




\begin{tabular}{|c|c|c|c|c|c|c|c|c|c|c|}
\hline \multicolumn{2}{|c|}{ Participant } & \multirow{3}{*}{$\begin{array}{c}\text { Score } \\
123 \\
\end{array}$} & \multirow{2}{*}{\multicolumn{2}{|c|}{ FNIR }} & \multirow{2}{*}{\multicolumn{2}{|c|}{ Identification }} & \multirow{2}{*}{\multicolumn{2}{|c|}{ Enrollment }} & \multirow{2}{*}{\multicolumn{2}{|c|}{ RAM }} \\
\hline \multirow{2}{*}{$\frac{\text { Letter }}{\mathrm{Q}}$} & \multirow{2}{*}{$\frac{\text { Sub. \# }}{1}$} & & & & & & & & & \\
\hline & & & 2 & 0.0021 & 22 & 65.02 & 20 & 6.84 & 2 & 7.54 \\
\hline I & 2 & 110 & 1 & 0.0015 & 17 & 46.13 & 30 & 15.33 & 21 & 108.71 \\
\hline$Q$ & 2 & 94 & 3 & 0.0022 & 19 & 53.40 & 20 & 6.84 & 1 & 7.54 \\
\hline I & 1 & 92 & 3 & 0.0022 & 13 & 37.19 & 25 & 12.96 & 5 & 49.68 \\
\hline $\mathrm{L}$ & 1 & 89 & 12 & 0.0054 & 4 & 10.48 & 2 & 2.23 & 22 & 119.77 \\
\hline $\mathrm{V}$ & 1 & 87 & 7 & 0.0036 & 15 & 38.93 & 7 & 2.40 & 9 & 63.53 \\
\hline $\mathrm{V}$ & 2 & 81 & 7 & 0.0036 & 18 & 52.80 & 7 & 2.40 & 8 & 63.53 \\
\hline $\mathrm{D}$ & 2 & 76 & 5 & 0.0024 & 21 & 63.05 & 24 & 8.28 & 13 & 79.43 \\
\hline $\mathrm{E}$ & 1 & 72 & 15 & 0.0063 & 2 & 6.12 & 11 & 3.85 & 14 & 79.72 \\
\hline $\mathrm{D}$ & 1 & 48 & 6 & 0.0031 & 20 & 61.24 & 14 & 4.28 & 12 & 79.43 \\
\hline $\bar{G}$ & 1 & 41 & 18 & 0.0106 & 1 & 3.89 & 18 & 5.62 & 26 & 156.12 \\
\hline $\mathrm{L}$ & 2 & 40 & 14 & 0.0062 & 6 & 20.78 & 1 & 0.66 & 27 & 177.49 \\
\hline $\mathrm{O}$ & 1 & 39 & 13 & 0.0057 & 14 & 38.34 & 3 & 2.40 & 20 & 101.00 \\
\hline $\mathrm{J}$ & 1 & 37 & 16 & 0.0068 & 7 & 26.15 & 5 & 2.40 & 18 & 101.00 \\
\hline $\mathrm{O}$ & 2 & 29 & 11 & 0.0051 & 23 & 68.46 & 3 & 2.40 & 19 & 101.00 \\
\hline $\mathrm{G}$ & 2 & 29 & 17 & 0.0084 & 11 & 34.55 & 18 & 5.62 & 25 & 156.12 \\
\hline $\mathrm{J}$ & 2 & 10 & 9 & 0.0047 & 24 & 69.28 & 5 & 2.40 & 17 & 101.00 \\
\hline $\mathrm{E}$ & 2 & 8 & 10 & 0.0049 & 10 & 33.09 & 15 & 4.38 & 28 & 317.99 \\
\hline$M$ & 1 & 4 & 27 & 0.0904 & 8 & 27.15 & 28 & 13.53 & 7 & 57.53 \\
\hline $\mathrm{F}$ & 2 & 4 & 26 & 0.0901 & 12 & 36.36 & 26 & 13.49 & 11 & 77.02 \\
\hline $\mathrm{H}$ & 1 & 0 & 23 & 0.0349 & 25 & 70.38 & 9 & 3.31 & 16 & 86.46 \\
\hline $\mathrm{M}$ & 2 & -1 & 25 & 0.0882 & 16 & 45.91 & 28 & 13.53 & 6 & 57.53 \\
\hline $\mathrm{H}$ & 2 & -2 & 24 & 0.0361 & 26 & 73.06 & 9 & 3.31 & 15 & 86.46 \\
\hline$S$ & 2 & -4 & 22 & 0.0190 & 27 & 82.13 & 22 & 7.83 & 24 & 150.35 \\
\hline $\mathrm{C}$ & 1 & -7 & 30 & NA & 3 & 6.40 & 12 & 4.20 & 3 & 46.49 \\
\hline$S$ & 1 & -19 & 21 & 0.0160 & 29 & 83.33 & 22 & 7.83 & 23 & 150.35 \\
\hline $\mathrm{F}$ & 1 & -26 & 28 & 0.0910 & 9 & 27.27 & 26 & 13.49 & 10 & 77.02 \\
\hline C & 2 & -29 & 29 & NA & 5 & 10.70 & 12 & 4.20 & 4 & 46.49 \\
\hline $\mathrm{U}$ & 1 & -49 & 20 & 0.0139 & 30 & 83.62 & 16 & 4.82 & 29 & 440.67 \\
\hline $\mathrm{U}$ & 2 & -49 & 19 & 0.0124 & 28 & 82.40 & 17 & 4.83 & 30 & 540.59 \\
\hline
\end{tabular}

Table 95: Tabulation of operationally-ranked results for Class B - Left and Right Slap. Letter refers to the participant's letter code found on the footer of this page. Sub. \# is an identifier used to differentiate between the two submissions each participant could make. Score refers to the sum of the scoring equations shown in Appendix L. The color bands are indicative of the $80^{\text {th }}$ percentile, $55^{\text {th }}$ percentile, and below of the Score column. The FNIR column was computed at the score threshold that gave FPIR $=10^{-3}$. NA indicates that the operations required to produce the value could not be performed. The Identification column shows the time used to perform a search over an enrollment set of 3000000 , as seen in Table 19. The Enrollment column shows the time used to create a search template to be used for a query, as seen in Table 77. Identification and Enrollment durations are reported in seconds, but were originally recorded to microsecond precision. RAM refers to the sum of the resident set sizes of the stage one identification processes over all compute nodes after returning from the identification stage one initialization method, as seen in Table 63. RAM is reported in gigabytes, where 1 GB is equal to 1073741824 bytes. The number to the left of a value provides the value's column-wise ranking, with the best performance shaded in green and the worst in pink. The table is sorted on descending Score.

\begin{tabular}{l|l|l|l|ll|l}
$\mathrm{C}=$ afis team & $\mathrm{D}=3 \mathrm{M}$ Cogent & $\mathrm{E}=$ Neurotechnology & $\mathrm{F}=$ Papillon & $\mathrm{G}=$ Dermalog & $\mathrm{H}=$ Hisign Bio-Info Institute \\
$\mathrm{I}=$ NEC & $\mathrm{J}=$ Sonda & $\mathrm{K}=$ Tiger IT & $\mathrm{L}=$ Innovatrics & $\mathrm{M}=$ SPEX & $\mathrm{O}=$ ID Solutions \\
$\mathrm{P}=\mathrm{id} 3$ & $\mathrm{Q}=$ Morpho & $\mathrm{S}=$ Decatur Industries & $\mathrm{T}=$ BIO-key & $\mathrm{U}=$ Aware & $\mathrm{V}=$ AA Technology
\end{tabular}




\begin{tabular}{|c|c|c|c|c|c|c|c|c|c|c|}
\hline \multicolumn{2}{|c|}{ Participant } & \multirow{3}{*}{$\begin{array}{c}\text { Score } \\
132 \\
\end{array}$} & \multirow{2}{*}{\multicolumn{2}{|c|}{ FNIR }} & \multirow{2}{*}{\multicolumn{2}{|c|}{ Identification }} & \multirow{2}{*}{\multicolumn{2}{|c|}{ Enrollment }} & \multirow{2}{*}{\multicolumn{2}{|c|}{ RAM }} \\
\hline \multirow{2}{*}{$\frac{\text { Letter }}{\mathrm{I}}$} & \multirow{2}{*}{$\frac{\text { Sub. \# }}{1}$} & & & & & & & & & \\
\hline & & & 2 & 0.0012 & 7 & 19.07 & 25 & 16.52 & 5 & 49.68 \\
\hline$Q$ & 1 & 129 & 2 & 0.0012 & 14 & 42.92 & 20 & 8.92 & 1 & 7.54 \\
\hline $\mathrm{Q}$ & 2 & 119 & 2 & 0.0012 & 24 & 66.01 & 20 & 8.92 & 2 & 7.54 \\
\hline $\mathrm{D}$ & 2 & 114 & 2 & 0.0012 & 18 & 46.70 & 24 & 11.86 & 13 & 79.43 \\
\hline I & 2 & 109 & 1 & 0.0009 & 16 & 43.86 & 30 & 19.36 & 21 & 108.71 \\
\hline $\mathrm{L}$ & 1 & 90 & 10 & 0.0031 & 5 & 14.50 & 2 & 3.05 & 22 & 119.79 \\
\hline $\mathrm{V}$ & 2 & 83 & 7 & 0.0024 & 19 & 49.30 & 7 & 3.36 & 9 & 63.53 \\
\hline$E$ & 1 & 72 & 16 & 0.0043 & 2 & 6.76 & 13 & 5.34 & 14 & 79.73 \\
\hline G & 1 & 71 & 18 & 0.0062 & 1 & 4.26 & 18 & 7.90 & 25 & 156.12 \\
\hline $\mathrm{V}$ & 1 & 71 & 9 & 0.0027 & 10 & 34.96 & 7 & 3.36 & 8 & 63.53 \\
\hline $\mathrm{D}$ & 1 & 52 & 6 & 0.0020 & 17 & 45.15 & 17 & 6.16 & 12 & 79.43 \\
\hline $\mathrm{L}$ & 2 & 41 & 11 & 0.0033 & 9 & 28.59 & 1 & 0.88 & 27 & 177.48 \\
\hline $\mathrm{O}$ & 1 & 39 & 15 & 0.0041 & 11 & 35.64 & 5 & 3.36 & 19 & 101.00 \\
\hline $\mathrm{J}$ & 1 & 36 & 17 & 0.0049 & 8 & 25.38 & 3 & 3.35 & 18 & 101.00 \\
\hline $\mathrm{O}$ & 2 & 31 & 13 & 0.0035 & 21 & 60.02 & 5 & 3.36 & 20 & 101.00 \\
\hline $\mathrm{J}$ & 2 & 27 & 11 & 0.0033 & 22 & 60.13 & 3 & 3.35 & 17 & 101.00 \\
\hline $\mathrm{E}$ & 2 & 20 & 7 & 0.0024 & 15 & 43.61 & 16 & 5.93 & 28 & 317.95 \\
\hline G & 2 & 7 & 14 & 0.0040 & 6 & 16.26 & 18 & 7.90 & 26 & 156.12 \\
\hline $\mathrm{M}$ & 1 & 1 & 26 & 0.0543 & 13 & 38.87 & 28 & 18.11 & 6 & 57.53 \\
\hline $\mathrm{M}$ & 2 & -8 & 25 & 0.0515 & 23 & 65.91 & 28 & 18.11 & 6 & 57.53 \\
\hline $\mathrm{C}$ & 1 & -8 & 30 & NA & 3 & 7.92 & 11 & 5.13 & 3 & 46.49 \\
\hline $\mathrm{U}$ & 2 & -17 & 22 & 0.0141 & 25 & 75.28 & 14 & 5.73 & 30 & 540.60 \\
\hline $\mathrm{S}$ & 1 & -18 & 20 & 0.0108 & 29 & 87.60 & 22 & 10.31 & 24 & 150.35 \\
\hline $\mathrm{H}$ & 1 & -19 & 23 & 0.0203 & 26 & 82.66 & 9 & 4.37 & 15 & 86.46 \\
\hline$S$ & 2 & -20 & 21 & 0.0136 & 30 & 88.46 & 22 & 10.31 & 23 & 150.35 \\
\hline $\mathrm{H}$ & 2 & -21 & 24 & 0.0204 & 27 & 86.56 & 9 & 4.37 & 16 & 86.46 \\
\hline $\mathrm{C}$ & 2 & -28 & 29 & NA & 4 & 10.21 & 11 & 5.13 & 4 & 46.49 \\
\hline $\mathrm{F}$ & 1 & -29 & 27 & 0.0591 & 12 & 37.56 & 26 & 18.07 & 10 & 77.02 \\
\hline $\mathrm{F}$ & 2 & -33 & 27 & 0.0591 & 20 & 49.34 & 26 & 18.07 & 10 & 77.02 \\
\hline $\mathrm{U}$ & 1 & -49 & 19 & 0.0099 & 28 & 86.60 & 15 & 5.82 & 29 & 440.68 \\
\hline
\end{tabular}

Table 96: Tabulation of operationally-ranked results for Class B - Identification Flats. Letter refers to the participant's letter code found on the footer of this page. Sub. \# is an identifier used to differentiate between the two submissions each participant could make. Score refers to the sum of the scoring equations shown in Appendix L. The color bands are indicative of the $80^{t h}$ percentile, $55^{\text {th }}$ percentile, and below of the Score column. The FNIR column was computed at the score threshold that gave FPIR $=10^{-3}$. NA indicates that the operations required to produce the value could not be performed. The Identification column shows the time used to perform a search over an enrollment set of 3000000 , as seen in Table 19. The Enrollment column shows the time used to create a search template to be used for a query, as seen in Table 78. Identification and Enrollment durations are reported in seconds, but were originally recorded to microsecond precision. RAM refers to the sum of the resident set sizes of the stage one identification processes over all compute nodes after returning from the identification stage one initialization method, as seen in Table 63. RAM is reported in gigabytes, where 1 GB is equal to 1073741824 bytes. The number to the left of a value provides the value's column-wise ranking, with the best performance shaded in green and the worst in pink. The table is sorted on descending Score.

\begin{tabular}{l|l|l|l|ll|l}
$\mathrm{C}=$ afis team & $\mathrm{D}=3 \mathrm{M}$ Cogent & $\mathrm{E}=$ Neurotechnology & $\mathrm{F}=$ Papillon & $\mathrm{G}=$ Dermalog & $\mathrm{H}=$ Hisign Bio-Info Institute \\
$\mathrm{I}=$ NEC & $\mathrm{J}=$ Sonda & $\mathrm{K}=$ Tiger IT & $\mathrm{L}=$ Innovatrics & $\mathrm{M}=$ SPEX & $\mathrm{O}=$ ID Solutions \\
$\mathrm{P}=\mathrm{id} 3$ & $\mathrm{Q}=$ Morpho & $\mathrm{S}=$ Decatur Industries & $\mathrm{T}=$ BIO-key & $\mathrm{U}=$ Aware & $\mathrm{V}=$ AA Technology
\end{tabular}




\begin{tabular}{|c|c|c|c|c|c|c|c|c|c|c|}
\hline \multicolumn{2}{|c|}{ Participant } & \multirow{3}{*}{$\begin{array}{c}\text { Score } \\
133 \\
\end{array}$} & \multirow{2}{*}{\multicolumn{2}{|c|}{ FNIR }} & \multirow{2}{*}{\multicolumn{2}{|c|}{ Identification }} & \multirow{2}{*}{\multicolumn{2}{|c|}{ Enrollment }} & \multirow{2}{*}{\multicolumn{2}{|c|}{ RAM }} \\
\hline Letter & Sub.\# & & & & & & & & & \\
\hline $\mathrm{Q}$ & 1 & & 2 & 0.0011 & 9 & 36.86 & 20 & 9.11 & 2 & 13.33 \\
\hline I & 2 & 114 & 1 & 0.0010 & 10 & 38.28 & 30 & 16.99 & 11 & 110.22 \\
\hline $\mathrm{D}$ & 2 & 108 & 2 & 0.0011 & 26 & 74.36 & 24 & 13.66 & 15 & 132.37 \\
\hline $\mathrm{Q}$ & 2 & 102 & 4 & 0.0013 & 8 & 35.35 & 20 & 9.11 & 1 & 13.33 \\
\hline $\mathrm{L}$ & 1 & 101 & 16 & 0.0102 & 3 & 17.51 & 1 & 3.48 & 23 & 192.19 \\
\hline $\mathrm{I}$ & 1 & 78 & 4 & 0.0013 & 16 & 52.41 & 29 & 16.67 & 4 & 84.42 \\
\hline $\mathrm{E}$ & 1 & 74 & 14 & 0.0088 & 2 & 12.77 & 11 & 5.20 & 14 & 127.39 \\
\hline $\mathrm{J}$ & 1 & 66 & 12 & 0.0047 & 12 & 43.57 & 5 & 3.62 & 20 & 181.00 \\
\hline $\mathrm{V}$ & 1 & 55 & 7 & 0.0024 & 14 & 47.02 & 7 & 3.66 & 13 & 121.80 \\
\hline G & 1 & 51 & 23 & 0.0368 & 1 & 7.71 & 18 & 8.32 & 25 & 274.35 \\
\hline $\mathrm{V}$ & 2 & 51 & 7 & 0.0024 & 19 & 62.26 & 7 & 3.66 & 12 & 121.79 \\
\hline $\mathrm{L}$ & 2 & 39 & 15 & 0.0095 & 6 & 23.53 & 1 & 3.48 & 27 & 274.95 \\
\hline $\mathrm{O}$ & 1 & 35 & 9 & 0.0025 & 23 & 68.54 & 3 & 3.61 & 19 & 181.00 \\
\hline $\mathrm{C}$ & 2 & 32 & 24 & 0.0711 & 5 & 18.38 & 13 & 5.80 & 10 & 92.86 \\
\hline $\mathrm{O}$ & 2 & 31 & 10 & 0.0027 & 24 & 73.41 & 3 & 3.61 & 22 & 181.00 \\
\hline $\mathrm{H}$ & 2 & 23 & 19 & 0.0275 & 20 & 65.36 & 9 & 5.16 & 18 & 144.02 \\
\hline $\mathrm{H}$ & 1 & 21 & 20 & 0.0276 & 21 & 68.07 & 9 & 5.16 & 17 & 144.02 \\
\hline D & 1 & 11 & 6 & 0.0015 & 25 & 73.93 & 17 & 7.10 & 16 & 132.37 \\
\hline $\mathrm{J}$ & 2 & 9 & 10 & 0.0027 & 28 & 77.75 & 6 & 3.62 & 21 & 181.00 \\
\hline $\mathrm{G}$ & 2 & 3 & 20 & 0.0276 & 7 & 23.69 & 18 & 8.32 & 26 & 274.35 \\
\hline $\mathrm{M}$ & 2 & 2 & 27 & 0.0826 & 13 & 46.70 & 25 & 15.83 & 8 & 90.93 \\
\hline $\mathrm{F}$ & 1 & 1 & 25 & 0.0734 & 15 & 51.69 & 27 & 15.87 & 7 & 90.93 \\
\hline $\mathrm{F}$ & 2 & -1 & 25 & 0.0734 & 18 & 60.86 & 27 & 15.87 & 6 & 90.93 \\
\hline $\mathrm{U}$ & 2 & -7 & 17 & 0.0155 & 22 & 68.30 & 15 & 5.91 & 29 & 811.45 \\
\hline $\mathrm{U}$ & 1 & -10 & 18 & 0.0163 & 27 & 74.94 & 15 & 5.91 & 29 & 811.45 \\
\hline$S$ & 1 & -21 & 22 & 0.0311 & 29 & 86.56 & 22 & 10.87 & 24 & 260.37 \\
\hline $\mathrm{M}$ & 1 & -21 & 28 & 0.0934 & 11 & 43.37 & 25 & 15.83 & 5 & 90.93 \\
\hline $\mathrm{E}$ & 2 & -25 & 13 & 0.0048 & 17 & 52.91 & 12 & 5.20 & 28 & 573.25 \\
\hline $\mathrm{C}$ & 1 & -35 & 30 & NA & 4 & 18.30 & 13 & 5.80 & 9 & 92.86 \\
\hline S & 2 & -94 & 29 & 0.1680 & 30 & 91.74 & 22 & 10.87 & 3 & 61.04 \\
\hline
\end{tabular}

Table 97: Tabulation of operationally-ranked results for Class C - Ten-Finger Plain-to-Plain. Letter refers to the participant's letter code found on the footer of this page. Sub. \# is an identifier used to differentiate between the two submissions each participant could make. Score refers to the sum of the scoring equations shown in Appendix L. The color bands are indicative of the $80^{t h}$ percentile, $55^{t h}$ percentile, and below of the Score column. The FNIR column was computed at the score threshold that gave FPIR $=10^{-3}$. NA indicates that the operations required to produce the value could not be performed. The Identification column shows the time used to perform a search over an enrollment set of 5000000 , as seen in Table 20. The Enrollment column shows the time used to create a search template to be used for a query, as seen in Table 79. Identification and Enrollment durations are reported in seconds, but were originally recorded to microsecond precision. $R A M$ refers to the sum of the resident set sizes of the stage one identification processes over all compute nodes after returning from the identification stage one initialization method, as seen in Table 64. RAM is reported in gigabytes, where 1 GB is equal to 1073741824 bytes. The number to the left of a value provides the value's column-wise ranking, with the best performance shaded in green and the worst in pink. The table is sorted on descending Score.

\begin{tabular}{l|l|l|l|ll|l}
$\mathrm{C}=$ afis team & $\mathrm{D}=3 \mathrm{M}$ Cogent & $\mathrm{E}=$ Neurotechnology & $\mathrm{F}=$ Papillon & $\mathrm{G}=$ Dermalog & $\mathrm{H}=$ Hisign Bio-Info Institute \\
$\mathrm{I}=$ NEC & $\mathrm{J}=$ Sonda & $\mathrm{K}=$ Tiger IT & $\mathrm{L}=$ Innovatrics & $\mathrm{M}=$ SPEX & $\mathrm{O}=$ ID Solutions \\
$\mathrm{P}=\mathrm{id} 3$ & $\mathrm{Q}=$ Morpho & $\mathrm{S}=$ Decatur Industries & $\mathrm{T}=$ BIO-key & $\mathrm{U}=$ Aware & $\mathrm{V}=$ AA Technology
\end{tabular}




\begin{tabular}{|c|c|c|c|c|c|c|c|c|c|c|}
\hline \multicolumn{2}{|c|}{ Participant } & \multirow{3}{*}{$\begin{array}{c}\text { Score } \\
124 \\
\end{array}$} & \multirow{2}{*}{\multicolumn{2}{|c|}{ FNIR }} & \multirow{2}{*}{\multicolumn{2}{|c|}{ Identification }} & \multirow{2}{*}{\multicolumn{2}{|c|}{ Enrollment }} & \multirow{2}{*}{\multicolumn{2}{|c|}{ RAM }} \\
\hline \multirow{2}{*}{$\frac{\text { Letter }}{\mathrm{I}}$} & \multirow{2}{*}{$\frac{\text { Sub. \# }}{2}$} & & & & & & & & & \\
\hline & & & 2 & 0.0014 & 7 & 30.82 & 24 & 18.73 & 11 & 137.03 \\
\hline I & 1 & 115 & 1 & 0.0013 & 15 & 54.56 & 25 & 20.23 & 4 & 113.80 \\
\hline Q & 2 & 101 & 2 & 0.0014 & 25 & 74.22 & 17 & 13.06 & 2 & 20.22 \\
\hline V & 1 & 90 & 5 & 0.0017 & 10 & 40.74 & 9 & 8.50 & 17 & 234.03 \\
\hline $\mathrm{D}$ & 1 & 84 & 4 & 0.0015 & 17 & 58.92 & 21 & 17.39 & 10 & 132.40 \\
\hline $\mathrm{L}$ & 2 & 73 & 14 & 0.0083 & 5 & 20.25 & 3 & 4.36 & 26 & 367.82 \\
\hline $\bar{E}$ & 1 & 71 & 18 & 0.0106 & 2 & 8.63 & 12 & 9.92 & 16 & 191.66 \\
\hline$Q$ & 1 & 69 & 5 & 0.0017 & 26 & 74.40 & 17 & 13.06 & 1 & 20.22 \\
\hline $\mathrm{L}$ & 1 & 66 & 17 & 0.0097 & 8 & 31.68 & 3 & 4.36 & 21 & 280.49 \\
\hline $\mathrm{C}$ & 2 & 55 & 15 & 0.0085 & 6 & 21.97 & 13 & 10.69 & 15 & 183.36 \\
\hline C & 1 & 55 & 16 & 0.0094 & 4 & 20.15 & 13 & 10.69 & 14 & 183.36 \\
\hline $\mathrm{V}$ & 2 & 51 & 8 & 0.0019 & 18 & 65.07 & 9 & 8.50 & 18 & 234.03 \\
\hline G & 1 & 49 & 24 & 0.0447 & 1 & 7.78 & 19 & 15.50 & 20 & 261.29 \\
\hline $\mathrm{J}$ & 1 & 44 & 13 & 0.0051 & 9 & 33.02 & 5 & 6.69 & 22 & 303.13 \\
\hline $\mathrm{O}$ & 1 & 34 & 11 & 0.0034 & 14 & 54.42 & 6 & 6.71 & 25 & 303.13 \\
\hline G & 2 & 33 & 21 & 0.0333 & 3 & 19.46 & 19 & 15.50 & 19 & 261.29 \\
\hline $\mathrm{O}$ & 2 & 32 & 9 & 0.0033 & 21 & 69.30 & 6 & 6.71 & 23 & 303.13 \\
\hline $\mathrm{J}$ & 2 & 31 & 9 & 0.0033 & 19 & 67.98 & 8 & 6.74 & 24 & 303.13 \\
\hline $\mathrm{U}$ & 2 & 15 & 22 & 0.0351 & 24 & 72.48 & 1 & 2.87 & 28 & 806.17 \\
\hline$M$ & 2 & 6 & 27 & 0.0716 & 13 & 48.80 & 26 & 20.89 & 5 & 130.29 \\
\hline $\mathrm{E}$ & 2 & 4 & 12 & 0.0050 & 11 & 42.73 & 11 & 9.91 & 30 & 930.48 \\
\hline $\mathrm{D}$ & 2 & 3 & 7 & 0.0018 & 27 & 74.97 & 30 & 30.47 & 9 & 132.37 \\
\hline $\mathrm{F}$ & 1 & 2 & 25 & 0.0536 & 16 & 55.79 & 28 & 20.92 & 7 & 130.29 \\
\hline $\mathrm{U}$ & 1 & -3 & 23 & 0.0358 & 28 & 82.61 & 1 & 2.87 & 28 & 806.17 \\
\hline $\mathrm{F}$ & 2 & -3 & 25 & 0.0536 & 20 & 68.20 & 28 & 20.92 & 6 & 130.29 \\
\hline $\mathrm{H}$ & 2 & -18 & 19 & 0.0199 & 29 & 84.50 & 15 & 12.17 & 12 & 144.02 \\
\hline $\mathrm{H}$ & 1 & -18 & 20 & 0.0201 & 30 & 84.51 & 15 & 12.17 & 13 & 144.02 \\
\hline $\mathrm{M}$ & 1 & -29 & 28 & 0.0783 & 12 & 48.08 & 26 & 20.89 & 8 & 130.29 \\
\hline $\mathrm{S}$ & 2 & -56 & 30 & 0.2462 & 23 & 70.77 & 22 & 17.57 & 3 & 78.28 \\
\hline$S$ & 1 & -96 & 29 & 0.0860 & 22 & 69.71 & 22 & 17.57 & 27 & 382.88 \\
\hline
\end{tabular}

Table 98: Tabulation of operationally-ranked results for Class C - Ten-Finger Rolled-to-Rolled. Letter refers to the participant's letter code found on the footer of this page. Sub. \# is an identifier used to differentiate between the two submissions each participant could make. Score refers to the sum of the scoring equations shown in Appendix L. The color bands are indicative of the $80^{t h}$ percentile, $55^{t h}$ percentile, and below of the Score column. The FNIR column was computed at the score threshold that gave FPIR $=10^{-3}$. The Identification column shows the time used to perform a search over an enrollment set of 5000000 , as seen in Table 20. The Enrollment column shows the time used to create a search template to be used for a query, as seen in Table 80. Identification and Enrollment durations are reported in seconds, but were originally recorded to microsecond precision. RAM refers to the sum of the resident set sizes of the stage one identification processes over all compute nodes after returning from the identification stage one initialization method, as seen in Table 65. RAM is reported in gigabytes, where 1 GB is equal to 1073741824 bytes. The number to the left of a value provides the value's column-wise ranking, with the best performance shaded in green and the worst in pink. The table is sorted on descending Score.

\begin{tabular}{l|l|l|l|ll|l}
$\mathrm{C}=$ afis team & $\mathrm{D}=3 \mathrm{M}$ Cogent & $\mathrm{E}=$ Neurotechnology & $\mathrm{F}=$ Papillon & $\mathrm{G}=$ Dermalog & $\mathrm{H}=$ Hisign Bio-Info Institute \\
$\mathrm{I}=$ NEC & $\mathrm{J}=$ Sonda & $\mathrm{K}=$ Tiger IT & $\mathrm{L}=$ Innovatrics & $\mathrm{M}=$ SPEX & $\mathrm{O}=$ ID Solutions \\
$\mathrm{P}=\mathrm{id} 3$ & $\mathrm{Q}=$ Morpho & $\mathrm{S}=$ Decatur Industries & $\mathrm{T}=$ BIO-key & $\mathrm{U}=$ Aware & $\mathrm{V}=$ AA Technology
\end{tabular}

